\section{Nonradiological Liquid Effluent Monitoring Program FY 1991 Annual Report}

\section{J. Peterson-Wright}

T. R. Meachum

J. J. Einerson

Published June 1992
Idaho National Engineering Laboratory EG\&G idaho, Inc. Idaho Falls, Idaho 83415

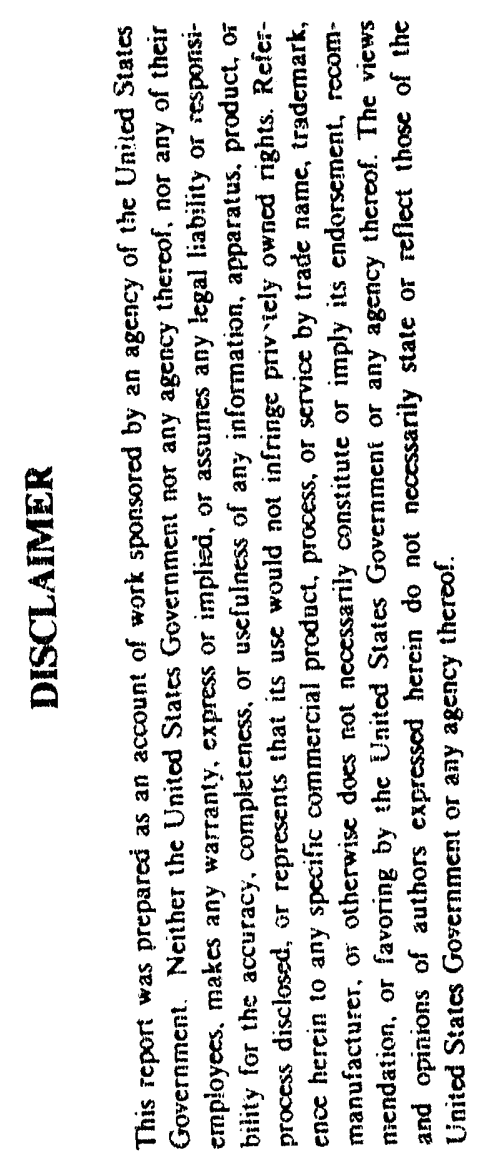




\begin{abstract}
A monitoring program for nonradioactive parameters and pollutants in liquid effluents was initiated in October 1985 for facilities operated by EG\&G Idaho, Inc., for the U.S. Department of Energy at the Idaho National Engineering Laboratory. Program design and implementation are discussed in this report. Design and methodologies for sampling, analysis, and data management are also discussed. Monitoring results for 12 liquid eflluent streams from fiscal year 1987 through fiscal year 1991 are presented with emphasis on fiscal year 1991 (October 1990 through September 1991) activities.
\end{abstract}




\section{ACKNOWLEDGMENTS}

We would like to give a special acknowledgment to EG\&G Idaho facility managers, whose cooperation and assistance are critical to the success of a viable monitoring program. 


\section{CONTENTS}

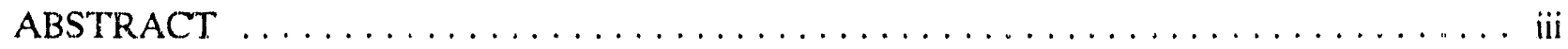

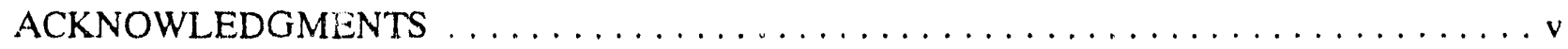

ACRONYMS AND ABBREVIATIONS $\ldots \ldots \ldots \ldots \ldots \ldots \ldots \ldots \ldots \ldots \ldots \ldots \ldots \ldots \ldots$

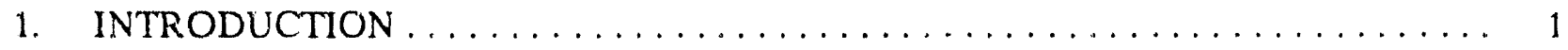

2. SAMPLING DESIGN AND METHODOLOGY $\ldots \ldots \ldots \ldots \ldots \ldots \ldots \ldots \ldots$

3. ANALYTICAL DESIGN AND METHODOLOGY $\ldots \ldots \ldots \ldots \ldots \ldots \ldots \ldots$

4. DATA MANAGEMENT AND ASSESSMENT' $\ldots \ldots \ldots \ldots \ldots \ldots \ldots \ldots \ldots \ldots$

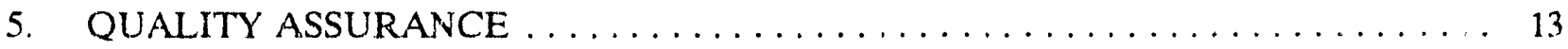

6. EFFLUENT STREAM DESCRIPTIONS AND DATA $\ldots \ldots \ldots \ldots \ldots \ldots \ldots \ldots$

6.1 Stream $001-$ TRA Effluent to Cold Waste Pond $\ldots \ldots \ldots \ldots \ldots \ldots \ldots \ldots$

6.2 Stream 002-TRA Eflluent to Chemical Leaching Pond ............. 15

6.3 Stream $003-$ TRA Effluent to Retention Basin $\ldots \ldots \ldots \ldots \ldots \ldots \ldots \ldots$

6.4 Stream 004-TRA Effluent to STP Trickling Filter Pond $\ldots \ldots \ldots \ldots \ldots \ldots$

6.5 Stream 008--TAN TSF Effluent from Sewage Treatment Plant ........... 16

6.6 Stream 009-TAN TSF Effluent to Drainage Pond ............... 17

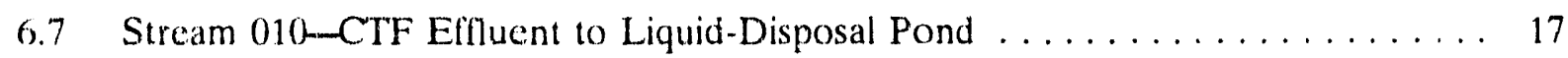

6.8 Stream 013 -CFA Effluent from CLRF $\ldots \ldots \ldots \ldots \ldots \ldots \ldots \ldots \ldots$

0.9 Stream 014 CFA Effluent to Sewage Treatment Plant . . . . . . . . . . 18

6.10 Stream 015-_CFA Oil/Water Separator Effluent $\ldots \ldots \ldots \ldots \ldots \ldots \ldots \ldots \ldots 19$

6.11 Stream 016 -IRC Waste Effluent $\ldots \ldots \ldots \ldots \ldots \ldots \ldots \ldots \ldots \ldots \ldots$

6.12 Stream $017-$ WCB Waste Eflnuent $\ldots \ldots \ldots \ldots \ldots \ldots \ldots \ldots \ldots \ldots$

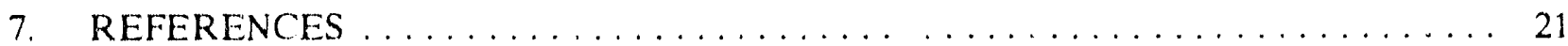

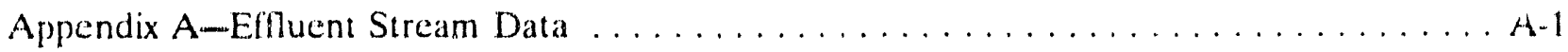

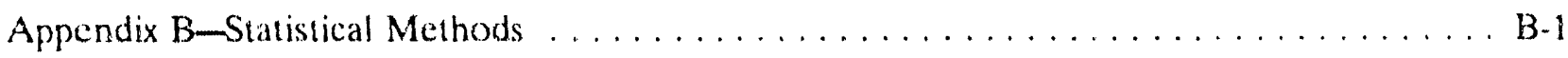




\section{TABLES}

1. Pertinent regulatory codes and orders. $\ldots \ldots \ldots \ldots \ldots \ldots \ldots \ldots \ldots \ldots \ldots \ldots \ldots$

2. Identification of effluent streams sampled during FY $1991 \ldots \ldots \ldots \ldots \ldots \ldots \ldots$

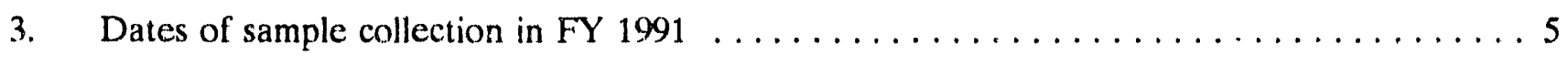

4. Analyte parameters for effluent streams sampled during FY $1991 \ldots \ldots \ldots \ldots \ldots \ldots \ldots$

5. Analyte parameters and methods. ${ }^{\mathrm{a}} \ldots \ldots \ldots \ldots \ldots \ldots \ldots \ldots \ldots \ldots \ldots$

6. Reporting limits. .............................. 9

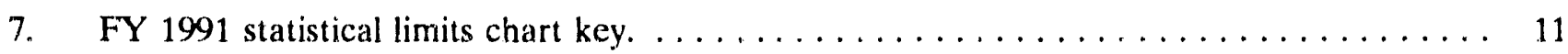

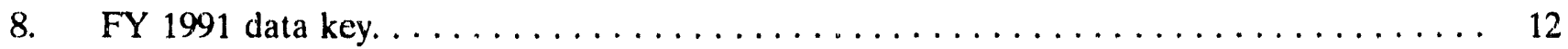

9. Holding times for effluent streanı samples. $\ldots \ldots \ldots \ldots \ldots \ldots \ldots \ldots \ldots \ldots \ldots$ 


\section{ACRONYMS AND ABBREVIATIONS}

\begin{tabular}{|c|c|}
\hline $\mathrm{AA}$ & atomic adsorption \\
\hline CFA & Central Facilities Area \\
\hline CFR & Code of Federal Regulations \\
\hline CLP & Contract Laboratory Program \\
\hline CLRF & Central Laundry and Respirator Facility \\
\hline $\mathrm{CTF}$ & Contairment Test Facility \\
\hline DOE & Department of Energy \\
\hline DOE-ID & DOE Idaho Field Office \\
\hline USEPA & United States Environmental Protection Agency \\
\hline FY & fiscal year \\
\hline GC/MS & gas chromatography/mass spectrometry \\
\hline GFAAS & graphite furnace atomic absorption spectrometry \\
\hline IC & ion chromatography \\
\hline ICP-AES & inductively coupled plasma-atomic emission spectrometry \\
\hline IDAPA & Idaho Administrative Procedures Act \\
\hline INEL & Idaho National Engineering Laboratory \\
\hline IRC & INEL Research Center \\
\hline NPDES & National Pollutant Discharge Elimination System \\
\hline PBF & Power Burst Facility \\
\hline RCRA & Resource Conservation and Recovery Act \\
\hline SOW & statement of work \\
\hline STP & sewage treatment plant \\
\hline
\end{tabular}


TAN Test Area North

TDS total dissolved solids

TOC total organic carbon

TRA Test Reactor Area

TSF Technical Support Facility

TSS total suspended solids

VOC volatile organic compound

WCB Willow Creek Building 


\section{Nonradiological Liquid Effluent Monitoring Program FY 1991 Annual Report}

\section{INTRODUCTION}

The Nonradiological Liquid Effluent Monitoring Program was instituted by EG\&G Idaho, Inc. (EG\&G Idaho), in fiscal year 1986 (FY 1986). Its purpose is to provide environmental monitoring for nonradioactive parameters and pollutants in liquid waste effluents generated within facilities operated by EG\&G Idaho at the Idaho National Engineering Laboratory (INEL). This effort involves sampling, analysis, and data interpretation carried out under a rigorous quality assurance program.

Before the implementation of this program, there had been no cohesive sampling and analysis effort directed towards nonradioactive species in the liquid effluents generated by various operations at the INEL. However, in 1984, an agreement between the Department of Energy (DOE) and the United States Environmental Protection Agency (USEPA) mandated the establishment of nonradiological environmental monitoring at DOE facilities to ensure compliance with applicable Federal, state, and local regulations, and to ensure the protection of human health and the environment. Each DOE facility is unique, and the level of monitoring effort is determined on a case-by-case basis, consistent with regulatory requirements and the degree of environmental assurance that activities at that particular site require.

DOE and DOE Idaho Field Office (DOE-II)) orders were issued requiring that a comprehensive effluent monitoring plan be developed and implemented at the INEL. Pertinent regulatory codes and orders are listed in Table 1. EG\&G Idaho's Safety organization developed a set of preliminary facility monitoring plans, which identified effluent sampling locations and parameters that delineated minimum criteria for program operation.

Effluent monitoring is the collection and analysis of samples and other measurements for the purpose of establishing the type and concentration of pollutants in liquid discharges from facilities. Because of the technical expertise and analytical equipment necessary to operate an USEPA-auditable program, EG\&G Idaho's Safety organization decided that the most practical approach to implementing the nonradiological liquid effluent monitoring portion of the preliminary facility monitoring plans would be to have this monitoring conducted on a Site-wide basis by a single organization. This led to the formation of the Nonradiological Liquid Effluent Monitoring Program, administered by the Environmental Monitoring Unit. The objective of the program is to monitor, evaluate, and report onsite discharges of liquid effluents in the vicinity of DOE sites to assess the levels of pollutants and their possible impact on the public and the environment. 
Table 1. Pertinent regulatory codes and orders.

Source

DOE Order 5480.1

DOE Order 5484.1

Chapter III

40 CFR 260

Executive Order

12088

IDAPA 16.01 .2200

IDAPA 16.01 .2420 .02

IDAPA 16.01 .2420 .04

DOE Order 5400.1

Chapter IV

City Order Section 8,

Chapter 7, and

Industrial Wastewater Acceptance Forms

for INEL Research Center
"Environmental, Safety, and Health

Protection Program"

"Environmental Protection, Safety, and Protection Information

Reporting Requirements"

"Hazardous Waste Management

System: General"

"Federal Compliance with Pollution Control Standards"

March 3, 1987

January 5, 1985

November 20, 1987

"General Environmental Protection Program"

November 9, 1991

Issue of permit

(January 1991) 


\section{SAMPLING DESIGN AND METHODOLOGY}

Liquid effluent streams identified in the preliminary facility monitoring plans issued by Safety were used as a basis for determining which effluents were included in the initial monitoring effort of FY 1986. This "monitoring plan" contained the rationale and design criteria for the monitoring program, extent and frequency of monitoring and measurements, procedures for laboratory analyses, quality assurance requirements, and program implementation. The data set on which these monitoring plans were based was extremely limited at that time, and continuing modifications have been made as the database has expanded. Effluent streams that were sampled during FY 1991 are listed in Table 2. A brief description of each effluent stream is provided in Section 6.

The sample collection program reflects specific facility needs. The sampling schedule for each effluent stream was designed to provide the maximum amount of data from the limited number of sampling excursions possible during the fiscal year. The initial sampling design required individual effluent streams to be grab-sampled once each quarter, beginning in October 1985, for three consecutive days, resulting in a total of 12 sampling excursions per effluent per year. Sampling was conducted at midweek (Tuesday, Wednesday, Thursday) during periods when the facilities were in a normal operation mode. This approach was intended to allow evaluation of short-term and long-term variations in normal effluent stream composition. For those effluents where composite samplers were operational, composite as well as grab samples were obtained to allow for more effective evaluation and selection of sampling methodologies. Stream 3 was sampled by compositor only, as grab sampling at that location was deemed impractical.

The theoretical statistical implications of the original sampling schedule (a nested design with days fixed) were reviewed, and it was decided to change the schedule design, beginning with the third quarter of FY 1986 (April 1986). The statisticians involved in the design process believed that a better estimate of the day-to-day variation of the effluent streams could be obtained by considering it a random effect from a normal disiribution; thus, they decided that the sampling days for each effluent stream should be selected at random. This stratified random approach to sampling is in agreement with current sampling theory (Pitard 1989). The sampling schedule for FY 1991 is presented in Table 3.

The sampling strategy was designed to provide accurate measurements of the concentration of pollutants in the effluent discharges. Sampling frequency and type are determined by considering the purpose for which the data are obtained. Locations are chosen, when practical, at the point at which the data most closely represent what is being released. Effluents discharging under a permit are monitored at the point where the applicable standards apply.

Field measurements taken during each sampling excursion included effluent temperature, pH, and conductivity. All field measurements were taken on separate grab samples, rather than on samples designated for laboratory analyses. 
Table 2. Identification of effluent streams sampled during FY 1991.

\begin{tabular}{ll}
\hline Stream number & \multicolumn{1}{c}{ Location } \\
\hline 001 & Test Reactor Area (TRA) effluent to cold waste pond (MTR-612) \\
002 & TRA effluent to chemical leaching pond (MTR-631) \\
003 & TRA effluent to retention basin (TRA-636) and monitoring shelter \\
& (MTR-712) \\
004 & TRA effluent to sewage treatment plant (STP) trickling filter pond \\
& (TRA-732) \\
008 & Test Area North (TAN)/Technical Support Facility (TSF) effluent to \\
009 & STP (TAN-623) \\
010 & Containment Test Facility (CTF) effluent to liquid disposal pond \\
013 & Central Facilities Area (CFA) effluent from Central Laundry and \\
& Respirator Facility (CFA-617) \\
014 & CFA STP effluent to drain field \\
015 & CFA effluent to oil/water separator (CFA-655) \\
016 & INEL Research Center (IRC) waste effluent \\
017 & Willow Creek Building (WCB) waste effluent \\
&
\end{tabular}


Table 3. Dates of sample collection in FY 1991.

\begin{tabular}{|c|c|c|c|c|}
\hline \multirow[b]{2}{*}{ Stream number } & \multicolumn{4}{|c|}{ Sampling dates } \\
\hline & Quarter 1 & Quarter 2 & Quarter 3 & Quarter 4 \\
\hline \multirow[t]{3}{*}{001} & $10 / 30 / 90$ & $1 / 03 / 91$ & $4 / 09 / 91$ & $7 / 03 / 91$ \\
\hline & NS & $2 / 07 / 91$ & $5 / 29 / 91$ & $8 / 29 / 91$ \\
\hline & $12 / 06 / 90$ & $3 / 26 / 91$ & NS & $9 / 18 / 91$ \\
\hline \multirow[t]{3}{*}{002} & $10 / 30 / 90$ & $1 / 03 / 91$ & $4 / 09 / 91$ & NS \\
\hline & NS & NS & $5 / 29 / 91$ & NS \\
\hline & $12 / 06 / 90$ & $3 / 26 / 91$ & $6 / 12 / 91$ & NS \\
\hline \multirow[t]{3}{*}{003} & $10 / 30 / 90$ & $1 / 03 / 91$ & NS & NS \\
\hline & NS & NS & $5 / 29 / 91$ & NS \\
\hline & $12 / 06,90$ & $3 / 26 / 91$ & $6 / 12 / 91$ & NS \\
\hline \multirow[t]{3}{*}{004} & $10 / 30 / 90$ & $1 / 03 / 91$ & $4 / 09 / 91$ & $7 / 03 / 91$ \\
\hline & $11 / 29 / 90$ & $2 / 07 / 91$ & $5 / 29 / 91$ & $8 / 29 / 91$ \\
\hline & $12 / 06 / 90$ & $3 / 26 / 91$ & $6 / 12 / 91$ & $9 / 18 / 91$ \\
\hline 005 & -- & -- & -- & $7 / 09 / 91$ \\
\hline \multirow[t]{3}{*}{008} & $10 / 04 / 90$ & $1 / 08 / 91$ & $4 / 03 / 91$ & $731 / 91$ \\
\hline & $11 / 13 / M 0$ & $2 / 26 / 91$ & $5 / 21 / 91$ & $8 / 15 / 91$ \\
\hline & $12 / 11 / 90$ & $3 / 14 / 91$ & $6 / 05 / 91$ & $9 / 11 / 91$ \\
\hline \multirow[t]{3}{*}{009} & $10 / 04 / 90$ & $1 / 08 / 91$ & $4 / 03 / 91$ & $7 / 31 / 91$ \\
\hline & $11 / 13 / 90$ & $2 / 26 / 91$ & $5 / 21 / 91$ & $8 / 15 / 91$ \\
\hline & $12 / 11 / 90$ & $3 / 14 / 91$ & $6 / 05 / 91$ & $9 / 11 / 91$ \\
\hline \multirow[t]{3}{*}{010} & $10 / 04 / 90$ & $1 / 08 / 91$ & $4 / 03 / 91$ & NS \\
\hline & $11 / 13 / 90$ & $2 / 26 / 91$ & $5 / 21 / 91$ & NS \\
\hline & $12 / 11 / 90$ & $3 / 14 / 91$ & NS & NS \\
\hline \multirow[t]{3}{*}{013} & $10 / 10 / 90$ & $1 / 29 / 91$ & $4 / 16 / 91$ & $7 / 16 / 91$ \\
\hline & $11 / 08 / 90$ & $2 / 12 / 91$ & $5 / 22 / 91$ & $8 / 21 / 91$ \\
\hline & $12 / 18 / 90$ & $3 / 12 / 91$ & $6 / 26 / 91$ & NS \\
\hline \multirow[t]{3}{*}{014} & $10 / 10 / \times 0$ & $1 / 29 / 91$ & $4 / 16 / 91$ & $7 / 16 / 91$ \\
\hline & $11 / 08 / 90$ & $2 / 12 / 91$ & $5 / 22 / 91$ & $8 / 21 / 91$ \\
\hline & $12 / 18 / 90$ & $3 / 12 / 91$ & $6 / 26 / 91$ & $9 / 18 / 91$ \\
\hline \multirow[t]{3}{*}{015} & $10 / 10 / 90$ & NS & & NS7/16/91 \\
\hline & $11,08 / 90$ & NS & NS & $8 / 21 / 91$ \\
\hline & ivS & NS & $6 / 26 / 91$ & $9 / 18 / 91$ \\
\hline \multirow[t]{3}{*}{016} & $10 / 17 / 90$ & $1 / 24 / 91$ & $4 / 25 / 910$ & $7 / 23 / 90$ \\
\hline & $11 / 19 / 90$ & $2 / 20 / 91$ & $5 / 14 / 91$ & $8 / 14 / 40$ \\
\hline & $12 / 13 / 90$ & $3 / 21 / 91$ & $6 / 18 / 91$ & NS \\
\hline \multirow[t]{3}{*}{017} & $10 / 17 / 90$ & $1 / 24 / 91$ & $4 / 25 / 910$ & $7 / 23 / \% 0$ \\
\hline & $11 / 19 / 90$ & $2 / 20 / 91$ & $5 / 14 / 91$ & $8 / 14 / 90$ \\
\hline & $12 / 13 / 90$ & $3 / 21 / 91$ & $6 / 18 / 91$ & NS \\
\hline
\end{tabular}




\section{ANALYTICAL DESIGN AND METHODOLOGY}

Parameters of interest for each liquid effnent stream were specified in the preliminary facility monitoring plans and were used as a basis to begin characterization of the effluent streams for the initial sampling effort in FY 1986. Parameters monitored in FY 1991 (see Table 4) were changed from previous years to accommodate new regulations, orders, and codes, and to reflect the changing processes at the INEI.

Standard methods are used to analyze samples whenever such methods are required by regulatory programs. Analyses not required by regulations are conducted as determined by site-specific conditions. All methods used in the analysis of the effluent samples are USEPA methods (USEPA 1980) or approved equivalents (see Table 5). Selection of the specific method used for a particular parameter is dependent on the sample matrix and on laboratory capability. Because matrix effects can intluence the precision, accuracy, and achievable detection limits of the analysis (see Table 6), it is vital that these efficts be minimized by selection of the proper analytical methodology.

The metals data (including hexavalent chromium) for this report were obtained by either graphite furnace atomic absorption spectrometry (GFAAS) or by inductively coupled plasma-atomic emission spectrometry (ICP-AES). Mercury was determined using cold vapor atomic absorption spectrometry (CVAAS). Anions data (including fuoride, chloride, nitrate, phosphate, bromide, and sulfate) were determined by ion chromatography (IC) methods. Total dissolved solids (TDS) and total suspended solids (TSS) data were obtained by gravimetric techniques. Total organic carbon (TOC) data were determined by persulfate oxidation and ultraviolet detection, and cyanide was determined by distillation followed by colorimetric analysis. Volatile organic compounds were determined by gas chromatography/mass spectrometry (GC/MS). 
Table 4. Analyte parameters for effluent streams sampled during FY 1991.

Stream number

001

002

003

004

008

009

010

013

014

015

016

017
Analyte parameters

Metals ${ }^{\mathrm{a}}$, anions ${ }^{\mathrm{b}}$, TOC $^{\mathrm{c}}$

Metals, anions, TOC, 'TDS ${ }^{\mathrm{d}}$

Metals, anions, TOC

Metals, anions, TOC

Metals, anions, TOC

Metals, anions, TOC

Metals, anions, TOC

Metals, anions, TOC, TDS, TSS ${ }^{e}$

Metals, anions, TOC

Metals, anions, TOC

Metals, anions, cyanide, TOC, TDS, hexavalent chromium, TSS, VOC ${ }^{f}$

Metals, anions, cyanide, TOC, TDS, hexavalent chromium, TSS, VOC silver, nickel, and thallium.
b. Anions include: fluoride, chloride, nitrate, phosphate, and sulfate.
c. TOC - total urganic carbon.
d. TDS - total dissolved solids.
e. TSS - total suspended solids.
f. VOC - volatile organic compounds.

a. Metals include: arsenic, barium, cadmium, chromium, copper, lead, mercury, selenium, zinc, 
Table 5. Analyte parameters and methods. ${ }^{a}$

\begin{tabular}{|c|c|c|c|c|c|}
\hline & \multirow[b]{2}{*}{ Parameter } & \multicolumn{2}{|c|}{ Preparation method } & \multicolumn{2}{|c|}{ Analysis method } \\
\hline & & ICP-AES & GFAAS & ICP-AES & GFAA \\
\hline \multicolumn{6}{|l|}{ Metals } \\
\hline & Arsenic & 200.7 & 206.2 & 200.7 & 206.2 \\
\hline & Barium & 200.7 & -. & 200.7 & $\ldots$ \\
\hline & Cadmium & 200.7 & -. & 200.7 & -. \\
\hline & Chromium & 200.7 & 218.2 & 200.7 & 218.2 \\
\hline & Lead & 200.7 & 239.2 & 200.7 & 239.2 \\
\hline & Nickel & 200.7 & -- & 200.7 & 239.2 \\
\hline & Selenium & 200.7 & 270.2 & 200.7 & 270.2 \\
\hline & Silver & 200.7 & $\ldots$ & 200.7 & -. \\
\hline & Mercury & -. & $245.1^{b}$ & $\ldots$ & $245.1^{\mathrm{b}}$ \\
\hline & Thallium & 200.7 & 279.2 & 200.7 & 279.2 \\
\hline & Zinc & 200.7 & -- & 200.7 & -. \\
\hline
\end{tabular}

Anions

Fluoride

Not applicable

300.0

Chloride

Nitrate

Phosphate

Sulfate

TOC

Not applicable

415.1

Not applicable

160.1

TSS

Not applicable

160.2

Hexavalent chromium

EPA SW-846,

EPA SW-846,

Method 7196

Method 7196

$\mathrm{pH}$

Not applicable

150.1

120.1

335.2.

Cyanide (total)

Not applicable

3352

EPA SW-846

VOCs

Not applicable

Method 8240

a. Unless otherwise slated, all methods cited in this table are from EPA-600 4-79-020, Method for Chemical Analysis of Water and Wastes (USEPA 1980). Methods 200.7 and 300.0 are additions to this methods manual. Depending on the sample matrix, equivalent EPA-approved methods in EPA SW-846 and CLP SOW No. 785 were sometimes used.

b. Cold vapor atomic absorption spectrometry (CVAAS). 
Table 6. Reporting limits.

Parameter

Metals

Silver

Arsenic

Barium

Cadınium

Chromium

Copper

Mercury

Nickel

Lead

Selenium

Thallium

Zinc

Chromium (hexavalent)
Instrument detection limit ${ }^{\text {a }}$

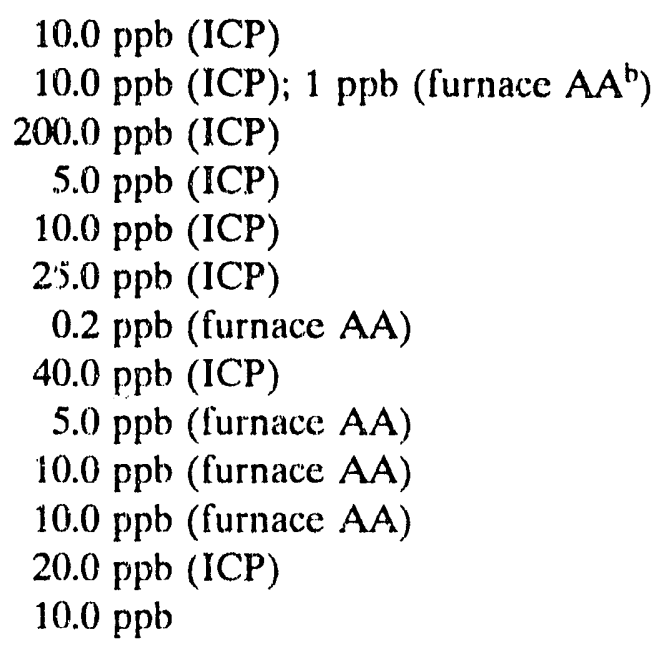

Anions

Chloride

Fluoride

$1.0 \mathrm{ppm}(\mathrm{IC})$

Nitrate

Phosphate

$0.5 \mathrm{ppm}$ (IC)

$1.0 \mathrm{ppm}$ (IC)

$0.1 \mathrm{ppm}$ (IC)

1.0 ppr: (IC)

Sulfate

$1.0 \mathrm{ppm}$

$10.0 \mathrm{ppm}$

5.0 ppm

Cyanide

$20.0 \mathrm{ppb}$

a. Instrument detection limit is the minimum analyte concentration level that can be discriminated from background. For most instrumentation, the quantitation limit is somewhat higher.

b. AA - atomic adsorption. 


\section{DATA MANAGEMENT AND ASSESSMENT}

The inherent variability associated with field and laboratory activities must be well defined in order for monitoring data to be effectively interpreted. The sampling and analysis scheme developed for the Nonradiological Liquid Effluent Monitoring Program was designed to provide data for a statistical model yielding information about the source and magnitude of variability in effluent stream composition.

The total variability in the measurement of an analyte pa:ameter is the sum of two variance components: day-to-day variability of the effluent itself, and variation between replicate samples collected on the same day (i.e., variation due to sampling). In the model, the sources of variability are assumed to be random effects. Because the field replicates are nested in sampling days, and days are sandomly chosen, the overall statistical model is a nested, random effects model.

The sampling and analysis variance components represent the inherent variation in a data point and, as long as they are quantified, are important to the eflluent monitoring program as quality control checks on the sampling and analytical processes. The variance attributable to the stream itself is of primary importance in characterization and monitoring of an effluent, because it reflects a change in the character of an effluent.

It is expected that the main contributor to analyte level variability will be the variation of effluent stream composition from day to day. If the variance due to field replicates is high (sampling variance), this may reflect the inhomogeneous character of the stream or indicate that the sampling method did not obtain representative samples.

Statistical limits charts present the monthly concentration data for each effluent stream along with two statistical confidence levels. These statistical limits charts are presented for each parameter in each stream in Appendix A. Statistical confidence limits are calculated for each parameter, based on the estimate of the variance of the analyte concentrations around the combined mean for FY 1987 and FY 1988. The symbols used to define the statistical limits chart parameters are summarized in Tatle 7. The lowest line of the statistical limits chart is the analyte concentration mean for FY 1987 through FY 1990. In general, the Level 1 control limit (the middle line on the statistical limits chart) is set at the upper $95 \%$ confidence limit on the individual measurements. Thus, a measurement exceeding the Level 1 control limit has a 1 in $20(5 \%)$ chance of being attributable to random fluctuations in concentration levels, based on the FY 1987 through FY 1990 results. The Level 2 statistical confidence limit (the cop line on the statistical limits chart) is set at the upper $99 \%$ confidence limit on the individual measurements and thus, has only a 1 in $100(1 \%)$ chance of being exceeded because of random fluctuations in actual concentrations. Values that exceed the Level 2 statistical confidence limit generally indicate a deviation irom the normal stream characteristics for the analyte in question. Botin upper and lower statistical confidence limits are represented for $\mathrm{pH}$; both limits are at Level 2 .

Note that an excursion above the Level 2 limit does not imply that regulatory standards have been exceeded, but does indicate a deviation from normal operating conditions, a situation that should be investigated. The statistical limits chart can help identify potential problems at a correctable stage. 
Table 7. FY 1991 statistical limits chart key.

\begin{tabular}{cl}
\hline Symbol & \multicolumn{1}{c}{ Explanation } \\
\hline "X" & First replicate sample \\
$" \Delta "$ & Second replicate sample \\
"-n" & Mean \\
$"-. "$ & Level 1 \\
$"-.-"$ & Level 2 \\
\hline
\end{tabular}

The statistical analysis developed for the Nonradiological Liquid Effluent Monitoring Program was designed to indicate the source and magnitude of variability of effluent stream composition. The total variability in the actual sample concentration of an analyte parameter (sampling variability) is treated as the sum of two varianze components: day-to-day variability of the effluent itself, and variation (for any reason) between replicate samples collected on the same day. This information is presented as quarterly means and variance components.

Table 8 explains the codes used for summarizing the annual data. Day-to-day variability is listed in the STD DAY column and variability between replicates is listed in the STD REP column. The total variability for a quarterly mean is given in the STD MEAN column.

Data from FY 1987 through FY 1990 were used to set individual stream statistical confidence limits.

Samples for which the analyte concentration is less than the minimum amount detectable by the analytical method are treated as missing data points in the statistical analysis of variance. This is done because of considerable disagreement concerning statistically valid methods for dealing with values below the practical quantitation limit. For the purposes of effluent monitoring, it was deemed to be more defensible to place a high bias on the mean by deleting the values below the practical quantitation limit, as opposed to placing a low bias on the mean by equating the value below the practical quantitation limits with zero. 
Table 8. FY 1991 data key.

Code

Explanation

QTR

Quarter, given as $\mathrm{XXqY}$, where $\mathrm{XX}$ is fiscal year and $\mathrm{Y}$ is quarter designation

MEAN

Quarterly mean concentration

STD MEAN

Standard error of quarterly mean

STD DAY

Standard deviation associated with the day of sampling (day-to-day variation)

STD REP

Standard deviation associated with field replicates within a day

N

Total possible number of field replicates

NMISS

Total number of missing data points

NLTD

Number of values that were below the practical quantitation limit

"X"

Value below the practical quantitation limit

"."

Missing data (e.g., not sampled) 


\section{QUALITY ASSURANCE}

A quality assurance (QA) program addressing analytical concerns is designed to ensure that data of a known quatity are produced by the analyses. The Nonradiological Liquid Effluent Monitoring QA Program follows the guidance provided in QAMS-(005/80 as a best management practice. Quality control practices are used to identify and resolve any problems caused by possible matrix effects, contamination, or instrument malfunction. The QA program for the Nonradiological Liquid Effluent Monitoring Program is commensurate with its nature and complexity in accordance with DOE Order 5400.1. Issues such as minimum criteria for accuracy, precision, completeness, minimum training/qualifications of personnel, organization and responsibilities, corrective action (in case of an out-of-control operation), calibration and standardization of analytical instrumentation, sampling and analytical methodology, sample and data tracking, chain of custody, and audit/evaluation programs are addressed and documented in the Effluent Monitoring Handbook for Operational Facilities (EG\&G Idaho 1992). The 7-7-7 rule is currently used by the USEPA in the analysis of water and wastewater samples (USEPA 1977). This means that any wastewater study iequires the analysis of a minimum of seven samples, seven blanks, and seven spiked blanks before inferences concerning the usability of the data can be made.

The observation of sample holding times for each analyte parameter of interest is crucial to the production of data of known quality. The holding time is the maximum amount of time that a sample may be stored between time of collection and analysis, and still permit meaningful analytical results. Holding times for eflluent stream samples, as presented in Table 9, meet USEPA regulatory guidelines.

Table 9. Holding times for eflluent stream samples.

Parameter

Metals

Mercury

Anions: shloride, sulfate, fluoride

Anions: nitrate, phosphate

Cyanide

TOC

TDS/TSS

VOCs

$\mathrm{pH}$

Conductivity

Dissolved oxygen

Hexavalent chromium
Holding time

6 months
28 days
28 days
48 hours
14 days
28 days
7 days
14 days
Field measurement
Field measurement
Field measurement
24 hours




\section{EFFLUENT STREAM DESCRIPTIONS AND DATA}

Water is used at INEL facilities in a variety of processes and operations. The final liquid effluents released to the environment are composed of discharges from diverse sources. In many cases, the impact of water usage by a given facility process on influent water quality is minimal, 6 eating relatively clean wastewater effluents that are roughly comparab.e in quality to the influent source. In other cases, however, wastewater effluents contain pollutants characteristic of particular processes. In order for effluent composition to be effectively interpreted, the characteristics of typical contributing sources must be assessed. Because of the great diversity of effluents from INEL facilities, the nature of the contributing sources for each effluent must be considered in stream-specific interpretation of data.

There are two major classes of liquid effluents from EG\&G Idaho facilities: those generated by numerous contributing sources within a facility, and those generated by a single source (i.e., a unique process or operation). For effluents generated by numerous contributing sources within a facility (i.e., nonspecific sources), operations have relatively little effect on water quality. Annual mean concentrations of individual pollutants usually lie in a relatively narrow range, well below regulated leveis. General trends can often be attributed to a unique contributing source or operating history.

For a single-source effluent, a change associated with its source has a more direct impact on the observed character of the effluent than does a comparable change in a stream generated by nonspecific sources. A single-source effluent may be homogeneous or highly variable, depending on the nature of its source. Thus, interpretation of data from a single-source effluent requires consideration of the contributing source. Unless the contributing sources are of similar nature, stream-10-stream comparisons with other single-source effluents are generally not useful.

In Sections 6.1 through 6.12, each liquid effluent strearn is described, and data are discussed on a stream-by-stream basis. Whenever possible, the observed data are correlated with the known operating history of the facility or the known character of the contributing sources. Comparisons with similar effluents are made whenever practical.

DOE Order 5480 prohibits the discharge of Resource Conservation and Recovery Act (RCRA) characteristic hazardous waste. All site effluents are below the regulatory limits for characteristically hazardous RCRA metals. Effluent Streams 16 and 17 discharge into the City of Idaho Falls sewer system, and are thus included under the City's National Pollutant Discharge Elimination System (NPDES) permit. There were no detected discharges above the City of Iditho Falls sewer regulations.

\subsection{Stream 001-TRA Effluent to Cold Waste Pond}

This effluent is generated by the cold waste drains within the Test Reactor Area (TRA). The cold drains are located throughout TRA, including laboratories and craft shops. Maintenance cleaning waste, and floor and yard drains are examples of intermittent TRA discharges that might be expected to alter water quality parameters during normal operation. The cold waste pond also receives secondary cooling water from the reactor when it is in operation. The cold waste effluents collect at the cold well sump and sampling station, and are pumped out to the cold waste leaching 
pond, which is located outside the TRA fence. Data collected from FY 1987 through FY 1991 indicate that the TRA cold waste effluent is homogenous. The pH range for field data collected in FY 1991 was 2.8 to 9.0 .

All RCRA metal concent:ations are below the applicarle regulatory limits.

Tables A-1 through A-4 list the data for FY 1991 for Stream 1. Table A-5 lists the statistical confidence limits for Stream 1, and Table A-6 lists the quarterly means and variance components for FY 1991. The statistical limits charts in Figures A-1 through A-18 present the statistical confidence limits and monthly concentration data for each analyte detected for Stream 1 for FY 1988 through FY 1991.

\subsection{Stream 002-TRA Effluent to Chemical Leaching Pond}

The TRA effluent to the chemical leaching pond is generated by water treatment processes at the TRA demineralizer facility. The ion-exchange process uses electrically charged resin beads to attract and adsorb oppositely charged ions from the water until the resin's exchange sites are filled with ions from the water. When thie exchange capacity of the resin is saturated, the resin bed is regenerated by rinsing the resin with an appropriate chemical solution. Cation-exchange regeneration, which uses sulfuric acid as a regenerant, is carried out approximately every other day, and produces nearly 600 gal of wastewater. Anion-exchange regeneration uses a sodium-hydroxide regenerant, and is performed approximately every third day, yielding 12,000 gal of wastewater. Ion-exchange regeneration waste streams typically contain mineral salts removed from the water, excess regenerant chemicals, and rinse waters from the regeneration process. Specific waste stream constituents anticipated in regeneration wastewater include calcium, sodium and magnesium salts, iron, copper, zinc, aluminum, manganese, potassium, chlorides, sulfates, sulfuric acid, and sodium hydroxide.

Data collected from FY 1987 through FY 1991 for this stream are consistent with the nature of the stream's source. The largest degree of variability is associated with the pH parameter. This is aot unexpected, because the effluent includes cation and anion regenerant, and the discharges may be quite acidic or basic. Water quality parameters determined from FY 1987 through FY 1991 are consistent with the large quantities of dissolved salts in demineralizer effluents. The high annual mean conductivity $(26,000 \mu \mathrm{S})$ and high annual dissolved solids $(23,000 \mathrm{mg} / \mathrm{L})$ result from the elevated levels of dissolved salts and free ions introduced during the regeneration process. The field $\mathrm{pH}$ measurement range for FY 1991 was 2.2 to 10.5.

All RCRA metal concentrations are below the applicable regulatory limits.

Tables A-7 through A-9 list the data for FY 1991 for Stream 2. Table A-10 lists the statistical confidence limits for Stream 2, and Table A-11 lists the quarterly means and variance components for FY 1987 through FY 1991. The statistical limits charts in Figures A-19 through A-38 present the statistical confidence limits and monthly concentration data for each analyte detected for Stream 2 for FY 1988 through FY 1991. 


\subsection{Stream 003-TRA Effluent to Retention Basin}

This effluent is generated by low-level radioactive waste drains at TRA. These drains are located throughout TRA in laboratories and craft shops, and within the reactor facilities. The effluent streams collect at the retention basin inlet. When the retention basin is full, the effluent is automatically pumped to a leaching pond that is located outside the TRA fence.

Composite data collected from FY 1987 through FY 1991 indicate that the wastewater discharged to the retention basin does not contain any RCRA characteristic metals above the regulatory limits.

The $\mathrm{pH}$ is decreasing and can be attributed to the waste minimization steps taken to decrease the amount of "cold" effluents discharged to the retention basin. Therefore, the effluent is not diluted as much, resulting in higher concentration of the parameters and a more acidic effluent stream. The field measurement $\mathrm{pH}$ range for FY 1991 was 4.0 to 9.2 .

Tables A-12 through A-15 list the data for FY 1991 for Stream 3. Table A-16 lists the statistical confidence limits for Stream 3, and Table A-17 lists the quarterly means and variance components for FY 1987 through FY 1991. The statistical limits charts in Figures A-39 through A-51 present the statistical confidence limits and monthly concentration data for each analyte detect:d for Stream 3 for FY 1988 through FY 1991.

\subsection{Stream 004-TRA Effluent to STP Trickling Filter Pond}

The TRA effluent to the sewage treatment plant (STP) trickling filter pond receives discharged wastewater from sanitary sewer drains throughout the TRA area. Grab sample data collected from FY 1987 through FY 1991 are comparable to data obtained for the three other STP effluents on site. The field measurement $\mathrm{pH}$ range for FY 1991 was 6.6 to 8.4 .

All RCRA metal concentrations were below regulatory limits.

Tables A-18 through A-21 list the data for FY 1991 for Stream 4. Table A-22 lists the statistical confidence limits for Stream 4, and Table A-2.3 lists the quarterly means and variance components for FY 1987 through FY 1991. The statistical limits charts in Figures A-52 through A-68 present the statistical confidence limits and monthly concentration data for each analyte detected for Stream 4 for FY 1988 through FY 1991.

\subsection{Stream 008-TAN TSF Effluent from Sewage Treatment Plant}

The Test Area North (TAN) Technical Support Facility (TSF) TRA effluent from the STP trickling filter pond receives discharged wastewater from sanitary sewer drains throughout the TRA area. Grab sample data collected from FY 1987 through FY 1991 are comparable to data obtained for the three other STP effluents on site. 
Data collected from FY 1987 through FY 1991 support characterization of the TAN TSF effluent as a homogeneous wastewater stream. The field $\mathrm{pH}$ measurement range for FY 1991 was 6.2 to 8.1 .

All RCRA metals are below regulatory limits.

Tables A-24 through A-27 list the data for FY 1991 for Stream 8. Table A-28 lists the statistical confidence limits for Stream 8 , and Table A-29 lists the quarterly means and variance components for FY 1987 through FY 1991. The statistical limits charts in Figures A-69 through A-86 present the statistical confidence limits and monthly concentration data for each analyte detected for Stream 8 for FY 1988 through FY 1991.

\subsection{Stream 009-TAN TSF Effluent to Drainage Pond}

The TAN TSF effluent to the drainage pond receives wastewater discharges from a variety of contributing sources. Boiler blowdown, such as that generated in the Service Building, is expected to contribute inorganic salts coricentrated from feedwater (calcium and magnesium salts, chlorides, and sulfates), corrosion products (metal oxides), and any chemical additives. Wastewater from the demineralizer system is expected to contribute mineral salts from makeup water, and excess regenerant chemicals (sodium hydroxide and sulfuric acid). Low-level radioactive waste drains, such as those found in TAN-615, TAN-633, and TAN-607, are not expected to contribute significant levels of pollutants to the effluent stream. Data from FY 1987 through FY 1991 are generally consistent with these anticipated discharges.

The TAN TSF effluent receives intermittent discharges from a number of contributing sources, which result in an inhomogeneous discharge. This variability is most apparent for the water quality parameters of $\mathrm{pH}$ and conductivity. The high conductivity level can be attributed to the soluble salts introduced from the boiler blowdown and demineralizer regenerant discharges. The demineralizer wastewater may also contribute to the observed variance in $\mathrm{pH}$. The field $\mathrm{pH}$ measurement range for FY 1991 data was 6.7 to 9.0 .

All RCRA metal concentrations are below regulatory limits.

Tables A-30 through A-33 list the data for FY 1991 for Stream 9. Table A-34 lists the statistical confidence limits for Stream 9, and Table A-35 lists the quarterly means and variance components for FY 1987 and FY 1991. The statistical limits charts in Figures A-87 through A-105 present the statistical confidence limits and monthly concentration data for each analyte detected for Stream 9 for FY 1988 through FY 1991.

\subsection{Stream 010-CiF Effluent to Liquid-Disposal Pond}

The Containment Test Facility (CTF) effluent to the liquid disposal pond receives wastewater from low-level radioactive waste drains and sumps throughout the CTF area. Characterization of data collected from FY 1987 through FY 1991 indicates that this is a homogeneous effluent stream. Although the CTF is now inactive, analyte concentrations have not decreased, which indicates that 
past operations had minimal impact on water quality. Monitoring was discontinued at this location in June of 1991. The field measurement $\mathrm{pH}$ range for FY 1991 was 6.9 to 8.5.

All RCRA metal concentrations are below regulatory limits.

Tables A-36 through A-38 list the data for FY 1991 for Stream 10. Table A-39 lists the statistical confidence limits for Stream 10, and Table A-40 lists the quarterly means and variance components for FY 1987 through FY 1991. The statistical limits charts in Figures A-106 through A-124 present the statistical confidence limits and monthly concentration data for each analyte .detected for Stream 10 for FY 1988 through FY 1991.

\subsection{Stream 013-CFA Effluent from CLRF}

Effluent characterization data collected for Stream 13 from FY 1987 through FY 1991 are consistent with the operations and processes that generate wastewater at the Central Laundry and Respirator Facility.

The discharged effluent is generated by routine maintenance and laundry operations, and may contain radioactive and nonradioactive contaminants. Composition of the effluent stream is quite variable, which is expected since a limited number of sources introduce contaminants and with minimal dilution.

Water quality parameters associated with this effluent are consistent with typical laundry and decontamination operations. The annual. mean $\mathrm{pH}$ is slightly basic (9.0). Dilution factors, uneven work distribution in the laundry, and variable chemical-use rates all contribute to the observed inhomogeneity of this waste stream. Concentrations of anions in the laundry effluent reflect the types of cleaning agents used in the facility. The field $\mathrm{pH}$ measurement range was 8.0 to 9.7 .

All RCRA metal concentrations are below regulatory limits.

Tables A-41 through A-44 list the data for FY 1991 for Stream 13. Table A-45 lists the statistical confidence limits for Stream 13, and Table A-46 lists the quarterly means and variance components for FY 1987 through FY 1991. The statistical limits charts in Figures A-125 through A-145 present the statistical confidence limits and monthly concentration data for each analyte detected for Stream 13 for FY 1988 through FY 1991.

\subsection{Stream 014 CFA Effluent to Sewage Treatment Plant}

The Central Facilities Area (CFA) effluent to the STP receives wastewater from sanitary sewer drains throughout the CFA. These drains have major potential for affecting the chemical characteristics of the overall CFA effluent. A number of unique discharge sources exist, including chemical laboratories, the craft shops, the cafeteria, the warehouse, photographic services, vehicles services, the laundry facility, and the dispensary. Data collected from FY 1987 through FY 1991 support the characterization of this stream as homogenous, but with a larger standard day-to-day variance than other effluents on site. The field measurement $\mathrm{pH}$ range for FY 1991 was 7.0 to 9.5. 
All RCRA metal concentrations are below regulatory limits.

Tables A-47 through A-50 list the data for FY 1991 for Stream 14. Table A-51 lists the statistical confidence limits for Stream 14, and Table A-52 lists the quarterly means and variance components for FY 1987 through FY 1991. The statistical limits charts in Figures A-146 through A-164 present the statistical confidence limits and monthly concentration data for each analyte detected for Stream 14 for FY 1988 through FY 1991.

\subsection{Stream 015-CFA Oil/Water Separator Effluent}

The oil/water separator collects nonpoint-source waste oils and water associated with the floor drains and vehicle maintenance areas of CFA-664. Data collected from FY 1987 through FY 1991 support historical trends in contaminant concentrations. The field $\mathrm{pH}$ measurement range for FY 1991 was 7.7 to 8.8 .

All RCRA metal concentrations are below regulatory limits.

Tables A-53 through A-56 list the data for FY 1991 for Stream 15. Table A-57 lists the statistical confidence limits for Stream 15, and Table A-58 lists the quarterly means and variance components for FY 1987 through FY 1991. The statistical limits charts in Figures A-165 through A-183 present the statistical confidence limits and monthly concentration data for each analyte detected for Stream 15 for FY 1988 through FY 1991.

\subsection{Stream 016-IRC Waste Effluent}

This effluent is generated by drain discharges from all laboratories and discharge from the mechanical room. The field $\mathrm{pH}$ measurement range for FY 1991 was 6.3 to 8.2. There were no discharges containing concentrations above the regulations of the City of Idaho Falls.

Tables A-59 through A-61 list the data for FY 1991 for Stream 16. Tables A-62 through A-66 list the statistical confidence limits for Stream 16, and Table A-67 lists the quarterly means and variance components for FY 1987 through FY 1991. The statistical limits charts in Figures A-184 through A-203 present the statistical confidence limits and monthly concentration data for each analyte detected for Stream 16 for FY 1988 through FY 1991.

\subsection{Stream 017-WCB Waste Effluent}

This effluent is generated by the wastewater from the Willow Creek Building (WCB). Contributing sources are the discharge from Micrographics, Reprographics, the Print Shop sinks and drains, and the x-ray photoprocessor in Medical. In most respects, the overall WCB wastewater effluent is quite characteristic of the known contributing sources. The field measurement $\mathrm{pH}$ range for FY 1991 was 7.0 to 9.0 .

Tables A-68 through A-71 list the data for FY 1991 for Stream 17. Tables A-12 through A-76 list the statistical confidence limits for Stream 17, and Table A-77 lists the quarterly means and variance components for FY 1987 through FY 1991. The statistical limits charts in Figures A-204 
through A-225 present the statistical confidence limits and monthly concentration data for each analyte detected for Stream 17 for FY 1988 through FY 1991. 


\section{REFERENCES}

EG\&G Idaho, 1992, Effluent Monitoring Handbook for Operational Facilities, EGG-ER-8044, current revision.

Pitard, Francis F., 1989, Pierre Gy's Sampling Theory and Sampling Practice, CRC Press, Inc.

USEPA, 1977, Handbook for Analytical Quality Control in Water and Wastewater Laboratories, USEPA Environmental Monitoring and Support Laboratory, Cincinnati, Ohio, March.

USEPA, 1980, Methods for Chemical Analysis of Water and Wastes, EPA-600/4-79-020, USEPA Monitoring and Support Laboratory, Cincinnati, Ohio, March. 
Appe rdix A

\section{Effluent Stream Data}




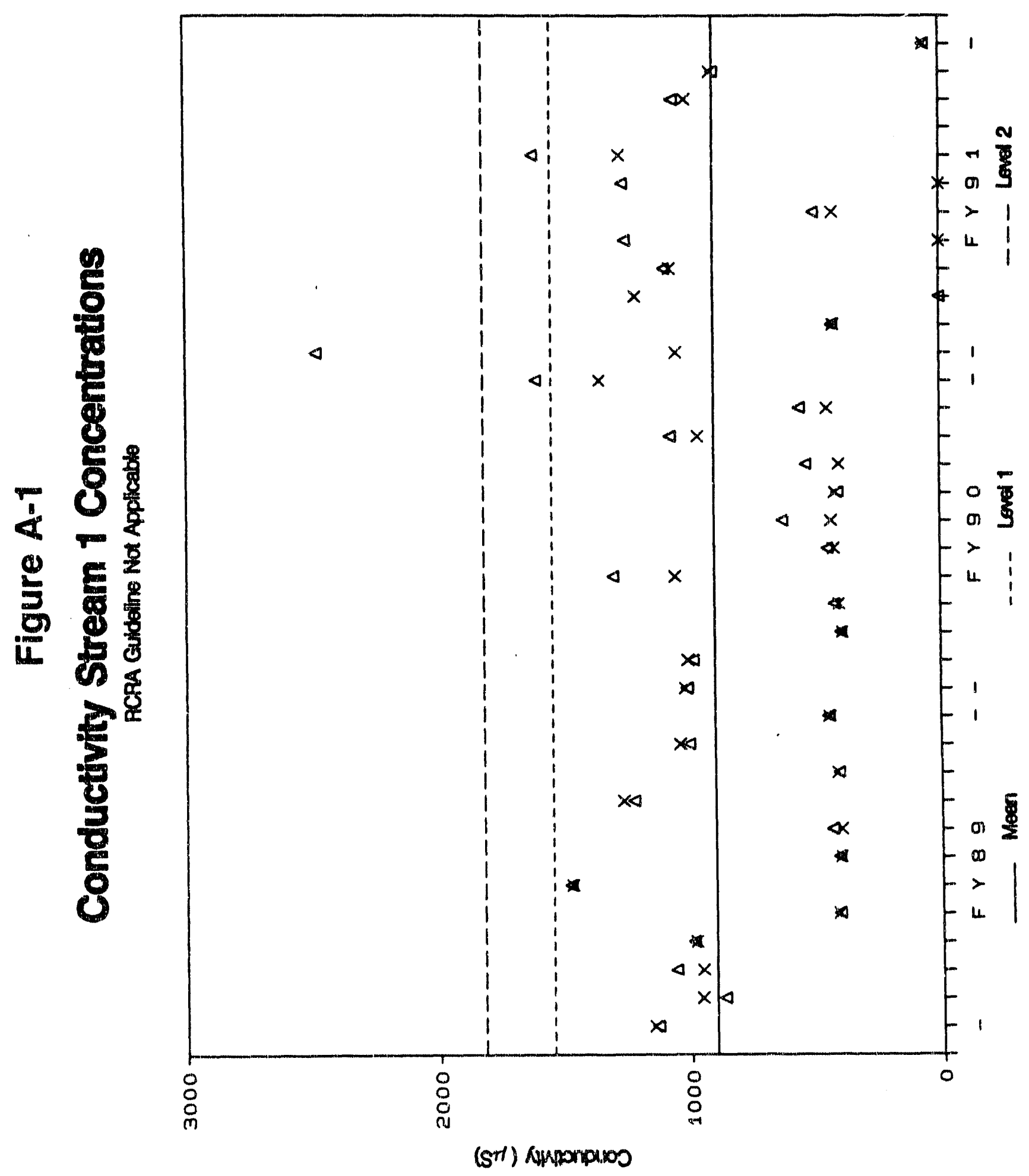




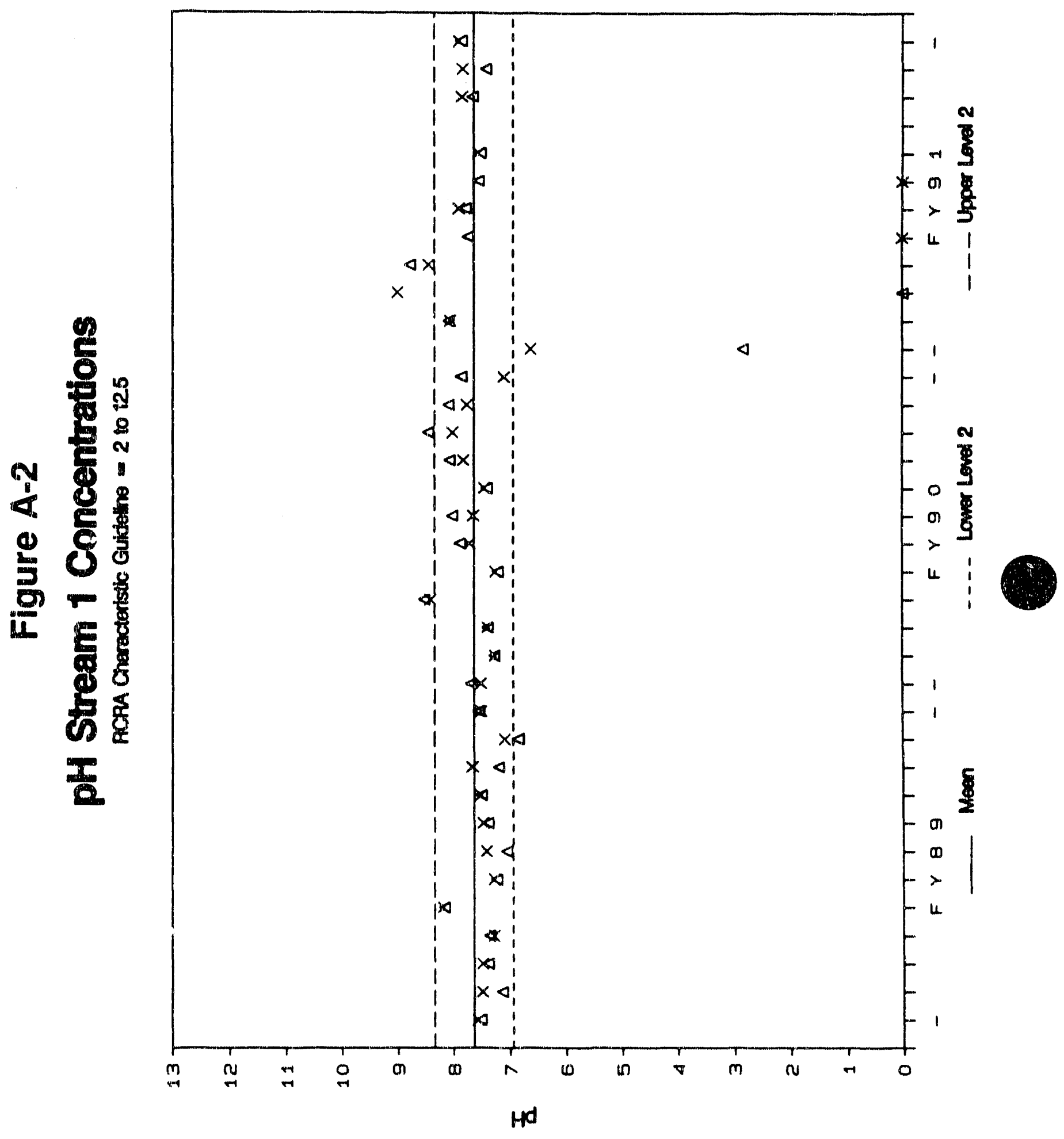

- 


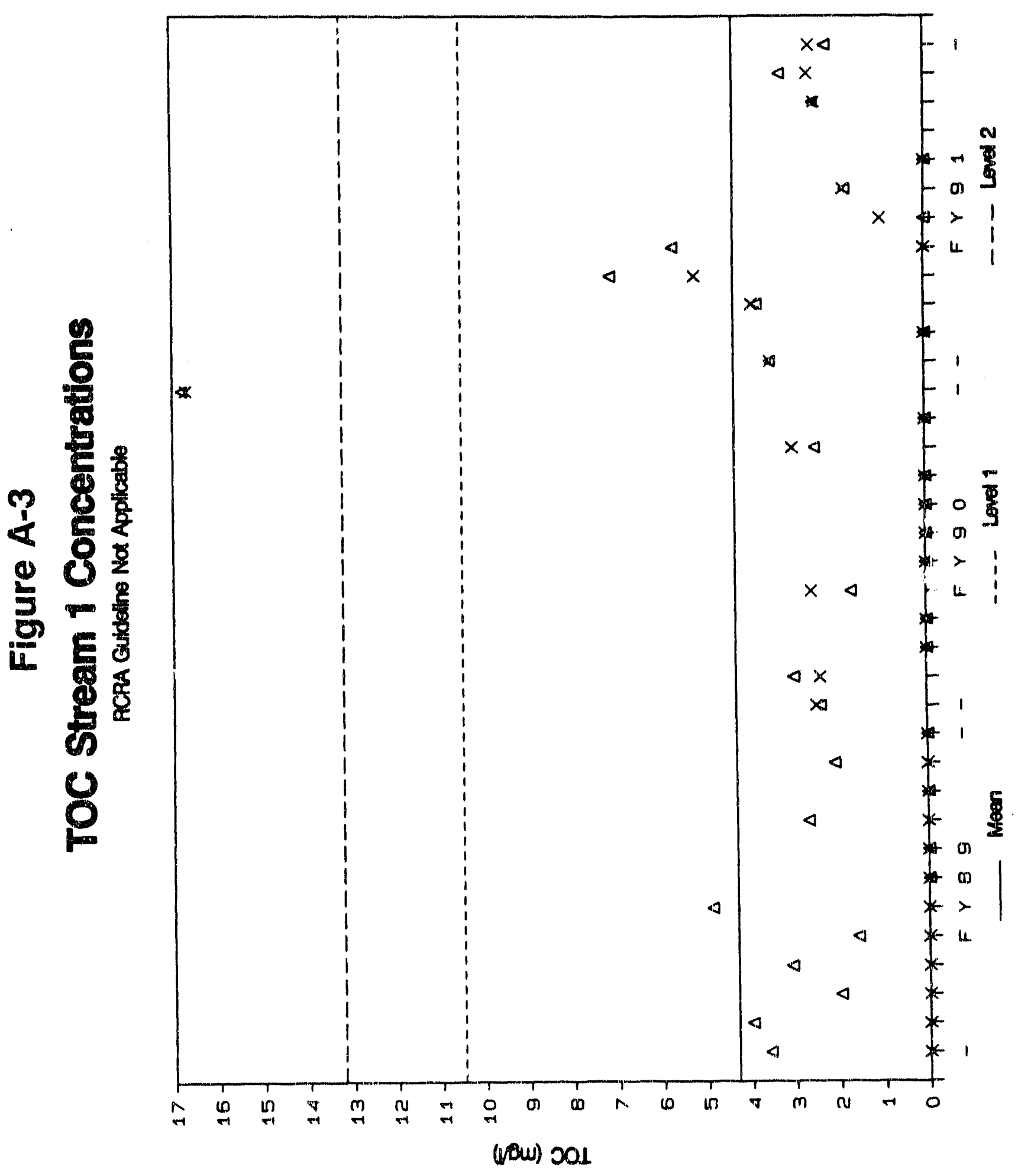




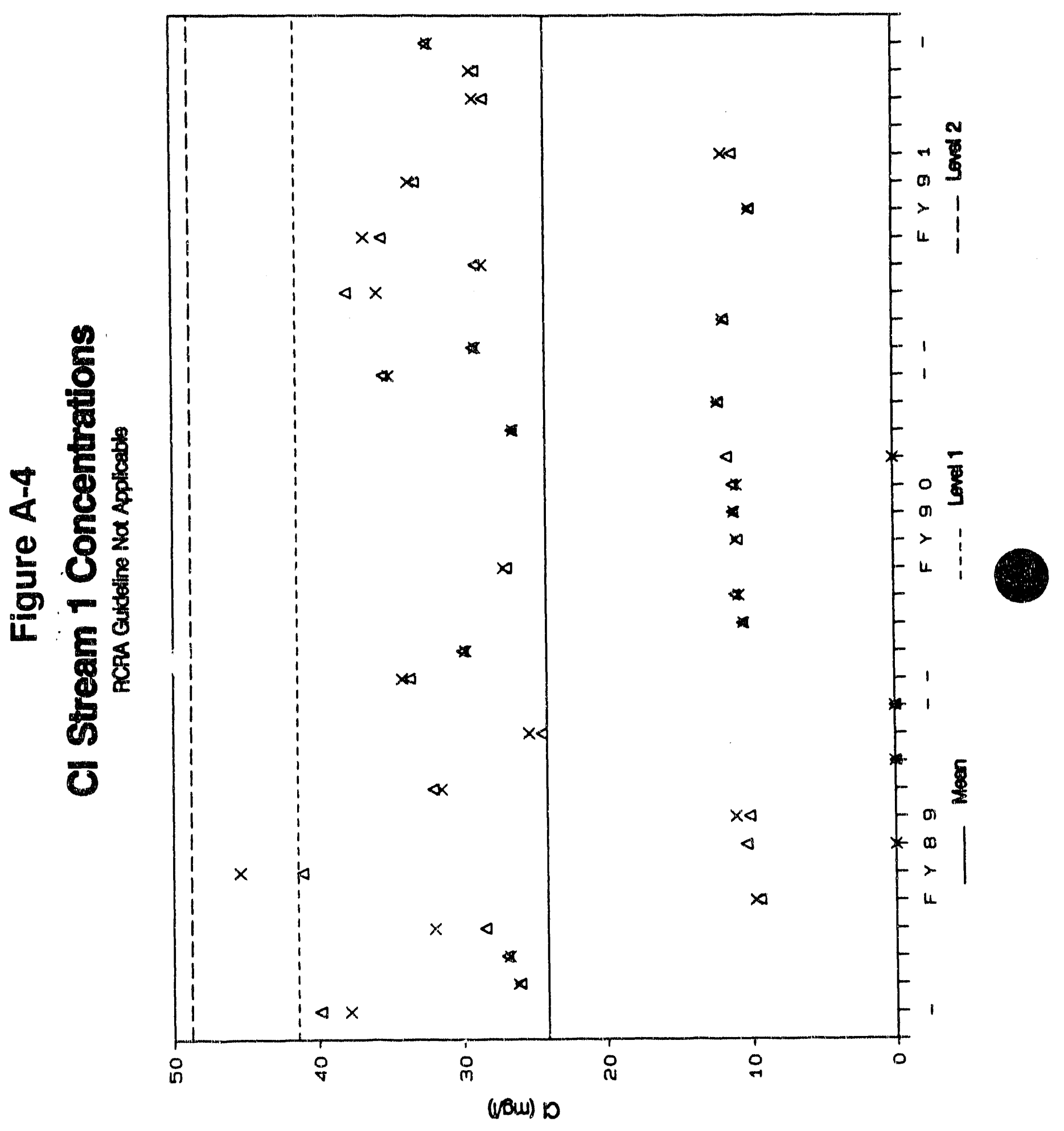

产 


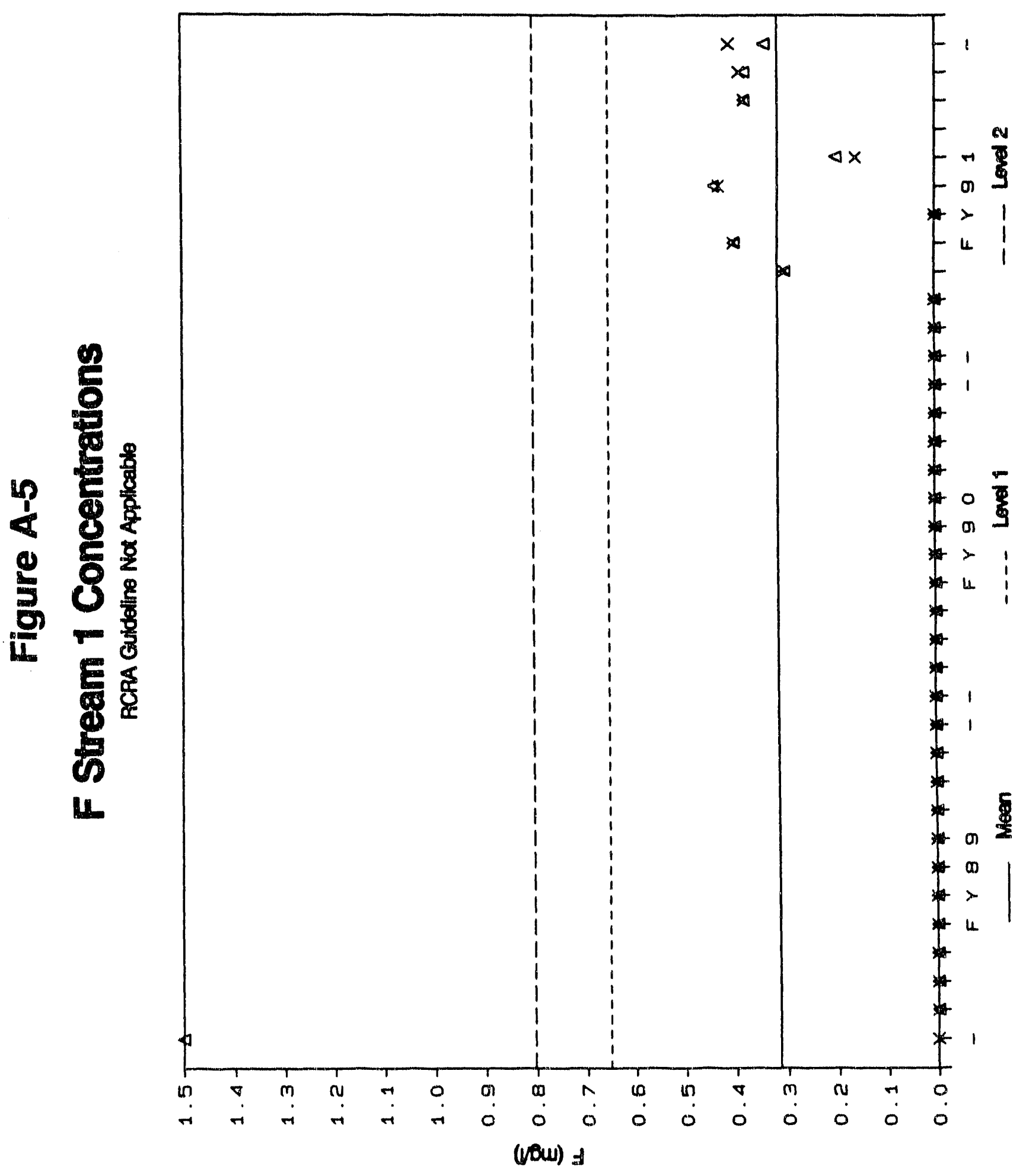




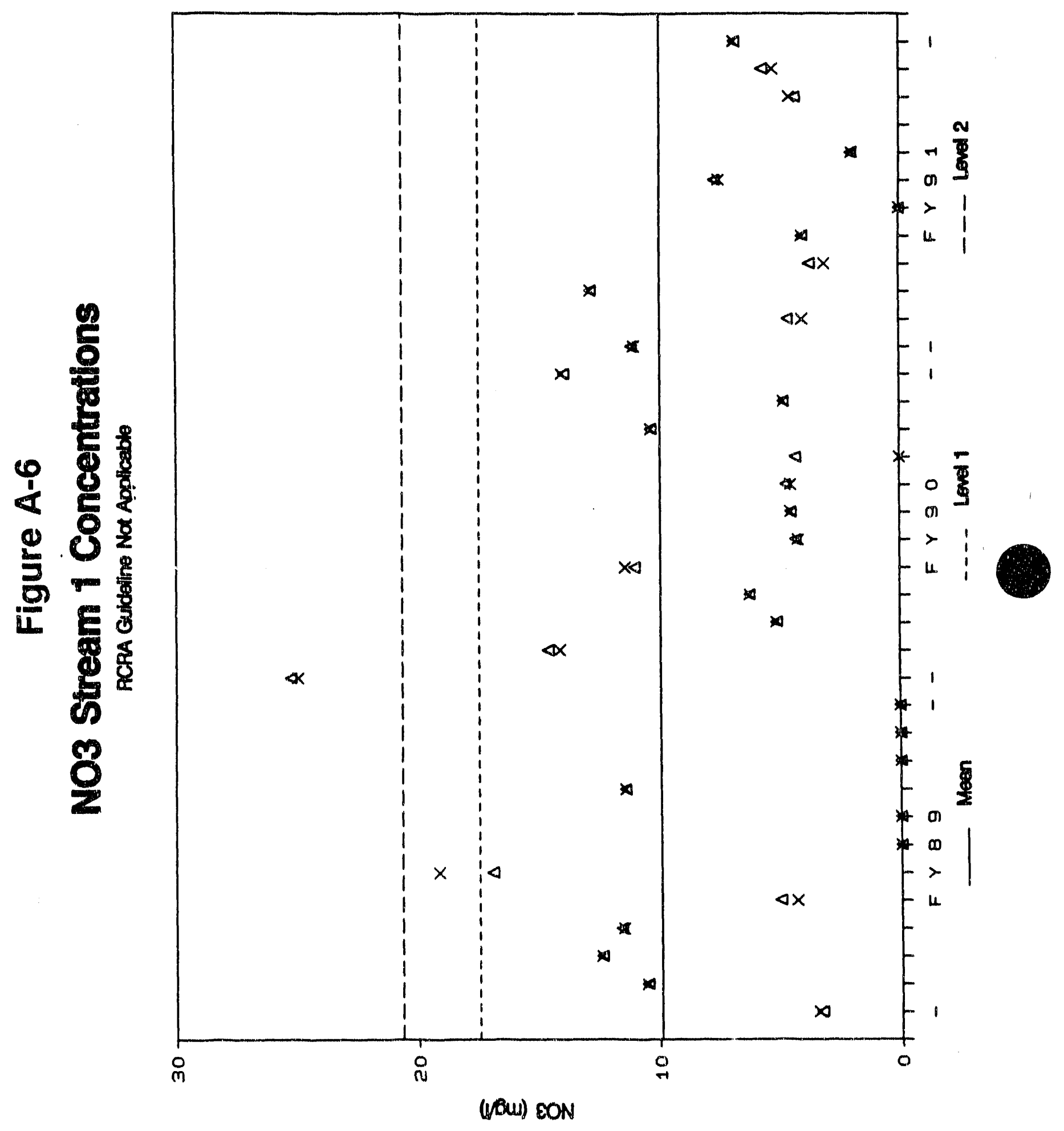




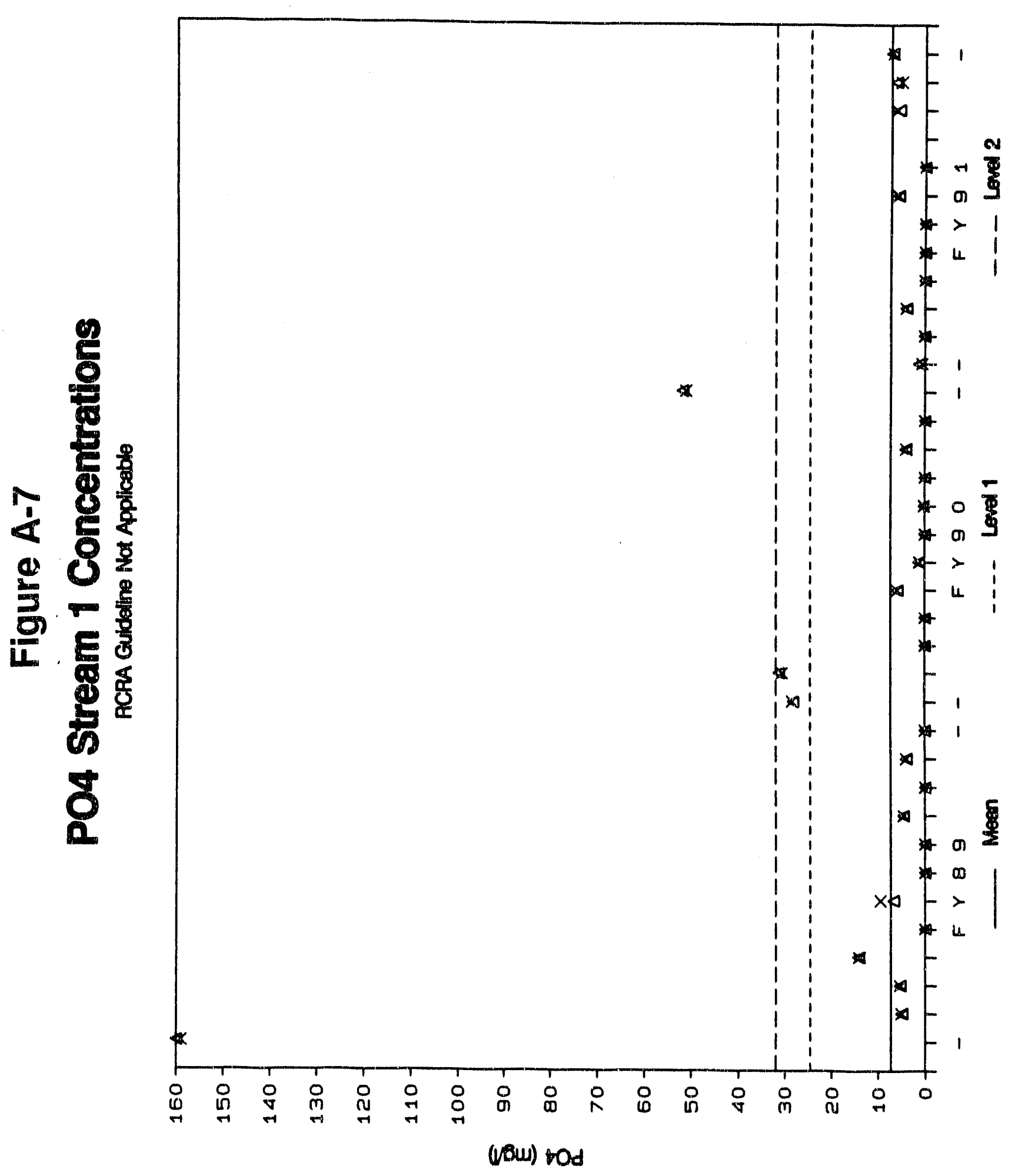




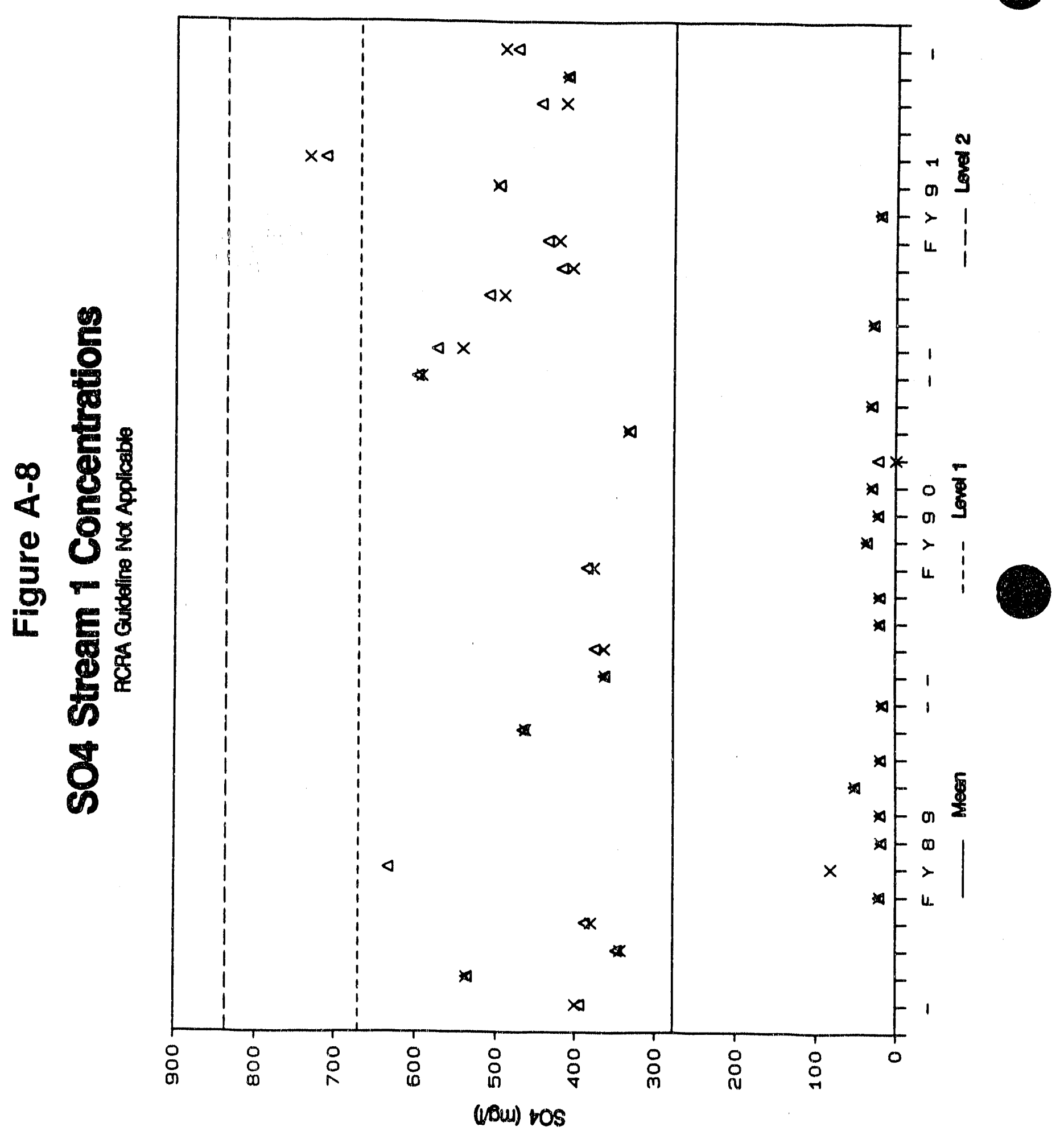




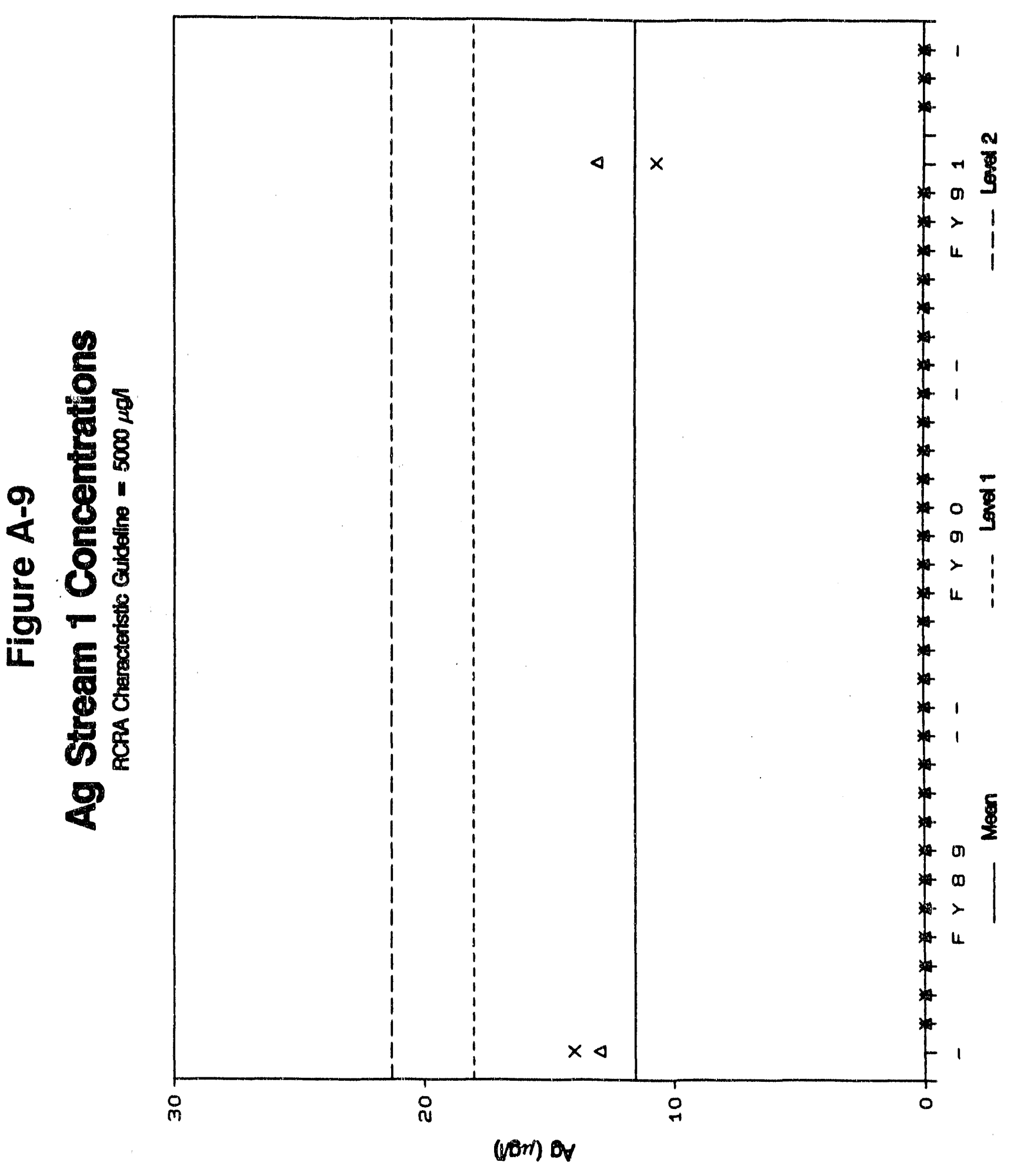




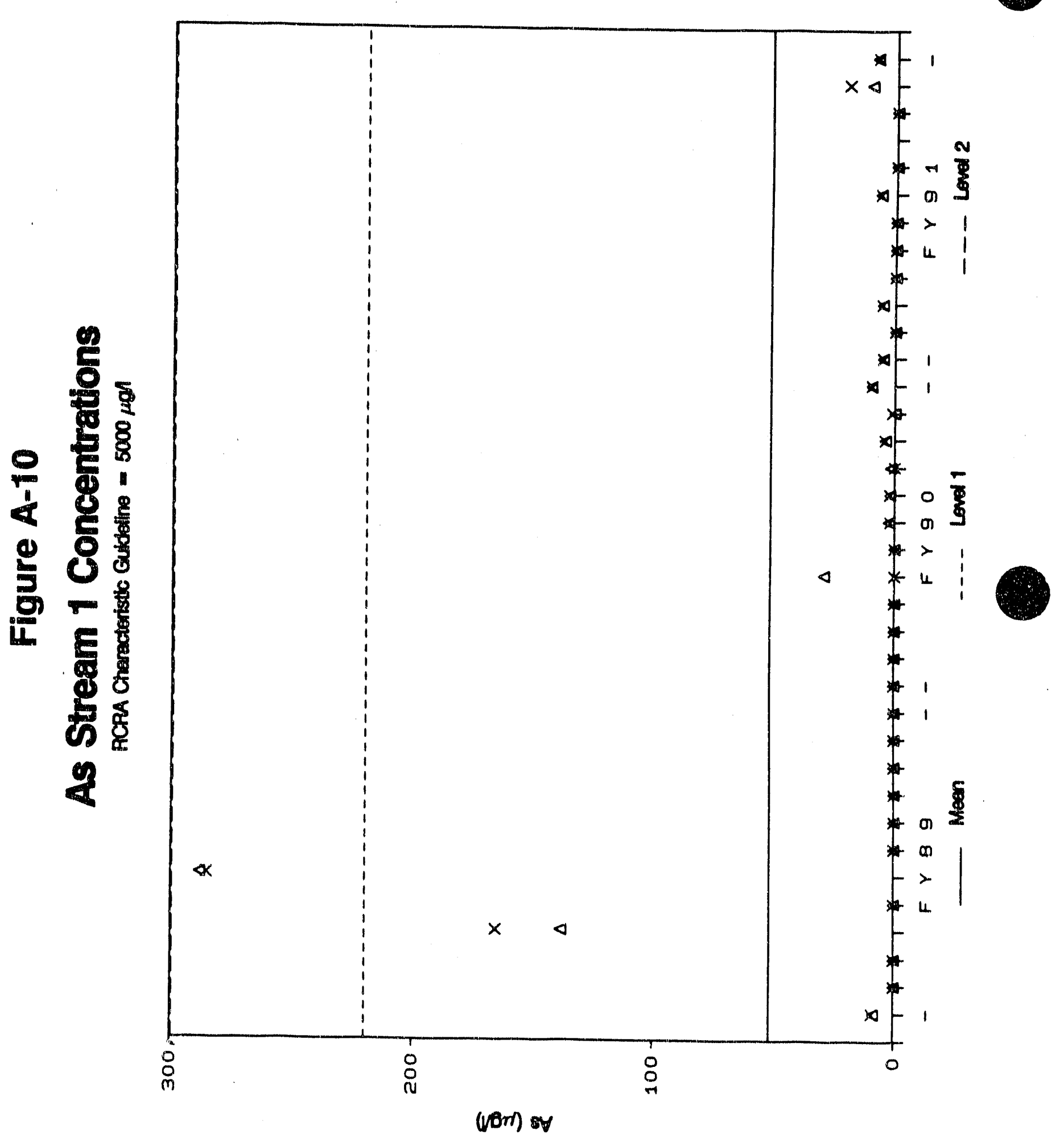




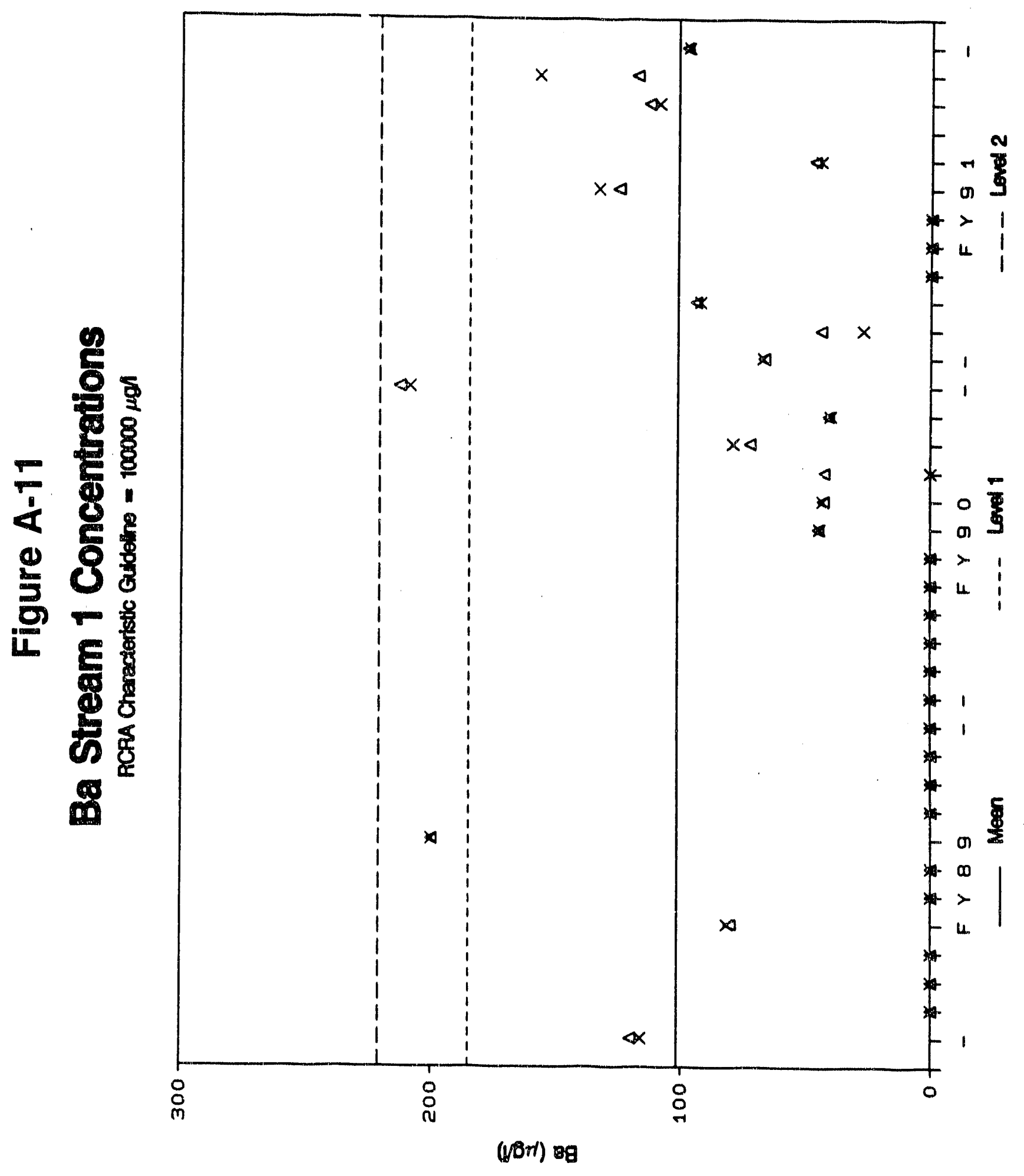




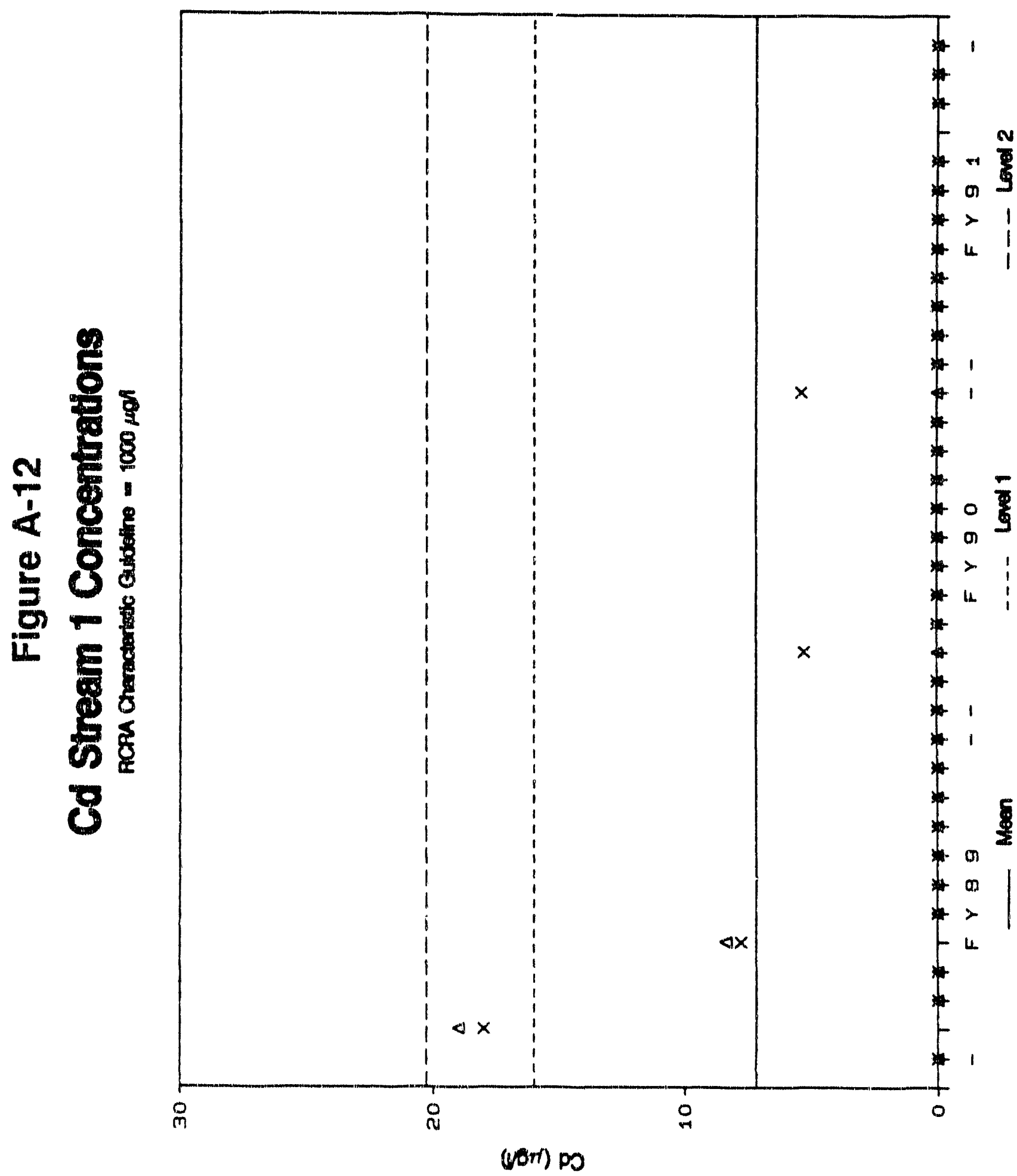

A- 14 


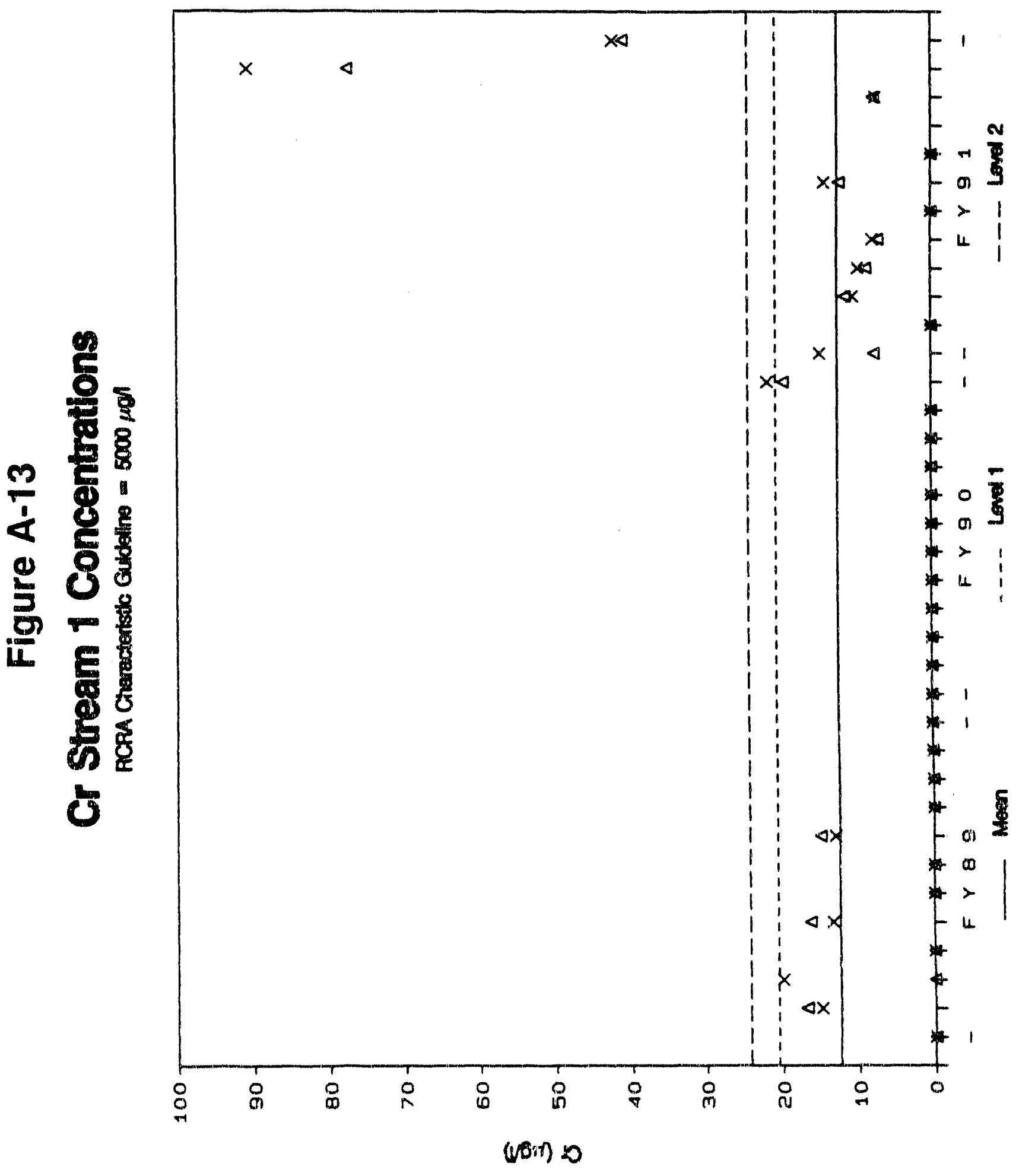

A- 15 


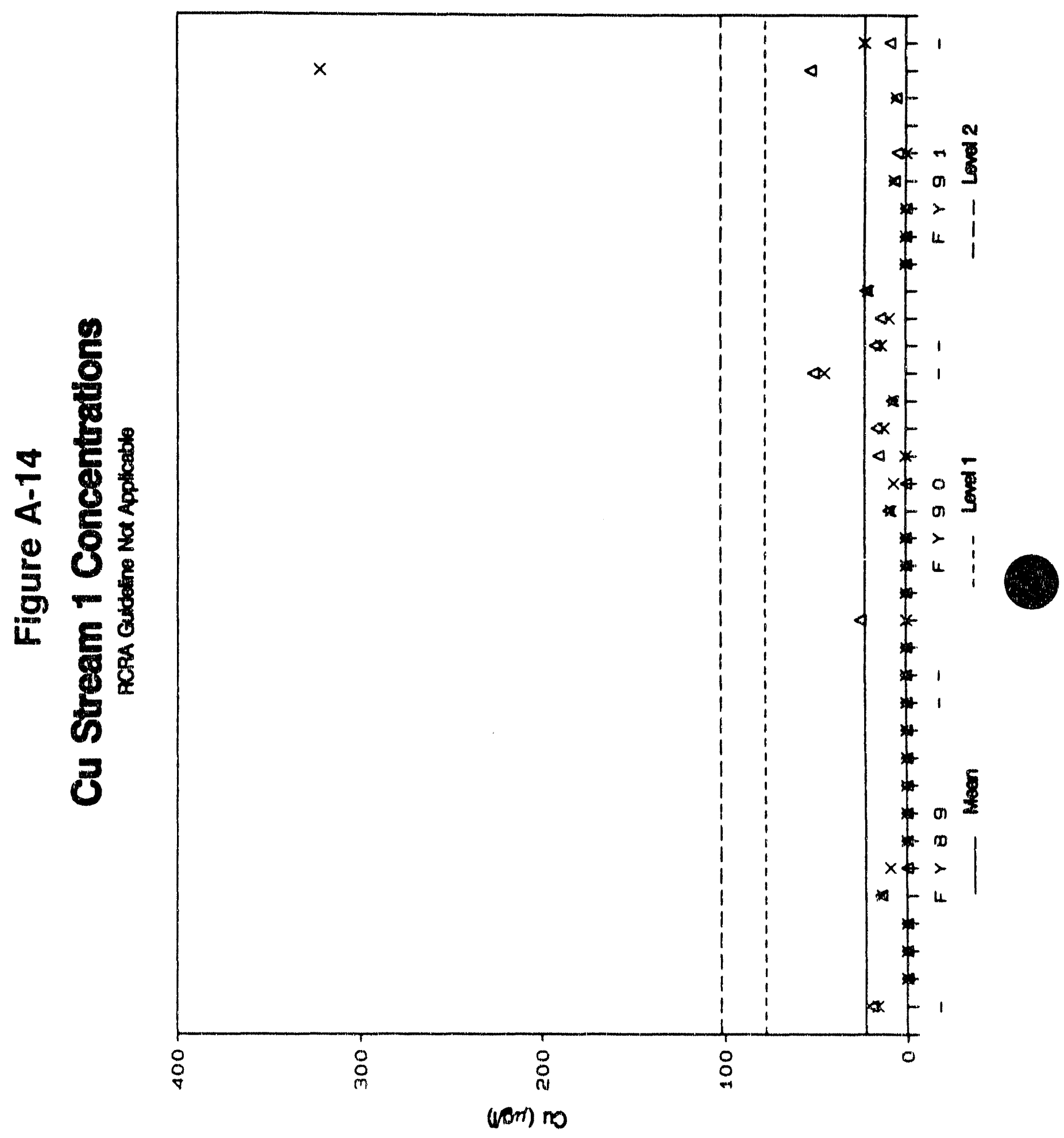

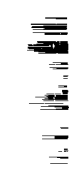
A- 16 


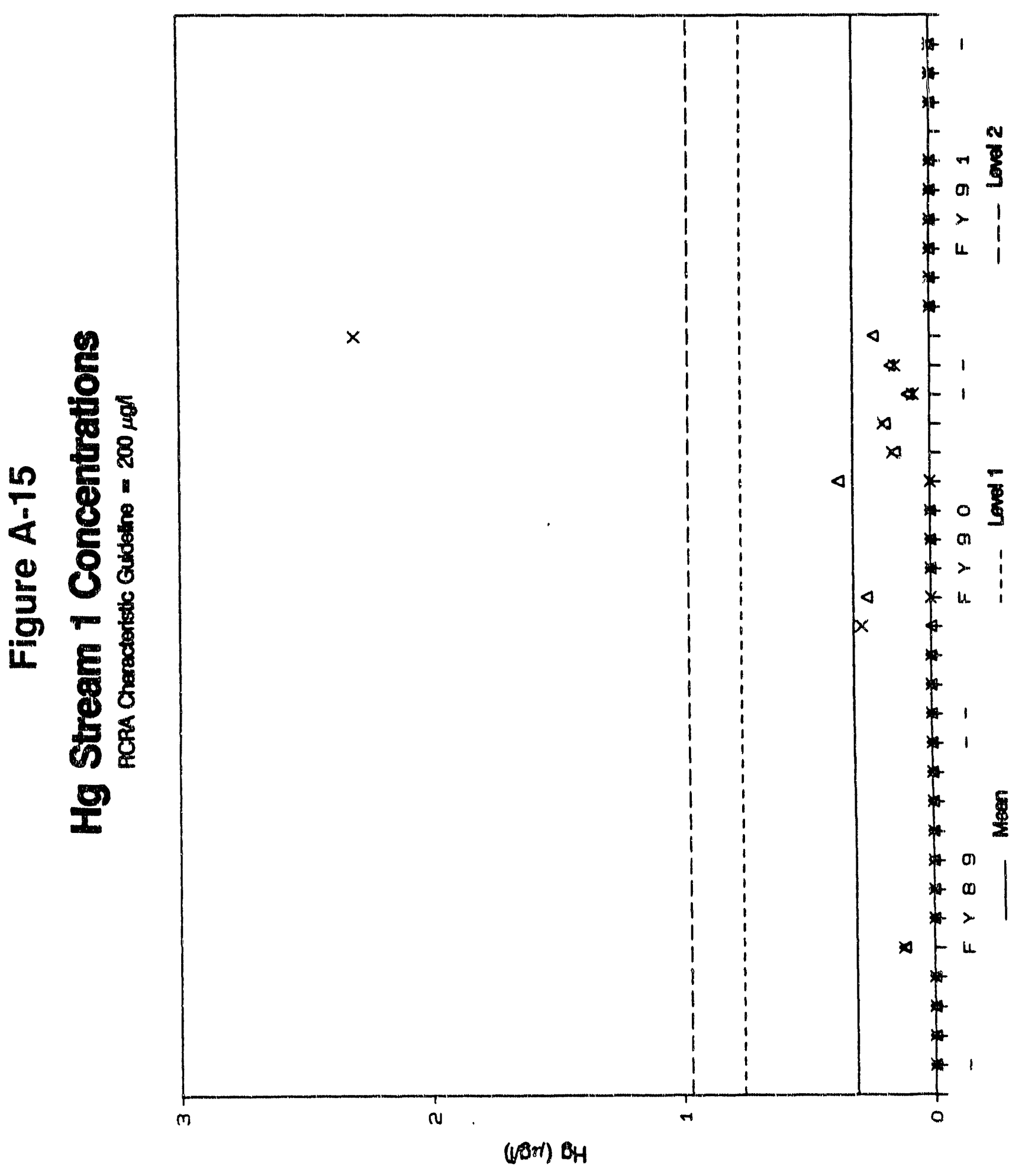




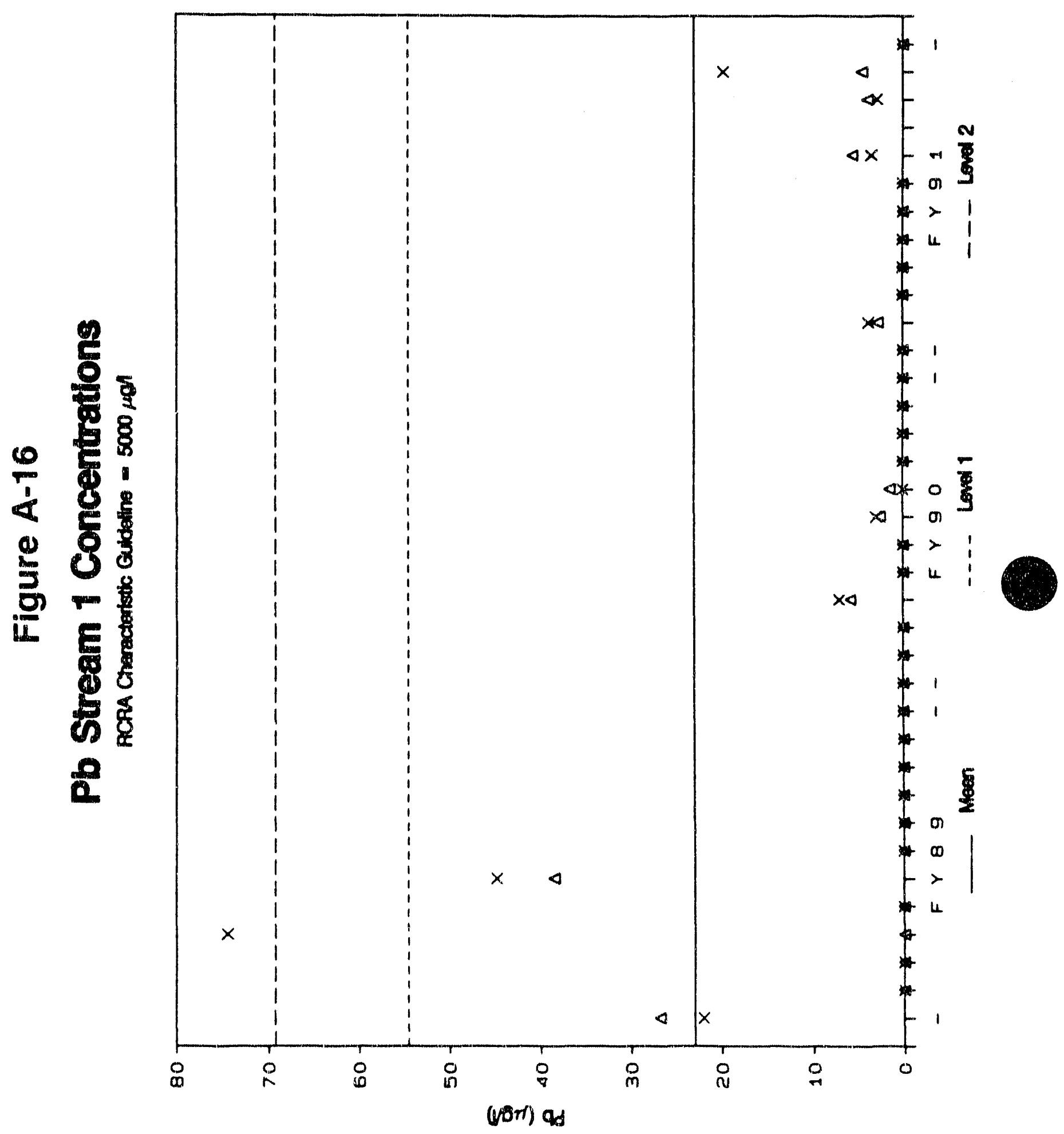




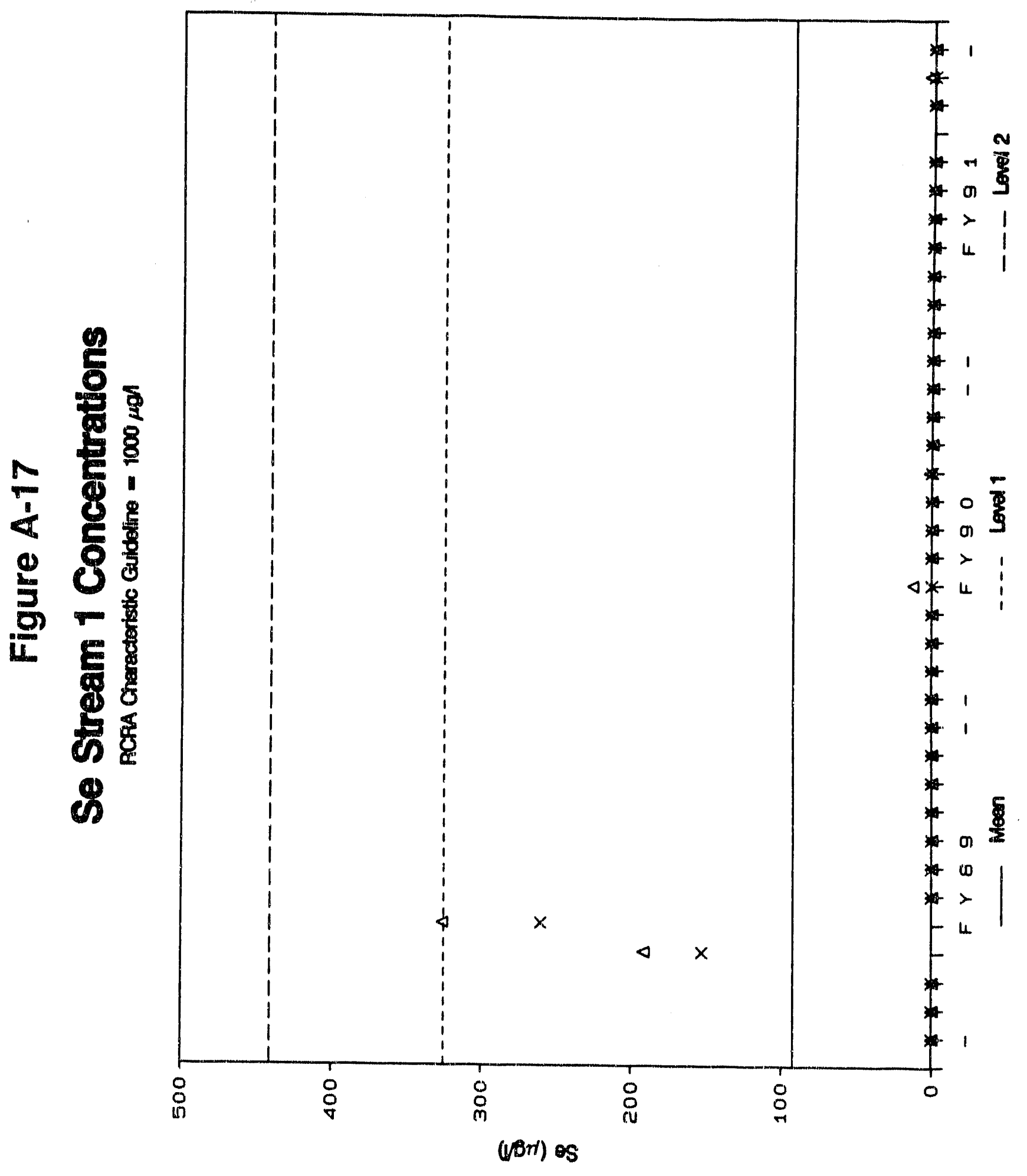

A- 19 

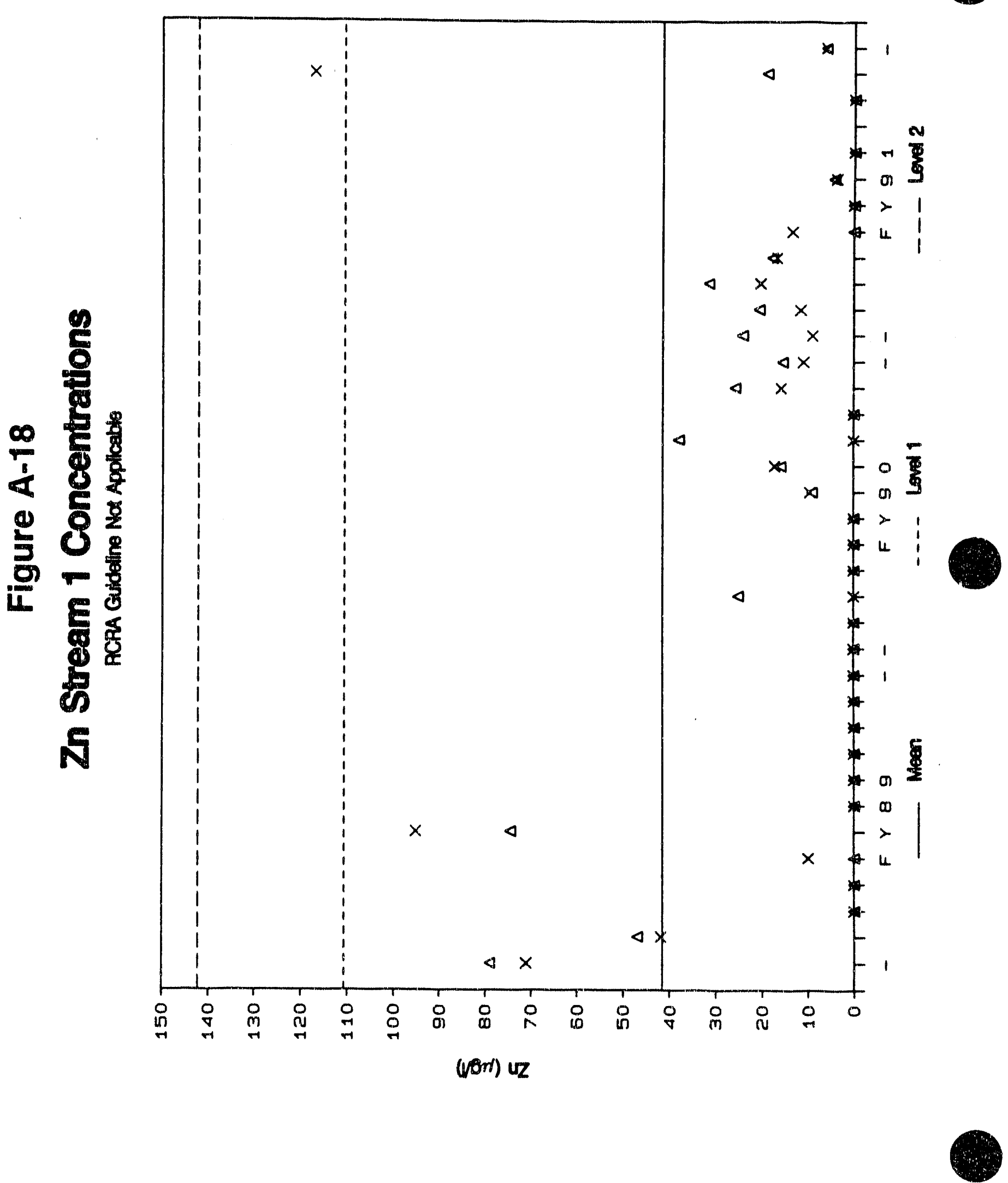


\section{Table A-1}

FY91 ENVIRONMENTAL MONITORING DATA

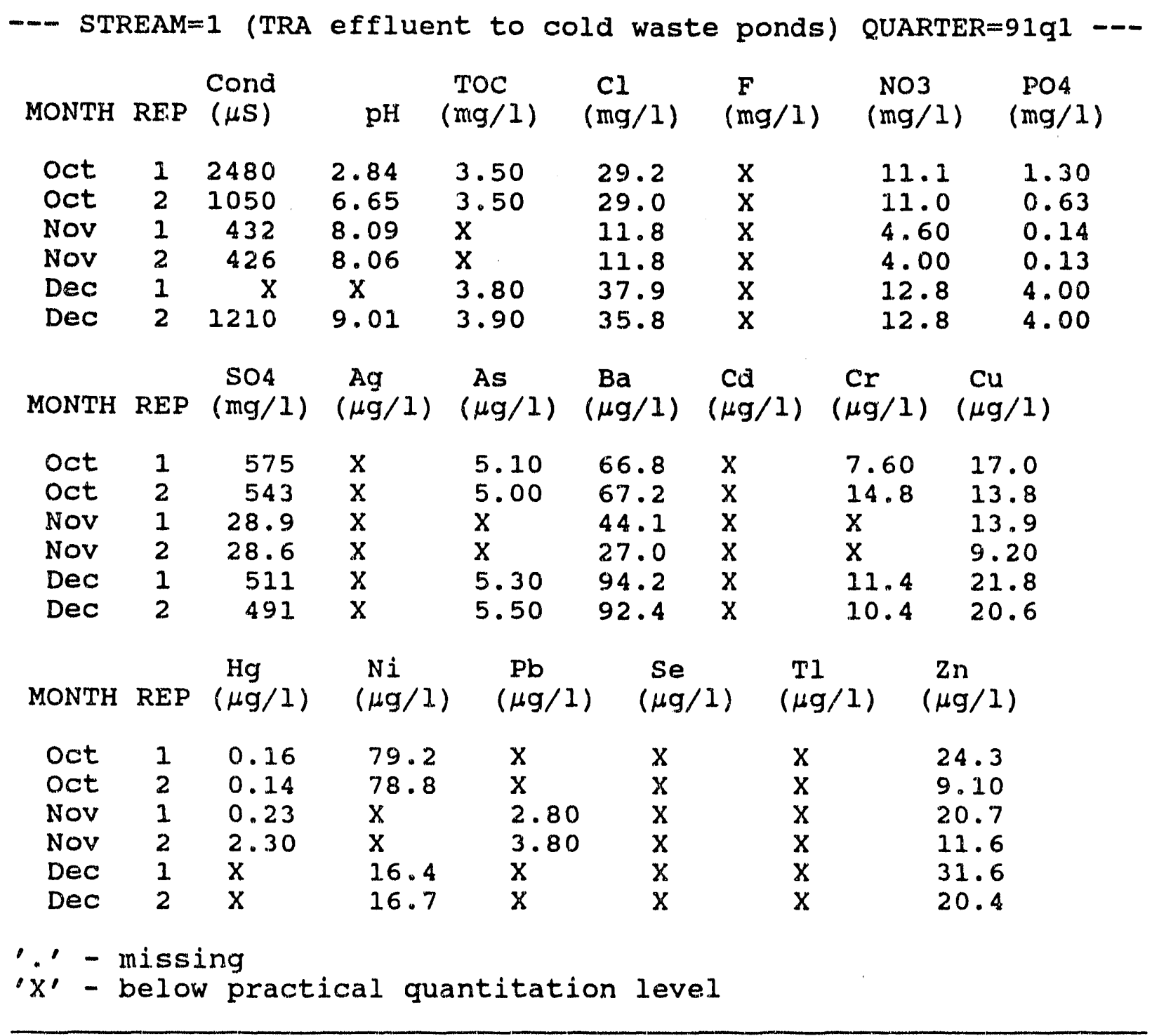




\section{Table A-2}

FY91 ENVIRONMENTAL MONITORING DATA

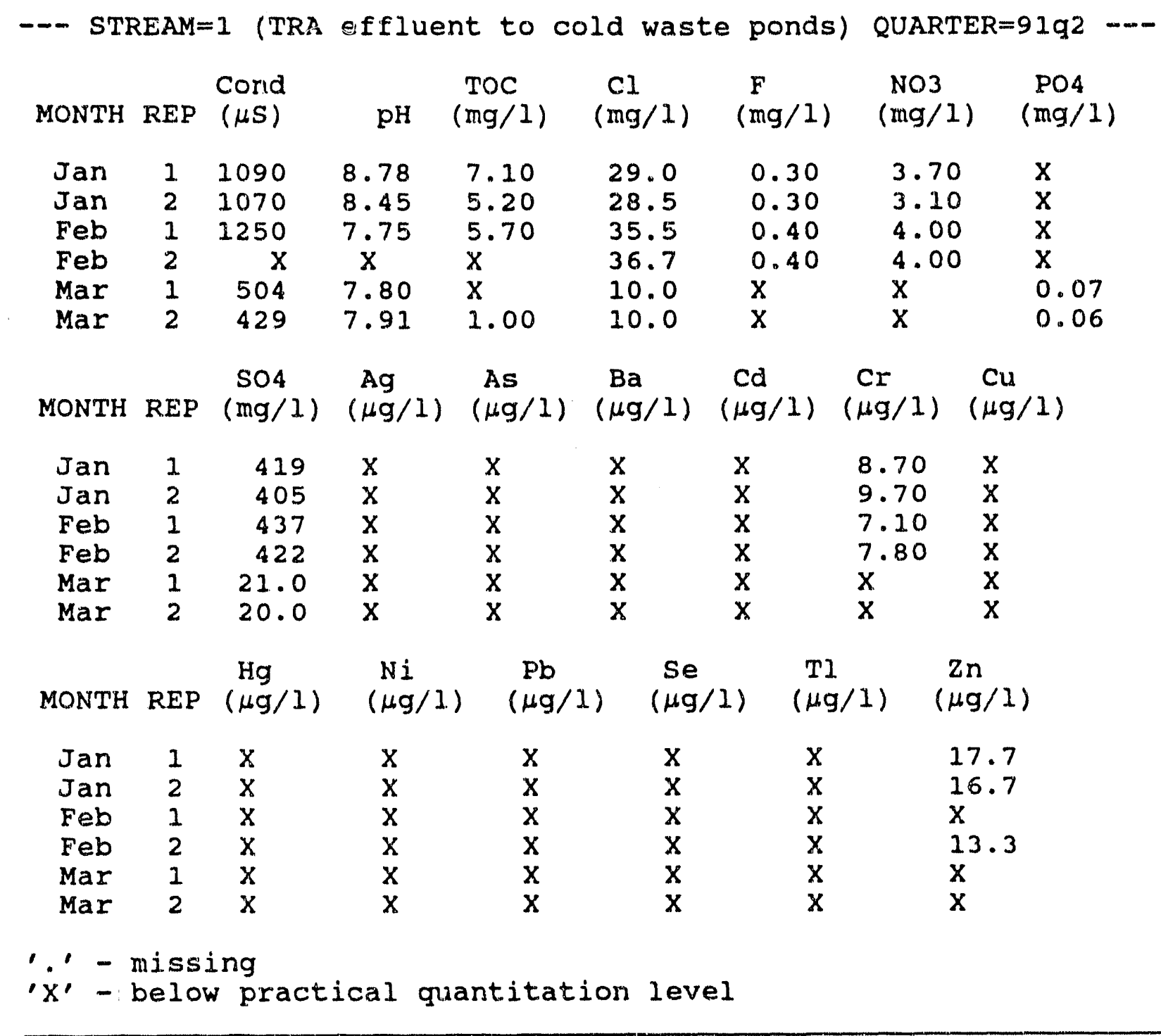




\section{Table A-3}

FY91 ENVIRONMENTAL MONITORING DATA

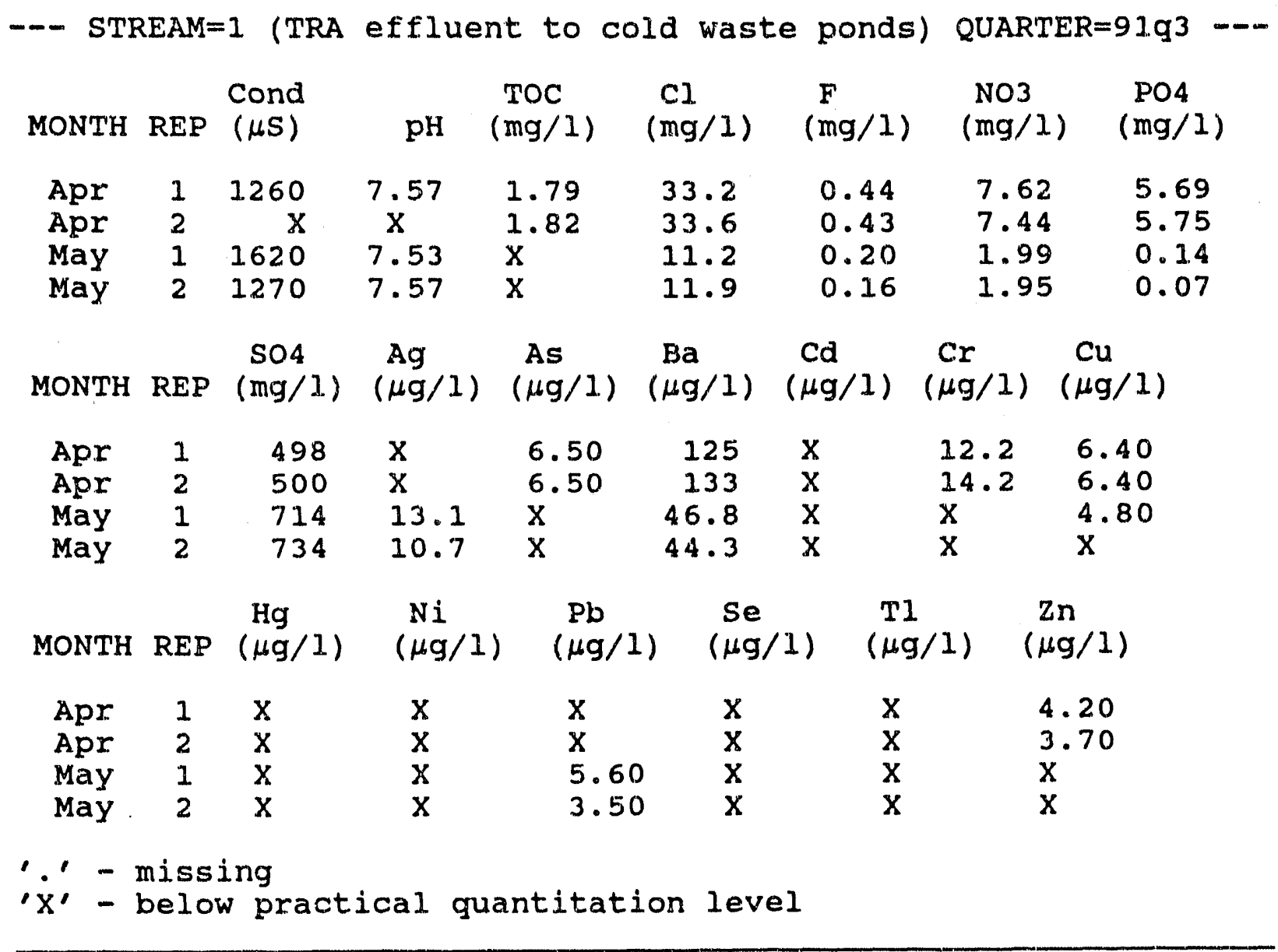


Table A-4

FY91 ENVIRONMENTAL MONITORING DATA

-- STREAM=1 (TRA effluent to cold waste ponds) QUARTER=91q4

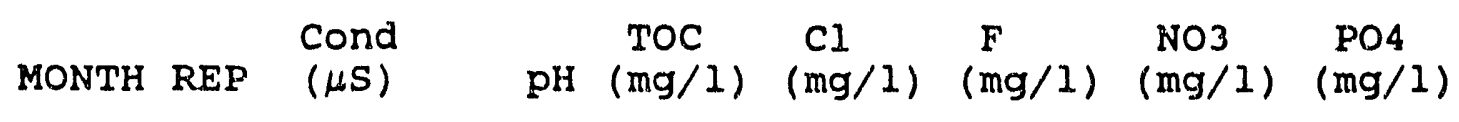

$\begin{array}{lrrrrrrrr}\text { Jul. } & 1 & 1060.0 & 7.67 & 2.51 & 28.4 & 0.38 & 4.32 & 5.63 \\ \text { Jul } & 2 & 1010.0 & 7.85 & 2.47 & 29.1 & 0.38 & 4.54 & 6.00 \\ \text { Aug } & 1 & 900.0 & 7.43 & 3.24 & 29.0 & 0.38 & 5.65 & 6.22 \\ \text { Aug } & 2 & 910.0 & 7.84 & 2.62 & 29.3 & 0.39 & 5.23 & 5.17 \\ \text { Sep } & 1 & 64.2 & 7.86 & 2.21 & 32.4 & 0.34 & 6.78 & 7.10 \\ \text { Sep } & 2 & 62.0 & 7.90 & 2.58 & 32.2 & 0.41 & 6.80 & 6.85\end{array}$

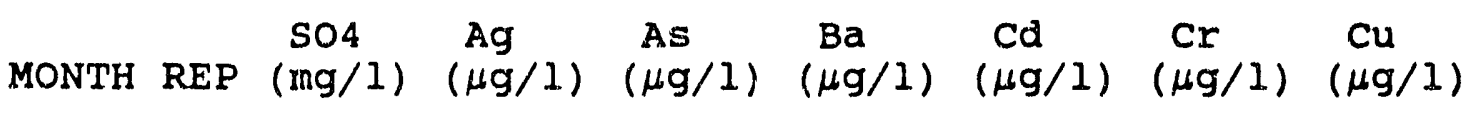

$\begin{array}{lllllrlrr}\text { Jul } & 1 & 446 & X & X & 113 & X & 7.70 & 5.50 \\ \text { JuI } & 2 & 414 & X & X & 109 & X & 7.40 & 5.50 \\ \text { Aug } & 1 & 413 & X & 10.5 & 118 & X & 77.4 & 52.3 \\ \text { Aug } & 2 & 412 & X & 20.1 & 157 & X & 90.6 & 322 \\ \text { Sep } & 1 & 476 & X & 8.30 & 98.1 & X & 40.9 & 9.00 \\ \text { Sep } & 2 & 491 & X & 7.90 & 97.3 & X & 42.2 & 22.5\end{array}$

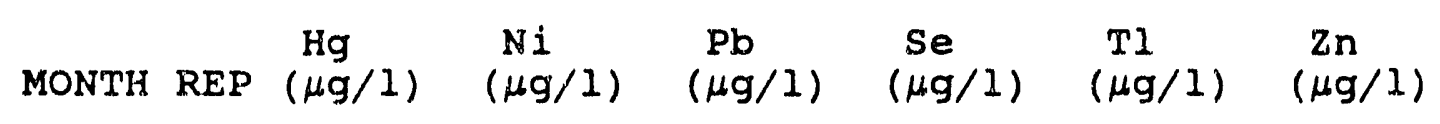

\begin{tabular}{|c|c|c|c|c|c|c|c|}
\hline Jul & 1 & $x$ & $x$ & 3.90 & $x$ & $x$ & $X$ \\
\hline Jul & 2 & $\mathrm{x}$ & $\mathrm{x}$ & 2.90 & $x$ & $x$ & $x$ \\
\hline Aug & 1 & $x$ & $x$ & 4.50 & 2.70 & $\mathrm{X}$ & 19.0 \\
\hline Aug & 2 & $X$ & 17.7 & 19.7 & $\mathrm{x}$ & $\mathrm{X}$ & 117 \\
\hline sep & 1 & $x$ & 28.0 & $x$ & $x$ & $x$ & 6.10 \\
\hline Sep & 2 & $\mathrm{x}$ & 27.1 & $x$ & $x$ & $\mathrm{X}$ & 6.10 \\
\hline
\end{tabular}

'. - missing

' $X$ ' - below practical quantitation level 
Table A-5

SUMMARY STATISTICS

STREAM=1 (TRA effluent to cold waste ponds)

\begin{tabular}{|c|c|c|c|c|c|c|c|}
\hline ANALY & TE & $\begin{array}{l}\times 87-F Y 90 \\
\text { MEAN }\end{array}$ & STD & $\mathbf{N}$ & LEVEL1 & LEVEL2 & GUIDELINE \\
\hline Cond & $(\mu \mathrm{S})$ & 900 & 387 & 94 & 1550 & 1820 & $\mathrm{~N} / \mathrm{A}$ \\
\hline $\mathrm{pH}$ & & 7.65 & 0.26 & 91. & $\begin{array}{ll}1 & 7.12 \\
u & 8.17\end{array}$ & $\begin{array}{ll}1 & 6.95 \\
u & 8.34\end{array}$ & $\begin{array}{ll}1 & 6.5 \\
u & 8.5\end{array}$ \\
\hline TOC & $(\mathrm{mg} / \mathrm{l})$ & 4.31 & 3.62 & 40 & 10.5 & 13.2 & $N / A$ \\
\hline $\mathrm{Cl}$ & $(\mathrm{mg} / \mathrm{l})$ & 24.1 & 10.4 & 87 & 41.4 & 48.8 & $N / A$ \\
\hline $\mathbf{F}$ & $(m g / I)$ & 0.31 & 0.19 & 27 & 0.65 & 0.80 & $\mathrm{~N} / \mathrm{A}$ \\
\hline NO3 & $(\mathrm{mg} / \mathrm{l})$ & 9.90 & 4.49 & 78 & 3.7 .4 & 20.6 & $\mathrm{~N} / \mathrm{A}$ \\
\hline PO4 & $(\mathrm{mg} / \mathrm{l})$ & 7.29 & 10.3 & 66 & 24.6 & 32.0 & $\mathrm{~N} / \mathrm{A}$ \\
\hline SO4 & $(m g / 1)$ & 278 & 235 & 92 & 671 & 837 & $\mathrm{~N} / \mathrm{A}$ \\
\hline Ag & $(\mu g / I)$ & 11.6 & 3.44 & 12 & 18.0 & 21.3 & 5000 \\
\hline Ás & $(\mu g / 1)$ & 51.4 & 94.8 & 19 & 220 & 300 & 5000 \\
\hline $\mathrm{Ba}$ & $(\mu g / 1)$ & 101 & 49.6 & 59 & 185 & 221 & 100,000 \\
\hline $\mathrm{cd}$ & $(\mu g / 1)$ & 7.18 & 4.88 & 16 & 16.0 & 20.3 & 1000 \\
\hline $\mathrm{Cr}$ & $(\mu g / 1)$ & 12.4 & 4.73 & 31 & 20.6 & 24.2 & 5000 \\
\hline $\mathrm{Cu}$ & $(\mu g / I)$ & 22.4 & 31.7 & 29 & 77.3 & 102 & $\mathrm{~N} / \mathrm{A}$ \\
\hline $\mathrm{Hg}$ & $(\mu g / 1)$ & 0.31 & 0.26 & 26 & 0.76 & 0.97 & 200 \\
\hline $\mathrm{Ni}$ & $(\mu \mathrm{g} / 1)$ & & & & & & $\mathrm{N} / \mathrm{A}$ \\
\hline $\mathrm{Pb}$ & $(\mu g / I)$ & 23.0 & 17.9 & 21 & 54.5 & 69.3 & 5000 \\
\hline $\mathrm{Se}$ & $(\mu g / 1)$ & 92.0 & 127 & 14 & 325 & 441 & 1000 \\
\hline TI & $(\mu g / 1)$ & 172 & 153 & 6 & 505 & 729 & $N / A$ \\
\hline $\mathrm{Zn}$ & $(\mu g / 1)$ & 41.7 & 39.6 & 25 & 111 & 142 & $N / A$ \\
\hline
\end{tabular}


Table A-6

QUARTERLY MEANS AND VARIANCE COMPONENTS

STREAM=1 (TRA effluent to cold waste ponds)

\begin{tabular}{|c|c|c|c|c|c|c|c|c|c|}
\hline \multicolumn{2}{|c|}{ ANALYTE } & QTR & MEAN & $\begin{array}{r}\text { STD } \\
\text { MEAN }\end{array}$ & $\begin{array}{l}\text { STD } \\
\text { DAY }\end{array}$ & $\begin{array}{l}\text { STD } \\
\text { REP }\end{array}$ & $\mathbf{N}$ & $\begin{array}{r}\mathrm{N} \\
\text { MISS }\end{array}$ & $\stackrel{N}{N}$ \\
\hline cond & $(\mu s)$ & $\begin{array}{l}91 q 1 \\
91 q 2 \\
91 q 3 \\
91 q 4\end{array}$ & $\begin{array}{r}1119 \\
869 \\
1383 \\
668\end{array}$ & $\begin{array}{r}434 \\
251 \\
\cdot \quad 305\end{array}$ & $\begin{array}{r}489 \\
417 \\
\cdot \quad 527\end{array}$ & $\begin{array}{r}717 \\
39.2 \\
20.8\end{array}$ & $\begin{array}{l}5 \\
5 \\
3 \\
6\end{array}$ & $\begin{array}{l}1 \\
1 \\
0 \\
0\end{array}$ & $\begin{array}{l}0 \\
0 \\
3 \\
0\end{array}$ \\
\hline $\mathrm{pH}$ & & $\begin{array}{l}91 q 1 \\
91 q 2 \\
91 q 3 \\
91 q 4\end{array}$ & $\begin{array}{l}6.93 \\
8.14 \\
7.56 \\
7.76\end{array}$ & $\begin{array}{l}1.33 \\
0.29 \\
0.07\end{array}$ & $\begin{array}{l}1.70 \\
0.47 \\
0.00\end{array}$ & $\begin{array}{l}1.91 \\
0.17 \\
0.18\end{array}$ & $\begin{array}{l}5 \\
5 \\
3 \\
6\end{array}$ & $\begin{array}{l}1 \\
1 \\
0 \\
0\end{array}$ & $\begin{array}{l}0 \\
0 \\
3 \\
0\end{array}$ \\
\hline TOC & $(\mathrm{mg} / \mathrm{l})$ & $\begin{array}{l}91 q 1 \\
91 q 2 \\
91 q^{3} \\
91 q 4\end{array}$ & $\begin{array}{l}3.67 \\
2.17 \\
1.81 \\
2.60\end{array}$ & $\begin{array}{l}0.17 \\
1.99 \\
0.17\end{array}$ & $\begin{array}{l}0.25 \\
1.33 \\
0.19\end{array}$ & $\begin{array}{l}0.05 \\
4.51 \\
0.29\end{array}$ & $\begin{array}{l}4 \\
6 \\
2 \\
6\end{array}$ & $\begin{array}{l}0 \\
0 \\
0 \\
0\end{array}$ & $\begin{array}{l}2 \\
0 \\
4 \\
0\end{array}$ \\
\hline$C l$ & $(\mathrm{mg} / \mathrm{l})$ & $\begin{array}{l}91 q 1 \\
91 q 2 \\
91 q 3 \\
91 q^{4}\end{array}$ & $\begin{array}{l}25.9 \\
25.0 \\
22.0 \\
30.1\end{array}$ & $\begin{array}{l}7.40 \\
7.77 \\
10.9 \\
1.12\end{array}$ & $\begin{array}{l}12.8 \\
13.5 \\
15.4 \\
1.93\end{array}$ & $\begin{array}{l}0.86 \\
0.53 \\
0.40 \\
0.32\end{array}$ & $\begin{array}{l}6 \\
6 \\
4 \\
6\end{array}$ & $\begin{array}{l}0 \\
0 \\
0 \\
0\end{array}$ & $\begin{array}{l}0 \\
0 \\
0 \\
0\end{array}$ \\
\hline$F$ & $(\mathrm{mg} / \mathrm{l})$ & $\begin{array}{l}91 q 1 \\
91 q 2 \\
91 q 3 \\
91 q 4\end{array}$ & $\begin{array}{l}x \\
0.07 \\
0.31 \\
0.38\end{array}$ & $\begin{array}{l}0.29 \\
0.13 \\
0.00\end{array}$ & $\begin{array}{l}0.49 \\
0.18 \\
0.00\end{array}$ & $\begin{array}{l}0.00 \\
0.02 \\
0.03\end{array}$ & $\begin{array}{l}0 \\
6 \\
4 \\
6\end{array}$ & $\begin{array}{l}0 \\
0 \\
0 \\
0\end{array}$ & $\begin{array}{l}6 \\
0 \\
0 \\
0\end{array}$ \\
\hline NO3 & $(\mathrm{mg} / \mathrm{I})$ & $\begin{array}{l}91 q 1 \\
91 q 2 \\
91 q^{3} \\
91 q^{4}\end{array}$ & $\begin{array}{l}9.38 \\
3.70 \\
4.75 \\
5.55\end{array}$ & $\begin{array}{l}2.59 \\
0.30 \\
2.78 \\
0.68\end{array}$ & $\begin{array}{l}4.48 \\
0.37 \\
3.93 \\
1.18\end{array}$ & $\begin{array}{l}0.25 \\
0.30 \\
0.09 \\
0.19\end{array}$ & $\begin{array}{l}6 \\
4 \\
4 \\
6\end{array}$ & $\begin{array}{l}0 \\
2 \\
0 \\
0\end{array}$ & $\begin{array}{l}0 \\
0 \\
0 \\
0\end{array}$ \\
\hline $\mathrm{PO} 4$ & $(\mathrm{mg} / \mathrm{I})$ & $\begin{array}{l}91 q 1 \\
91 q 2 \\
91 q 3 \\
91 q 4\end{array}$ & $\begin{array}{l}1.70 \\
0.07 \\
2.91 \\
6.16\end{array}$ & $\begin{array}{l}1.17 \\
2.81 \\
0.41\end{array}$ & $\begin{array}{l}2.03 \\
\dot{3} .97 \\
0.63\end{array}$ & $\begin{array}{l}0.27 \\
0.04 \\
0.47\end{array}$ & $\begin{array}{l}6 \\
2 \\
4 \\
6\end{array}$ & $\begin{array}{l}0 \\
0 \\
0 \\
0\end{array}$ & $\begin{array}{l}0 \\
4 \\
0 \\
0\end{array}$ \\
\hline SO4 & $(\mathrm{mg} / \mathrm{l})$ & $\begin{array}{l}91 q 1 \\
91 q 2 \\
91 q 3 \\
91 . q^{4}\end{array}$ & $\begin{array}{l}363 \\
287 \\
612 \\
442\end{array}$ & $\begin{array}{r}168 \\
134 \\
113 \\
21.4\end{array}$ & $\begin{array}{r}291 \\
231 \\
159 \\
35.6\end{array}$ & $\begin{array}{l}15.4 \\
8.39 \\
10.0 \\
14.4\end{array}$ & $\begin{array}{l}6 \\
6 \\
4 \\
6\end{array}$ & $\begin{array}{l}0 \\
0 \\
0 \\
0\end{array}$ & $\begin{array}{l}0 \\
0 \\
0 \\
0\end{array}$ \\
\hline Ag & $(\mu g / 1)$ & $\begin{array}{l}91 q 1 \\
91 q 2 \\
91 q 3 \\
91 q 4\end{array}$ & $\begin{array}{l}x \\
x \\
11.9 \\
x\end{array}$ & $\dot{.}$ & $\begin{array}{l}\dot{0} \\
\dot{.}\end{array}$ & $\dot{.}$ & $\begin{array}{l}0 \\
0 \\
2 \\
0\end{array}$ & $\begin{array}{l}0 \\
0 \\
0 \\
0\end{array}$ & $\begin{array}{l}6 \\
6 \\
4 \\
6\end{array}$ \\
\hline
\end{tabular}




\section{Table A-6 (continued)}

QUARTERLY MEANS AND VARIANCE COMPONENTS

STREAM=1 (TRA effluent to cold waste ponds)

(continued)

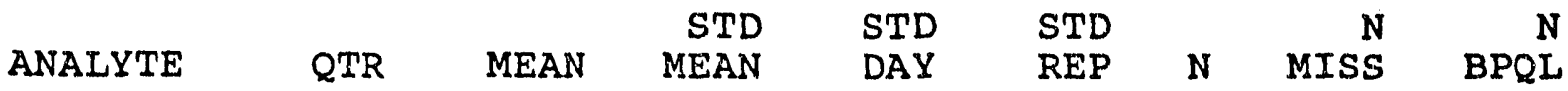

As $(\mu g / 1) \quad 91 q 1$

$91 \mathrm{q} 2$

$91 q 3$

9194

$\begin{array}{lllllll}5.22 & 0.17 & 0.23 & 0.11 & 4 & 0 & 2 \\ x & \cdot & \dot{0} & \dot{1} & 0 & 0 & 6 \\ 6.50 & \dot{5} & \dot{5} & \dot{3} & 2 & 0 & 4 \\ 6.63 & 5.48 & 9.07 & 3.94 & 6 & 0 & 0\end{array}$

$\mathrm{Ba}(\mu \mathrm{g} / 1) \quad 91 \mathrm{q}$

$91 q^{2}$

$91 q^{3}$

$91 q 4$

\begin{tabular}{|c|c|c|c|}
\hline 65.3 & 16.7 & 28.5 & 7.02 \\
\hline $\mathrm{X}$ & & • & \\
\hline $\begin{array}{r}87.3 \\
115\end{array}$ & $\begin{array}{l}41.7 \\
11.7\end{array}$ & $\begin{array}{l}58.9 \\
16.8\end{array}$ & $\begin{array}{l}4.19 \\
16.0\end{array}$ \\
\hline
\end{tabular}

$\begin{aligned} \mathrm{Cd} \quad(\mu \mathrm{g} / 1) \quad & 91 \mathrm{1} 1 \\ & 91 \mathrm{~g}\end{aligned}$

9193

9194

$\mathrm{X}$
$\mathrm{X}$
$\mathrm{X}$

C. $(\mu g / I)$

$91 q 1$

$91 q^{2}$

$\begin{array}{lllllll}11.1 & 0.15 & 0.00 & 3.63 & 4 & 0 & 2 \\ 2.22 & 6.13 & 10.6 & 0.50 & 6 & 0 & 0 \\ 13.2 & 0 & 5 & . & 2 & 0 & 4 \\ 44.4 & 22.1 & 38.1 & 5.42 & 6 & 0 & 0\end{array}$

$\mathrm{Cu} \quad(\mu \mathrm{g} / 1)$

9194

:

16.8

16.0

$$
2
$$

3

$\begin{array}{llllllll}91 q 1 & 16.1 & 2.80 & 4.56 & 2.37 & 6 & 0 & 0 \\ 91 q 2 & X & \cdot & \cdot & . & 0 & 0 & 6 \\ 9193 & 5.87 & . & . & . & 3 & 0 & 3 \\ 9194 & 69.5 & 58.9 & 65.9 & 110 & 6 & 0 & 0\end{array}$

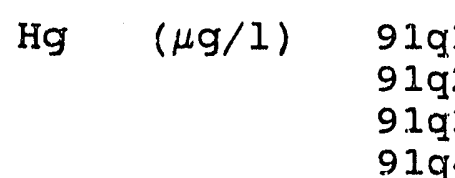

$\begin{array}{llllllll}91 q 1 & 0.19 & 0.04 & 0.06 & 0.01 & 4 & 0 & 2 \\ 91 q^{2} & X & . & . & . & 0 & 0 & 6 \\ 91 q_{3} & X & . & . & . & 0 & 0 & 6 \\ 9194 & X & . & . & . & 0 & 0 & 6\end{array}$

$\mathrm{Ni} \quad(\mu \mathrm{g} / \mathrm{l})$

$$
9191
$$

$91 q 2$

9193

9294

47.8
$\mathrm{X}$
$\mathrm{X}$
24.3

31.

44.2

0.25

$\begin{array}{lll}4 & 0 & 2 \\ 0 & 0 & 6 \\ 0 & 0 & 6 \\ 3 & 0 & 3\end{array}$

$\mathrm{Pb} \quad(\mu \mathrm{g} / 1) \quad 91 \mathrm{ql}$

$91 q 2$

$91 \mathrm{q} 3$

9194

3.30
$X$
4.55
4.50

:. 11

$\dot{:}$
5.59

$\begin{array}{ll}. & 2 \\ . & 0 \\ \dot{6} .22 & 2 \\ & 6\end{array}$

$\begin{array}{ll}0 & 4 \\ 0 & 6 \\ 0 & 4 \\ 0 & 0\end{array}$


Table A-6 (continued)

QUARTERLY MEANS AND VARIANCE COMPONENTS

STREAM=1 (TRA effluent to cold waste ponds) (continued)

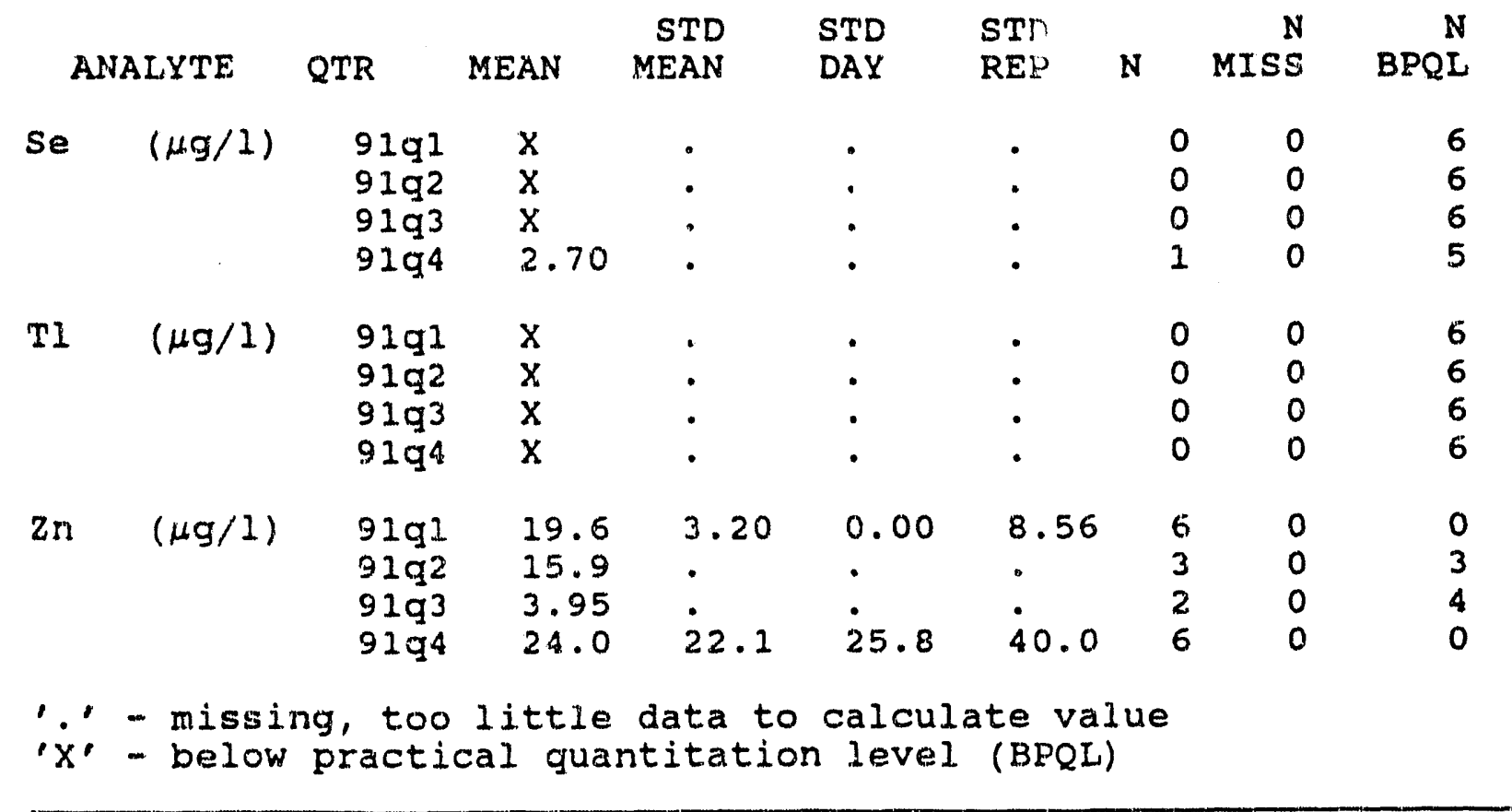




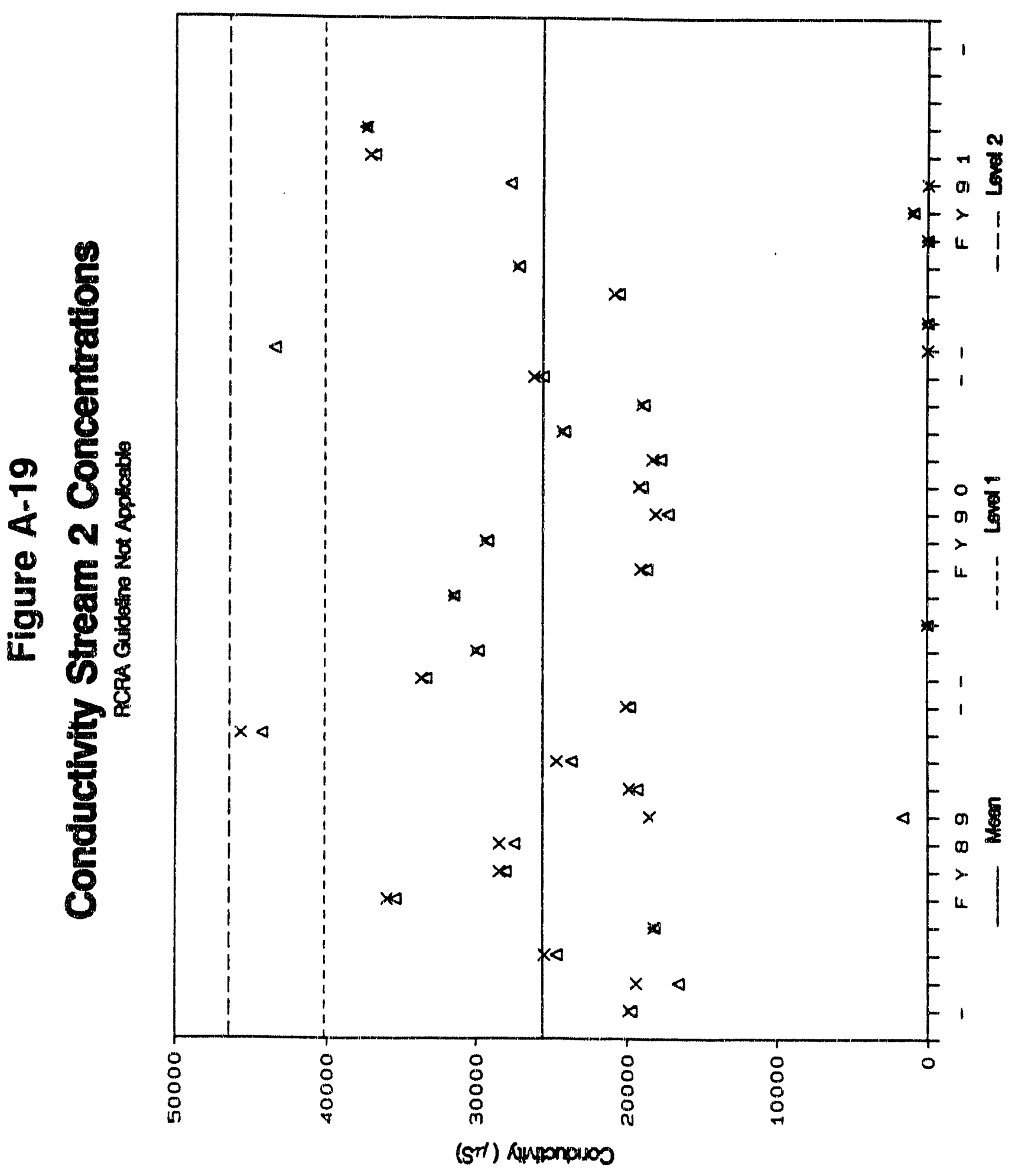




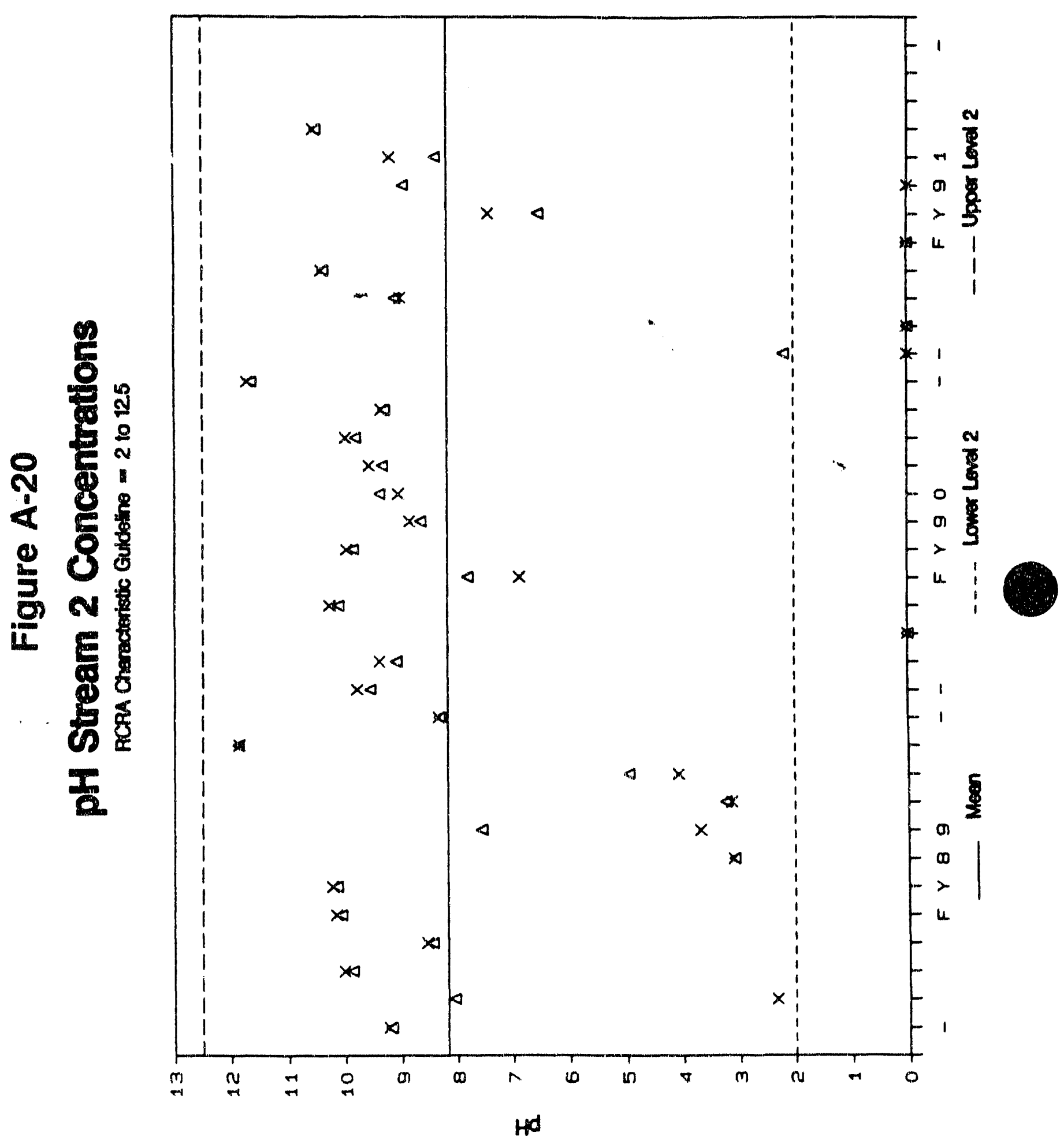




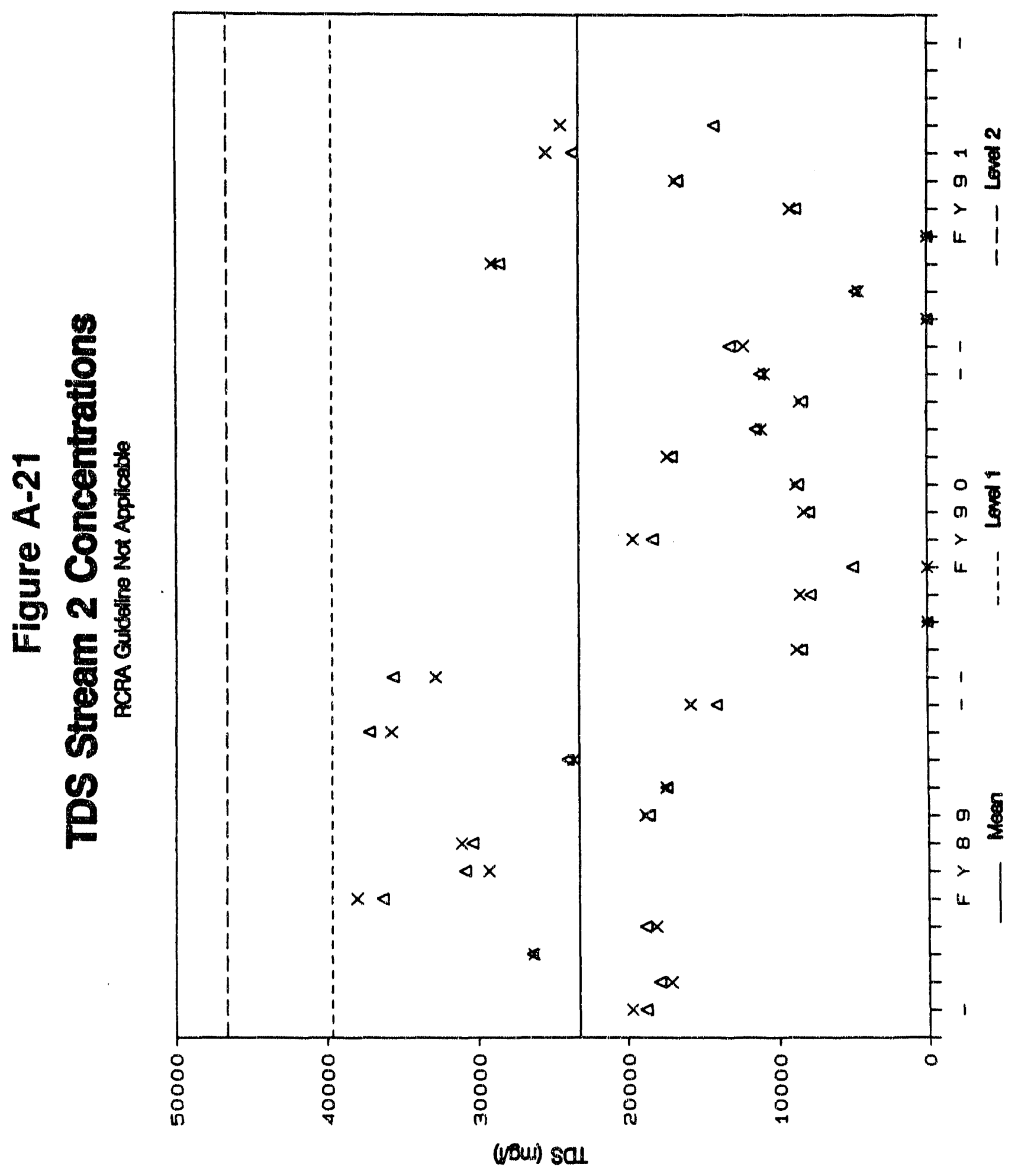




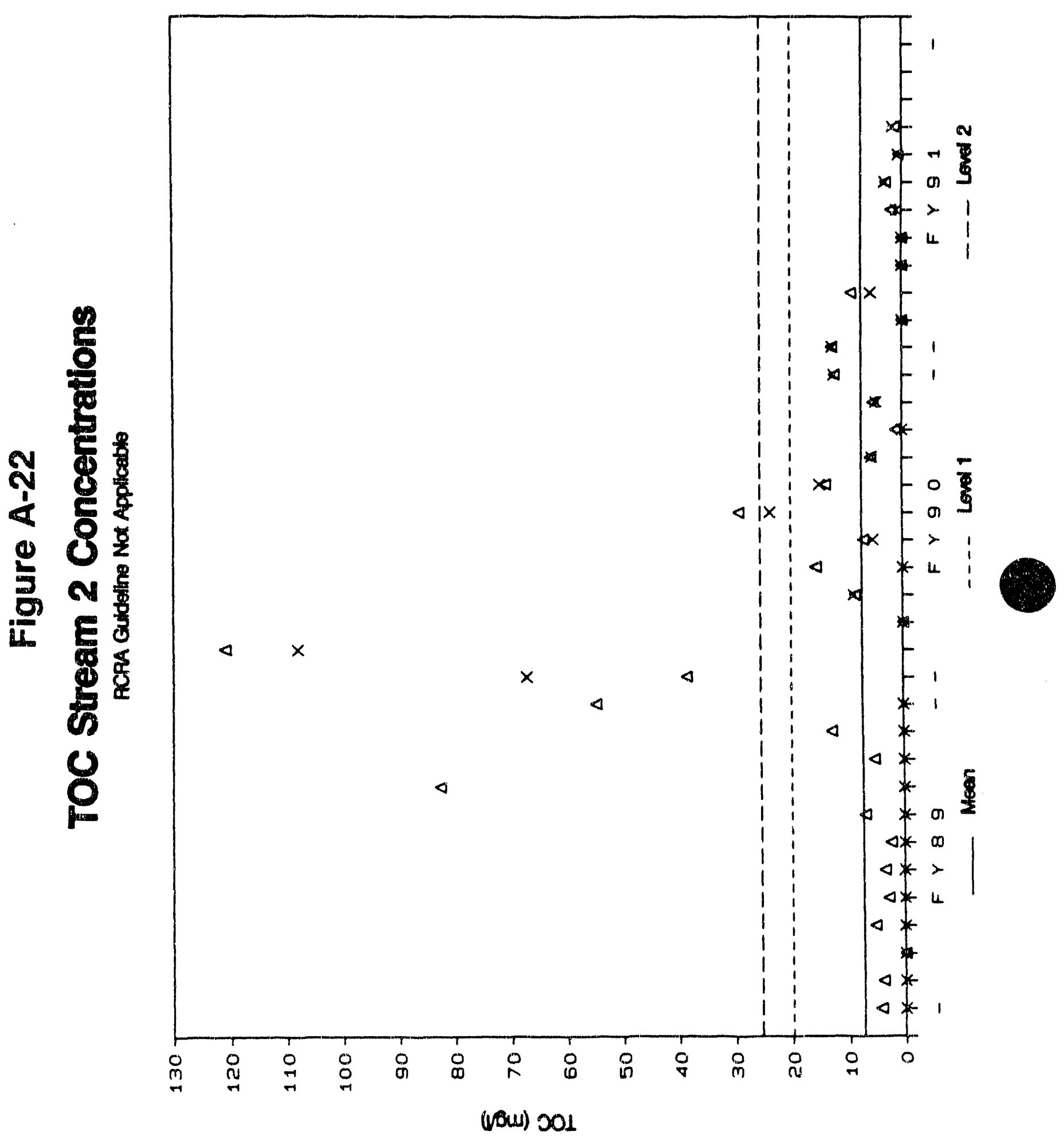




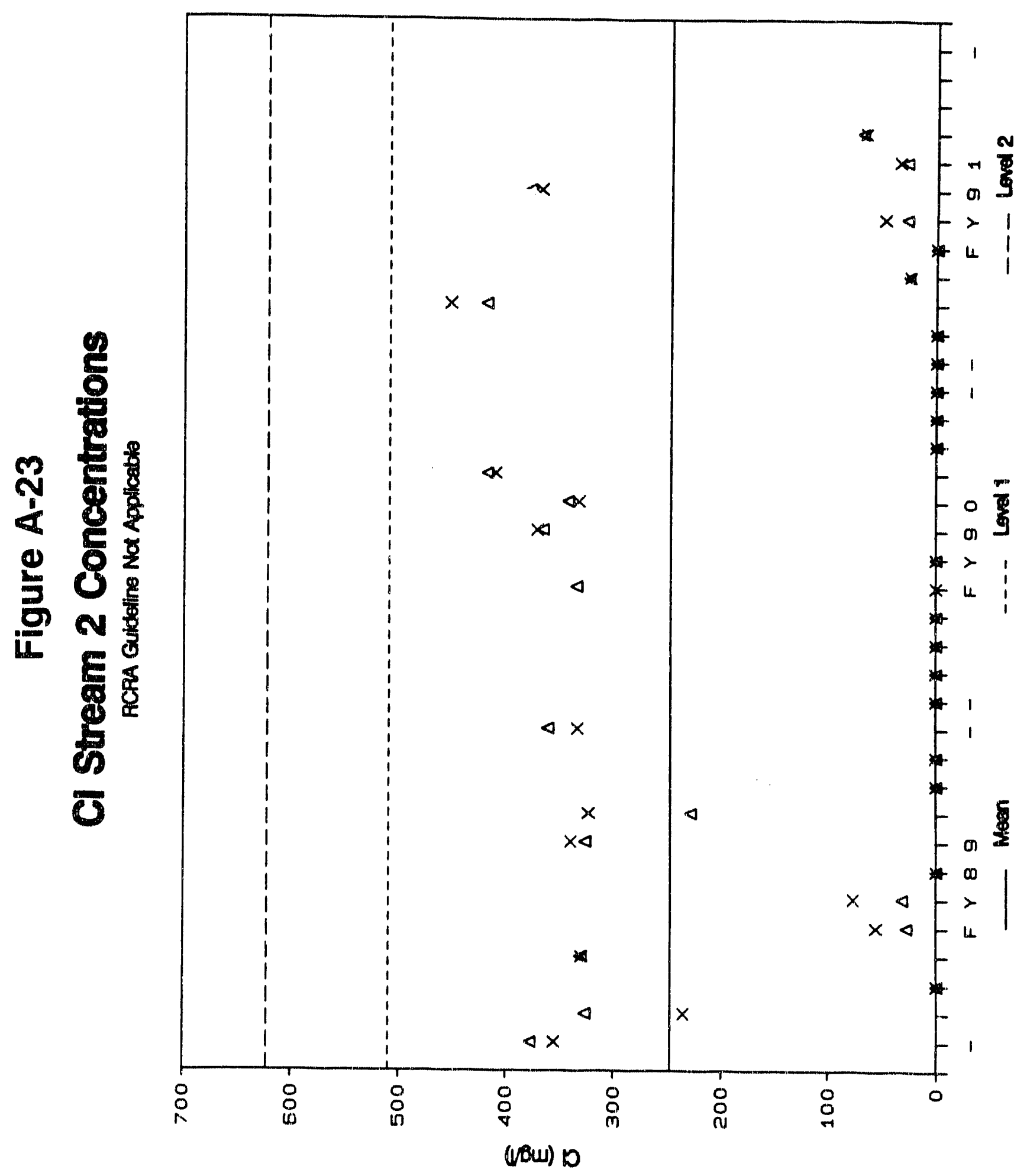




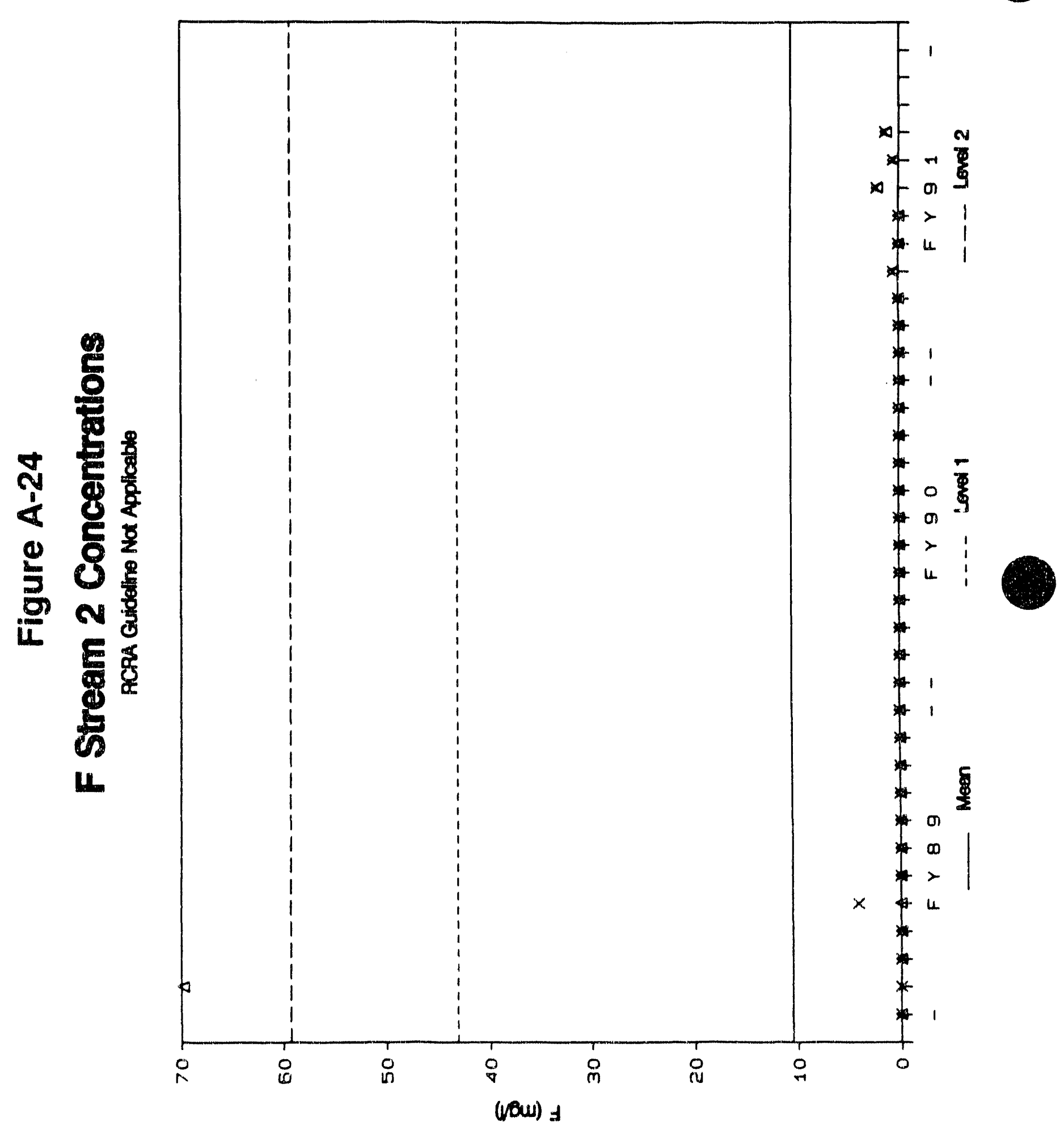




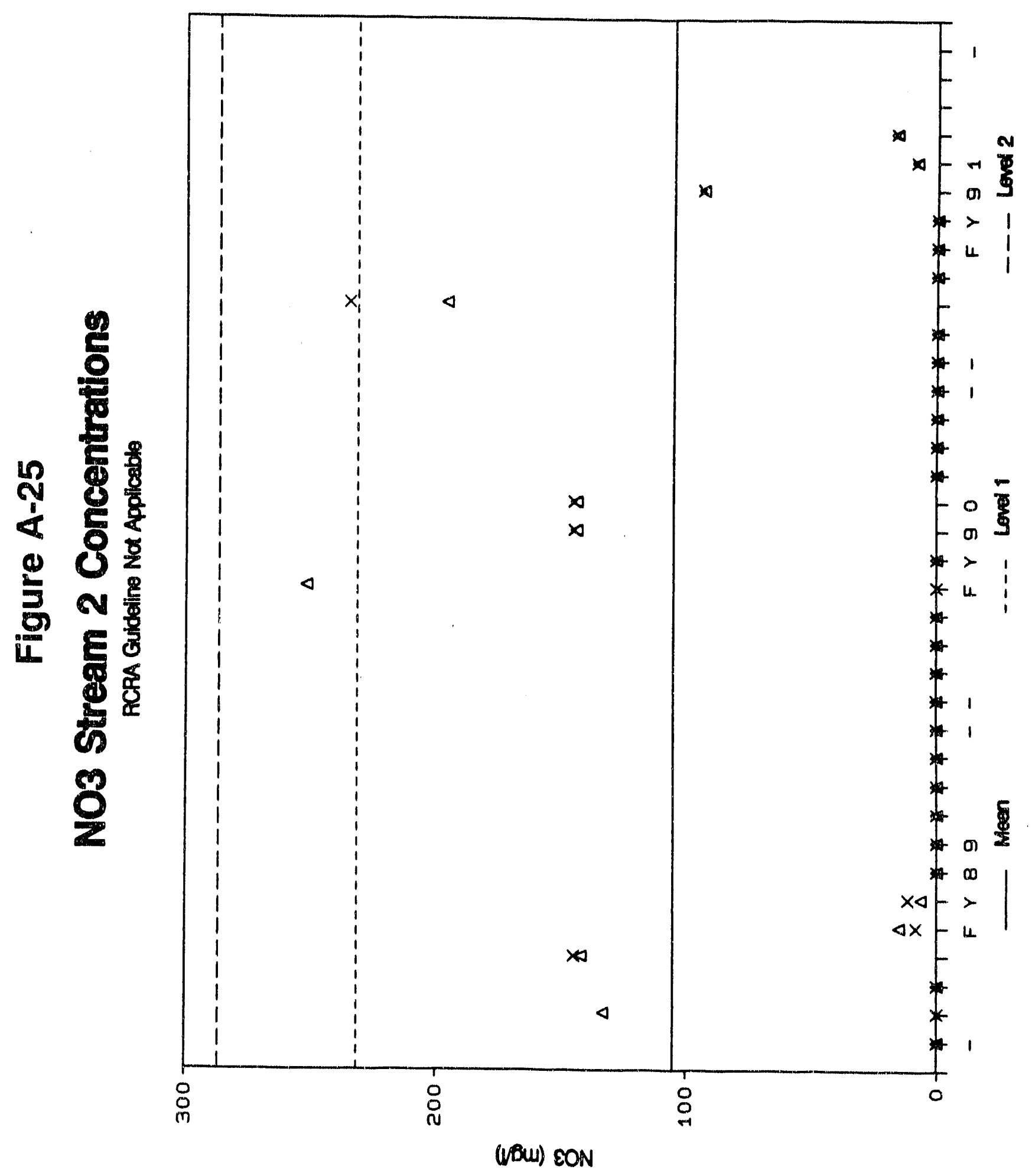




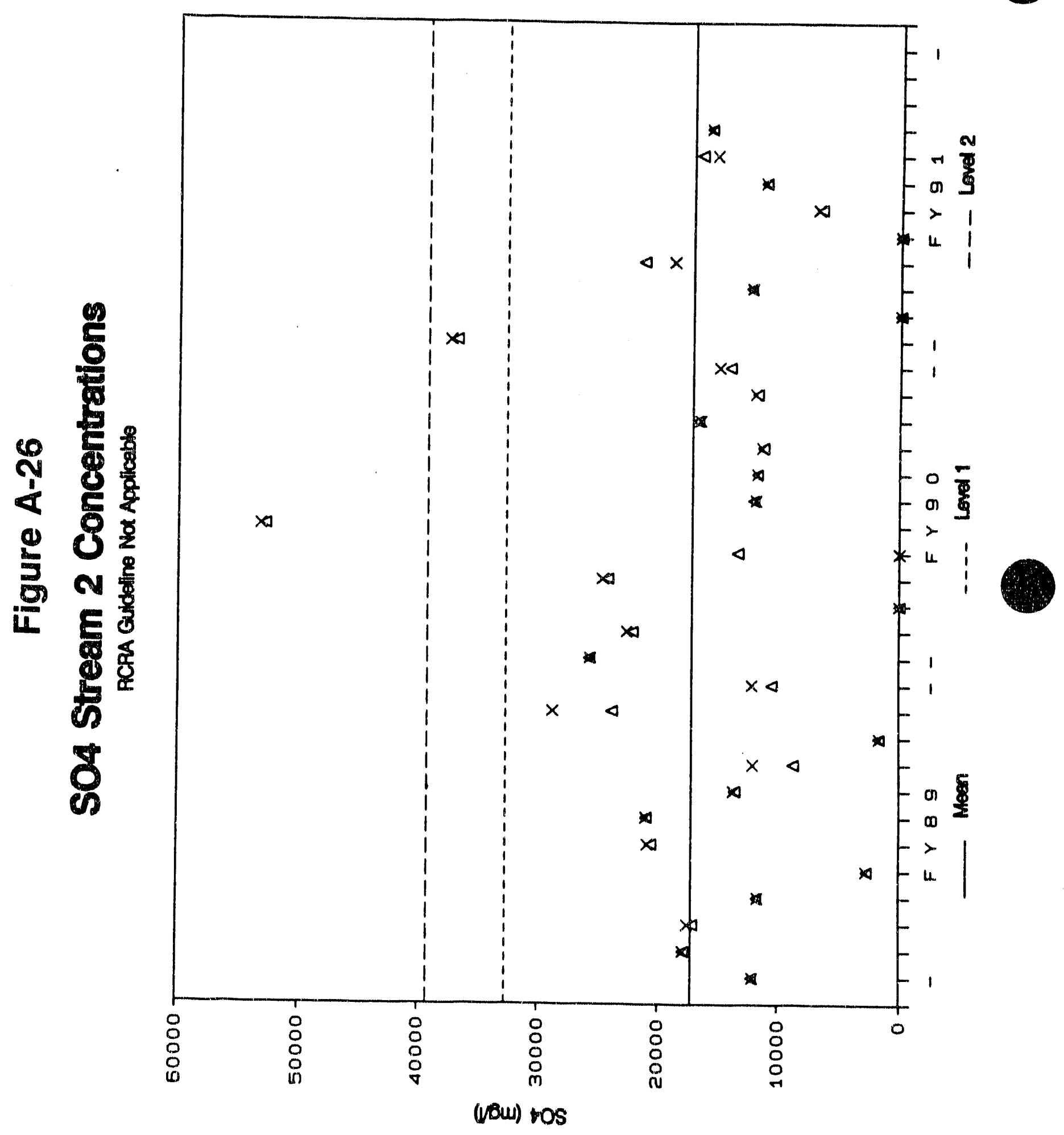




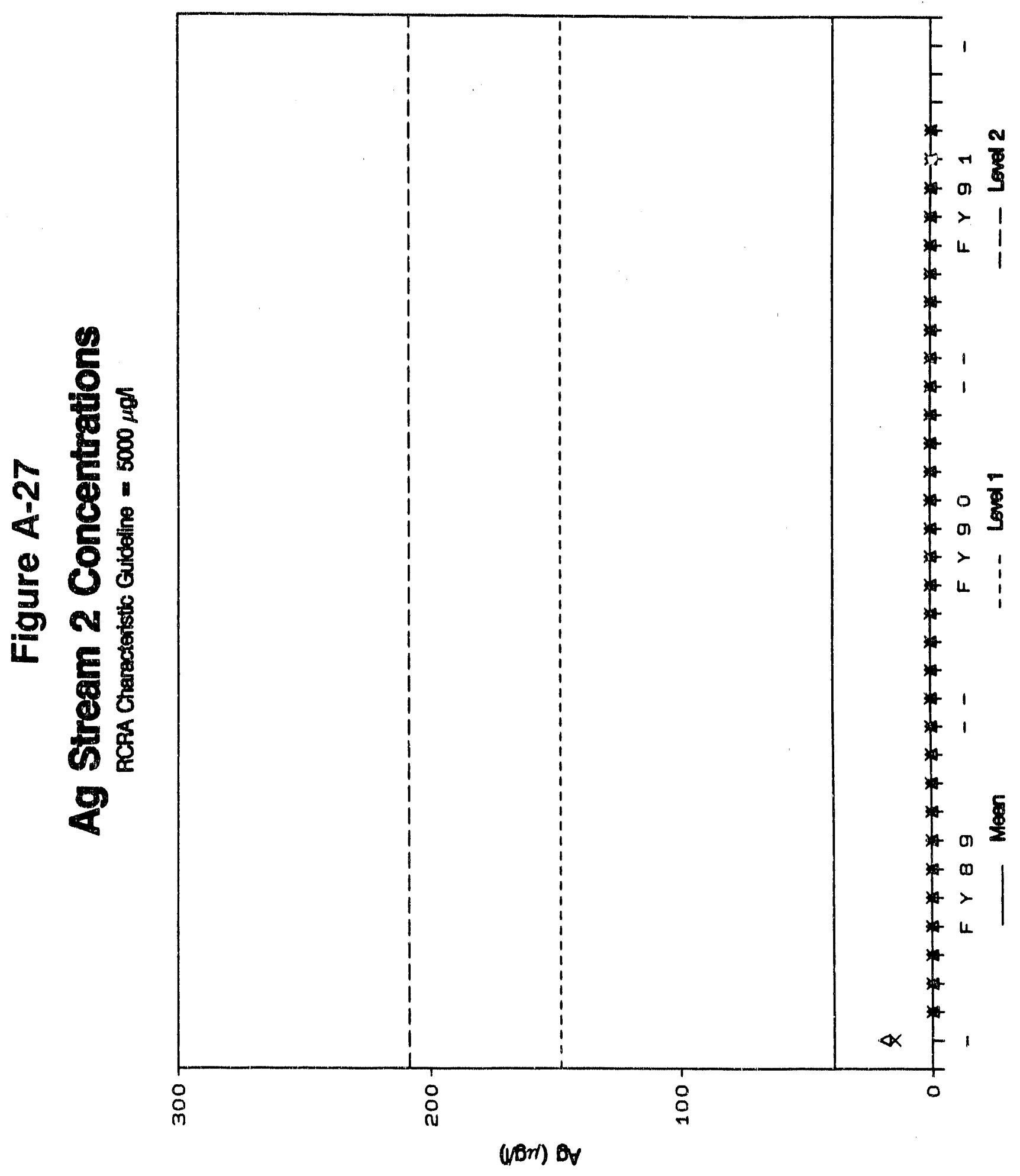




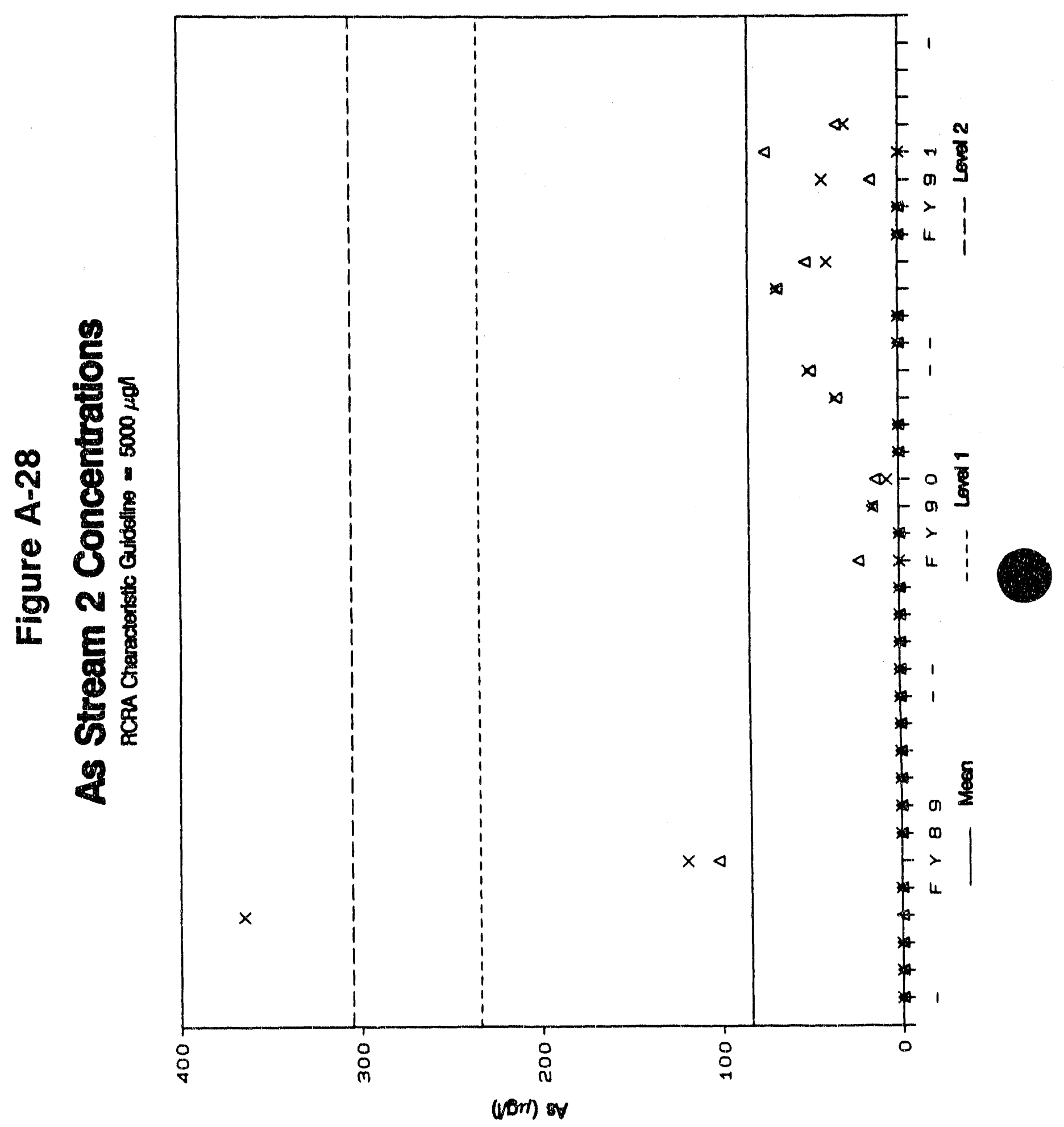




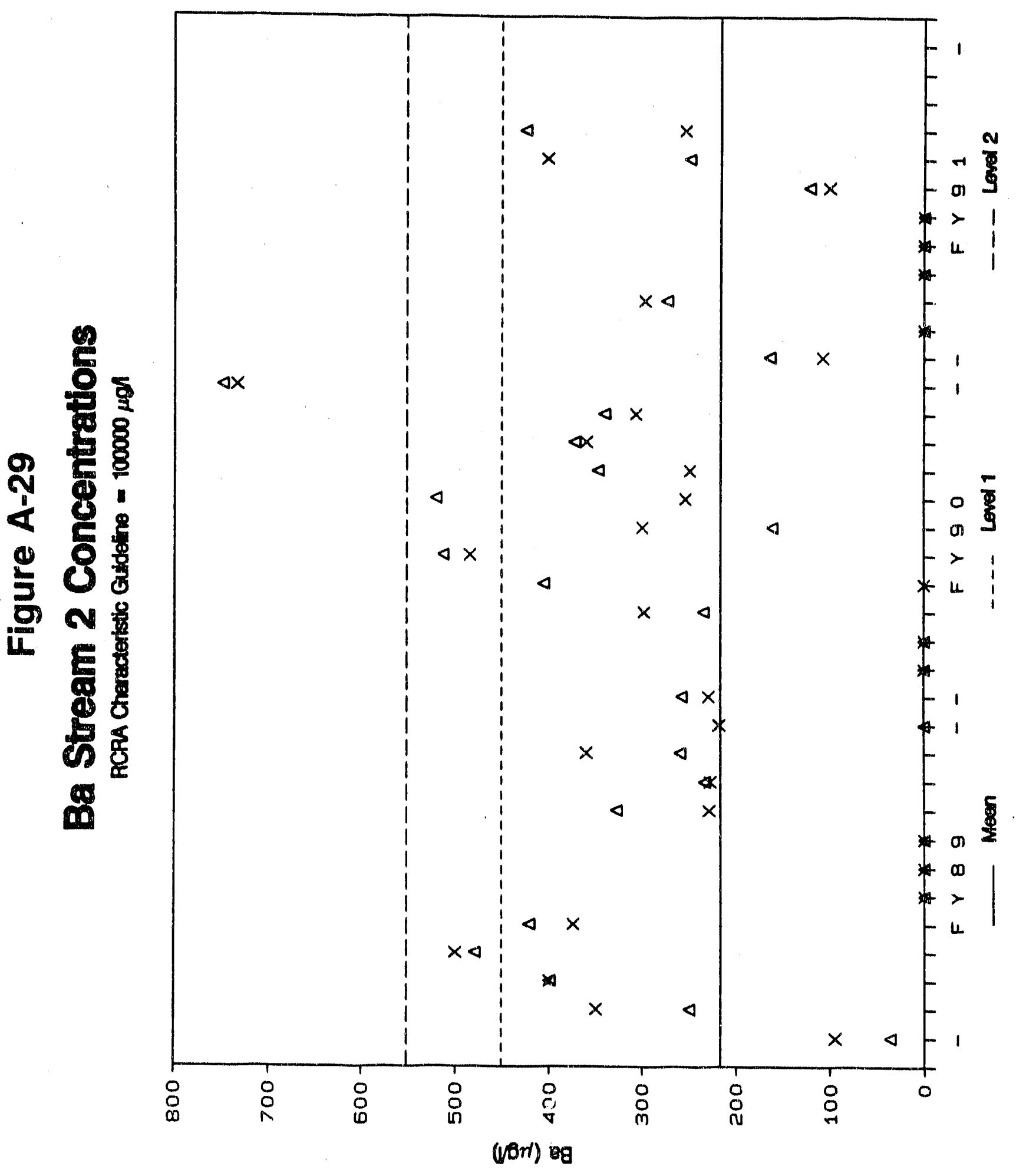




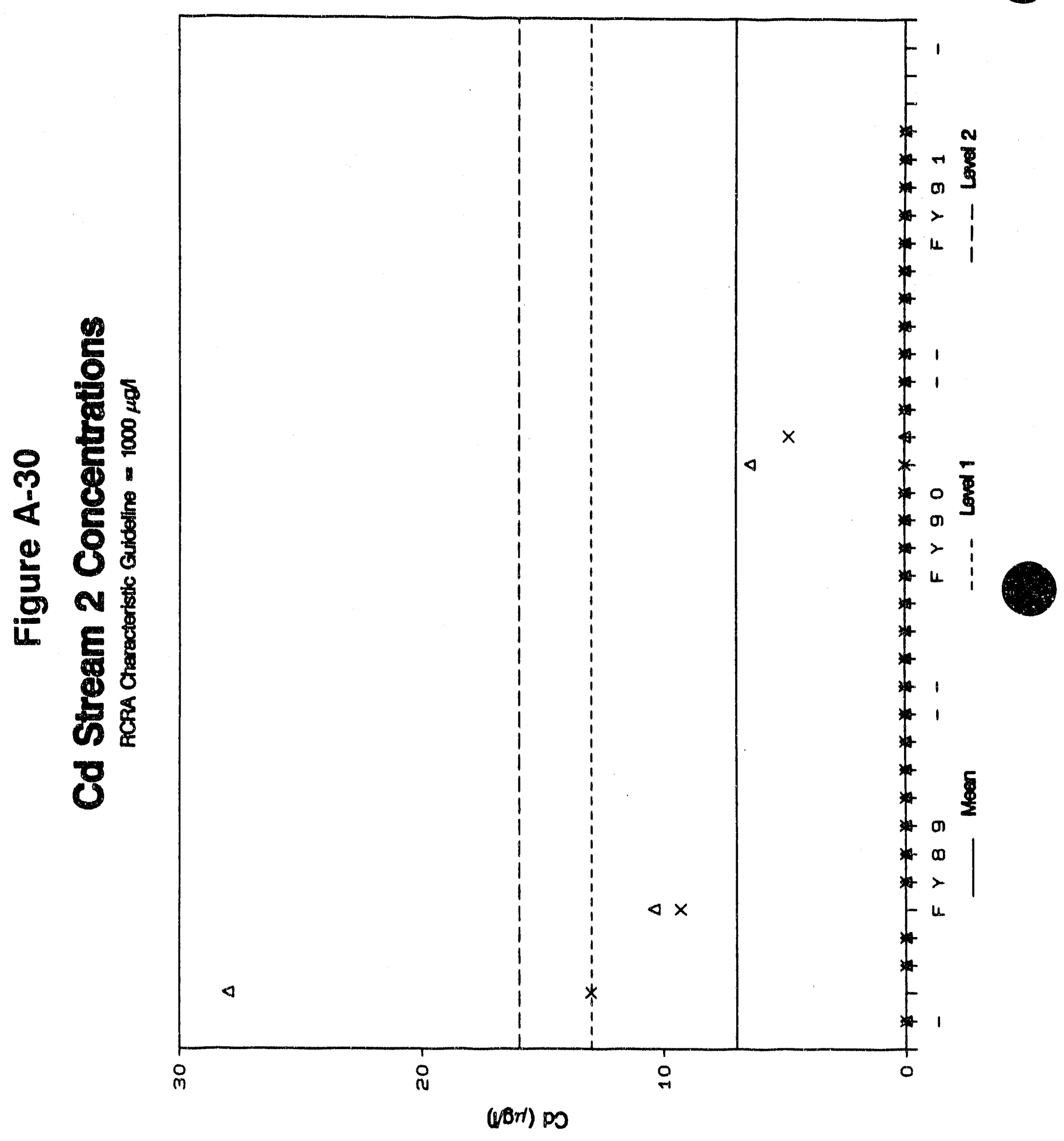




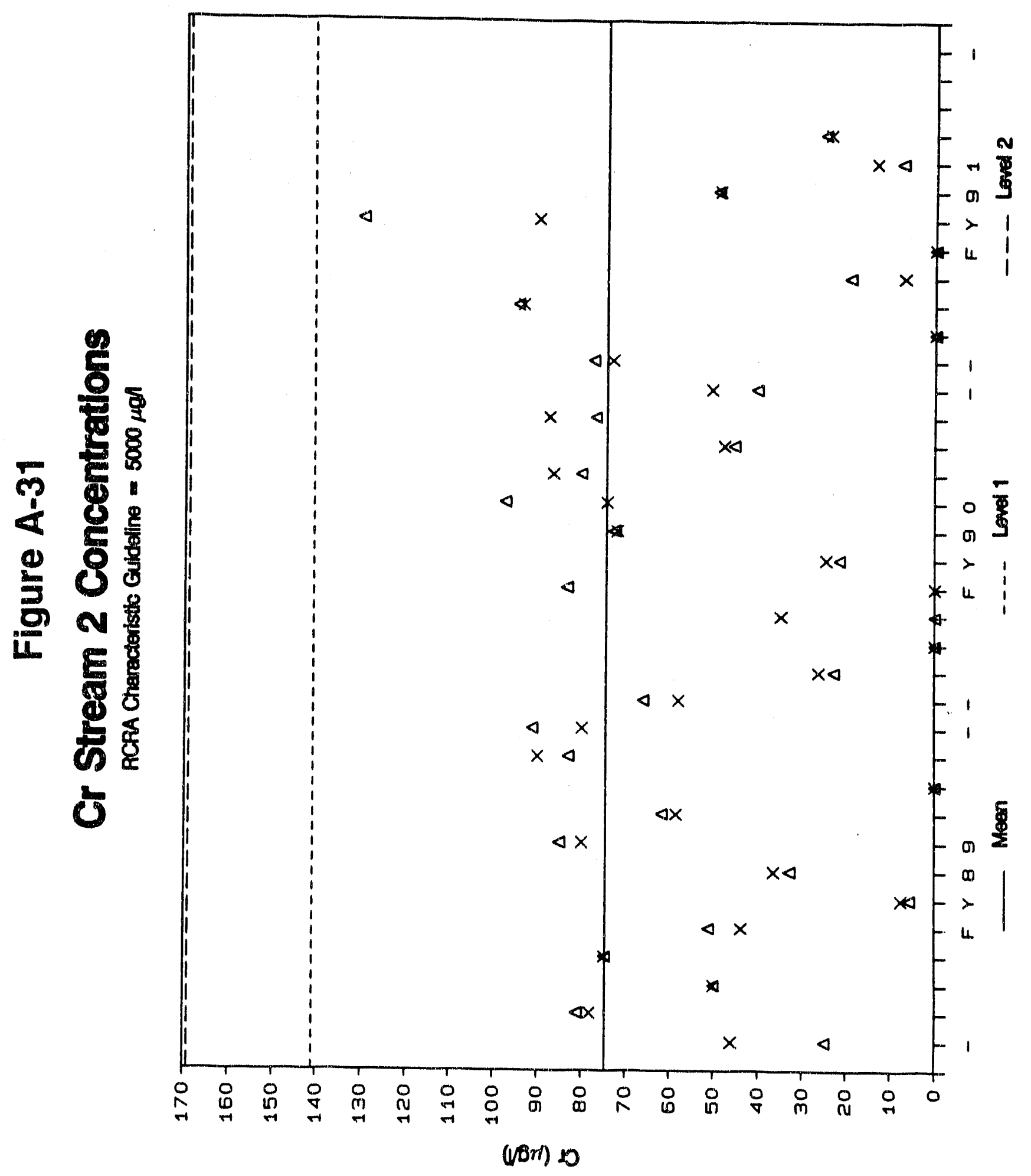




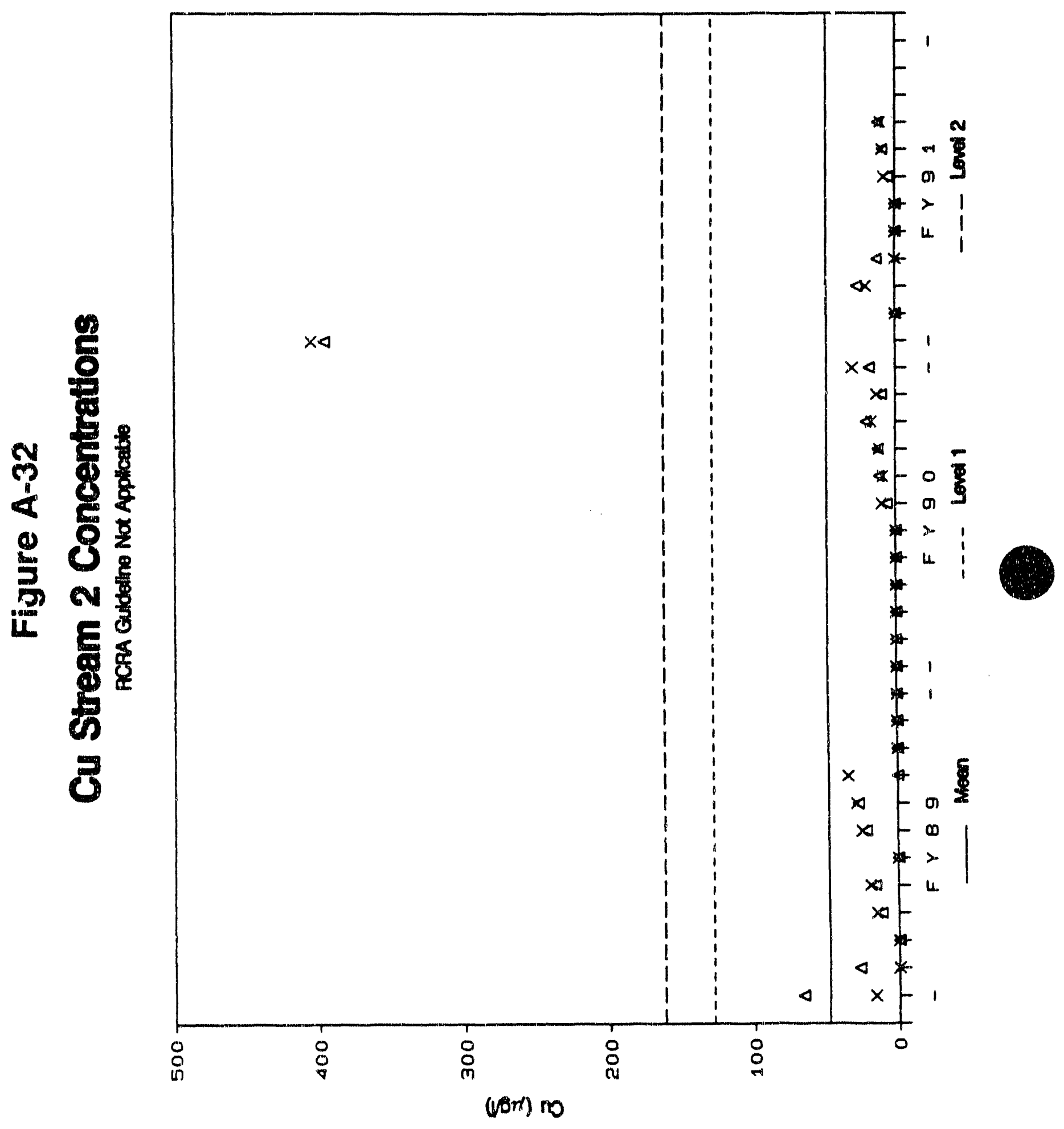




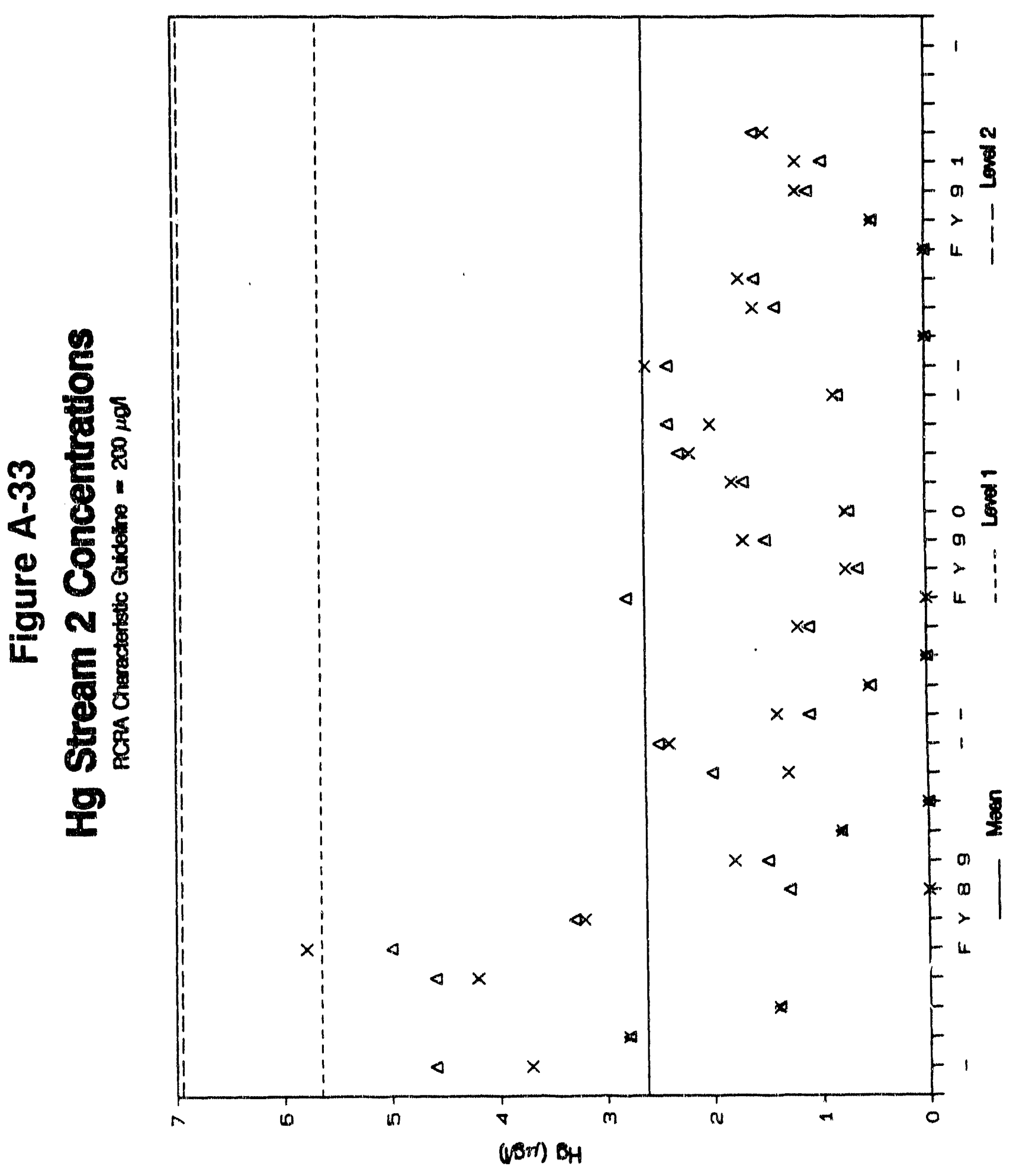




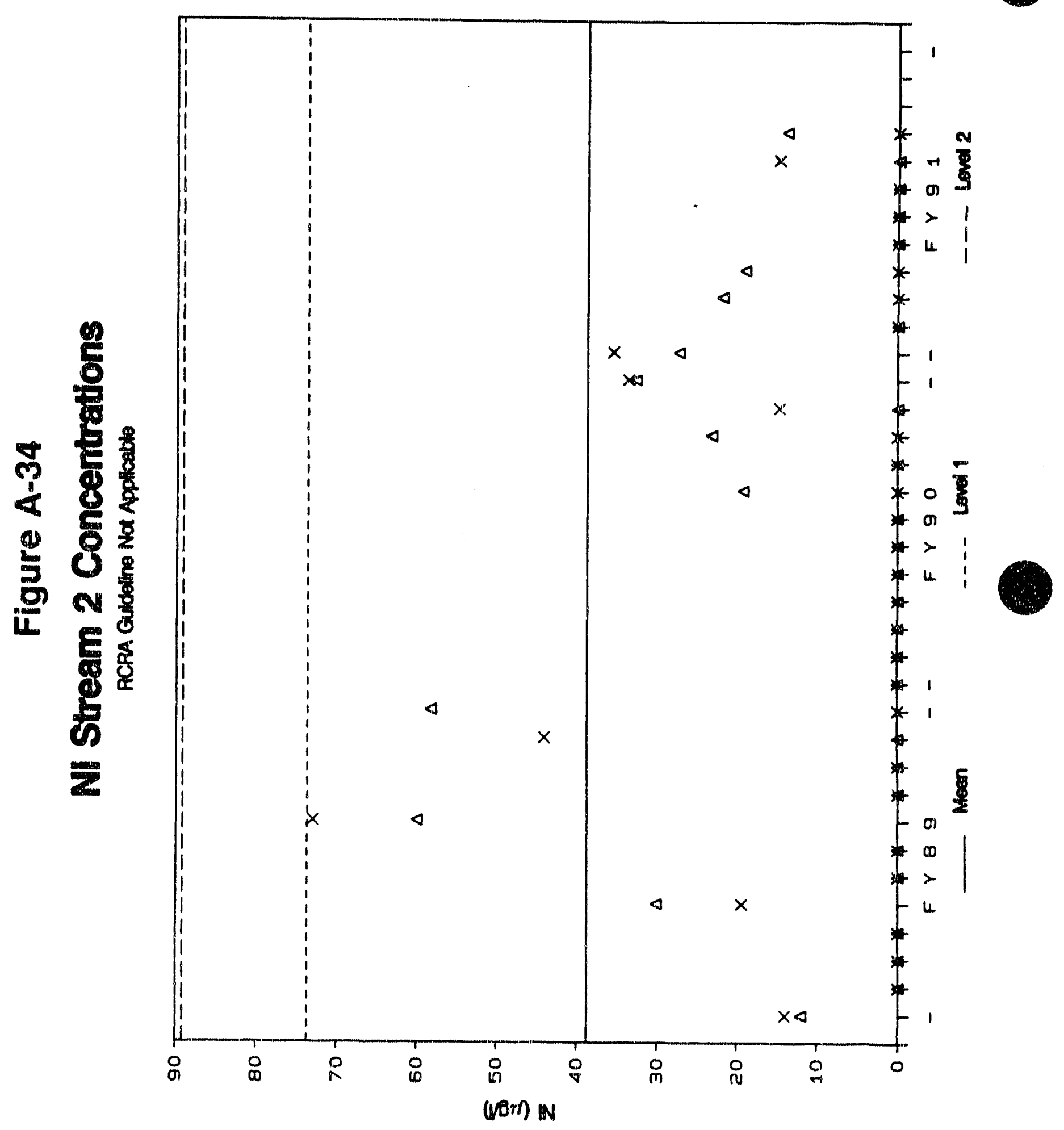




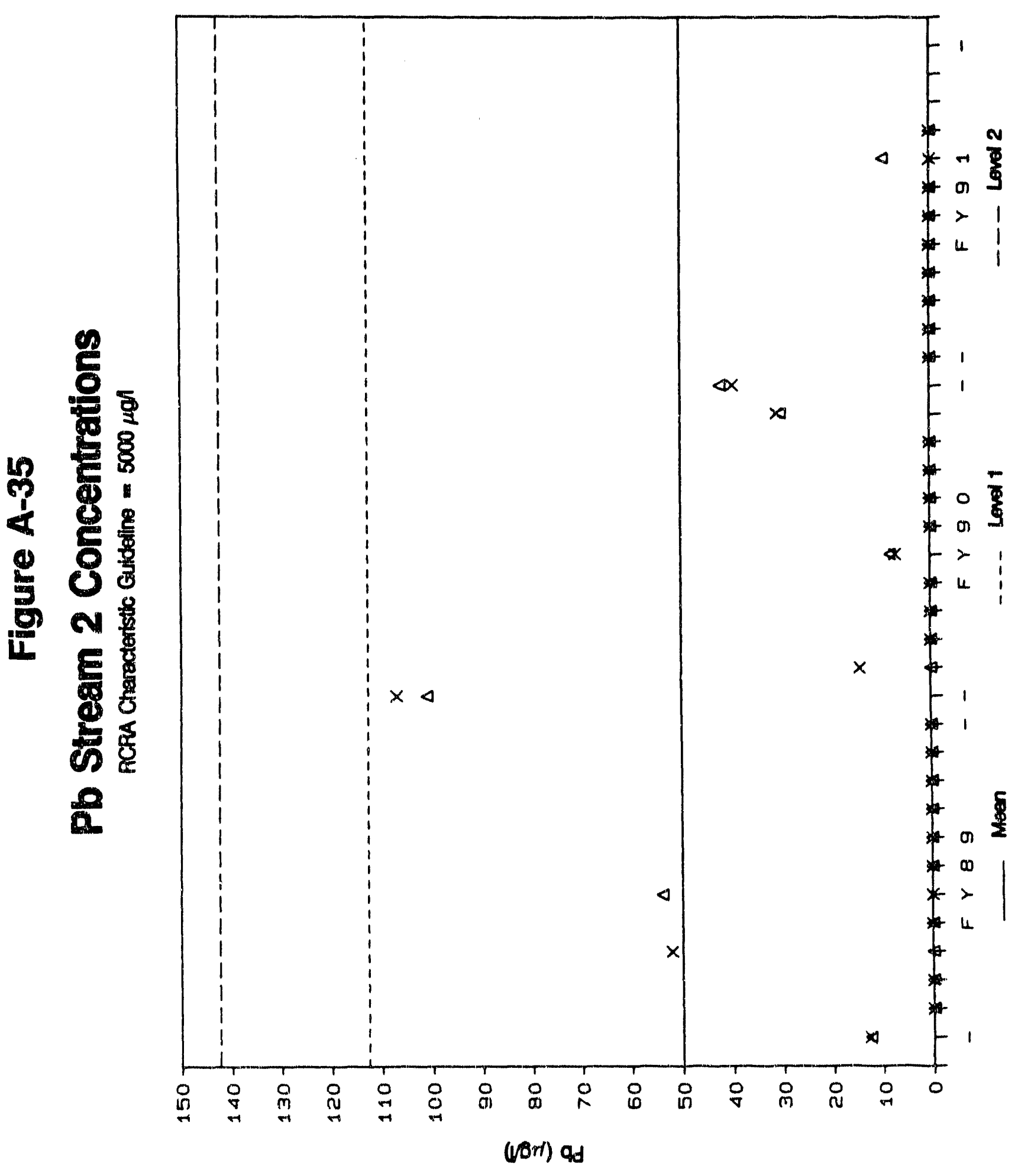




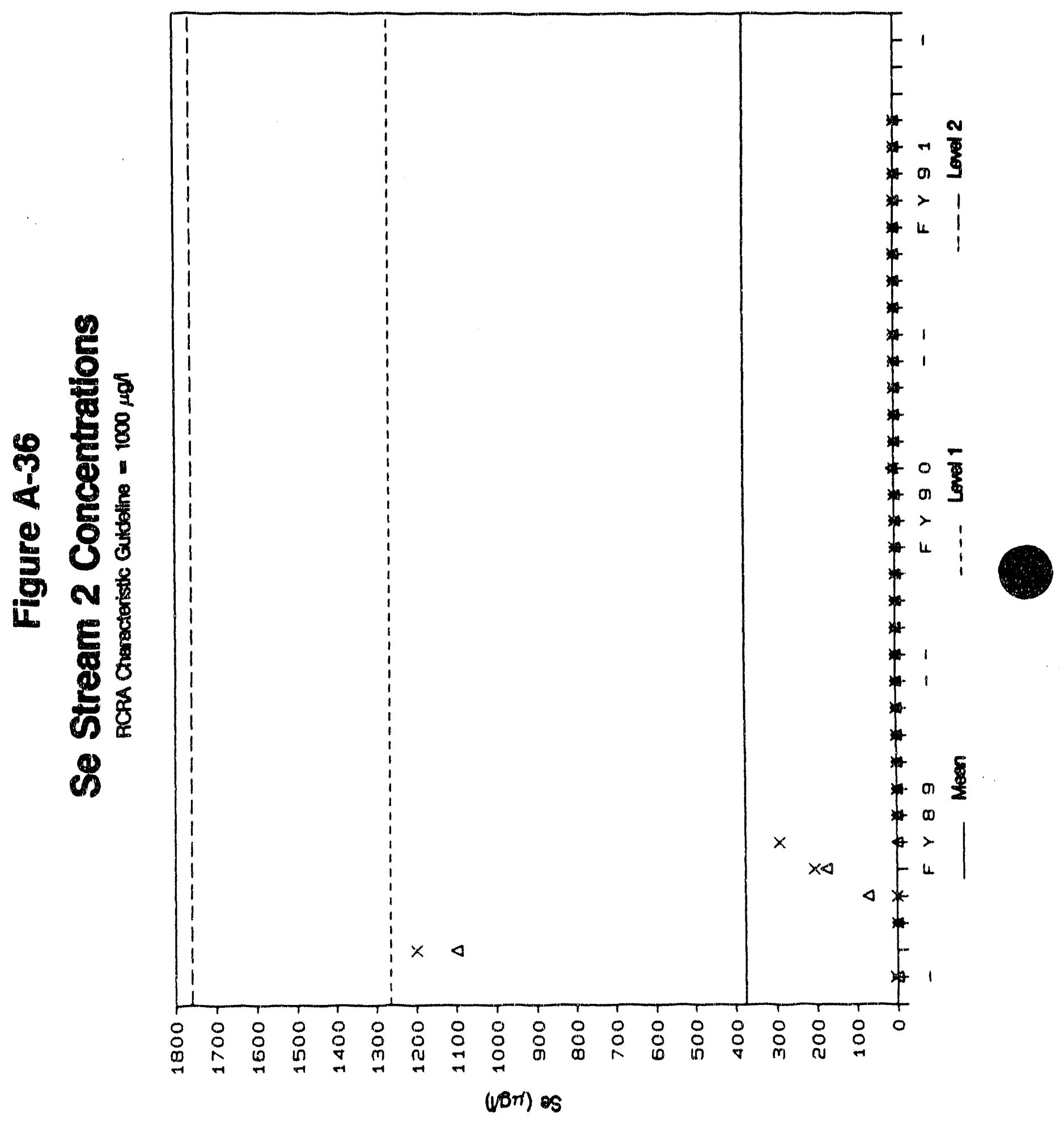




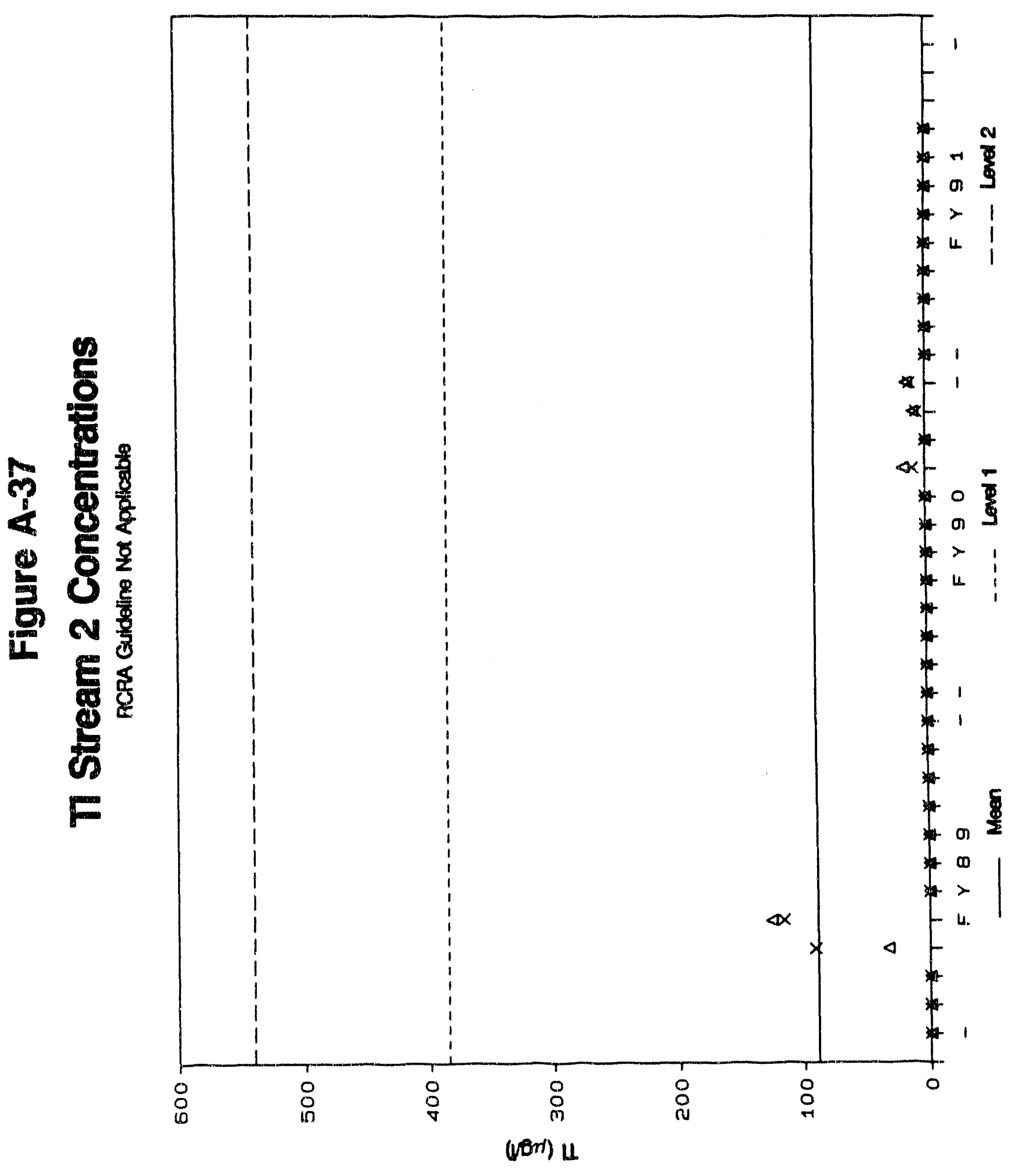




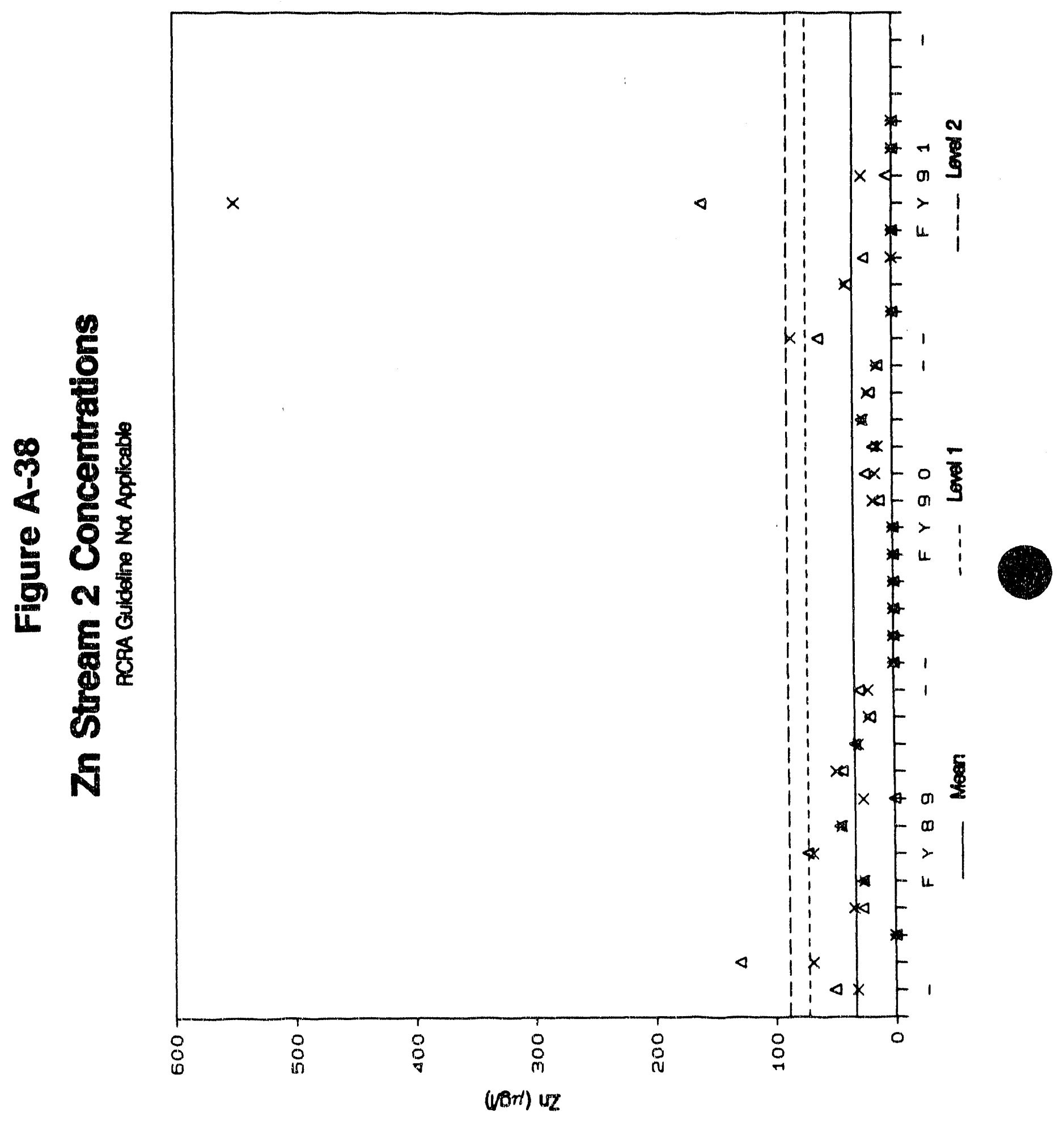




\section{Table A-7}

FY91 ENVIRONMENTAL MONITORING DATA

- STREAM=2 (TRA effluent to chemical leaching pond) QUARTER=91q1 MONTH REP $\begin{array}{llllllll}\text { Cond } & (\mu S) & \mathrm{TDS} & \mathrm{TOC} & \mathrm{Cl} & \mathrm{F} & \mathrm{NO3} & \mathrm{PO4} \\ (\mathrm{mg} / \mathrm{l}) & (\mathrm{mg} / \mathrm{l}) & (\mathrm{mg} / \mathrm{l}) & (\mathrm{mg} / \mathrm{l}) & (\mathrm{mg} / \mathrm{l}) & (\mathrm{mg} / \mathrm{l})\end{array}$

$\begin{array}{lrrrrlllll}\text { Oct } & 1 & 43500 & 2.18 & 13200 & 12.7 & \mathrm{X} & \mathrm{X} & \mathrm{X} & \mathrm{X} \\ \text { Oct } & 2 & \mathrm{X} & \mathrm{X} & 12300 & 12.6 & \mathrm{X} & \mathrm{X} & \mathrm{X} & \mathrm{X} \\ \text { Nov } & 1 & \mathrm{X} & \mathrm{X} & \mathrm{X} & \mathrm{X} & \mathrm{X} & \mathrm{X} & \mathrm{X} & \mathrm{X} \\ \text { Nov } & 2 & \mathrm{X} & \mathrm{X} & \mathrm{X} & \mathrm{X} & \mathrm{X} & \mathrm{X} & \mathrm{X} & \mathrm{X} \\ \text { Dec } & 1 & 20600 & 9.10 & 4850 & 9.10 & 419 & \mathrm{X} & 196 & \mathrm{X} \\ \text { Dec } & 2 & 20900 & 9.01 & 4640 & 5.60 & 453 & \mathrm{X} & 235 & \mathrm{X}\end{array}$

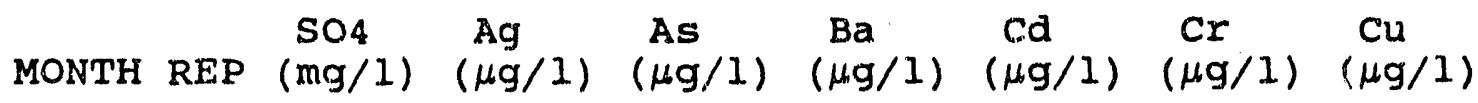

$\begin{array}{lrrllllll}\text { Oct } & 1 & 36900 & X & X & 165 & X & 77.4 & 396 \\ \text { Oct } & 2 & 37400 & X & X & 108 & X & 73.1 & 405 \\ \text { Nov } & 1 & X & X & X & X & X & X & X \\ \text { Nov } & 2 & X & X & X & X & X & X & X \\ \text { Dec } & 1 & 12400 & X & 68.0 & 274 & X & 94.7 & 27.3 \\ \text { Dec } & 2 & 12300 & X & 68.0 & 297 & X & 93.6 & 21.3\end{array}$

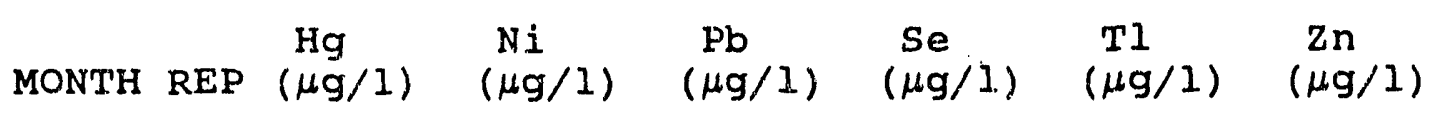

$\begin{array}{llllllll}\text { Oct } & 1 & 2.40 & 27.3 & X & X & X & 62.2 \\ \text { Oct } & 2 & 2.60 & 35.5 & X & X & X & 85.0 \\ \text { Nov } & 1 & X & X & X & X & X & X \\ \text { Nov } & 2 & X & X & X & X & X & X \\ \text { Dec } & 1 & 1.40 & 21.9 & X & X & X & 38.3 \\ \text { Dec } & 2 & 1.60 & X & X & X & X & 39.2\end{array}$

'.' - missing

' $X$ ' - below practical quantitation level 
FY91 ENVIRONMENTAL MONITORING DATA

- STREAM=2 (TRA effluent to chemical leaching pond) QUARTER=91q2 $\begin{array}{llllllll}\text { Cond } & \text { TDS } & \text { TOC } & \mathrm{Cl} & \mathrm{F} & \mathrm{NO} 3 & \mathrm{PO}\end{array}$ $\begin{array}{llllllll}(\mu S) & \mathrm{pH} & (\mathrm{mg} / \mathrm{l}) & (\mathrm{mg} / \mathrm{l}) & (\mathrm{mg} / \mathrm{l}) & (\mathrm{mg} / \mathrm{l}) & (\mathrm{mg} / \mathrm{l}) & (\mathrm{mg} / \mathrm{l})\end{array}$

$\begin{array}{lrrrrlllll}\text { Jan } & 1 & 27200 & 10.38 & 28500 & X & 25.8 & 0.50 & X & X \\ \text { Jan } & 2 & 27300 & 10.41 & 29100 & X & 24.5 & 0.50 & X & X \\ \text { Feb } & 1 & X & X & X & X & X & X & X & X \\ \text { Feb } & 2 & X & X & X & X & X & X & X & X \\ \text { Mar } & 1 & 981 & 6.54 & 8840 & 2.00 & 28.0 & X & X & 0.12 \\ \text { Mar } & 2 & 992 & 7.43 & 9230 & 1.00 & 48.0 & X & X & 0.12\end{array}$

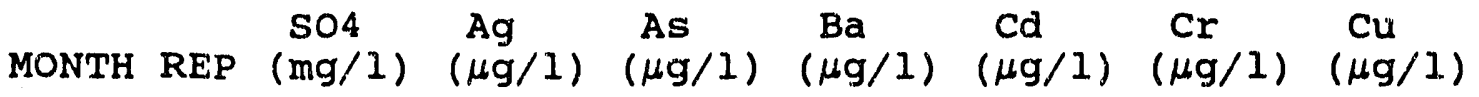

$\begin{array}{lrrrlllll}\text { Jan } & 1 & 21400 & X & 52.0 & X & X & 19.3 & 13.2 \\ \text { Jan } & 2 & 18800 & X & 40.0 & X & X & 6.90 & X \\ \text { Feb } & 1 & X & X & X & X & X & X & X \\ \text { Feb } & 2 & X & X & X & X & X & X & X \\ \text { Mar } & 1 & 6630 & X & X & X & X & 130 & X \\ \text { Mar } & 2 & 6900 & X & X & X & X & 90.0 & X\end{array}$

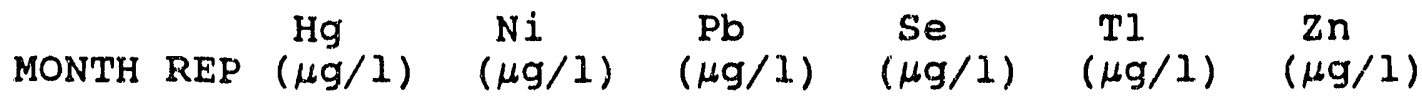

$\begin{array}{llllllll}\text { Jan } & 1 & 1.59 & 19.0 & X & X & X & 23.3 \\ \text { Jan } & 2 & 1.73 & X & X & X & X & X \\ \text { Feb } & 1 & X & X & X & X & X & X \\ \text { Feb } & 2 & X & X & X & X & X & X \\ \text { Mar } & 1 & 0.50 & X & X & X & X & 160 \\ \text { Mar } & 2 & 0.50 & X & X & X & X & 550\end{array}$

$\therefore$ - missing

' $X$ ' - below practical quantitation level 


\section{Table A-9}

FY91 ENVIRONMENTAL MONITORING DATA

- STREAM=2 (TRA effluent to chemical leaching pond) QUARTER=91q3 -

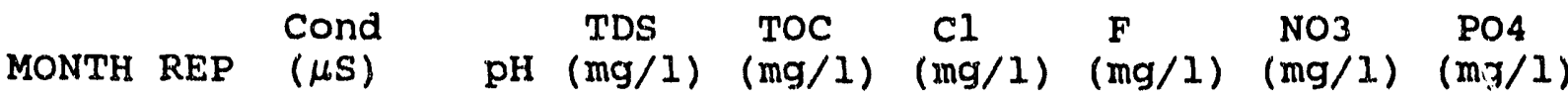

$\begin{array}{ccrrrrrrrr}\text { Apr } & 1 & 27800 & 8.96 & 16600 & 2.98 & 378 & 2.05 & 93.2 & 0.75 \\ \text { Apr } & 2 & X & X & 16800 & 3.12 & 368 & 2.11 & 93.4 & 0.87 \\ \text { May } & 1 & 36900 & 8.38 & 23600 & 0.66 & 28.8 & 0.54 & 8.30 & 0.21 \\ \text { May } & 2 & 37200 & 9.19 & 25400 & 0.70 & 35.0 & 0.52 & 8.48 & 0.20 \\ \text { Jun } & 1 & 37500 & 10.50 & 14200 & 1.40 & 69.0 & 1.26 & 16.5 & 0.69 \\ \text { Jun } & 2 & 37400 & 10.54 & 24400 & 1.78 & 66.1 & 1.30 & 16.5 & 0.66\end{array}$

$\begin{array}{cccccccc} & \mathrm{SO} 4 & \mathrm{Ag} & \mathrm{As} & \mathrm{Ba} & \mathrm{Cd} & \mathrm{Cr} & \mathrm{Cu} \\ \operatorname{MONTH} \operatorname{REP} & (\mathrm{mg} / 1) & (\mu \mathrm{g} / 1) & (\mu \mathrm{g} / 1) & (\mu \mathrm{g} / 1) & (\mu \mathrm{g} / 1) & (\mu \mathrm{g} / 1) & (\mu \mathrm{g} / 1)\end{array}$

$\begin{array}{lllllllll}\text { Apr } & 1 & 11200 & X & 15.9 & 122 & X & 49.3 & 5.30 \\ \text { Apr } & 2 & 11200 & X & 42.7 & 101 & X & 48.9 & 8.10 \\ \text { May } & 1 & 16600 & X & 74.4 & 249 & X & 7.60 & 9.20 \\ \text { May } & 2 & 15300 & X & X & 402 & X & 13.5 & 9.60 \\ \text { Jun } & 1 & 15800 & X & 34.9 & 425 & X & 25.2 & 12.5 \\ \text { Jun } & 2 & 15800 & X & 30.3 & 254 & X & 24.0 & 10.8\end{array}$

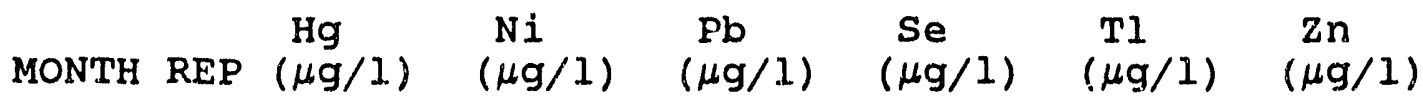

$\begin{array}{llllllll}\text { Apr } & 1 & 1.10 & X & X & X & X & 4.90 \\ \text { Apr } & 2 & 1.20 & X & X & X & X & 25.5 \\ \text { May } & 1 & 0.97 & X & 9.40 & X & X & X \\ \text { May } & 2 & 1.20 & 14.8 & X & X & X & X \\ \text { Jun } & 1 & 1.60 & 13.8 & X & X & X & X \\ \text { Jun } & 2 & 1.50 & X & X & X & X & X\end{array}$

'.' - missing

' $X$ ' - below practical quantitation level 


\section{Table A-10}

\section{SUMMARY STATISTICS}

STREAM=2 (TRA effluent to chemical leaching pond)

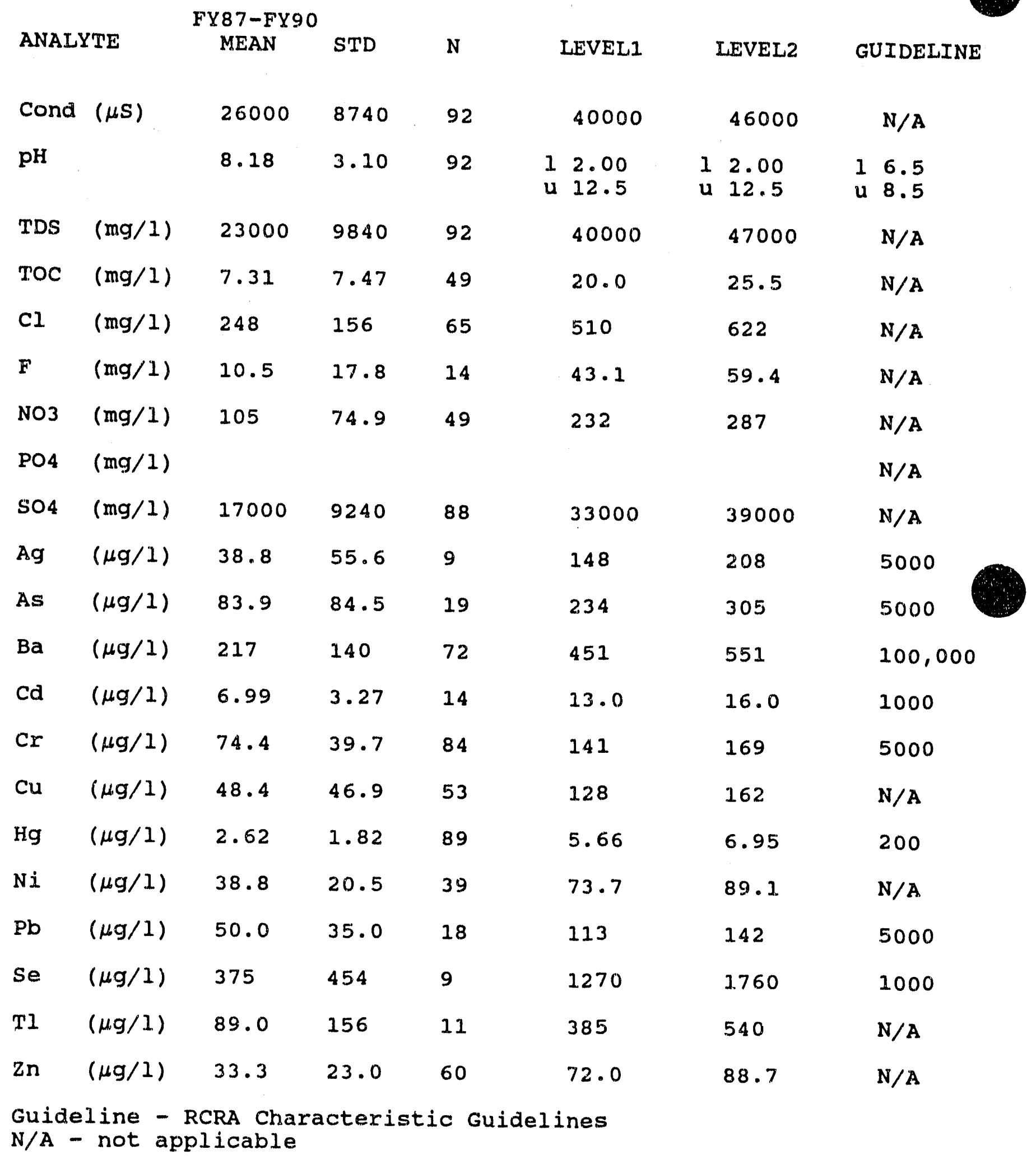




\section{Table A-11}

QUARTERLY MEANS AND VARIANCE COMPONENTS

- - STREAM=2 (TRA effluent to chemical leaching pond)

$\begin{array}{llllll} & & \text { STD } & \text { STD } & \text { STD } & N\end{array}$ N

Cond $(\mu S)$

91q1 28000

$9192 \quad 14000$

$\begin{array}{ll}9193 & 35000 \\ 9194 & x\end{array}$

$\begin{array}{ccccc}13000 & 19000 & 50.3 & 4 & 3 \\ 2838 & 4729 & 158 & 5 & 1 \\ 0 & . & . & 0 & 0\end{array}$

BPQL

$\mathrm{pH}$

$9191: 6.76$

$91 q^{2}$

$91 \mathrm{q} 3$

8.69

i. 70

9.510 .62

$\dot{2} .39 \quad 0.45$

$\begin{array}{lll}3 & 3 & 0 \\ 4 & 2 & 0\end{array}$

9194

$\mathrm{X}$

0.980 .41

51

$\operatorname{TDS}(\mathrm{mg} / \mathrm{l})$

$91 q$

8748

4003

-

- 0

0
0
0
6
0
0
0
6

$91 \mathrm{q} 2$

$91 \mathrm{q} 3$

19000

$9883 \quad 14000$

462

2293

2613

332

$91 \mathrm{q}$

$\operatorname{TOC}(\mathrm{mg} / 1)$

$\begin{array}{ll}91 \mathrm{q1} & 10.0 \\ 91 \mathrm{q} 2 & 1.50 \\ 91 \mathrm{q3}^{3} & 1.77 \\ 91 \mathrm{q} 4 & \mathrm{X}\end{array}$

2.65

3.54

$0.69 \quad 1.19$

4229

$\begin{array}{lll}4 & 2 & 0 \\ 4 & 2 & 0 \\ 6 & 0 & 0 \\ 0 & 0 & 6\end{array}$

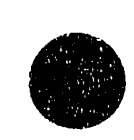

Tss (mg/I)

$91 q^{2}$

$91 q^{3}$

$91 \mathrm{q} 4$

32.0
$x^{595}$

:

1.75

4

0.17

2

4

0

Cl. $(\mathrm{mg} / \mathrm{l})$

$$
\begin{aligned}
& 9191 \\
& 9192 \\
& 91 q 3 \\
& 91 q 4
\end{aligned}
$$

436

436
31.6
157

6.42

$\begin{array}{rr}5.69 & 10.0 \\ 187 & 4.95\end{array}$

108

$\begin{array}{lll}2 & 2 & 2 \\ 4 & 2 & 0 \\ 6 & 0 & 0 \\ 0 & 0 & 6\end{array}$

$\mathrm{F}(\mathrm{mg} / \mathrm{l}) \quad 91 q 1 \mathrm{X}$

9192

$$
91 q^{3}
$$

$\mathrm{X}$

$91 q^{3}$
$91 q^{4}$

1.30

\begin{tabular}{|c|c|c|c|c|c|c|c|}
\hline $91 \mathrm{ql}$ & 216 & - & - & - & 2 & 2 & 2 \\
\hline $91 q^{2}$ & $X$ & - & - & . & 0 & 0 & 6 \\
\hline $91 q^{3}$ & 39.4 & 27.1 & 46.9 & 0.1 .2 & 6 & 0 & 0 \\
\hline $91 q^{4}$ & $\mathrm{X}$ & . & - & . & 0 & 0 & 6 \\
\hline
\end{tabular}

$\begin{array}{lll}\dot{0} & \dot{0} & \dot{0} \\ \dot{0.45} & \dot{0.77} & \dot{0.03} \\ & \dot{0} & \dot{ }\end{array}$

$\begin{array}{lll}0 & 2 & 4 \\ 2 & 0 & 4 \\ 6 & 0 & 0 \\ 0 & 0 & 6\end{array}$

No3 $(\mathrm{mg} / 1)$

$\mathrm{PO} 4(\mathrm{mg} / \mathrm{l}) \quad 91 \mathrm{ql}$

$\begin{array}{llllllll}91 q 1 & x & . & \cdot & . & 0 & 2 & 4 \\ 91 q 2 & 0.12 & \cdot & & \dot{0} & 2 & 0 & 4 \\ 91 q 3 & 0.56 & 0.18 & 0.32 & 0.05 & 6 & 0 & 0 \\ 91 q 4 & x & . & \cdot & . & 0 & 0 & 6\end{array}$

$\mathrm{SO} 4(\mathrm{mg} / \mathrm{I})$

$\begin{array}{rrrrrrrr}91 q 1 & 25000 & 12000 & 18000 & 255 & 4 & 2 & 0 \\ 91 q 2 & 13000 & 6666 & 9384 & 1275 & 4 & 2 & 0 \\ 91 q 3 & 14000 & 1559 & 2674 & 531 & 6 & 0 & 0 \\ 91 q 4 & X & . & . & . & 0 & 0 & 6\end{array}$




\section{Table A-11 (continued)}

\section{QUARTERLY MEANS AND VARIANCE COMPONENTS}

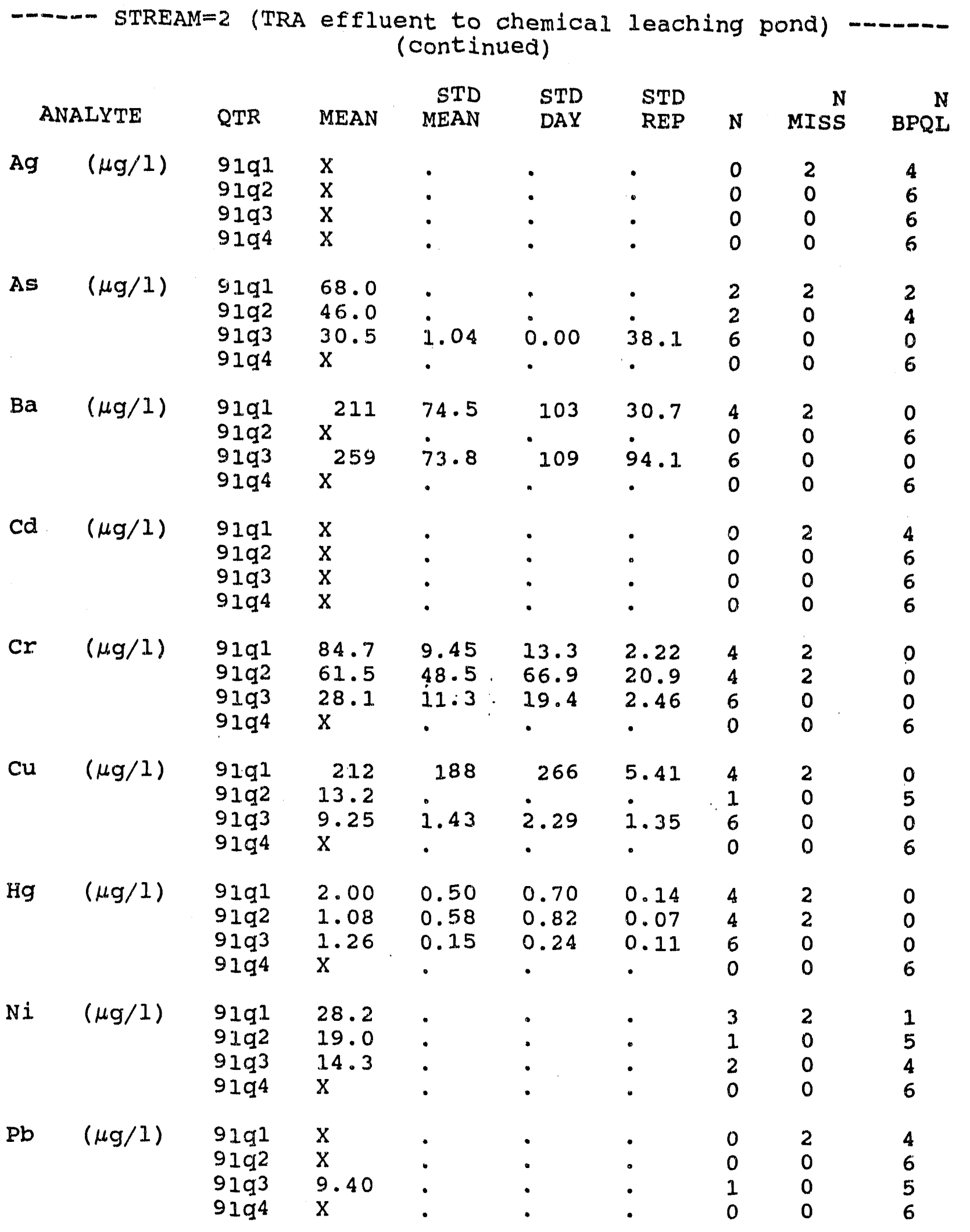


Table A-11 (continued)

QUARTERLY MEANS AND VARIANCE COMPONENTS

- - STREAM=2 (TRA effluent to chemical leaching pond) -...-(continued)

\begin{tabular}{|c|c|c|c|c|c|c|c|c|c|}
\hline \multicolumn{2}{|c|}{ ANALYTE } & QTR & MEAN & $\begin{array}{r}\text { STD } \\
\text { MEAN }\end{array}$ & $\begin{array}{l}\text { STD } \\
\text { DAY }\end{array}$ & $\begin{array}{l}\text { STD } \\
\text { REP }\end{array}$ & N & $\begin{array}{r}\mathrm{N} \\
\text { MISS }\end{array}$ & BPQI \\
\hline $\mathrm{Se}$ & $(\mu g / I)$ & $\begin{array}{l}91 q 1 \\
91 q 2 \\
91 q^{3} \\
91 q^{4}\end{array}$ & $\begin{array}{l}X \\
X \\
X \\
X\end{array}$ & $\begin{array}{l}\dot{\cdot} \\
\dot{\cdot}\end{array}$ & $\dot{.}$ & $\begin{array}{l}\dot{\bullet} \\
\dot{\bullet}\end{array}$ & $\begin{array}{l}0 \\
0 \\
0 \\
0\end{array}$ & $\begin{array}{l}2 \\
0 \\
0 \\
0\end{array}$ & $\begin{array}{l}4 \\
6 \\
6 \\
6\end{array}$ \\
\hline TI & $(\mu g / I)$ & $\begin{array}{l}91 q 1 \\
91 q 2 \\
91 q 3 \\
91 q 4\end{array}$ & $\begin{array}{l}X \\
X \\
X \\
X\end{array}$ & $\dot{\dot{\bullet}}$ & $\dot{\dot{ }}$ & $\begin{array}{l}\dot{\bullet} \\
\dot{\cdot}\end{array}$ & $\begin{array}{l}0 \\
0 \\
0 \\
0\end{array}$ & $\begin{array}{l}2 \\
0 \\
0 \\
0\end{array}$ & $\begin{array}{l}4 \\
6 \\
6 \\
6\end{array}$ \\
\hline $\mathrm{Zn}$ & $(\mu g / 1)$ & $\begin{array}{l}91 q 1 \\
91 q 2 \\
91 q^{3} \\
91 q^{4}\end{array}$ & $\begin{array}{r}56.2 \\
244 \\
15.2 \\
X\end{array}$ & $\begin{array}{l}17.4 \\
: \\
:\end{array}$ & $\begin{array}{l}23 \cdot 3 \\
\dot{0} \\
\dot{b}\end{array}$ & $\begin{array}{l}11.4 \\
: \\
:\end{array}$ & $\begin{array}{l}4 \\
3 \\
2 \\
0\end{array}$ & $\begin{array}{l}2 \\
0 \\
0 \\
0\end{array}$ & $\begin{array}{l}0 \\
3 \\
4 \\
6\end{array}$ \\
\hline$' x^{\prime}$ & $\begin{array}{l}\text { missi } \\
\text { below }\end{array}$ & $\begin{array}{l}\text { too } \\
\text { cti }\end{array}$ & $\begin{array}{l}\text { cle } \\
\text { qua }\end{array}$ & $\begin{array}{l}\text { ta } t \\
\text { tati }\end{array}$ & $1 \mathrm{Cl}$ & $\begin{array}{l}=e \text { va } \\
B P Q I\end{array}$ & & & \\
\hline
\end{tabular}




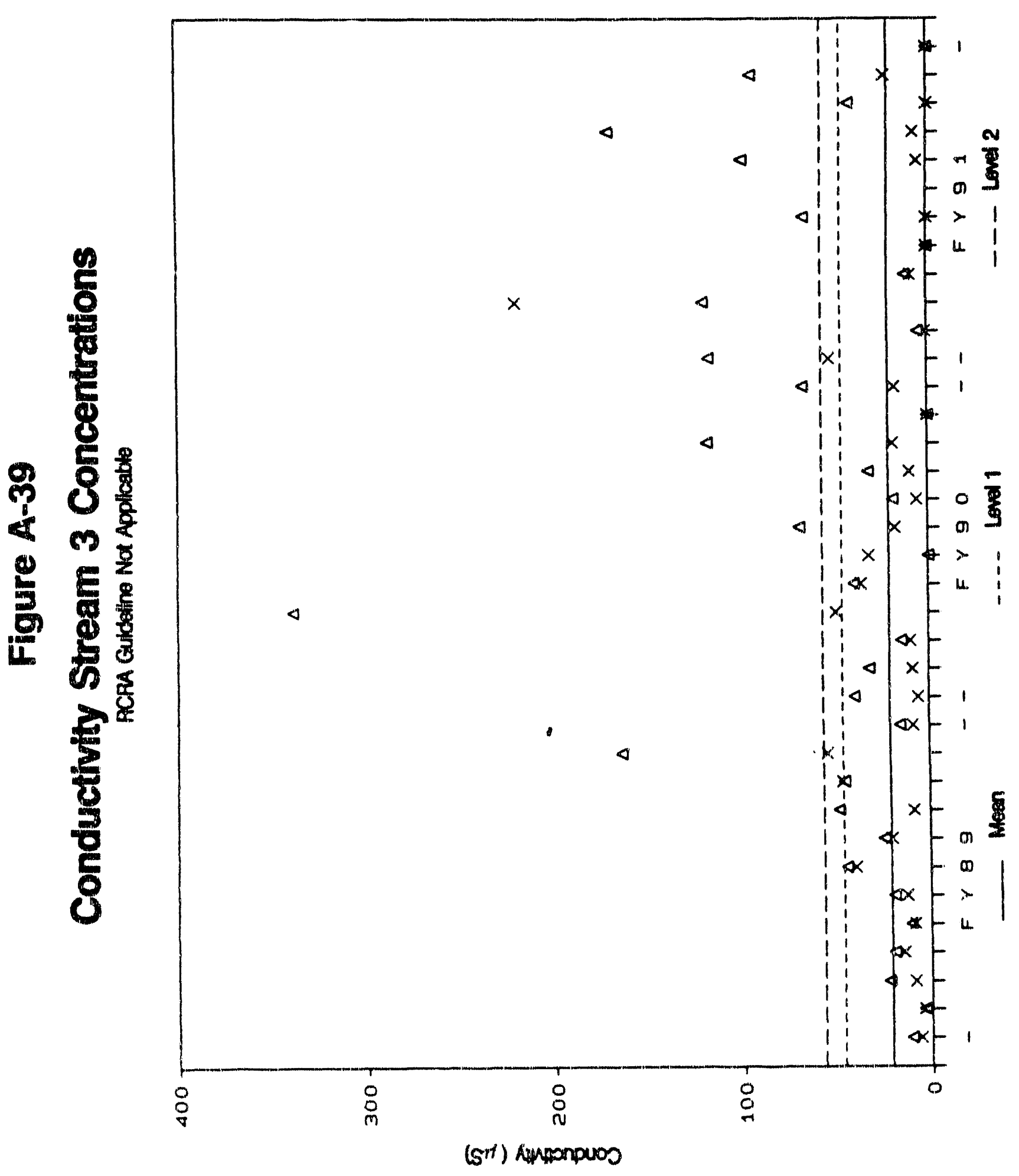




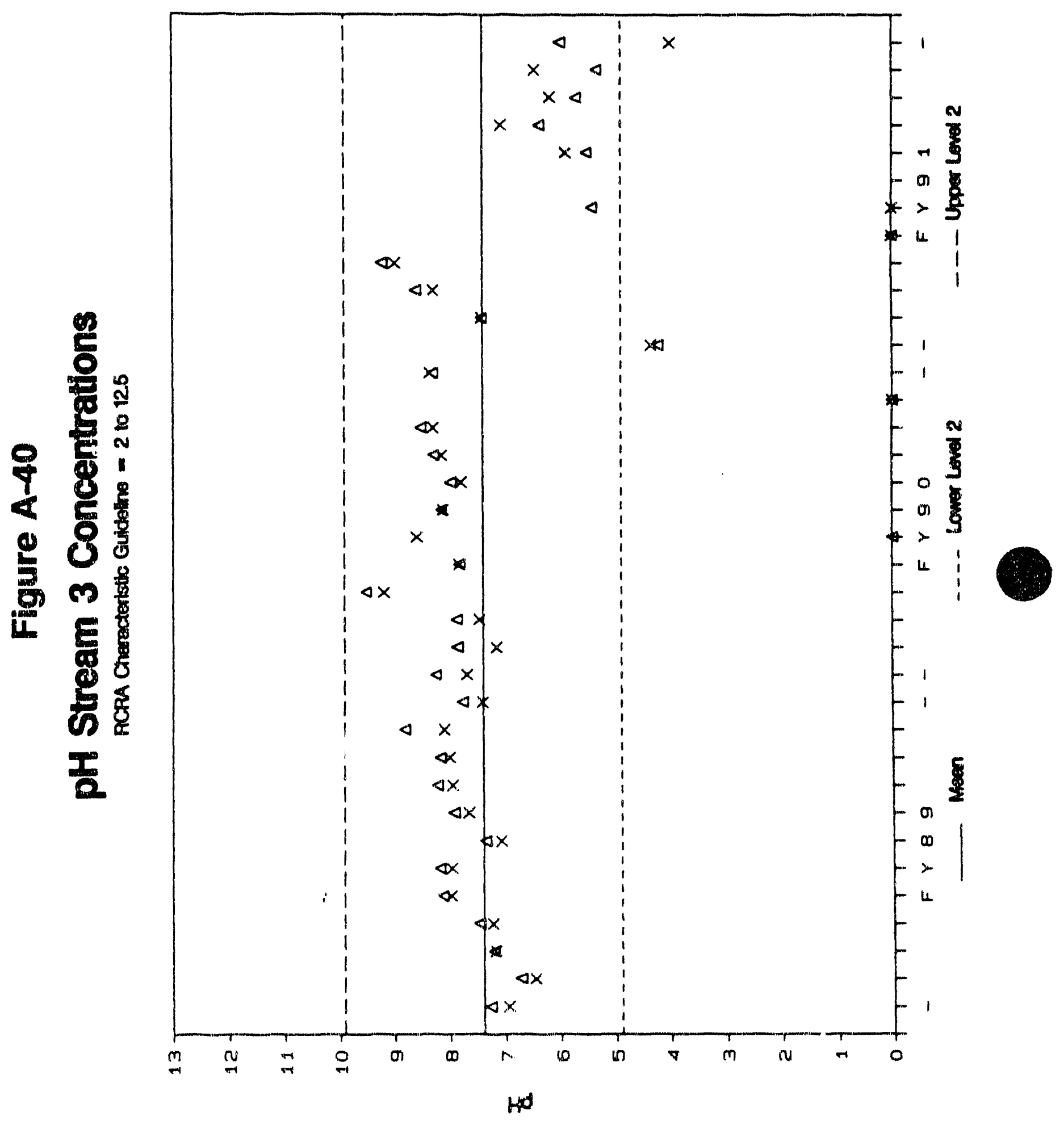




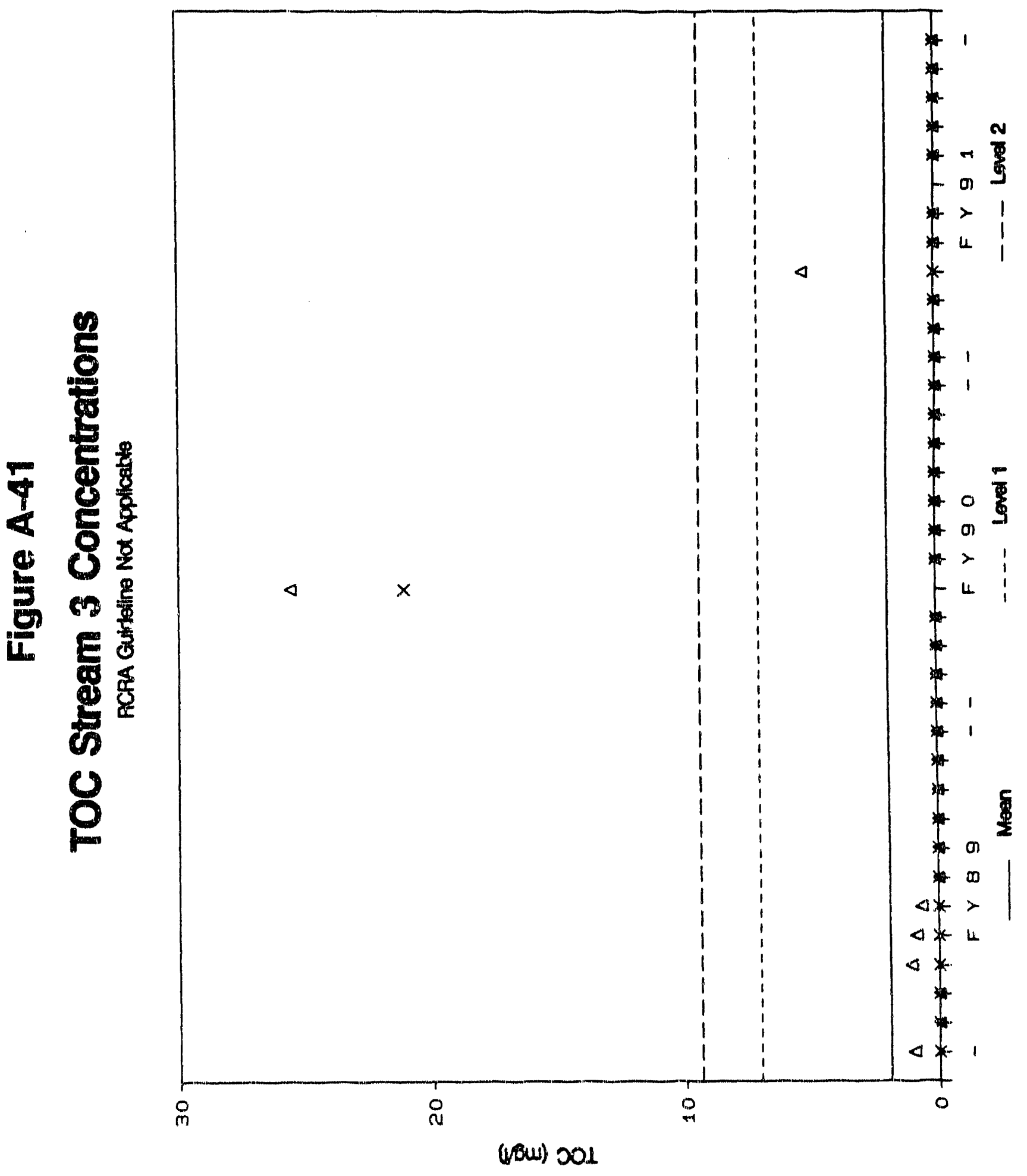




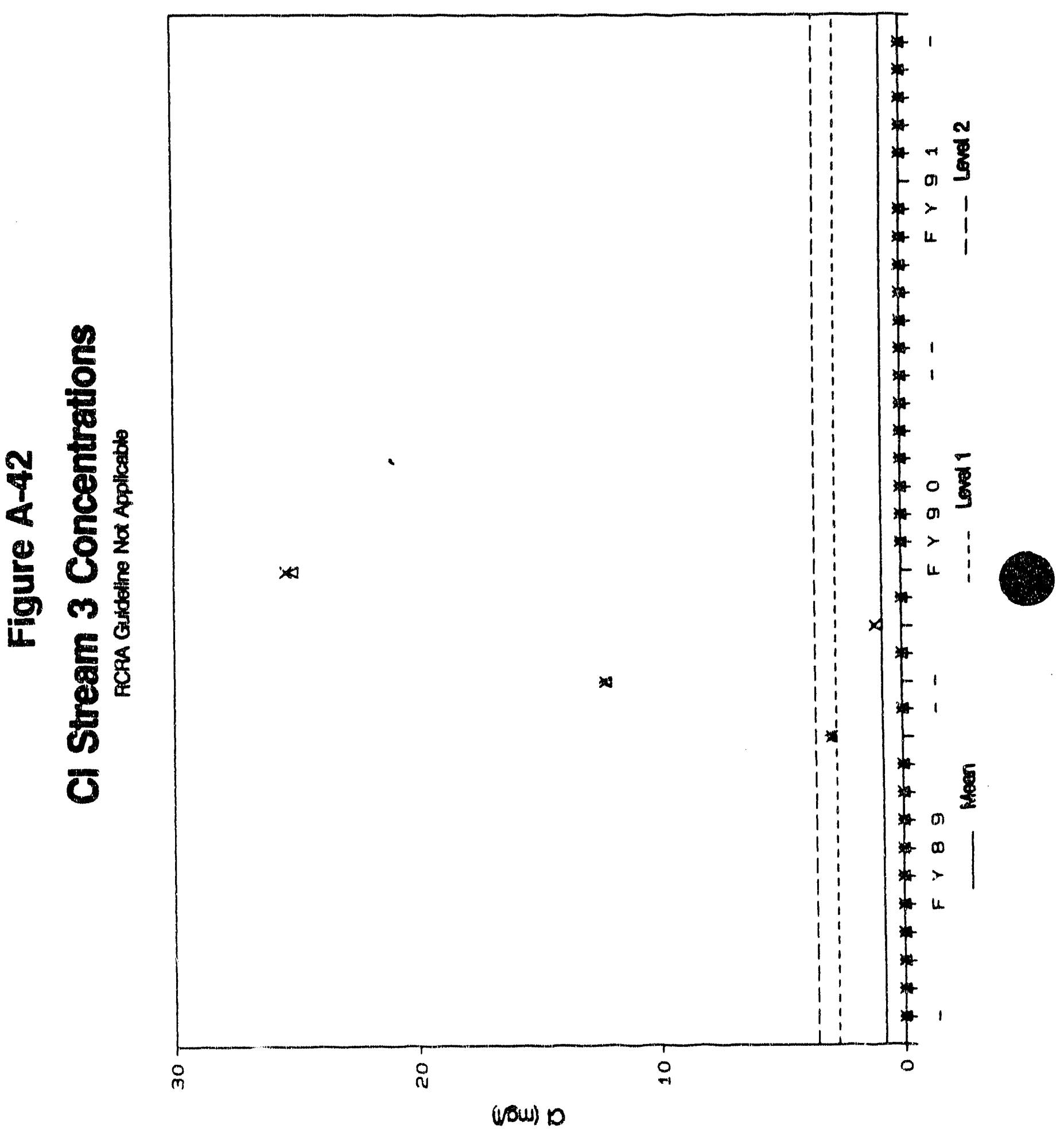




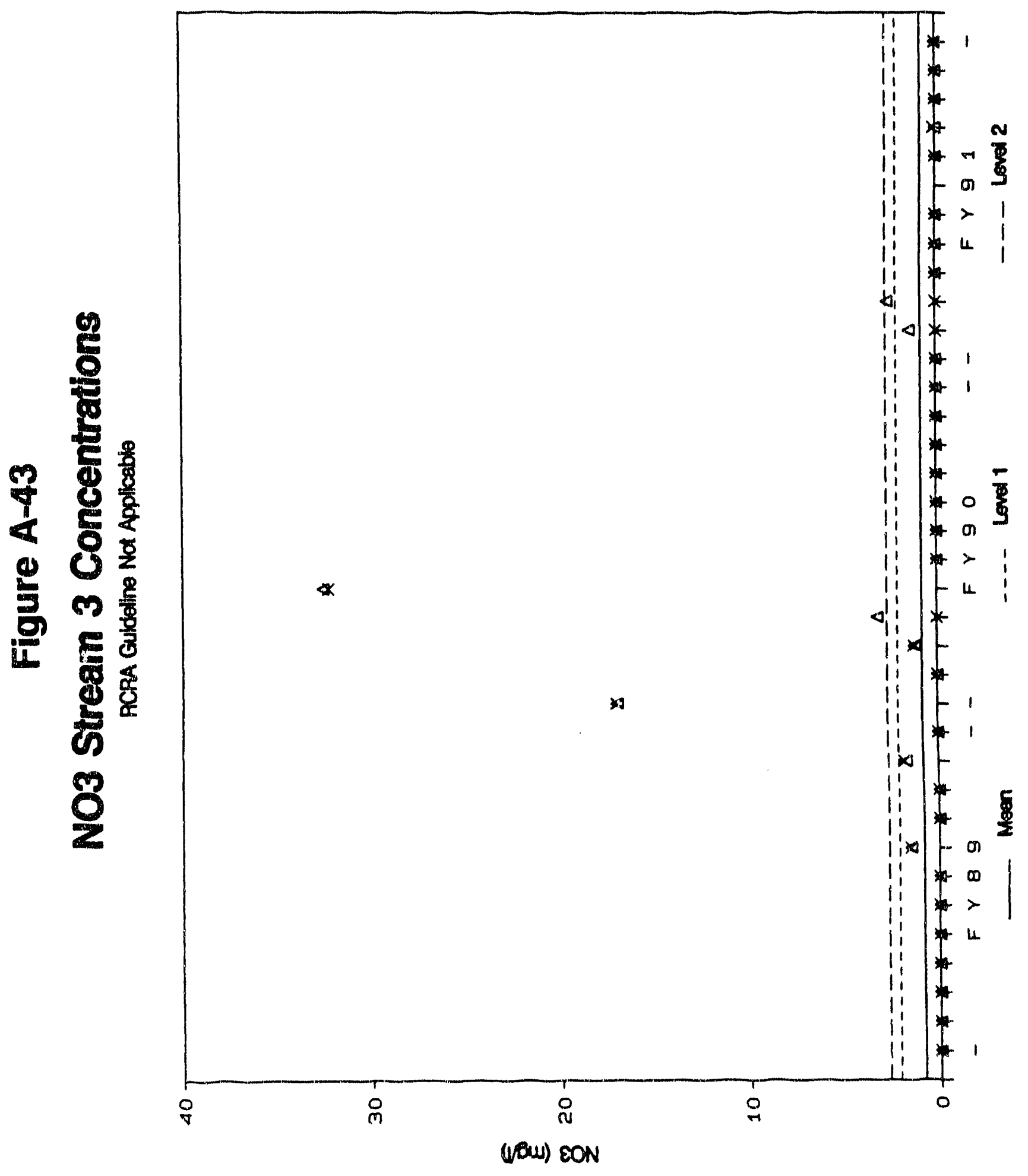




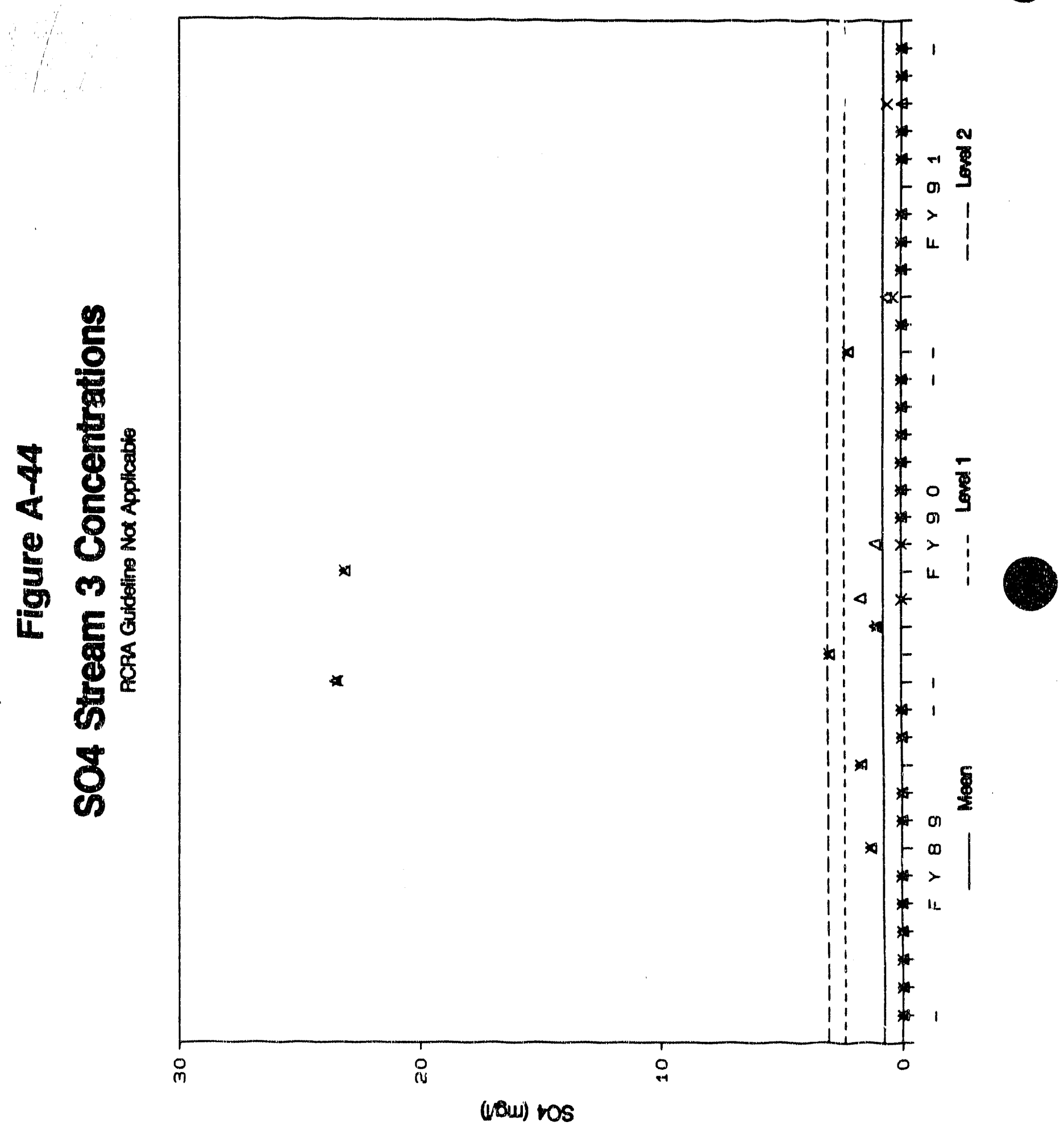




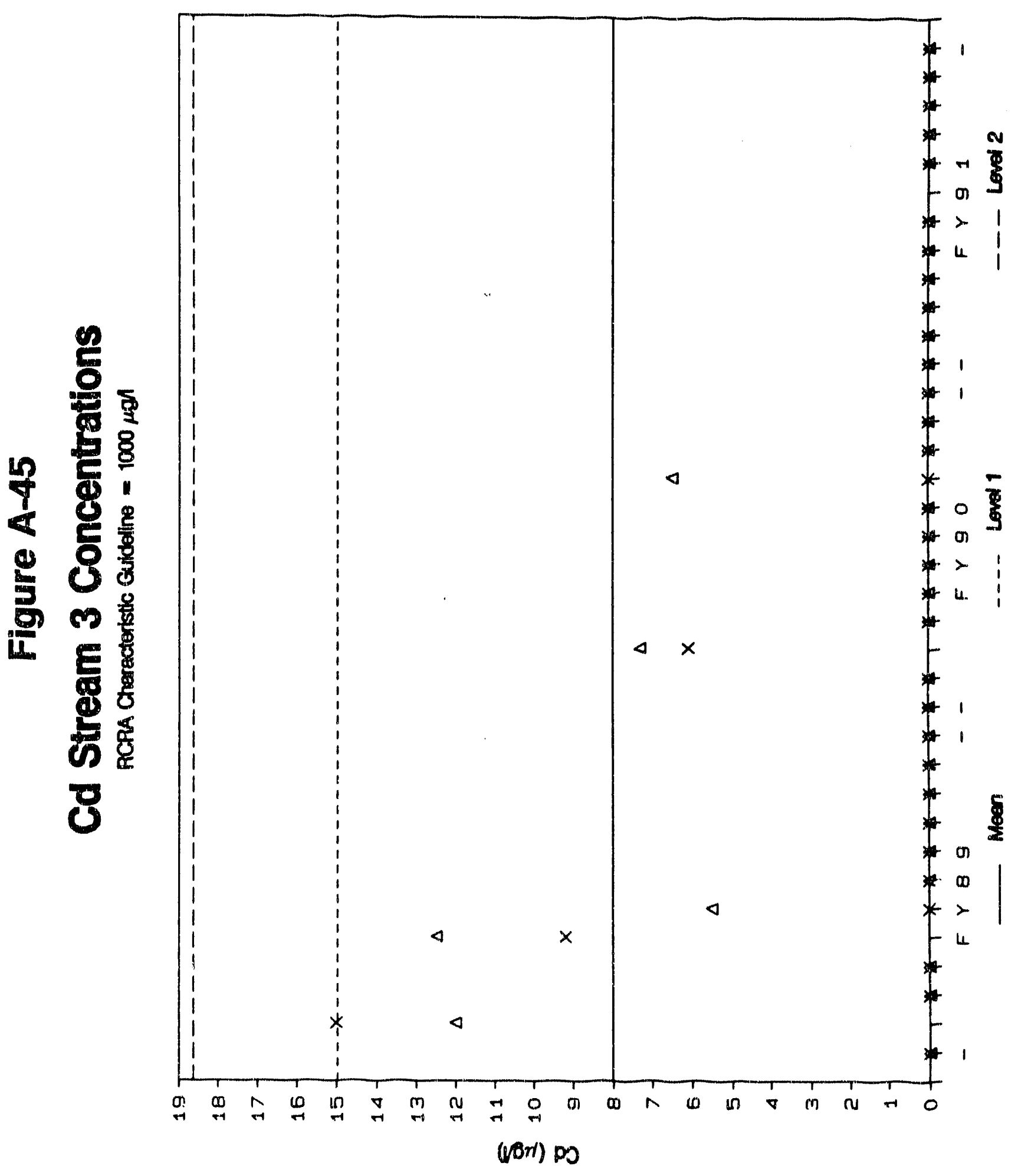




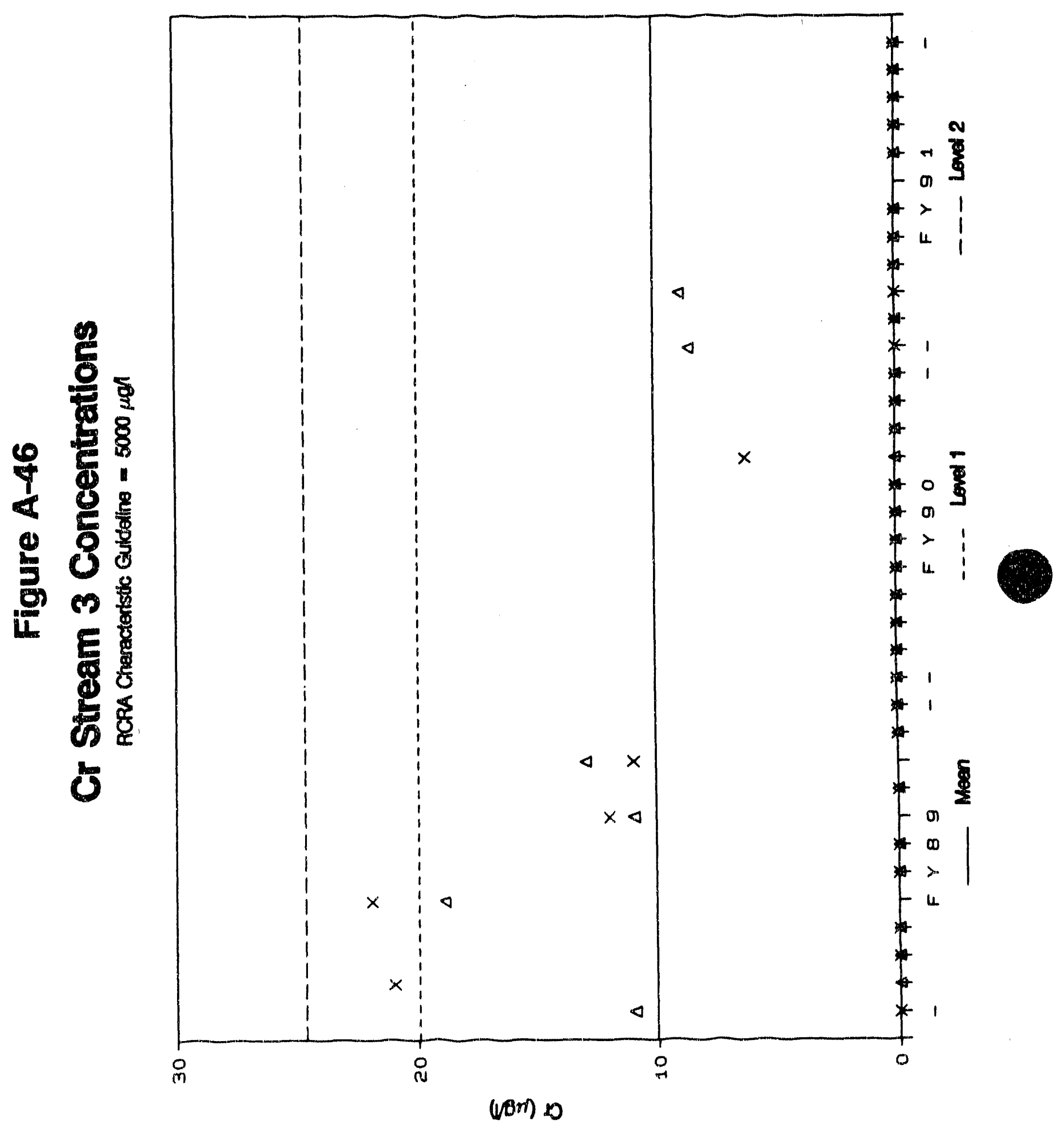

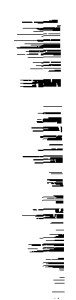




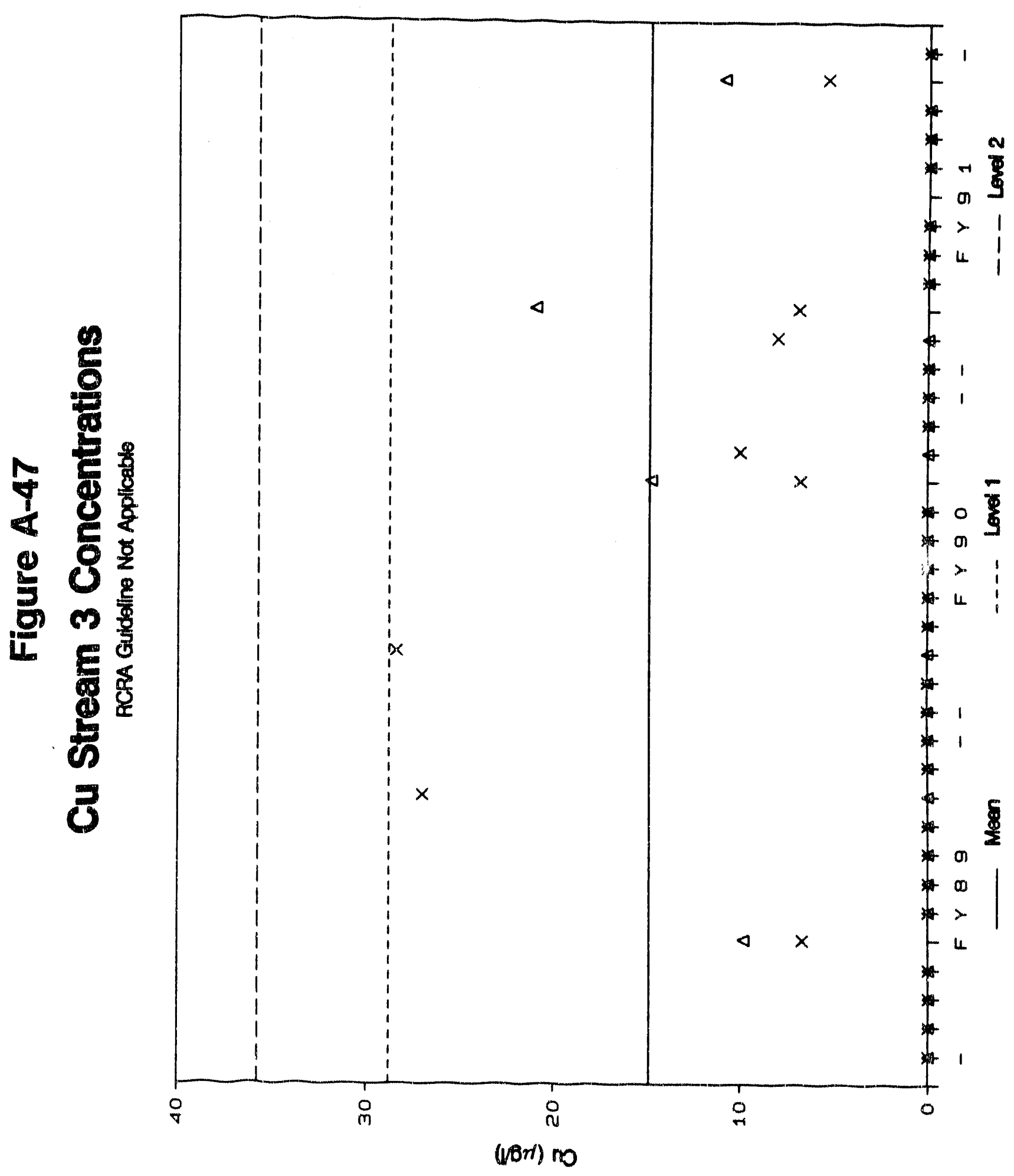




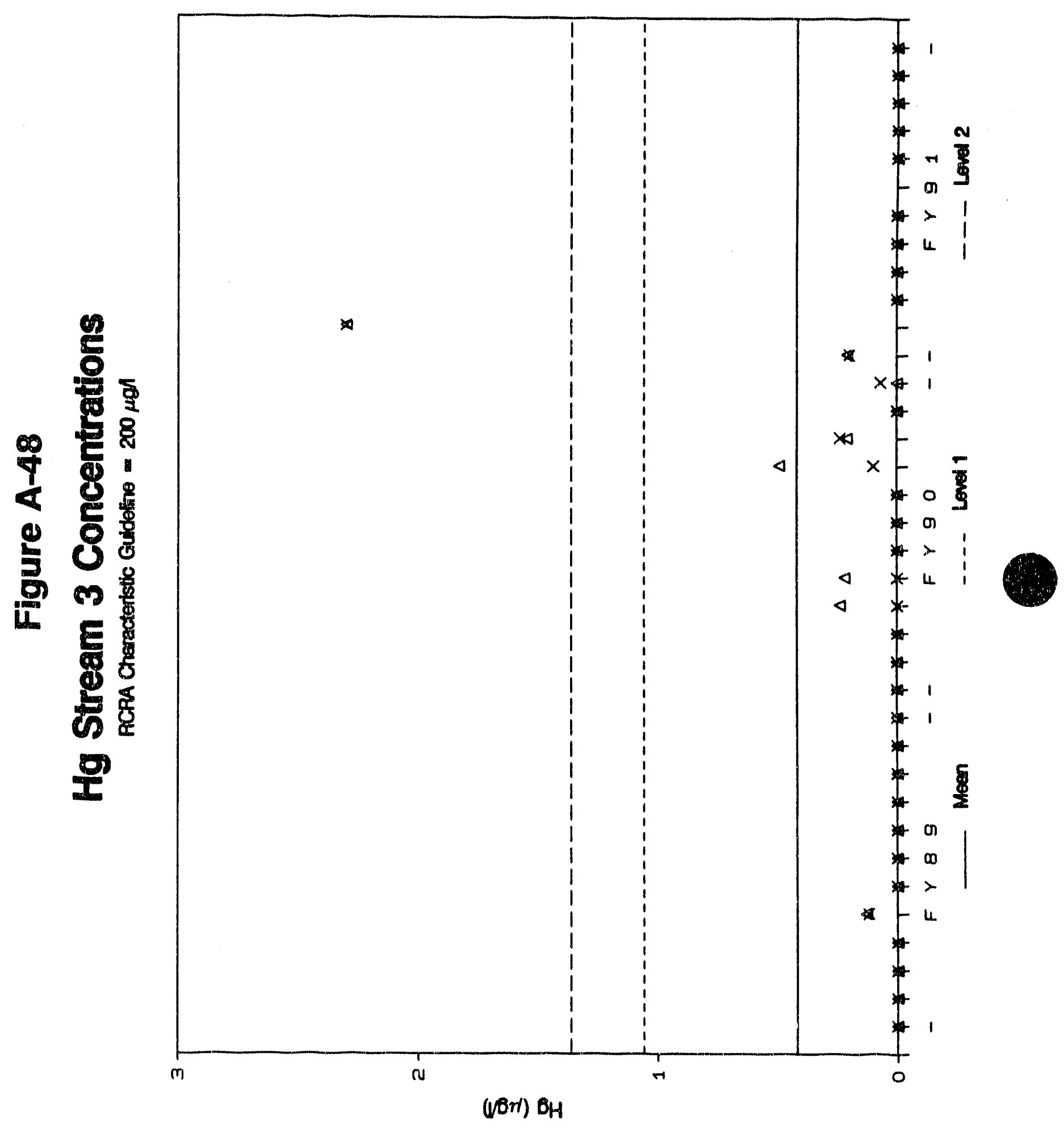




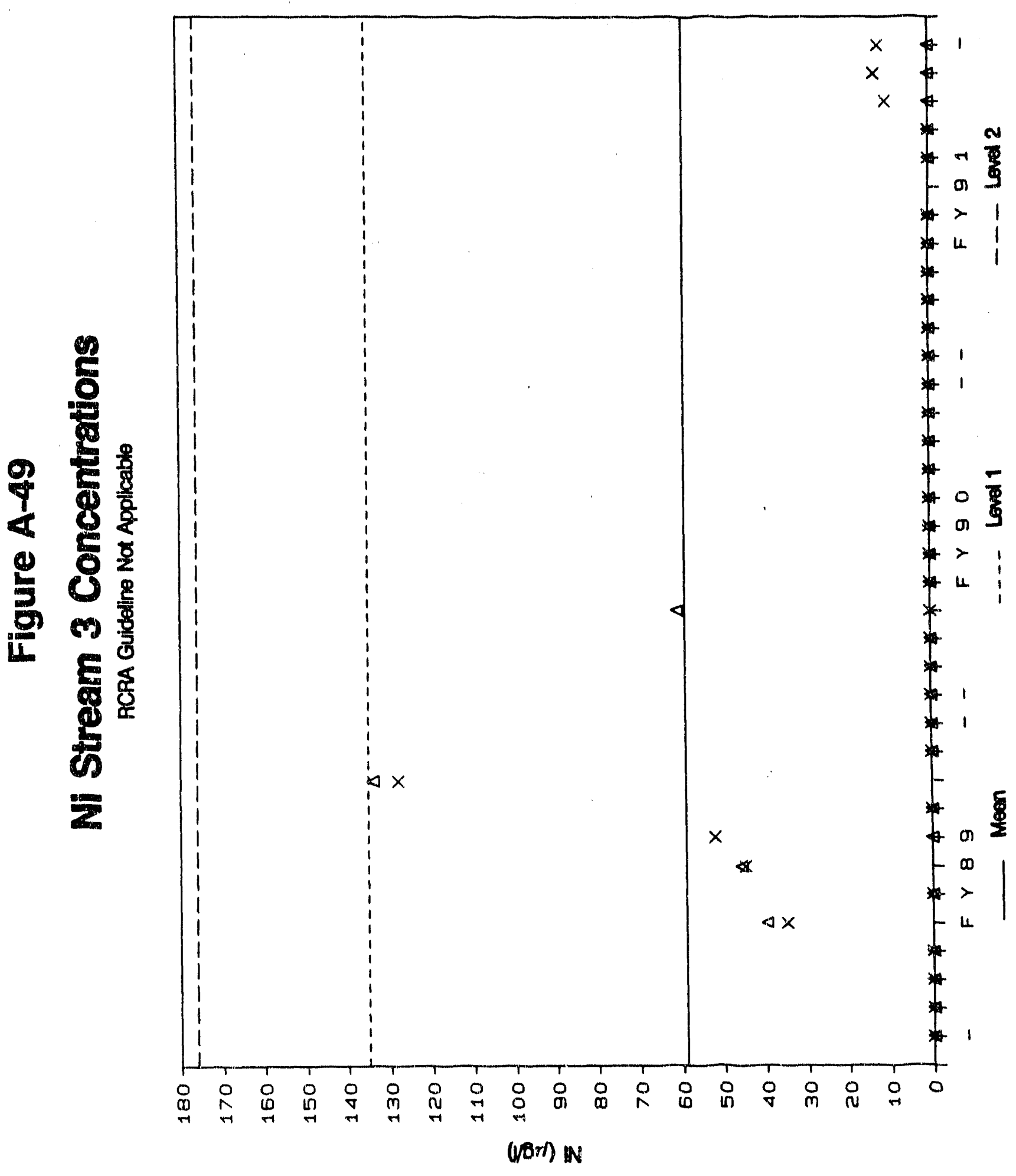

A- 67 


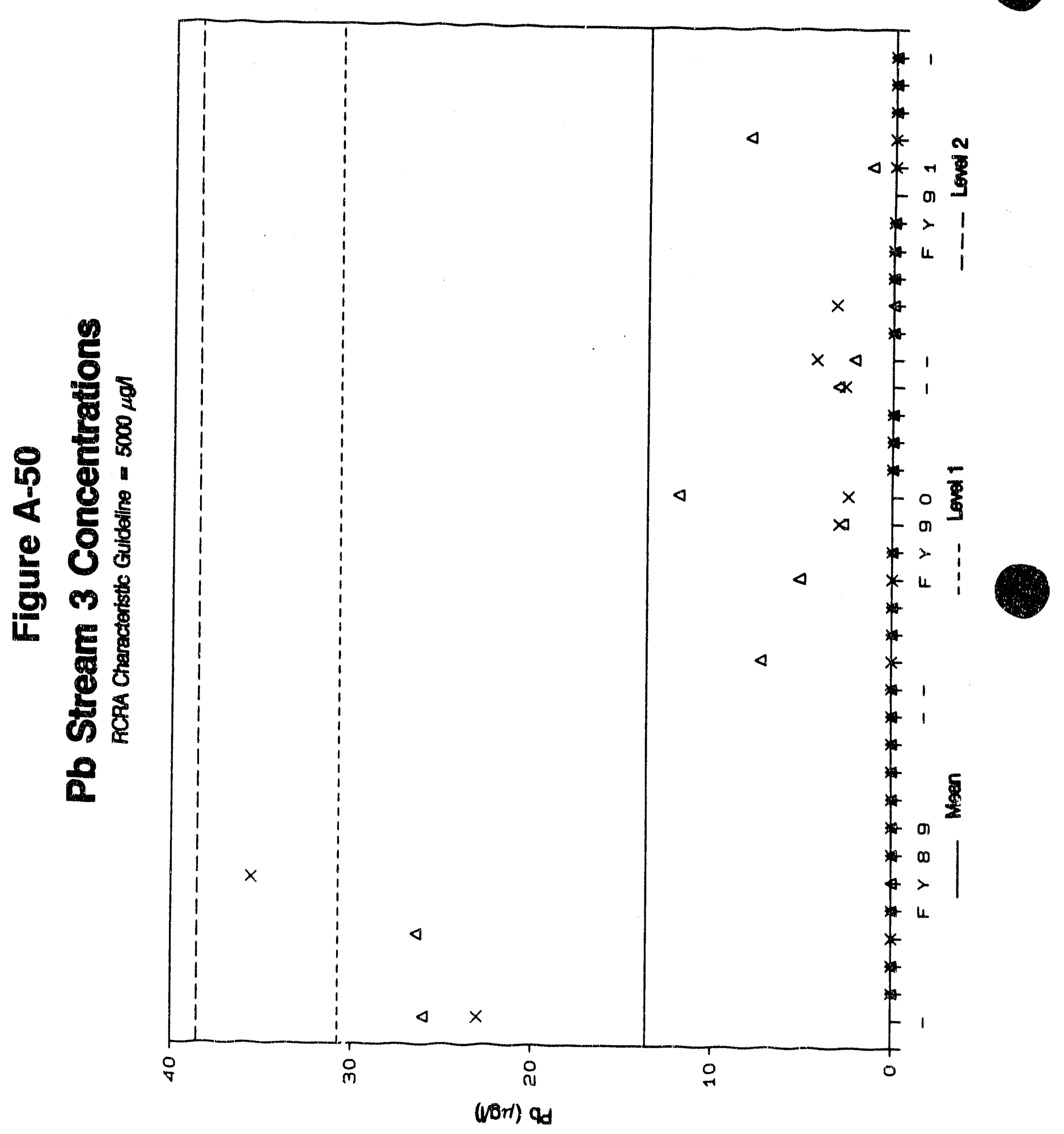




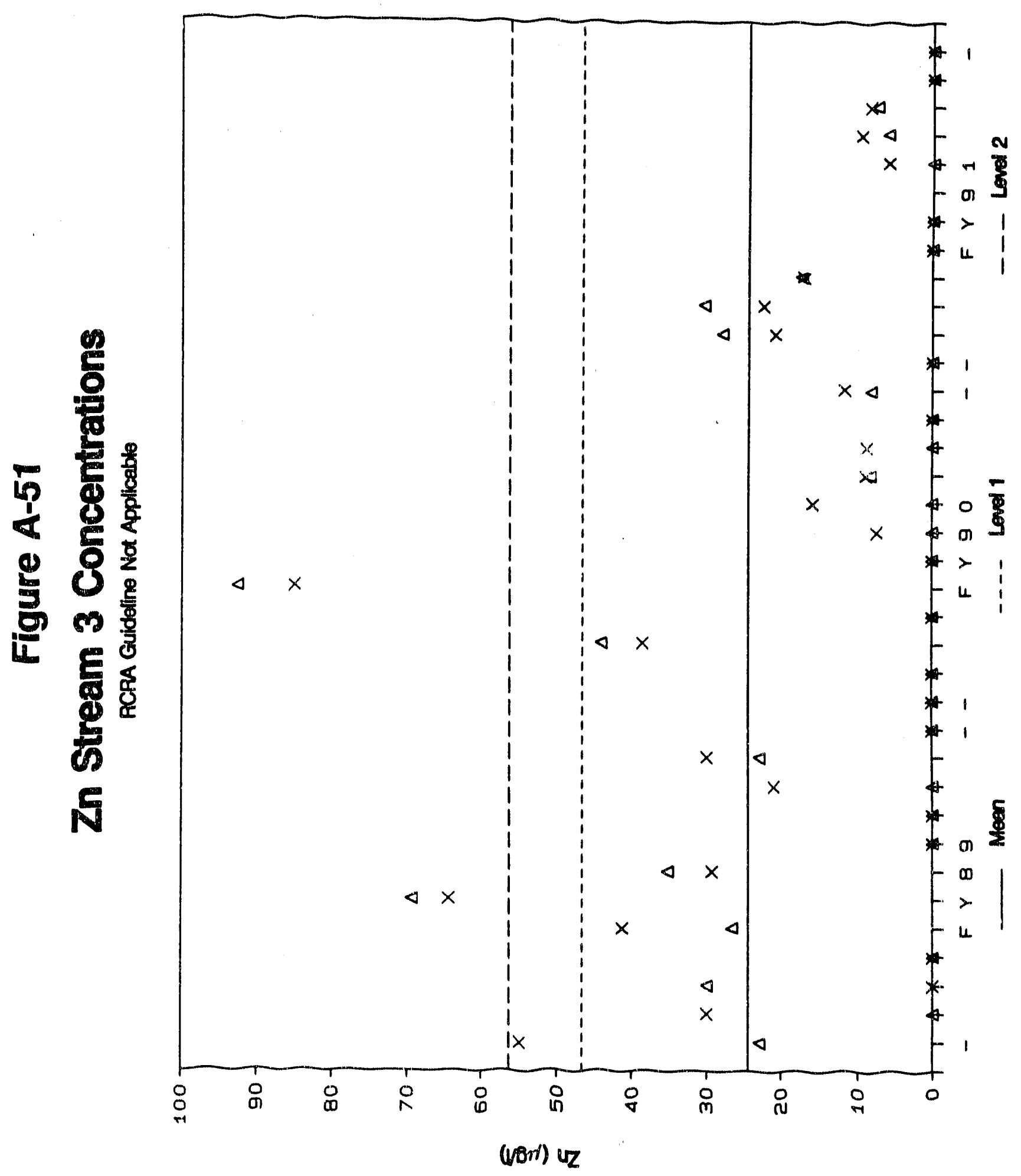


Table A-12

FY91. ENVIRONMENTAL MONITORING DATA

-- STREAM=3 (TRA effluent to retention basin) QUARTER=9191 -- -

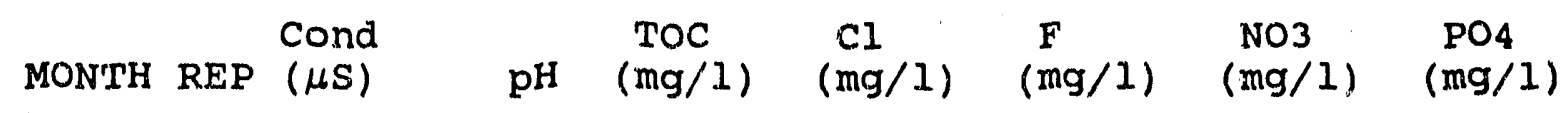

$\begin{array}{lrrrrllll}\text { Oct } & 1 & 117 & 4.22 & X & X & X & X & X \\ \text { Oct } & 2 & 53 & 4.35 & X & X & X & X & X \\ \text { Nov } & 1 & 5 & 7.43 & X & X & X & 1.40 & X \\ \text { Nov } & 2 & 1 & 7.43 & X & X & X & X & X \\ \text { Dec } & 1 & 120 & 8.62 & X & X & X & 2.60 & X \\ \text { Dec } & 2 & 220 & 8.30 & X & X & X & X & X\end{array}$

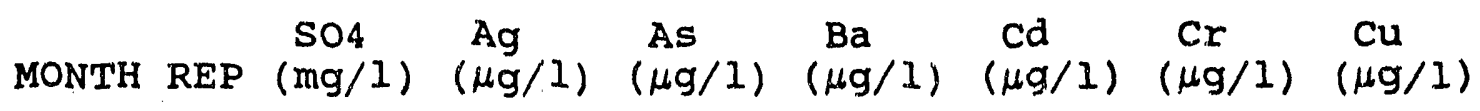

$\begin{array}{lllllllll}\text { Oct } & 1 & 2.20 & \mathrm{X} & \mathrm{X} & \mathrm{X} & \mathrm{X} & 8.60 & \mathrm{X} \\ \text { Oct } & 2 & 2.20 & 3.50 & \mathrm{X} & \mathrm{X} & \mathrm{X} & \mathrm{X} & \mathrm{X} \\ \text { Nov } & 1 & \mathrm{X} & \mathrm{X} & \mathrm{X} & \mathrm{X} & \mathrm{X} & \mathrm{X} & \mathrm{X} \\ \text { Nov } & 2 & \mathrm{X} & \mathrm{X} & \mathrm{X} & \mathrm{X} & \mathrm{X} & \mathrm{X} & 8.00 \\ \text { Dec } & 1 & 0.63 & \mathrm{X} & \mathrm{X} & \mathrm{X} & \mathrm{X} & 9.00 & 21.0 \\ \text { Dec } & 2 & 0.36 & \mathrm{X} & \mathrm{X} & \mathrm{X} & \mathrm{X} & \mathrm{X} & 6.90\end{array}$

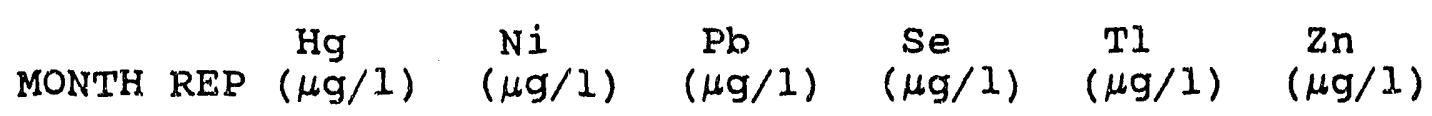

$\begin{array}{llllllll}\text { Oct } & 1 & 0.21 & \mathrm{X} & 2.20 & \mathrm{X} & \mathrm{X} & \mathrm{X} \\ \text { Oct } & 2 & 0.20 & \mathrm{X} & 4.30 & \mathrm{X} & \mathrm{X} & \mathrm{X} \\ \text { Nov } & 1 & 2.30 & \mathrm{X} & \mathrm{X} & \mathrm{X} & \mathrm{X} & 27.9 \\ \text { Nov } & 2 & 2.30 & \mathrm{X} & \mathrm{X} & \mathrm{X} & \mathrm{X} & 20.8 \\ \text { Dec } & 1 & \mathrm{X} & \mathrm{X} & \mathrm{X} & \mathrm{X} & \mathrm{X} & 30.4 \\ \text { Dec } & 2 & \mathrm{X} & \mathrm{X} & 3.20 & \mathrm{X} & \mathrm{X} & 22.5\end{array}$

$'$ ' - missing

' $\dot{x}$ ' - below practical quantitation level 
Table A-13

FY91 ENVIRONMENTAL MONITORING DATA

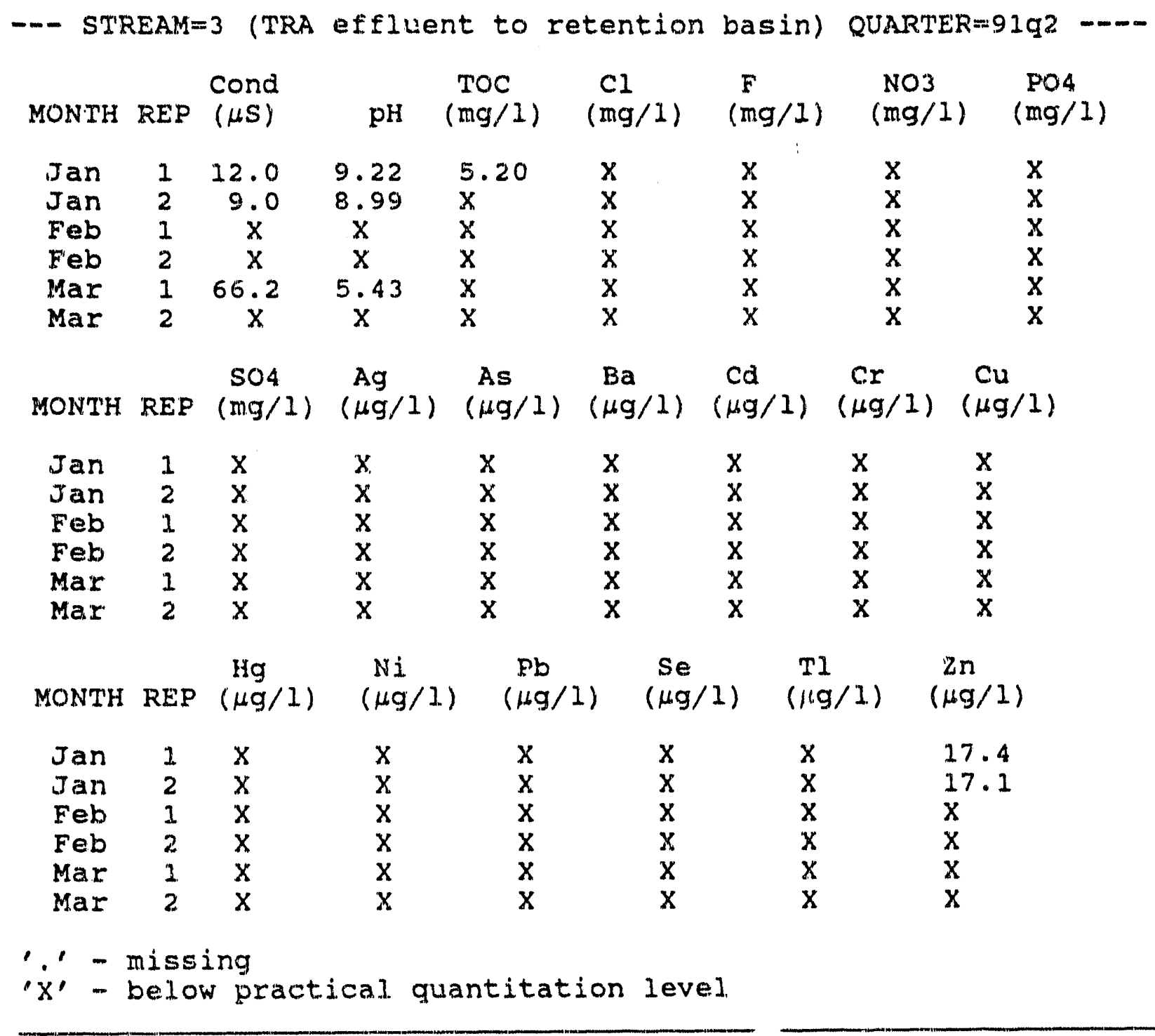


Table A-14

FY91 ENVIRONMENTAL MONITORING DATA

-.- STREAM=3 (TRA effluent to retention basin) Q(IARTER=9193 .....

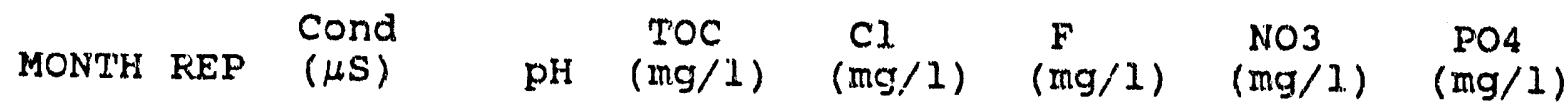

$\begin{array}{lrrrrllll}\text { May } & 1 & 98.9 & 5.53 & x & x & x & x & x \\ \text { May } & 2 & 5.2 & 5.90 & x & x & x & x & x \\ \text { Jun } & 1 & 170.0 & 6.38 & x & x & x & x & x \\ \text { Jun } & 2 & 7.3 & 7.07 & x & x & x & 0.11 & x\end{array}$

$\begin{array}{llllllll}\text { MONTH REP } & \mathrm{SO}^{2} & \mathrm{Ag} & \mathrm{As} & \mathrm{Ba} & \mathrm{Cd} & \mathrm{Cr} & \mathrm{Cu} \\ (\mu \mathrm{g} / 1) & (\mu g / 1) & (\mu g / 1) & (\mu \mathrm{g} / 1) & (\mu \mathrm{g} / 1) & (\mu \mathrm{g} / 1)\end{array}$

$\begin{array}{lllllllll}\text { May } & 1 & \mathrm{X} & \mathrm{X} & \mathrm{X} & 1.30 & \mathrm{X} & \mathrm{X} & \mathrm{x} \\ \text { May } & 2 & \mathrm{X} & \mathrm{X} & \mathrm{X} & \mathrm{X} & \mathrm{X} & \mathrm{X} & \mathrm{X} \\ \text { Jun } & 1 & \mathrm{X} & \mathrm{X} & \mathrm{X} & \mathrm{X} & \mathrm{X} & \mathrm{X} & \mathrm{X} \\ \text { Jun } & 2 & \mathrm{X} & \mathrm{X} & \mathrm{X} & \mathrm{X} & \mathrm{X} & \mathrm{X} & \mathrm{X}\end{array}$

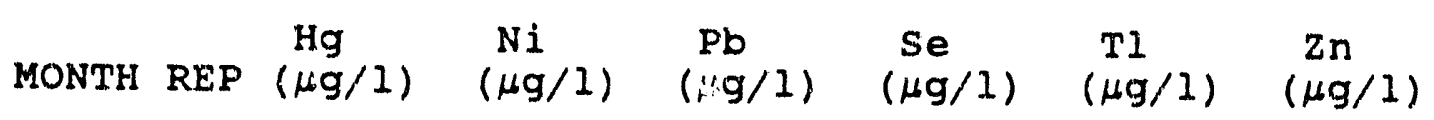

$\begin{array}{llllllll}\text { May } & 1 & X & X & 1.30 & X & X & X \\ \text { May } & 2 & X & X & X & X & X & 5.90 \\ \text { Jun } & 1 & X & X & 8.10 & X & X & 6.00 \\ \text { Jun } & 2 & X & X & X & X & X & 9.60\end{array}$

$'$ ' - missing

' $X$ ' - below practical quantitation level 


\section{Table A-15}

FY91 ENVIRONMENTAL MONITORING DATA

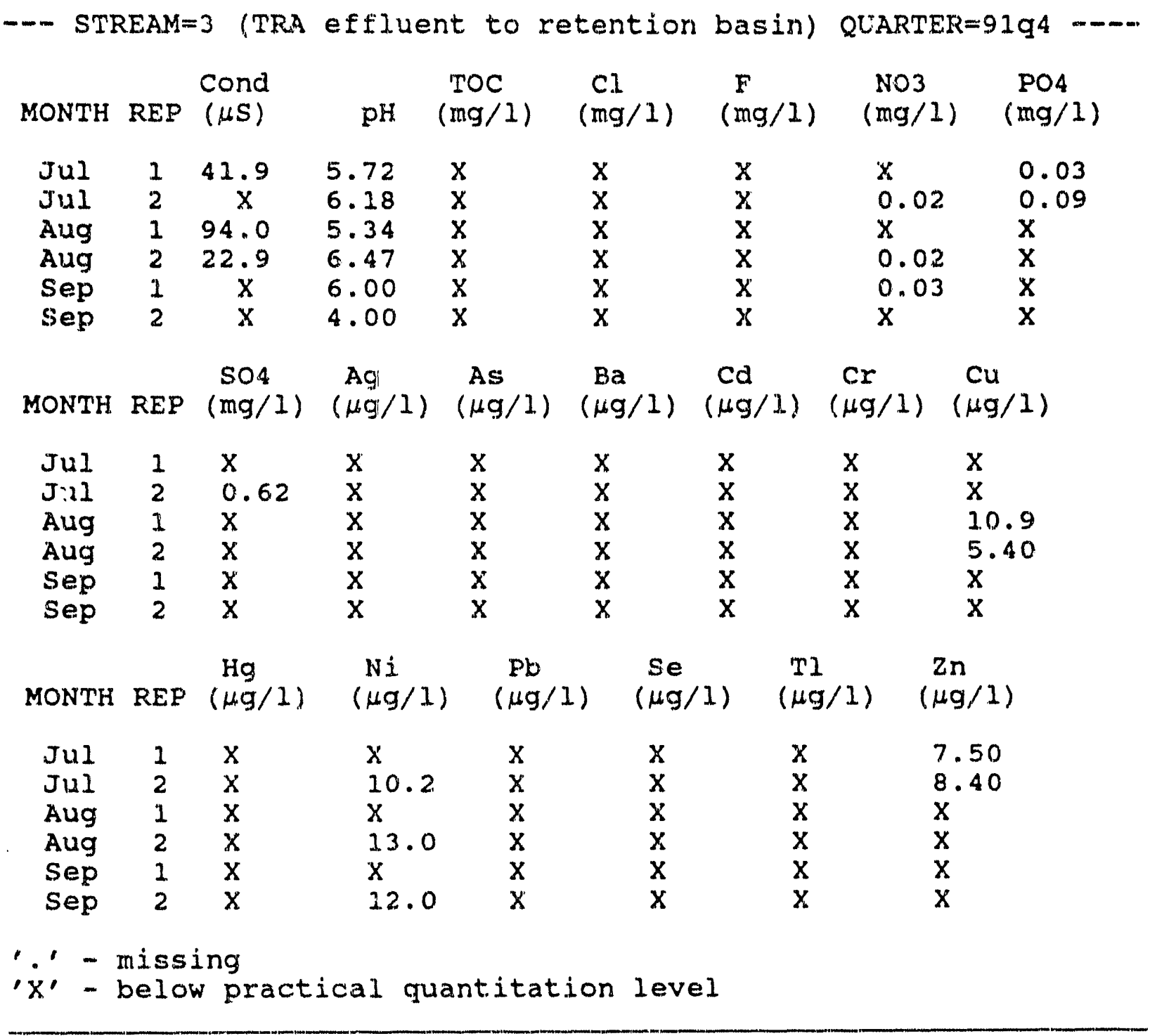




\section{Table A-16}

SUMMARY STATISTICS

STREAM=3 (TRA effluent to retention basin)

\begin{tabular}{|c|c|c|c|c|c|c|c|}
\hline \multicolumn{2}{|c|}{ ANALY'IE } & \multicolumn{2}{|c|}{ FY87-FY90 } & N & LEVELI & LEVEL2 & GUIDELINE \\
\hline Cond & $(\mu S)$ & 21.4 & 15.0 & 82 & 46.6 & 57.3 & $N / A$ \\
\hline $\mathrm{pH}$ & & 7.41 & 0.95 & 88 & $\begin{array}{ll}1 & 5.52 \\
\mathrm{u} & 9.31\end{array}$ & $\begin{array}{ll}1 & 4.90 \\
\text { u } & 9.92\end{array}$ & $\begin{array}{ll}1 & 6.5 \\
\text { u } & 8.5\end{array}$ \\
\hline TOC & $(\mathrm{mg} / \mathrm{l})$ & 1.92 & 2.88 & 21 & 7.01 & 9.37 & $\mathrm{~N} / \mathrm{A}$ \\
\hline $\mathrm{Cl}$ & $(m g / 1)$ & 0.79 & 1.11 & 27 & 2.72 & 3.59 & $N / A$ \\
\hline$F$ & $(\mathrm{mg} / \mathrm{I})$ & & & & & & $N / A$ \\
\hline NO3 & $(\mathrm{mg} / \mathrm{l})$ & 0.82 & 0.74 & 26 & 2.11 & 2.70 & $N / A$ \\
\hline PO4 & $(\mathrm{mg} / \mathrm{l})$ & & & & & & $N / A$ \\
\hline SO4 & $(\mathrm{mg} / \mathrm{l})$ & 0.77 & 0.92 & 29 & 2.36 & 3.07 & $N / A$ \\
\hline Ag & $(\mu \mathrm{g} / 1)$ & & & & & & 5000 \\
\hline As & $(\mu \mathrm{g} / 1)$ & & & & & & 5000 \\
\hline $\mathrm{Ba}$ & $(\mu g / 1)$ & & & & & & 100,000 \\
\hline$c d$ & $(\mu g / 1)$ & 8.01 & 3.68 & 11 & 15.0 & 18.6 & 1000 \\
\hline $\mathrm{Cr}$ & $(\mu \mathrm{g} / 1)$ & 10.1 & 5.54 & 18 & 20.0 & 24.7 & 5000 \\
\hline $\mathrm{Cu}$ & $(\mu \mathrm{g} / 1)$ & 14.9 & 7.51 & 23 & 28.8 & 35.8 & $\mathrm{~N} / \mathrm{A}$ \\
\hline $\mathrm{Hg}$ & $(\mu g /])$ & 0.42 & 0.36 & 18 & 1.06 & 1.36 & 200 \\
\hline $\mathrm{Ni}$ & $(\mu g / 1)$ & 59.0 & 39.6 & 10 & 135 & 176 & $N / A$ \\
\hline $\mathrm{Pb}$ & $(\mu g / 1)$ & 13.6 & 9.78 & 24 & 30.7 & 38.6 & 5000 \\
\hline se & $(\mu \mathrm{g} / 1)$ & & & & & & 1000 \\
\hline Tl & $(\mu g / 1)$ & & & & & & $\mathrm{N} / \mathrm{A}$ \\
\hline $2 n$ & $(\mu g / 1)$ & 24.5 & 13.0 & 39 & 46.6 & 56.4 & $\mathrm{~N} / \mathrm{A}$ \\
\hline
\end{tabular}


Table A-17

QUARTERLY MEANS AND VARIANCE COMPONENTS

STREAM=3 (TRA effluent to retention basin)

\begin{tabular}{|c|c|c|c|c|c|c|c|c|}
\hline ANALYTE & QTR & MEAN & $\begin{array}{r}\text { STD } \\
\text { MEAN }\end{array}$ & $\begin{array}{l}\text { STD } \\
\text { DAY }\end{array}$ & $\begin{array}{l}\text { STD } \\
\text { REP }\end{array}$ & $\mathrm{N}$ & MISS & $\underset{\text { BPQI }}{N}$ \\
\hline Cond $(\mu S)$ & $\begin{array}{l}91 q 1 \\
91 q 2 \\
91 q 3 \\
91 q 4\end{array}$ & $\begin{array}{l}86.0 \\
29.1 \\
70.3 \\
52.9\end{array}$ & $\begin{array}{l}48.2 \\
\dot{18.3} \\
.\end{array}$ & $\begin{array}{l}76.1 \\
0.00 \\
\dot{0}\end{array}$ & $\begin{array}{l}48.5 \\
\dot{93.9} \\
\dot{r}\end{array}$ & $\begin{array}{l}6 \\
3 \\
4 \\
3\end{array}$ & $\begin{array}{l}0 \\
0 \\
0 \\
0\end{array}$ & $\begin{array}{l}0 \\
3 \\
0 \\
3\end{array}$ \\
\hline $\mathrm{pH}$ & $\begin{array}{l}91 q 1 \\
91 q 2 \\
91 q^{3} \\
91 q^{4}\end{array}$ & $\begin{array}{l}6.72 \\
7.88 \\
6.22 \\
5.62\end{array}$ & $\begin{array}{l}1.26 \\
\dot{0} .51 \\
0.31\end{array}$ & $\begin{array}{l}2.17 \\
0.66 \\
0.00\end{array}$ & $\begin{array}{l}0.14 \\
0.39 \\
0.96\end{array}$ & $\begin{array}{l}6 \\
3 \\
4 \\
6\end{array}$ & $\begin{array}{l}0 \\
0 \\
0 \\
0\end{array}$ & $\begin{array}{l}0 \\
3 \\
0 \\
0\end{array}$ \\
\hline
\end{tabular}

TOC $(\mathrm{mg} / \mathrm{l}) \quad 91 \mathrm{gl} \times$

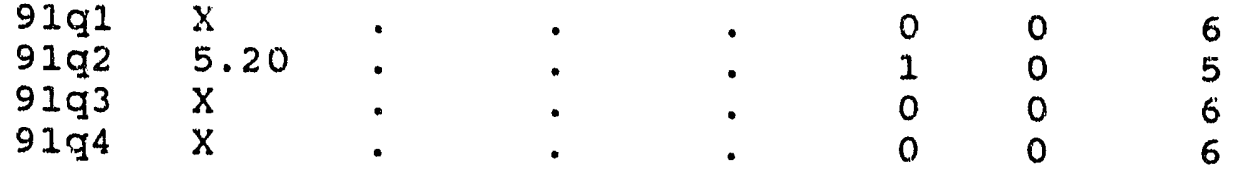

Cl $(\mathrm{mg} / 1)$ 9lql $\mathrm{x}$

$\cdot 0.0000$

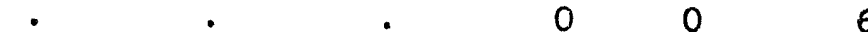

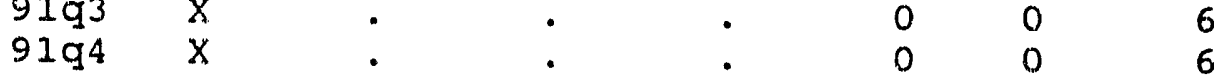

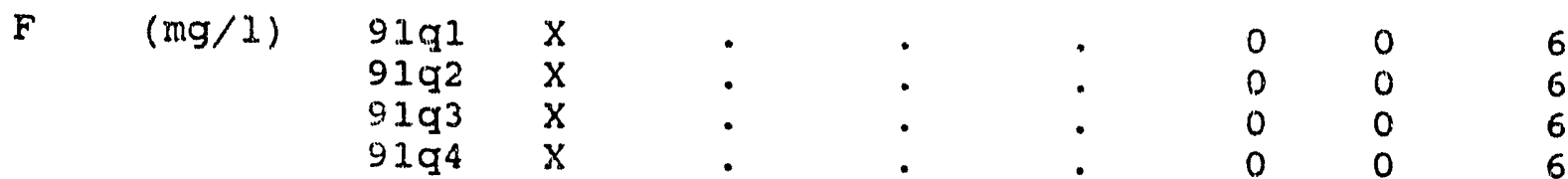

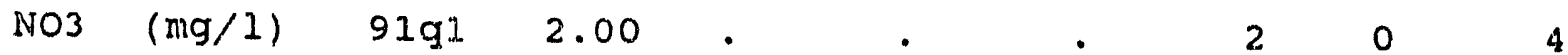

$91 q^{2} \times \quad . \quad . \quad 00006$

$\begin{array}{lllllllll}9193 & 0.11 & . & . & . & 1 & 0 & 5\end{array}$

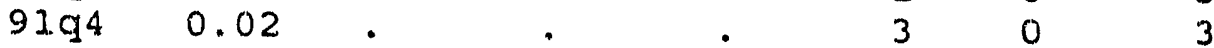

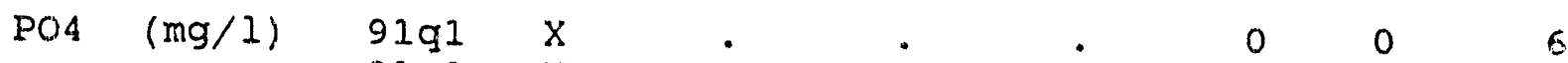

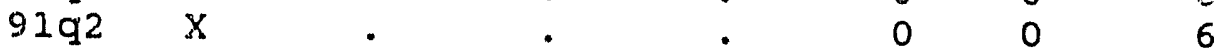

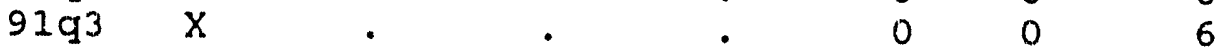

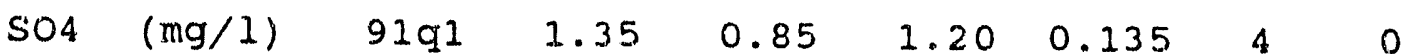

$9192 \times$

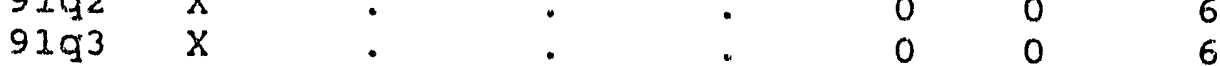

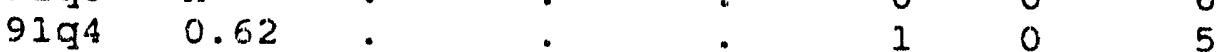

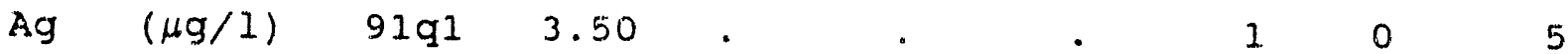

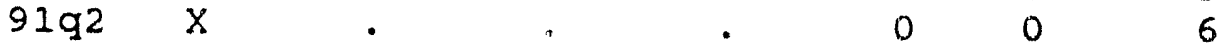

$\begin{array}{lllllll}9193 & X & \cdot & \cdot & 0 & 0 & 6\end{array}$

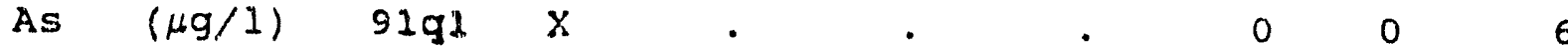

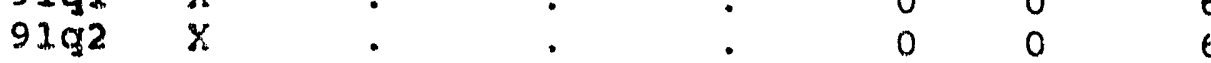

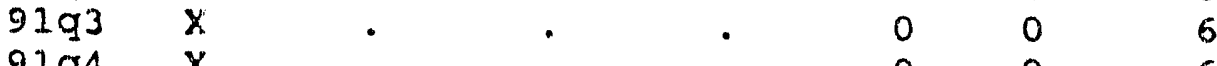

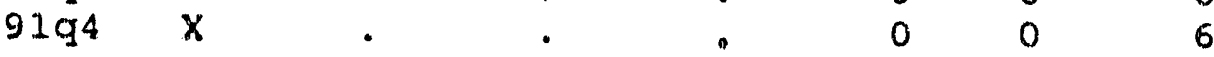


Table A-17 (continued)

QUARTERLY MEANS AND VARIANCE COMPONENTS

STREAM=3 (TRA effluent to retention basin) (continued)

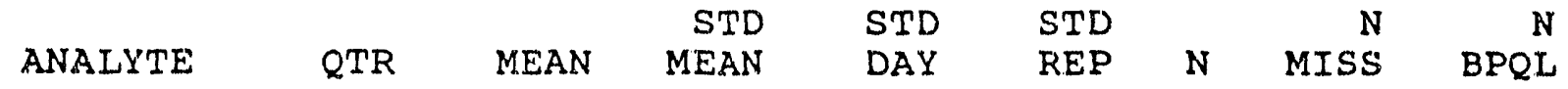

Ba

$\mathrm{Ba} \quad(\mu g / 1)$

$\begin{array}{ll}9191 & x \\ 9192 & x \\ 9193 & 1.30 \\ 9194 & x\end{array}$

$\operatorname{cd}(\mu \mathrm{g} / \mathrm{I})$

$91 q 1 \quad x$

$91 \mathrm{q}^{2}$

$91 \mathrm{q}^{3}$

9194

$\mathrm{X}$
$\mathrm{X}$
$\mathrm{X}$
$\mathrm{X}$

$\operatorname{cr}(\mu g / 1)$

$91 q 1$

9192

9193

9194

8.80
$x$
$x$
$x$

$\mathrm{Cu} \quad(\mu \mathrm{g} / 1) \quad 91 \mathrm{ql}$

$91 \mathrm{q} 2$

9193

9194

12.0
$X$
$X$
8.15

$\mathrm{Hg} \quad(\mu \mathrm{g} / \mathrm{l})$

$9191 \quad 1.25 \quad 1.05$

$91 q 2$

9193

91.94

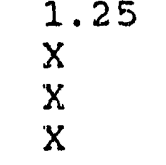

$\mathrm{Ni} \quad(\mu \mathrm{g} / 1) \quad 91 \mathrm{qI}$

9192

$91 q^{3}$

9194

$\mathrm{X}$
$\mathrm{X}$
$\mathrm{X}$

11.7

$\begin{array}{ll}0 & 0 \\ 0 & 0 \\ 1 & 0 \\ 0 & 0\end{array}$

6
6
5
6

6

$\begin{array}{lll}0 & 0 & 6 \\ 0 & 0 & 6 \\ 0 & 0 & 6 \\ 0 & 0 & 6\end{array}$

$\mathrm{Pb} \quad(\mu \mathrm{g} / 1)$

$91 q 1$
$91 q 2$
9193
9194

3.23
$x$
4.70
$x$

se $(\mu g / 1)$

9191
9192
9193
9194

$\mathrm{X}$
$\mathrm{X}$
$\mathrm{X}$
$\mathrm{X}$

TI $(\mu g / 1) \quad 91 q 1$

$$
91 \mathrm{~g}^{2}
$$

9193

9194

$x$
$X$
$x$
$x$




\section{Table A-17 (continued)}

\section{QUARTERLY MEANS AND VARIANCE COMPONENTS}

STREAM=3 (TRA effluent to retention basin) (continued)

\begin{tabular}{|c|c|c|c|c|c|c|c|c|c|}
\hline & ALYTE & QTR & MEAN & $\begin{array}{r}\text { STD } \\
\text { MEAN }\end{array}$ & $\begin{array}{l}\text { STD } \\
\text { DAY }\end{array}$ & $\begin{array}{l}\text { STD } \\
\text { REF }\end{array}$ & $\mathrm{N}$ & $\begin{array}{r}\mathrm{N} \\
\text { MISS }\end{array}$ & $\underset{B P Q L}{N}$ \\
\hline $\mathrm{zn}$ & $(\mu g / l)$ & $\begin{array}{l}91 q 1 \\
91 q^{2} \\
91 q^{3} \\
91 q^{4}\end{array}$ & $\begin{array}{l}25.4 \\
17.3 \\
7.17 \\
7.95\end{array}$ & $\begin{array}{l}1.05 \\
\dot{0} \\
\dot{0}\end{array}$ & $\begin{array}{l}0.00 \\
\dot{0} \\
\text {. }\end{array}$ & $\begin{array}{l}5.31 \\
: \\
:\end{array}$ & $\begin{array}{l}4 \\
2 \\
3 \\
2\end{array}$ & $\begin{array}{l}0 \\
0 \\
0 \\
0\end{array}$ & $\begin{array}{l}2 \\
4 \\
3 \\
4\end{array}$ \\
\hline
\end{tabular}




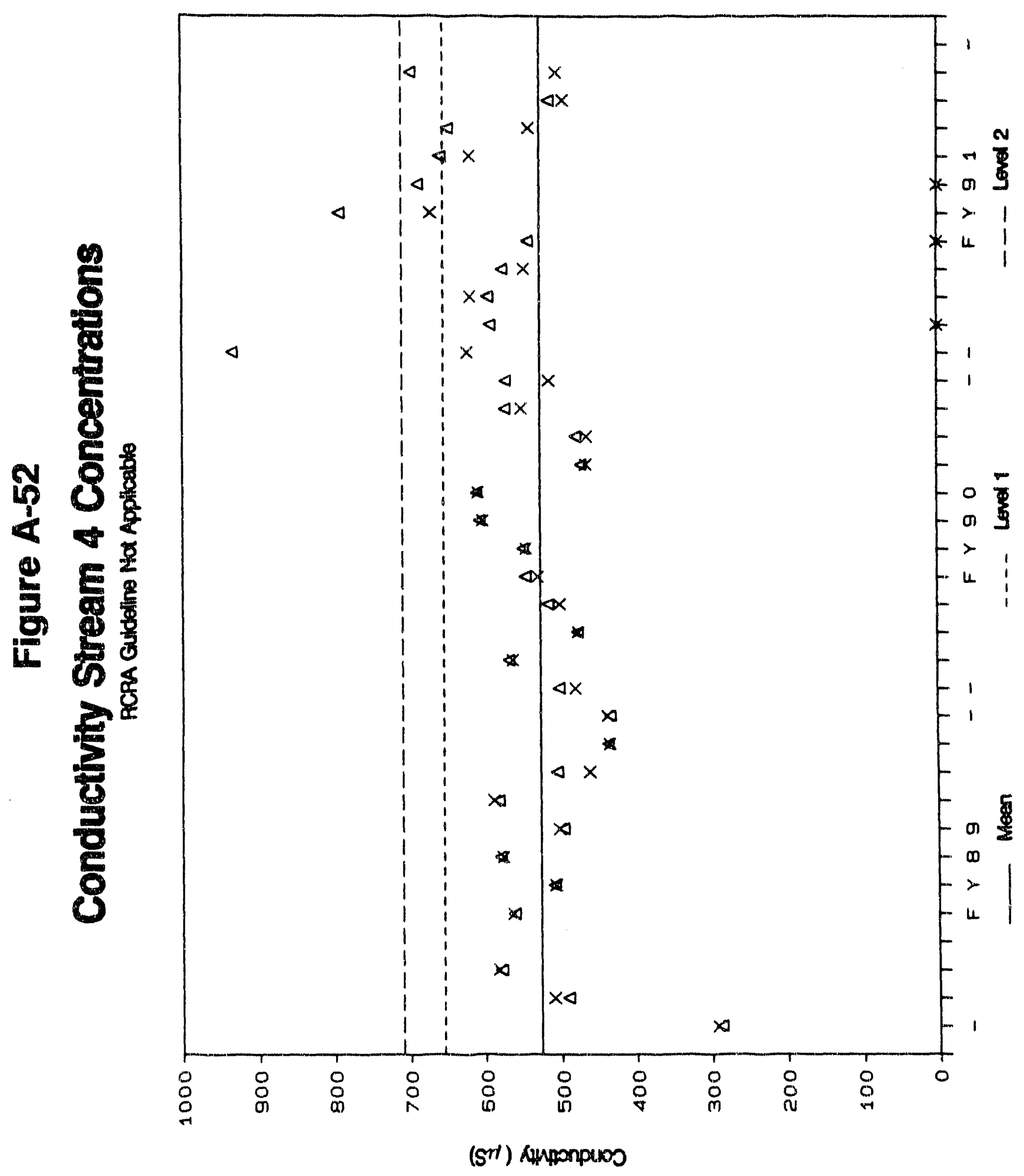




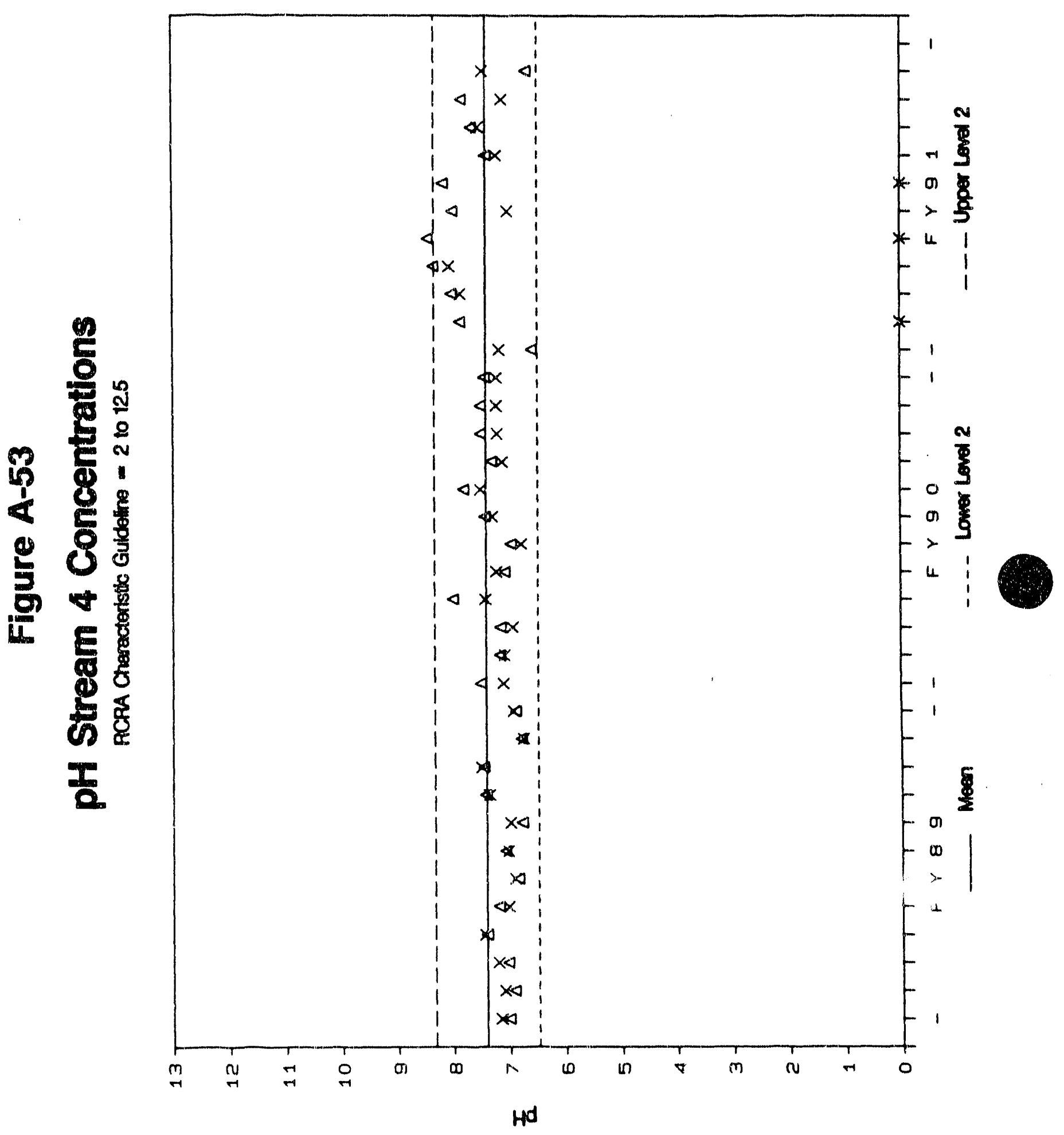




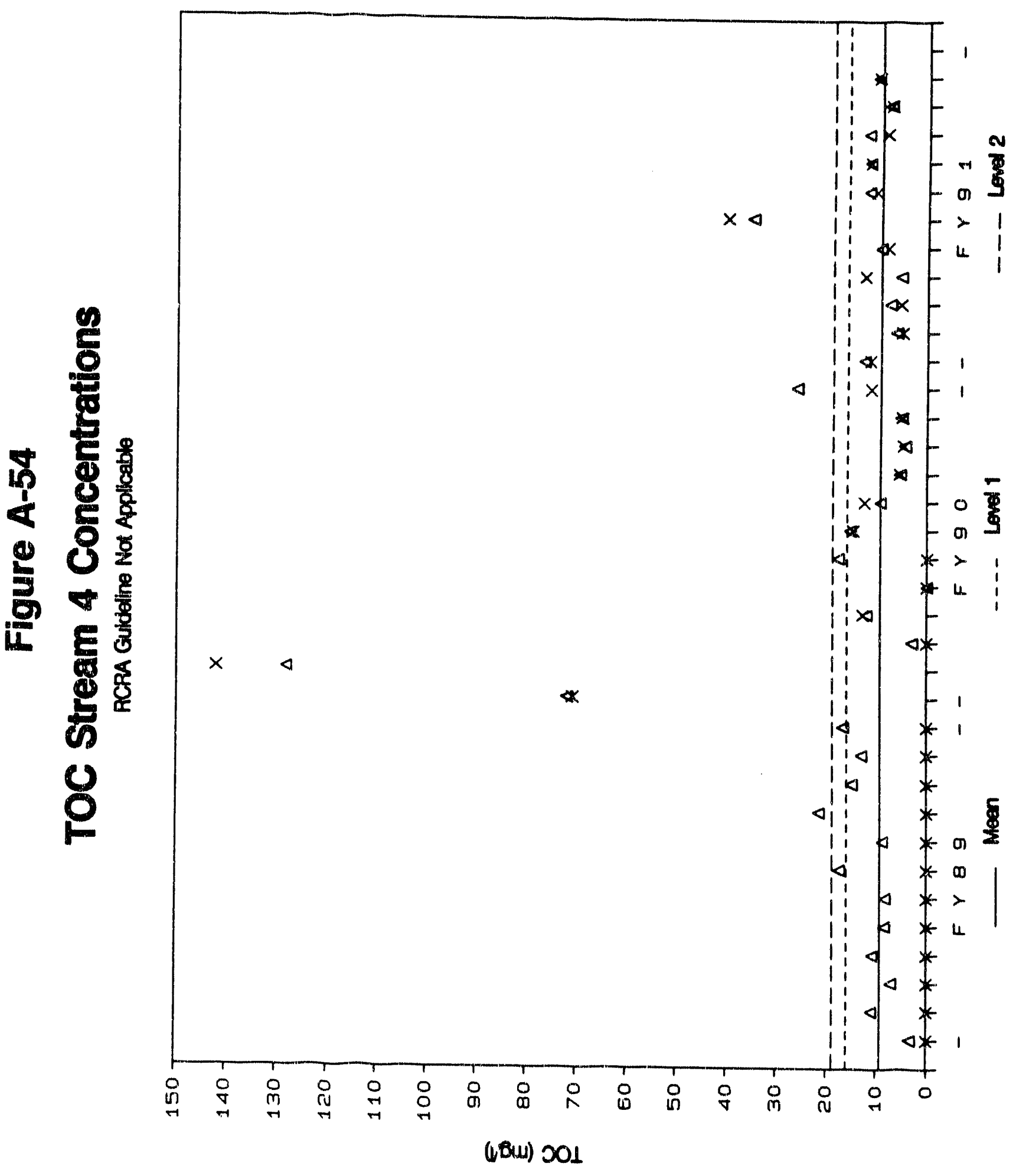




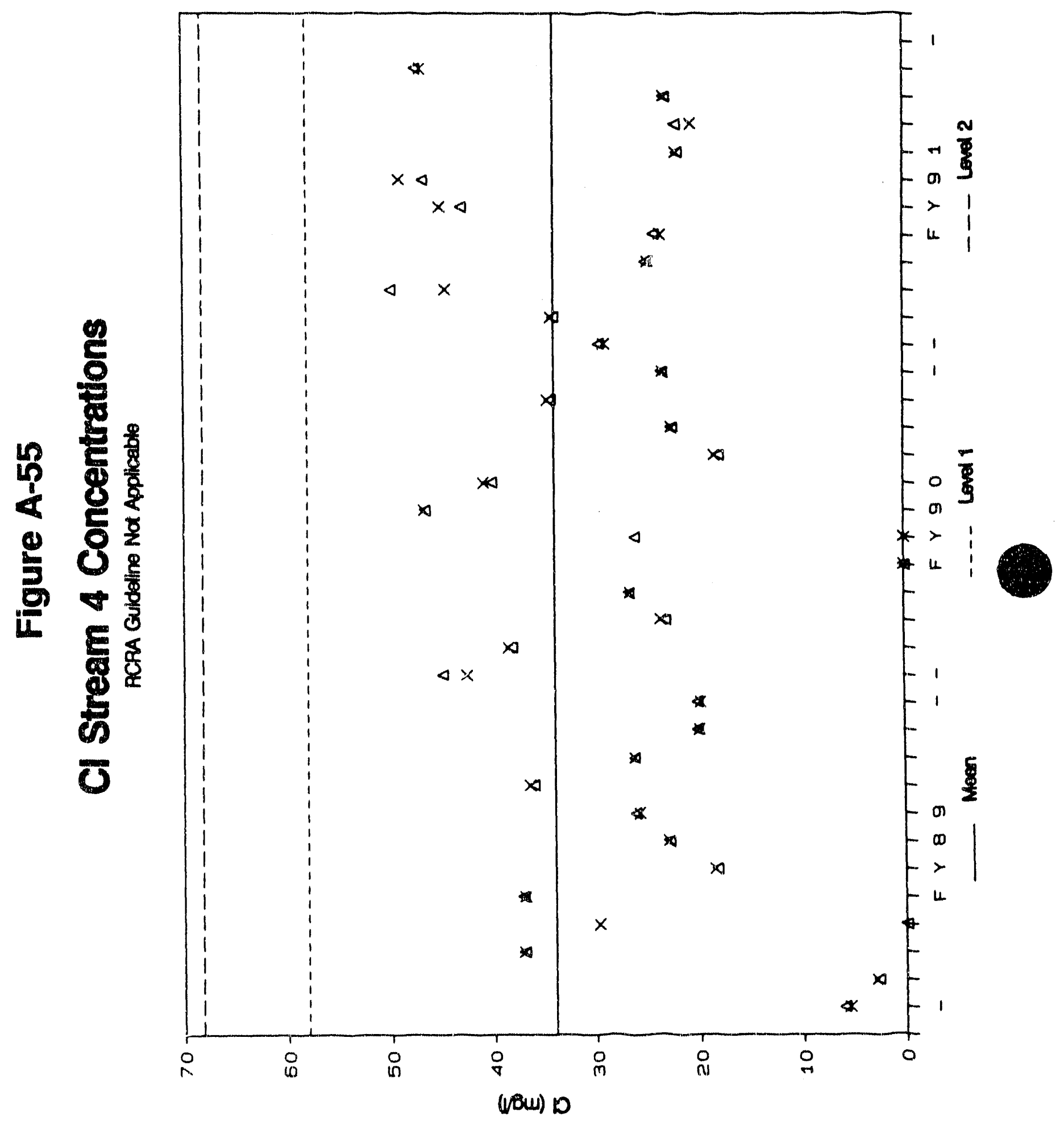




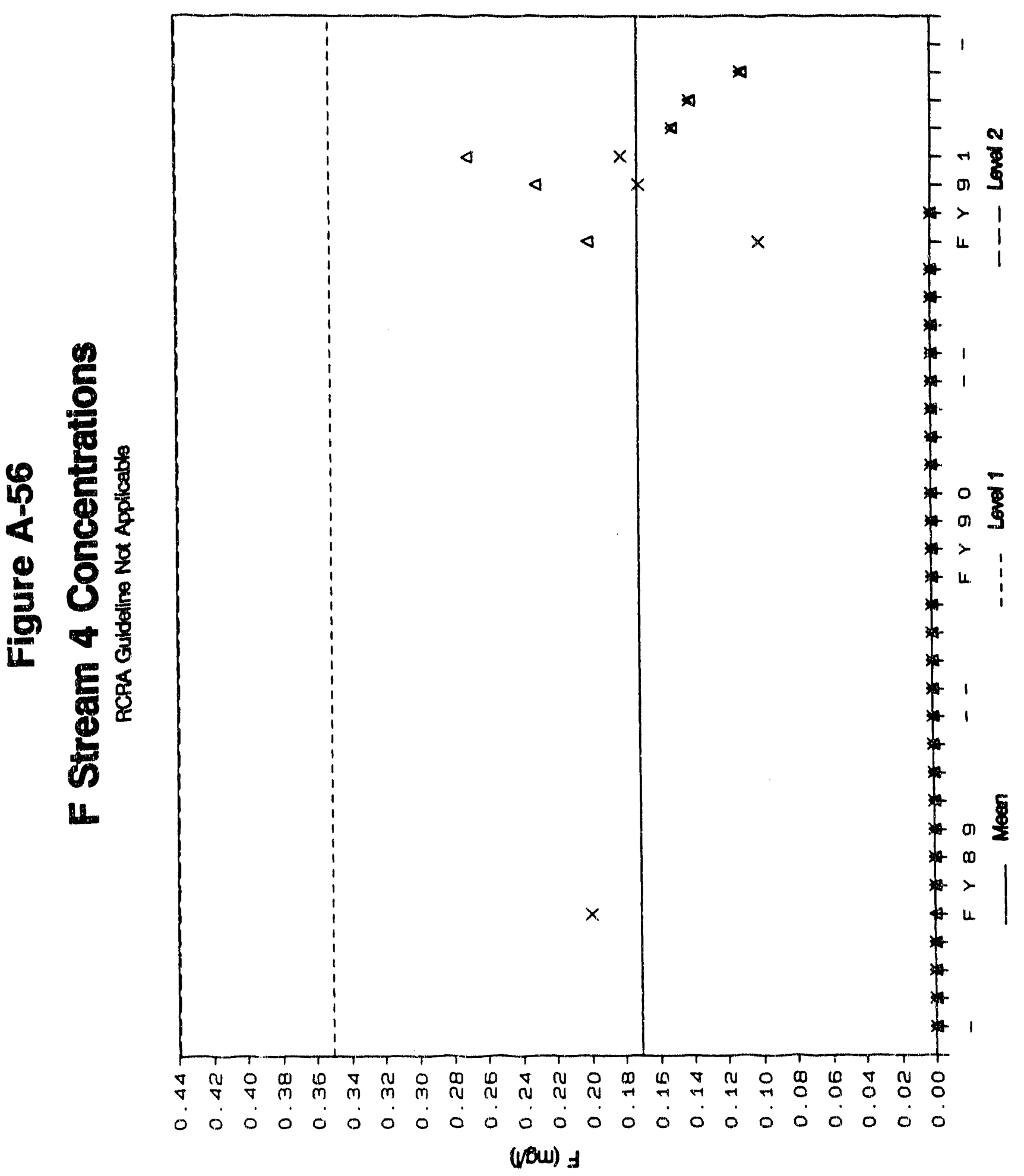




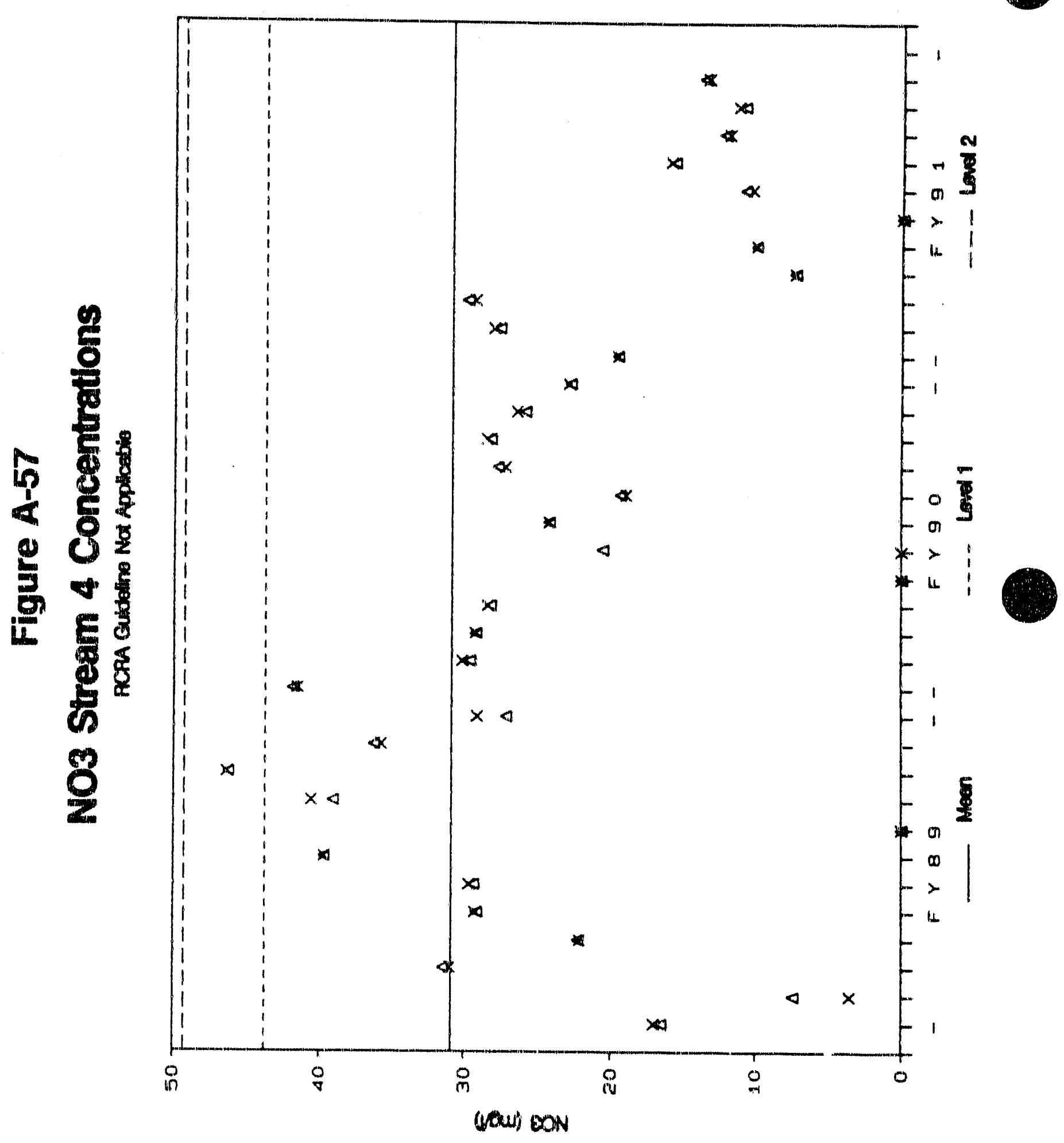




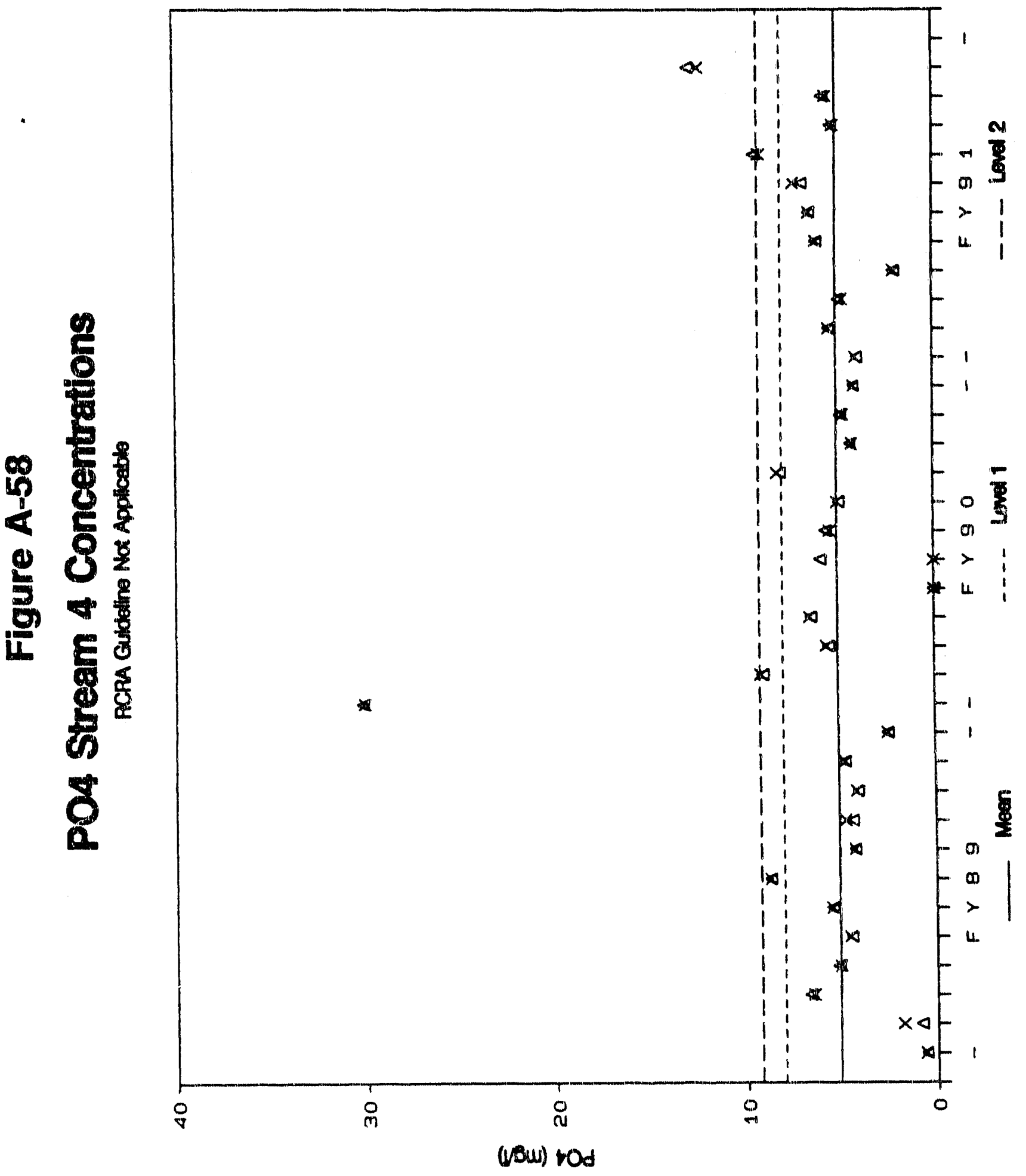




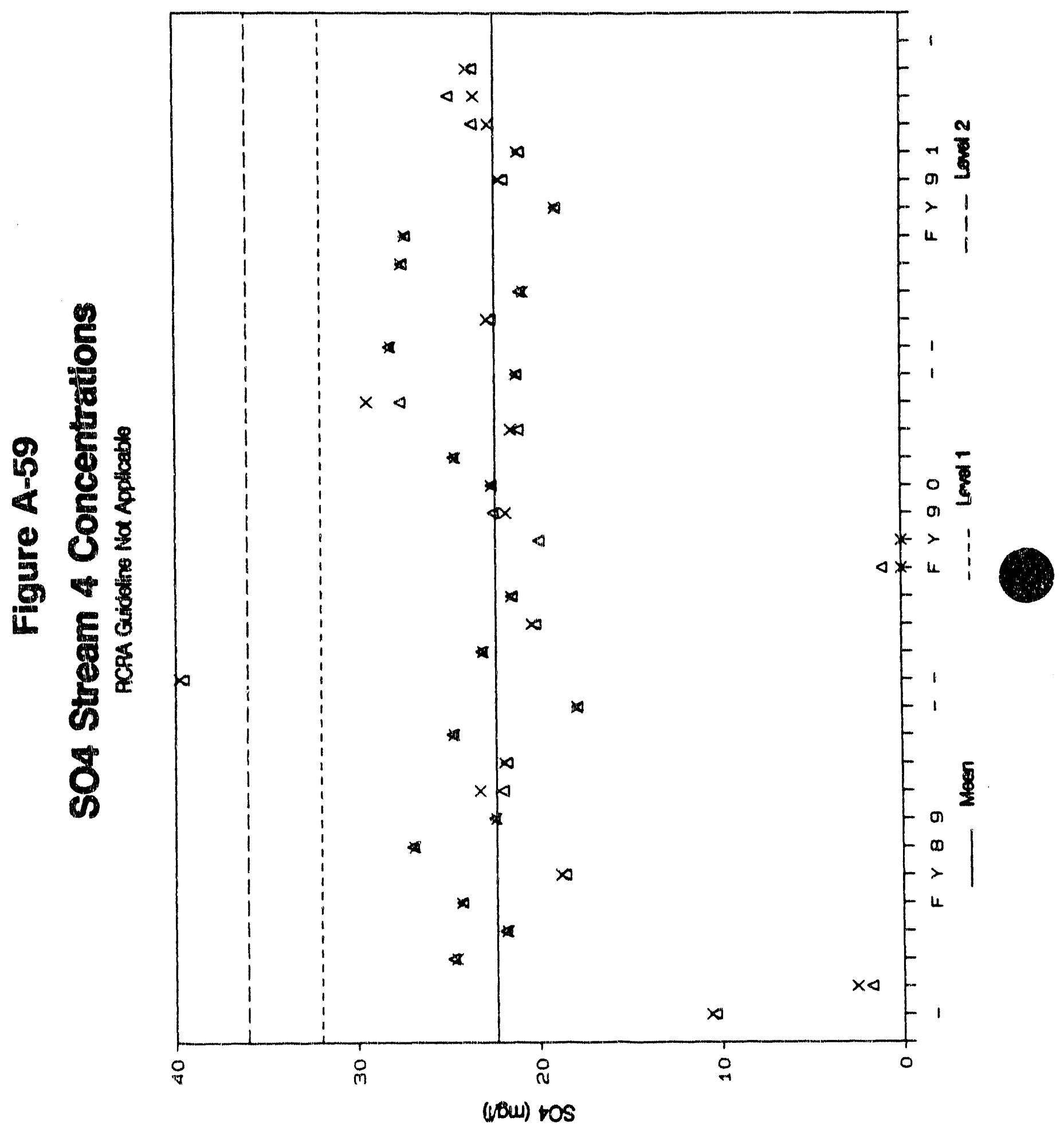




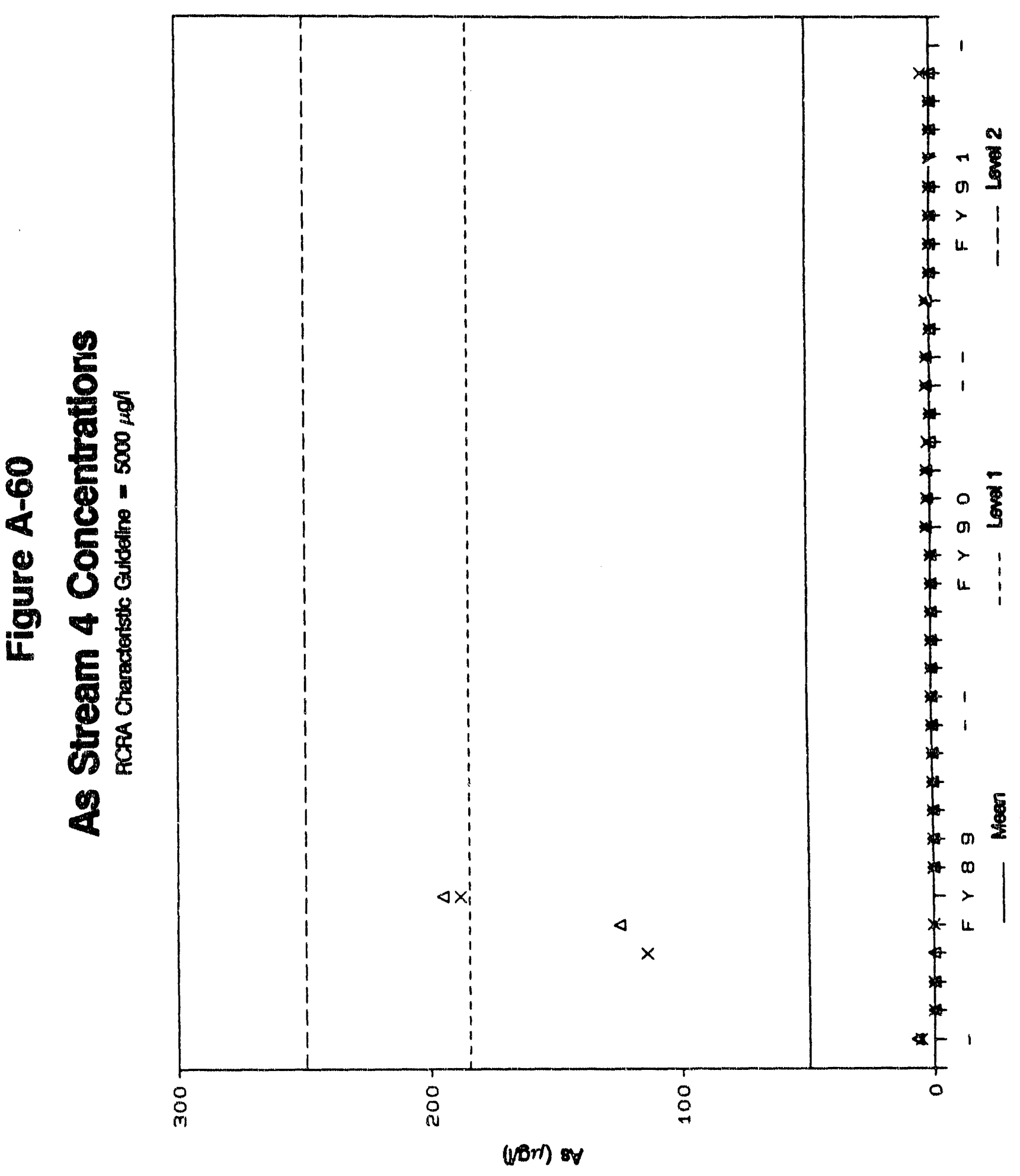




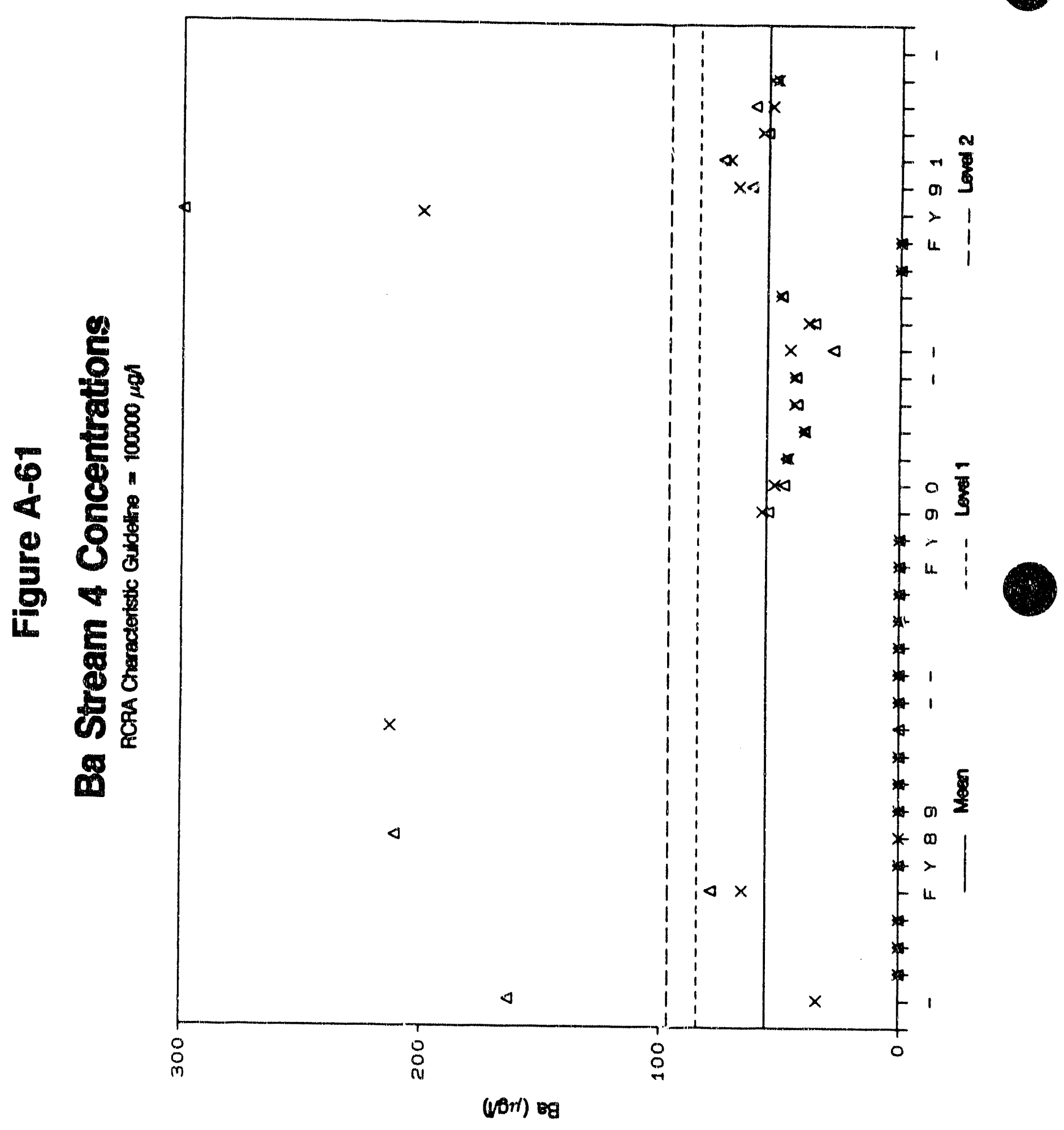




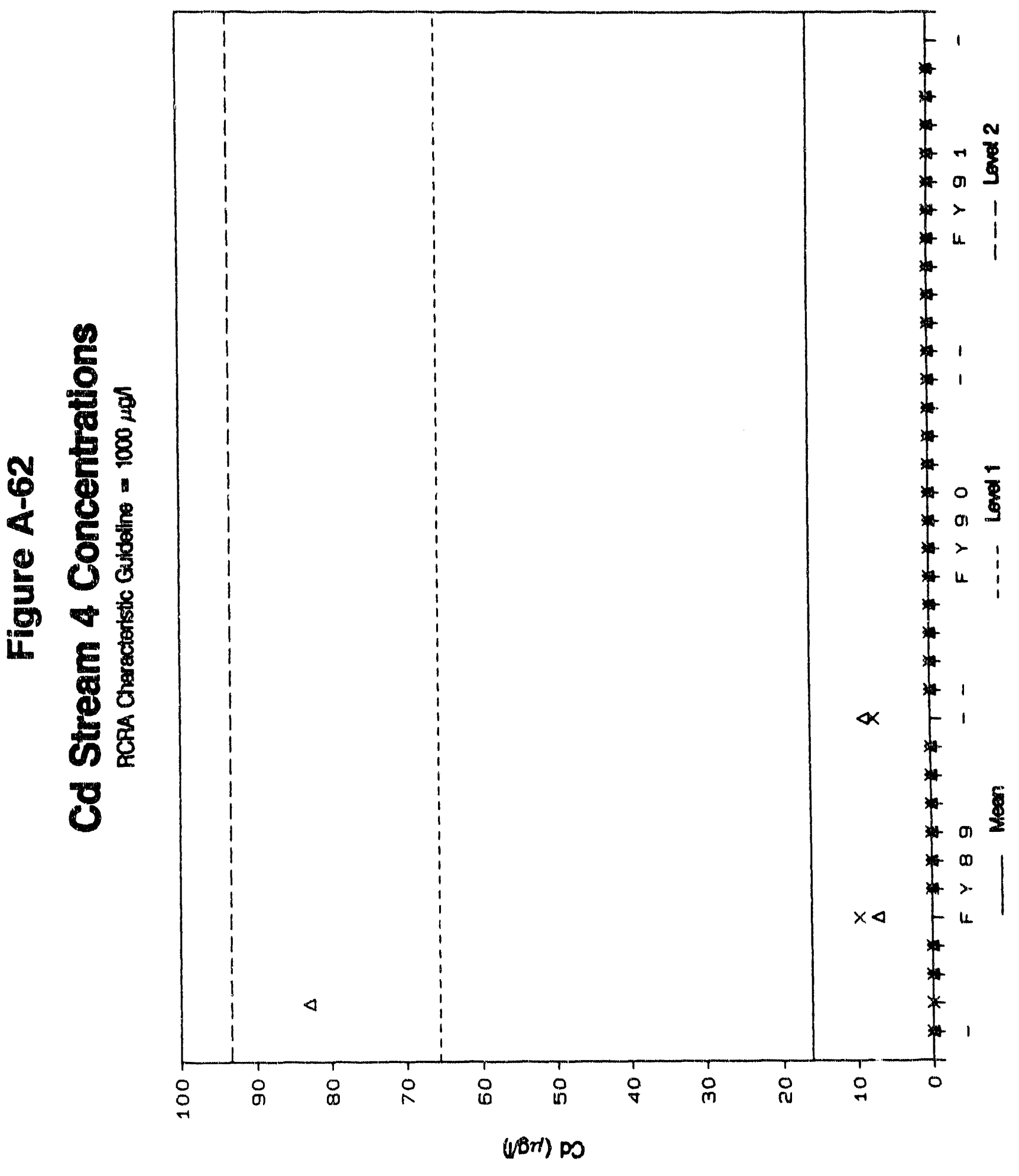




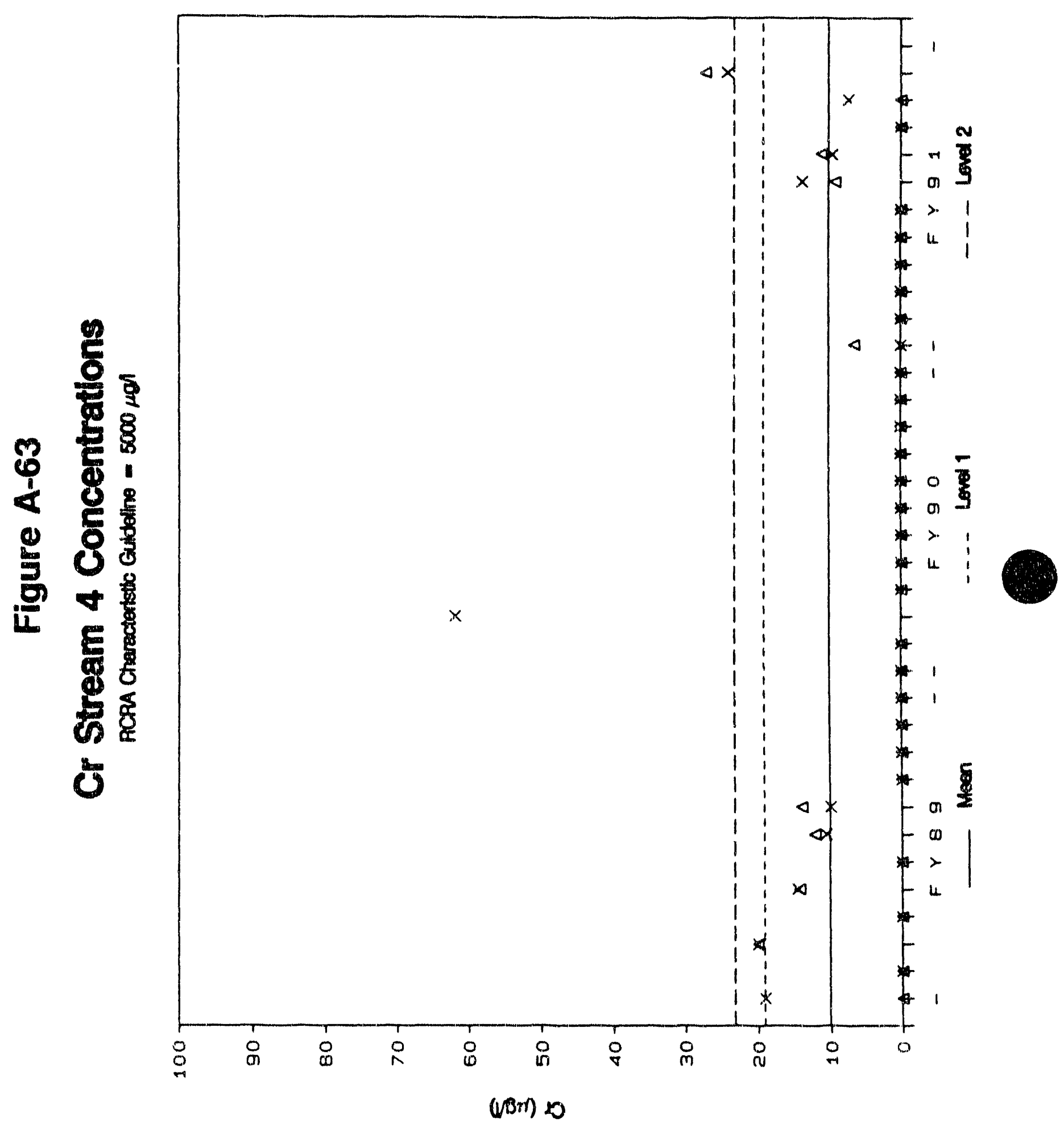




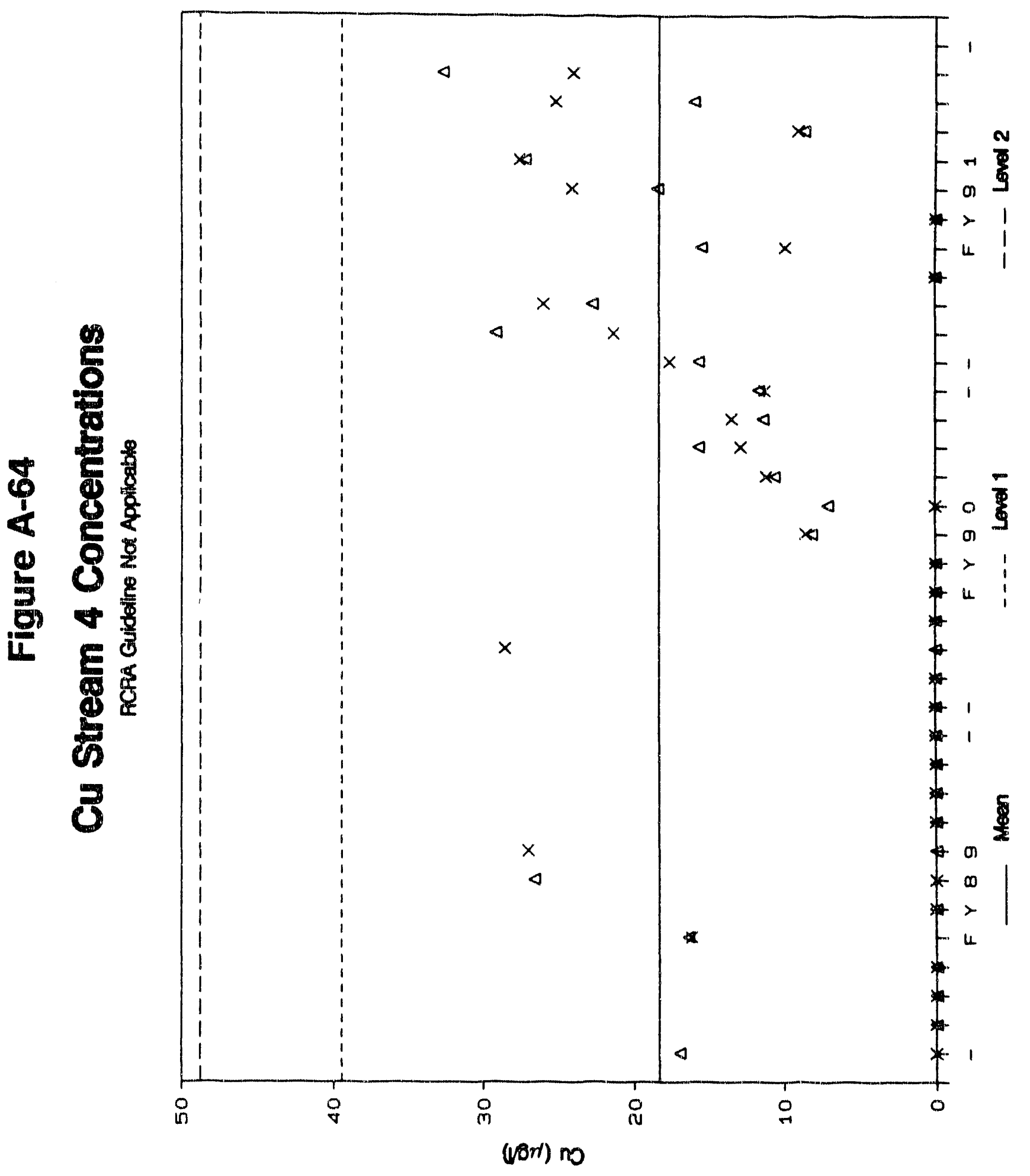




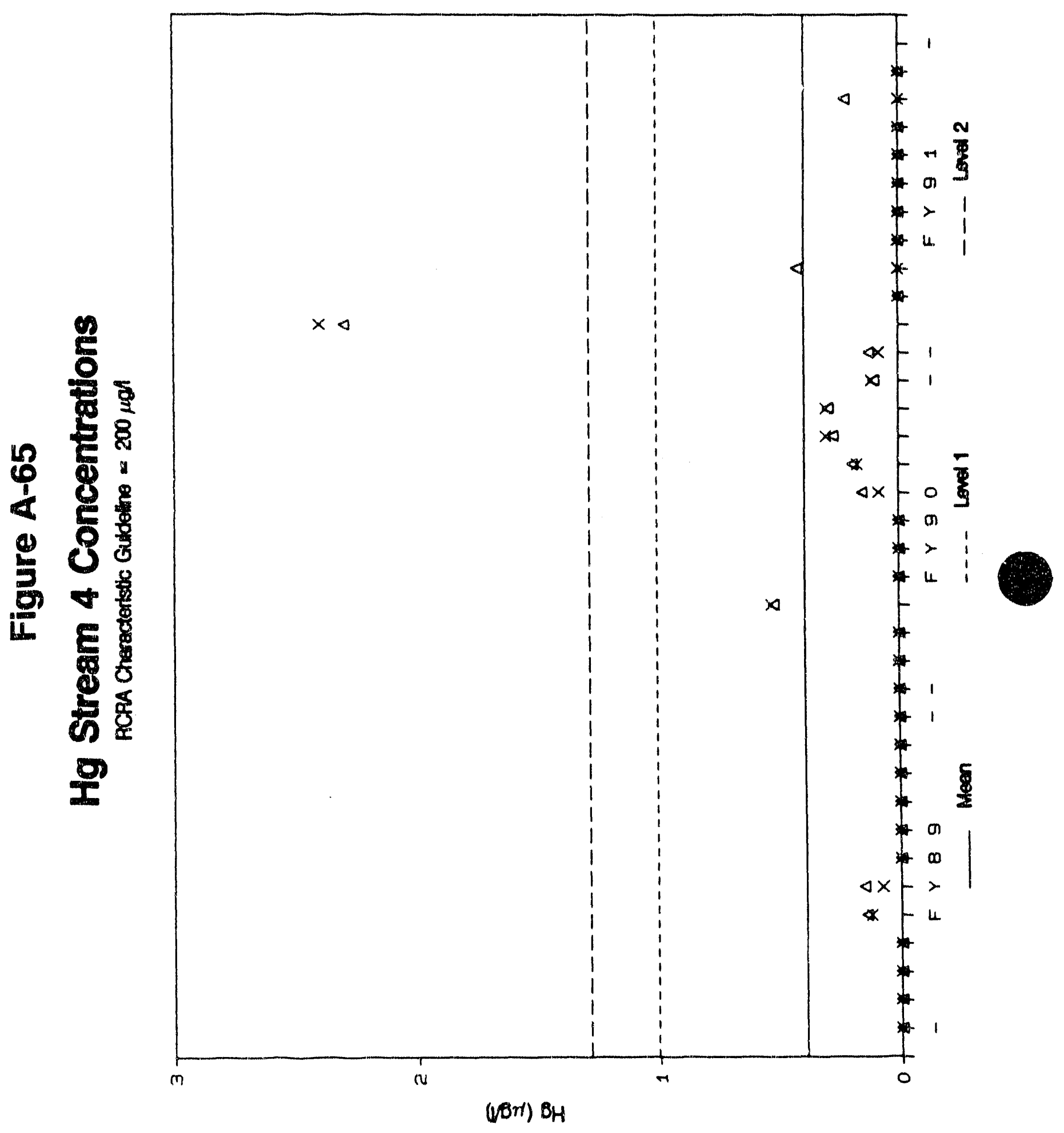




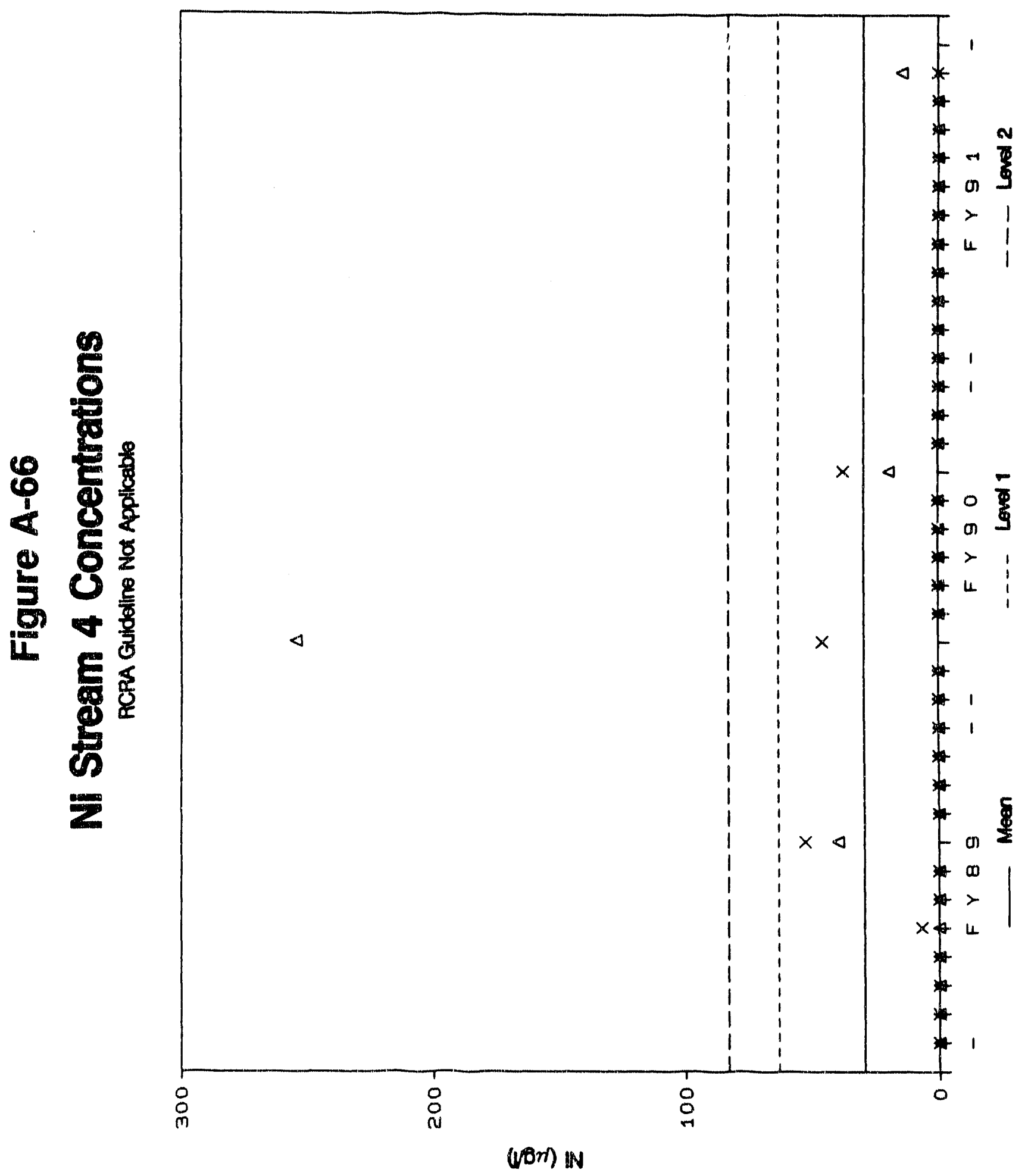




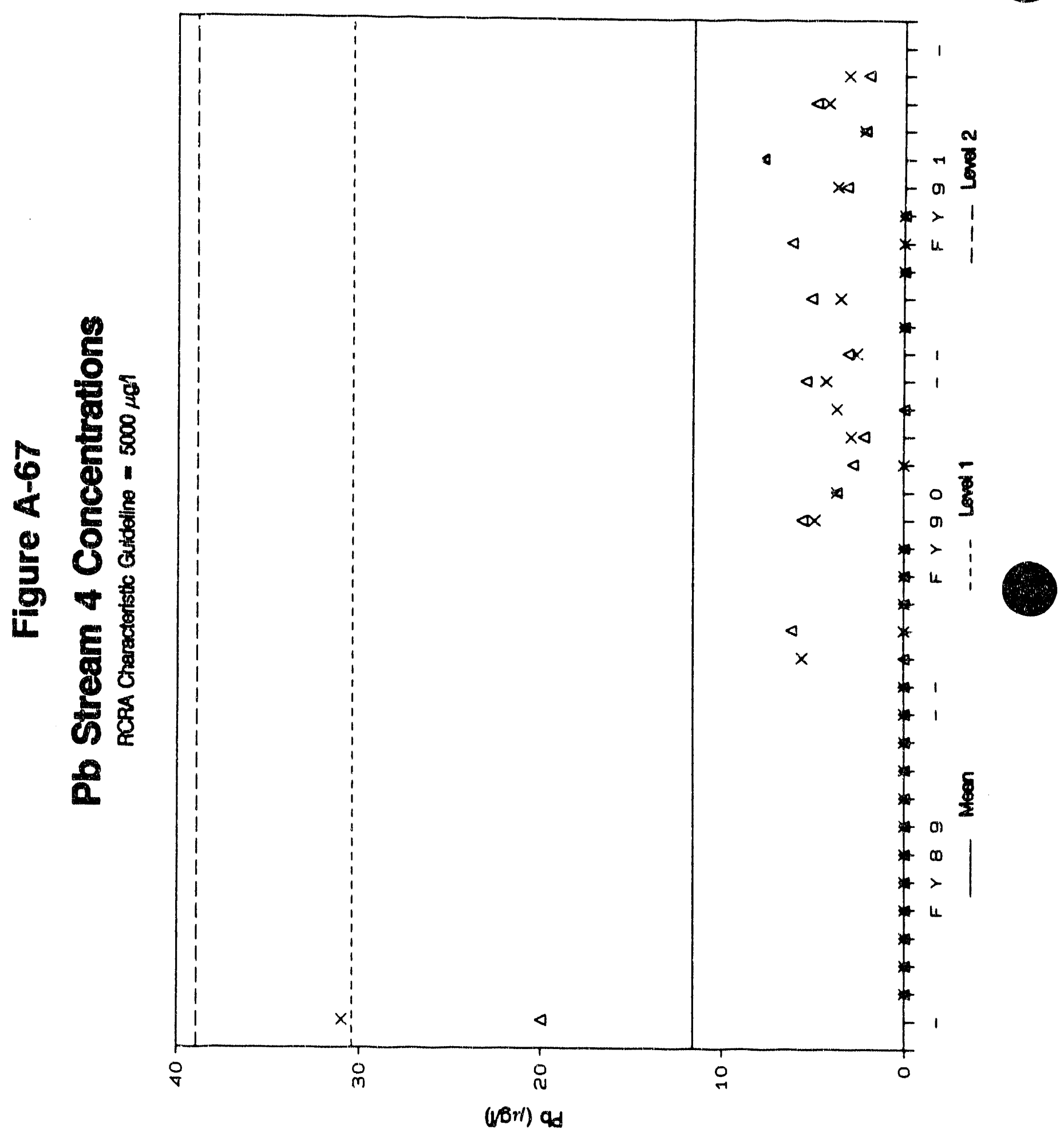




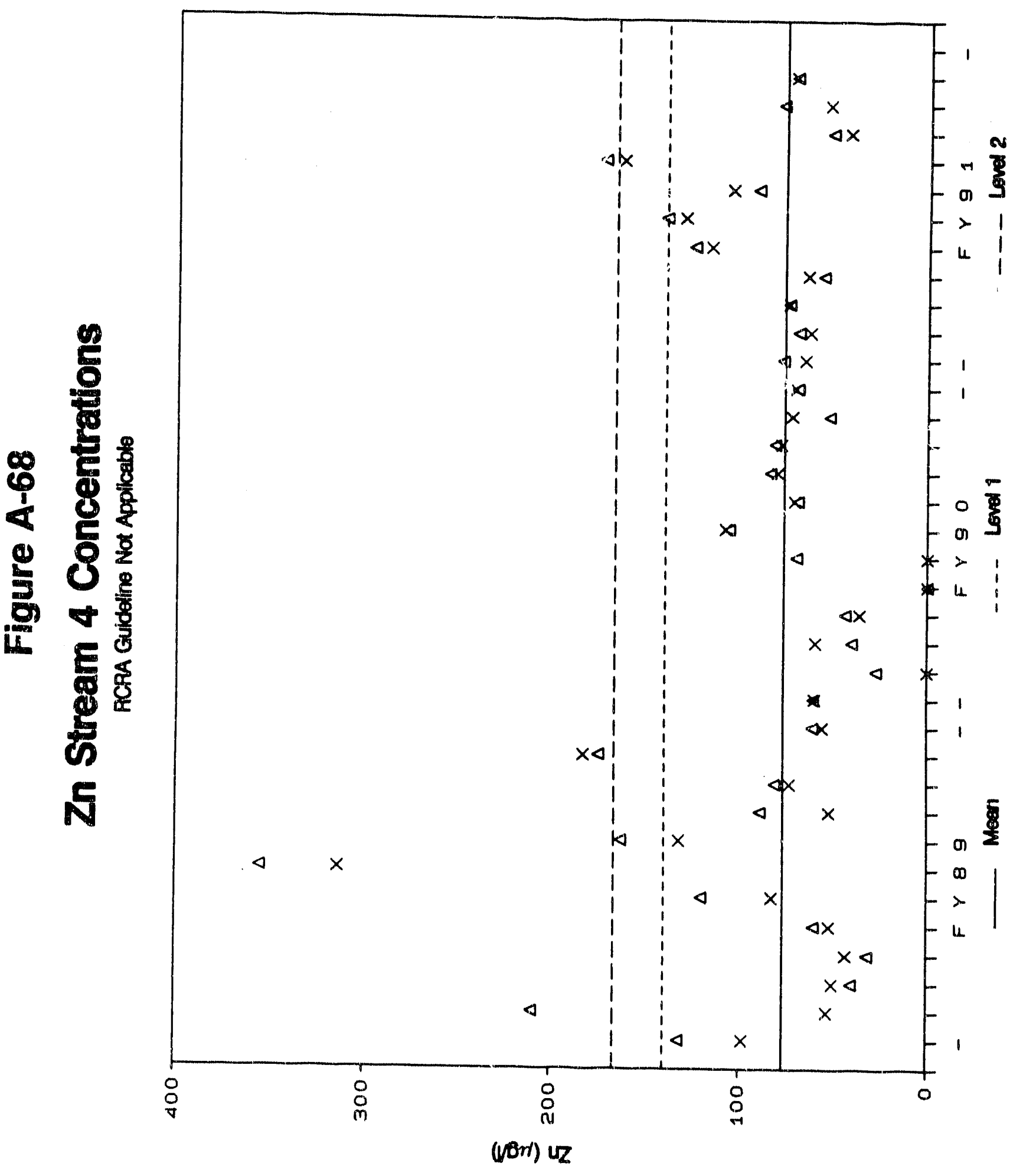




\section{Table A-18}

FY91 ENVIRONMENTAL MONITORING DATA

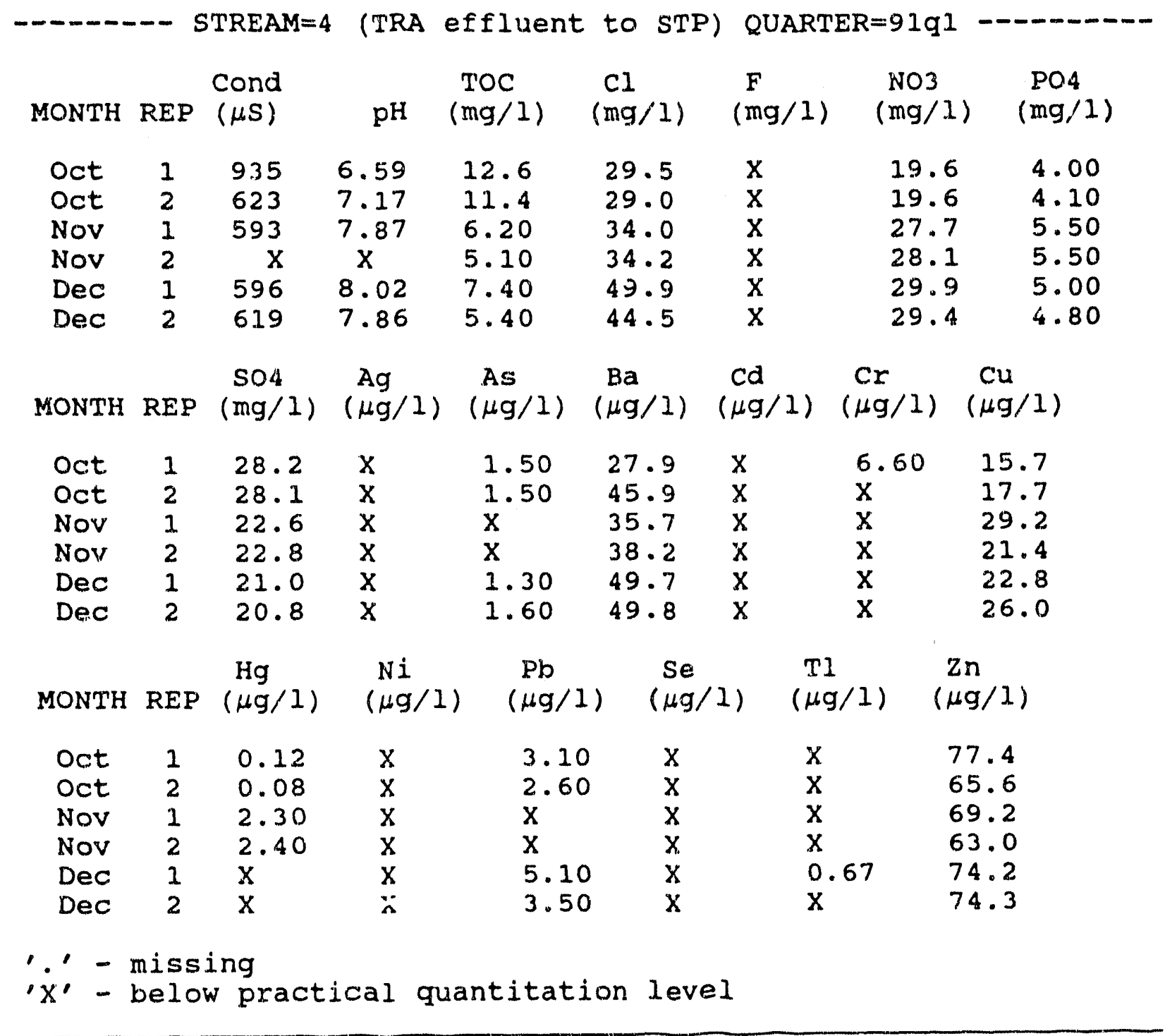




\section{Table A-19}

FY91 ENVIRONMENTAL MONITORING DATA

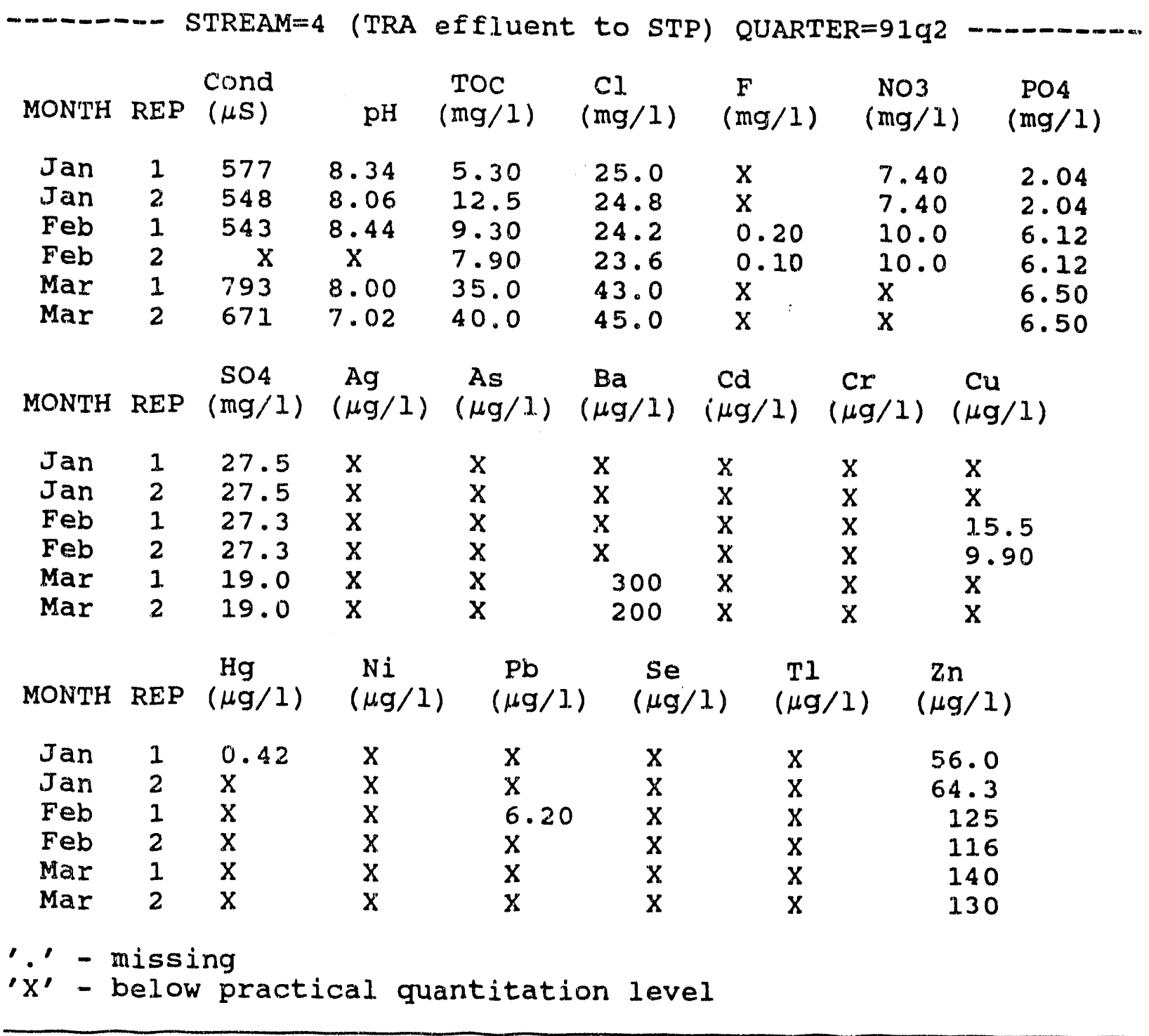


Table A-20

FY91 ENVIRONMENTAL MONITORING DATA

STREAM=4 (TRA effluent to STP) QUARTER=91q3

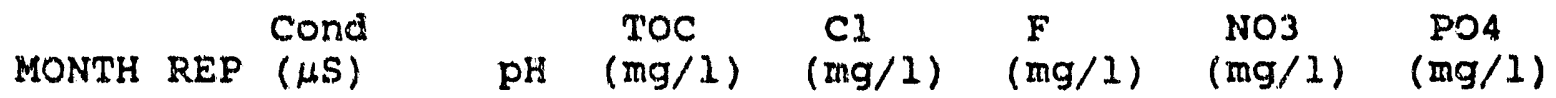

$\begin{array}{lllllllll}\text { Apr } & 1 & 687 & 8.17 & 11.8 & 46.7 & 0.23 & 10.8 & 6.88 \\ \text { Apr } & 2 & X & X & 10.3 & 49.0 & 0.17 & 1.0 .3 & 7.34 \\ \text { May } & 1 & 659 & 7.41 & 11.7 & 22.0 & 0.27 & 15.7 & 9.42 \\ \text { May } & 2 & 619 & 7.22 & 11.6 & 22.1 & 0.18 & 16.0 & 9.18 \\ \text { Jun } & 1 & 649 & 7.66 & 12.0 & 22.2 & 0.15 & 12.2 & 5.35 \\ \text { Jun } & 2 & 541 & 7.54 & 8.28 & 20.6 & 0.15 & 12.0 & 5.26\end{array}$

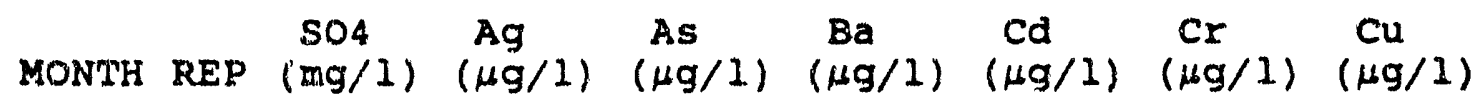

$\begin{array}{lllllllll}\text { Apr } & 1 & 21.9 & 5.90 & X & 62.4 & X & 9.20 & 18.5 \\ \text { Apr } & 2 & 22.1 & X & X & 68.0 & X & 13.8 & 24.1 \\ \text { May } & 1 & 21.0 & X & X & 74.5 & X & 11.1 & 27.2 \\ \text { May } & 2 & 21.1 & X & X & 71.5 & X & 9.60 & 27.6 \\ \text { Jun } & 1 & 23.6 & X & X & 56.1 & X & X & 8.70 \\ \text { Jun } & 2 & 22.7 & X & X & 57.7 & X & X & 9.10\end{array}$

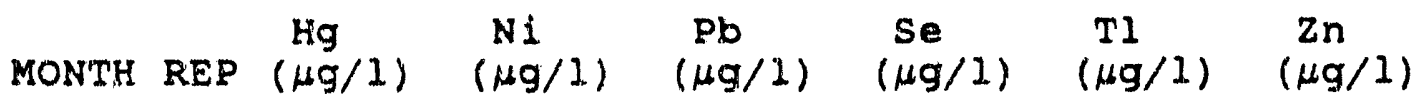

$\begin{array}{lllllllr}\text { Apr } & 1 & \mathrm{X} & \mathrm{X} & 3.20 & \mathrm{X} & \mathrm{X} & 91.2 \\ \text { Apr } & 2 & \mathrm{X} & \mathrm{X} & 3.70 & \mathrm{X} & \mathrm{X} & 105 \\ \text { May } & 1 & \mathrm{X} & \mathrm{X} & 7.80 & \mathrm{X} & \mathrm{X} & 173 \\ \text { May } & 2 & \mathrm{X} & \mathrm{X} & 7.80 & \mathrm{X} & \mathrm{X} & 163 \\ \text { Jun } & 1 & \mathrm{X} & \mathrm{X} & 2.20 & \mathrm{X} & \mathrm{X} & 51.8 \\ \text { Jun } & 2 & \mathrm{X} & \mathrm{X} & 2.20 & \mathrm{X} & \mathrm{X} & 42.3\end{array}$

$'$ ' - missing

' $x$ ' - below practical quantitation level 


\section{Table A-21}

FY91 ENVIRONMENTAL MONITORING DATA

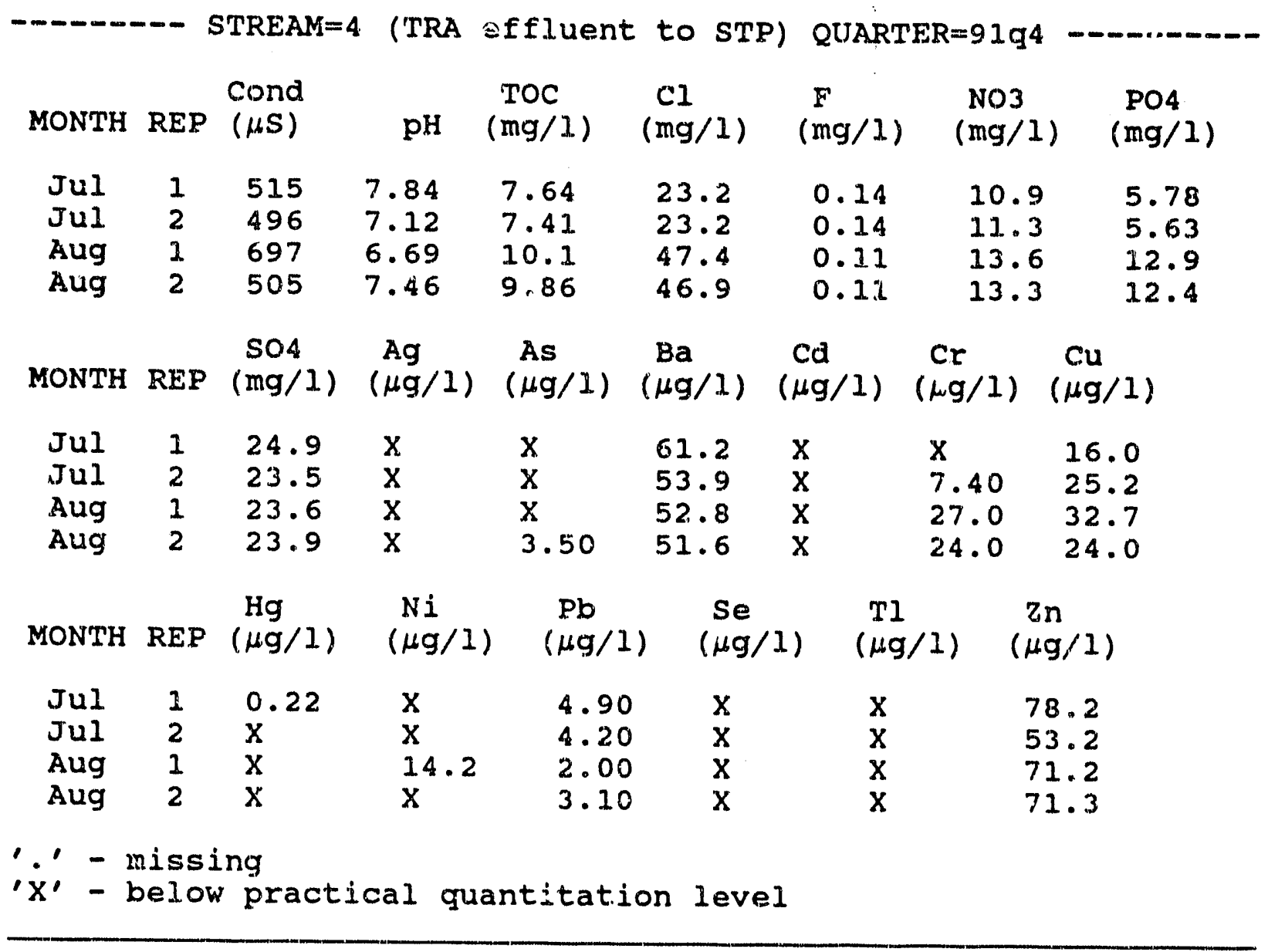


Table A-22

SUMMARY STATISTICS

STREAM=4 (TRA effluent to STP)

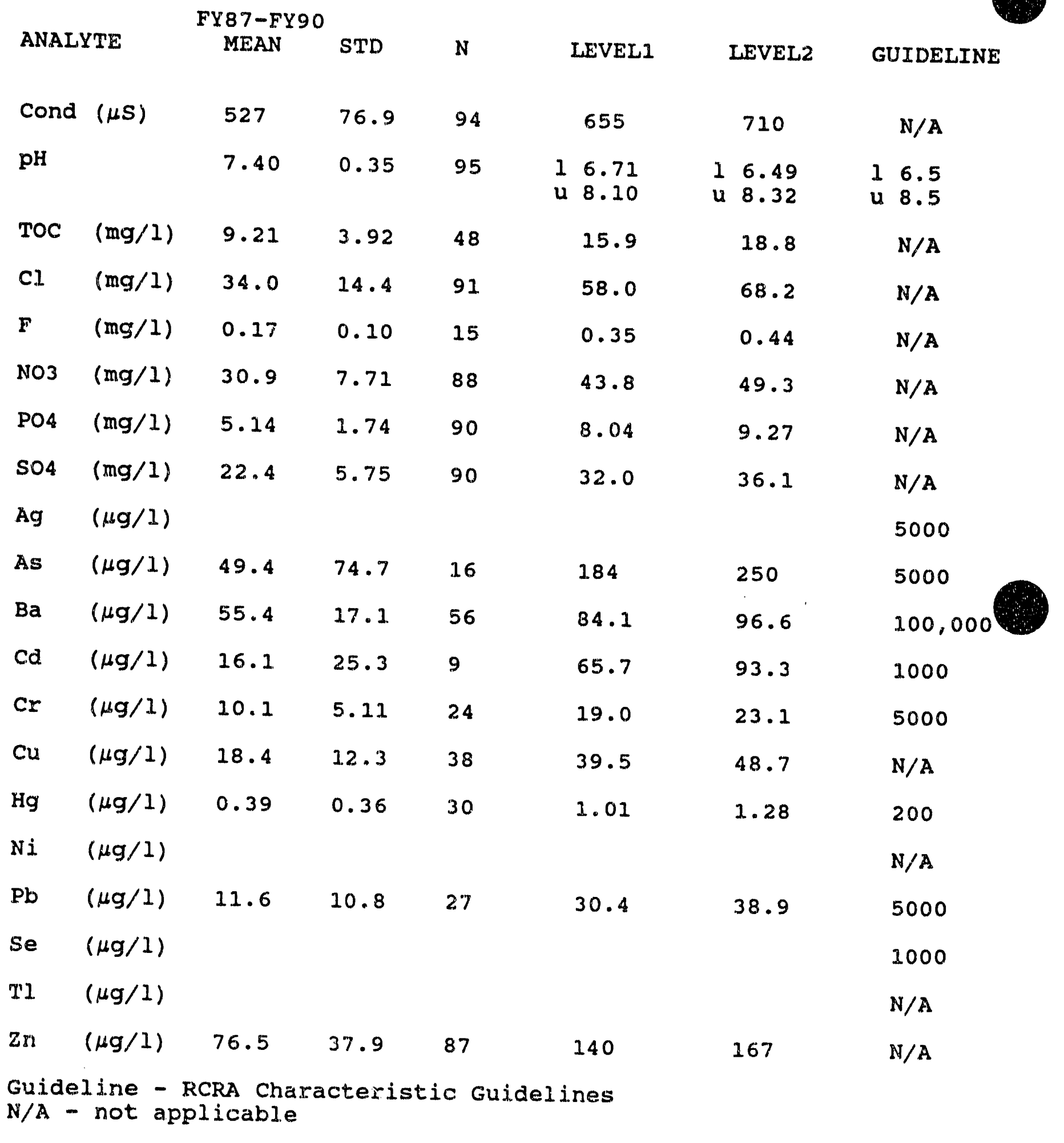


Table A-23

QUARTERLY MEANS AND VARIANCE COMPONENTS

STREAM=4 (TRA effluent to STP)

\begin{tabular}{|c|c|c|c|c|c|c|c|c|c|}
\hline \multicolumn{2}{|c|}{ ANALYTE } & \multirow{2}{*}{$\begin{array}{l}\text { QTR } \\
91 q 1 \\
91 q 2 \\
91 q 3 \\
91 q 4\end{array}$} & \multirow{2}{*}{$\begin{array}{c}\text { MEAN } \\
673 \\
626 \\
631 \\
553\end{array}$} & \multirow{2}{*}{$\begin{array}{r}\text { STD } \\
\text { MEAN } \\
60.0 \\
64.1 \\
24.0 \\
47.7\end{array}$} & \multirow{2}{*}{$\begin{array}{l}\text { STD } \\
\text { DAY } \\
0.00 \\
96.1 \\
0.00 \\
0.00\end{array}$} & \multirow{2}{*}{$\begin{array}{r}\text { STD } \\
\text { REP } \\
\\
156 \\
62.7 \\
57.6 \\
96.5\end{array}$} & \multirow{2}{*}{$\begin{array}{l}N \\
5 \\
5 \\
5 \\
4\end{array}$} & \multirow{2}{*}{$\begin{array}{c}\text { N } \\
\text { MISS } \\
1 \\
1 \\
1 \\
0\end{array}$} & \multirow{2}{*}{$\begin{array}{c}\mathrm{N} \\
\text { BPQL } \\
0 \\
0 \\
0 \\
0\end{array}$} \\
\hline Cond & $(\mu S)$ & & & & & & & & \\
\hline $\mathrm{pH}$ & & $\begin{array}{l}91 q 1 \\
91 q^{2} \\
91 q^{3} \\
91 q^{4}\end{array}$ & $\begin{array}{l}7.50 \\
7.97 \\
7.60 \\
7.28\end{array}$ & $\begin{array}{l}0.38 \\
0.28 \\
0.23 \\
0.20\end{array}$ & $\begin{array}{l}0.59 \\
0.27 \\
0.38 \\
0.00\end{array}$ & $\begin{array}{l}0.30 \\
0.51 \\
0.11 \\
0.53\end{array}$ & $\begin{array}{l}5 \\
5 \\
5 \\
4\end{array}$ & $\begin{array}{l}1 \\
1 \\
1 \\
0\end{array}$ & $\begin{array}{l}0 \\
0 \\
0 \\
0\end{array}$ \\
\hline TOC & $(\mathrm{mg} / \mathrm{I})$ & $\begin{array}{l}91 q 1 \\
91 q^{2} \\
91 q^{3} \\
91 q^{4}\end{array}$ & $\begin{array}{l}8.02 \\
18.3 \\
10.9 \\
8.75\end{array}$ & $\begin{array}{l}2.00 \\
9.58 \\
0.44 \\
1.23\end{array}$ & $\begin{array}{l}3.39 \\
16.4 \\
0.00 \\
1.73\end{array}$ & $\begin{array}{l}1.05 \\
3.62 \\
1.64 \\
0.17\end{array}$ & $\begin{array}{l}6 \\
6 \\
6 \\
4\end{array}$ & $\begin{array}{l}0 \\
0 \\
0 \\
0\end{array}$ & $\begin{array}{l}0 \\
0 \\
0 \\
0\end{array}$ \\
\hline $\mathrm{Cl}$ & $(\mathrm{mg} / \mathrm{I})$ & $\begin{array}{l}91 q 1 \\
91 q 2 \\
91 q^{3} \\
91 q^{4}\end{array}$ & $\begin{array}{l}36.8 \\
30.9 \\
30.4 \\
35.2\end{array}$ & $\begin{array}{l}5.36 \\
6.54 \\
8.71 \\
12.0\end{array}$ & $\begin{array}{l}9.15 \\
11.3 \\
15.1 \\
16.9\end{array}$ & $\begin{array}{l}2.22 \\
0.86 \\
1.14 \\
0.25\end{array}$ & $\begin{array}{l}6 \\
6 \\
6 \\
4\end{array}$ & $\begin{array}{l}0 \\
0 \\
0 \\
0\end{array}$ & $\begin{array}{l}0 \\
0 \\
0 \\
0\end{array}$ \\
\hline$F$ & $(\mathrm{mg} / \mathrm{l})$ & $\begin{array}{l}91 q 1 \\
91 q 2 \\
91 q^{3} \\
91 q^{4}\end{array}$ & $\begin{array}{l}x \\
0.15 \\
0.19 \\
0.13\end{array}$ & $\begin{array}{l}\dot{0} \\
0.02 \\
0.02\end{array}$ & $\begin{array}{l}\dot{0} \\
\dot{0} .02 \\
0.02\end{array}$ & $\begin{array}{l}\dot{0} \\
0.04 \\
0.00\end{array}$ & $\begin{array}{l}0 \\
2 \\
6 \\
4\end{array}$ & $\begin{array}{l}0 \\
0 \\
0 \\
0\end{array}$ & $\begin{array}{l}6 \\
4 \\
0 \\
0\end{array}$ \\
\hline No3 & $(\mathrm{mg} / 1)$ & $\begin{array}{l}91 q^{2} \\
91 q^{2} \\
91 q^{3} \\
91 q^{4}\end{array}$ & $\begin{array}{l}25.7 \\
8.70 \\
12.8 \\
12.3\end{array}$ & $\begin{array}{l}3.10 \\
1.30 \\
1.57 \\
1.17\end{array}$ & $\begin{array}{l}5.37 \\
1.84 \\
2.71 \\
1.65\end{array}$ & $\begin{array}{l}0.26 \\
0.00 \\
0.26 \\
0.25\end{array}$ & $\begin{array}{l}6 \\
4 \\
6 \\
4\end{array}$ & $\begin{array}{l}0 \\
2 \\
0 \\
0\end{array}$ & $\begin{array}{l}0 \\
0 \\
0 \\
0\end{array}$ \\
\hline PO4 & $(\mathrm{mg} / \mathrm{l})$ & $\begin{array}{l}91 q^{1} \\
91 q^{2} \\
91 q^{3} \\
91 q^{4}\end{array}$ & $\begin{array}{l}4.82 \\
4.89 \\
7.24 \\
9.17\end{array}$ & $\begin{array}{l}0.42 \\
1.43 \\
1.16 \\
3.46\end{array}$ & $\begin{array}{l}0.73 \\
2.47 \\
1.99 \\
4.90\end{array}$ & $\begin{array}{l}0.09 \\
0.00 \\
0.22 \\
0.26\end{array}$ & $\begin{array}{l}6 \\
6 \\
6 \\
4\end{array}$ & $\begin{array}{l}0 \\
0 \\
0 \\
0\end{array}$ & $\begin{array}{l}0 \\
0 \\
0 \\
0\end{array}$ \\
\hline So: & $(\mathrm{mg} / \mathrm{l})$ & $\begin{array}{l}91 q 1 \\
91 q 2 \\
91 q 3 \\
91 q 4\end{array}$ & $\begin{array}{l}23.9 \\
24.6 \\
22.1 \\
24.0\end{array}$ & $\begin{array}{l}2.18 \\
2.80 \\
0.61 \\
0.23\end{array}$ & $\begin{array}{l}3.77 \\
4.85 \\
1.02 \\
0.00\end{array}$ & $\begin{array}{l}0.12 \\
0.00 \\
0.38 \\
0.72\end{array}$ & $\begin{array}{l}6 \\
6 \\
6 \\
4\end{array}$ & $\begin{array}{l}0 \\
0 \\
0 \\
0\end{array}$ & $\begin{array}{l}0 \\
0 \\
0 \\
0\end{array}$ \\
\hline Ag & $(\mu g / 1)$ & $\begin{array}{l}91 q 1 \\
91 q^{2} \\
91 q^{3} \\
91 q^{4}\end{array}$ & $\begin{array}{l}x \\
x \\
5.90 \\
x\end{array}$ & $\dot{.}$ & $\dot{.}$ & $\dot{\dot{.}}$ & $\begin{array}{l}0 \\
0 \\
1 \\
0\end{array}$ & $\begin{array}{l}0 \\
0 \\
0 \\
0\end{array}$ & $\begin{array}{l}6 \\
6 \\
5 \\
6\end{array}$ \\
\hline
\end{tabular}




\section{Table A-23 (continued)}

QUARTERLY MEANS AND VARIANCE COMPONENTS

STREAM $=4$

(TRA effluent to STP)

(continued)

\begin{tabular}{|c|c|c|c|c|c|c|c|c|}
\hline \multicolumn{2}{|c|}{ ANALYTE } & QTR & MEAN & $\begin{array}{r}\text { STD } \\
\text { MEAN }\end{array}$ & $\begin{array}{l}\text { STD } \\
\text { DAY }\end{array}$ & $\begin{array}{l}\text { STD } \\
\text { REP }\end{array}$ & $\mathrm{N}$ & MISS \\
\hline As & $(\mu g / I)$ & $\begin{array}{l}91 q 1 \\
91 q^{2} \\
91 q^{3} \\
91 q^{4}\end{array}$ & $\begin{array}{l}1.47 \\
x \\
x \\
3.50\end{array}$ & $\begin{array}{l}0.03 \\
\dot{.} \\
\dot{0}\end{array}$ & $\begin{array}{l}0.00 \\
\dot{.} \\
.\end{array}$ & $\begin{array}{l}0.15 \\
\dot{0} \\
\dot{0}\end{array}$ & $\begin{array}{l}4 \\
0 \\
0 \\
1\end{array}$ & $\begin{array}{l}0 \\
0 \\
0 \\
0\end{array}$ \\
\hline $\mathrm{Ba}$ & $(\mu g / I)$ & $\begin{array}{l}91 q^{1} \\
91 q^{2} \\
91 q^{3} \\
91 q^{4}\end{array}$ & $\begin{array}{r}41.2 \\
250 \\
65.0 \\
54.9\end{array}$ & $\begin{array}{l}4.28 \\
\dot{4} .65 \\
2.67\end{array}$ & $\begin{array}{l}5.23 \\
7.83 \\
2.73\end{array}$ & $\begin{array}{l}7.42 \\
\dot{2} .67 \\
3.70\end{array}$ & $\begin{array}{l}6 \\
2 \\
6 \\
4\end{array}$ & $\begin{array}{l}0 \\
0 \\
0 \\
0\end{array}$ \\
\hline$c d$ & $(\mu g / I)$ & $\begin{array}{l}91 q 1 \\
91 q 2 \\
91 q 3 \\
91 q 4\end{array}$ & $\begin{array}{l}X \\
X \\
X \\
X\end{array}$ & $\dot{:}$ & $\begin{array}{l}\dot{.} \\
\dot{.}\end{array}$ & $\begin{array}{l}\dot{.} \\
\dot{\cdot}\end{array}$ & $\begin{array}{l}0 \\
0 \\
0 \\
0\end{array}$ & $\begin{array}{l}0 \\
0 \\
0 \\
0\end{array}$ \\
\hline $\mathrm{Cr}$ & $(\mu g / I)$ & $\begin{array}{l}91 q 1 \\
91 q 2 \\
91 q 3 \\
91 q^{4}\end{array}$ & $\begin{array}{l}6.60 \\
x \\
5.62 \\
19.5\end{array}$ & $\begin{array}{l}\dot{5} .32 \\
\dot{5}\end{array}$ & $\dot{\dot{9}} .11$ & $\dot{i} .98$ & $\begin{array}{l}1 \\
0 \\
6 \\
3\end{array}$ & $\begin{array}{l}0 \\
0 \\
0 \\
0\end{array}$ \\
\hline $\mathrm{Cu}$ & $(\mu g / 1)$ & $\begin{array}{l}91 q 1 \\
91 q 2 \\
91 q^{3} \\
91 q^{4}\end{array}$ & $\begin{array}{l}22.1 \\
12.7 \\
19.2 \\
24.5\end{array}$ & $\begin{array}{l}2.73 \\
5.44 \\
3.87\end{array}$ & $\begin{array}{l}4.01 \\
\dot{9} .29 \\
3.16\end{array}$ & $\begin{array}{l}3.54 \\
\dot{2} .30 \\
6.33\end{array}$ & $\begin{array}{l}6 \\
2 \\
6 \\
4\end{array}$ & $\begin{array}{l}0 \\
0 \\
0 \\
0\end{array}$ \\
\hline $\mathrm{Hg}$ & $(\mu g / 1)$ & $\begin{array}{l}91 q 1 \\
91 q 2 \\
91 q 3 \\
91 q 4\end{array}$ & $\begin{array}{l}1.22 \\
0.42 \\
x \\
0.22\end{array}$ & $\begin{array}{l}1.12 \\
: \\
\cdot\end{array}$ & $\begin{array}{l}1.59 \\
: \\
.\end{array}$ & $\begin{array}{l}0.054 \\
. \\
.\end{array}$ & $\begin{array}{l}4 \\
1 \\
0 \\
1\end{array}$ & $\begin{array}{l}0 \\
0 \\
0 \\
0\end{array}$ \\
\hline $\mathrm{Ni}$ & $(\mu g / l)$ & $\begin{array}{l}9.1 q 1 \\
91 q 2 \\
91 q 3 \\
91 q 4\end{array}$ & $\begin{array}{l}X \\
X \\
X \\
14.2\end{array}$ & $\dot{.}$ & $\dot{\dot{ }}$ & $\dot{\dot{ }}$ & $\begin{array}{l}0 \\
0 \\
0 \\
1\end{array}$ & $\begin{array}{l}0 \\
0 \\
0 \\
0\end{array}$ \\
\hline
\end{tabular}

$\begin{array}{llll}\mathrm{Pb} & (\mu \mathrm{g} / 1) \quad 91 \mathrm{ql} & 3.58\end{array}$

$91 q^{2}$

6.20

0.73

0.84

0.84

4

$\begin{array}{ll}0 & 2 \\ 0 & 5 \\ 0 & 0 \\ 0 & 0\end{array}$

Se $(\mu g / 1)$

$91 q^{3}$

4.48

i. 70

2. 94

0.20

3.55

1.00

1.34

0.65

1
4

N

PQL

$91 q 1$

$\begin{array}{ll}91 q 2 & x \\ 91 q^{3} & x \\ 91 q 4 & x\end{array}$

$\mathrm{x}$
$\mathrm{X}$
$\mathrm{X}$
$\mathrm{X}$




\section{Table A-23 (continued)}

\section{QUARTERLY MEANS AND VARIANCE COMPONENTS}

\section{STREAM=4 (TRA effluent to STP)}

(continued)

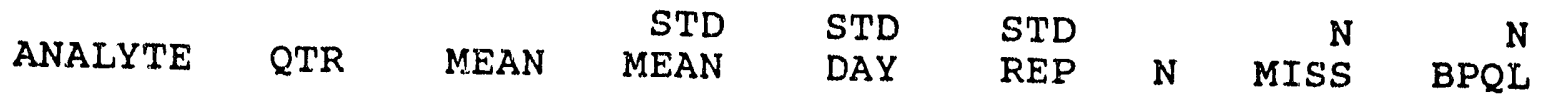

\begin{tabular}{|c|c|c|c|c|c|c|c|}
\hline $\mathrm{Tl} \quad(\mu \mathrm{g} / \mathrm{I})$ & $\begin{array}{l}91 q 1 \\
91 q 2 \\
91 q 3 \\
91 q 4\end{array}$ & $\begin{array}{l}0.67 \\
x \\
x \\
x\end{array}$ & $\dot{\bullet}$ & • & $\begin{array}{l}\circ \\
\circ\end{array}$ & $\begin{array}{l}1 \\
0 \\
0 \\
0\end{array}$ & $\begin{array}{l}0 \\
0 \\
0 \\
0\end{array}$ \\
\hline
\end{tabular}

$\begin{array}{lrrrrrrrr}\mathrm{Zn} \quad(\mu \mathrm{g} / \mathrm{l}) & 91 \mathrm{q} 1 & 70.6 & 2.39 & 1.54 & 5.44 & 6 & 0 & 0 \\ & 9192 & 105 & 22.9 & 39.4 & 6.45 & 6 & 0 & 0 \\ & 9193 & 104 & 35.1 & 60.5 & 7.97 & 6 & 0 & 0 \\ & 9194 & 68.5 & 2.77 & 0.00 & 12.5 & 4 & 0 & 0\end{array}$

'́' - missing, too little data to calculate value

' $X$ ' - below practical quantitation level (BPQL) 


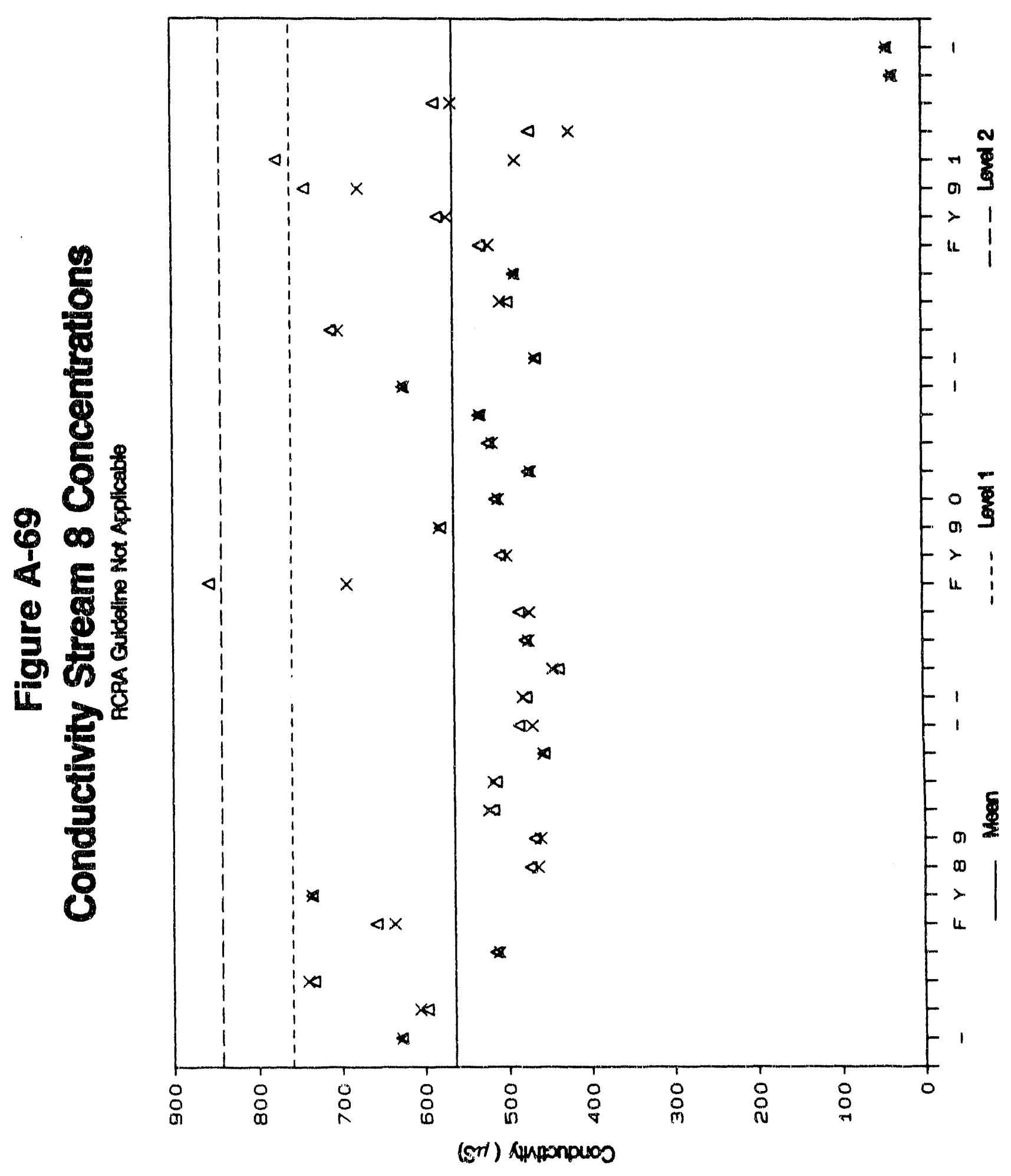




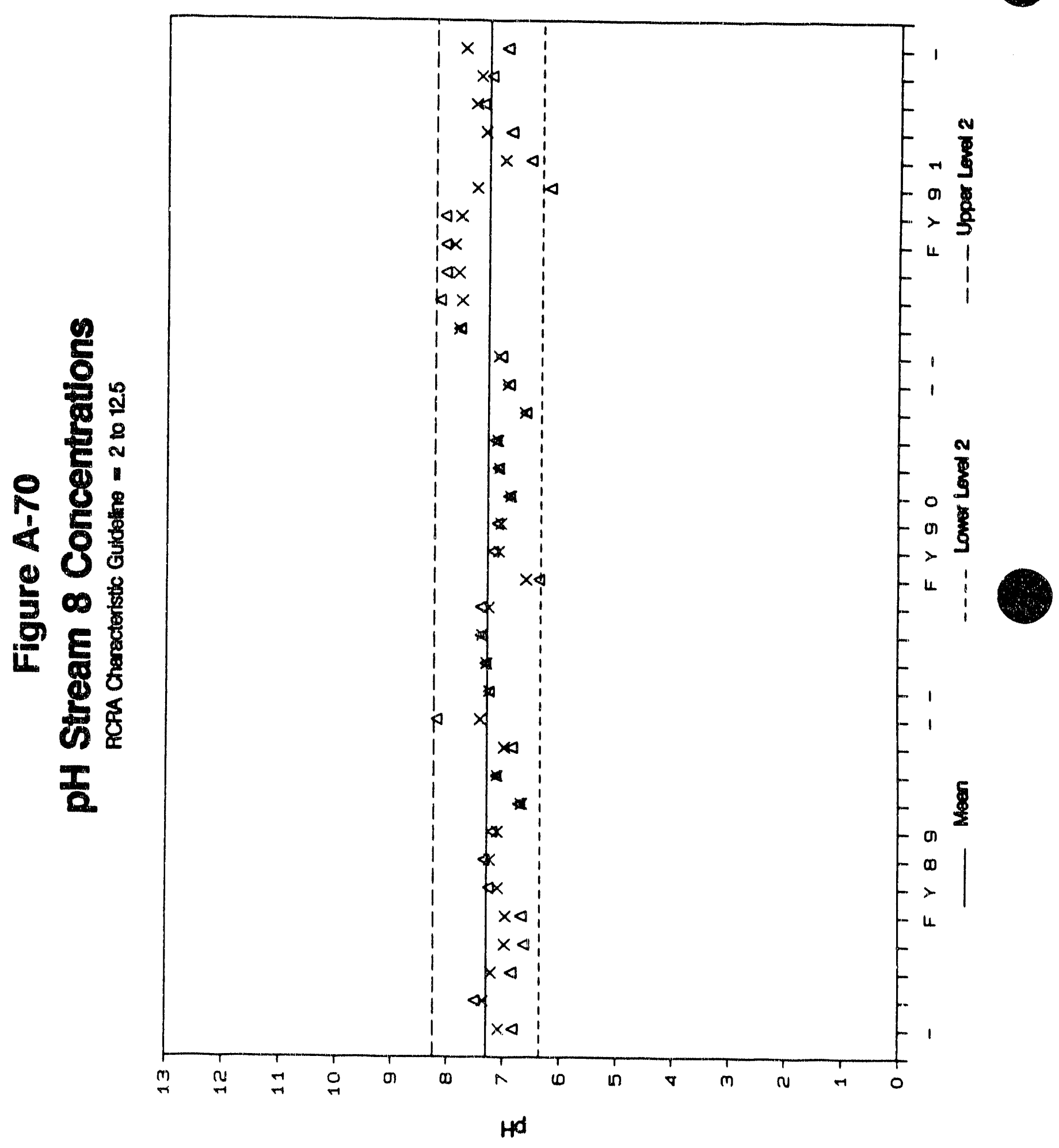




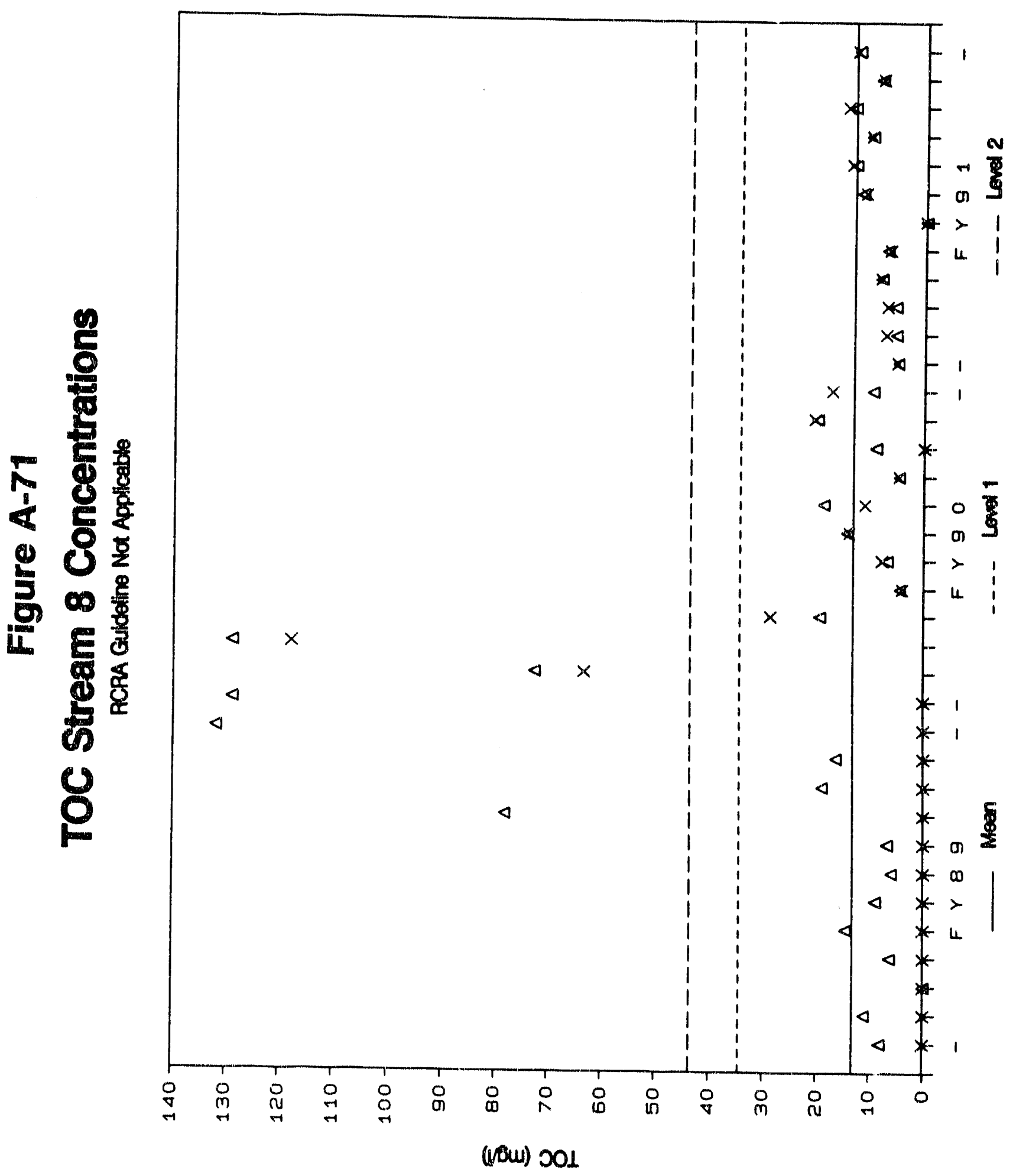




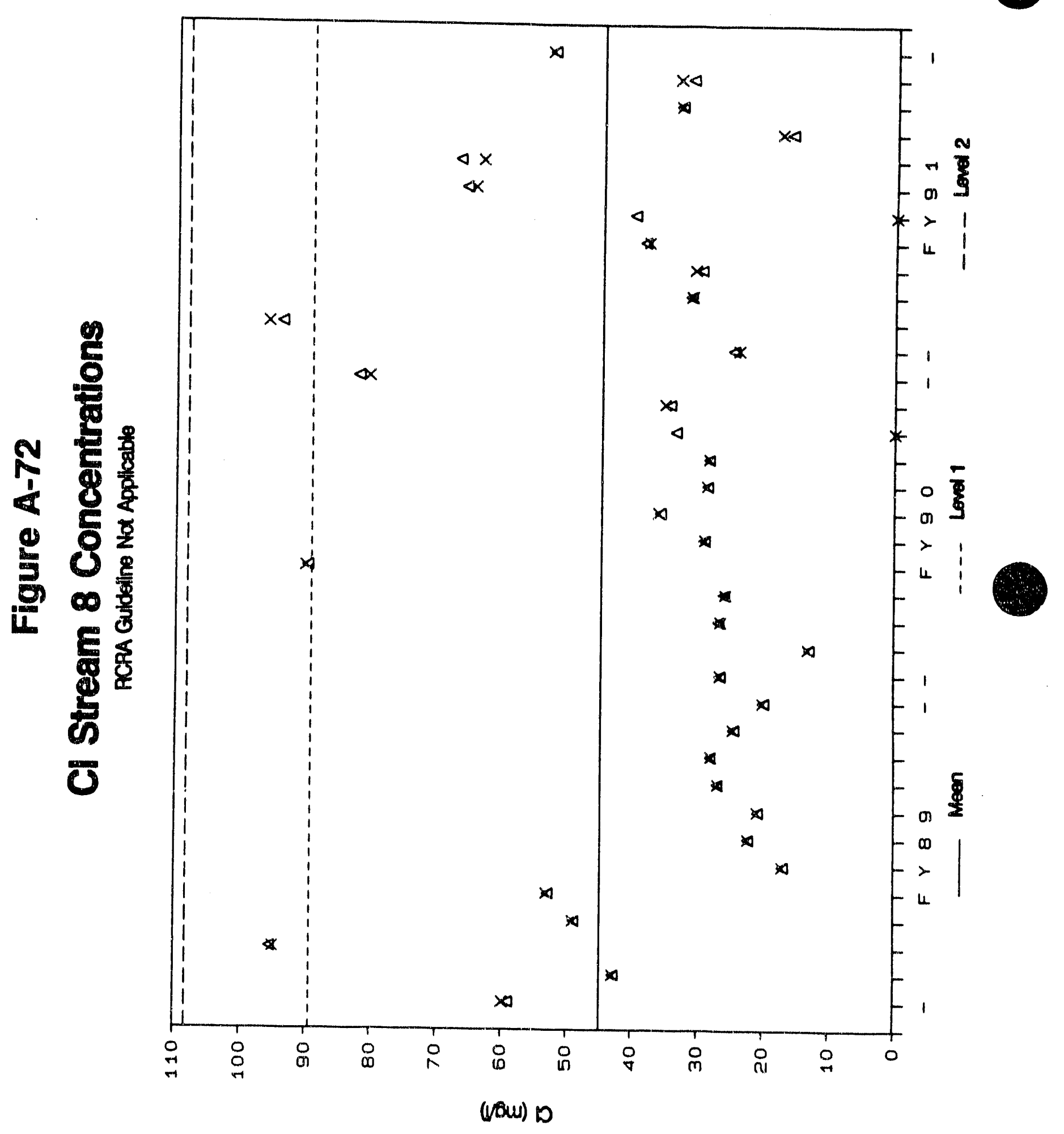




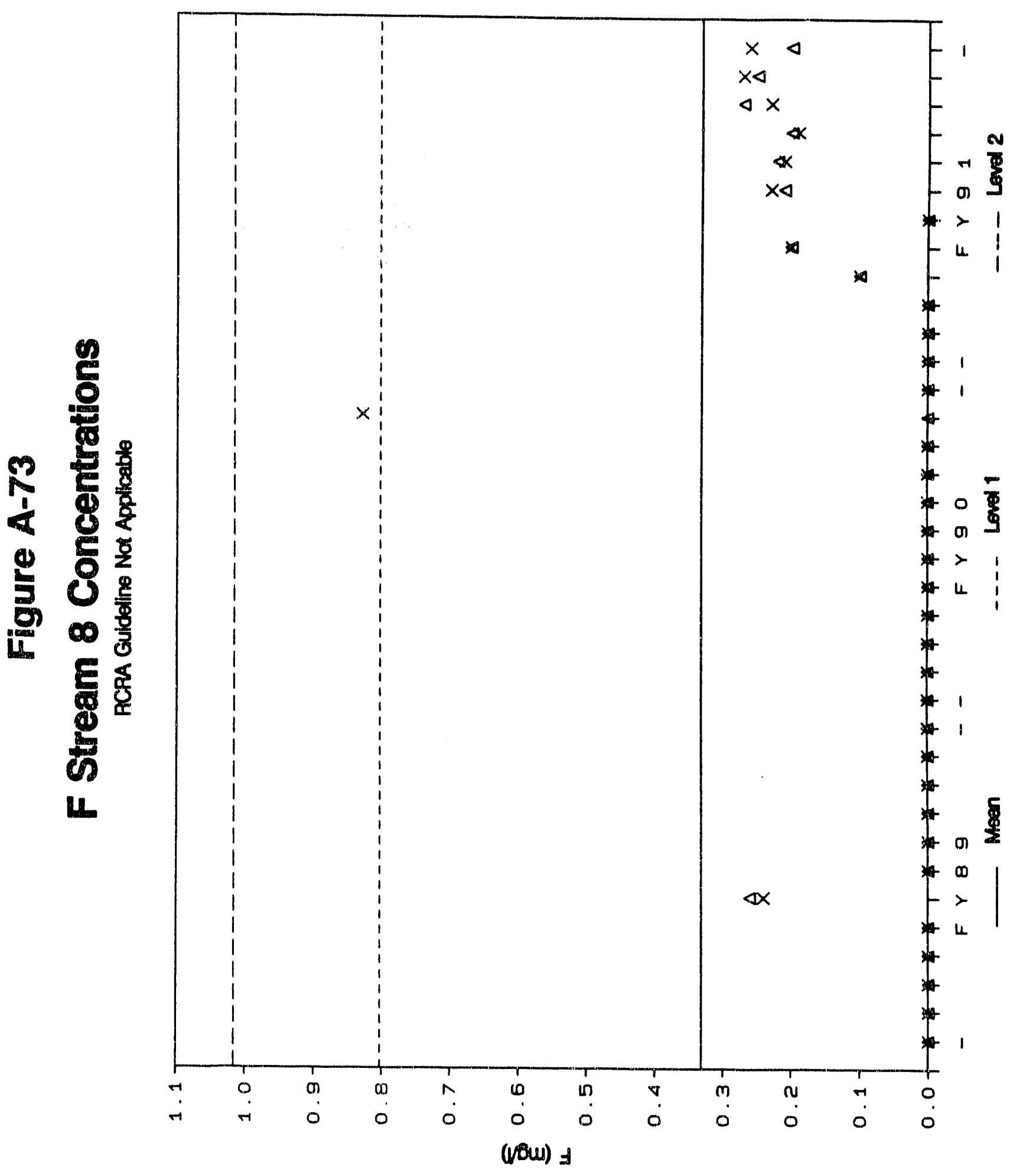




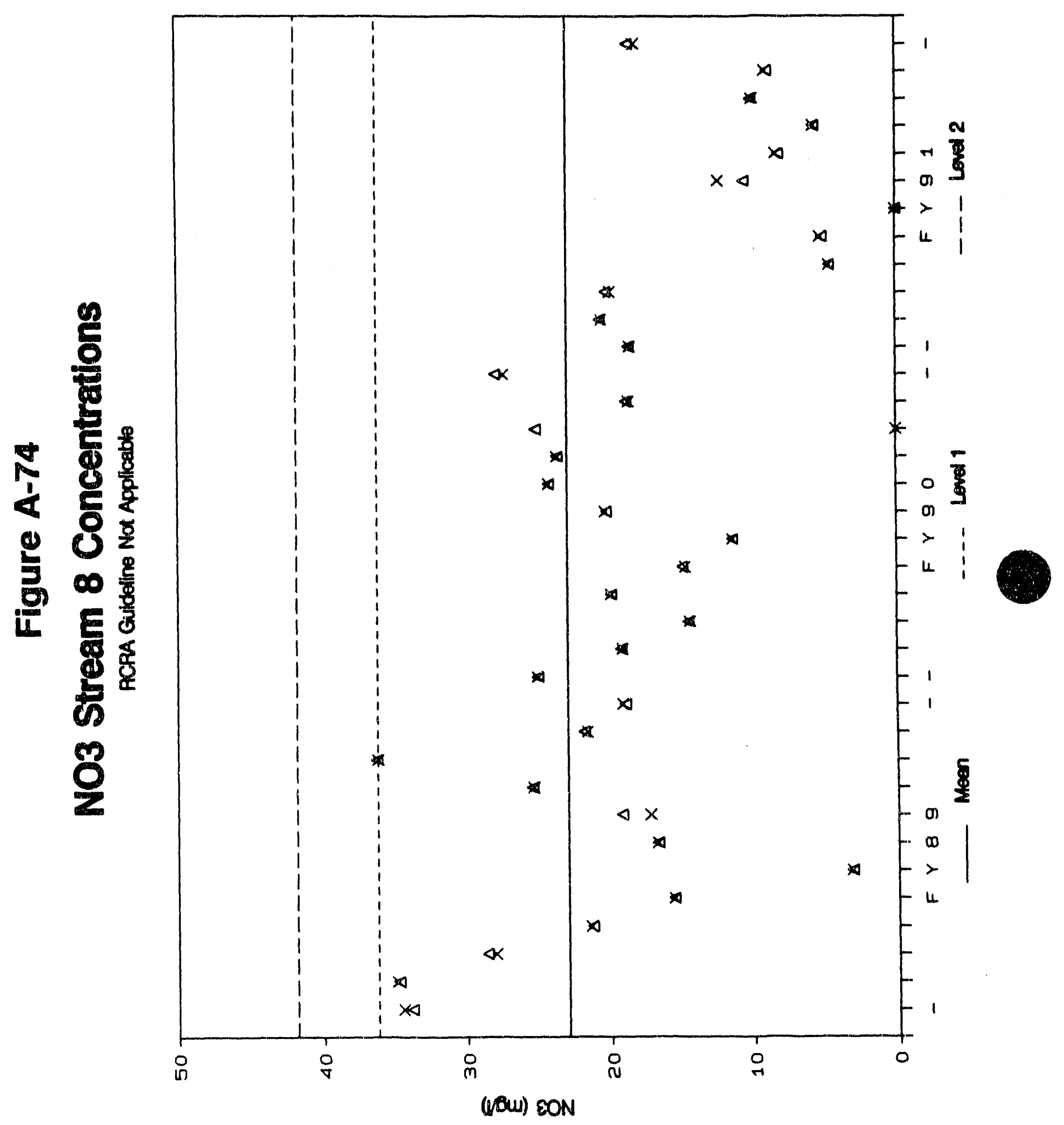




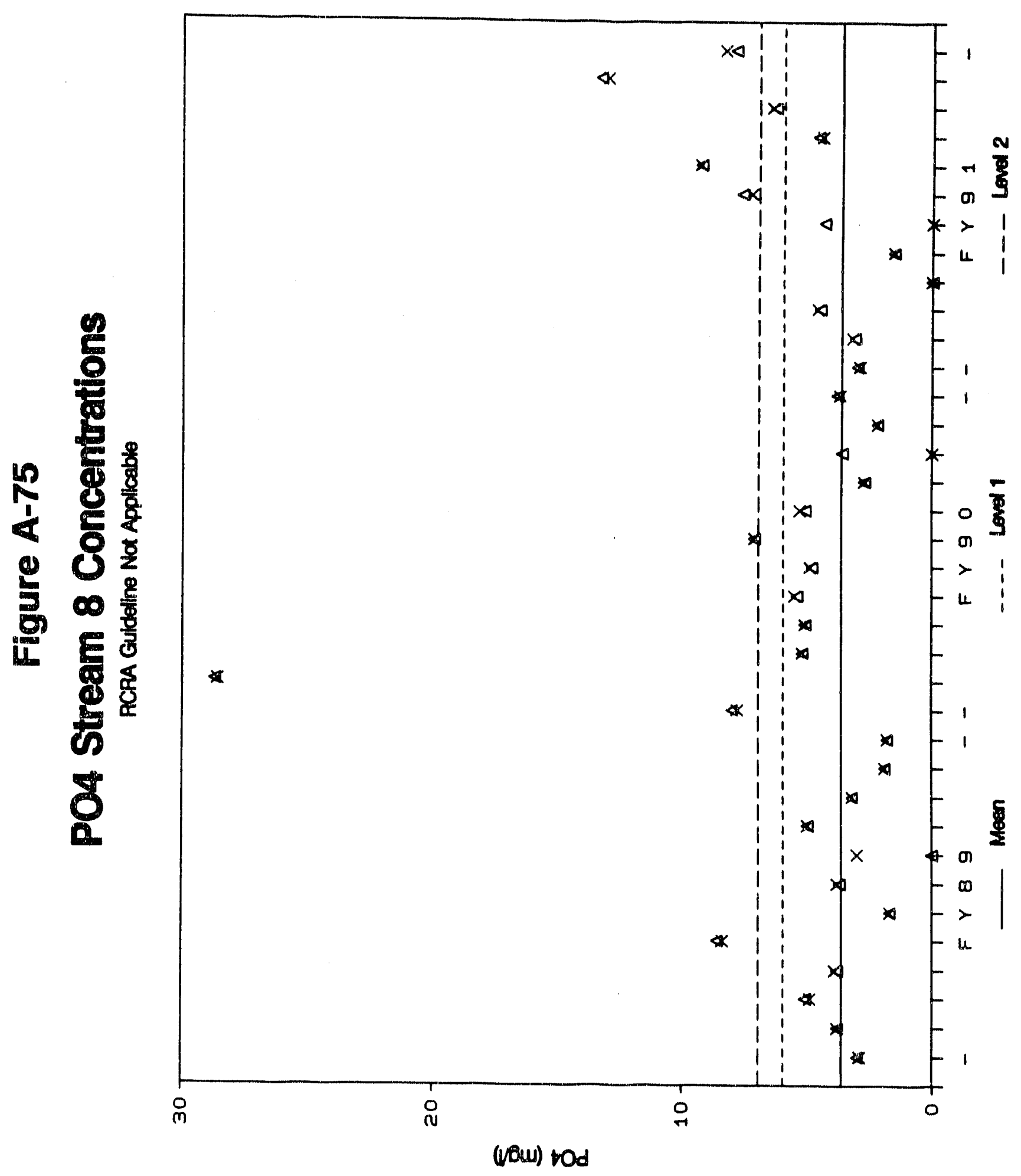




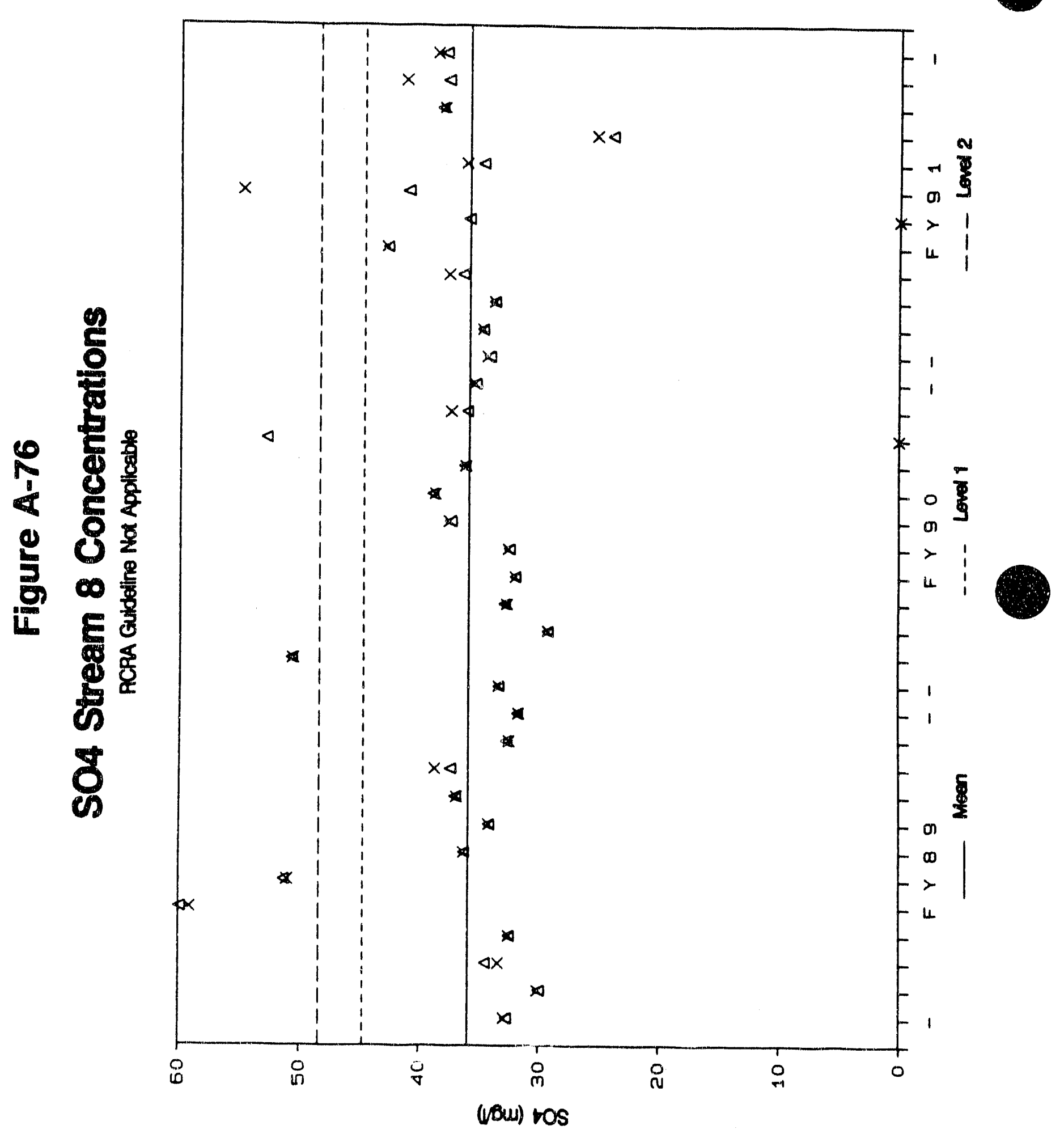




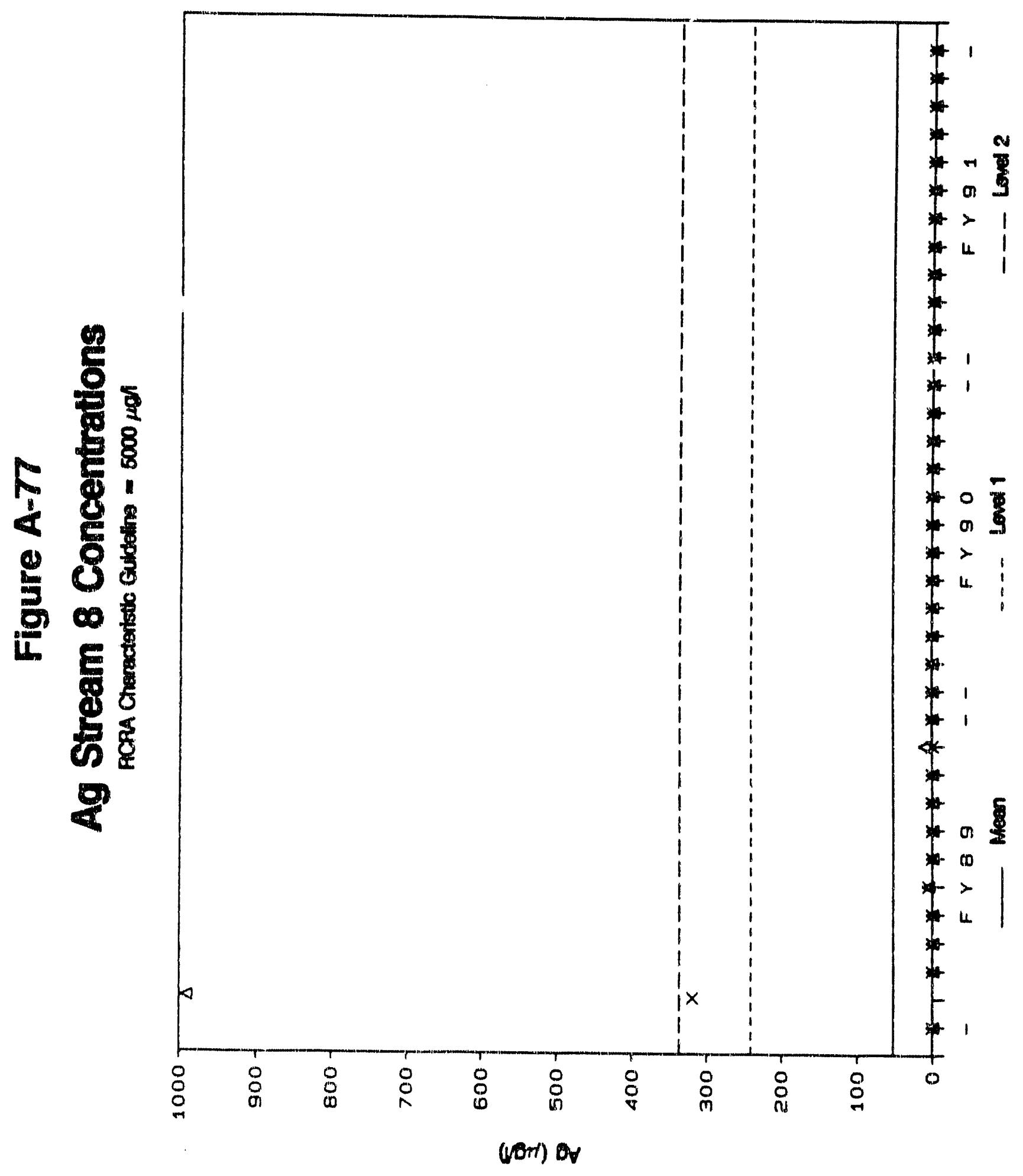




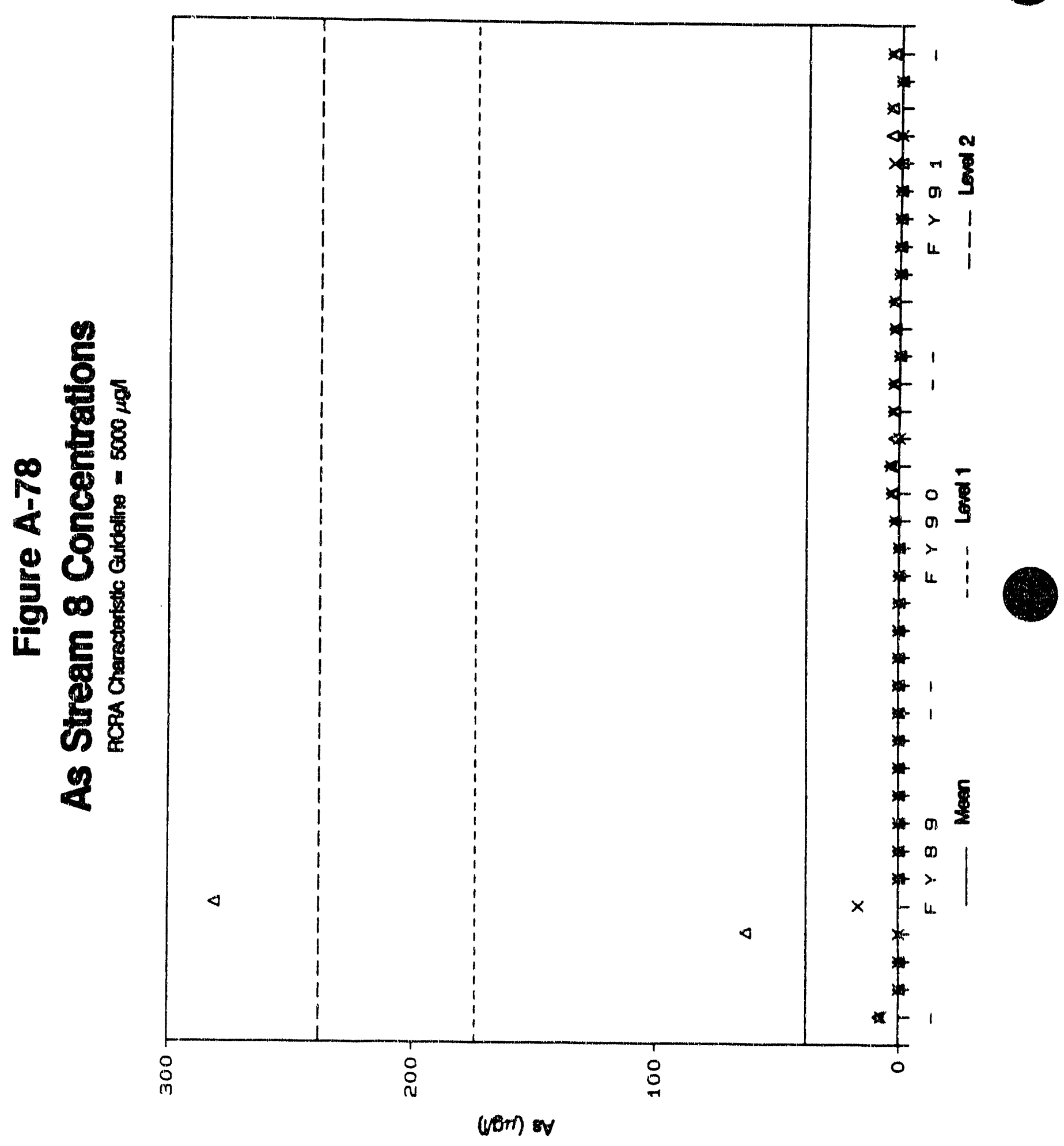




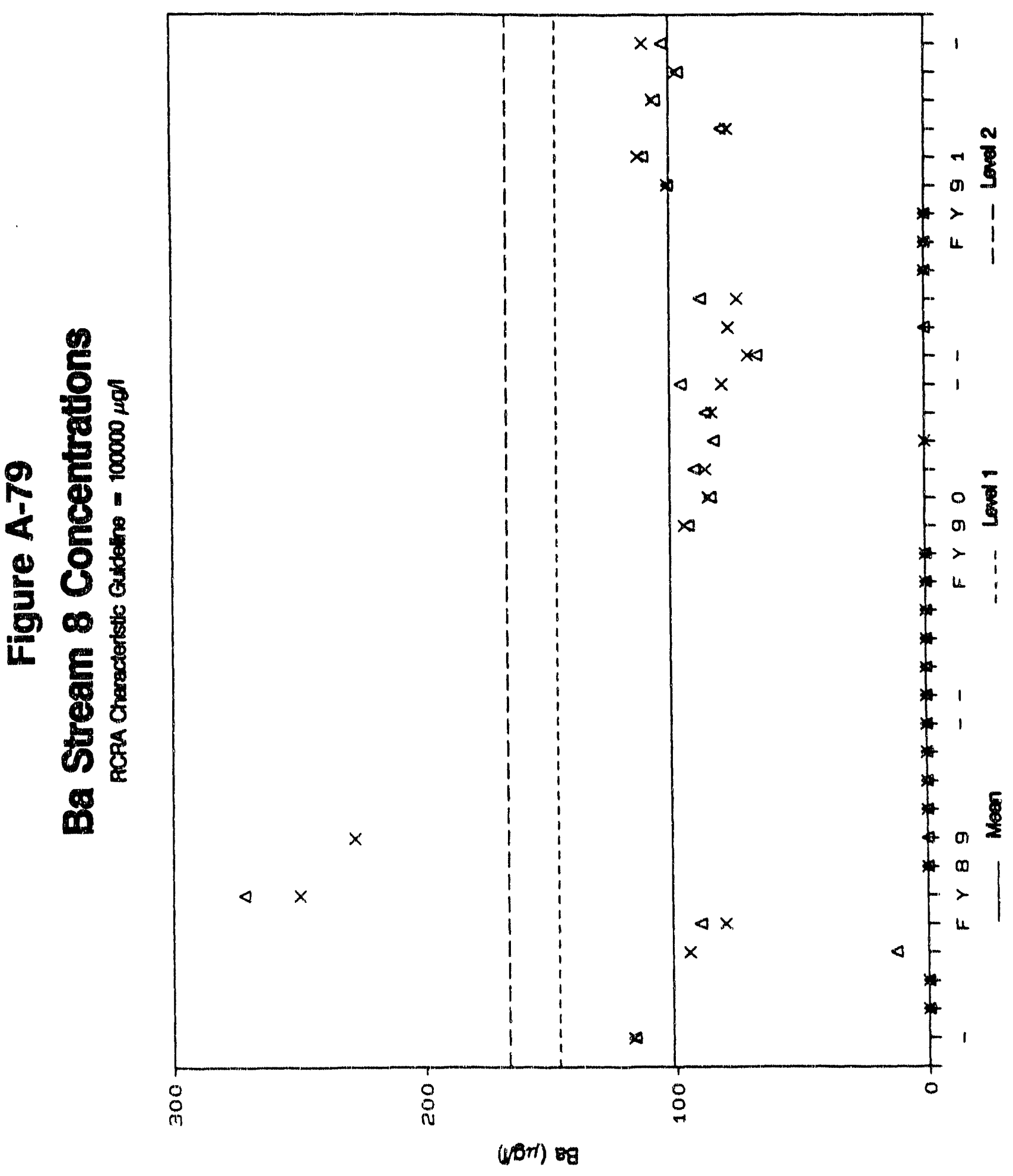

$A-115$ 


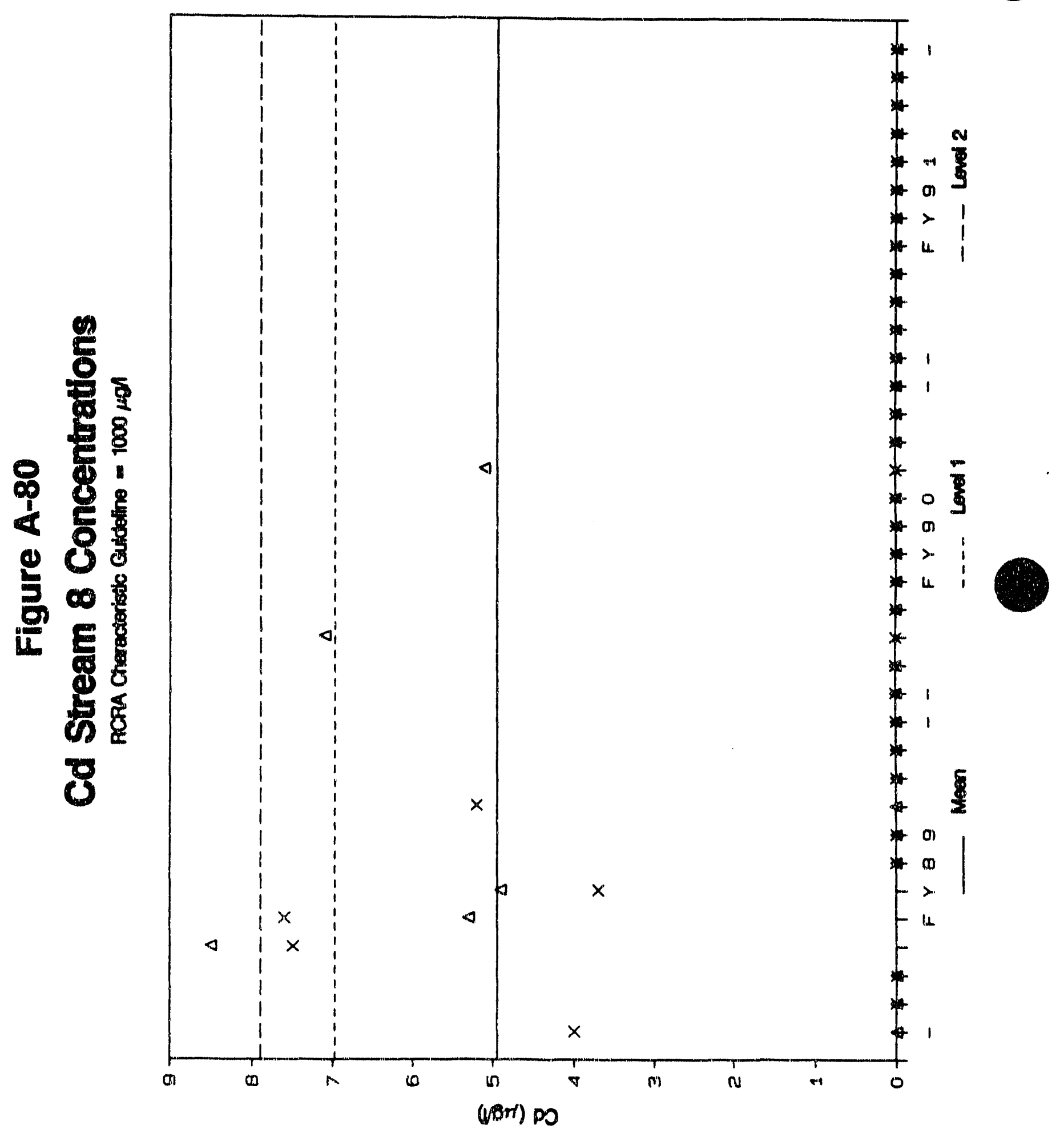




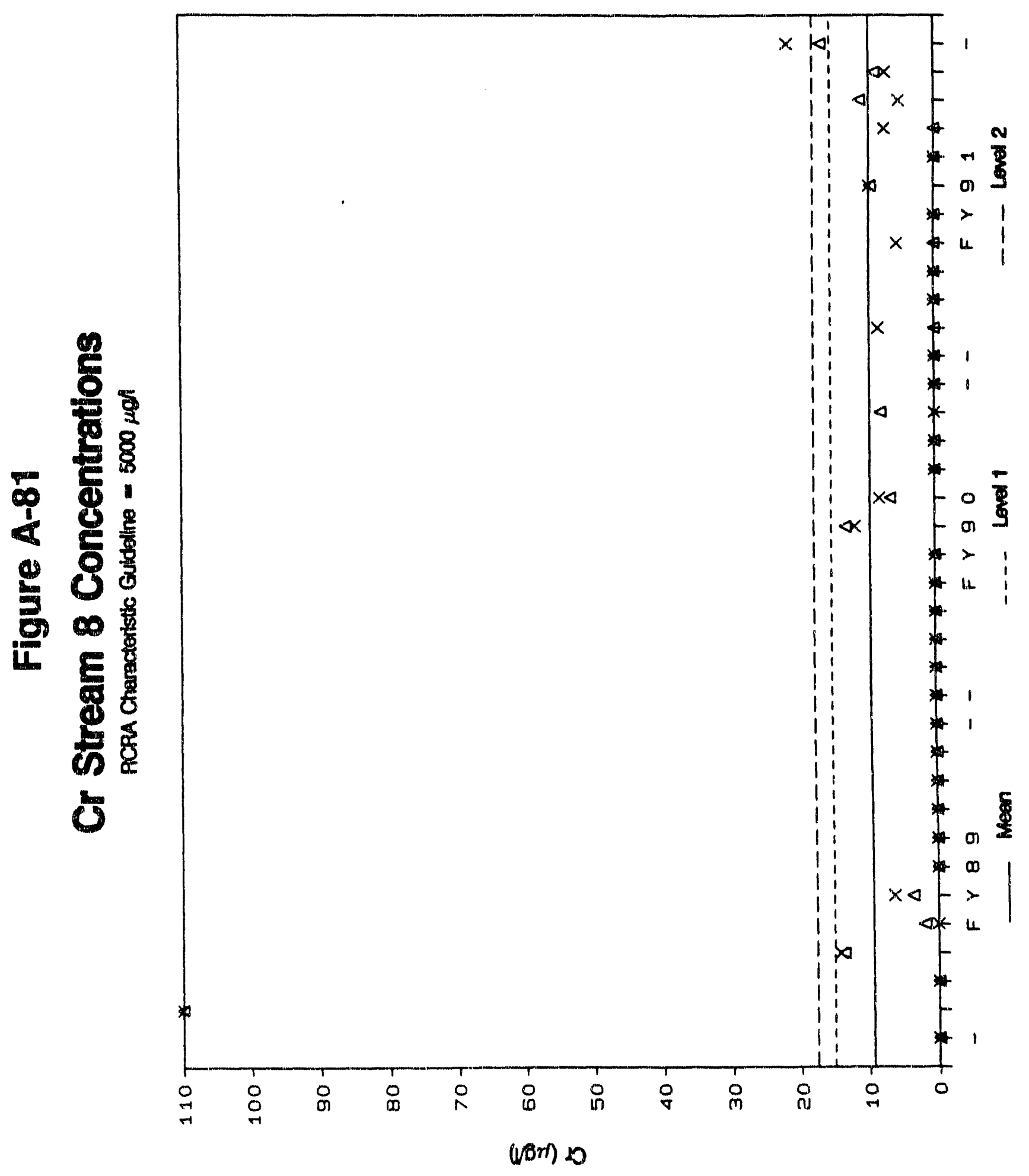




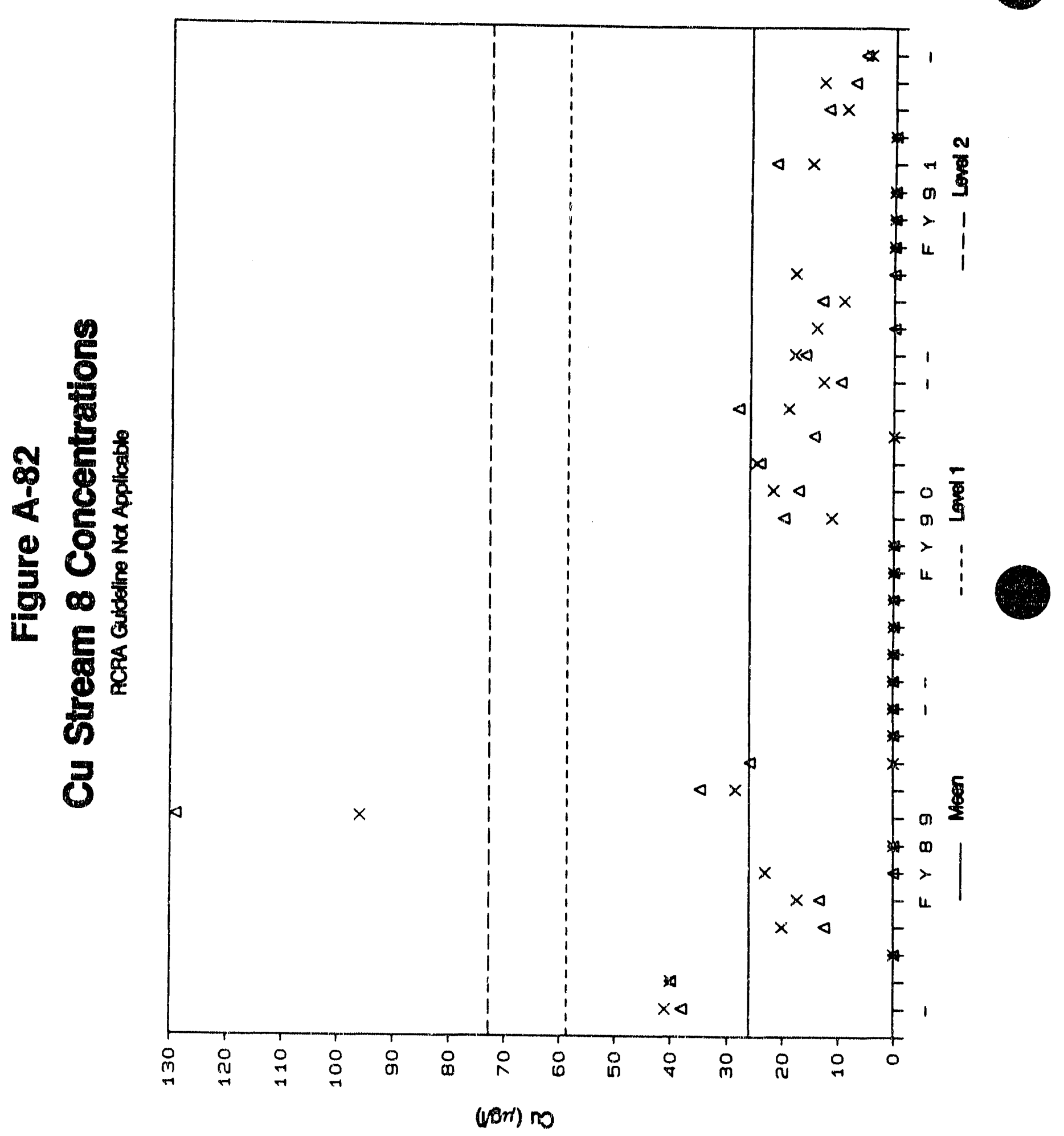




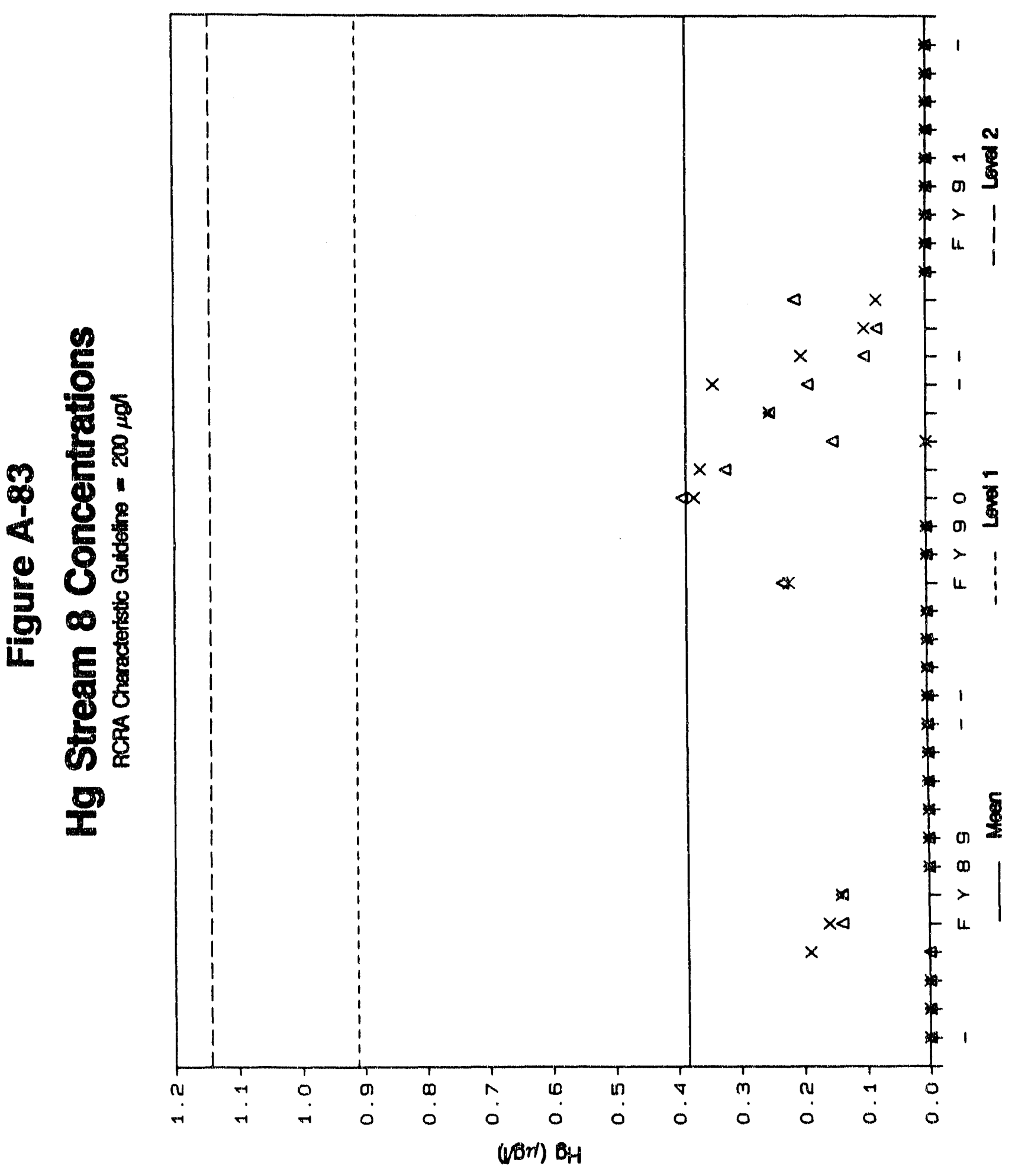




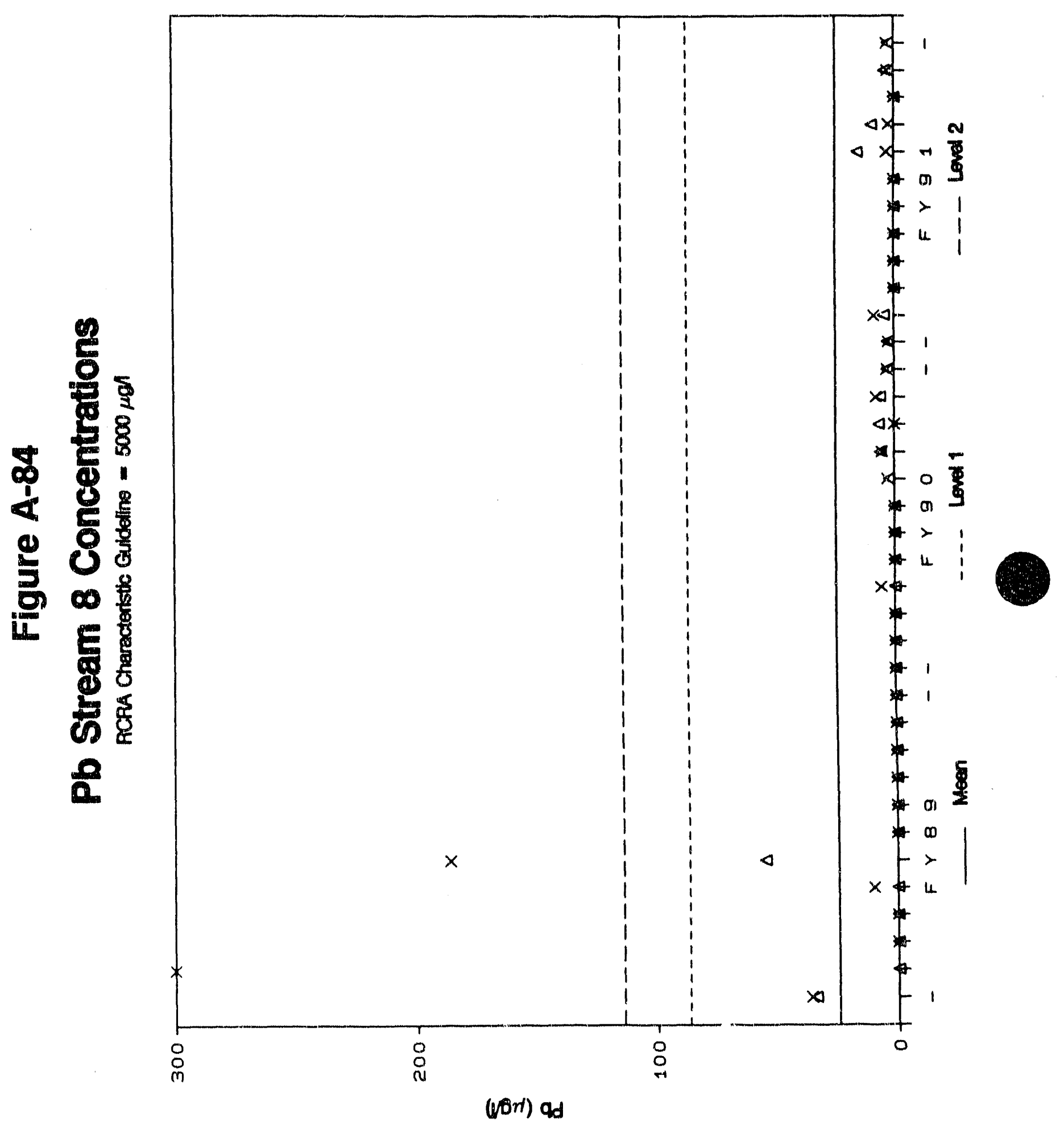




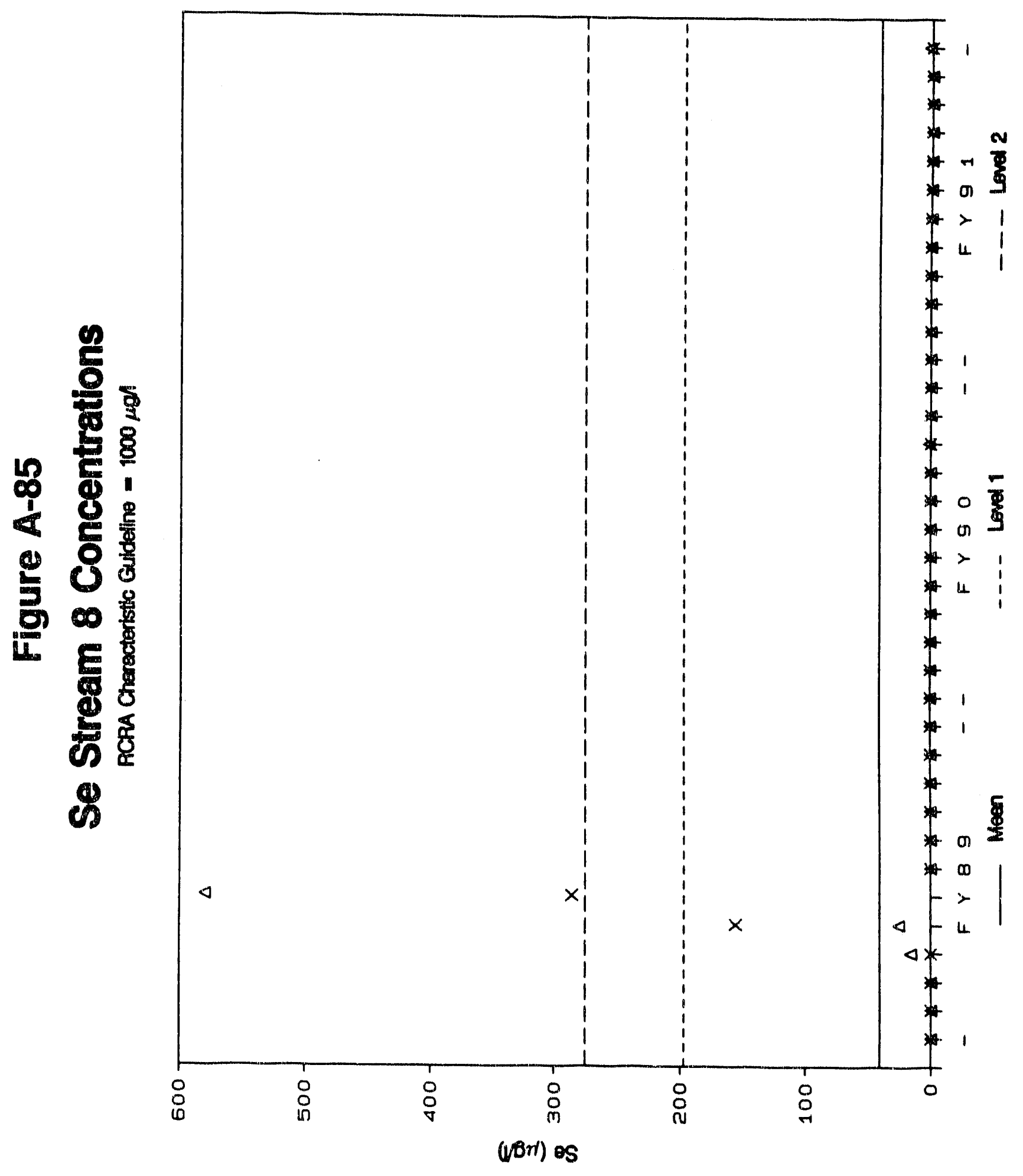




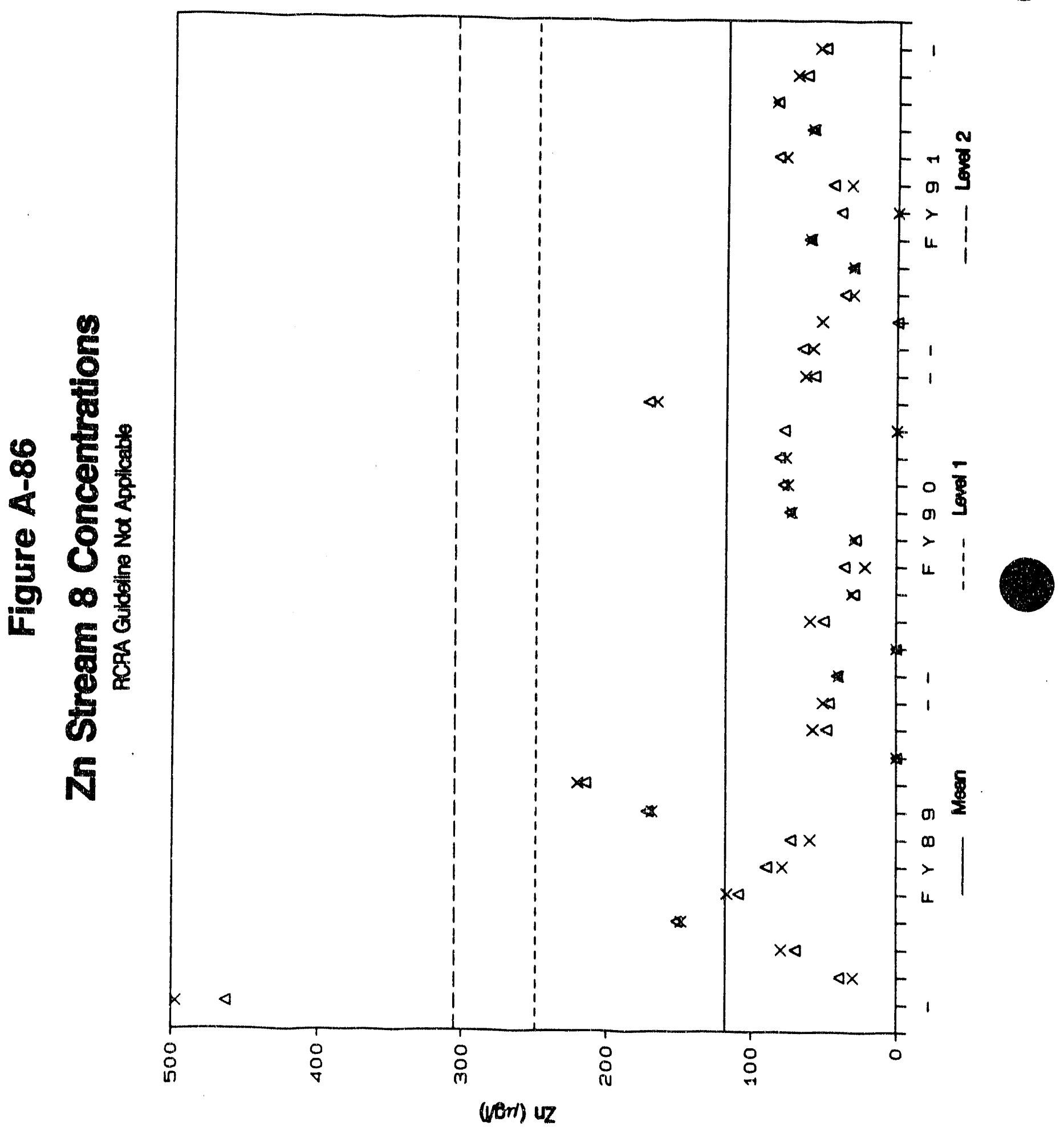




\section{Table A-24}

FY91 ENVIRONMENTAL MONITORING DATA

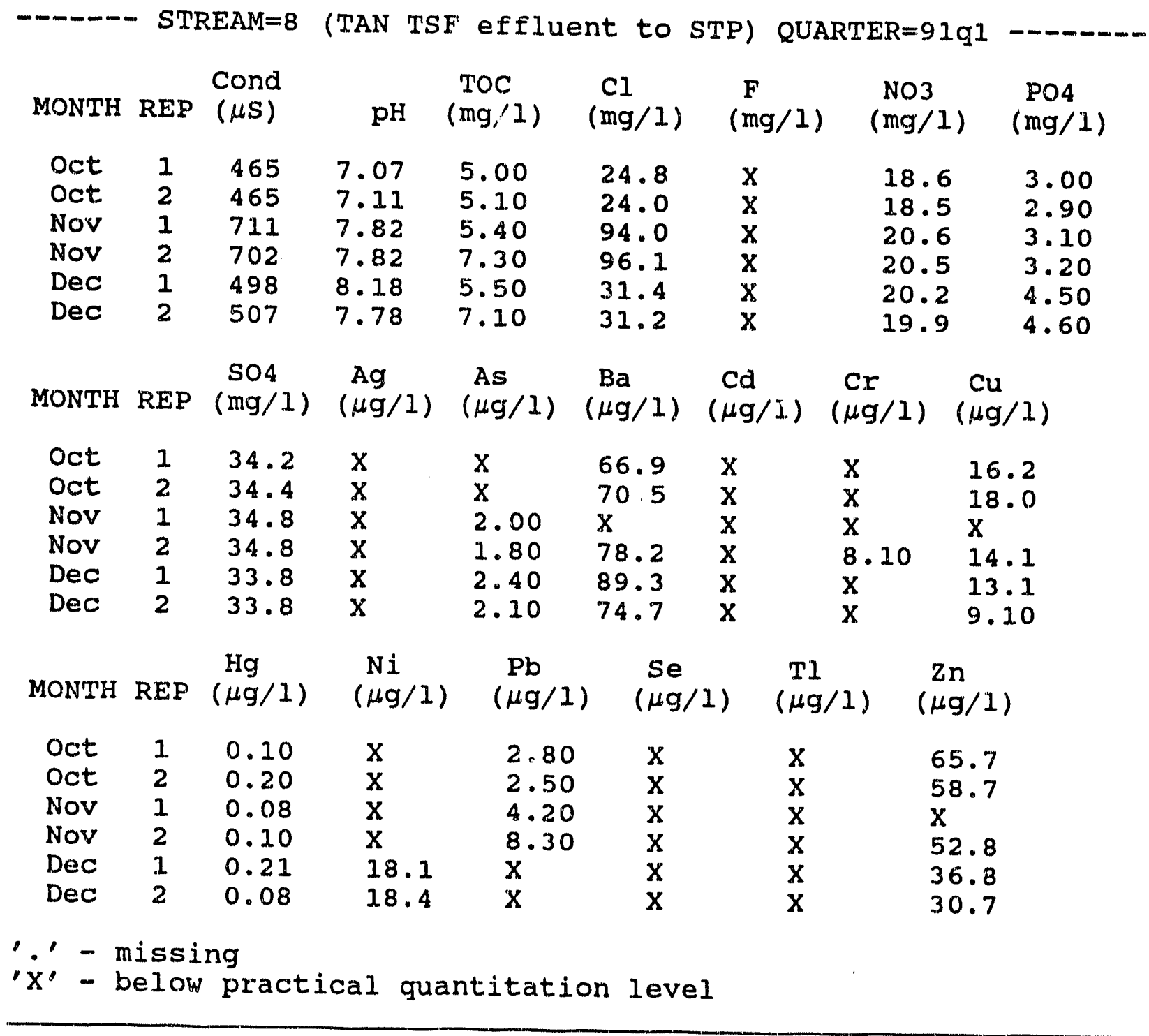




\section{Table A-25}

FY91 ENVIRONMENTAL MONITORING DATA

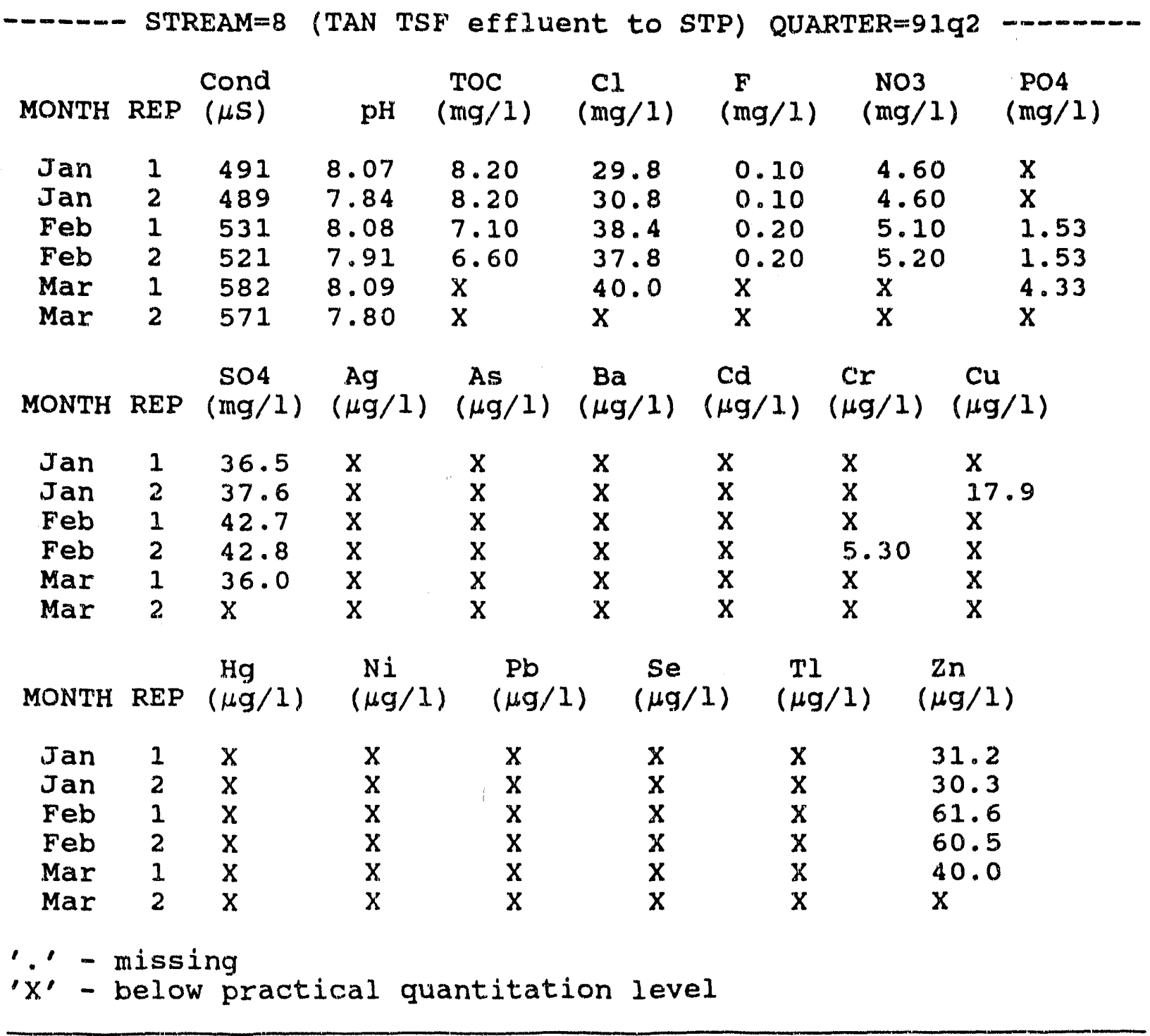




\section{Table A-26}

FY91. ENVIRONMENTAL MONITORING DATA

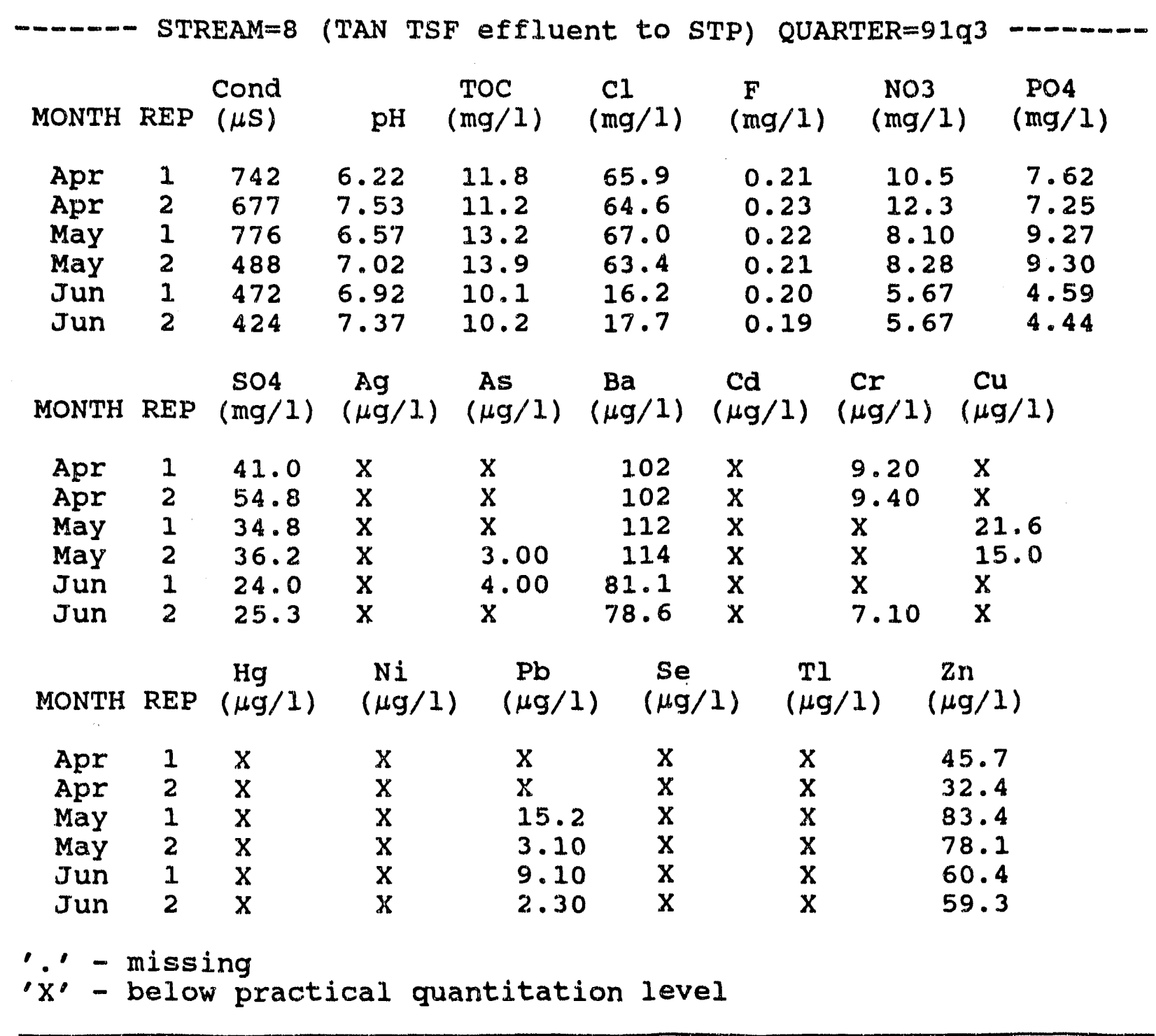




\section{Table A-27}

FY91 ENVIRONMENTAL MONITORING DATA

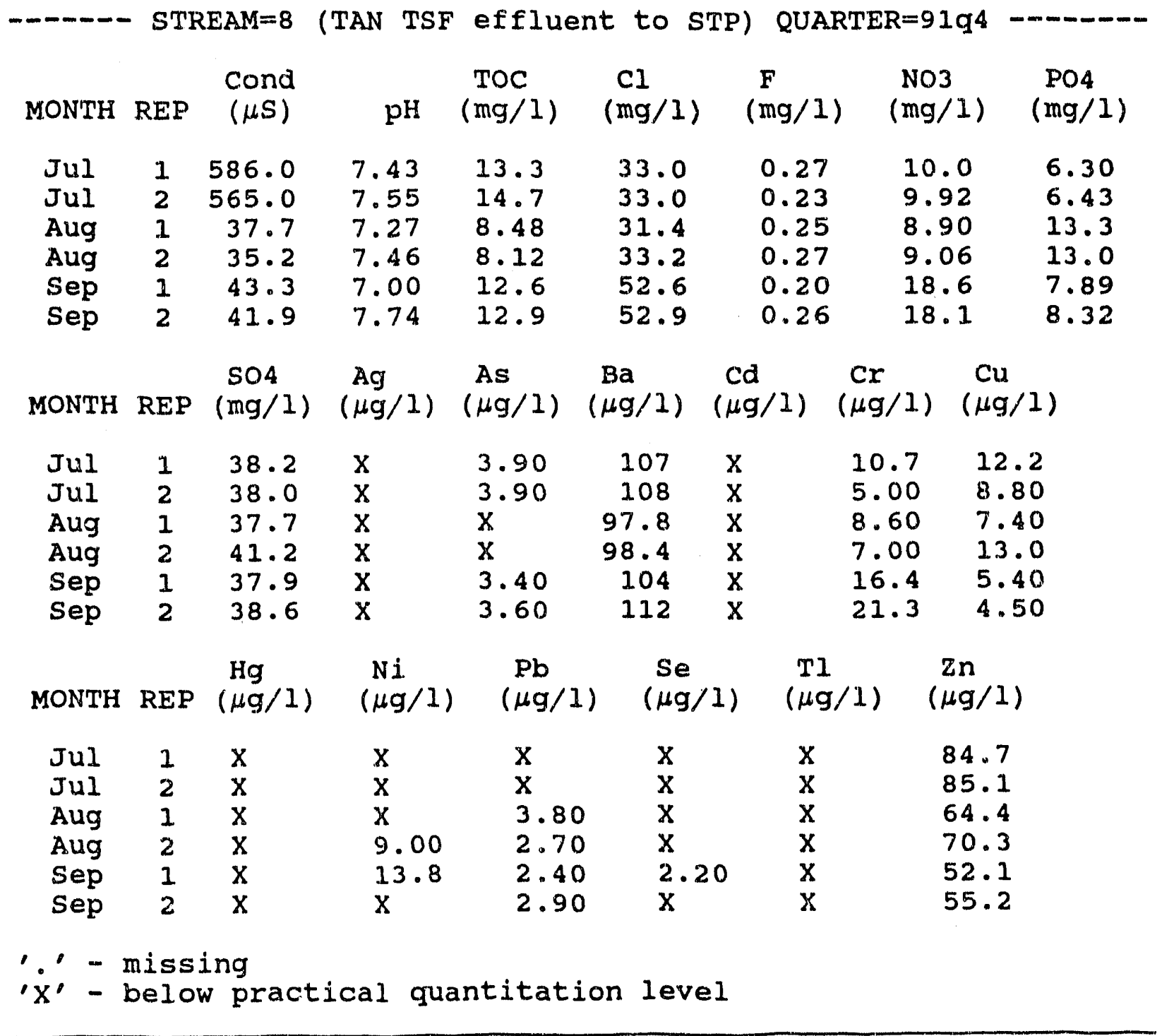




\section{Table A-28}

SUMMARY STATISTICS

STREAM $=8$ (TAN TSF effluent to STP)

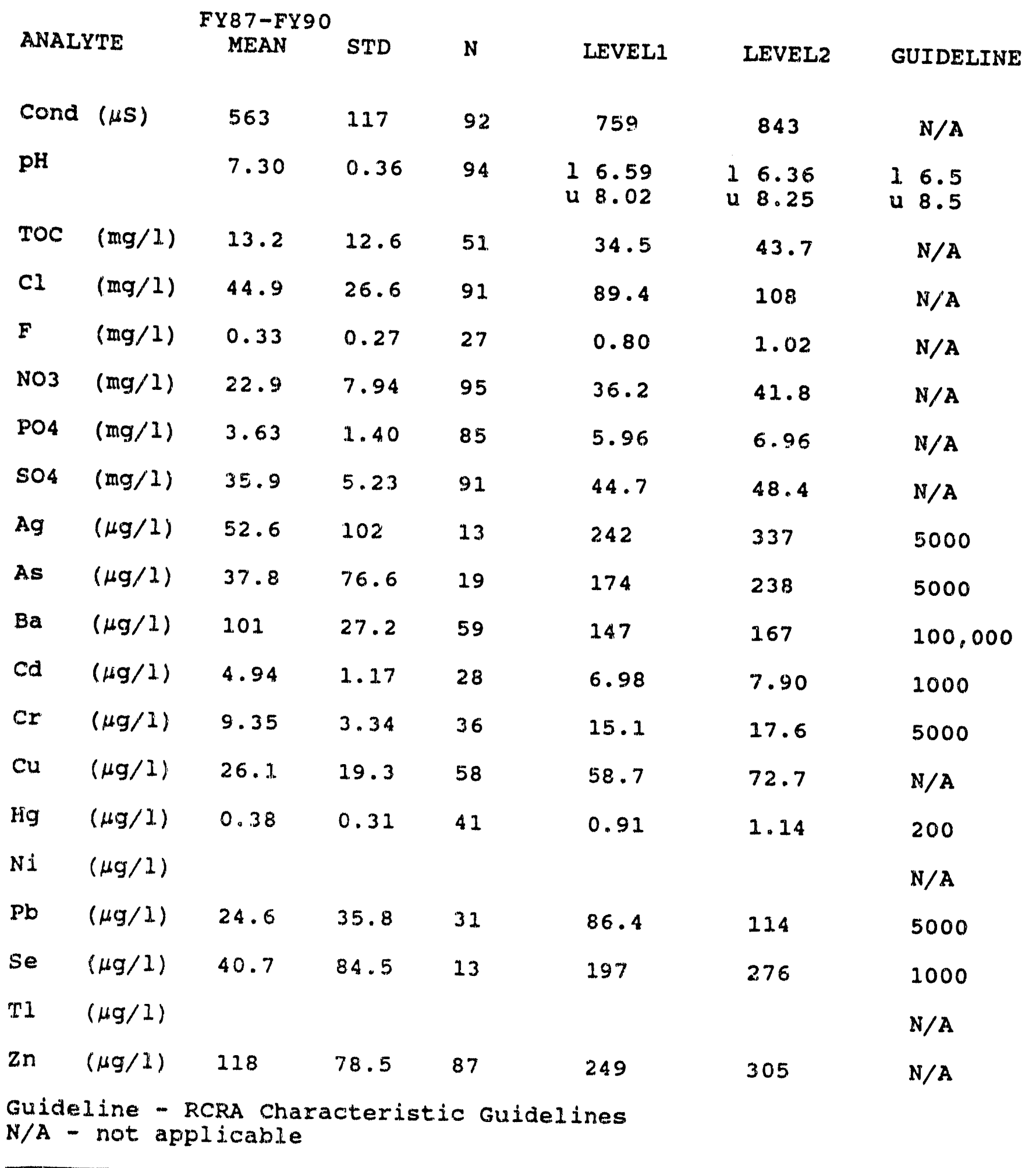


Table A-29

QUARTERLY MEANS AND VARIANCE COMPONENTS

STREAM =8 (TAN TSF effluent to STP)

\begin{tabular}{|c|c|c|c|c|c|c|c|c|c|}
\hline ANALY & TE & QTR & MEAN & $\begin{array}{r}\text { STD } \\
\text { MEAN }\end{array}$ & $\begin{array}{l}\text { STD } \\
\text { DAY }\end{array}$ & $\begin{array}{l}\text { STD } \\
\text { REP }\end{array}$ & $\mathrm{N}$ & $\begin{array}{r}\mathrm{N} \\
\text { MISS }\end{array}$ & BPC \\
\hline Cond & $(\mu S)$ & $\begin{array}{l}91 q 1 \\
91 q 2 \\
91 q 3 \\
91 q^{4}\end{array}$ & $\begin{array}{l}558 \\
531 \\
597 \\
218\end{array}$ & $\begin{array}{r}75.0 \\
25.1 \\
77.5 \\
179\end{array}$ & $\begin{array}{r}130 \\
43.2 \\
103 \\
309\end{array}$ & $\begin{array}{r}5.20 \\
6.12 \\
122 \\
8.65\end{array}$ & $\begin{array}{l}6 \\
6 \\
6 \\
6\end{array}$ & $\begin{array}{l}0 \\
0 \\
0 \\
0\end{array}$ & $\begin{array}{l}0 \\
0 \\
0 \\
0\end{array}$ \\
\hline $\mathrm{pH}$ & & $\begin{array}{l}91 q 1 \\
91 q^{2} \\
91 q^{3} \\
91 q 4\end{array}$ & $\begin{array}{l}7.63 \\
7.96 \\
6.94 \\
7.41\end{array}$ & $\begin{array}{l}0.27 \\
0.02 \\
0.11 \\
0.04\end{array}$ & $\begin{array}{l}0.46 \\
0.00 \\
0.00 \\
0.00\end{array}$ & $\begin{array}{l}0.16 \\
0.17 \\
0.59 \\
0.32\end{array}$ & $\begin{array}{l}6 \\
6 \\
6 \\
6\end{array}$ & $\begin{array}{l}0 \\
0 \\
0 \\
0\end{array}$ & $\begin{array}{l}0 \\
0 \\
0 \\
0\end{array}$ \\
\hline TOC & $(m g / I)$ & $\begin{array}{l}91 q 1 \\
91 q 2 \\
91 q^{3} \\
91 q 4\end{array}$ & $\begin{array}{l}5.90 \\
7.53 \\
11.7 \\
11.7\end{array}$ & $\begin{array}{l}0.43 \\
0.67 \\
0.99 \\
1.73\end{array}$ & $\begin{array}{l}0.17 \\
0.94 \\
1.69 \\
2.97\end{array}$ & $\begin{array}{l}1.01 \\
0.25 \\
0.38 \\
0.60\end{array}$ & $\begin{array}{l}6 \\
4 \\
6 \\
6\end{array}$ & $\begin{array}{l}0 \\
2 \\
0 \\
0\end{array}$ & $\begin{array}{l}0 \\
0 \\
0 \\
0\end{array}$ \\
\hline Cl & $(\mathrm{mg} / \mathrm{l})$ & $\begin{array}{l}91 q^{1} \\
91 q^{2} \\
91 q^{3} \\
91 q^{4}\end{array}$ & $\begin{array}{l}50.2 \\
35.4 \\
49.1 \\
39.3\end{array}$ & $\begin{array}{l}22.5 \\
3.14 \\
16.1 \\
6.70\end{array}$ & $\begin{array}{l}38.9 \\
5.22 \\
27.8 \\
11.6\end{array}$ & $\begin{array}{l}0.92 \\
0.58 \\
1.68 \\
0.75\end{array}$ & $\begin{array}{l}6 \\
5 \\
6 \\
6\end{array}$ & $\begin{array}{l}0 \\
1 \\
0 \\
0\end{array}$ & $\begin{array}{l}0 \\
0 \\
0 \\
0\end{array}$ \\
\hline $\mathrm{F}$ & $(\mathrm{mg} / \mathrm{l})$ & $\begin{array}{l}91 q 1 \\
91 q^{2} \\
91 q^{3} \\
91 q^{4}\end{array}$ & $\begin{array}{l}X \\
0.02 \\
0.21 \\
0.25\end{array}$ & $\begin{array}{l}\dot{0} .20 \\
0.01 \\
0.01\end{array}$ & $\begin{array}{l}0.33 \\
0.01 \\
0.00\end{array}$ & $\begin{array}{l}\dot{0} .00 \\
0.01 \\
0.03\end{array}$ & $\begin{array}{l}0 \\
5 \\
6 \\
6\end{array}$ & $\begin{array}{l}0 \\
1 \\
0 \\
0\end{array}$ & $\begin{array}{l}6 \\
0 \\
0 \\
0\end{array}$ \\
\hline NO3 & $(\mathrm{mg} / \mathrm{I})$ & $\begin{array}{l}91 q^{1} \\
91 q^{2} \\
91 q^{3} \\
91 q^{4}\end{array}$ & $\begin{array}{l}19.7 \\
4.87 \\
8.43 \\
12.4\end{array}$ & $\begin{array}{l}0.60 \\
0.27 \\
1.66 \\
2.98\end{array}$ & $\begin{array}{l}1.04 \\
0.39 \\
2.83 \\
5.15\end{array}$ & $\begin{array}{l}0.13 \\
0.05 \\
0.75 \\
0.21\end{array}$ & $\begin{array}{l}6 \\
4 \\
6 \\
6\end{array}$ & $\begin{array}{l}0 \\
2 \\
0 \\
0\end{array}$ & $\begin{array}{l}0 \\
0 \\
0 \\
0\end{array}$ \\
\hline PO4 & $(\mathrm{mg} / \mathrm{l})$ & $\begin{array}{l}91 q^{1} \\
91 q^{2} \\
91 q^{3} \\
91 q^{4}\end{array}$ & $\begin{array}{l}3.55 \\
2.46 \\
7.08 \\
9.20\end{array}$ & $\begin{array}{l}0.50 \\
\dot{1} .39 \\
2.03\end{array}$ & $\begin{array}{l}0.87 \\
\dot{2} .40 \\
3.51\end{array}$ & $\begin{array}{l}0.07 \\
0.16 \\
0.22\end{array}$ & $\begin{array}{l}6 \\
3 \\
6 \\
6\end{array}$ & $\begin{array}{l}0 \\
0 \\
0 \\
0\end{array}$ & $\begin{array}{l}0 \\
3 \\
0 \\
0\end{array}$ \\
\hline SO4 & $(\mathrm{mg} / \mathrm{l})$ & $\begin{array}{l}91 q 1 \\
91 q^{2} \\
91 q 3 \\
91 q^{4}\end{array}$ & $\begin{array}{l}34.3 \\
39.1 \\
36.0 \\
38.6\end{array}$ & $\begin{array}{l}0.29 \\
2.24 \\
6.72 \\
0.43\end{array}$ & $\begin{array}{l}0.50 \\
3.71 \\
10.9 \\
0.00\end{array}$ & $\begin{array}{l}0.08 \\
0.55 \\
5.69 \\
1.46\end{array}$ & $\begin{array}{l}6 \\
5 \\
6 \\
6\end{array}$ & $\begin{array}{l}0 \\
1 \\
0 \\
0\end{array}$ & $\begin{array}{l}0 \\
0 \\
0 \\
0\end{array}$ \\
\hline Ag & $(\mu g / I)$ & $\begin{array}{l}91 q 1 \\
91 q^{2} \\
91 q^{3} \\
91 q^{4}\end{array}$ & $\begin{array}{l}X \\
X \\
X \\
X\end{array}$ & $\dot{.}$ & $\dot{\dot{ }}$ & $\begin{array}{l}\dot{.} \\
\dot{.}\end{array}$ & $\begin{array}{l}0 \\
0 \\
0 \\
0\end{array}$ & $\begin{array}{l}0 \\
0 \\
0 \\
0\end{array}$ & $\begin{array}{l}6 \\
6 \\
6 \\
6\end{array}$ \\
\hline $\mathrm{As}$ & $(\mu \mathrm{g} / 1)$ & $\begin{array}{l}91 q 1 \\
91 q 2 \\
91 q^{3} \\
9194\end{array}$ & $\begin{array}{l}2.08 \\
X \\
3.50 \\
1.47\end{array}$ & $\begin{array}{l}0.17 \\
\dot{=} \\
2.24\end{array}$ & $\begin{array}{l}0.21 \\
\dot{=} \\
3.87\end{array}$ & $\begin{array}{l}0.18 \\
\dot{0} \\
0.08\end{array}$ & $\begin{array}{l}4 \\
0 \\
2 \\
6\end{array}$ & $\begin{array}{l}0 \\
0 \\
0 \\
0\end{array}$ & $\begin{array}{l}2 \\
6 \\
4 \\
0\end{array}$ \\
\hline
\end{tabular}


Table A-29 (continued)

QUARTERLY MEANS AND VARIANCE COMPONENTS

STREAM $=8$ (TAN TSF effluent to STP)

(continued)

\begin{tabular}{|c|c|c|c|c|c|c|c|c|c|}
\hline \multicolumn{2}{|c|}{ ANAIYTE } & QTR & MEAN & $\begin{array}{r}\text { STD } \\
\text { MEAN }\end{array}$ & $\begin{array}{l}\text { STD } \\
\text { DAY }\end{array}$ & $\begin{array}{l}\text { STD } \\
\text { REP }\end{array}$ & $\mathbf{N}$ & MISS & $\stackrel{\mathbf{N}}{\mathrm{BPQL}}$ \\
\hline $\mathrm{Ba}$ & $(\mu g / 1)$ & $\begin{array}{l}91 q 1 \\
91 q 2 \\
91 q 3 \\
91 q 4\end{array}$ & $\begin{array}{l}75.9 \\
\times \\
98.3 \\
1.05\end{array}$ & $\begin{array}{l}4.38 \\
\dot{9} .75 \\
3.22\end{array}$ & $\begin{array}{l}4.69 \\
16.9 \\
5.07\end{array}$ & $\begin{array}{l}7.52 \\
i .31 \\
3.30\end{array}$ & $\begin{array}{l}5 \\
0 \\
6 \\
6\end{array}$ & $\begin{array}{l}0 \\
0 \\
0 \\
0\end{array}$ & $\begin{array}{l}1 \\
6 \\
0 \\
0\end{array}$ \\
\hline $\mathrm{ca}$ & $(\mu g / 1)$ & $\begin{array}{l}91 q^{1} \\
91 q^{2} \\
91 q^{3} \\
91 q^{4}\end{array}$ & $\begin{array}{l}X \\
X \\
X \\
X\end{array}$ & $\begin{array}{l}\dot{.} \\
\dot{.}\end{array}$ & $\dot{.}$ & $\begin{array}{l}\dot{.} \\
\dot{.}\end{array}$ & $\begin{array}{l}0 \\
0 \\
0 \\
0\end{array}$ & $\begin{array}{l}0 \\
0 \\
0 \\
0\end{array}$ & $\begin{array}{l}6 \\
6 \\
6 \\
6\end{array}$ \\
\hline $\mathrm{Cr}$ & $(\mu g / 1)$ & $\begin{array}{l}91 q 1 \\
91 q 2 \\
91 q^{3} \\
91 q^{4}\end{array}$ & $\begin{array}{l}8.10 \\
5.30 \\
8.57 \\
11.5\end{array}$ & $\begin{array}{l}\dot{b} \\
\dot{3} .68\end{array}$ & $\begin{array}{l}\dot{\dot{ }} \\
\dot{5} .97\end{array}$ & $\begin{array}{l}\dot{\dot{3}} \\
\dot{3} .14\end{array}$ & $\begin{array}{l}1 \\
1 \\
3 \\
6\end{array}$ & $\begin{array}{l}0 \\
0 \\
0 \\
0\end{array}$ & $\begin{array}{l}5 \\
5 \\
3 \\
0\end{array}$ \\
\hline $\mathrm{Cu}$ & $(\mu g / I)$ & $\begin{array}{l}91 q 1 \\
91 q 2 \\
91 q^{3} \\
91 q 4\end{array}$ & $\begin{array}{l}14.1 \\
17.9 \\
18.3 \\
8.55\end{array}$ & $\begin{array}{l}1.98 \\
\dot{1} \\
1.80\end{array}$ & $\begin{array}{l}2.87 \\
\dot{2} \\
\dot{2} .47\end{array}$ & $\begin{array}{l}2.19 \\
\dot{2} \\
2.70\end{array}$ & $\begin{array}{l}5 \\
1 \\
2 \\
6\end{array}$ & $\begin{array}{l}0 \\
0 \\
0 \\
0\end{array}$ & $\begin{array}{l}1 \\
5 \\
4 \\
0\end{array}$ \\
\hline $\mathrm{Hg}$ & $(\mu g / I)$ & $\begin{array}{l}91 q 1 \\
91 q 2 \\
91 q 3 \\
91 q 4\end{array}$ & $\begin{array}{l}0.13 \\
x \\
x \\
x\end{array}$ & $\begin{array}{l}0.02 \\
\dot{:} \\
\dot{.}\end{array}$ & $\begin{array}{l}0.00 \\
\dot{\cdot} \\
\dot{\cdot}\end{array}$ & $\begin{array}{l}0.07 \\
\dot{\cdot} \\
\dot{.}\end{array}$ & $\begin{array}{l}6 \\
0 \\
0 \\
0\end{array}$ & $\begin{array}{l}0 \\
0 \\
0 \\
0\end{array}$ & $\begin{array}{l}0 \\
6 \\
6 \\
6\end{array}$ \\
\hline $\mathrm{Ni}$ & $(\mu g / 1)$ & $\begin{array}{l}91 q 1 \\
91 q^{2} \\
91 q^{3} \\
91 q^{4}\end{array}$ & $\begin{array}{l}18.3 \\
X \\
X \\
11.4\end{array}$ & $\begin{array}{l}\dot{ } \\
\dot{.}\end{array}$ & $\dot{.}$ & $\begin{array}{l}\dot{\cdot} \\
\dot{\cdot}\end{array}$ & $\begin{array}{l}2 \\
0 \\
0 \\
2\end{array}$ & $\begin{array}{l}0 \\
0 \\
0 \\
0\end{array}$ & $\begin{array}{l}4 \\
6 \\
6 \\
4\end{array}$ \\
\hline $\mathrm{Pb}$ & $(\mu g / I)$ & $\begin{array}{l}91 q 1 \\
91 q 2 \\
91 q 3 \\
91 q 4\end{array}$ & $\begin{array}{l}4.45 \\
X \\
4.28 \\
1.30\end{array}$ & $\begin{array}{l}1.80 \\
3.30 \\
1.66\end{array}$ & $\begin{array}{l}2.09 \\
4.07 \\
2.85\end{array}$ & $\begin{array}{l}2.06 \\
5.67 \\
0.49\end{array}$ & $\begin{array}{l}4 \\
0 \\
6 \\
6\end{array}$ & $\begin{array}{l}0 \\
0 \\
0 \\
0\end{array}$ & $\begin{array}{l}2 \\
6 \\
0 \\
0\end{array}$ \\
\hline se & $(\mu g / I)$ & $\begin{array}{l}91 q 1 \\
91 q 2 \\
91 q 3 \\
91 q 4\end{array}$ & $\begin{array}{l}X \\
X \\
X \\
2.20\end{array}$ & $\dot{:}$ & $\dot{.}$ & $\begin{array}{l}\dot{.} \\
\dot{.}\end{array}$ & $\begin{array}{l}0 \\
0 \\
0 \\
1\end{array}$ & $\begin{array}{l}0 \\
0 \\
0 \\
0\end{array}$ & $\begin{array}{l}6 \\
6 \\
6 \\
5\end{array}$ \\
\hline Tl & $(\mu g / 1)$ & $\begin{array}{l}91 q 1 \\
91 q 2 \\
91 q 3 \\
91 q 4\end{array}$ & $\begin{array}{l}X \\
X \\
X \\
X\end{array}$ & $\dot{\dot{.}}$ & $\begin{array}{l}\dot{.} \\
\dot{.}\end{array}$ & $\dot{.}$ & $\begin{array}{l}0 \\
0 \\
0 \\
0\end{array}$ & $\begin{array}{l}0 \\
0 \\
0 \\
0\end{array}$ & $\begin{array}{l}6 \\
6 \\
6 \\
6\end{array}$ \\
\hline
\end{tabular}


Table A-29 (continued)

QUARTERIY MEANS AND VARIANCE COMPONENTS

STREAM=8 (TAN TSF effluent to STP) (continued)

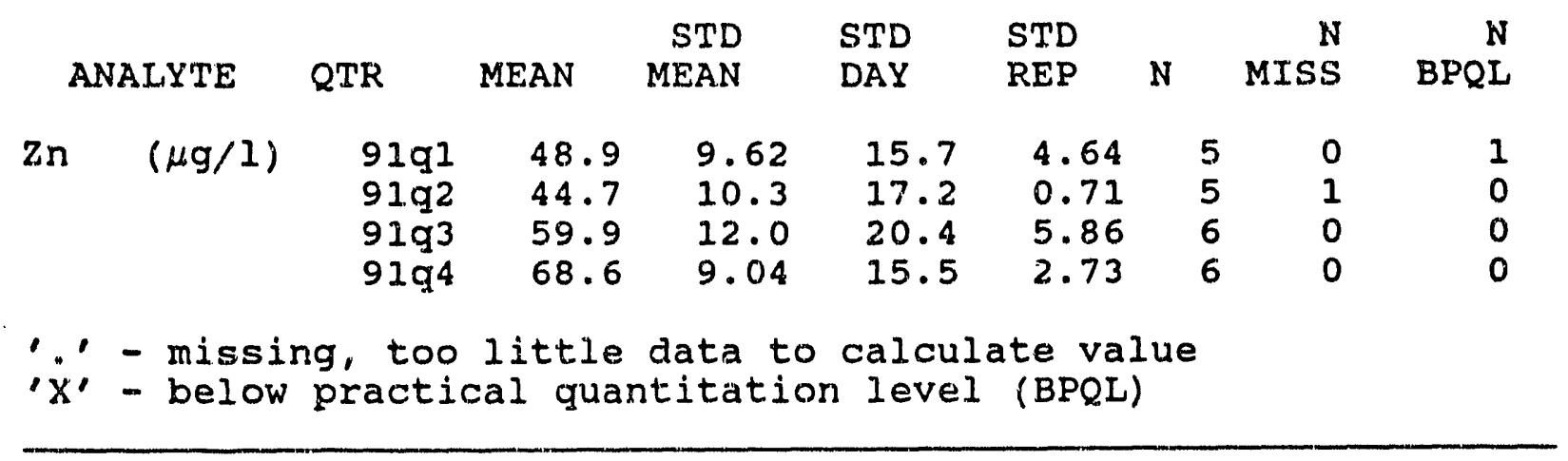




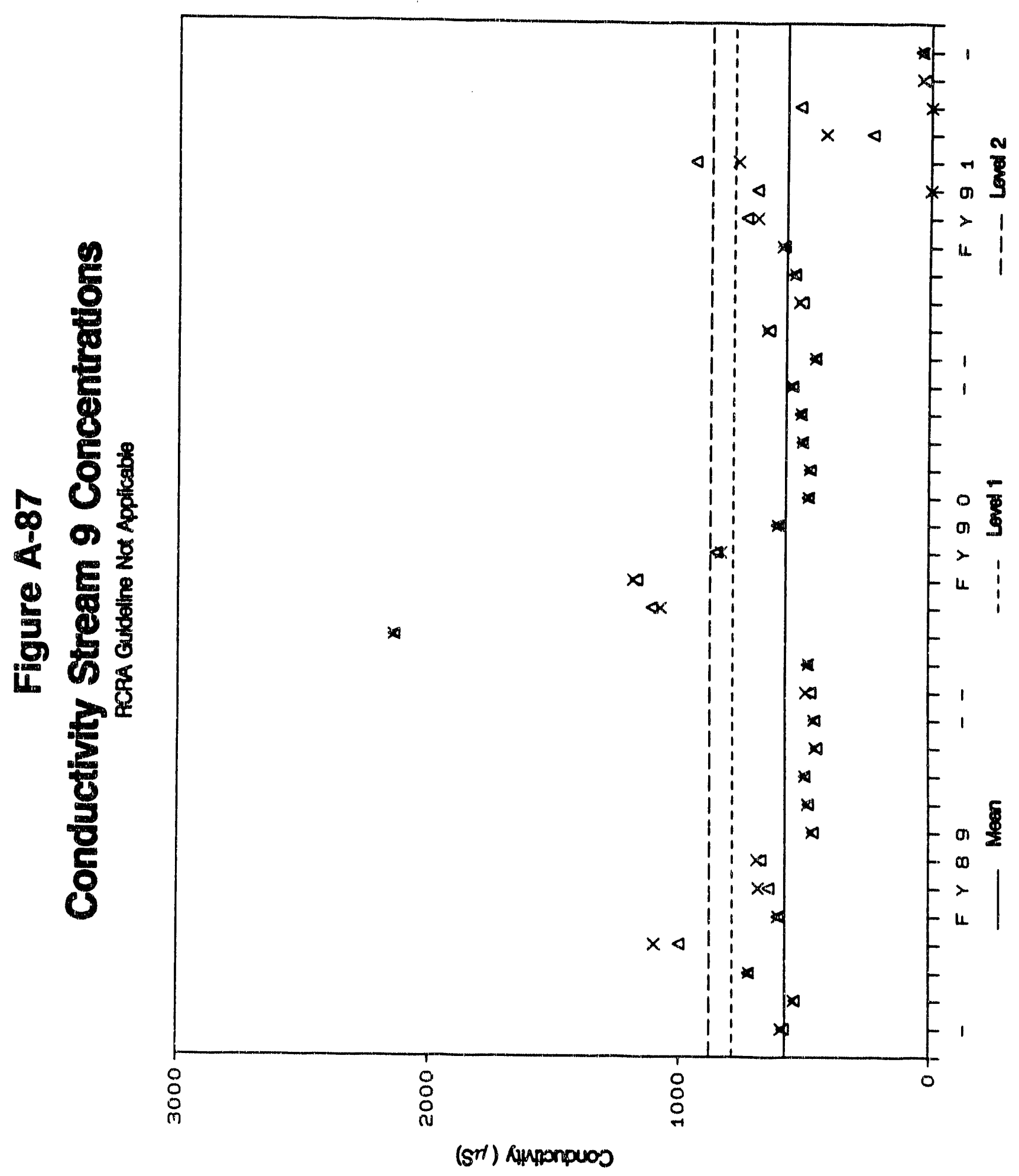




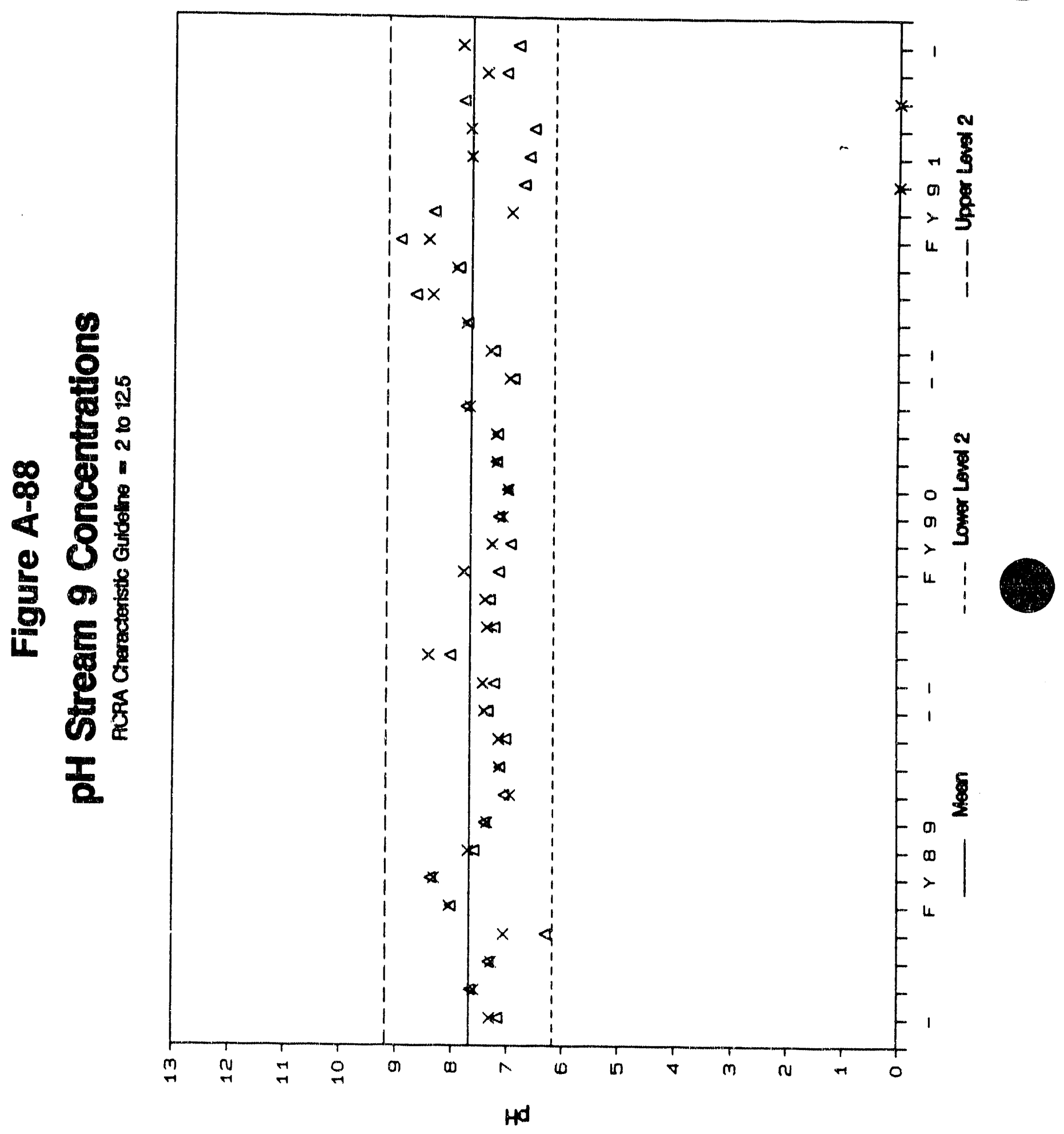




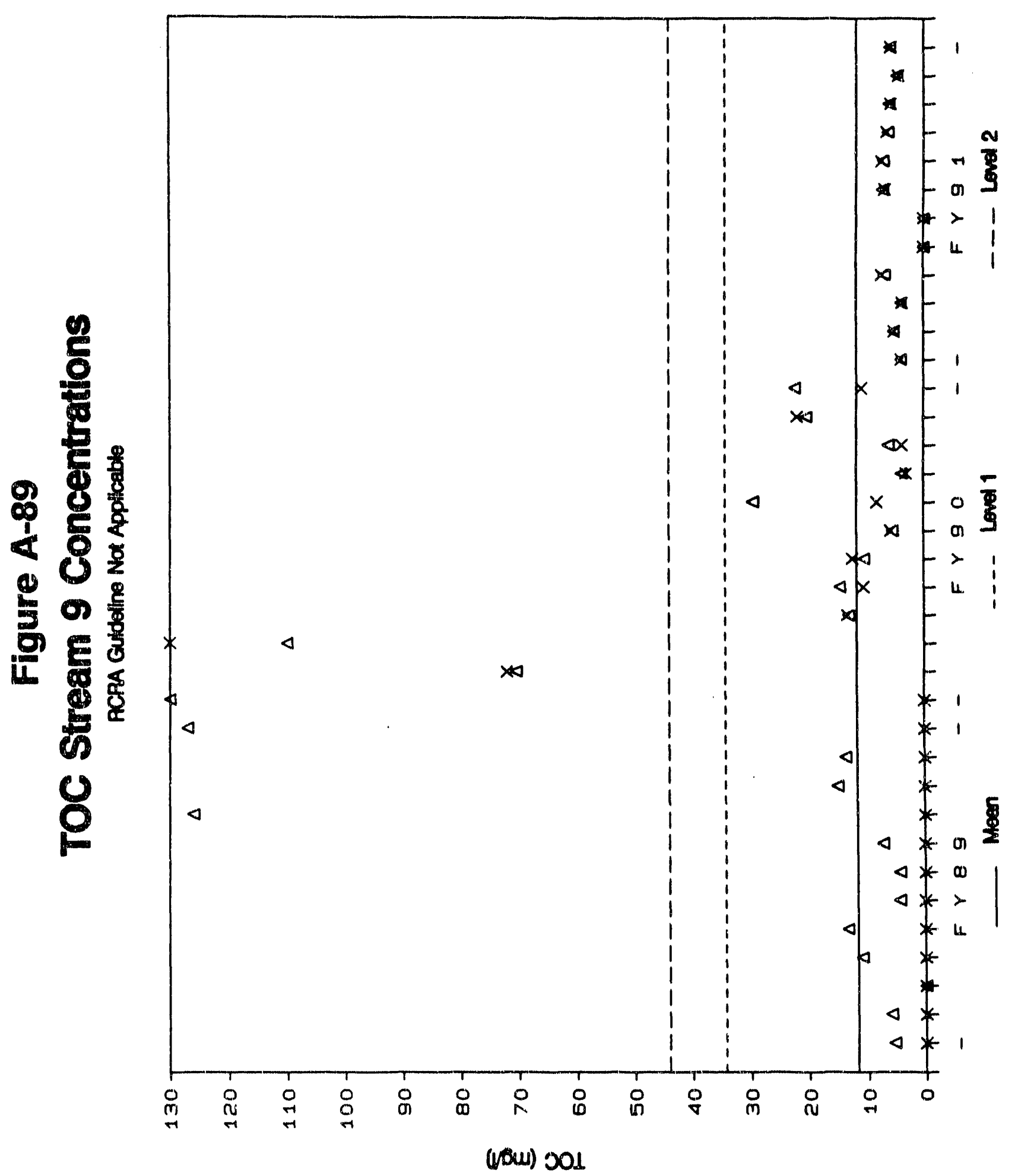




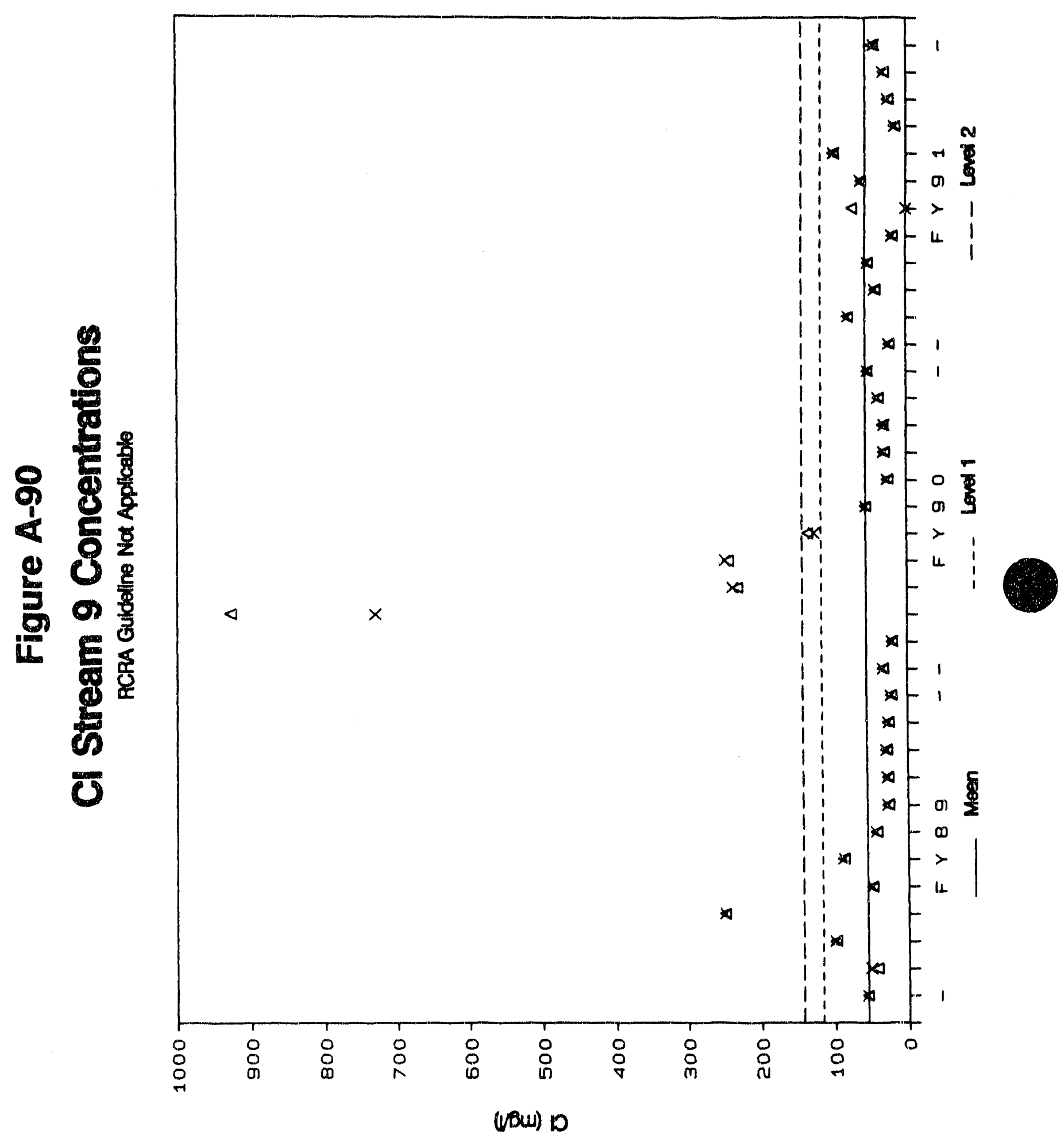




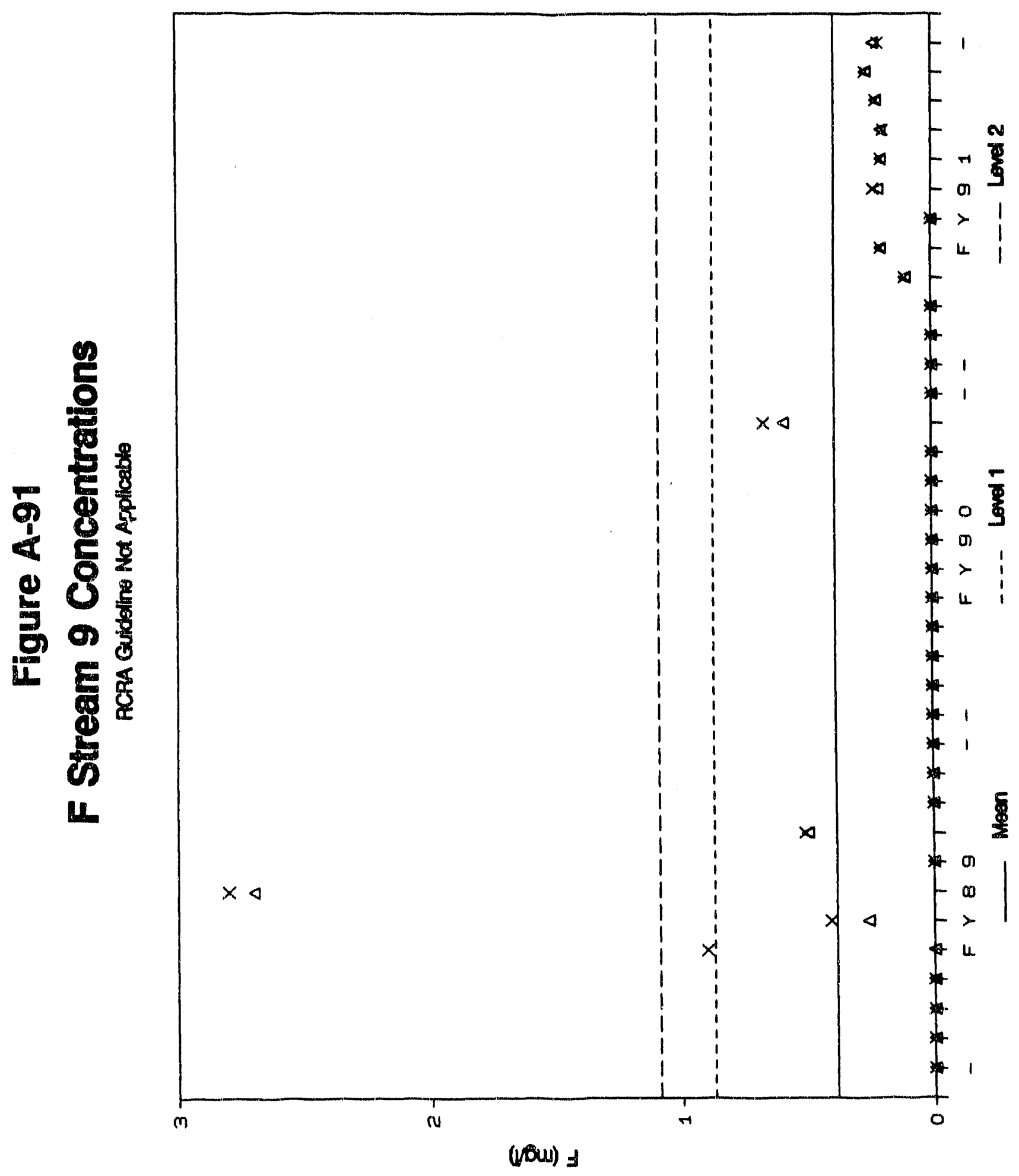

A- 135 


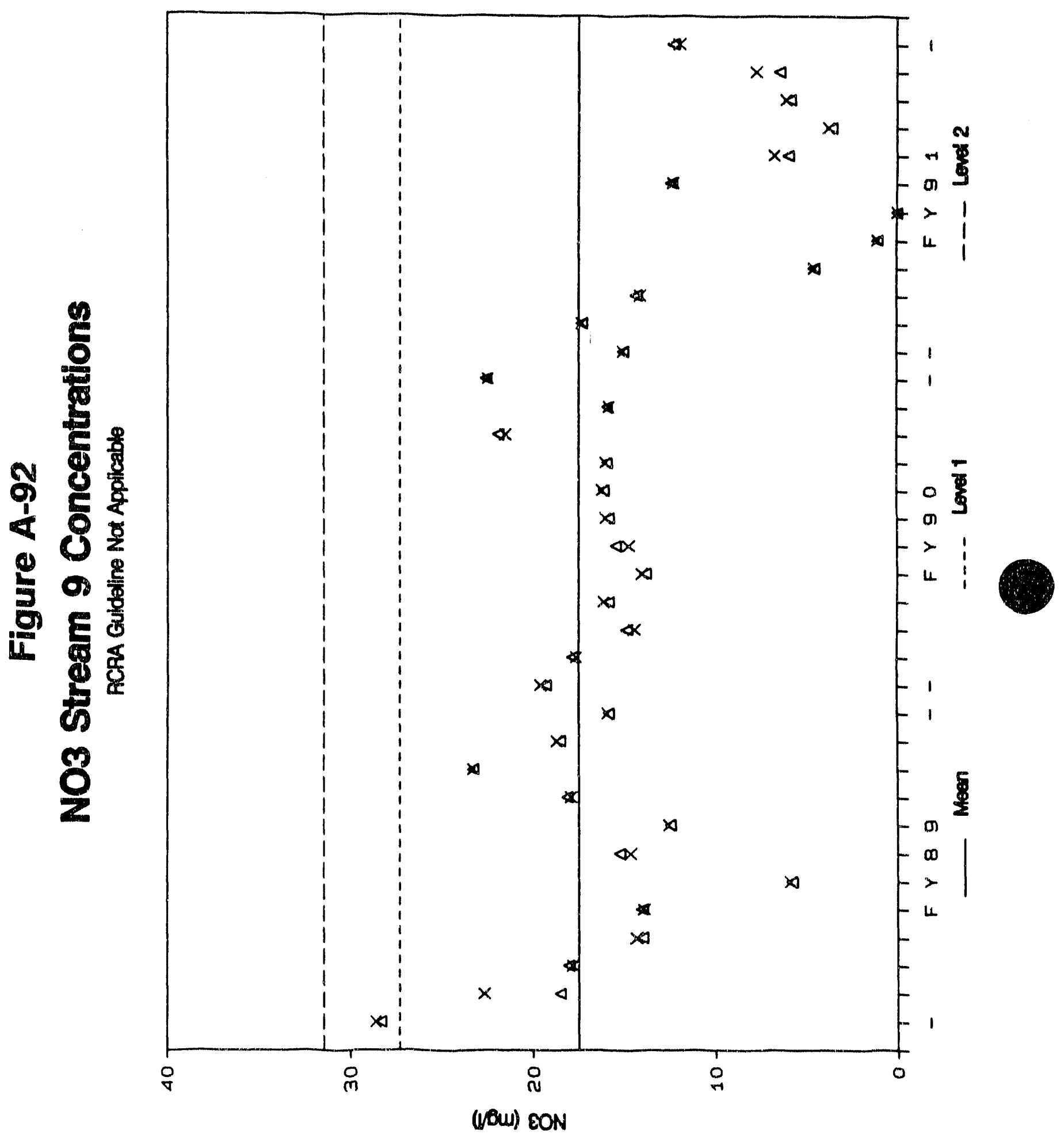




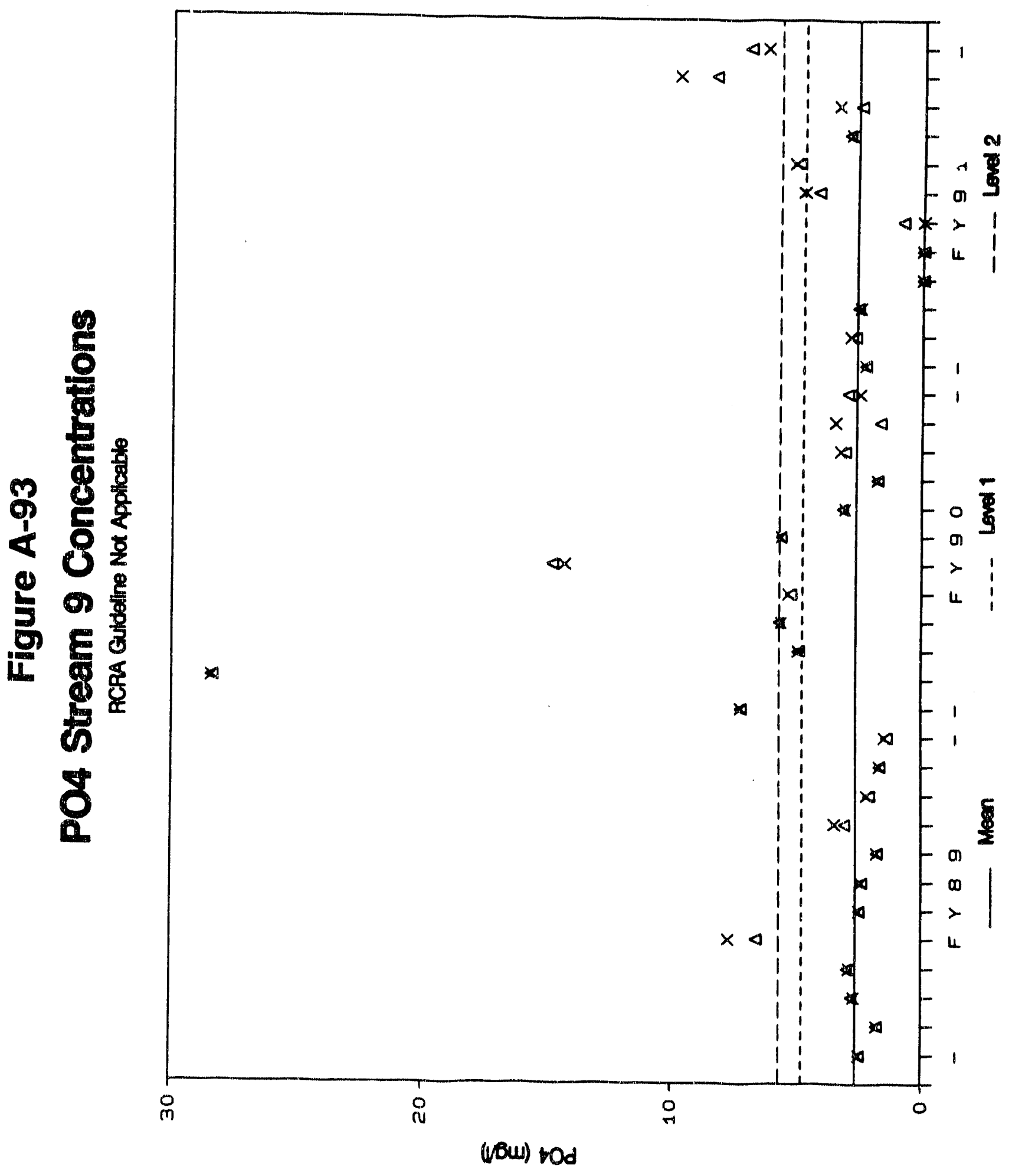




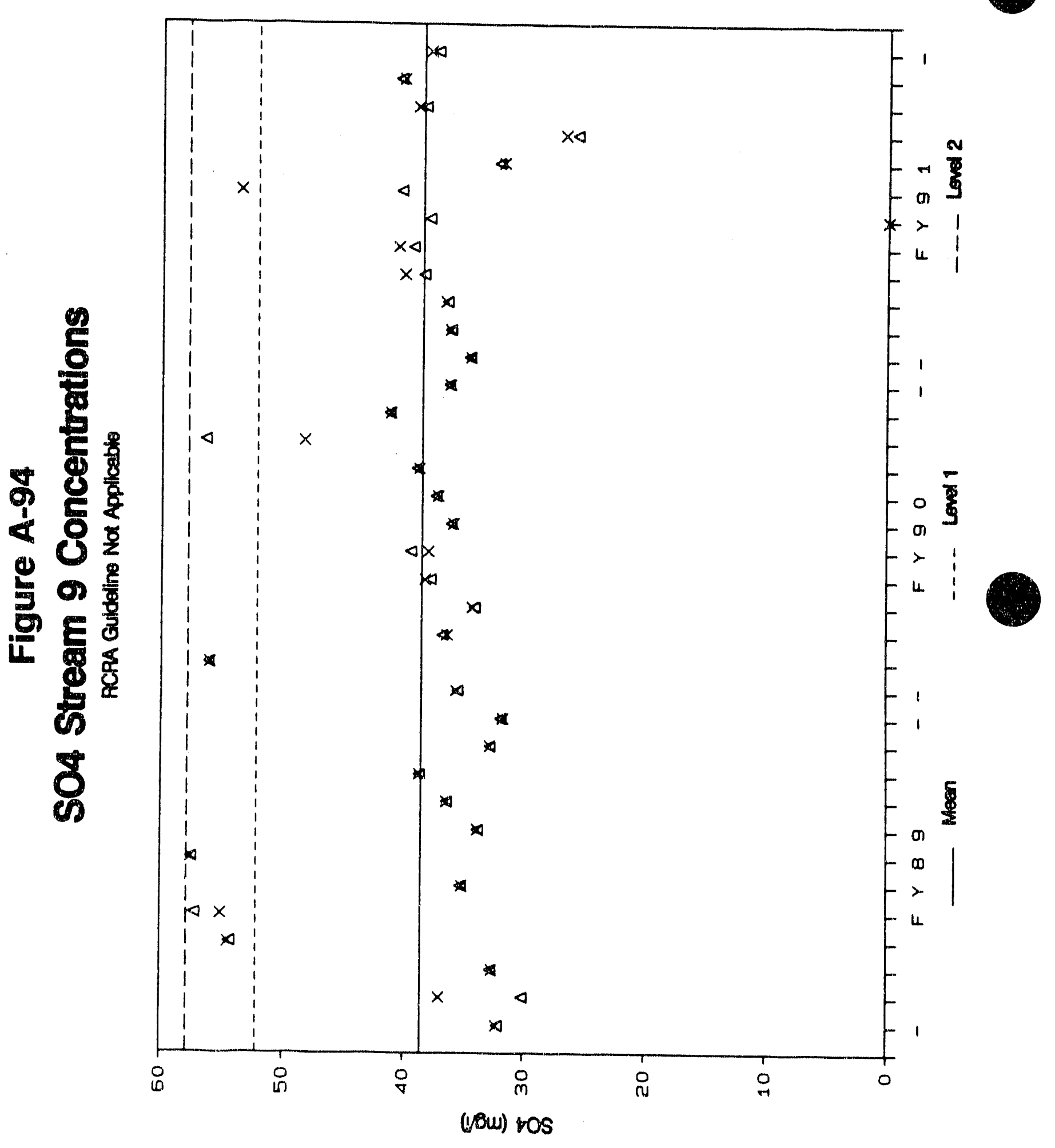




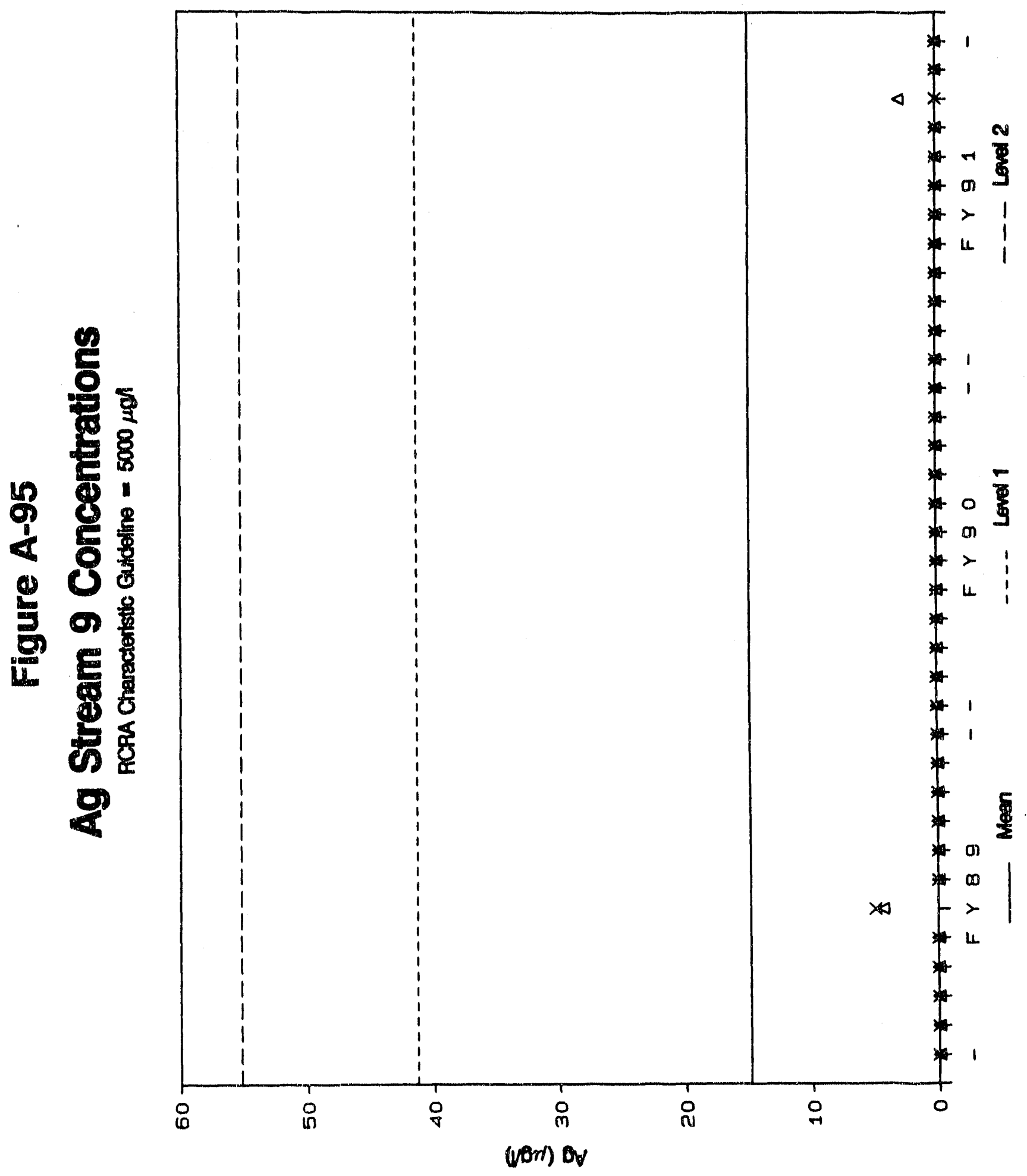




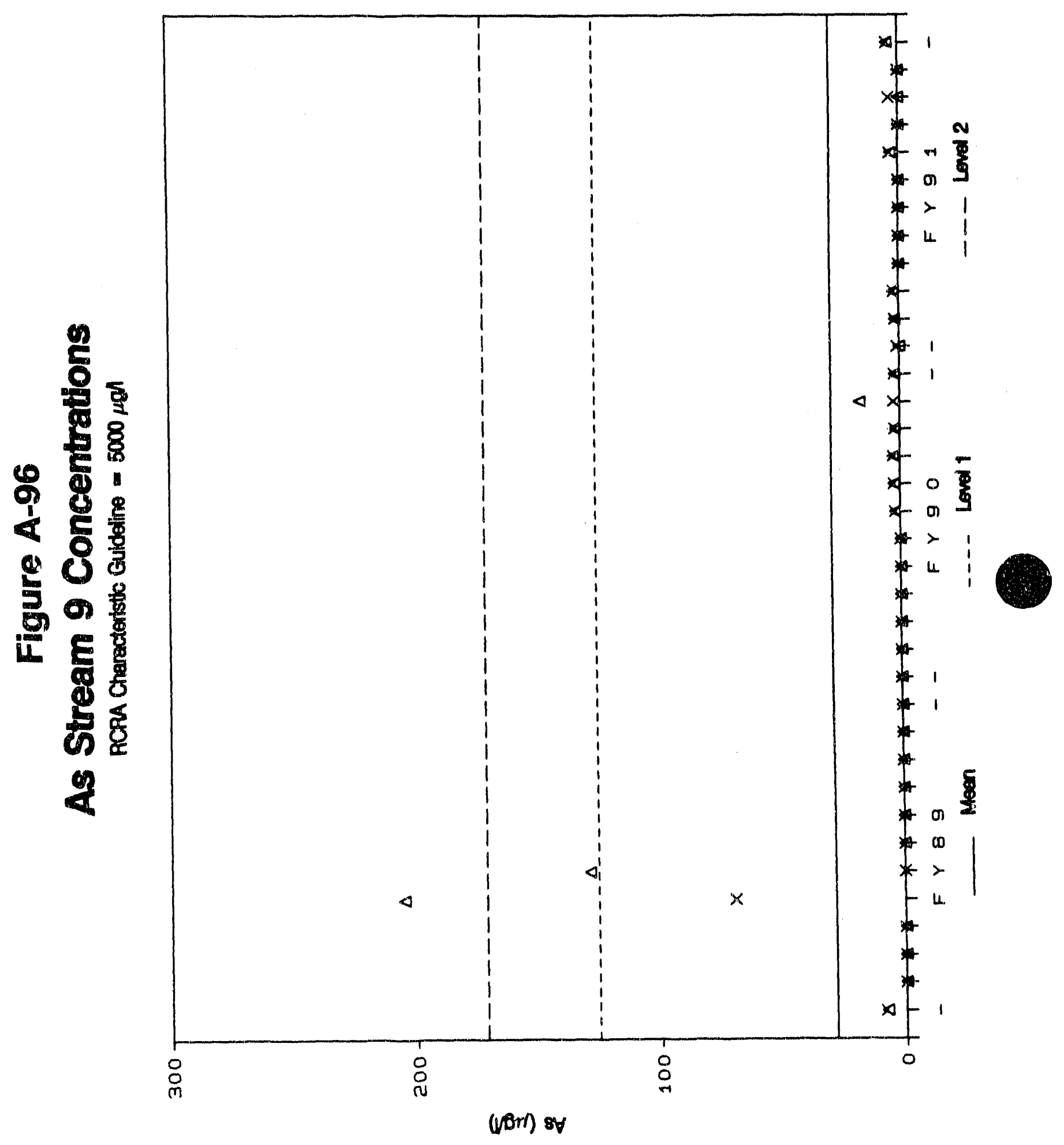




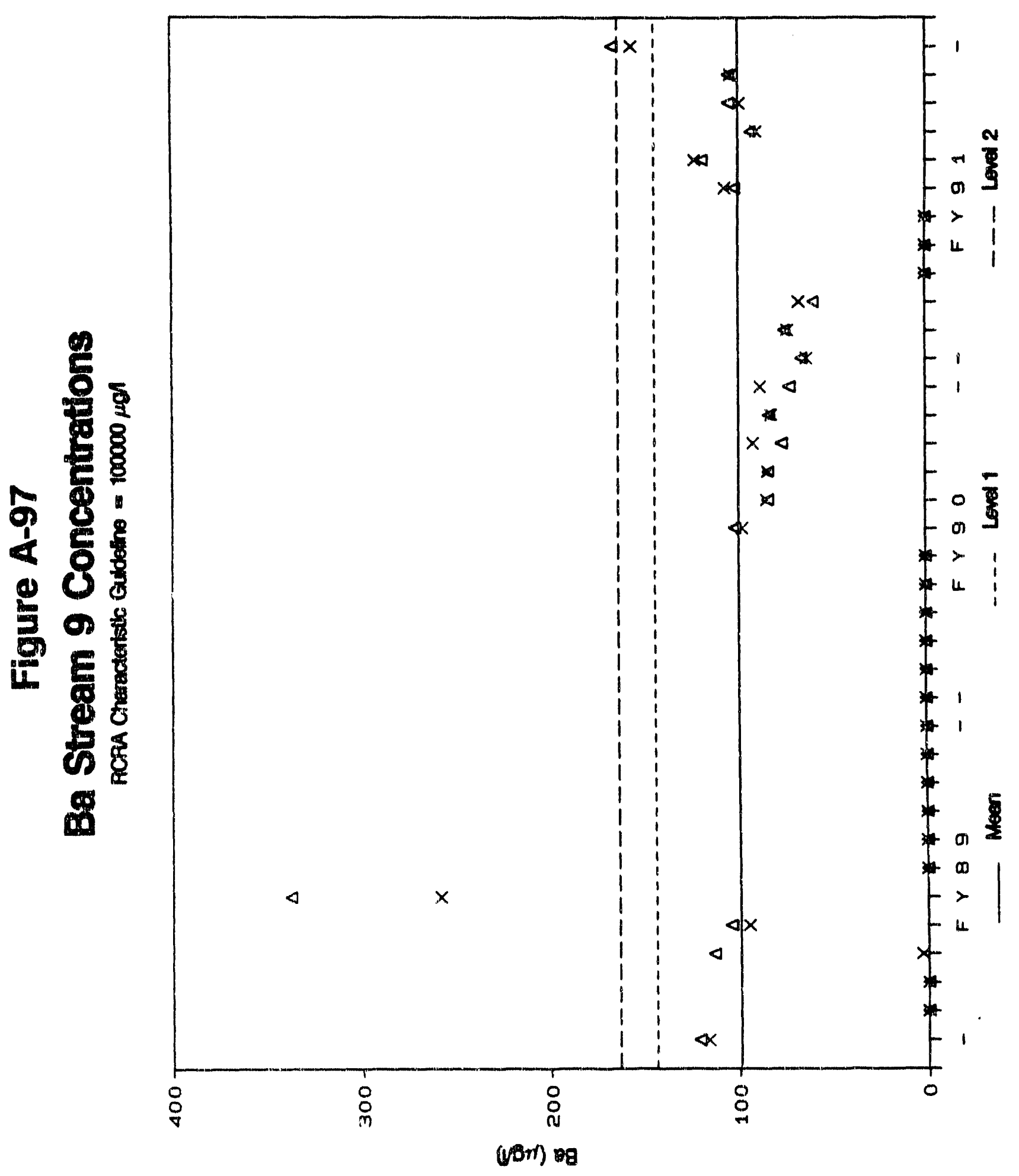




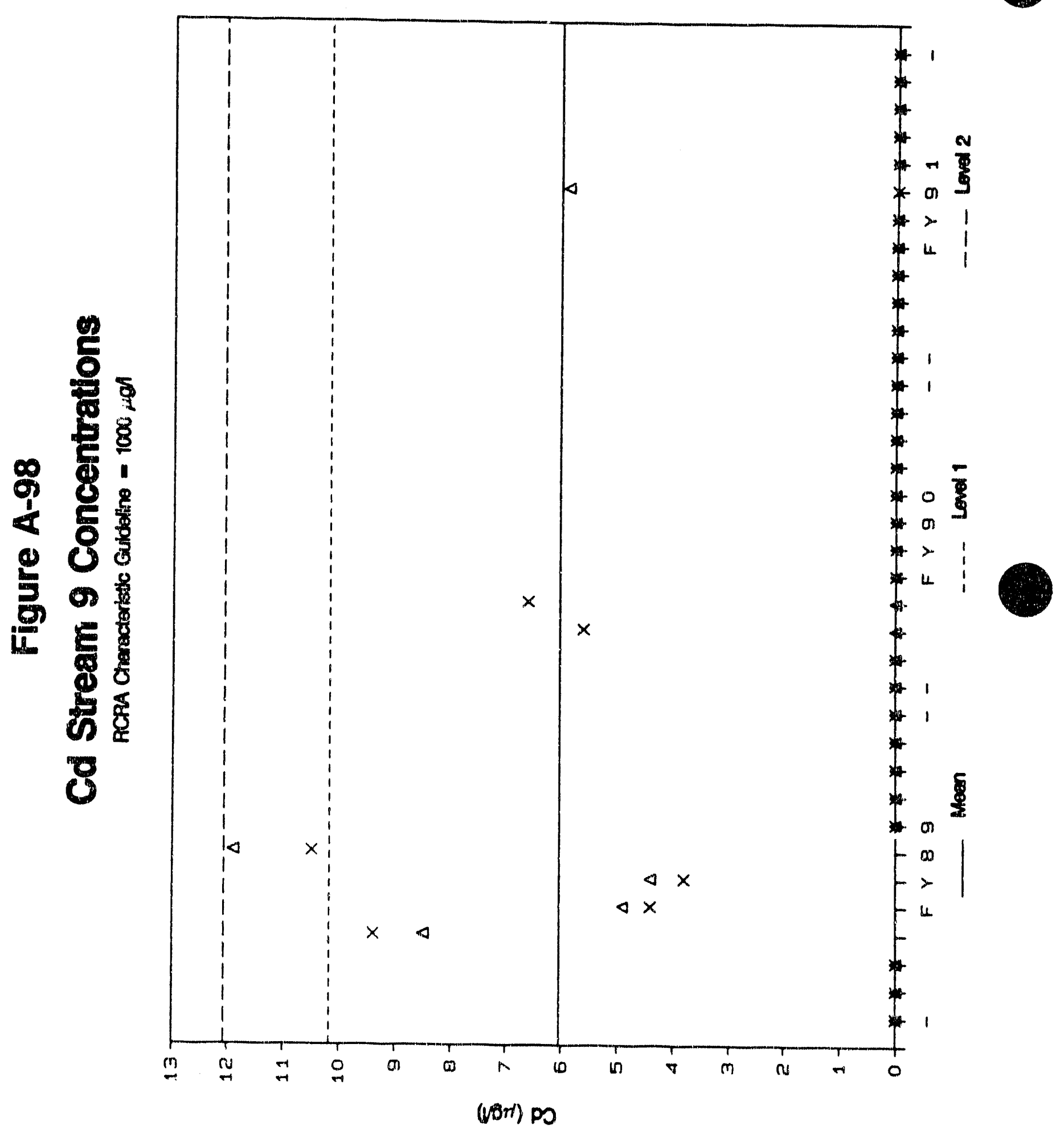

A- 142 


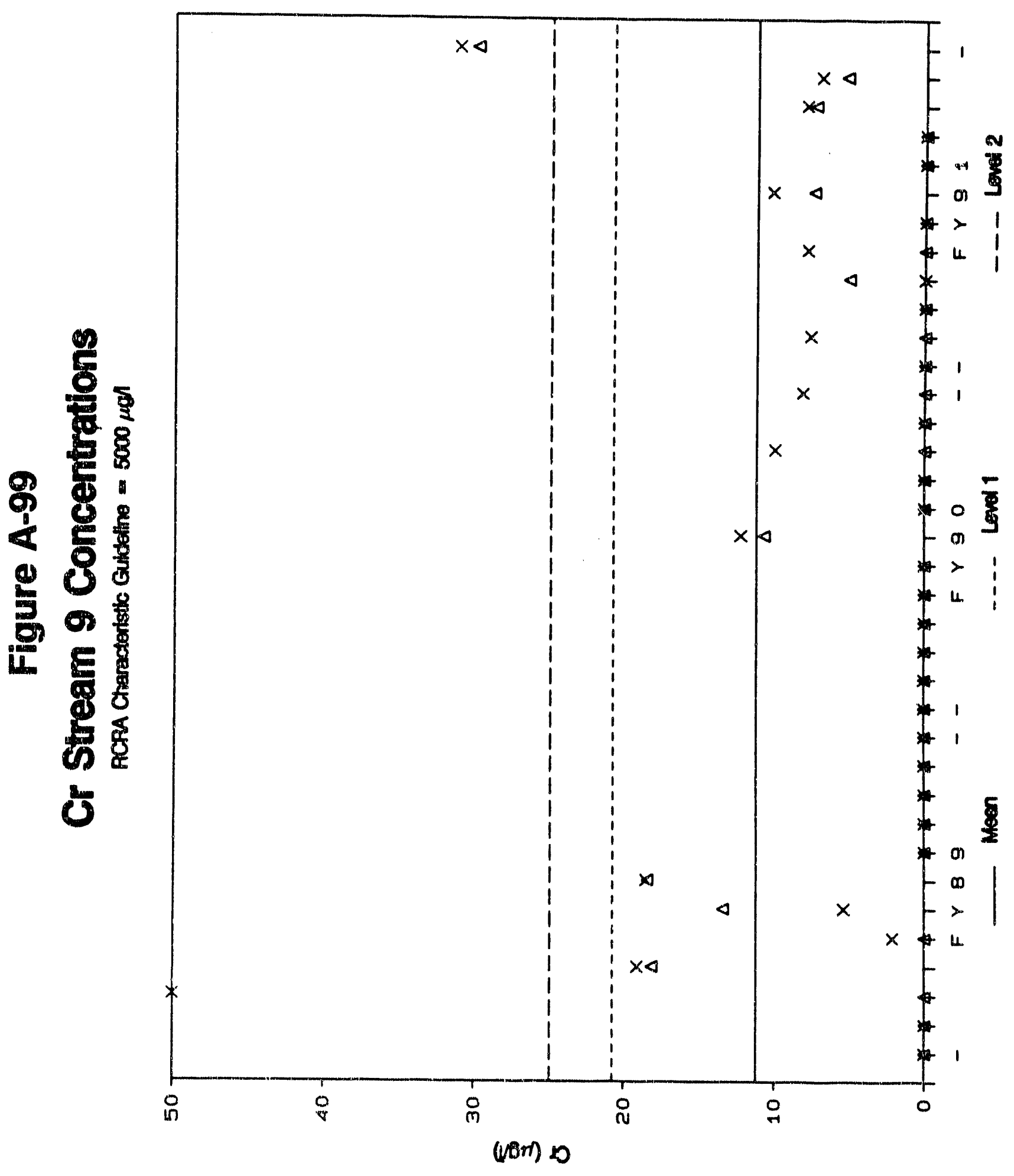




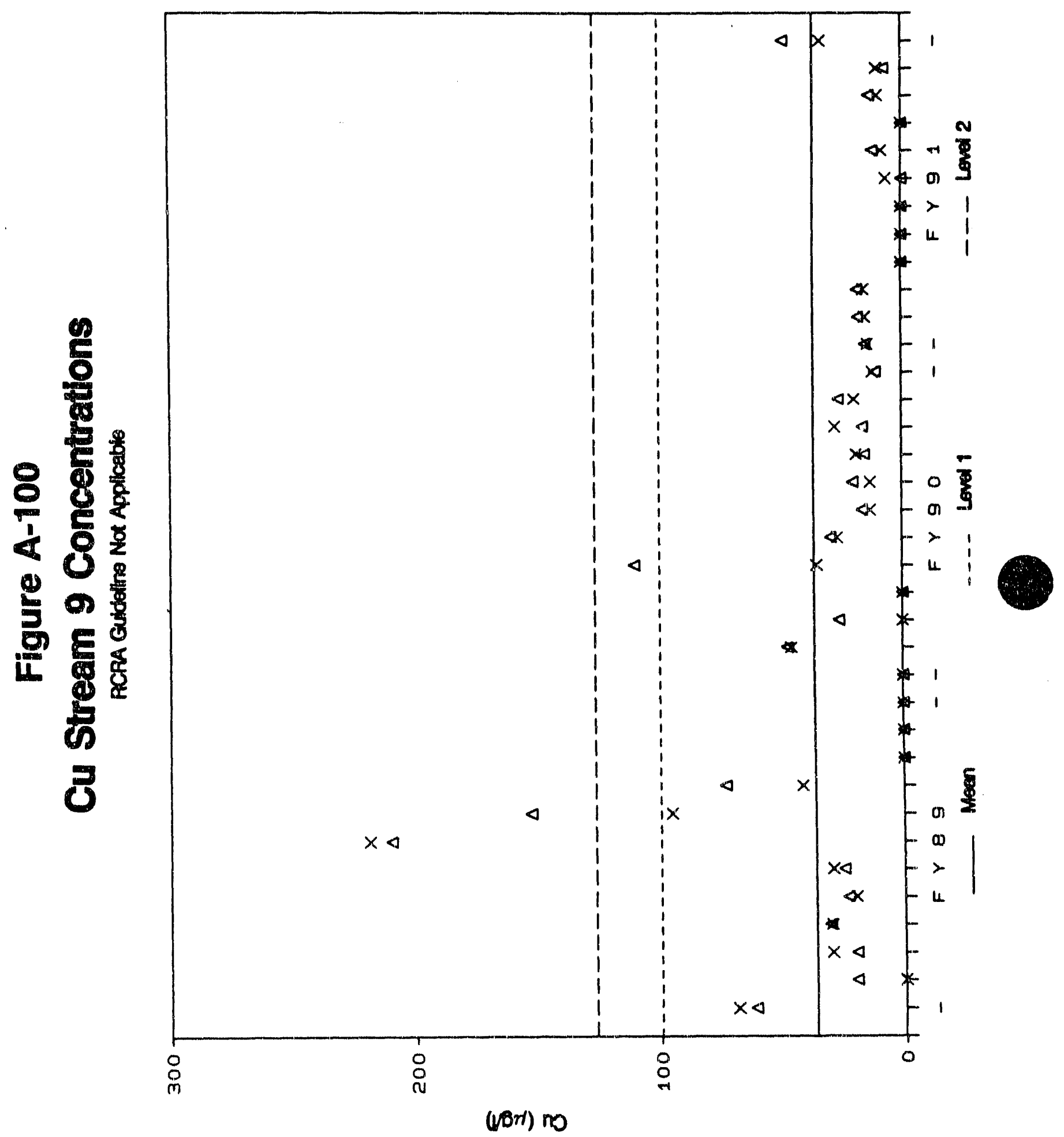




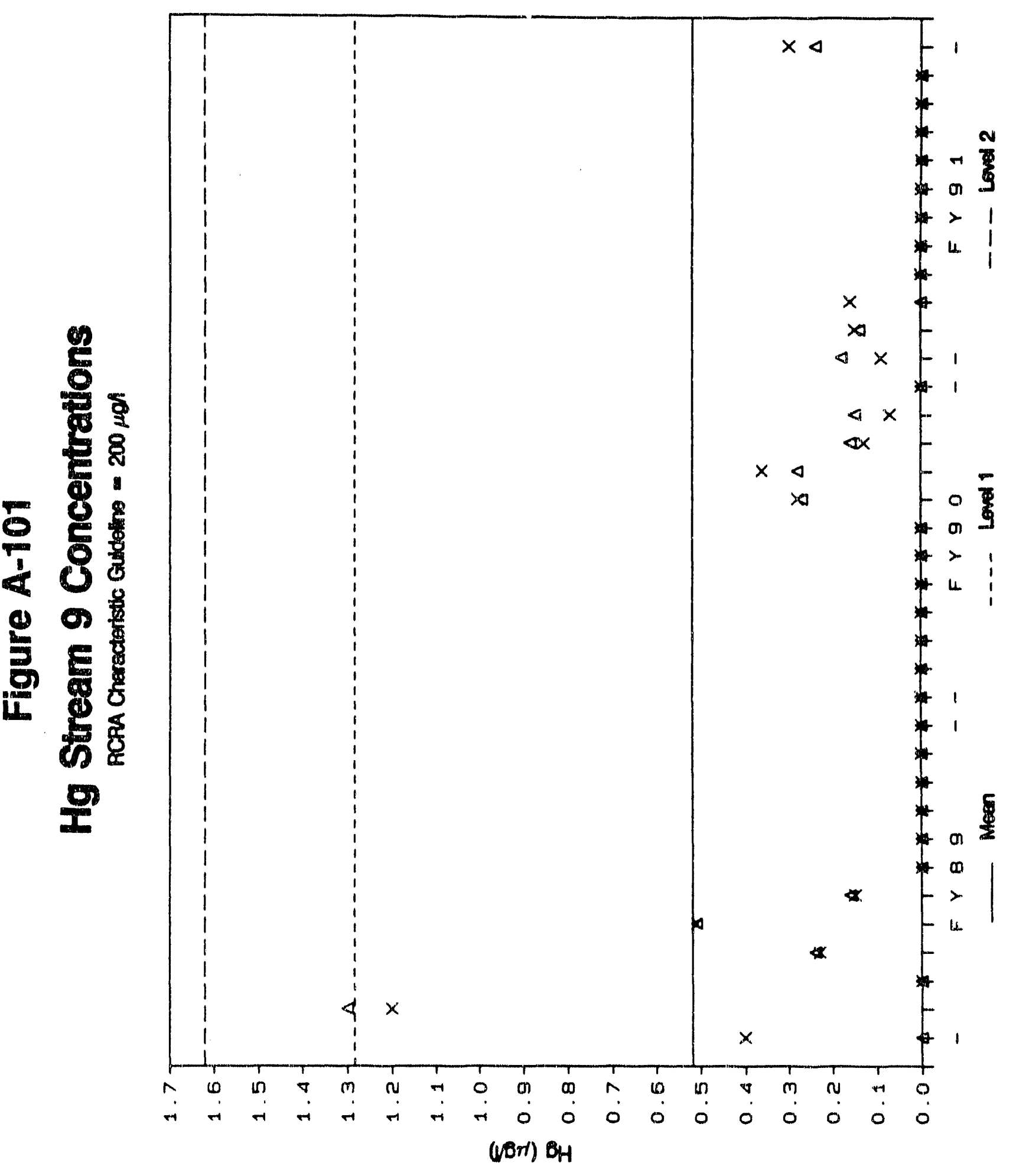




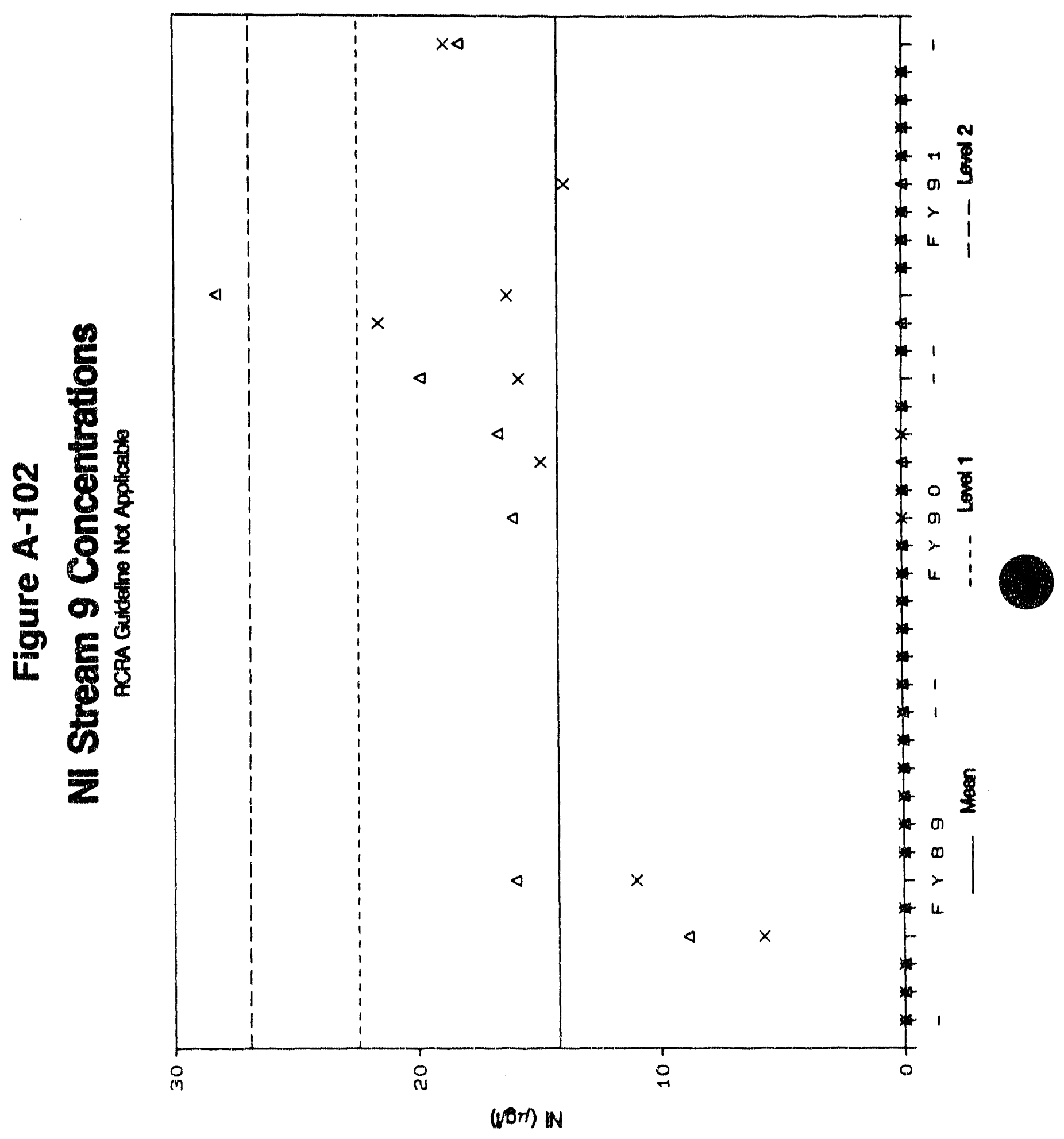




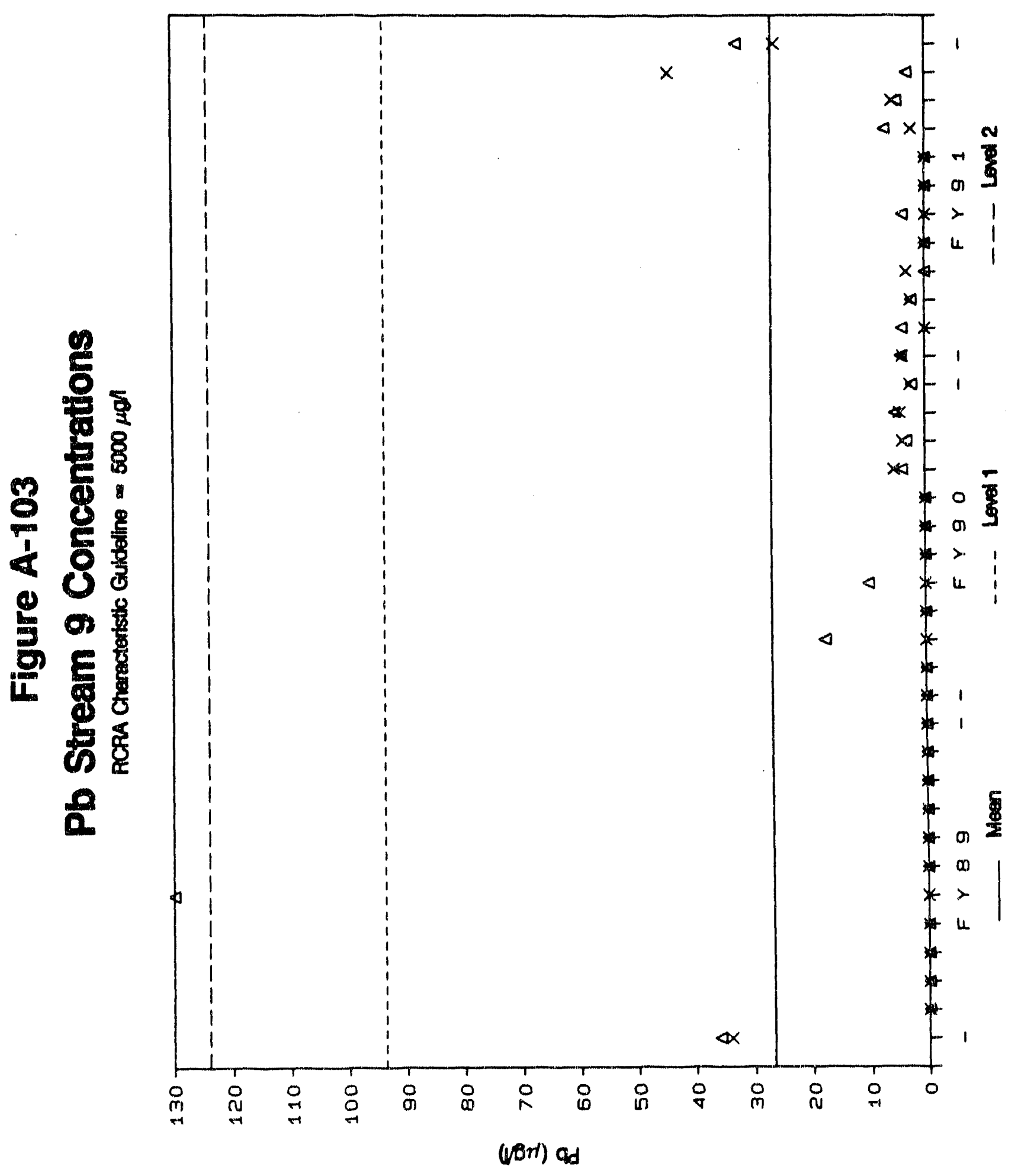




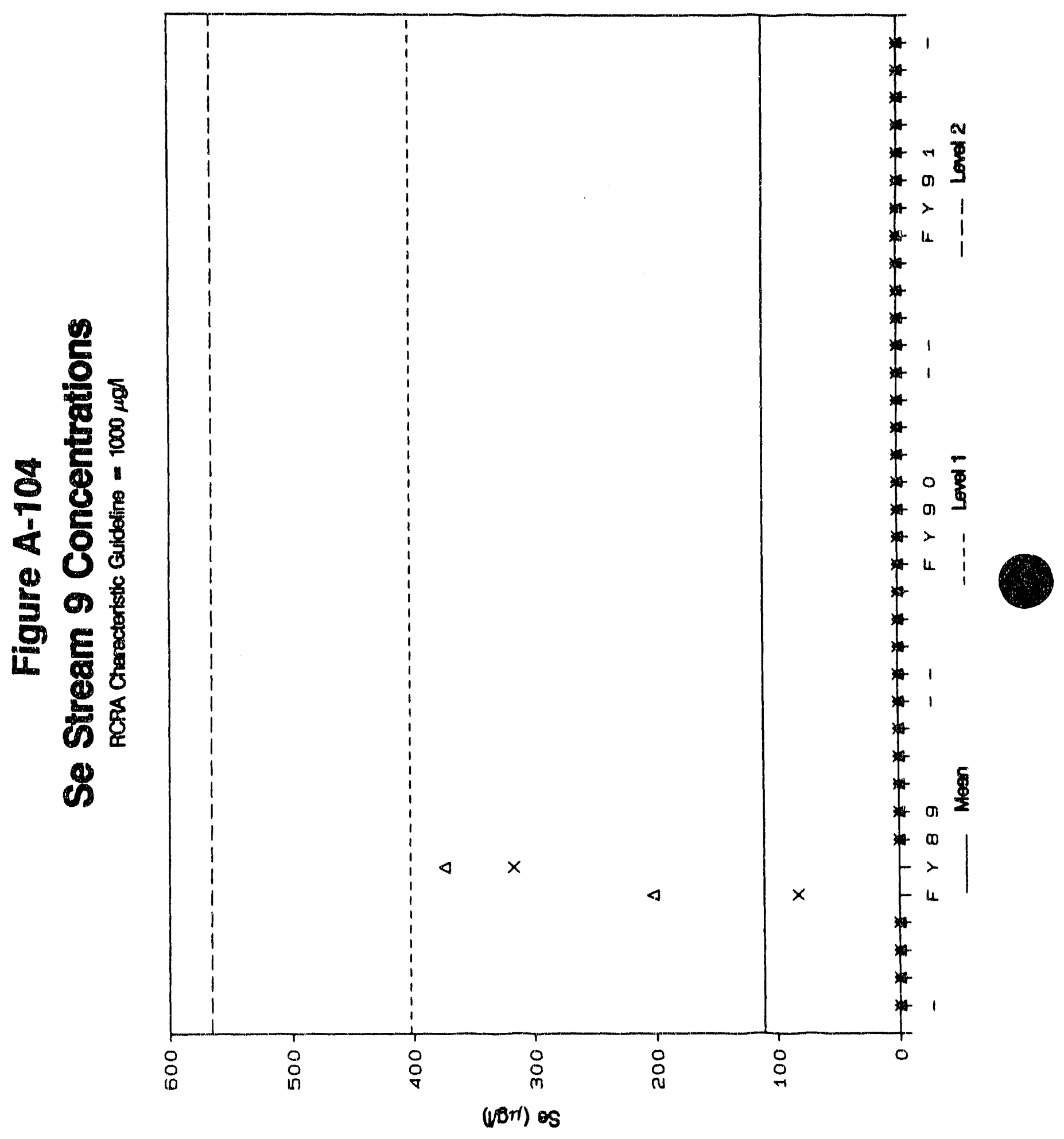




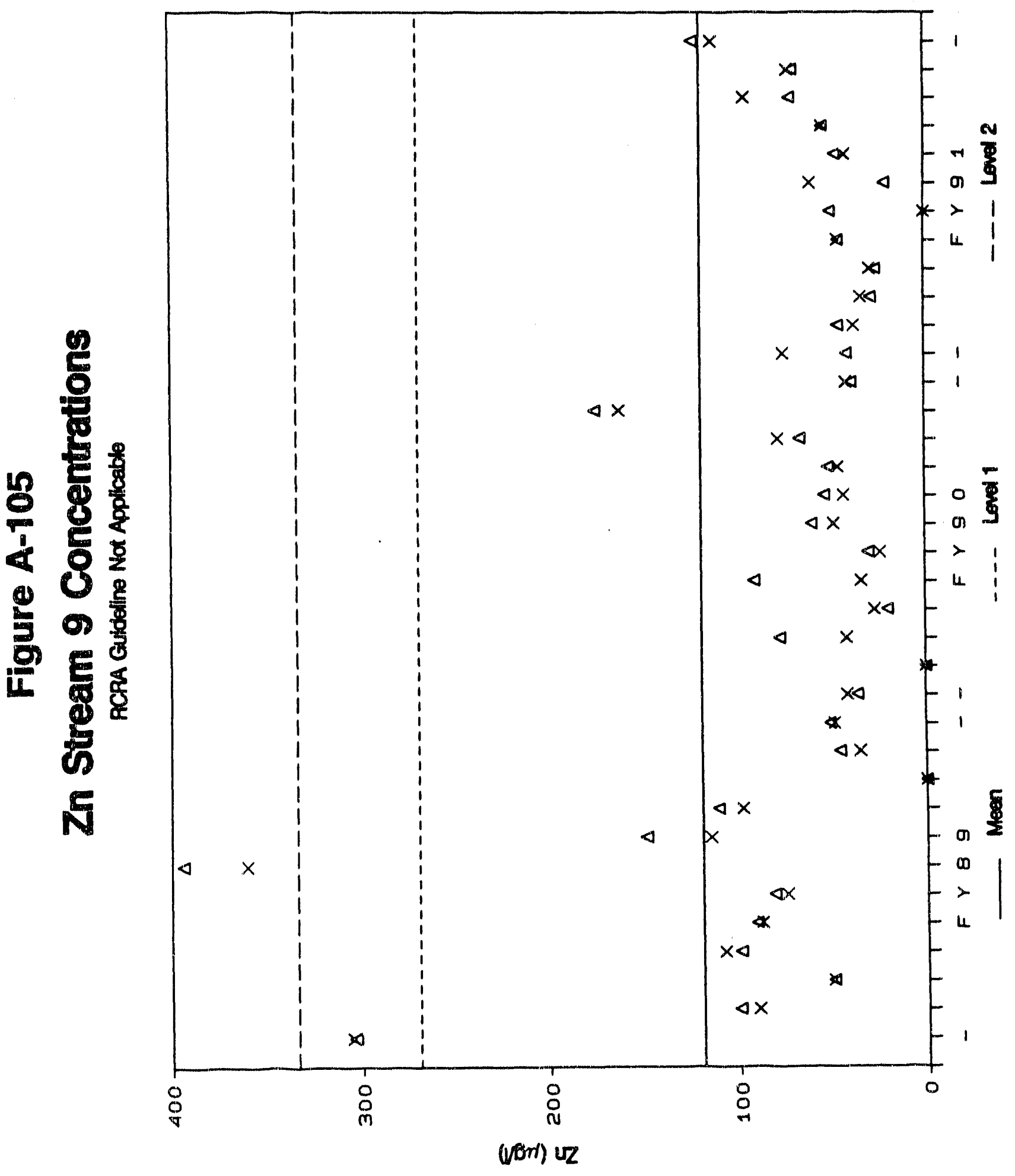




\section{Table A-30}

FY9I ENVIRONMENTAL MONITORING DATA

-- STREAM=9 (TAN TSF effluent to drainage pond) QUARTER=91q1 --

$\begin{array}{ccccccccc}\text { MONTH } & \text { REP } & \begin{array}{c}\text { Cond } \\ (\mu \mathrm{S})\end{array} & \mathrm{pH} & \begin{array}{c}\mathrm{TOC} \\ (\mathrm{mg} / \mathrm{l})\end{array} & \begin{array}{c}\mathrm{Cl} \\ (\mathrm{mg} / \mathrm{l})\end{array} & \begin{array}{c}\mathrm{F} \\ (\mathrm{mg} / 1)\end{array} & \begin{array}{c}\text { NO3 } \\ (\mathrm{mg} / \mathrm{l})\end{array} & \begin{array}{c}\text { PO4 } \\ (\mathrm{mg} / \mathrm{l})\end{array} \\ \text { OCt } & 1 & 457 & 7.27 & 4.00 & 23.6 & \mathrm{X} & 15.0 & 2.30 \\ \text { OCt } & 2 & 455 & 7.33 & 4.00 & 23.9 & \mathrm{X} & 15.0 & 2.30 \\ \text { NOV } & 1 & 642 & 7.75 & 5.30 & 80.4 & \mathrm{X} & 17.3 & 2.70 \\ \text { NoV } & 2 & 649 & 7.77 & 5.20 & 79.9 & \mathrm{X} & 17.3 & 2.90 \\ \text { DeC } & 1 & 510 & 8.68 & 4.00 & 42.3 & \mathrm{X} & 14.3 & 2.60 \\ \text { DeC } & 2 & 521 & 8.38 & 3.80 & 42.4 & \mathrm{X} & 14.1 & 2.50\end{array}$

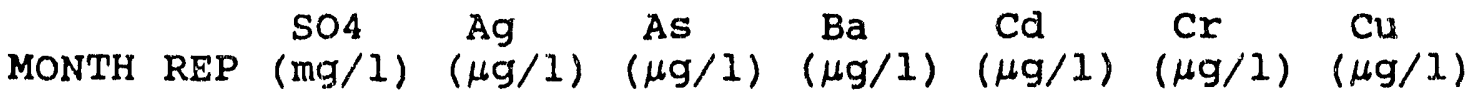

$\begin{array}{lllllllll}\text { Oct } & 1 & 34.6 & \mathrm{X} & \mathrm{X} & 66.0 & \mathrm{X} & \mathrm{X} & 15.2 \\ \text { Oct } & 2 & 34.5 & \mathrm{X} & 1.00 & 63.3 & \mathrm{X} & \mathrm{X} & 14.1 \\ \text { Nov } & 1 & 36.2 & \mathrm{X} & 1.90 & 74.7 & \mathrm{X} & \mathrm{X} & 18.0 \\ \text { Nov } & 2 & 36.2 & \mathrm{X} & 1.80 & 73.1 & \mathrm{X} & 7.70 & 15.2 \\ \text { Dec } & 1 & 36.5 & \mathrm{X} & 2.50 & 59.6 & \mathrm{X} & \mathrm{X} & 18.3 \\ \text { Dec } & 2 & 36.6 & \mathrm{X} & 2.30 & 67.1 & \mathrm{X} & \mathrm{X} & 15.7\end{array}$

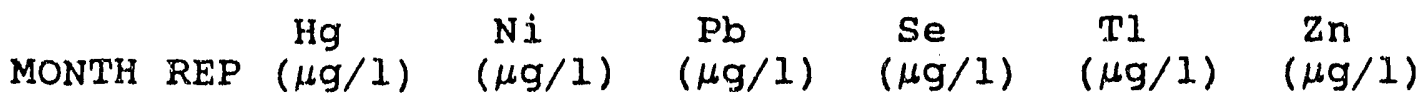

$\begin{array}{llllllll}\text { Oct } & 1 & 0.18 & \mathrm{X} & 4.30 & \mathrm{X} & \mathrm{X} & 41.3 \\ \text { Oct } & 2 & 0.09 & \mathrm{X} & 4.00 & \mathrm{X} & \mathrm{X} & 75.2 \\ \text { Nov } & 1 & 0.14 & \mathrm{X} & 4.10 & \mathrm{X} & \mathrm{X} & 45.9 \\ \text { Nov } & 2 & 0.15 & 21.6 & \mathrm{X} & \mathrm{X} & \mathrm{X} & 37.3 \\ \text { Dec } & 1 & \mathrm{X} & 28.3 & 2.50 & \mathrm{X} & \mathrm{X} & 28.3 \\ \text { Dec } & 2 & 0.16 & 16.3 & 2.60 & \mathrm{X} & \mathrm{X} & 33.5\end{array}$

$\therefore$ - missing

' $X$ ' - below practical quantitation level 


\section{Table A-31}

FY91 ENVIRONMENTAL MONITORING DATA

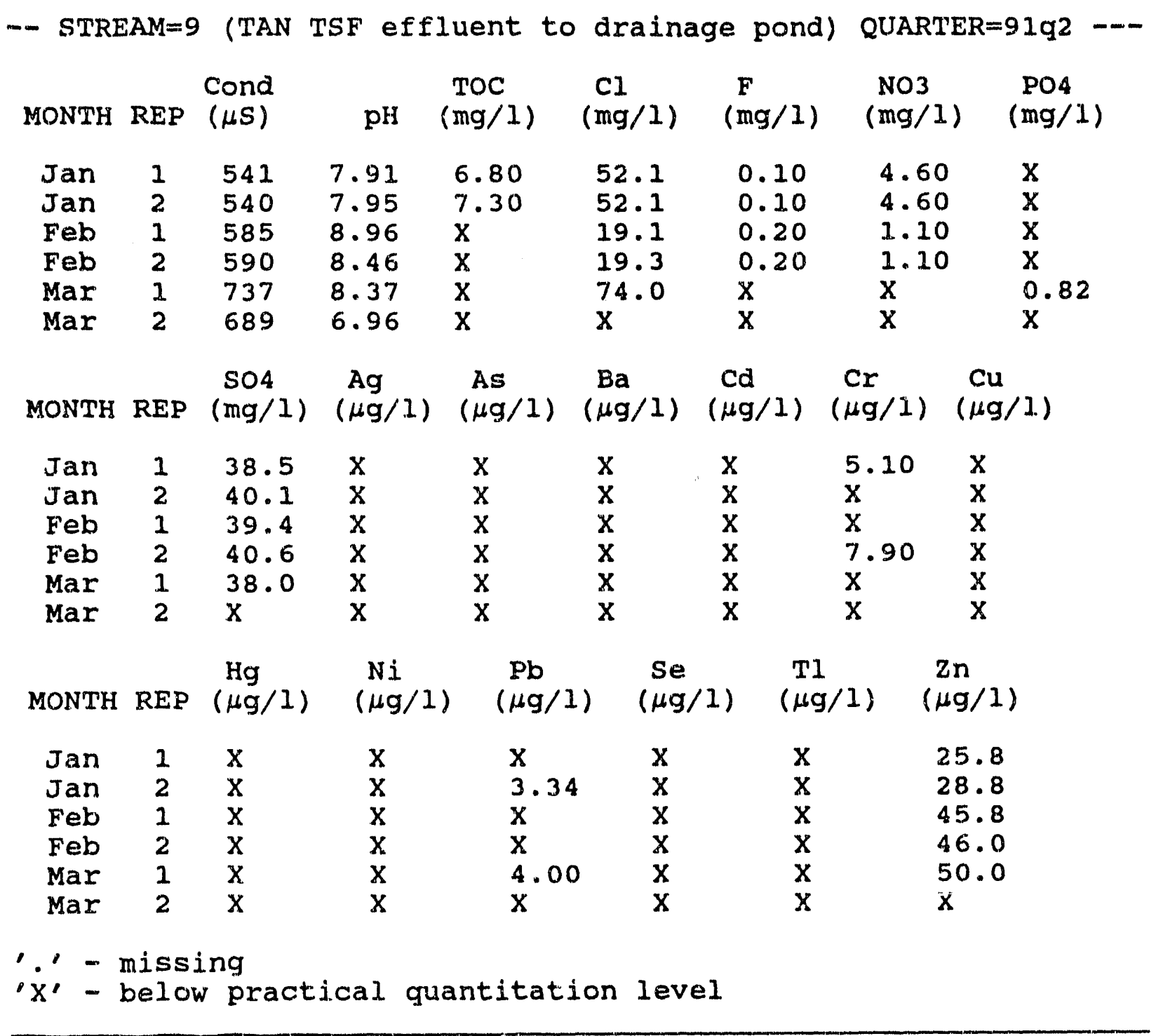




\section{Table A-32}

FY91 ENVIRONMENTAL MONITORING DATA

-- STREAM=9 (TAN TSF effluent to drainage pond) QUARTER=9193 ---

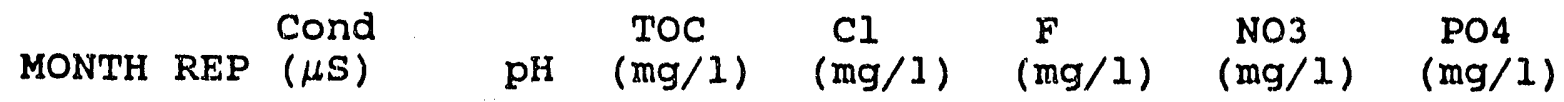

$\begin{array}{lrrrrrrrr}\text { Apr } & 1 & 694 & 6.75 & 7.17 & 61.2 & 0.21 & 12.3 & 4.22 \\ \text { Apr } & 2 & \mathrm{X} & \mathrm{X} & 6.80 & 61.9 & 0.23 & 12.2 & 4.77 \\ \text { May } & 1 & 940 & 6.66 & 7.02 & 100 & 0.20 & 6.00 & 4.99 \\ \text { May } & 2 & 771 & 7.69 & 7.38 & 98.8 & 0.20 & 6.78 & 5.11 \\ \text { Jun } & 1 & 233 & 6.57 & 6.26 & 15.8 & 0.20 & 3.63 & 3.00 \\ \text { Jun } & 2 & 418 & 7.71 & 6.49 & 15.8 & 0.19 & 3.81 & 2.91\end{array}$

$\begin{array}{cccccccc}\operatorname{MONTH} & \mathrm{REP} & \mathrm{Ag} & \mathrm{As} & \mathrm{Ba} & \mathrm{Cd} & \mathrm{Cr} & \mathrm{Cu} \\ (\mathrm{mg} / 1) & (\mu \mathrm{g} / 1) & (\mu \mathrm{g} / 1) & (\mu \mathrm{g} / 1) & (\mu \mathrm{g} / 1) & (\mu \mathrm{g} / 1) & (\mu \mathrm{g} / 1)\end{array}$

$\begin{array}{lllllrlll}\text { Apr } & 1 & 40.4 & X & X & 101 & 5.90 & 7.50 & X \\ \text { Apr } & 2 & 53.6 & X & X & 106 & X & 10.2 & 6.50 \\ \text { May } & 1 & 32.2 & X & 3.30 & 118 & X & X & 11.9 \\ \text { May } & 2 & 31.8 & X & 3.70 & 122 & X & X & 8.40 \\ \text { Jun } & 1 & 25.8 & X & X & 92.4 & X & X & X \\ \text { Jun } & 2 & 26.8 & X & X & 89.2 & X & X & X\end{array}$

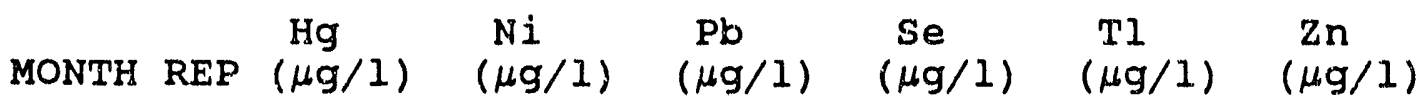

$\begin{array}{llllllll}\text { Apr } & 1 & X & X & X & X & X & 21.1 \\ \text { Apr } & 2 & X & 13.9 & X & X & X & 60.6 \\ \text { May } & 1 & X & X & X & X & X & 47.4 \\ \text { May } & 2 & X & X & X & X & X & 42.0 \\ \text { Jun } & 1 & X & X & 7.10 & X & X & 54.2 \\ \text { Jun } & 2 & X & X & 2.50 & X & X & 54.2\end{array}$

$\because '$ - missing

' $\mathrm{X}$ ' - below practical quantitation level 


\section{Table A-33}

FY91 ENVIRONMENTAL MONITORING DATA

\begin{tabular}{|c|c|c|c|c|c|c|c|c|c|c|}
\hline \multicolumn{2}{|c|}{ - STREAM=9 } & (TA & \multicolumn{2}{|c|}{ TSF effluent to } & \multicolumn{2}{|c|}{ drainage } & pond) & \multicolumn{3}{|c|}{ QUARTER $=91 \mathrm{q} 4$} \\
\hline MONTH & REP & $\begin{array}{l}\text { Cond } \\
(\mu S)\end{array}$ & $\mathrm{pH}$ & $\begin{array}{l}\text { TOC } \\
(\mathrm{mg} / \mathrm{l})\end{array}$ & $\begin{array}{l}\mathrm{cl} \\
(\mathrm{mg} / \mathrm{l})\end{array}$ & & $\begin{array}{l}\mathrm{F} \\
\mathrm{mg} / \mathrm{l})\end{array}$ & & 3 & $\begin{array}{c}\mathrm{PO} 4 \\
(\mathrm{mg} / \mathrm{l})\end{array}$ \\
\hline $\begin{array}{l}\text { Jul } \\
\text { Jul } \\
\text { Aug } \\
\text { Aug } \\
\text { Sep } \\
\text { Sep }\end{array}$ & $\begin{array}{l}1 \\
2 \\
1 \\
2 \\
1 \\
2\end{array}$ & $\begin{array}{c}523.0 \\
\times \\
25.3 \\
35.5 \\
42.0 \\
35.4\end{array}$ & $\begin{array}{l}7.84 \\
x \\
7.07 \\
7.42 \\
6.86 \\
7.86\end{array}$ & $\begin{array}{l}6.09 \\
5.82 \\
4.76 \\
4.49 \\
5.91 \\
5.78\end{array}$ & $\begin{array}{l}25.2 \\
25.8 \\
30.8 \\
31.6 \\
45.7 \\
44.0\end{array}$ & & $\begin{array}{l}0.22 \\
0.22 \\
0.26 \\
0.26 \\
0.23 \\
0.21\end{array}$ & & $\begin{array}{l}89 \\
11 \\
47 \\
75 \\
2 \\
9 \\
9\end{array}$ & $\begin{array}{l}2.55 \\
3.43 \\
8.32 \\
9.76 \\
6.95 \\
6.27\end{array}$ \\
\hline MONTH & REP & $\begin{array}{c}\mathrm{so4} \\
(\mathrm{mg} / \mathrm{I})\end{array}$ & $\frac{\mathrm{Ag}}{(\mu \mathrm{g} / 1)}$ & $\begin{array}{l}\text { As } \\
(\mu \mathrm{g} / 1)\end{array}$ & $\begin{array}{l}\mathrm{Ba} \\
(\mu \mathrm{g} / 1)\end{array}$ & $\frac{\mathrm{Cd}}{(\mu \mathrm{g})}$ & /1) & $\begin{array}{l}\mathrm{Cr} \\
(\mu \mathrm{g} / 1)\end{array}$ & $\begin{array}{l}\mathrm{Cl} \\
(\mu \mathrm{C}\end{array}$ & \\
\hline $\begin{array}{l}\text { Jul } \\
\text { Jul } \\
\text { Aug } \\
\text { Aug } \\
\text { Sep } \\
\text { Sep }\end{array}$ & $\begin{array}{l}1 \\
2 \\
1 \\
2 \\
1 \\
2\end{array}$ & $\begin{array}{l}38.4 \\
39.0 \\
40.5 \\
40.2 \\
37.4 \\
38.0\end{array}$ & $\begin{array}{l}2.90 \\
X \\
x \\
x \\
X \\
x\end{array}$ & $\begin{array}{l}X \\
3.90 \\
x \\
X \\
4.60 \\
4.60\end{array}$ & $\begin{array}{r}104 \\
98.4 \\
104 \\
103 \\
166 \\
156\end{array}$ & $\begin{array}{l}X \\
X \\
X \\
X \\
X \\
X\end{array}$ & & $\begin{array}{l}7.40 \\
8.00 \\
5.30 \\
7.00 \\
29.9 \\
31.1\end{array}$ & $\begin{array}{l}13 \\
10 \\
7 . \\
10 \\
48 \\
33\end{array}$ & \\
\hline MONTH & REP & $\begin{array}{l}\mathrm{Hg} \\
(\mu \mathrm{g} / 1)\end{array}$ & $\begin{array}{l}\mathrm{Ni} \\
(\mu \mathrm{g} / I)\end{array}$ & $\begin{array}{l}\mathrm{Pb} \\
(\mu \mathrm{g} / 1)\end{array}$ & $\begin{array}{c}\mathrm{Se} \\
(\mu \mathrm{g} / \mathrm{J}\end{array}$ & & $\stackrel{\mathrm{TI}}{(\mu \mathrm{g})}$ & & $\mathrm{g} / 1$ & \\
\hline $\begin{array}{l}\text { Jul } \\
\text { Jul } \\
\text { Aug } \\
\text { Aug } \\
\text { Sep } \\
\text { Sep }\end{array}$ & $\begin{array}{l}1 \\
2 \\
1 \\
2 \\
1 \\
2\end{array}$ & $\begin{array}{l}X \\
X \\
X \\
X \\
0.24 \\
0.30\end{array}$ & $\begin{array}{l}X \\
X \\
x \\
X \\
18.3 \\
18.9\end{array}$ & $\begin{array}{l}5.10 \\
5.80 \\
3.30 \\
44.3 \\
32.6 \\
26.1\end{array}$ & $\begin{array}{l}x \\
X \\
X \\
X \\
X \\
X\end{array}$ & & $\begin{array}{l}X \\
X \\
X \\
X \\
X \\
X\end{array}$ & & $\begin{array}{l}1.5 \\
5.5 \\
0.3 \\
2.5 \\
123 \\
113\end{array}$ & \\
\hline
\end{tabular}




\section{Table A-34}

SUMMARY STATISTICS

$$
\text { STREAM=9 (TAN TSF effluent to drainage pond) }
$$

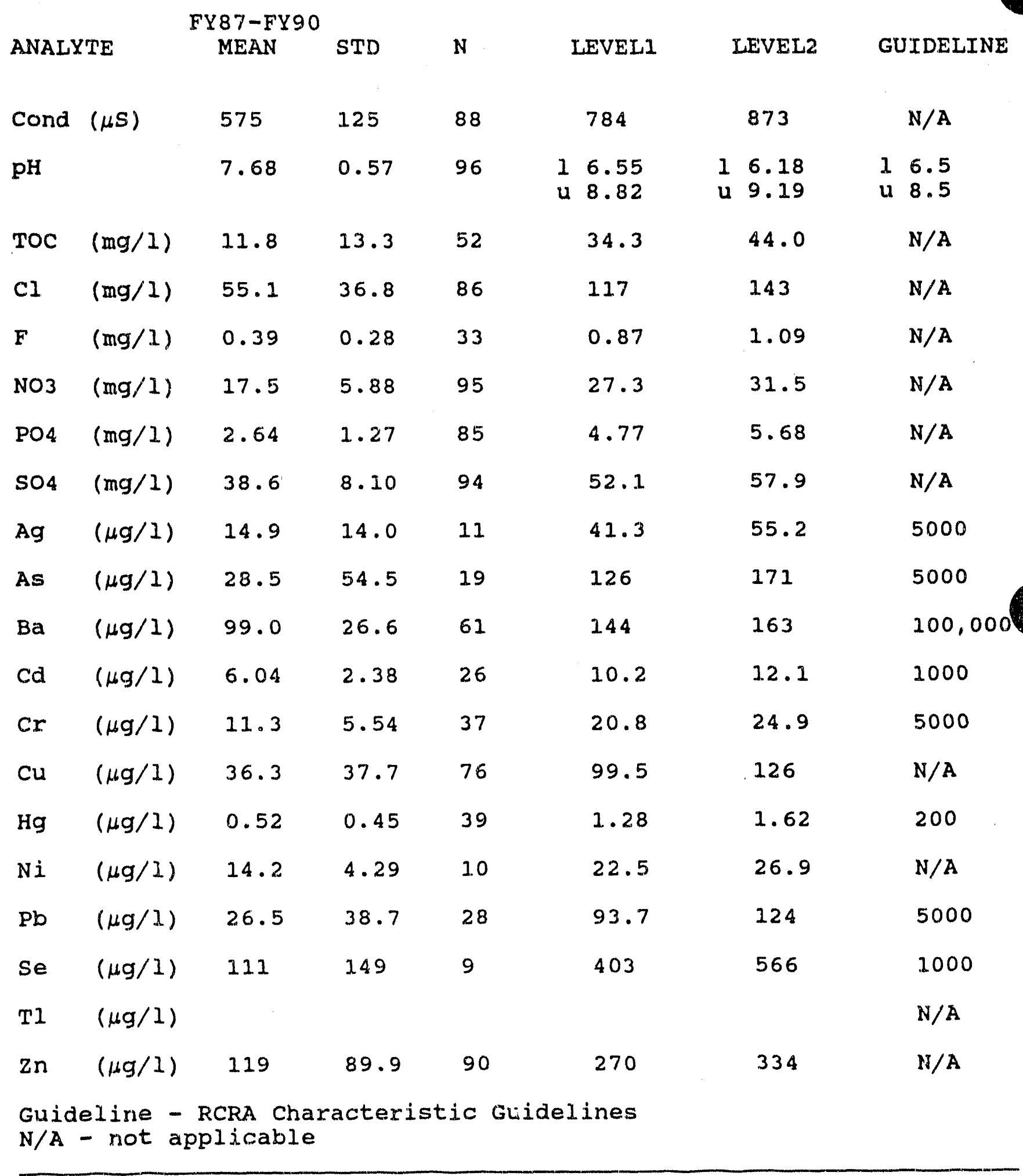




\section{Table A-35}

QUARTERLY MEANS AND VARIANCE COMPONENTS

STREAM $=9$ (TAN TSF effluent to drainage pond)

\begin{tabular}{|c|c|c|c|c|c|c|c|c|c|}
\hline \multicolumn{2}{|c|}{ ANALYTE } & QTR & MEAN & $\begin{array}{r}\text { STD } \\
\text { MEAN }\end{array}$ & $\begin{array}{l}\text { STD } \\
\text { DAY }\end{array}$ & $\begin{array}{l}\text { STD } \\
\text { REP }\end{array}$ & N & $\begin{array}{r}\mathrm{N} \\
\text { MISS }\end{array}$ & BPQI \\
\hline Cond & $(\mu S)$ & $\begin{array}{l}91 q 1 \\
91 q^{2} \\
91 q^{3} \\
91 q^{4}\end{array}$ & $\begin{array}{l}539 \\
614 \\
611 \\
132\end{array}$ & $\begin{array}{r}56.0 \\
51.5 \\
179 \\
147\end{array}$ & $\begin{array}{r}95.8 \\
88.1 \\
284 \\
244\end{array}$ & $\begin{array}{r}5.39 \\
19.7 \\
125 \\
6.07\end{array}$ & $\begin{array}{l}6 \\
6 \\
5 \\
5\end{array}$ & $\begin{array}{l}0 \\
0 \\
1 \\
1\end{array}$ & $\begin{array}{l}0 \\
0 \\
0 \\
0\end{array}$ \\
\hline $\mathrm{pH}$ & & $\begin{array}{l}91 q 1 \\
91 q 2 \\
91 q 3 \\
91 q^{4}\end{array}$ & $\begin{array}{l}7.86 \\
8.10 \\
7.08 \\
7.41\end{array}$ & $\begin{array}{l}0.36 \\
0.31 \\
0.02 \\
0.14\end{array}$ & $\begin{array}{l}0.62 \\
0.33 \\
0.00 \\
0.00\end{array}$ & $\begin{array}{l}0.13 \\
0.61 \\
0.77 \\
0.53\end{array}$ & $\begin{array}{l}6 \\
6 \\
5 \\
5\end{array}$ & $\begin{array}{l}0 \\
0 \\
1 \\
1\end{array}$ & $\begin{array}{l}0 \\
0 \\
0 \\
0\end{array}$ \\
\hline TOC & $(\mathrm{mg} / \mathrm{l})$ & $\begin{array}{l}91 q 1 \\
91 q^{2} \\
91 q^{3} \\
91 q^{4}\end{array}$ & $\begin{array}{l}4.38 \\
7.05 \\
6.85 \\
5.47\end{array}$ & $\begin{array}{l}0.43 \\
0.25 \\
0.43\end{array}$ & $\begin{array}{l}0.75 \\
0.40 \\
0.73\end{array}$ & $\begin{array}{l}0.09 \\
0.23 \\
0.17\end{array}$ & $\begin{array}{l}6 \\
2 \\
6 \\
6\end{array}$ & $\begin{array}{l}0 \\
0 \\
0 \\
0\end{array}$ & $\begin{array}{l}0 \\
4 \\
0 \\
0\end{array}$ \\
\hline Cl & $(\mathrm{mg} / \mathrm{l})$ & $\begin{array}{l}91 q 1 \\
91 q 2 \\
91 q 3 \\
91 q 4\end{array}$ & $\begin{array}{l}48.7 \\
43.3 \\
58.9 \\
33.8\end{array}$ & $\begin{array}{l}16.6 \\
15.9 \\
24.2 \\
5.74\end{array}$ & $\begin{array}{l}28.7 \\
26.6 \\
41.9 \\
9.93\end{array}$ & $\begin{array}{l}0.24 \\
0.10 \\
0.57 \\
0.81\end{array}$ & $\begin{array}{l}6 \\
5 \\
6 \\
6\end{array}$ & $\begin{array}{l}0 \\
1 \\
0 \\
0\end{array}$ & $\begin{array}{l}0 \\
0 \\
0 \\
0\end{array}$ \\
\hline$F$ & $(\mathrm{mg} / \mathrm{l})$ & $\begin{array}{l}91 q 1 \\
91 q 2 \\
91 q 3 \\
9194\end{array}$ & $\begin{array}{l}x \\
0.02 \\
0.21 \\
0.23\end{array}$ & $\begin{array}{l}0.20 \\
0.01 \\
0.01\end{array}$ & $\begin{array}{l}\dot{0} .33 \\
0.01 \\
0.02\end{array}$ & $\begin{array}{l}0.00 \\
0.01 \\
0.01\end{array}$ & $\begin{array}{l}0 \\
5 \\
6 \\
6\end{array}$ & $\begin{array}{l}0 \\
1 \\
0 \\
0\end{array}$ & $\begin{array}{l}6 \\
0 \\
0 \\
0\end{array}$ \\
\hline NO3 & $(\mathrm{mg} / \mathrm{l})$ & $\begin{array}{l}91 q 1 \\
91 q 2 \\
91 q 3 \\
91 q 4\end{array}$ & $\begin{array}{l}15.5 \\
2.85 \\
7.46 \\
8.39\end{array}$ & $\begin{array}{l}0.93 \\
1.75 \\
2.53 \\
1.86\end{array}$ & $\begin{array}{l}1.61 \\
2.47 \\
4.38 \\
3.19\end{array}$ & $\begin{array}{l}0.08 \\
0.00 \\
0.33 \\
0.55\end{array}$ & $\begin{array}{l}6 \\
4 \\
6 \\
6\end{array}$ & $\begin{array}{l}0 \\
2 \\
0 \\
0\end{array}$ & $\begin{array}{l}0 \\
0 \\
0 \\
0\end{array}$ \\
\hline PO4 & $(\mathrm{mg} / \mathrm{l})$ & $\begin{array}{l}91 q 1 \\
9192 \\
9193 \\
9194\end{array}$ & $\begin{array}{l}2.55 \\
0.82 \\
4.17 \\
6.21\end{array}$ & $\begin{array}{l}0.14 \\
0.63 \\
1.76\end{array}$ & $\begin{array}{l}0.24 \\
i .07 \\
3.00\end{array}$ & $\begin{array}{l}0.09 \\
0.23 \\
0.74\end{array}$ & $\begin{array}{l}6 \\
1 \\
6 \\
6\end{array}$ & $\begin{array}{l}0 \\
0 \\
0 \\
0\end{array}$ & $\begin{array}{l}0 \\
5 \\
0 \\
0\end{array}$ \\
\hline 504 & $(\mathrm{mg} / \mathrm{I})$ & $\begin{array}{l}9191 \\
9192 \\
9193 \\
9194\end{array}$ & $\begin{array}{l}35.8 \\
39.3 \\
35.1 \\
38.9\end{array}$ & $\begin{array}{l}0.62 \\
0.53 \\
6.17 \\
0.77\end{array}$ & $\begin{array}{l}1.07 \\
0.46 \\
9.99 \\
1.31\end{array}$ & $\begin{array}{l}0.06 \\
1.00 \\
5.41 \\
0.37\end{array}$ & $\begin{array}{l}6 \\
5 \\
6 \\
6\end{array}$ & $\begin{array}{l}0 \\
1 . \\
0 \\
0\end{array}$ & $\begin{array}{l}0 \\
0 \\
0 \\
0\end{array}$ \\
\hline $\mathrm{Ag}$ & $(\mu g / 1)$ & $\begin{array}{l}919^{2} \\
919^{2} \\
919^{3} \\
91 q^{4}\end{array}$ & $\begin{array}{l}X \\
X \\
X \\
2.90\end{array}$ & $\dot{.}$ & $\begin{array}{l}\cdot \\
\dot{\cdot} \\
\dot{\cdot}\end{array}$ & $\dot{.}$ & $\begin{array}{l}0 \\
0 \\
0 \\
1\end{array}$ & $\begin{array}{l}0 \\
0 \\
0 \\
0\end{array}$ & $\begin{array}{l}6 \\
6 \\
6 \\
5\end{array}$ \\
\hline
\end{tabular}




\section{Table A-35 (continued)}

QUARTERLY MEANS AND VARIANCE COMPONENTS

$$
\text { STREAM=9 (TAN TSF effluent to dirainage pond) }
$$
(continued)

\begin{tabular}{|c|c|c|c|c|c|c|c|c|c|}
\hline \multicolumn{2}{|c|}{ ANALYTE } & QTR & MEAN & $\begin{array}{r}\text { STD } \\
\text { MEAN }\end{array}$ & $\begin{array}{l}\text { STD } \\
\text { DAY }\end{array}$ & $\begin{array}{l}\text { STD } \\
\text { REP }\end{array}$ & $\mathrm{N}$ & MISS & $\mathrm{BPQ}$ \\
\hline As & $(u g / 1)$ & $\begin{array}{l}91 q 1 \\
91 q^{2} \\
91 q 3 \\
91 q 4\end{array}$ & $\begin{array}{l}1.90 \\
x \\
3.50 \\
4.37\end{array}$ & $\begin{array}{l}0.38 \\
\dot{5} \\
\dot{5}\end{array}$ & $\begin{array}{l}0.63 \\
\dot{0} \\
\dot{\cdot}\end{array}$ & $\begin{array}{l}0.11 \\
\dot{:} \\
\dot{0}\end{array}$ & $\begin{array}{l}5 \\
0 \\
2 \\
3\end{array}$ & $\begin{array}{l}0 \\
0 \\
0 \\
0\end{array}$ & $\begin{array}{l}1 \\
6 \\
4 \\
3\end{array}$ \\
\hline $\mathrm{Ba}$ & $(\mu g / 1)$ & $\begin{array}{l}91 q 1 \\
91 q 2 \\
91 q 3 \\
91 q^{4}\end{array}$ & $\begin{array}{l}67.3 \\
x \\
105 \\
122\end{array}$ & $\begin{array}{l}3.32 \\
\dot{8} .45 \\
19.6\end{array}$ & $\begin{array}{l}5.25 \\
14.5 \\
33.7\end{array}$ & $\begin{array}{l}3.32 \\
2.92 \\
4.70\end{array}$ & $\begin{array}{l}6 \\
0 \\
6 \\
6\end{array}$ & $\begin{array}{l}0 \\
0 \\
0 \\
0\end{array}$ & $\begin{array}{l}0 \\
6 \\
0 \\
0\end{array}$ \\
\hline$c d$ & $(\mu \mathrm{g} / 1)$ & $\begin{array}{l}91 q^{1} \\
91 q^{2} \\
91 q^{3} \\
91 q^{4}\end{array}$ & $\begin{array}{l}X \\
x \\
5.90 \\
x\end{array}$ & $\begin{array}{l}\dot{.} \\
\dot{\cdot}\end{array}$ & $\begin{array}{l}\dot{.} \\
\dot{.}\end{array}$ & $\begin{array}{l}\dot{\cdot} \\
\dot{\cdot}\end{array}$ & $\begin{array}{l}0 \\
0 \\
1 \\
0\end{array}$ & $\begin{array}{l}0 \\
0 \\
0 \\
0\end{array}$ & $\begin{array}{l}6 \\
6 \\
5 \\
6\end{array}$ \\
\hline $\mathrm{Cr}$ & $(\mu g / 1)$ & $\begin{array}{l}91 q 1 \\
91 q 2 \\
91 q 3 \\
91 q^{4}\end{array}$ & $\begin{array}{l}7.70 \\
6.50 \\
8.85 \\
14.8\end{array}$ & $\begin{array}{l}\dot{:} \\
\dot{7} .87\end{array}$ & $\begin{array}{l}\dot{ } \\
13.6\end{array}$ & $\begin{array}{l}\dot{\dot{0}} \\
\dot{0} .88\end{array}$ & $\begin{array}{l}1 \\
2 \\
2 \\
6\end{array}$ & $\begin{array}{l}0 \\
0 \\
0 \\
0\end{array}$ & $\begin{array}{l}5 \\
4 \\
4 \\
0\end{array}$ \\
\hline $\mathrm{Cu}$ & $(\mu g / 1)$ & $\begin{array}{l}91 q^{1} \\
91 q^{2} \\
91 q^{3} \\
91 q^{4}\end{array}$ & $\begin{array}{l}16.1 \\
x \\
8.93 \\
20.4\end{array}$ & $\begin{array}{l}0.73 \\
\dot{10.2}\end{array}$ & $\begin{array}{l}0.51 \\
\dot{17.1}\end{array}$ & $\begin{array}{l}1.62 \\
\dot{6} .49\end{array}$ & $\begin{array}{l}6 \\
0 \\
3 \\
6\end{array}$ & $\begin{array}{l}0 \\
0 \\
0 \\
0\end{array}$ & $\begin{array}{l}0 \\
6 \\
3 \\
0\end{array}$ \\
\hline $\mathrm{Hg}$ & $(\mu \mathrm{g} / \mathrm{I})$ & $\begin{array}{l}91 q 1 \\
91 q 2 \\
91 q 3 \\
91 q 4\end{array}$ & $\begin{array}{l}0.14 \\
x \\
X \\
0.27\end{array}$ & $\begin{array}{l}0.00 \\
. \\
.\end{array}$ & $\begin{array}{l}0.00 \\
\dot{0} \\
\dot{ }\end{array}$ & $\begin{array}{l}0.05 \\
\dot{.} \\
\dot{.}\end{array}$ & $\begin{array}{l}5 \\
0 \\
0 \\
2\end{array}$ & $\begin{array}{l}0 \\
0 \\
0 \\
0\end{array}$ & $\begin{array}{l}1 \\
6 \\
6 \\
4\end{array}$ \\
\hline $\mathrm{Ni}$ & $(\mu g / 1)$ & $\begin{array}{l}91 q 1 \\
91 q 2 \\
9193 \\
9194\end{array}$ & $\begin{array}{l}22.1 \\
\times \\
13.9 \\
18.6\end{array}$ & $\begin{array}{l}\dot{\bullet} \\
\dot{\cdot}\end{array}$ & $\dot{.}$ & $\begin{array}{l}\dot{.} \\
\dot{.}\end{array}$ & $\begin{array}{l}3 \\
0 \\
1 \\
2\end{array}$ & $\begin{array}{l}0 \\
0 \\
0 \\
0\end{array}$ & $\begin{array}{l}3 \\
6 \\
5 \\
4\end{array}$ \\
\hline $\mathrm{Pb}$ & $(\mu g / 1)$ & $\begin{array}{l}91 q 1 \\
91 q^{2} \\
91 q^{3} \\
91 q^{4}\end{array}$ & $\begin{array}{l}3.50 \\
3.67 \\
4.80 \\
1.9 .5\end{array}$ & $\begin{array}{l}0.58 \\
\dot{7} \\
7.22\end{array}$ & $\begin{array}{l}0.96 \\
\dot{3} .58\end{array}$ & $\begin{array}{l}0.1 .6 \\
i 6.9\end{array}$ & $\begin{array}{l}5 \\
2 \\
2 \\
6\end{array}$ & $\begin{array}{l}0 \\
0 \\
0 \\
0\end{array}$ & $\begin{array}{l}1 \\
4 \\
4 \\
0\end{array}$ \\
\hline Se & $(\mu g / 1)$ & $\begin{array}{l}91 q 1 \\
91 q^{2} \\
91 q^{3} \\
91 q^{4}\end{array}$ & $\begin{array}{l}X \\
X \\
X \\
X\end{array}$ & $\dot{.}$ & $\dot{\bullet}$ & $\dot{\bullet}$ & $\begin{array}{l}0 \\
0 \\
0 \\
0\end{array}$ & $\begin{array}{l}0 \\
0 \\
0 \\
0\end{array}$ & $\begin{array}{l}6 \\
6 \\
6 \\
6\end{array}$ \\
\hline
\end{tabular}




\section{Table A-35 (continued)}

\section{QUARTERLY MEANS AND VARIANCE COMPONENTS}

\section{STREAM $=9$ (TAN TSF effluent to drainage pond)}

(continued)

\begin{tabular}{|c|c|c|c|c|c|c|c|c|c|}
\hline \multicolumn{2}{|c|}{ ANALYTE } & QTR & MEAN & $\begin{array}{r}\text { STD } \\
\text { MEAN }\end{array}$ & $\begin{array}{l}\text { STD } \\
\text { DAY }\end{array}$ & $\begin{array}{l}\text { STD } \\
\text { REP }\end{array}$ & $\mathrm{N}$ & $\begin{array}{r}\mathrm{N} \\
\text { MISS }\end{array}$ & $\begin{array}{r}\text { N } \\
\text { BPQL }\end{array}$ \\
\hline T1 & $(\mu g / \lambda)$ & $\begin{array}{l}91 q 1 \\
91 q 2 \\
91 q^{3} \\
91 q 4\end{array}$ & $\begin{array}{l}x \\
x \\
x \\
x\end{array}$ & $\begin{array}{l}\dot{\bullet} \\
\dot{\bullet} \\
\dot{ }\end{array}$ & . & $\dot{.}$ & $\begin{array}{l}0 \\
0 \\
0 \\
0\end{array}$ & $\begin{array}{l}0 \\
0 \\
0 \\
0\end{array}$ & $\begin{array}{l}6 \\
6 \\
6 \\
6\end{array}$ \\
\hline $\mathrm{zn}$ & $(\mu g / 1)$ & $\begin{array}{l}91 q^{1} \\
91 q^{2} \\
91 q^{3} \\
91 q^{4}\end{array}$ & $\begin{array}{l}43.6 \\
39.3 \\
46.6 \\
91.0\end{array}$ & $\begin{array}{l}7.96 \\
7.42 \\
3.97 \\
14.0\end{array}$ & $\begin{array}{l}9.26 \\
12.3 \\
0.00 \\
23.0\end{array}$ & $\begin{array}{l}14.4 \\
1.50 \\
16.3 \\
10.7\end{array}$ & $\begin{array}{l}6 \\
5 \\
6 \\
6\end{array}$ & $\begin{array}{l}0 \\
1 \\
0 \\
0\end{array}$ & $\begin{array}{l}0 \\
0 \\
0 \\
0\end{array}$ \\
\hline$\dot{x}$ & $\begin{array}{l}\text { - missi } \\
\text { below }\end{array}$ & $\begin{array}{l}\text { ng, too } \\
\text { practi }\end{array}$ & $\begin{array}{l}\text { little } \\
\text { cal qua }\end{array}$ & $\begin{array}{c}\text { data } \\
\text { ntitat }\end{array}$ & $\begin{array}{l}\text { calc } \\
\text { lev }\end{array}$ & $\begin{array}{l}\text { te va } \\
\text { (BPQI }\end{array}$ & & & \\
\hline
\end{tabular}




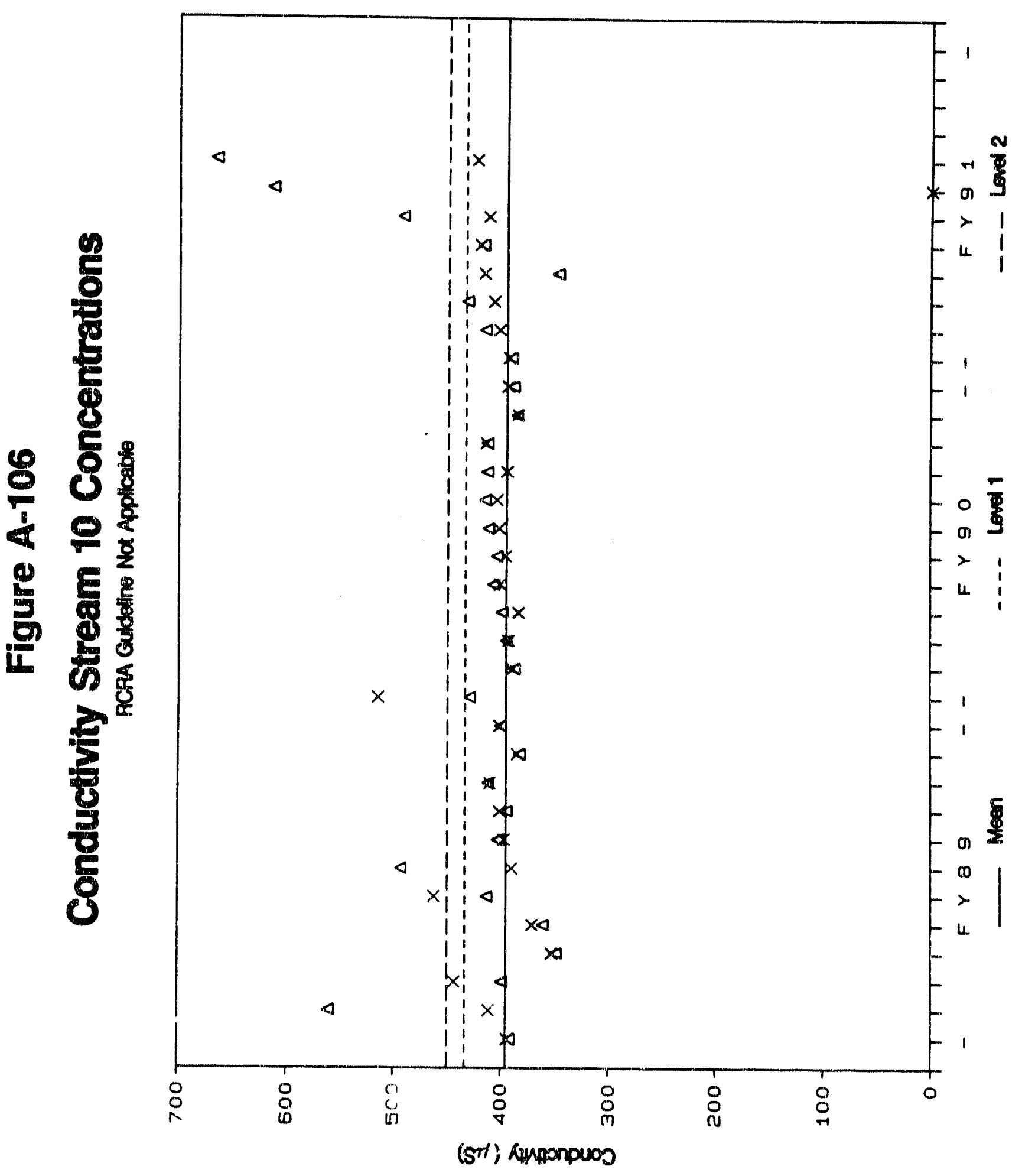




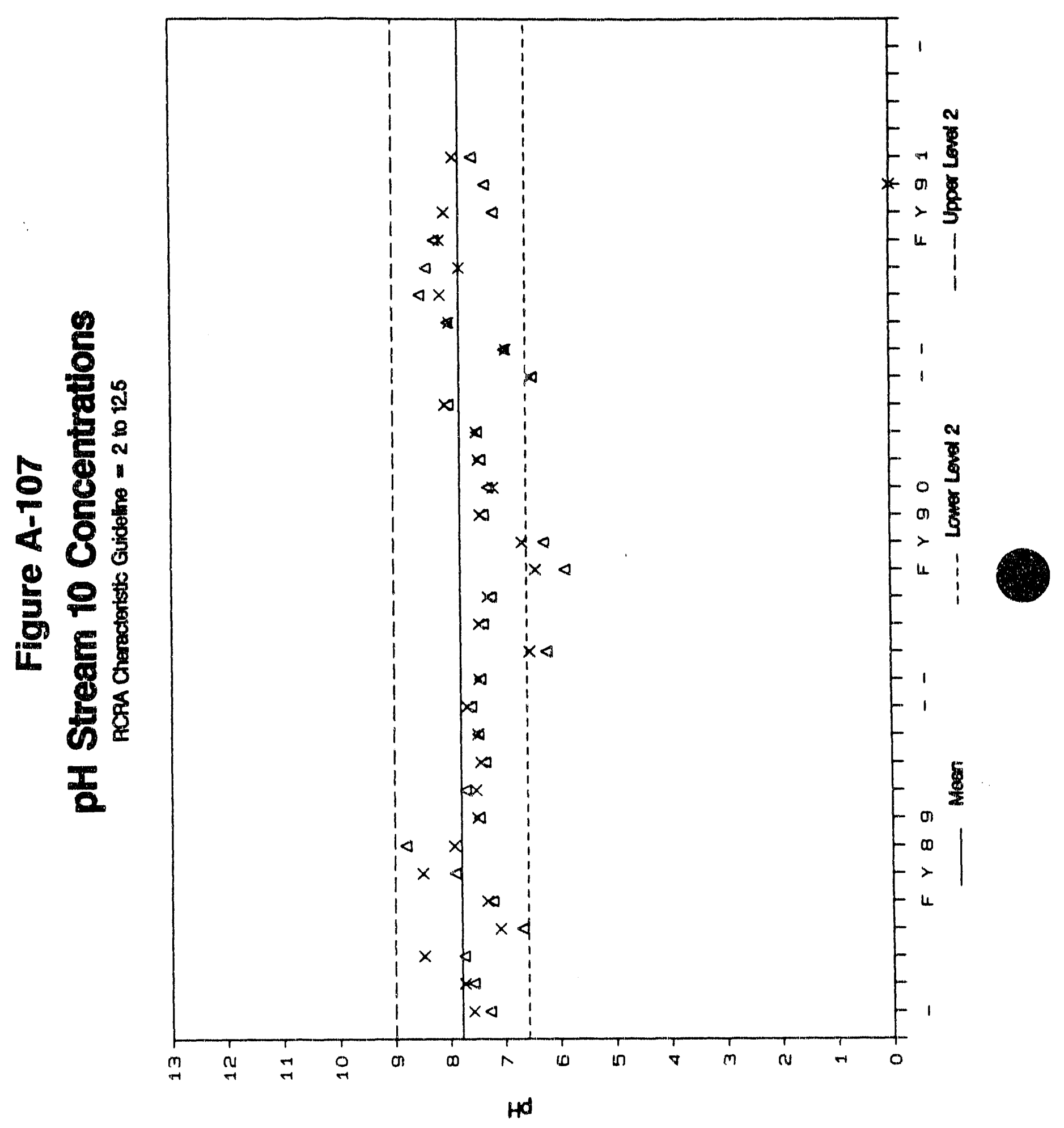




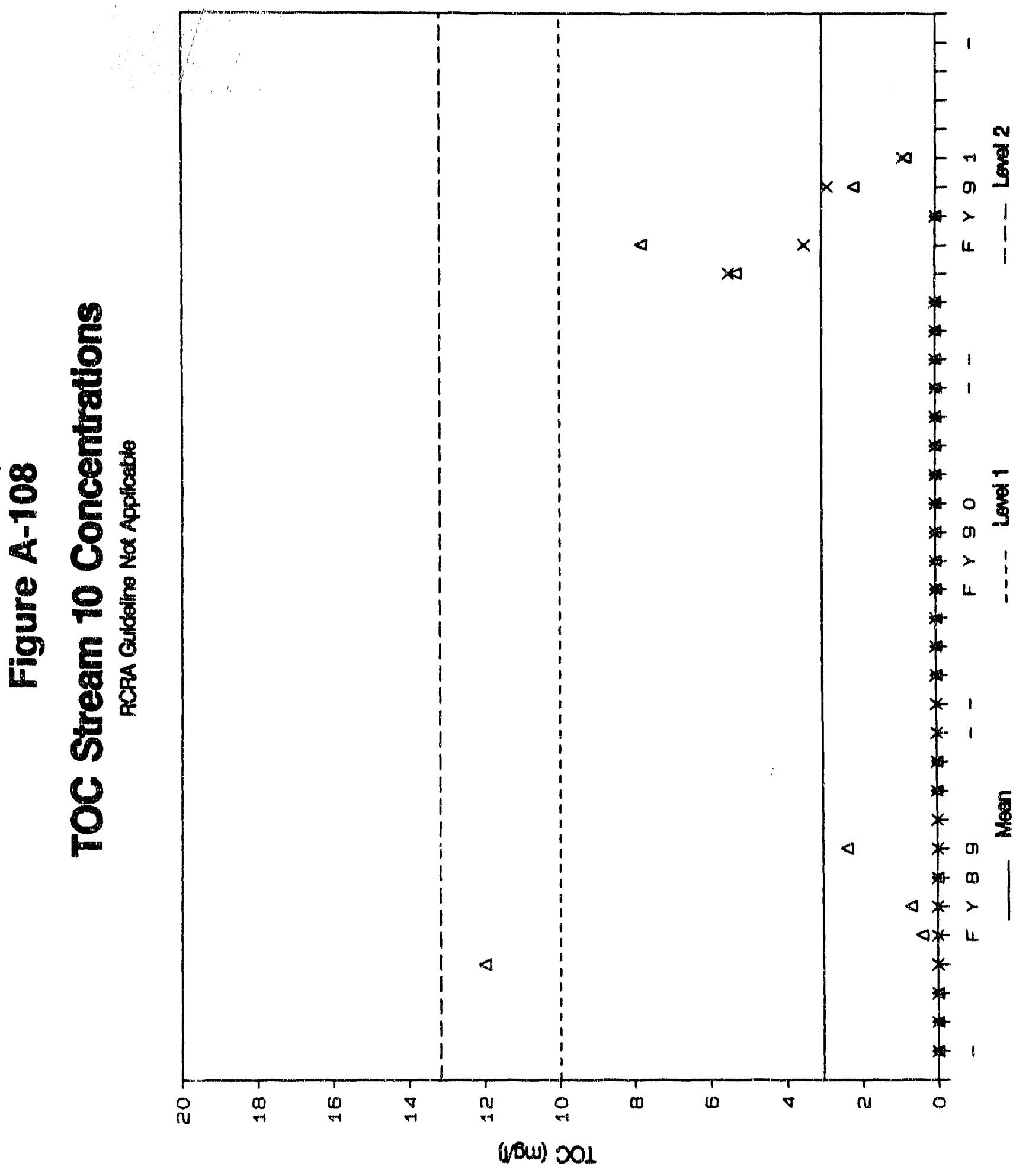




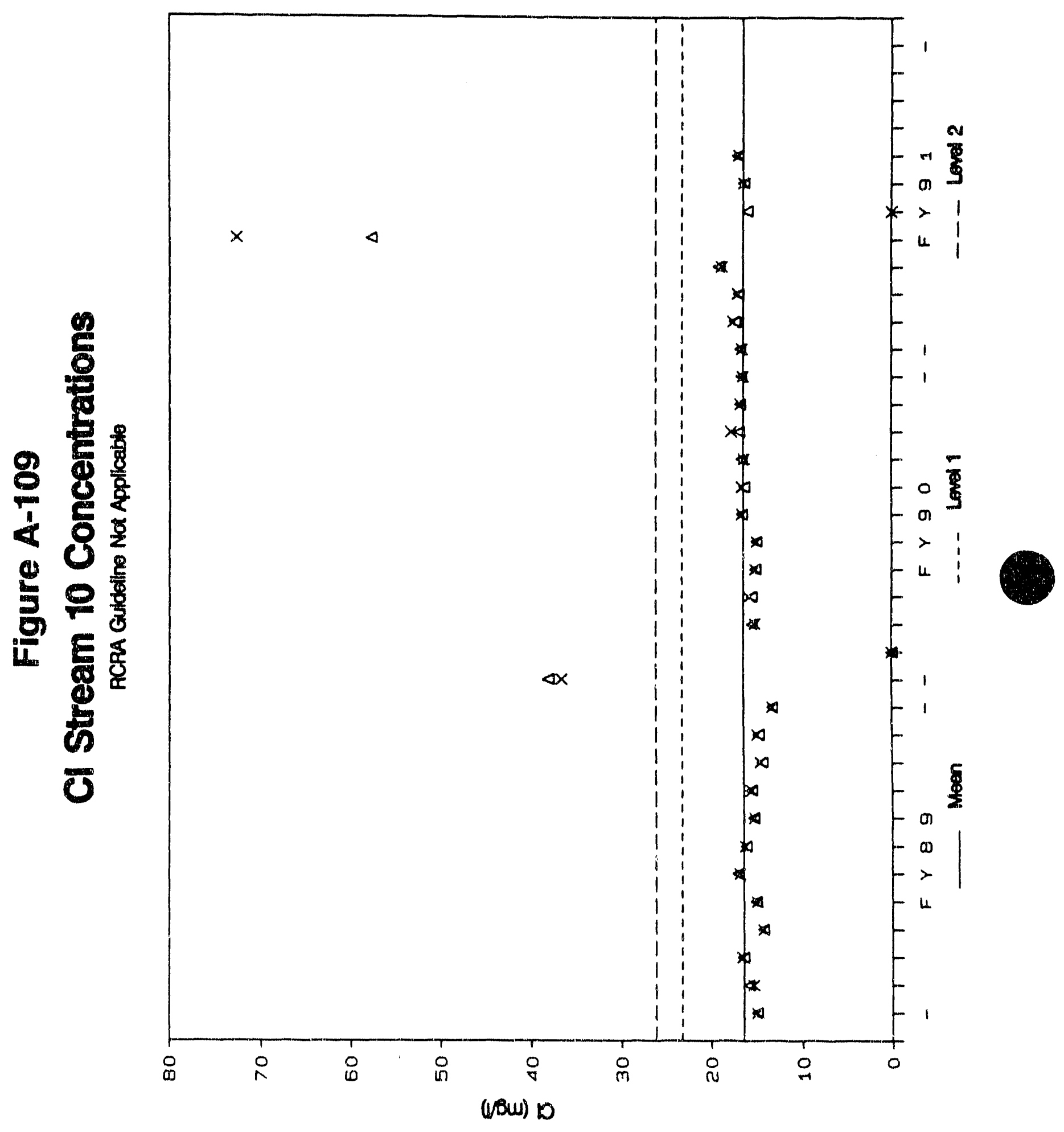




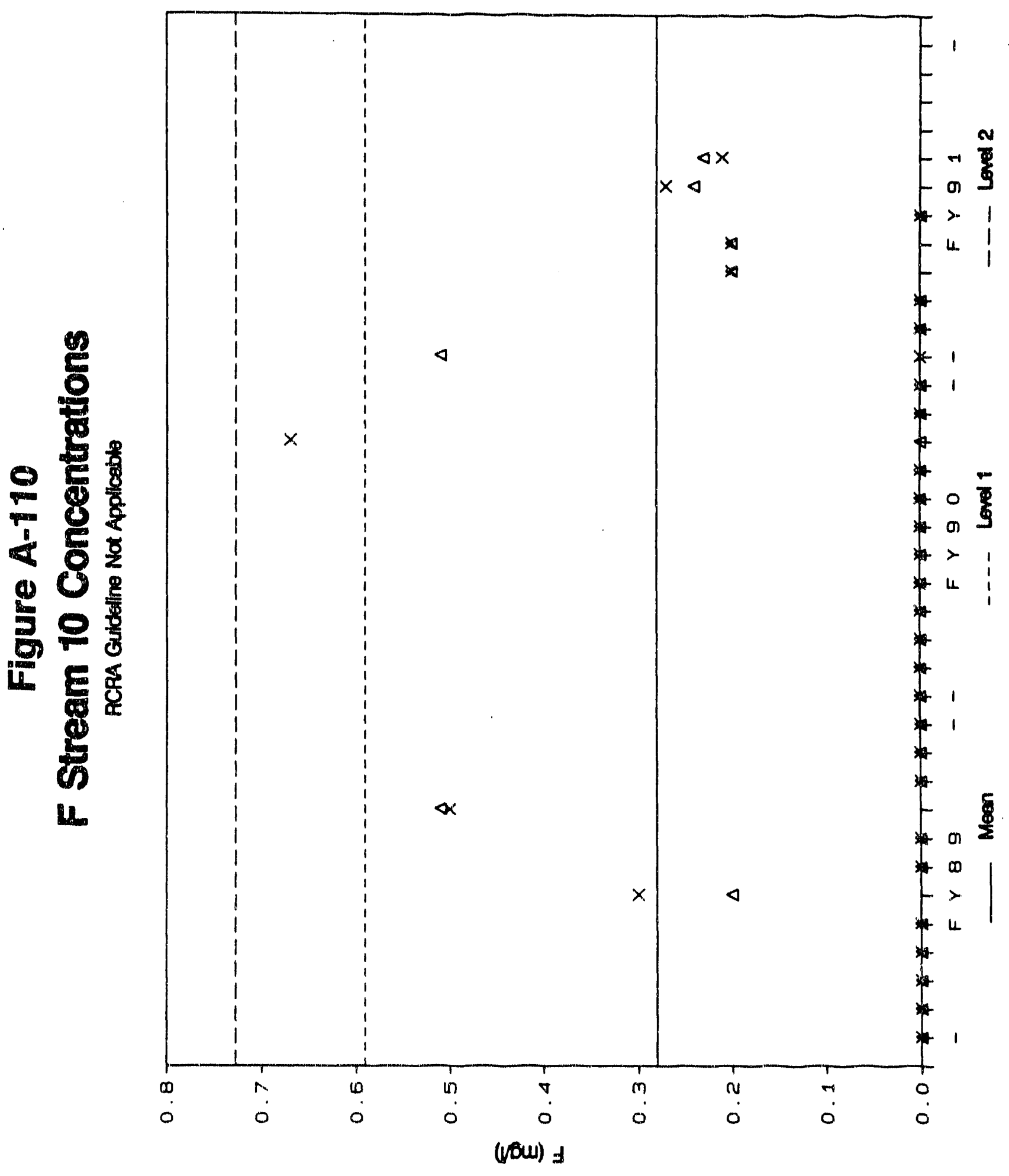




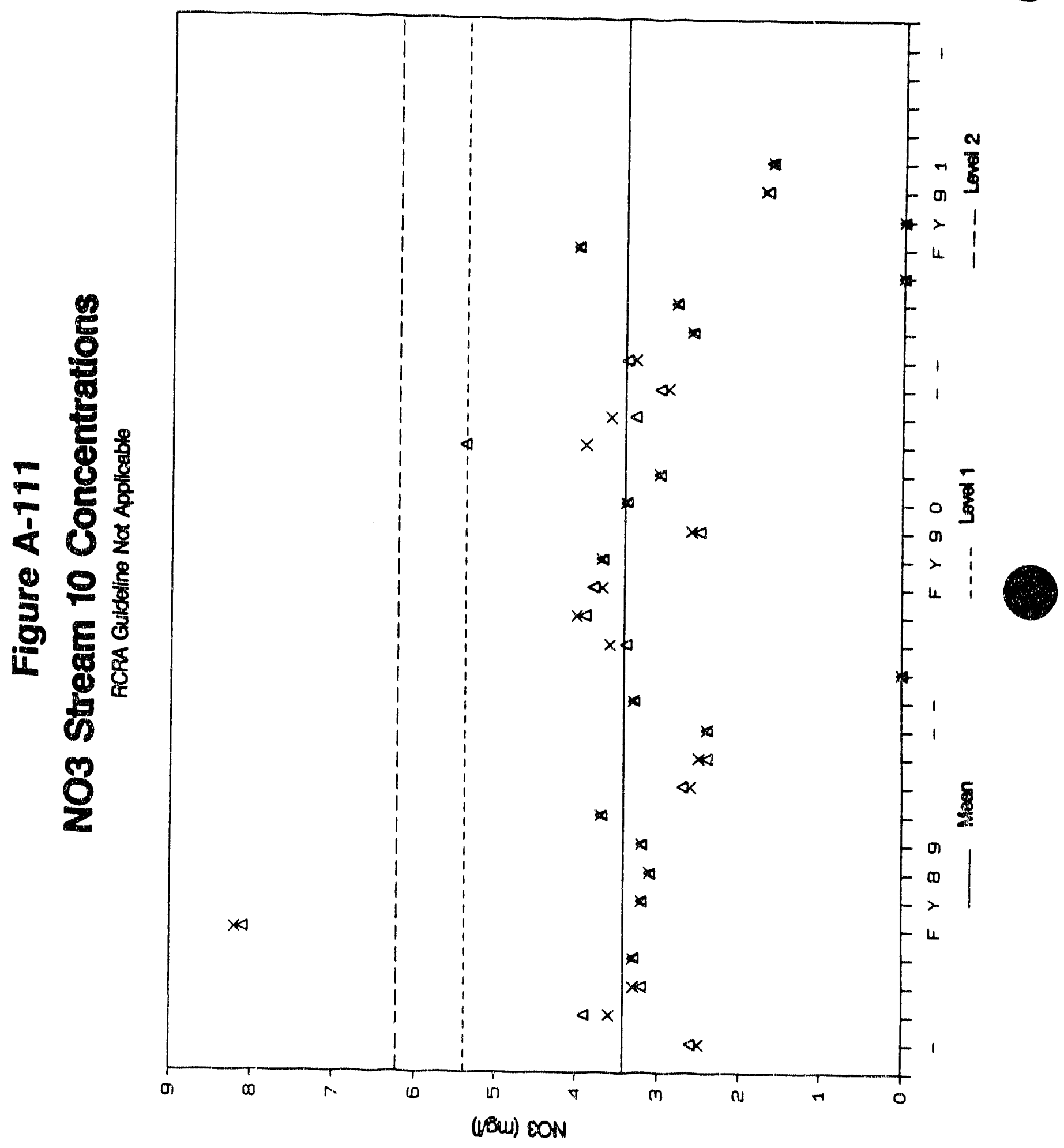




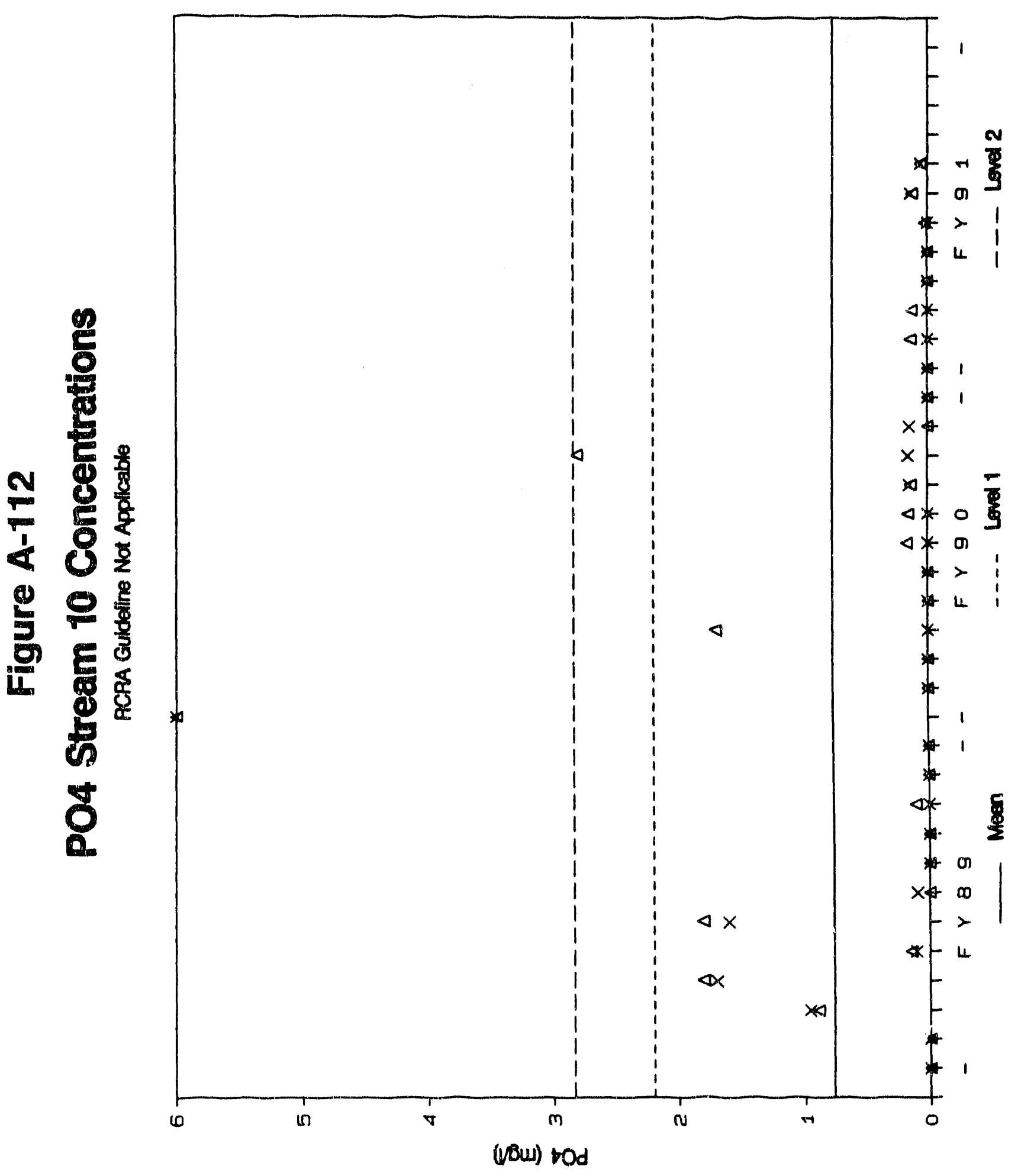




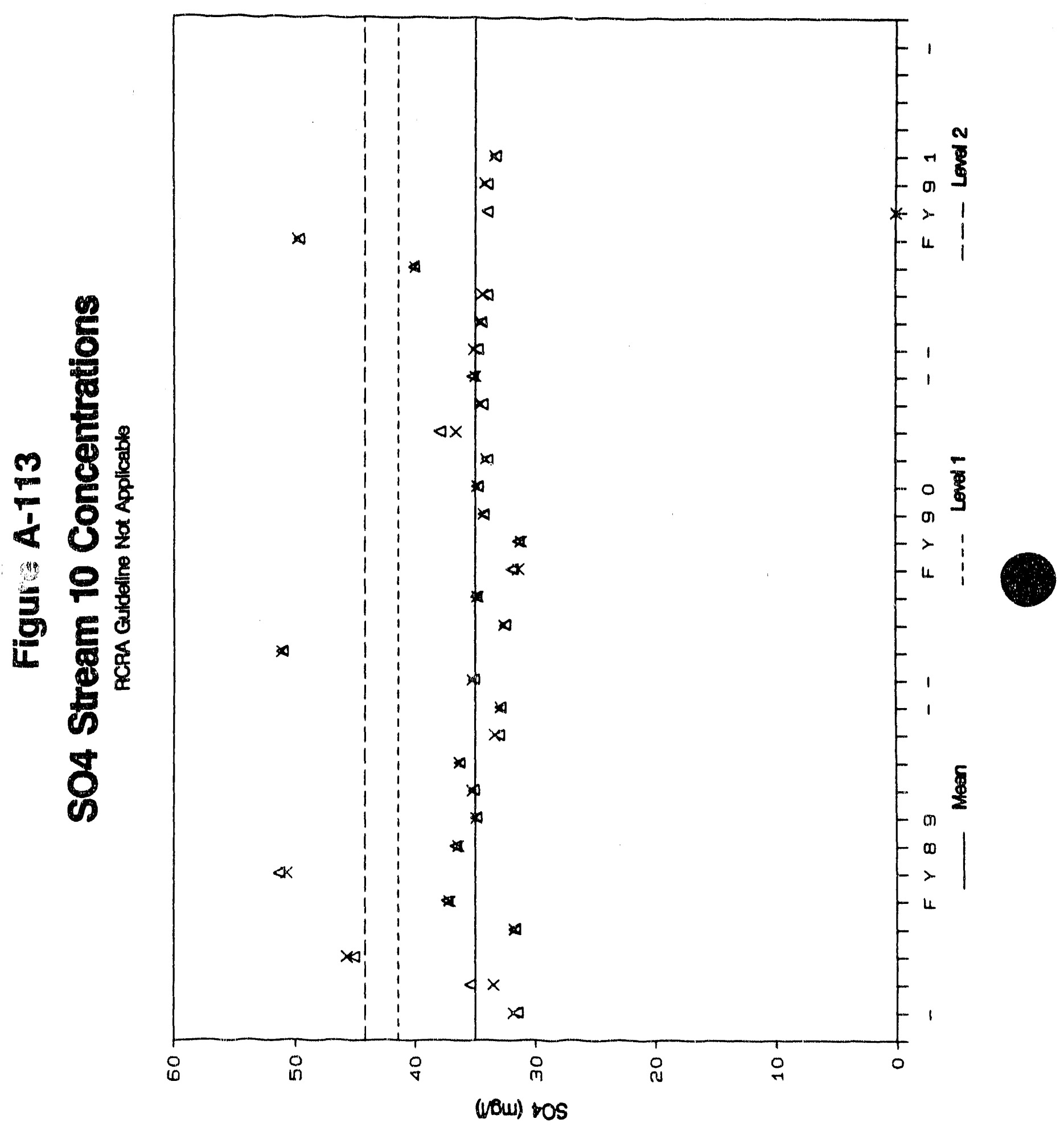




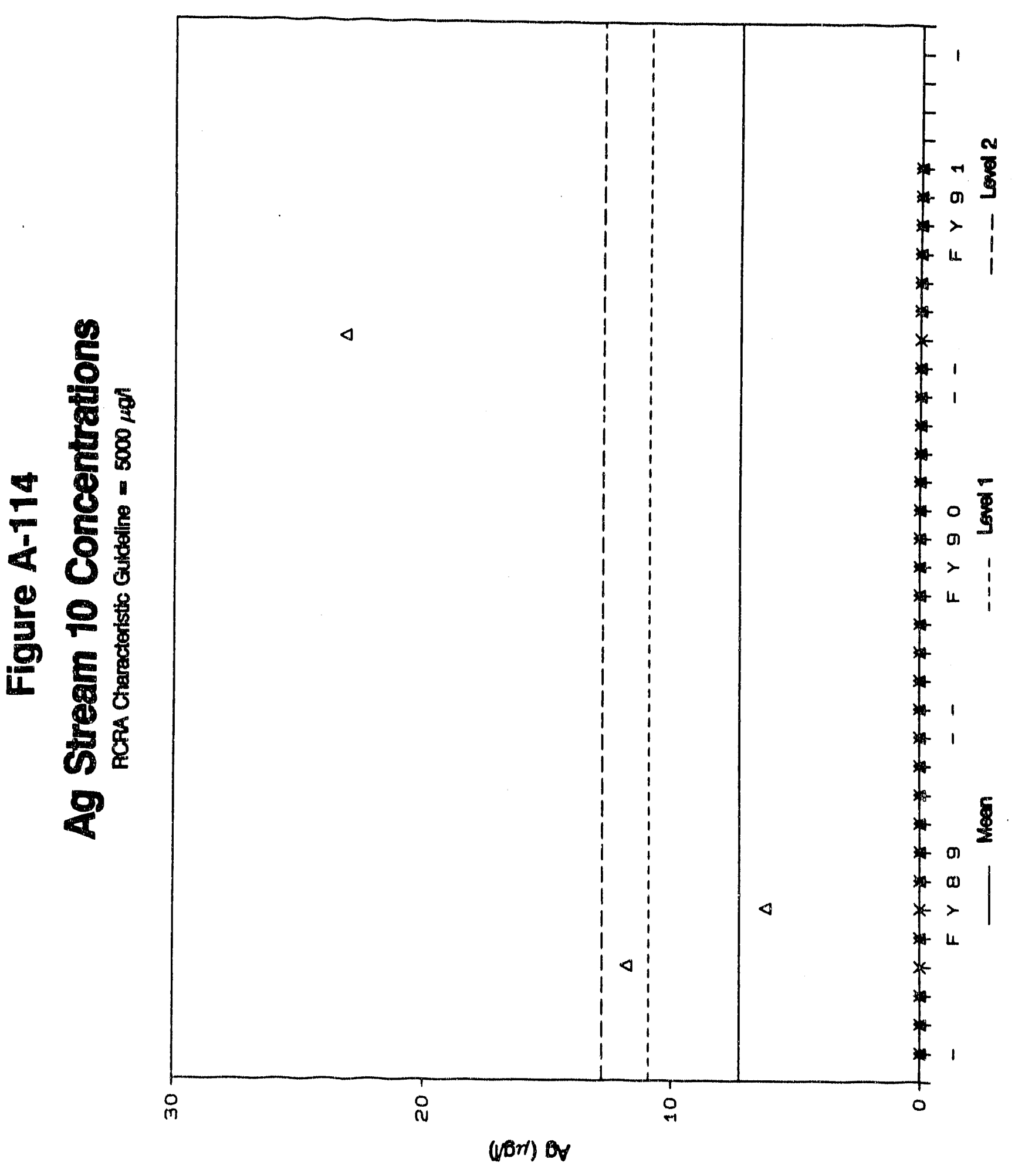




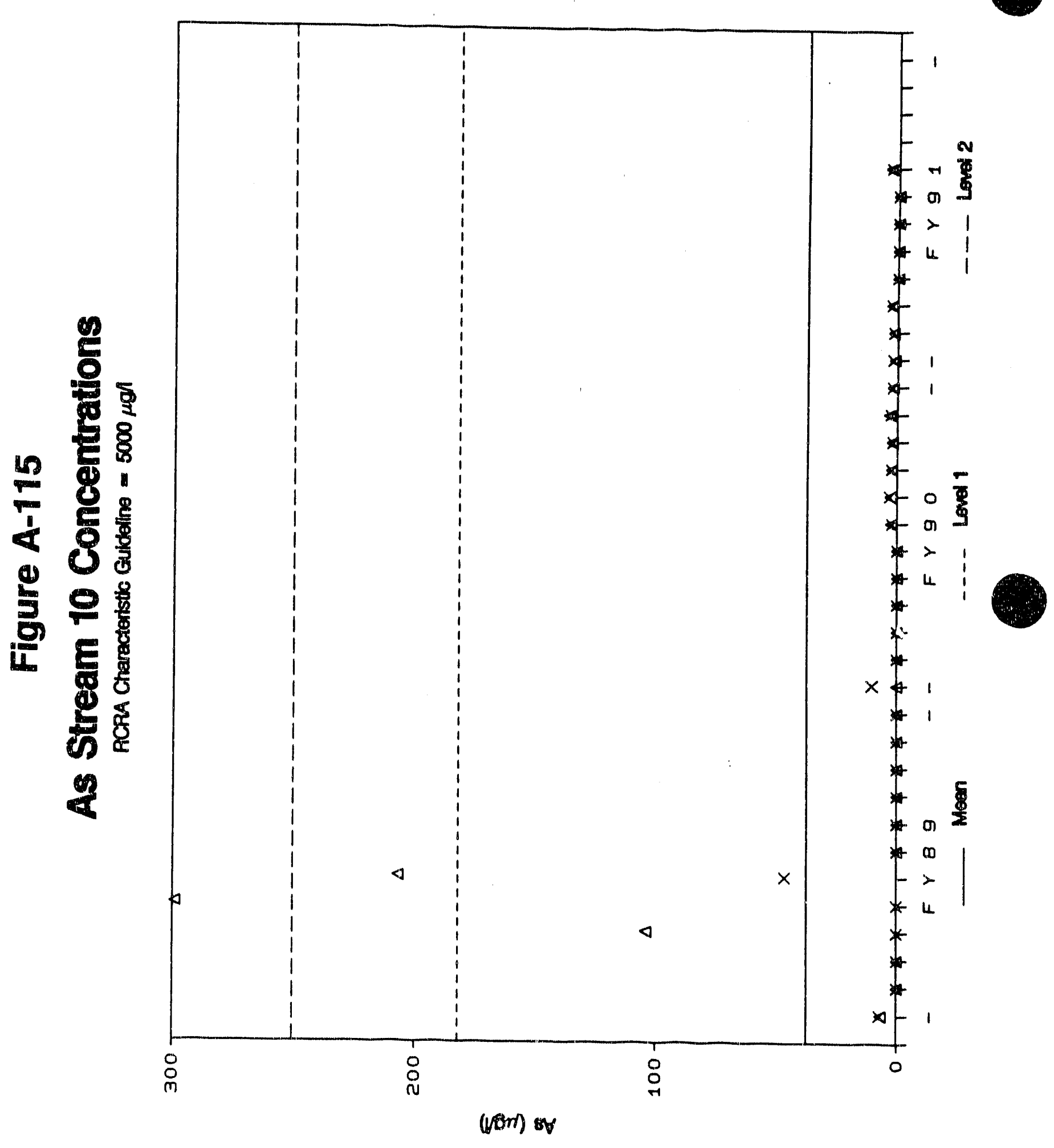




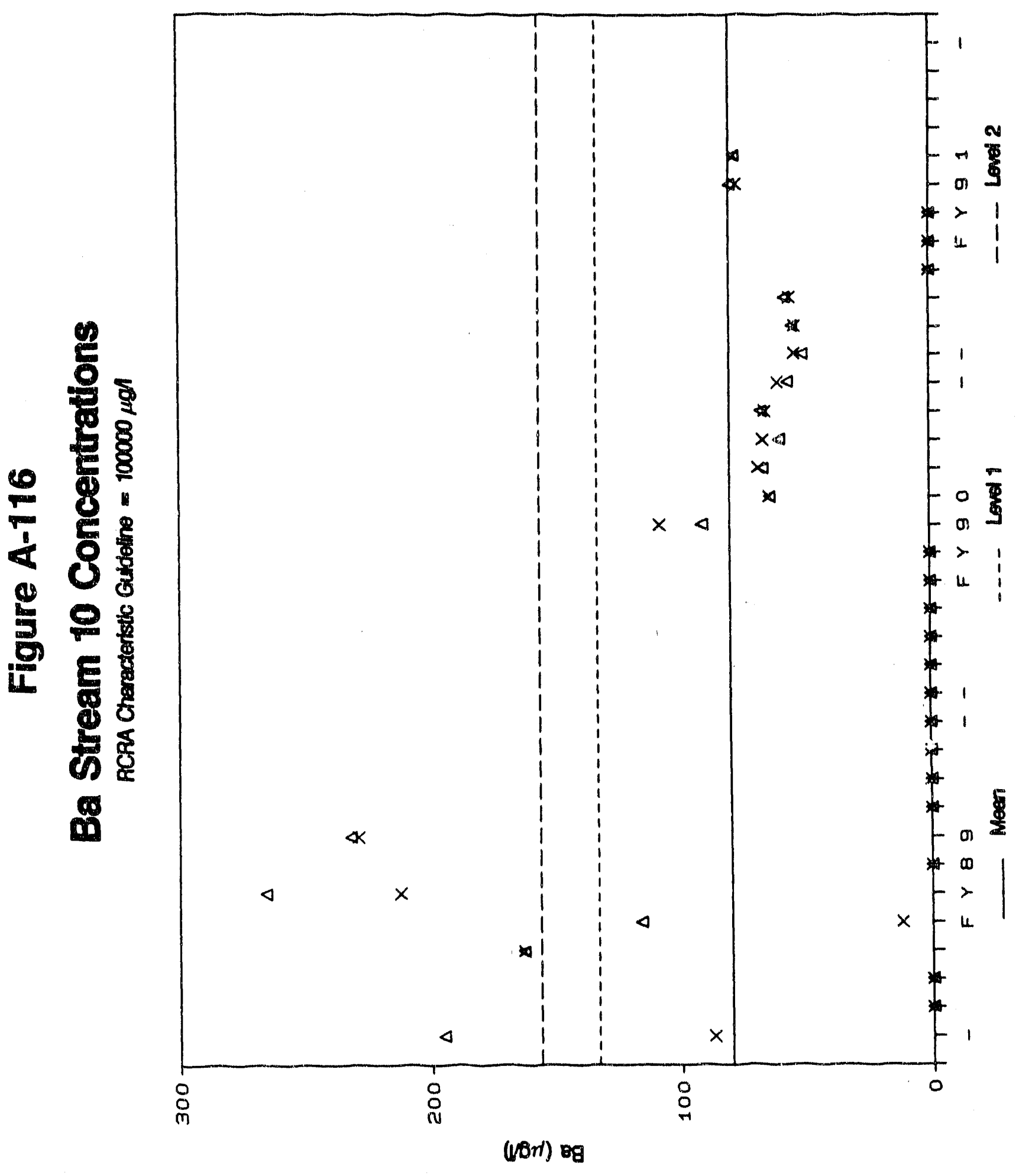

A-169

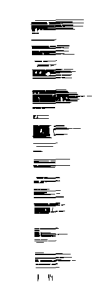




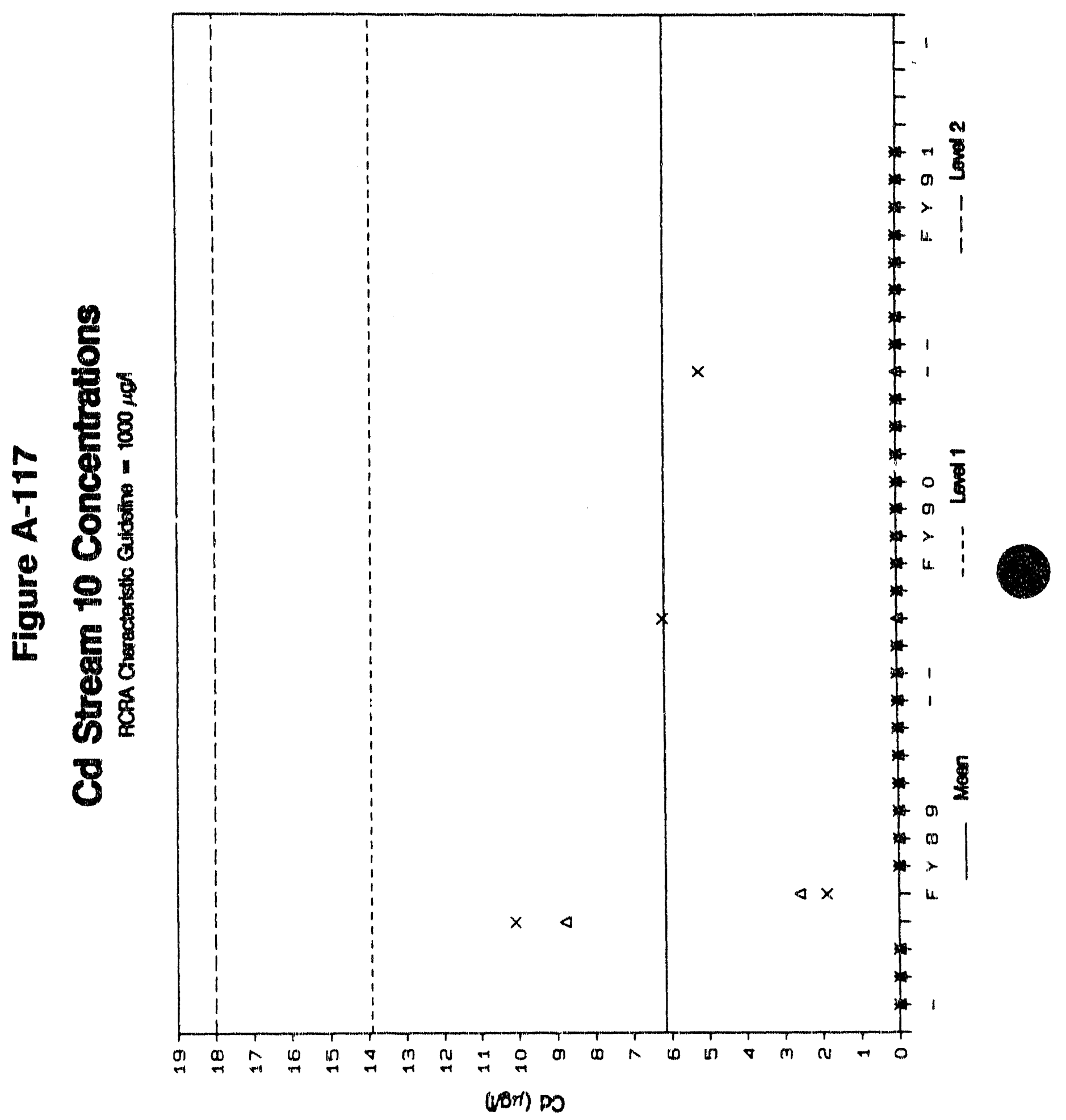




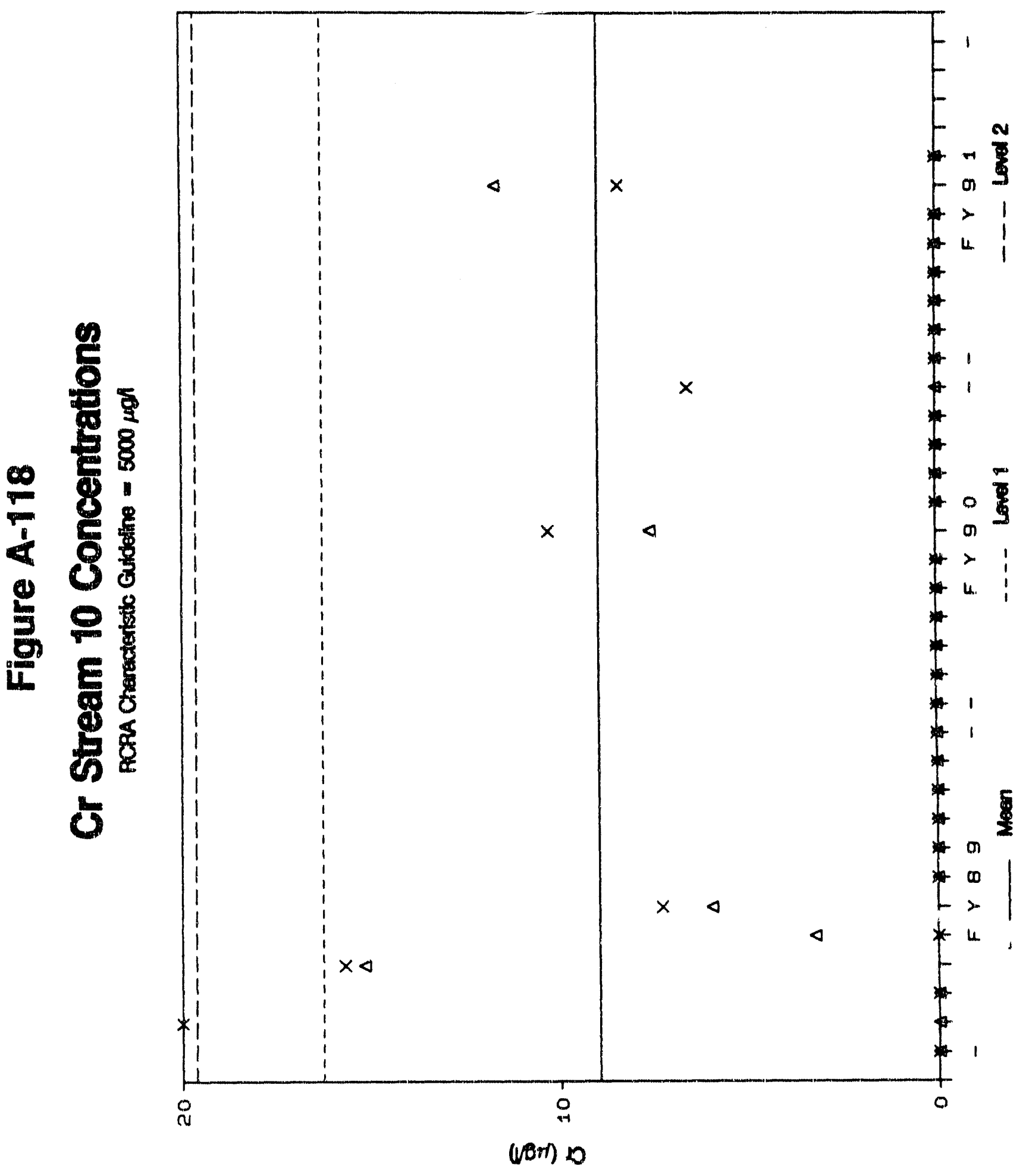




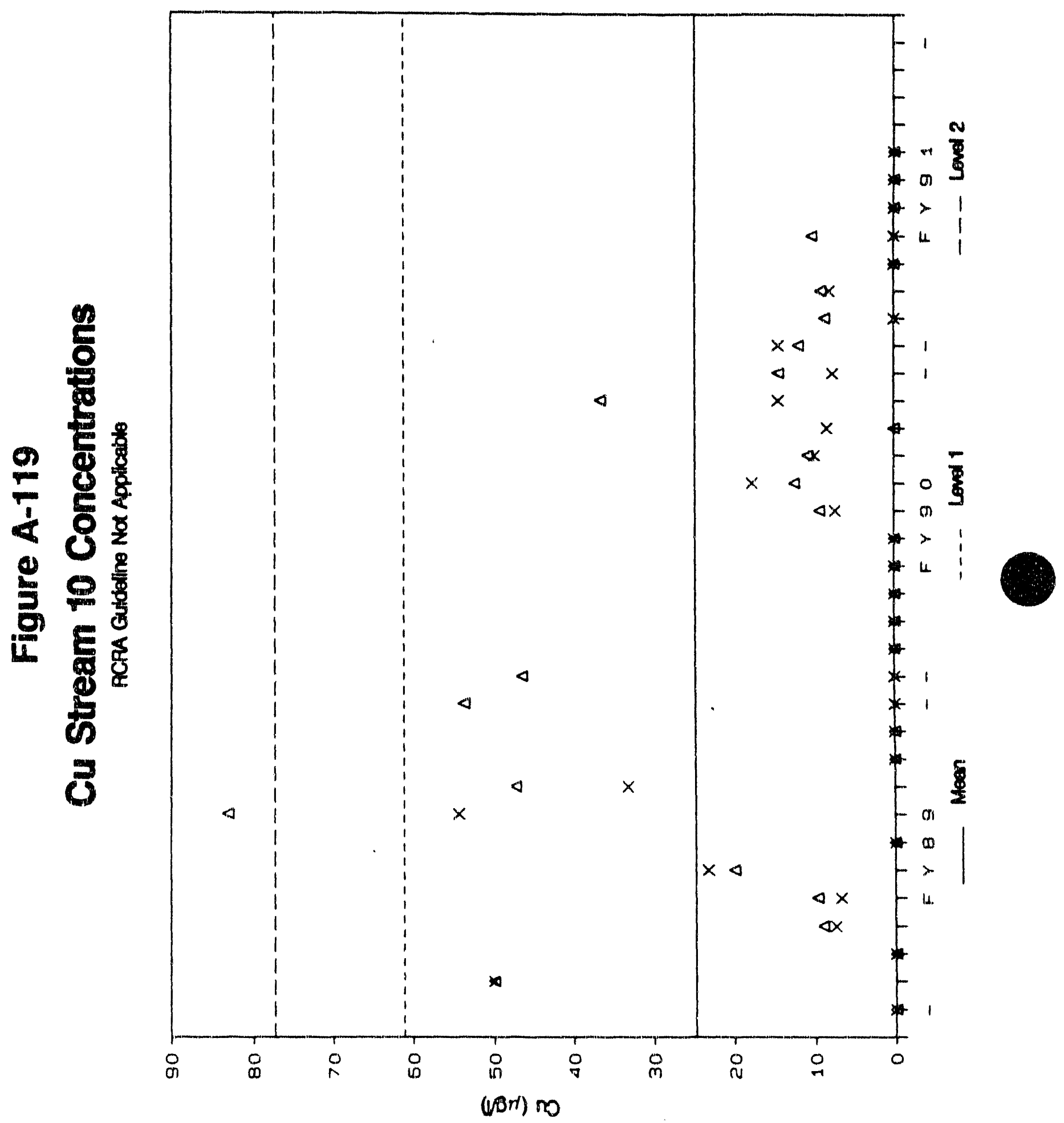




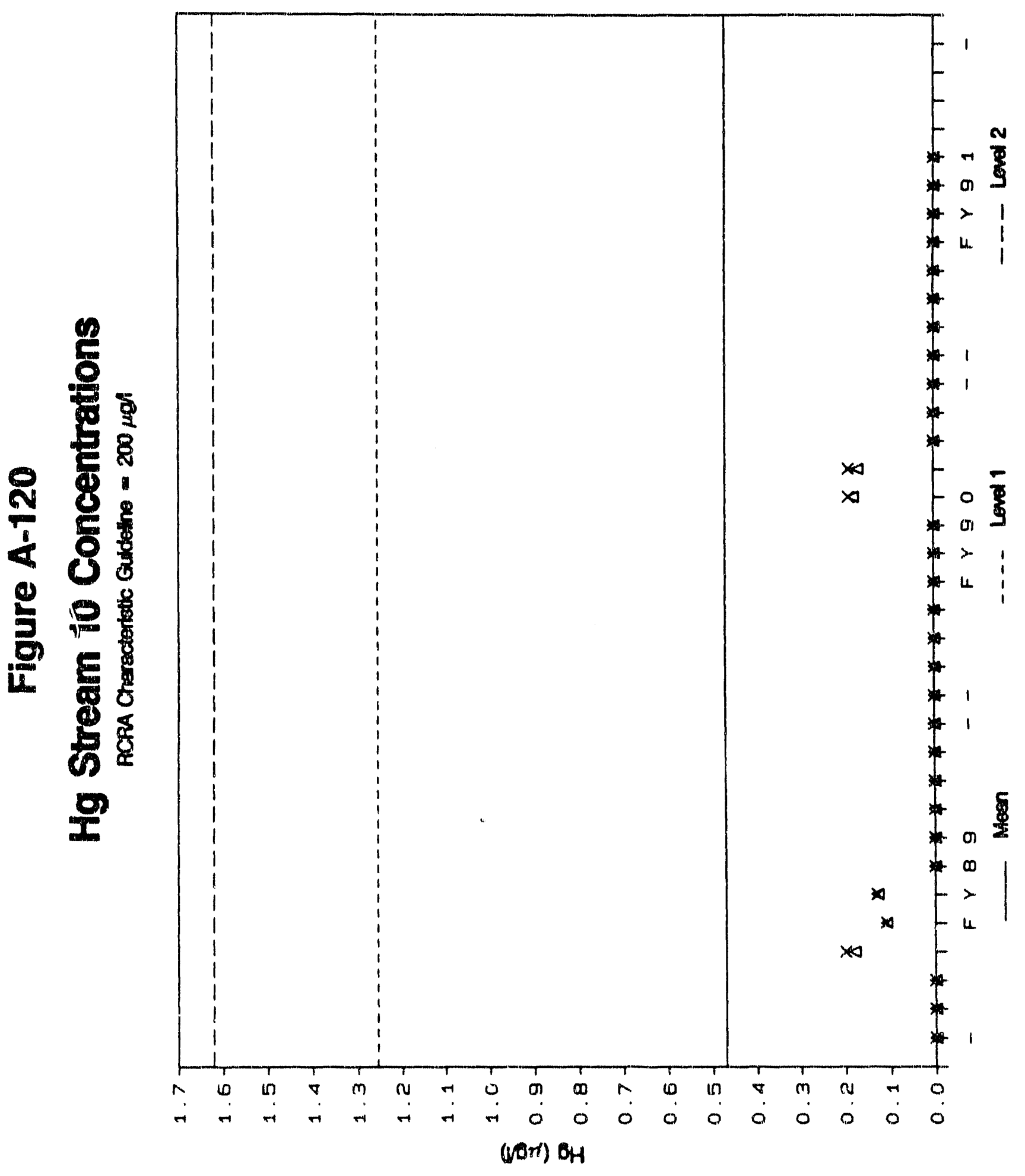




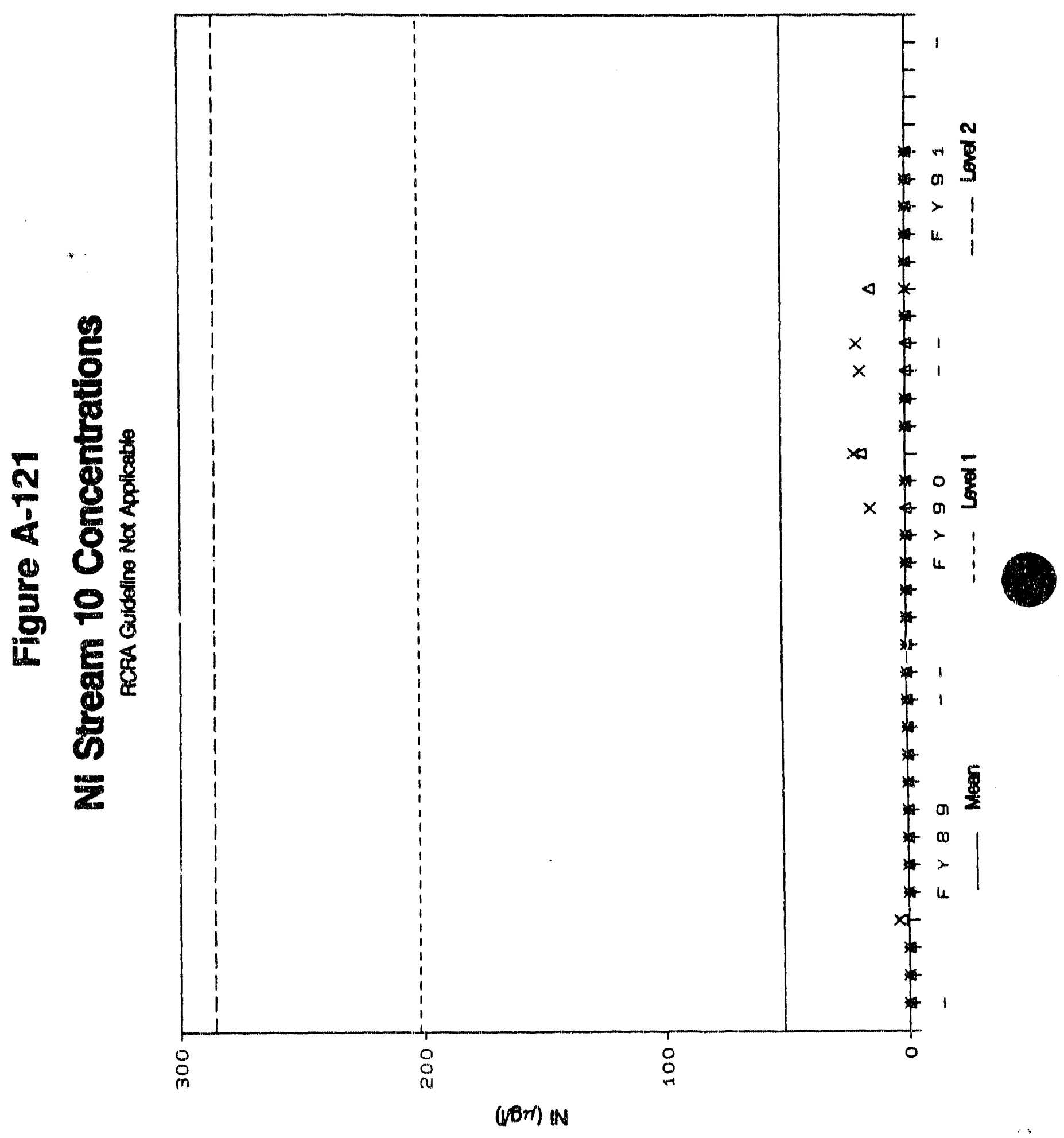




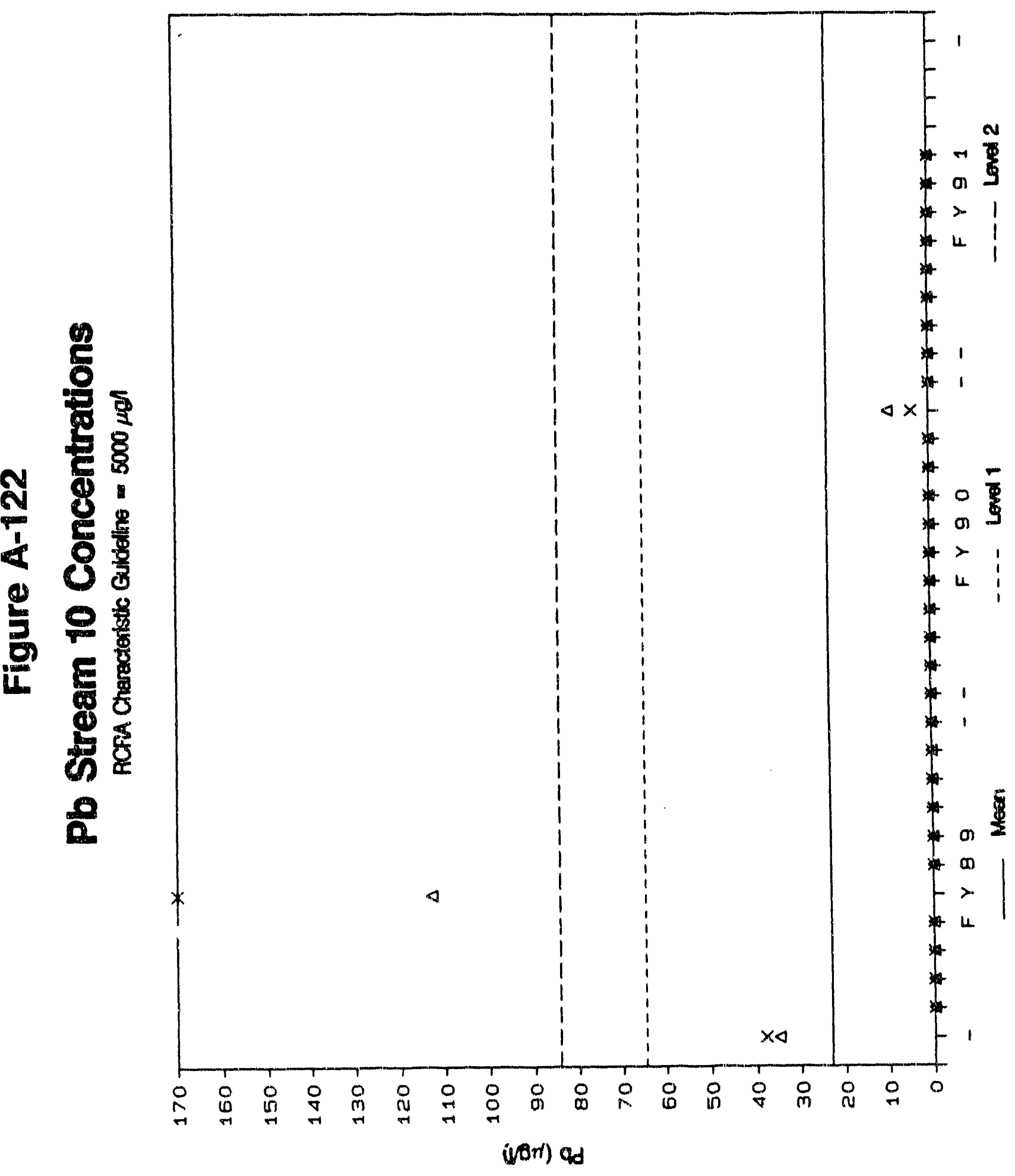

A- 175 


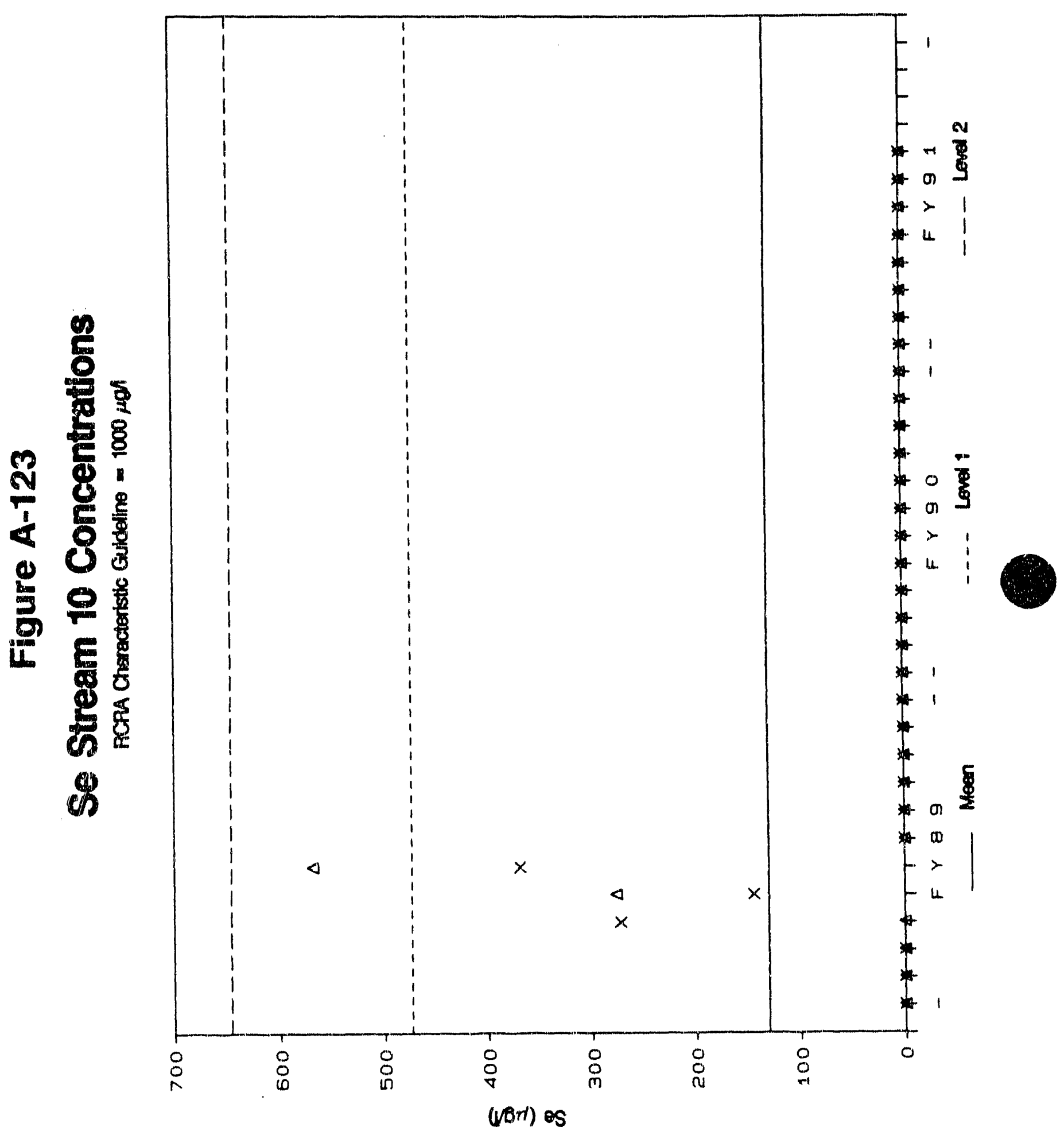




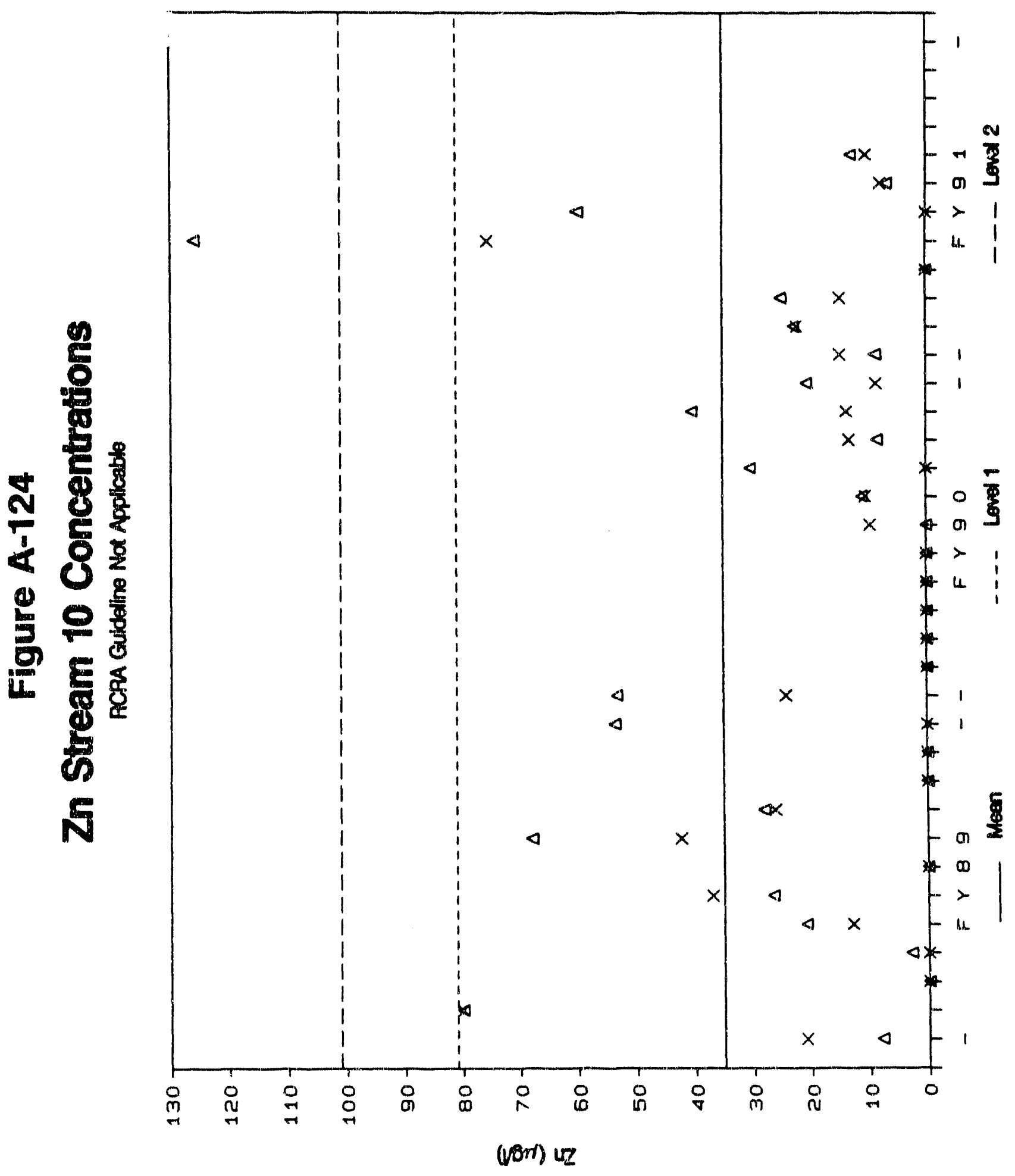

A- 117 


\section{Table A-36}

FY91 ENVIRONMENTAL MONITORING DATA

- STREAM=10 (CTF effluent to Iiquid disposal pond) QUARTER=91qI

$\begin{array}{lcccccccc}\text { MONTH } & \text { REP } & \begin{array}{c}\text { COnd } \\ (\mu \mathrm{S})\end{array} & \mathrm{pH} & \begin{array}{c}\mathrm{TOC} \\ (\mathrm{mg} / \mathrm{l})\end{array} & \begin{array}{c}\mathrm{Cl} \\ (\mathrm{mg} / 1)\end{array} & \begin{array}{c}\mathrm{F} \\ (\mathrm{mg} / 1)\end{array} & \begin{array}{c}\mathrm{NO3} \\ (\mathrm{mg} / 1)\end{array} & \begin{array}{c}\mathrm{PO4} \\ (\mathrm{mg} / 1)\end{array} \\ \text { OCt } & 1 & 391 & 6.97 & \mathrm{X} & 16.8 & 0.51 & 3.40 & \mathrm{X} \\ \text { Oct } & 2 & 393 & 6.94 & \mathrm{X} & 16.6 & \mathrm{X} & 3.30 & \mathrm{X} \\ \text { NOV } & 1 & 415 & 8.00 & \mathrm{X} & 17.4 & \mathrm{X} & 2.60 & 0.14 \\ \text { NOV } & 2 & 402 & 7.97 & \mathrm{X} & 17.6 & \mathrm{X} & 2.60 & \mathrm{X} \\ \text { Dec } & 1 & 432 & 8.51 & \mathrm{X} & 17.0 & \mathrm{X} & 2.80 & 0.13 \\ \text { Dec } & 2 & 407 & 8.13 & \mathrm{X} & 17.1 & \mathrm{X} & 2.80 & \mathrm{X}\end{array}$

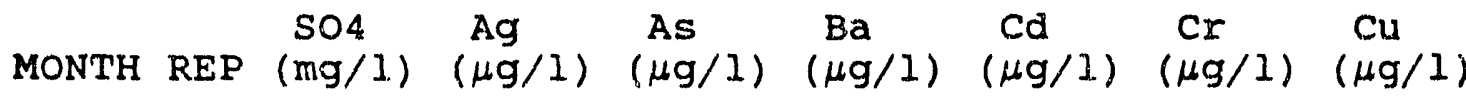

$\begin{array}{lllllllll}\text { Oct } & 1 & 34.8 & \mathrm{X} & 1.20 & 50.9 & \mathrm{X} & \mathrm{X} & 12.0 \\ \text { Oct } & 2 & 35.1 & \mathrm{X} & 2.00 & 54.1 & \mathrm{X} & \mathrm{X} & 14.5 \\ \text { Nov } & 1 & 34.5 & 23.2 & 1.70 & 55.0 & \mathrm{X} & \mathrm{X} & 8.70 \\ \text { Nov } & 2 & 34.5 & \mathrm{X} & 1.50 & 53.9 & \mathrm{X} & \mathrm{X} & \mathrm{X} \\ \text { Dec } & 1 & 34.0 & \mathrm{X} & 2.50 & 57.9 & \mathrm{X} & \mathrm{X} & 9.20 \\ \text { Dec } & 2 & 34.4 & \mathrm{X} & 2.50 & 55.9 & \mathrm{X} & \mathrm{X} & 8.10\end{array}$

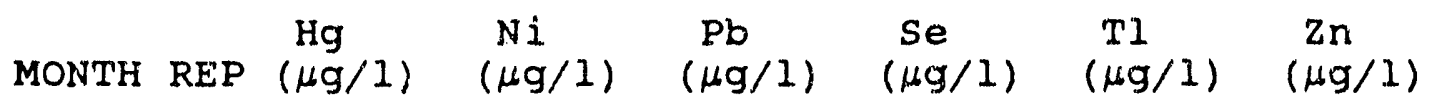

$\begin{array}{llllllll}\text { Oct } & 1 & \mathrm{X} & \mathrm{X} & \mathrm{X} & \mathrm{X} & \mathrm{X} & 8.70 \\ \text { Oct } & 2 & \mathrm{X} & 20.4 & \mathrm{X} & \mathrm{X} & \mathrm{X} & 14.8 \\ \text { Nov } & 1 & \mathrm{X} & \mathrm{X} & \mathrm{X} & \mathrm{X} & \mathrm{X} & 22.8 \\ \text { Nov } & 2 & \mathrm{X} & \mathrm{X} & \mathrm{X} & \mathrm{X} & \mathrm{X} & 22.3 \\ \text { Dec } & 1 & \mathrm{X} & 15.1 & \mathrm{X} & \mathrm{X} & \mathrm{X} & 25.0 \\ \text { Dec } & 2 & \mathrm{X} & \mathrm{X} & \mathrm{X} & \mathrm{X} & \mathrm{X} & 14.8\end{array}$

$' . '$ - missing

' $x$ ' - below practical quantitation level 


\section{Table A-37}

FY91 ENVIRONMENTAL MONITORING DATA

- STREAM=10 (CTF effluent to liquid disposal pond) QUARTER=91q2 -

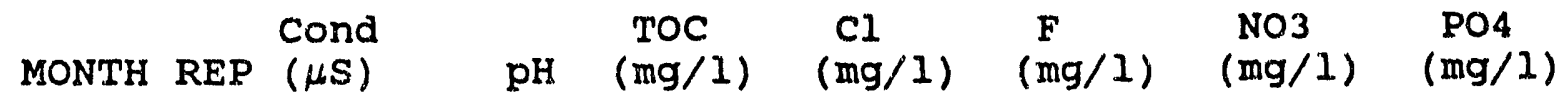

$\begin{array}{lllllllll}\text { Jan } & 1 & 348 & 8.39 & 5.30 & 19.1 & 0.20 & X & X \\ \text { Jan } & 2 & 416 & 7.78 & 5.50 & 18.8 & 0.20 & X & X \\ \text { Feb } & 1 & 417 & 8.24 & 7.80 & 57.7 & 0.20 & 4.00 & X \\ \text { Feb } & 2 & 420 & 8.14 & 3.50 & 72.7 & 0.20 & 4.00 & X \\ \text { Mar } & 1 & 492 & 7.16 & X & 16.0 & X & X & 0.03 \\ \text { Mar } & 2 & 412 & 8.05 & X & X & X & X & X\end{array}$

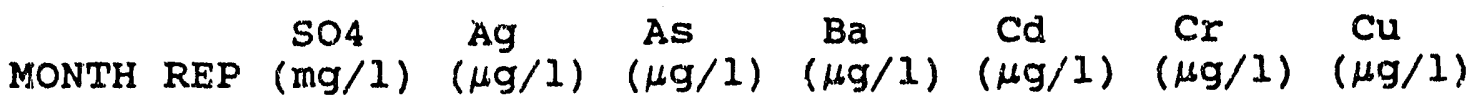

$\begin{array}{lllllllll}\text { Jan } & 1 & 40.1 & X & X & X & X & X & X \\ \text { Jan } & 2 & 40.0 & X & X & X & X & X & X \\ \text { Feb } & 1 & 49.7 & X & X & X & X & X & 10.3 \\ \text { Feb } & 2 & 49.8 & X & X & X & X & X & X \\ \text { Mar } & 1 & 34.0 & X & X & X & X & X & X \\ \text { Mar } & 2 & X & X & X & X & X & X & X\end{array}$

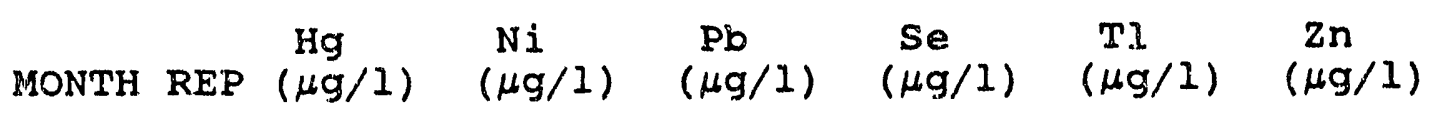

$\begin{array}{llllllll}\text { Jan } & 1 & X & X & X & X & X & X \\ \text { Jan } & 2 & X & X & X & X & X & X \\ \text { Feb } & 1 & X & X & X & X & X & 126 \\ \text { Feb } & 2 & X & X & X & X & X & 75.6 \\ \text { Mar } & 1 & X & X & X & X & X & 60.0 \\ \text { Mar } & 2 & X & X & X & X & X & X\end{array}$

$'$.' - missing

' $X$ ' - below practical quantitation level 


\section{Table A-38}

FY91 ENVIRONMENTAL MONITORING DATA

- STREAM=10 (CTF effluent to liquid disposal pond) QUARTER=91q3

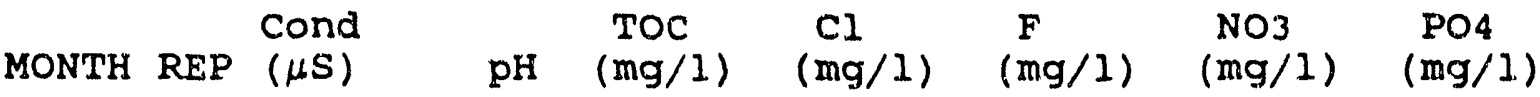

$\begin{array}{llccccccc}\text { Apr } & 1 & 614 & 7.31 & 2.20 & 16.4 & 0.24 & 1.69 & 0.13 \\ \text { Apr } & 2 & X & X & 2.89 & 16.4 & 0.27 & 1.71 & 0.14 \\ \text { May } & 1 & 666 & 7.55 & 0.79 & 17.1 & 0.23 & 1.64 & 0.06 \\ \text { May } & 2 & 423 & 7.89 & 0.86 & 17.0 & 0.21 & 1.62 & 0.06\end{array}$

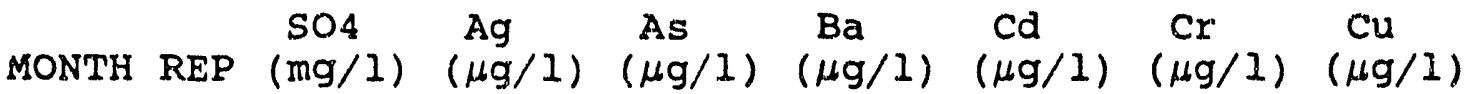

$\begin{array}{lllllllll}\text { Apr } & 1 & 34.0 & \mathrm{X} & \mathrm{X} & 79.6 & \mathrm{X} & 11.7 & \mathrm{X} \\ \text { Apr } & 2 & 34.2 & \mathrm{X} & \mathrm{X} & 77.0 & \mathrm{X} & 8.40 & \mathrm{X} \\ \text { May } & 1 & 33.3 & \mathrm{X} & 3.50 & 77.9 & \mathrm{X} & \mathrm{X} & \mathrm{X} \\ \text { May } & 2 & 33.4 & \mathrm{X} & 3.00 & 77.9 & \mathrm{X} & \mathrm{X} & \mathrm{X}\end{array}$

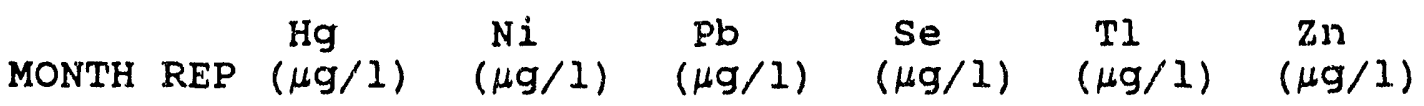

$\begin{array}{llllllll}\text { Apr } & 1 & X & X & X & X & X & 6.80 \\ \text { Apr } & 2 & X & X & X & X & X & 7.90 \\ \text { May } & 1 & X & X & X & X & X & 12.9 \\ \text { May } & 2 & X & X & X & X & X & 10.3\end{array}$

'. - missing

' $X '$ - below practical quantitation level 


\section{Table A-39}

SUMMARY STATISTICS

STREAM=10 (CTF effluent to liquid disposal pond)

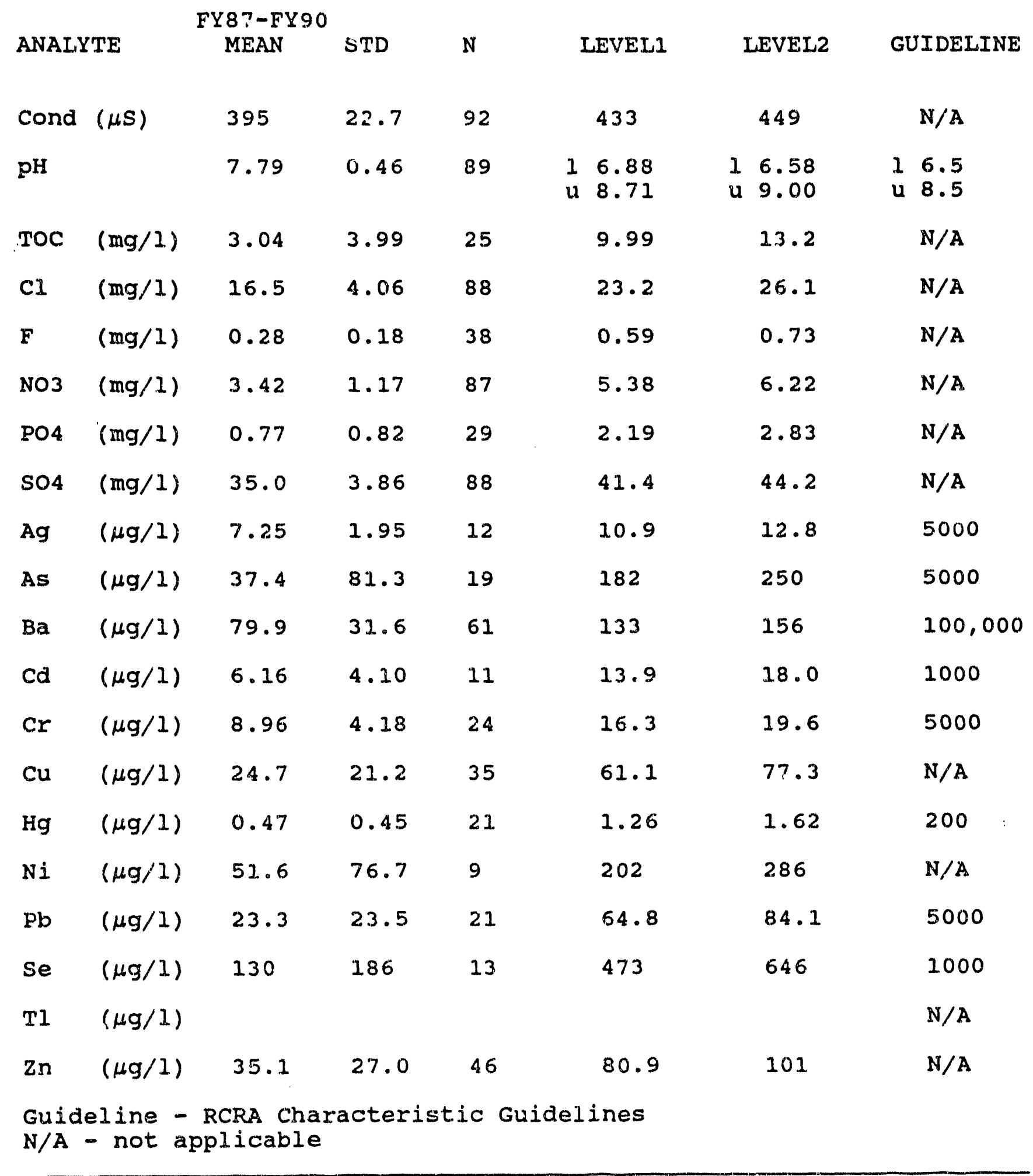




\section{Table A-40}

QUARTERLY MEANS AND VARIANCE COMPONENTS

STREAM=10 (CTE effIuent to liquid disposal pond)

$\begin{array}{lllllll} & & \text { STD } & \text { STD } & \text { STD } & N & N \\ \text { ANALYTE } & \text { MEAN } & \text { MEAN } & \text { DAY } & \text { REP } & N & \text { MISS } \\ \text { BPQL }\end{array}$

Cond ( $\mu S)$

$\begin{array}{lllll}9191 & 407 & 7.99 & 11.2 & 11.5\end{array}$

$9192 \quad 418 \quad 20.2 \quad 17.25011 .5 \quad 6 \quad 000$

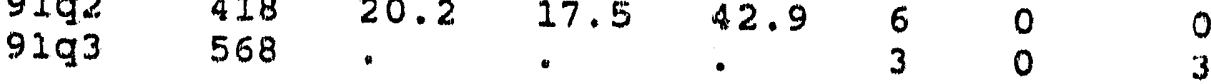

pH

$\begin{array}{llllllll}9193 & 7.75 & 0.41 & 0.70 & 0.16 & 6 & 0 & 0 \\ 9192 & 7.96 & 0.18 & 0.00 & 0.44 & 6 & 0 & 0 \\ 9193 & 7.58 & . & 0 & . & 3 & 0 & 3\end{array}$

$\operatorname{TOC}(\mathrm{mg} / 1) \quad 91 \mathrm{~g} 1 \mathrm{x}$

$\begin{array}{llllllll}9191 & x & & & 0 & 0 & 6 \\ 9192 & 5.53 & 0.13 & 0.00 & 2.15 & 4 & 2 & 0 \\ 9193 & 1.58 & 0.86 & 1.19 & 0.35 & 4 & 0 & 0\end{array}$

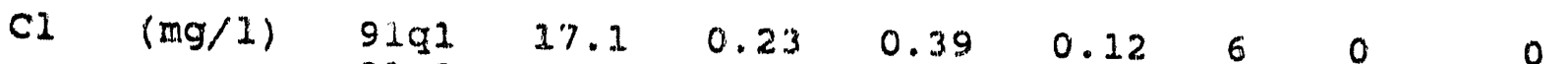

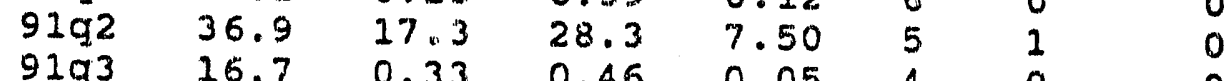

$\begin{array}{lllllllll} & (\mathrm{mg} / 1) & 91 \mathrm{gl} & 0.51 & & & & & \\ & 9192 & 0.06 & 0.21 & 0.35 & 0.00 & 5 & 1 & 5 \\ & 9193 & 0.24 & 0.02 & 0.02 & 0.02 & 4 & 0 & 0\end{array}$

$\begin{array}{lllllllll}\text { NO3 }(\mathrm{mg} / \mathrm{l}) & 91 \mathrm{gl} & 2.92 & 0.22 & 0.38 & 0.04 & 6 & 0 & 0 \\ & 9192 & 4.00 & 0.03 & 0.05 & 0.01 & 4 & 0 & 4 \\ & 9193 & 1.66 & 0.03 & 0.05 & 0.01 & 4 & 0 & 0\end{array}$

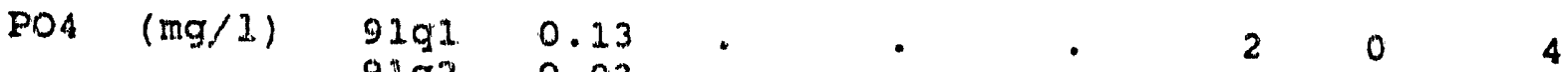

$\begin{array}{llllllll}9192 & 0.03 & 0.04 & 0 & 0 & 1 & 0 & 5 \\ 9193 & 0.10 & 0.04 & 0.05 & 0.01 & 4 & 0\end{array}$

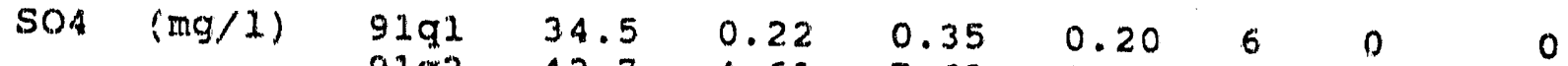

$\begin{array}{llllllll}9192 & 42.7 & 4.61 & 7.69 & 0.07 & 5 & 1 & 0 \\ 9193 & 33.7 & 0.37 & 0.52 & 0.11 & 4 & 0 & 0\end{array}$

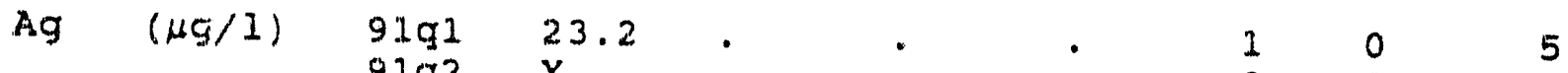

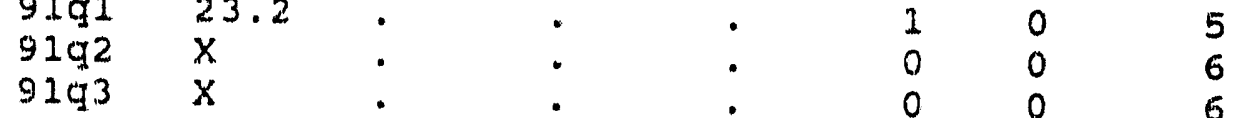

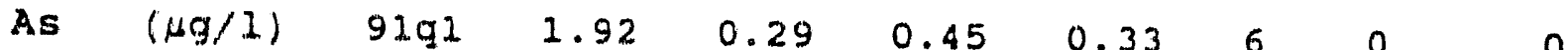

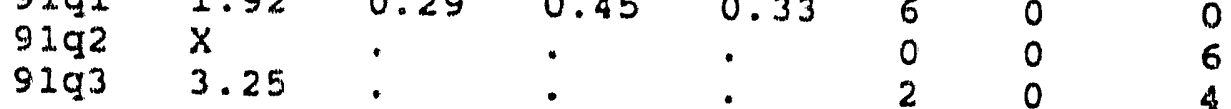

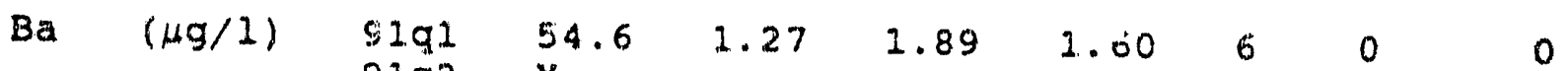

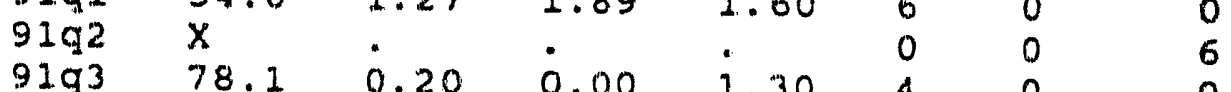

$\operatorname{Cd}(\mu \mathrm{g} / 1) \quad 91 \mathrm{gl} \quad \mathrm{x} \quad \ldots .00000$

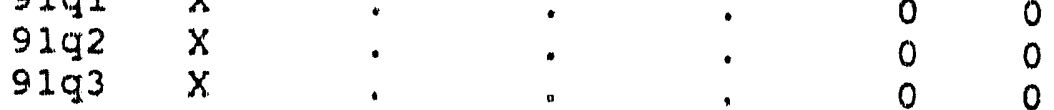

6 
Table A-40 (continued)

QUARTERLY MEANS AND VARIANCE COMPONENTS

...- STREAM=10 (CTF effluent to 1iquid disposal pond) (continued)

\begin{tabular}{|c|c|c|c|c|c|c|c|c|c|}
\hline \multicolumn{2}{|c|}{ ANALYTE } & QTR & MEAN & $\begin{array}{r}\text { STD } \\
\text { MEAN }\end{array}$ & $\begin{array}{l}\text { STD } \\
\text { DAY }\end{array}$ & $\begin{array}{l}\text { STD } \\
\text { REP }\end{array}$ & $\mathrm{N}$ & $\underset{\text { MISS }}{\mathrm{N}}$ & $B P Q$ \\
\hline$c r$ & $(\mu g / l)$ & $\begin{array}{l}91 q^{1} \\
91 q^{2} \\
919^{3}\end{array}$ & $\begin{array}{l}x \\
x \\
10.1\end{array}$ & $\dot{.}$ & $\dot{\bullet}$ & $\dot{\dot{ }}$ & $\begin{array}{l}0 \\
0 \\
2\end{array}$ & $\begin{array}{l}0 \\
0 \\
0\end{array}$ & $\begin{array}{l}6 \\
6 \\
4\end{array}$ \\
\hline $\mathrm{Cu}$ & $(\mu g / I)$ & $\begin{array}{l}9191 \\
9192 \\
9193\end{array}$ & $\begin{array}{l}10.5 \\
10.3 \\
x\end{array}$ & $\begin{array}{l}1.67 \\
\dot{.}\end{array}$ & $\begin{array}{l}2.59 \\
. \\
.\end{array}$ & $\begin{array}{l}1.37 \\
\cdot \\
\end{array}$ & $\begin{array}{l}5 \\
1 \\
0\end{array}$ & $\begin{array}{l}0 \\
0 \\
0\end{array}$ & $\begin{array}{l}1 \\
5 \\
6\end{array}$ \\
\hline $\mathrm{Hg}$ & $(\mu g / I)$ & $\begin{array}{l}91 q^{1} \\
91 q^{2} \\
91 q^{3}\end{array}$ & $\begin{array}{l}x \\
x \\
x\end{array}$ & $\dot{\bullet}$ & $\dot{.}$ & $\dot{.} \cdot$ & $\begin{array}{l}0 \\
0 \\
0\end{array}$ & $\begin{array}{l}0 \\
0 \\
0\end{array}$ & $\begin{array}{l}6 \\
6 \\
6\end{array}$ \\
\hline $\mathrm{Ni}$ & $(\mu g / I)$ & $\begin{array}{l}91 q 1 \\
91 q^{2} \\
91 q^{3}\end{array}$ & $\begin{array}{l}17.8 \\
x \\
x\end{array}$ & $\begin{array}{l}\dot{\bullet} \\
\dot{\cdot}\end{array}$ & $\dot{\dot{ }}$ & $\dot{\bullet}$ & $\begin{array}{l}2 \\
0 \\
0\end{array}$ & $\begin{array}{l}0 \\
0 \\
0\end{array}$ & $\begin{array}{l}4 \\
6 \\
6\end{array}$ \\
\hline $\mathrm{Pb}$ & $(\mu g / l)$ & $\begin{array}{l}919^{1} \\
91 q^{2} \\
91 . q^{3}\end{array}$ & $\begin{array}{l}x \\
x \\
x\end{array}$ & $\begin{array}{l}\dot{\bullet} \\
\dot{\bullet}\end{array}$ & $\dot{\bullet}$ & . & $\begin{array}{l}0 \\
0 \\
0\end{array}$ & $\begin{array}{l}0 \\
0 \\
0\end{array}$ & $\begin{array}{l}6 \\
6 \\
6\end{array}$ \\
\hline Se & $(\mu g / I)$ & $\begin{array}{l}9191 \\
9192 \\
919^{3}\end{array}$ & $\begin{array}{l}x \\
x \\
x\end{array}$ & $\dot{\bullet}$ & $\dot{\bullet}$ & $\dot{\bullet}$ & $\begin{array}{l}0 \\
0 \\
0\end{array}$ & $\begin{array}{l}0 \\
0 \\
0\end{array}$ & $\begin{array}{l}6 \\
6 \\
6\end{array}$ \\
\hline $\mathrm{Tl}$ & $(\mu g / I)$ & $\begin{array}{l}91 q 1 \\
91 q 2 \\
91 q^{3}\end{array}$ & $\begin{array}{l}x \\
x \\
x\end{array}$ & $\dot{\bullet}$ & $\dot{:}$ & $\dot{\dot{.}}$ & $\begin{array}{l}0 \\
0 \\
0\end{array}$ & $\begin{array}{l}0 \\
0 \\
0\end{array}$ & $\begin{array}{l}6 \\
6 \\
6\end{array}$ \\
\hline $\mathrm{Zn}$ & $(\mu g / 1)$ & $\begin{array}{l}91 q^{1} \\
91 q^{2} \\
91 q^{3}\end{array}$ & $\begin{array}{l}18.1 \\
87.2 \\
9.48\end{array}$ & $\begin{array}{l}3.25 \\
2.12\end{array}$ & $\begin{array}{l}4.46 \\
2.83\end{array}$ & $\begin{array}{l}4.86 \\
\dot{1} .41\end{array}$ & $\begin{array}{l}6 \\
3 \\
4\end{array}$ & $\begin{array}{l}0 \\
0 \\
0\end{array}$ & $\begin{array}{l}0 \\
3 \\
0\end{array}$ \\
\hline
\end{tabular}




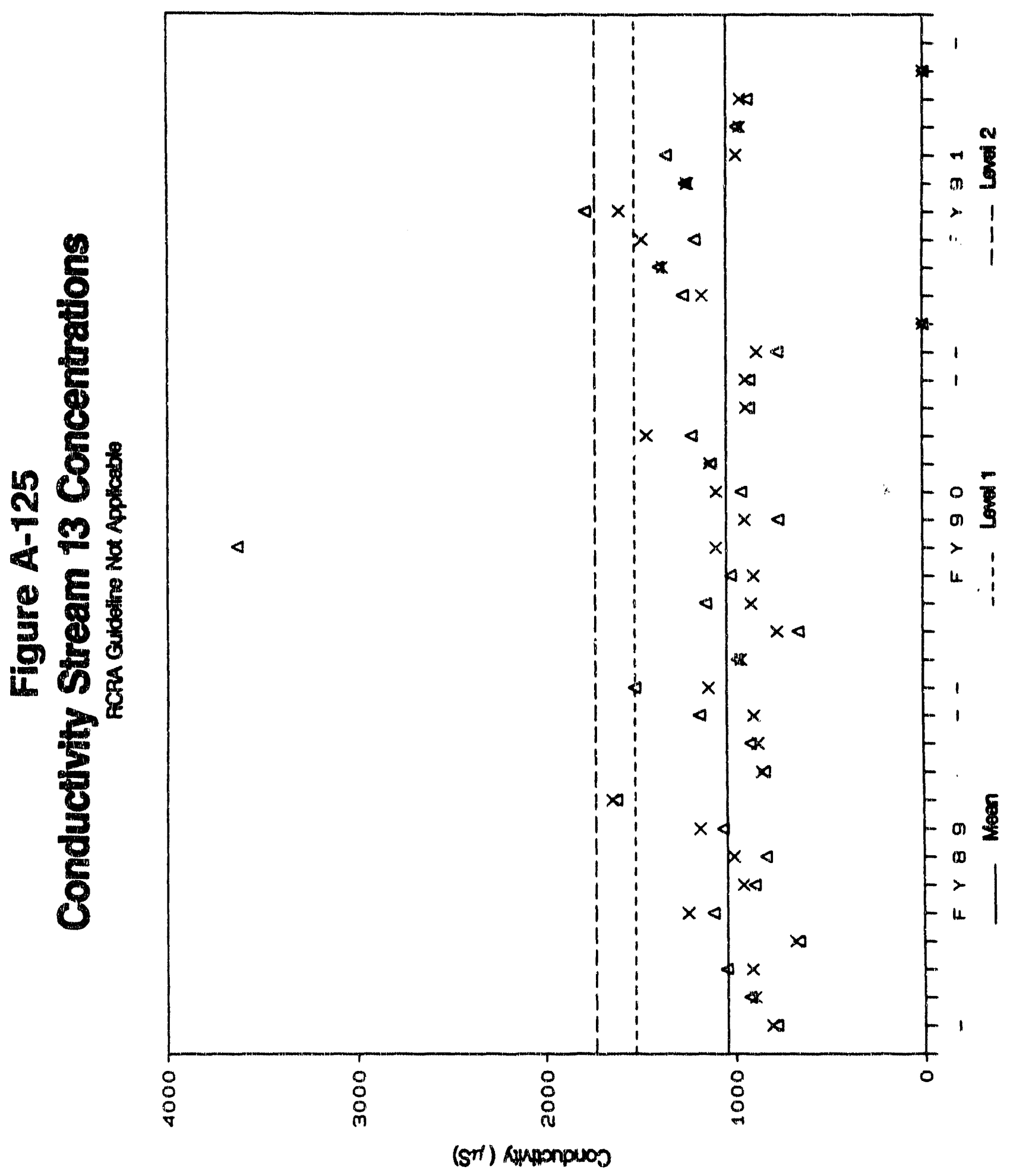

$\bar{A}-185$ 


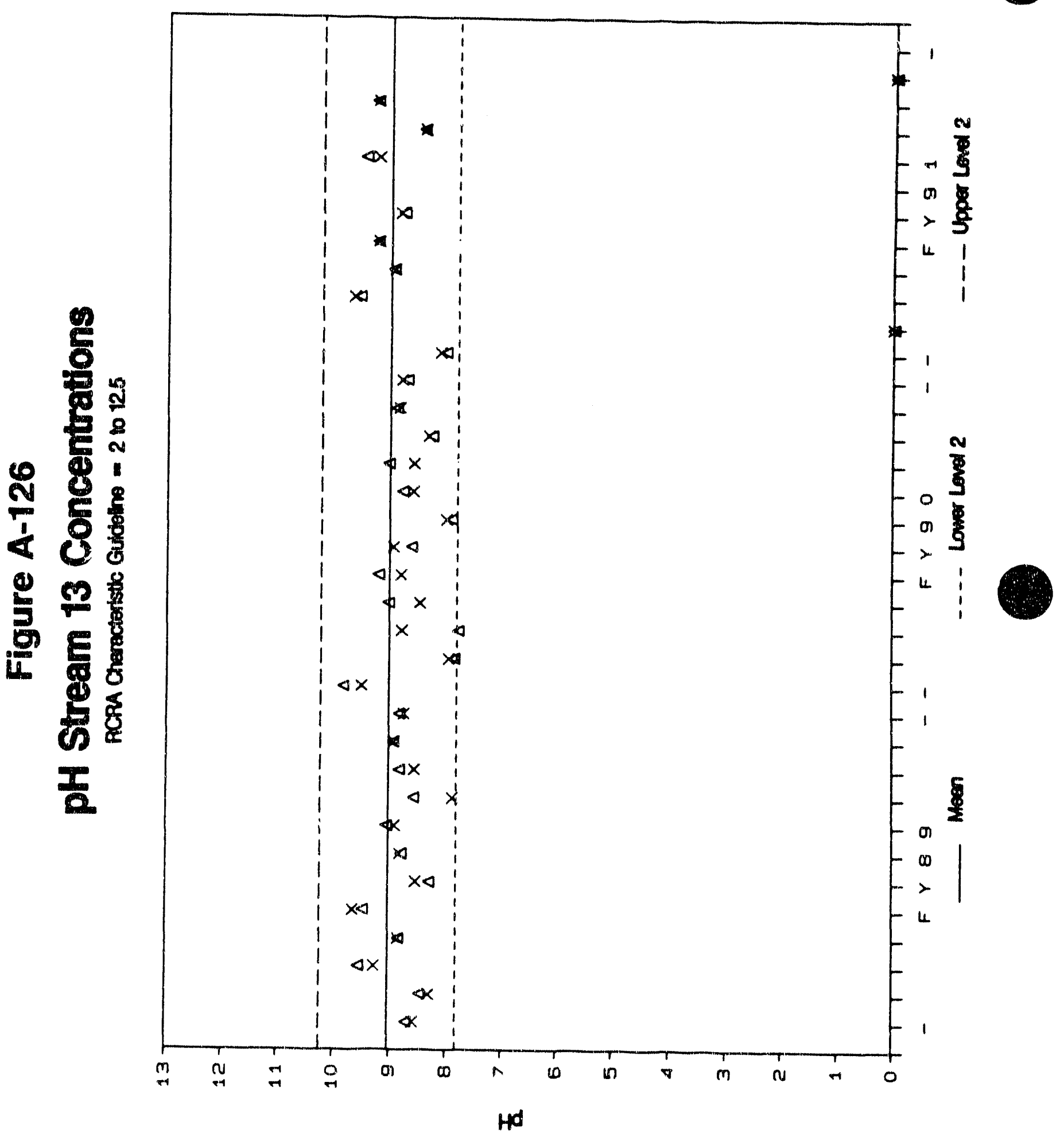




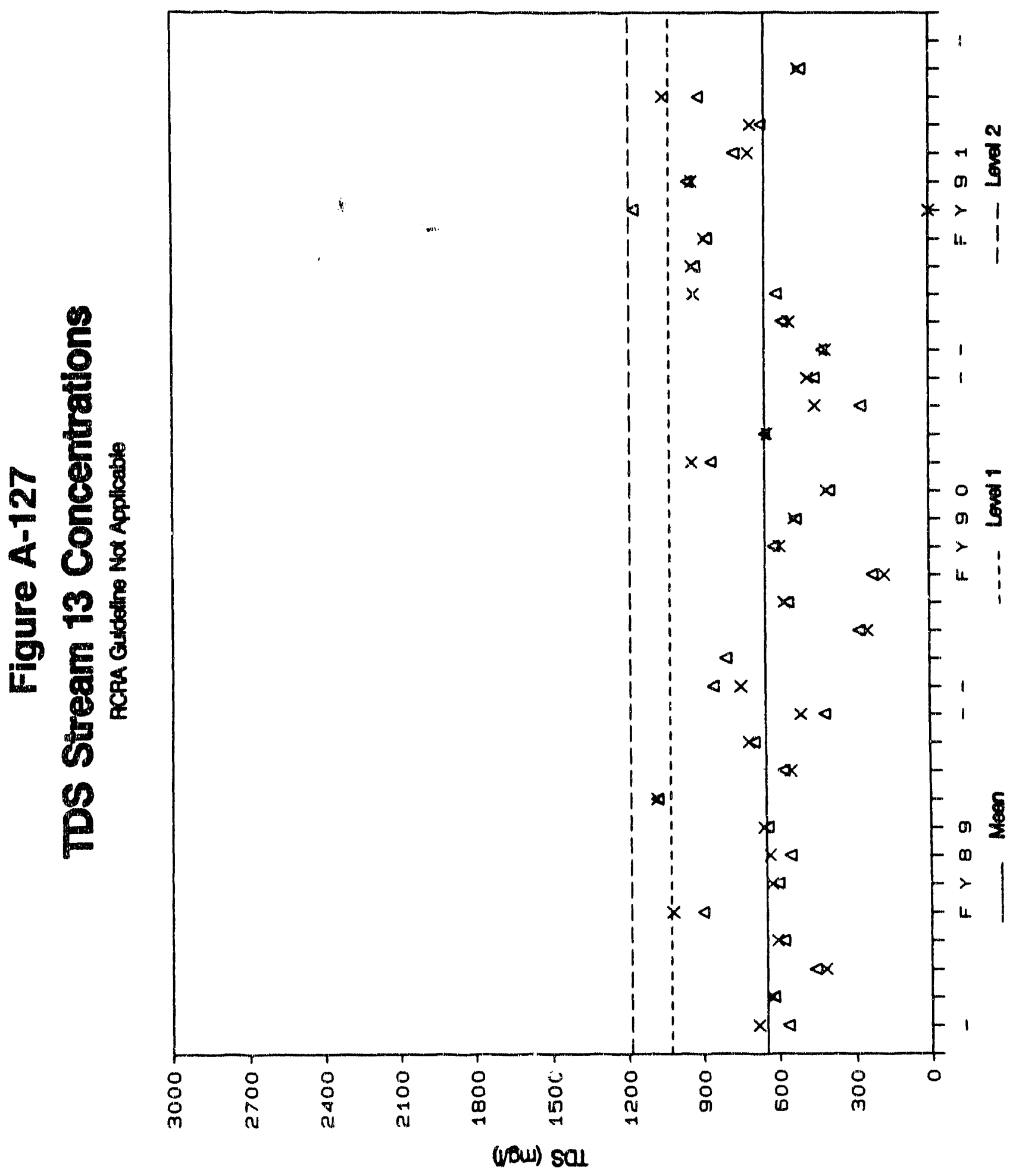




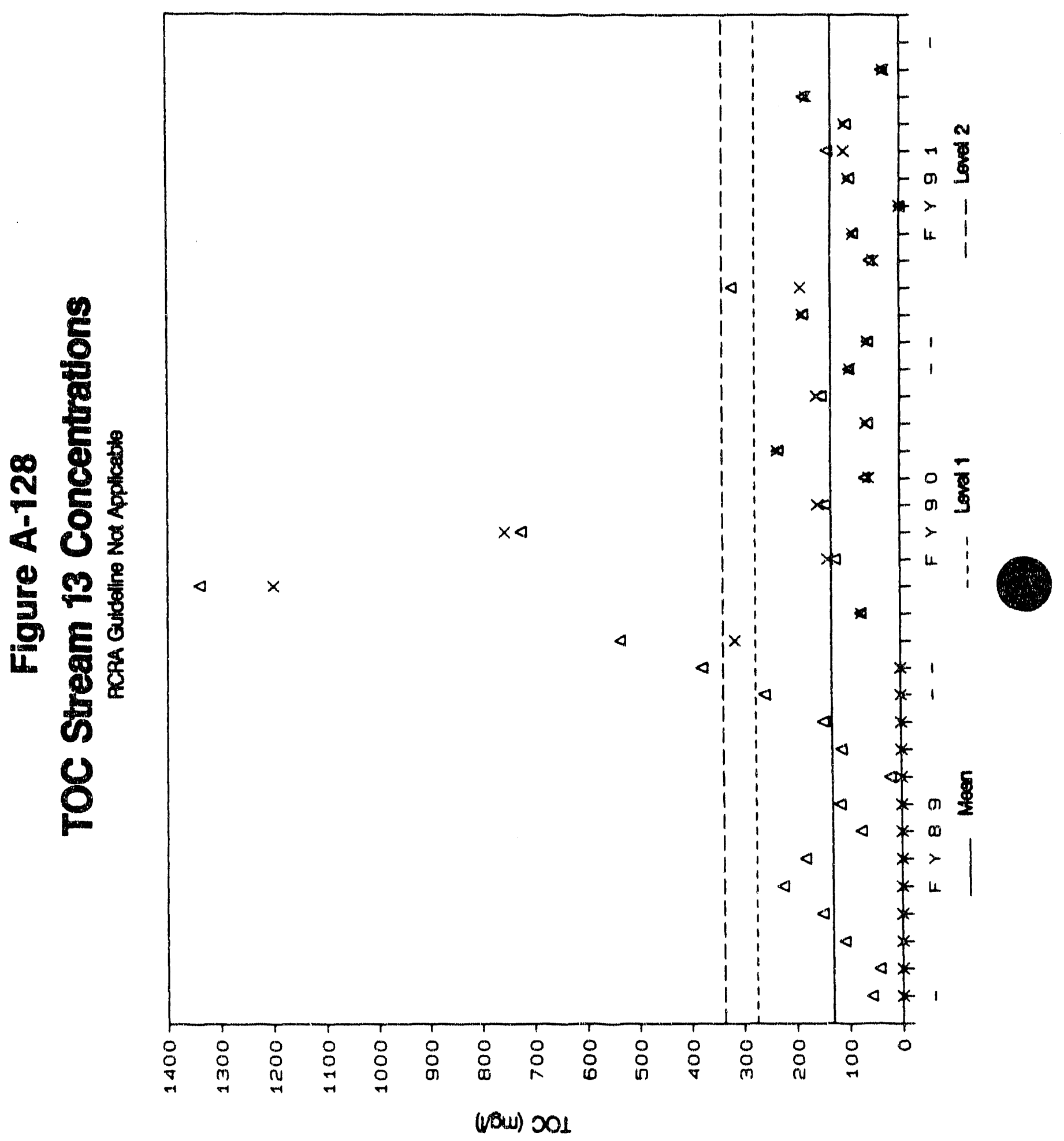




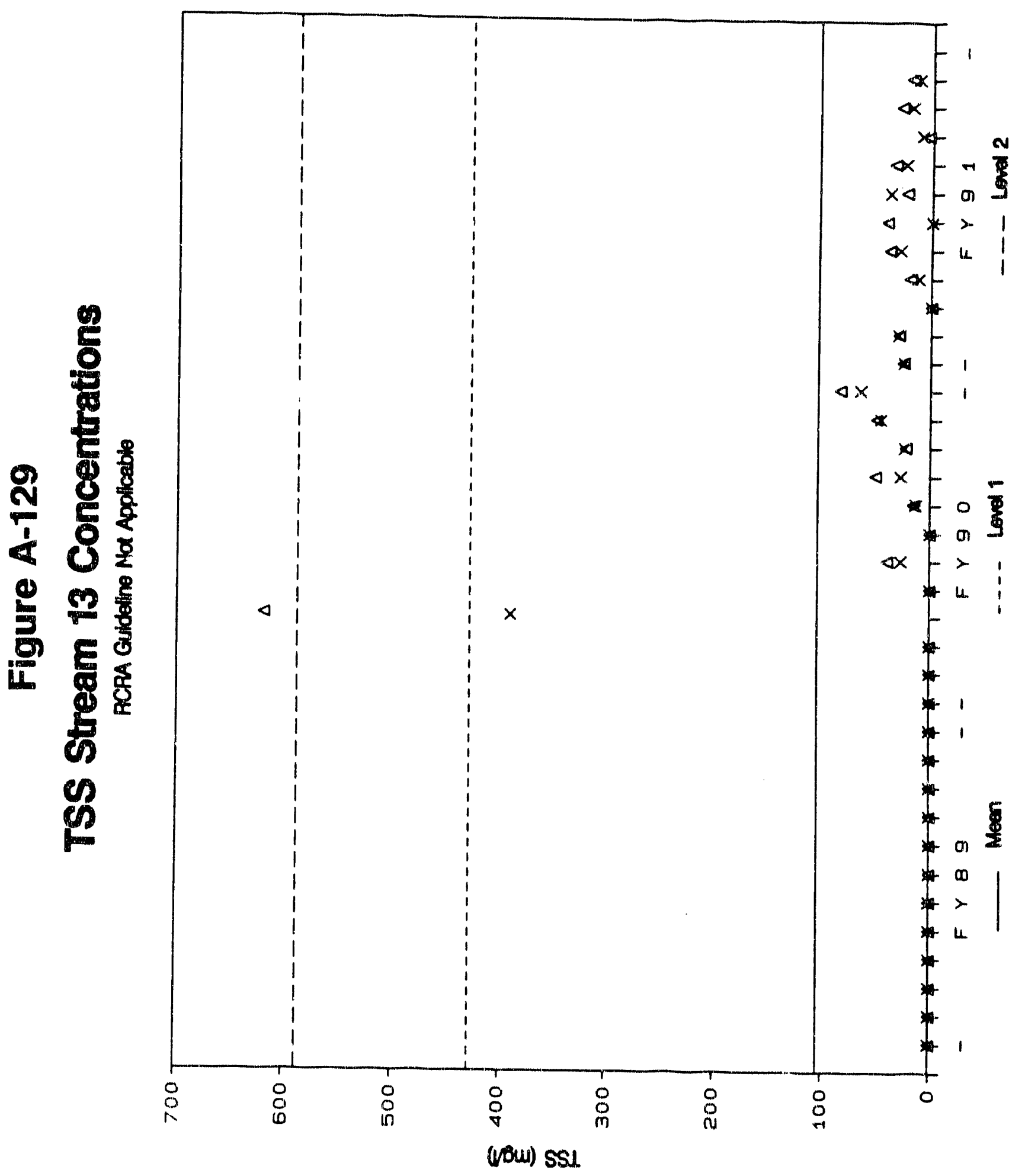




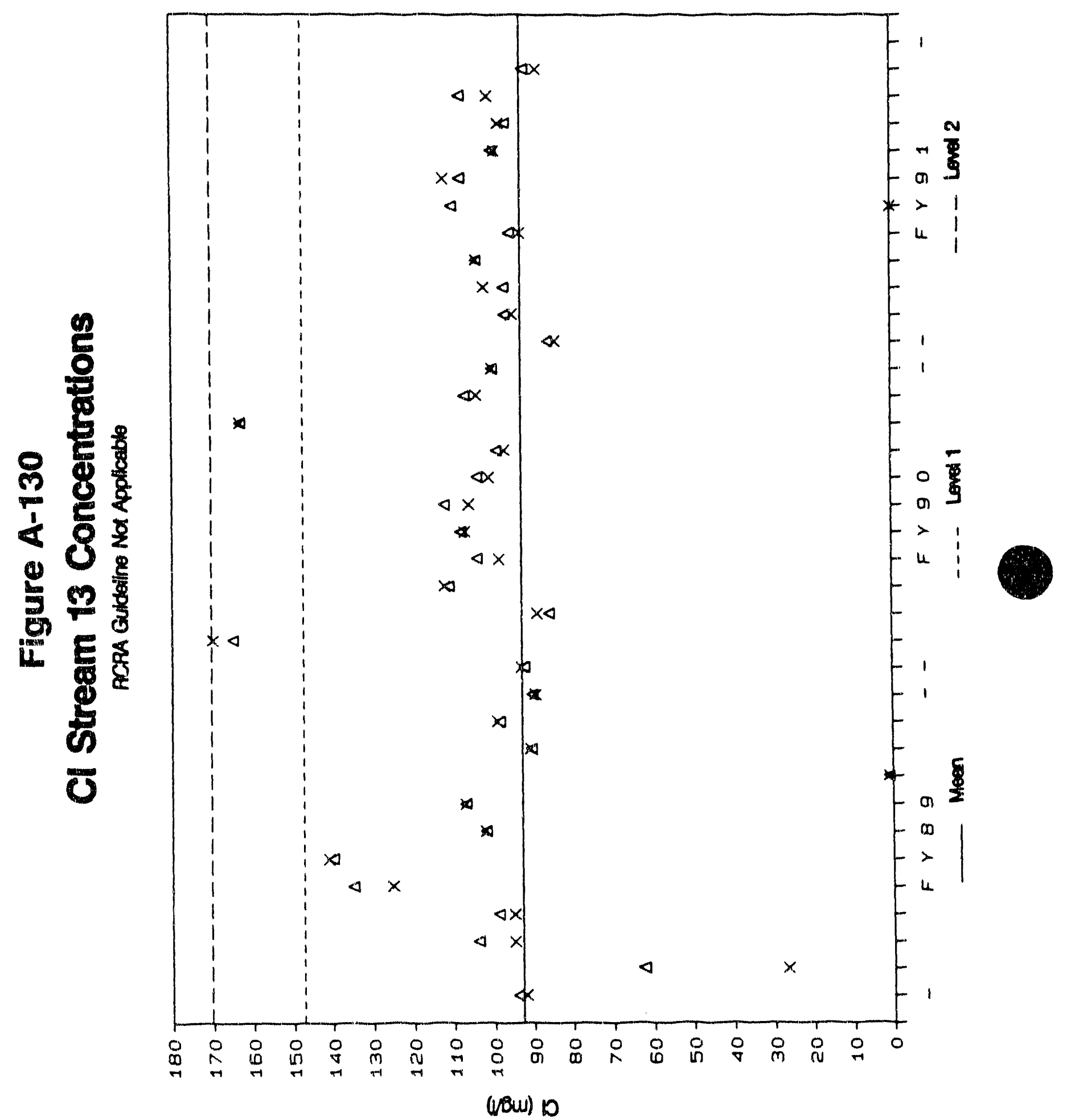




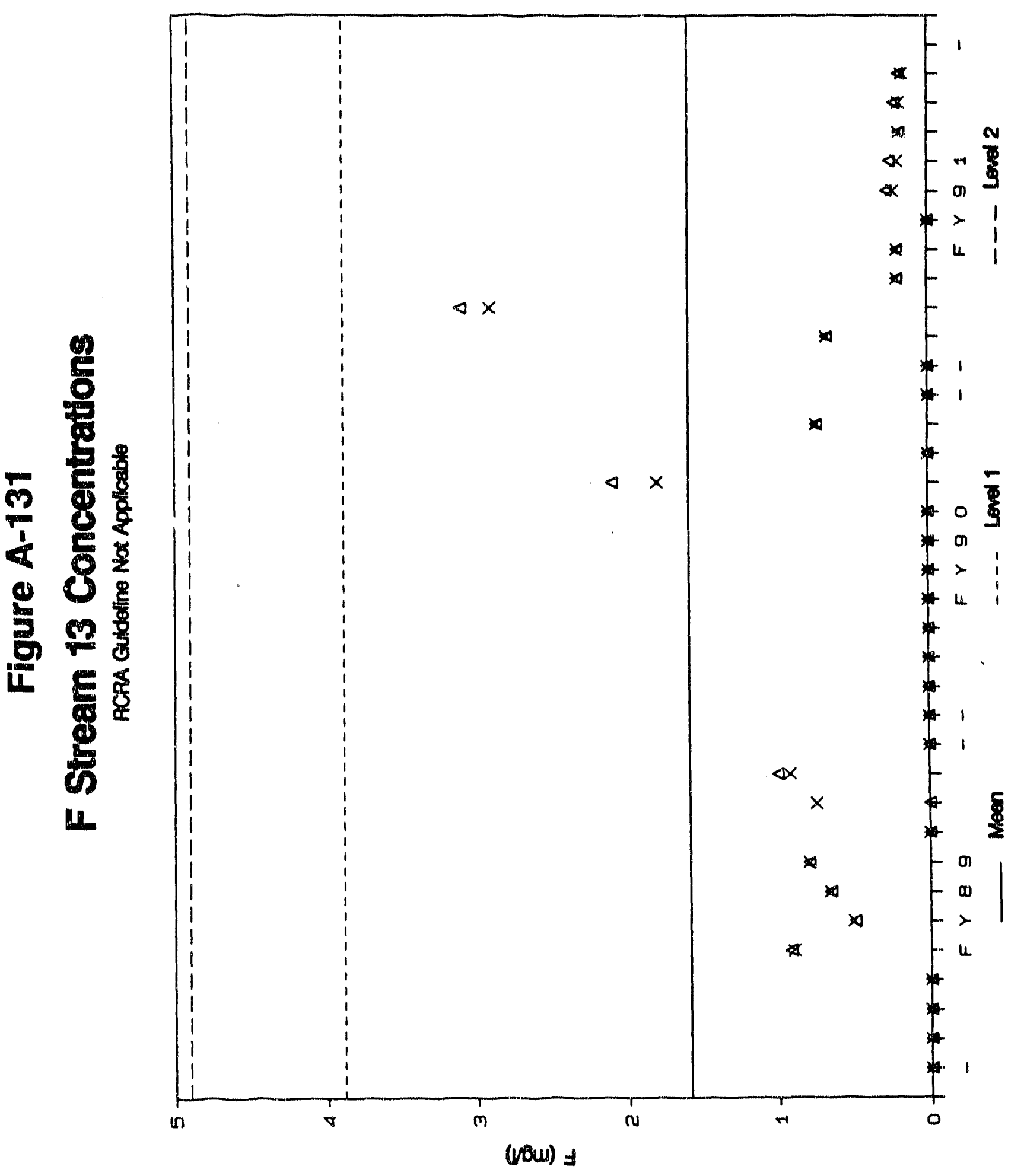




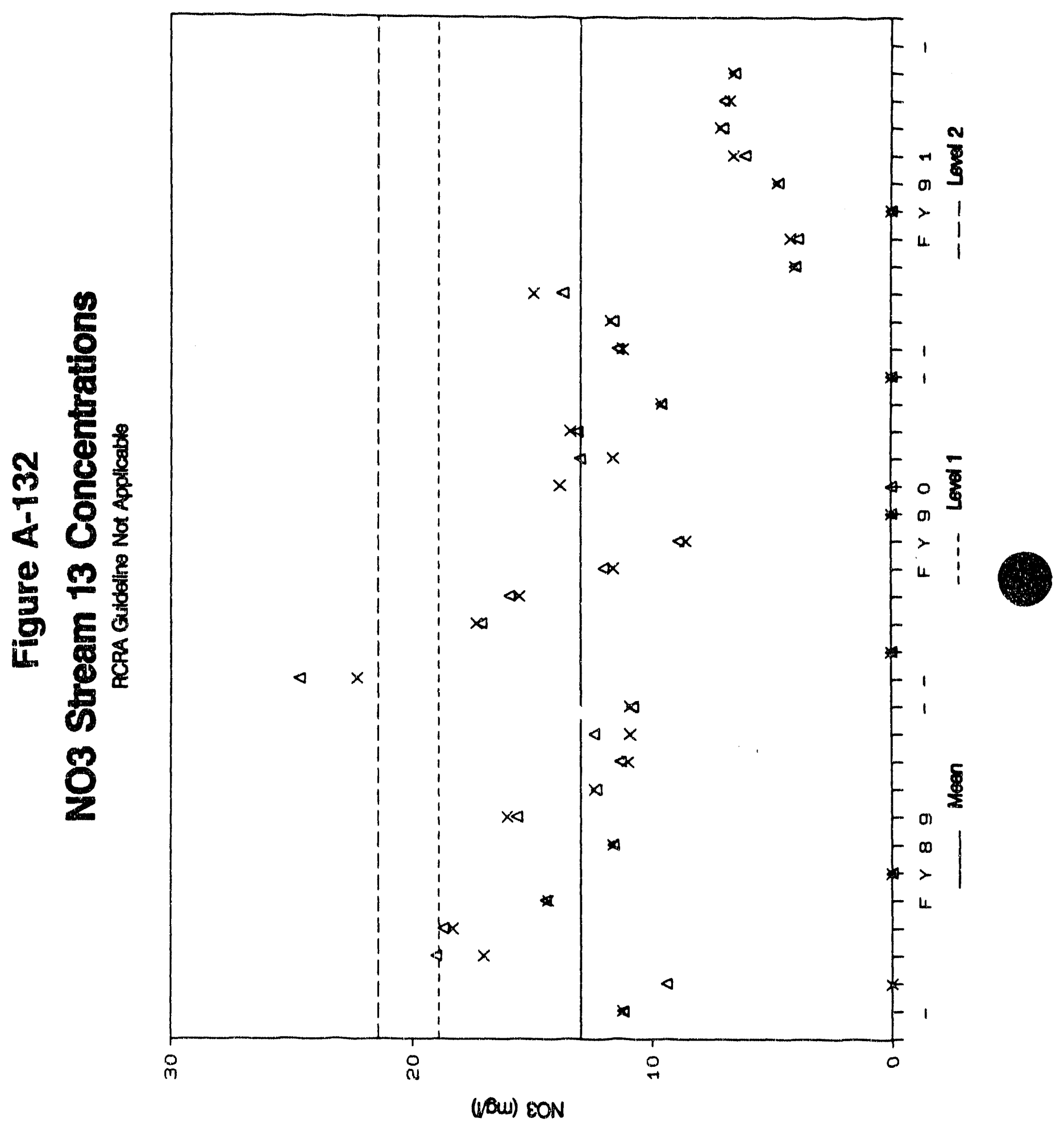




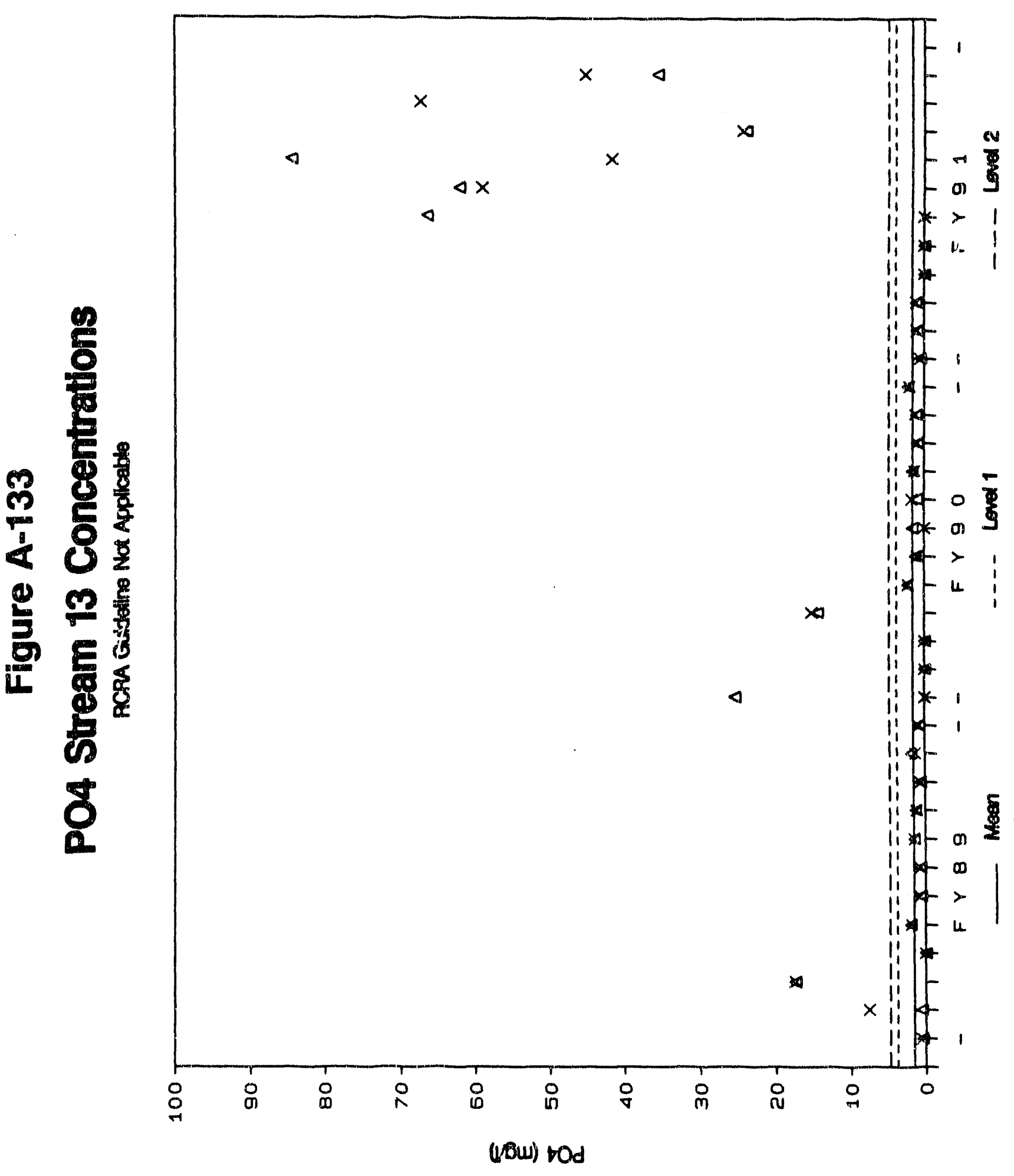




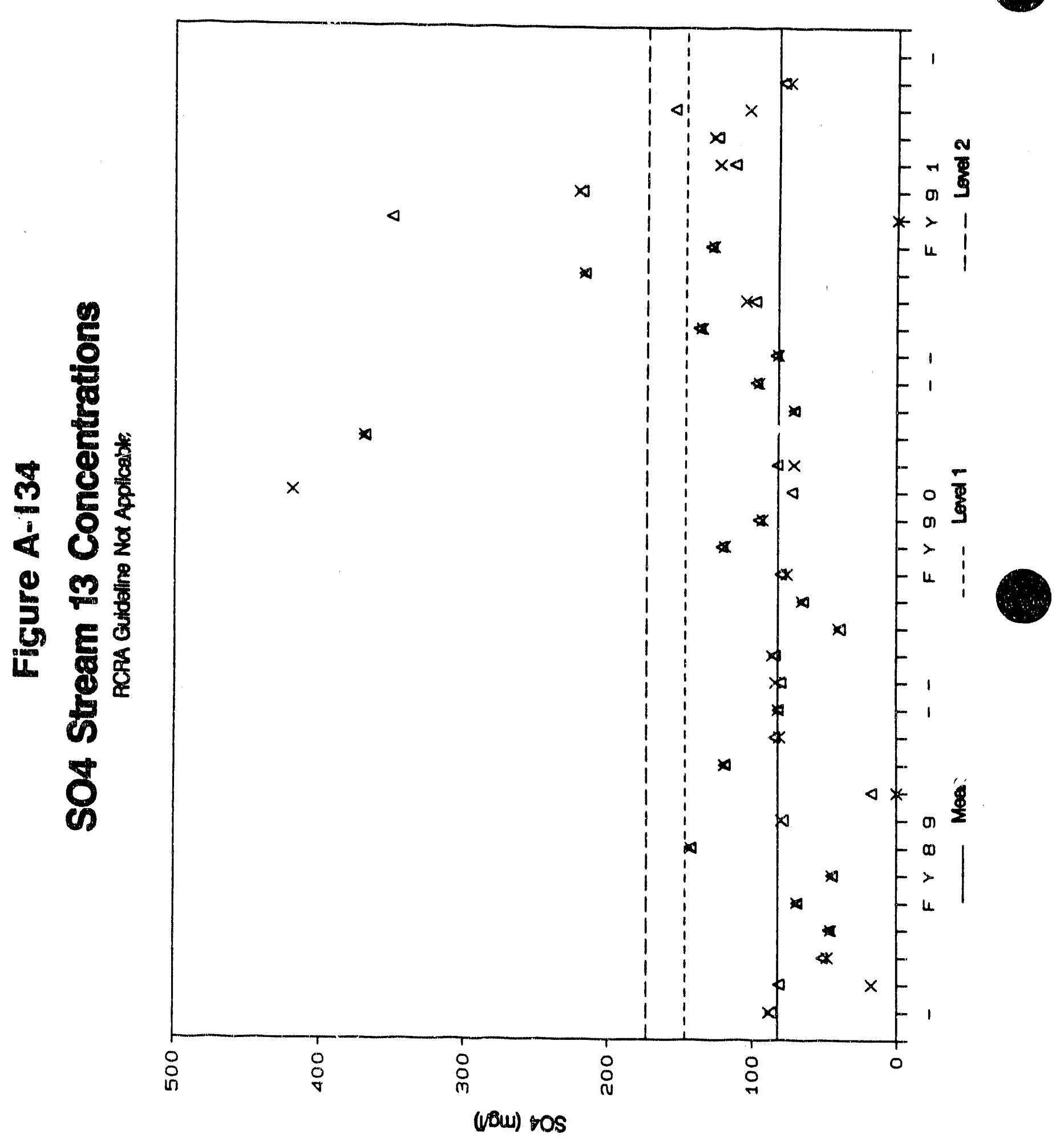

A- 194 


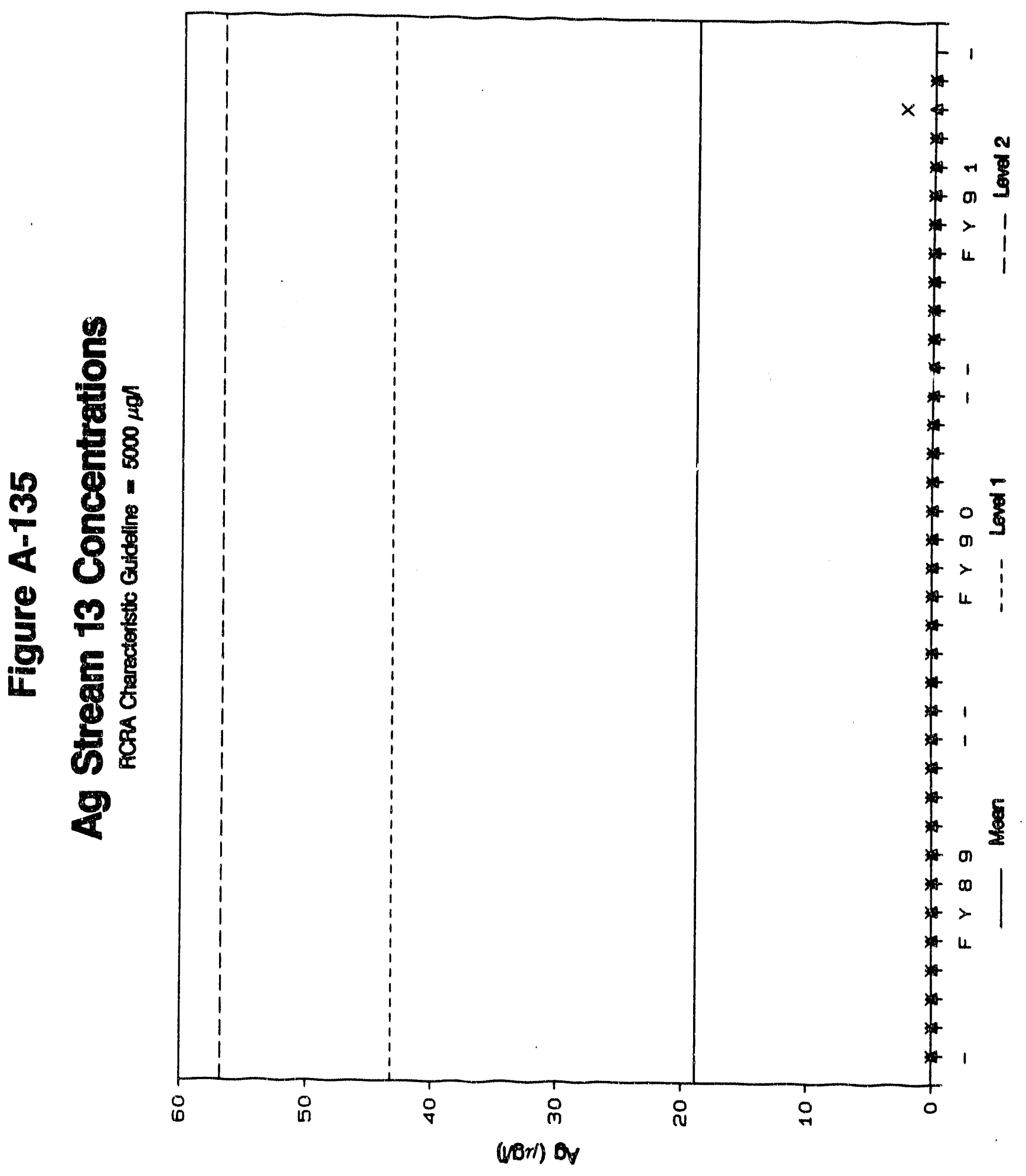




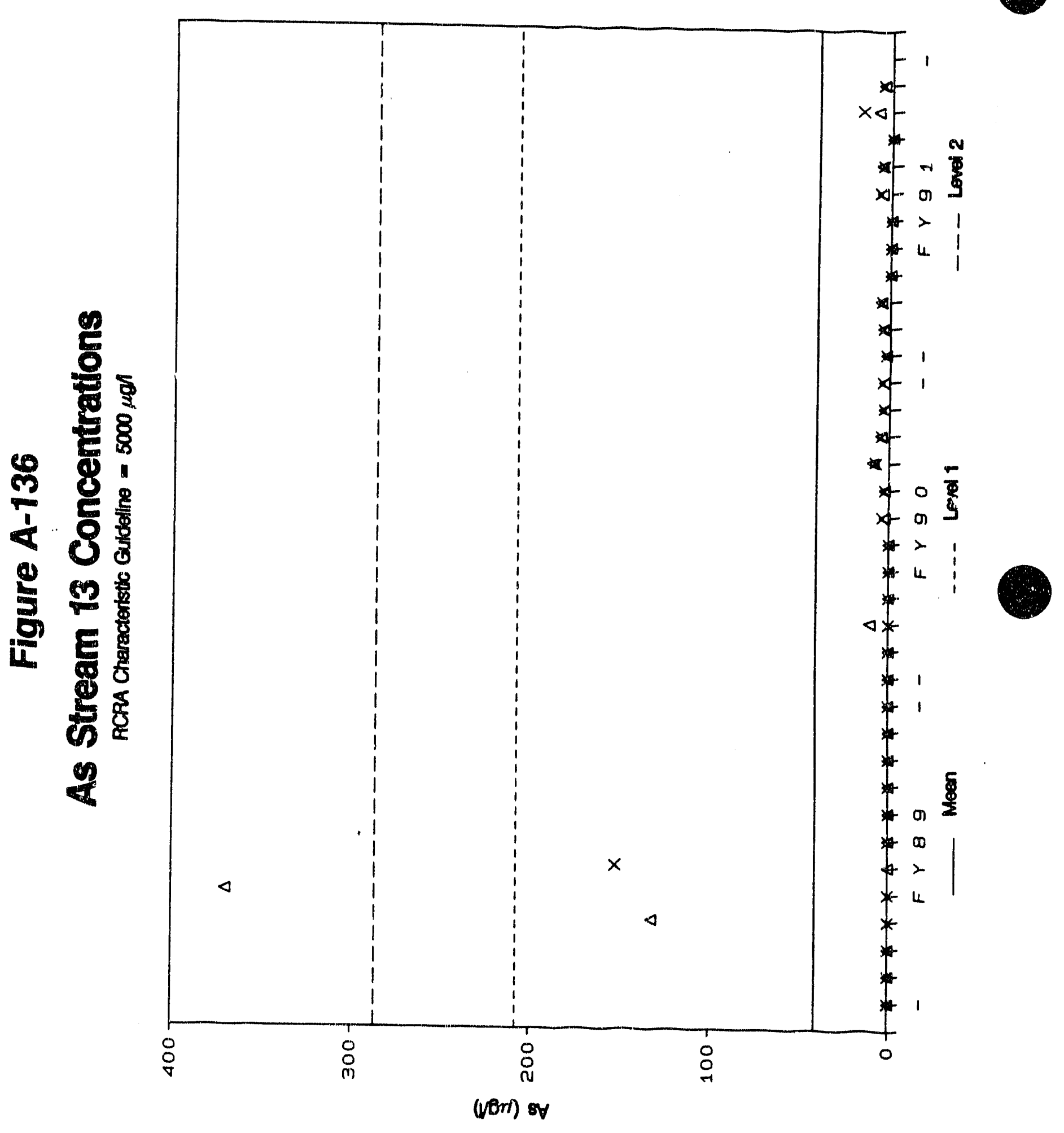




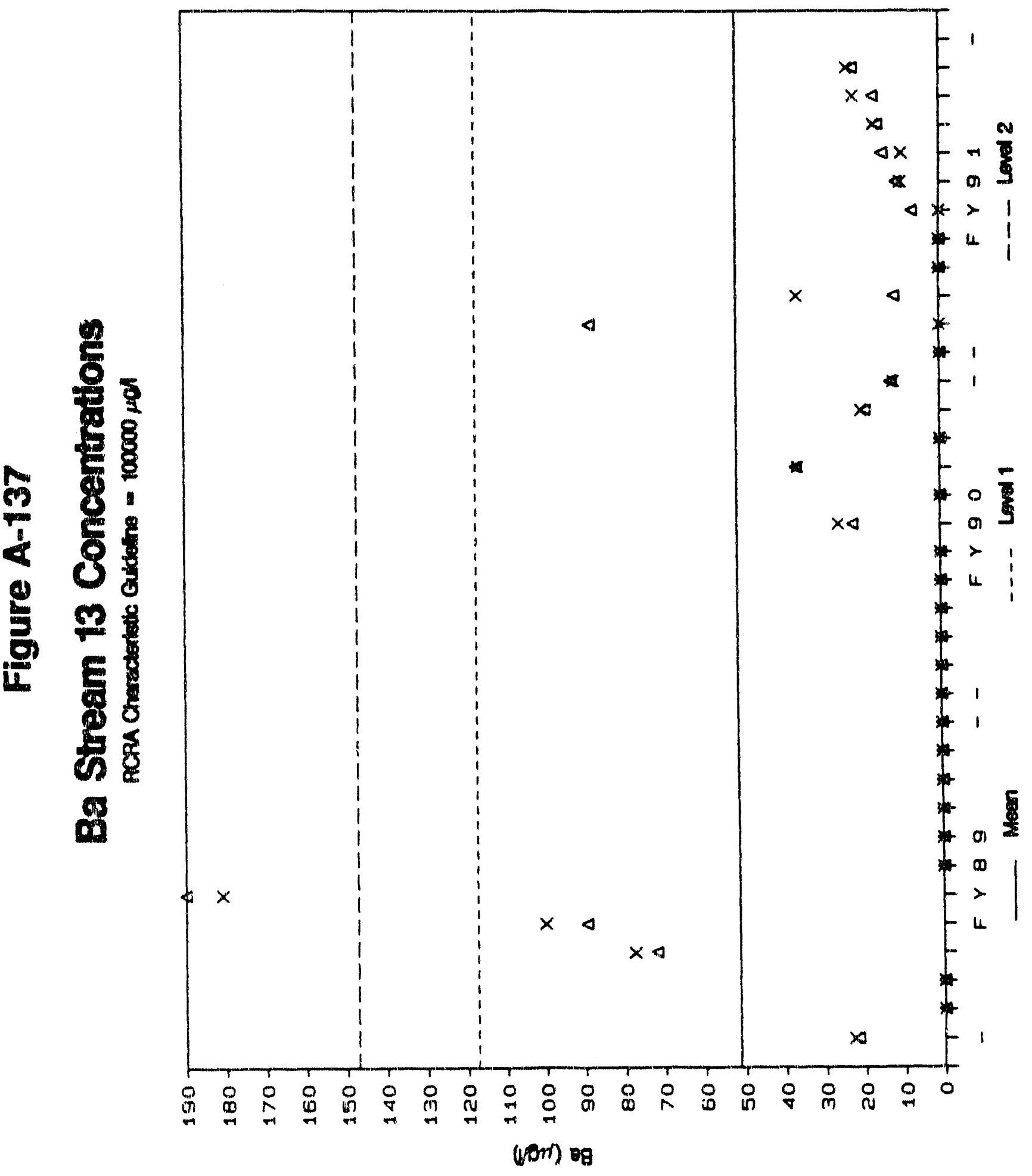




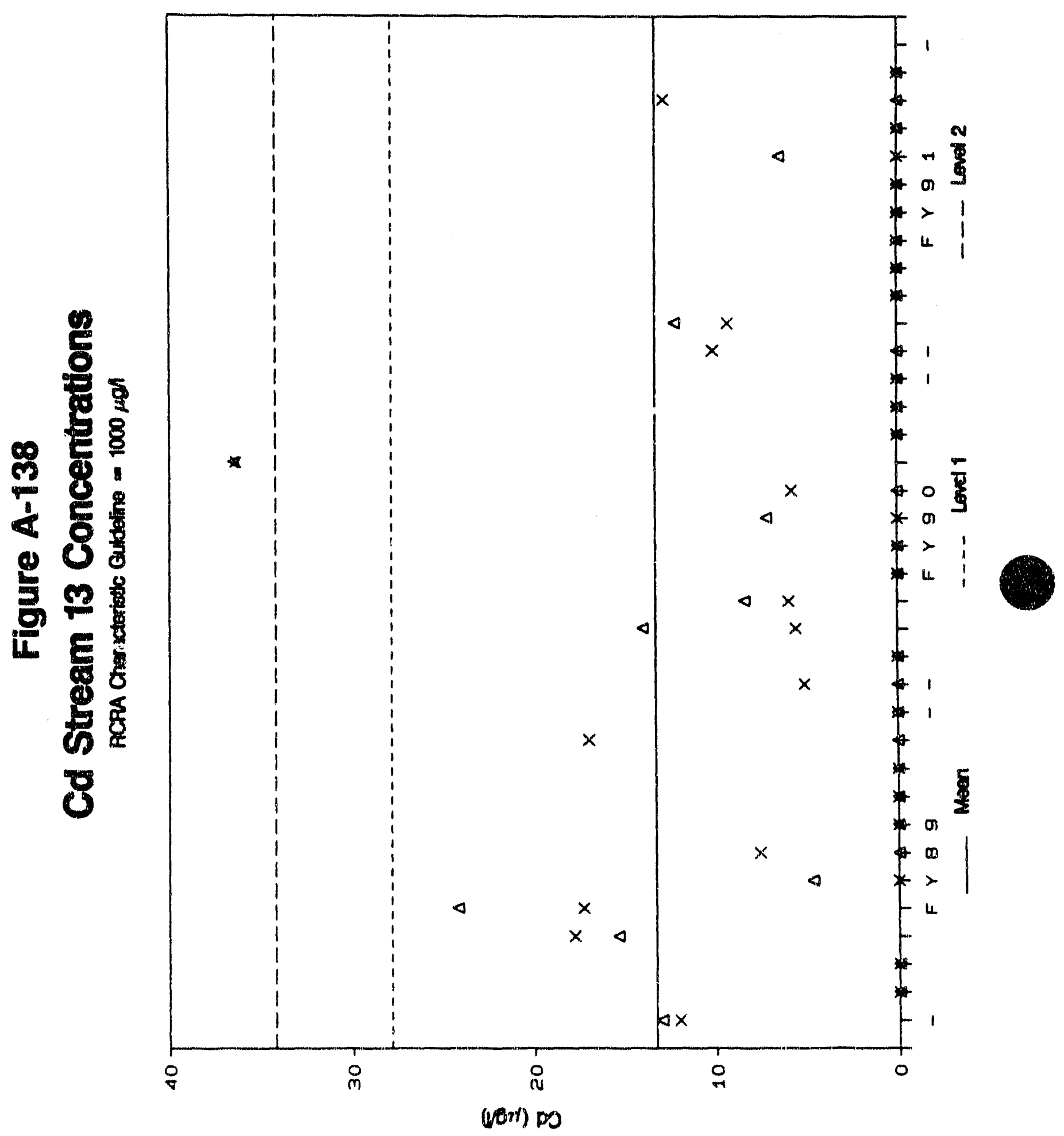




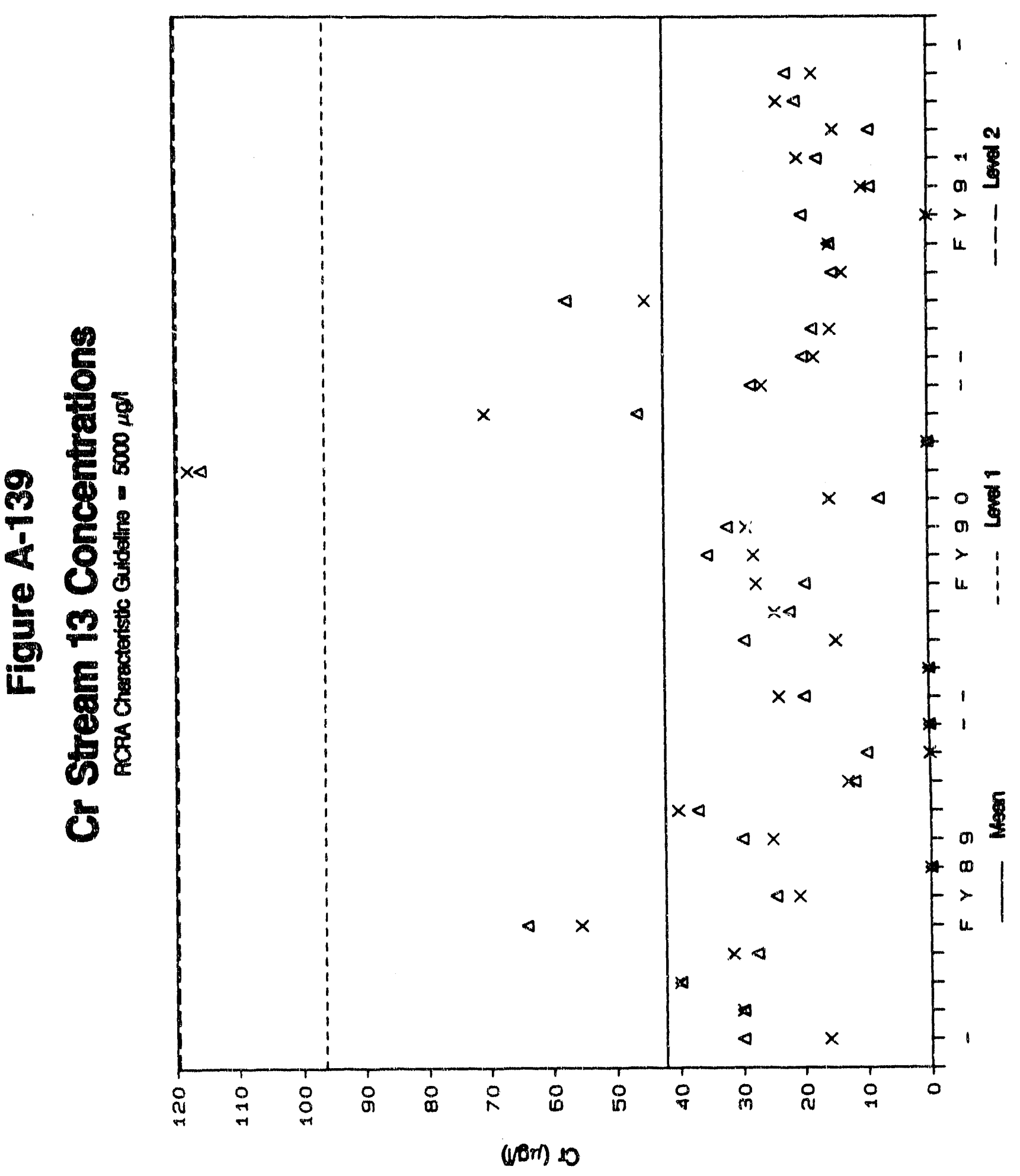




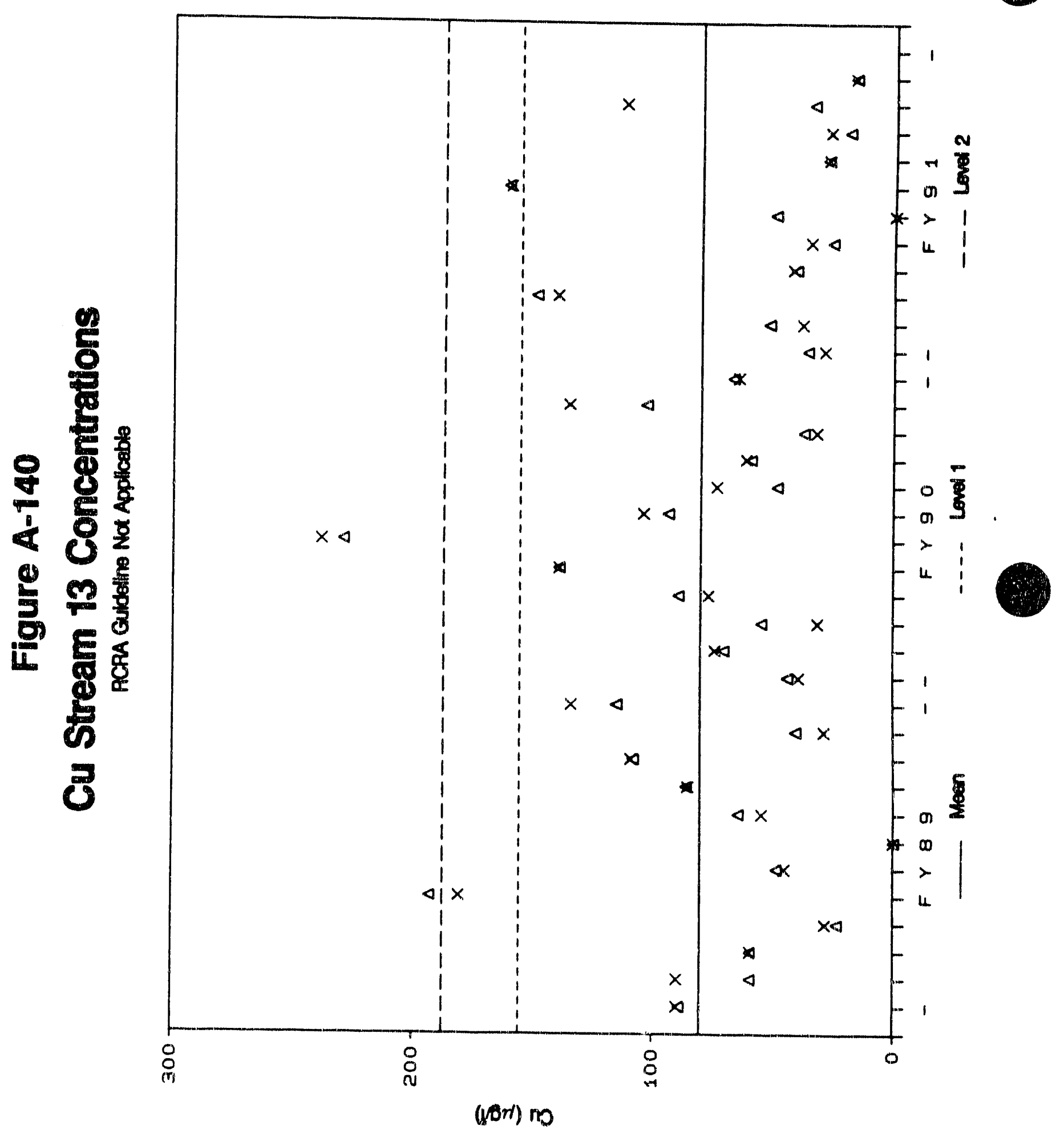




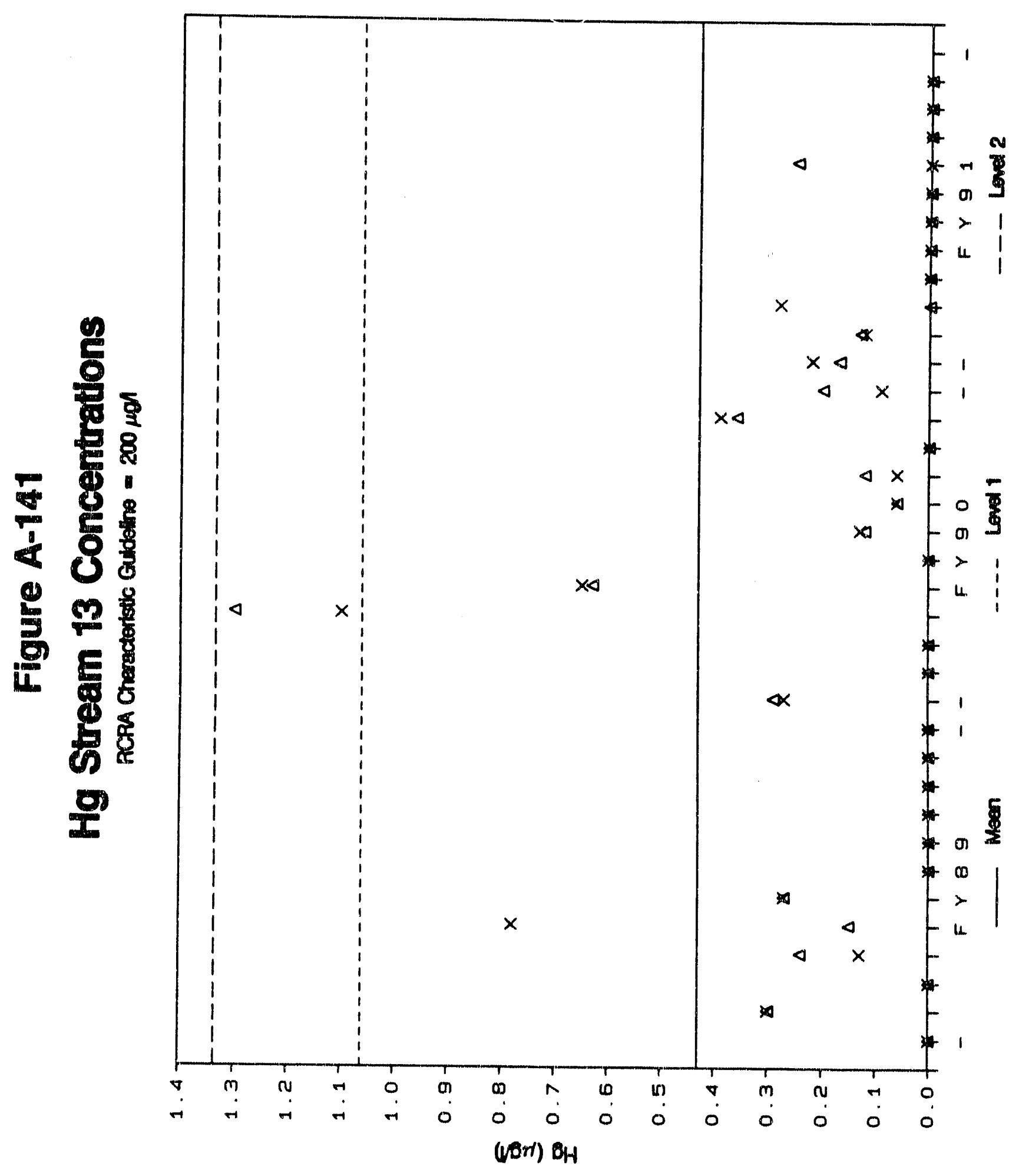




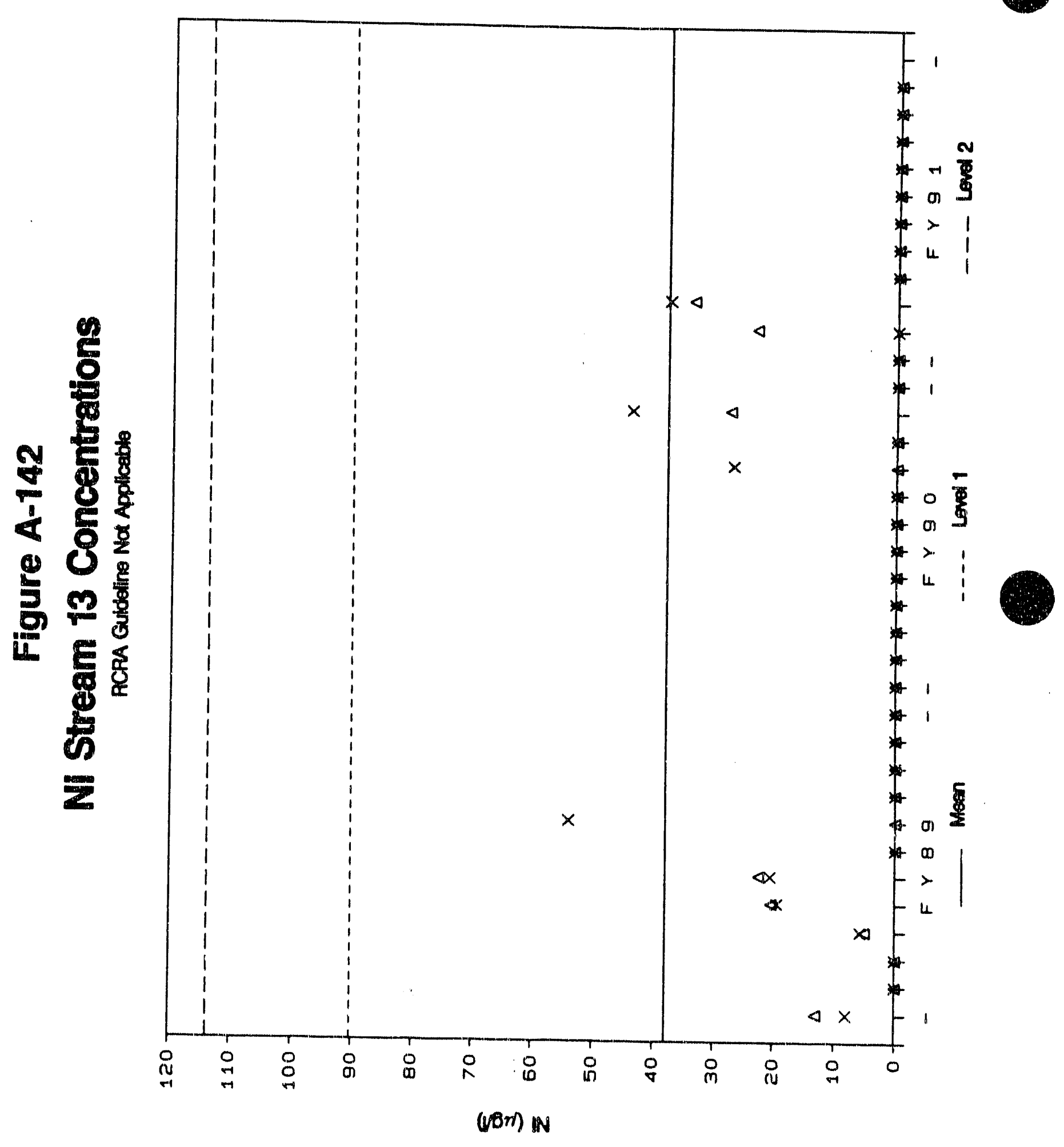




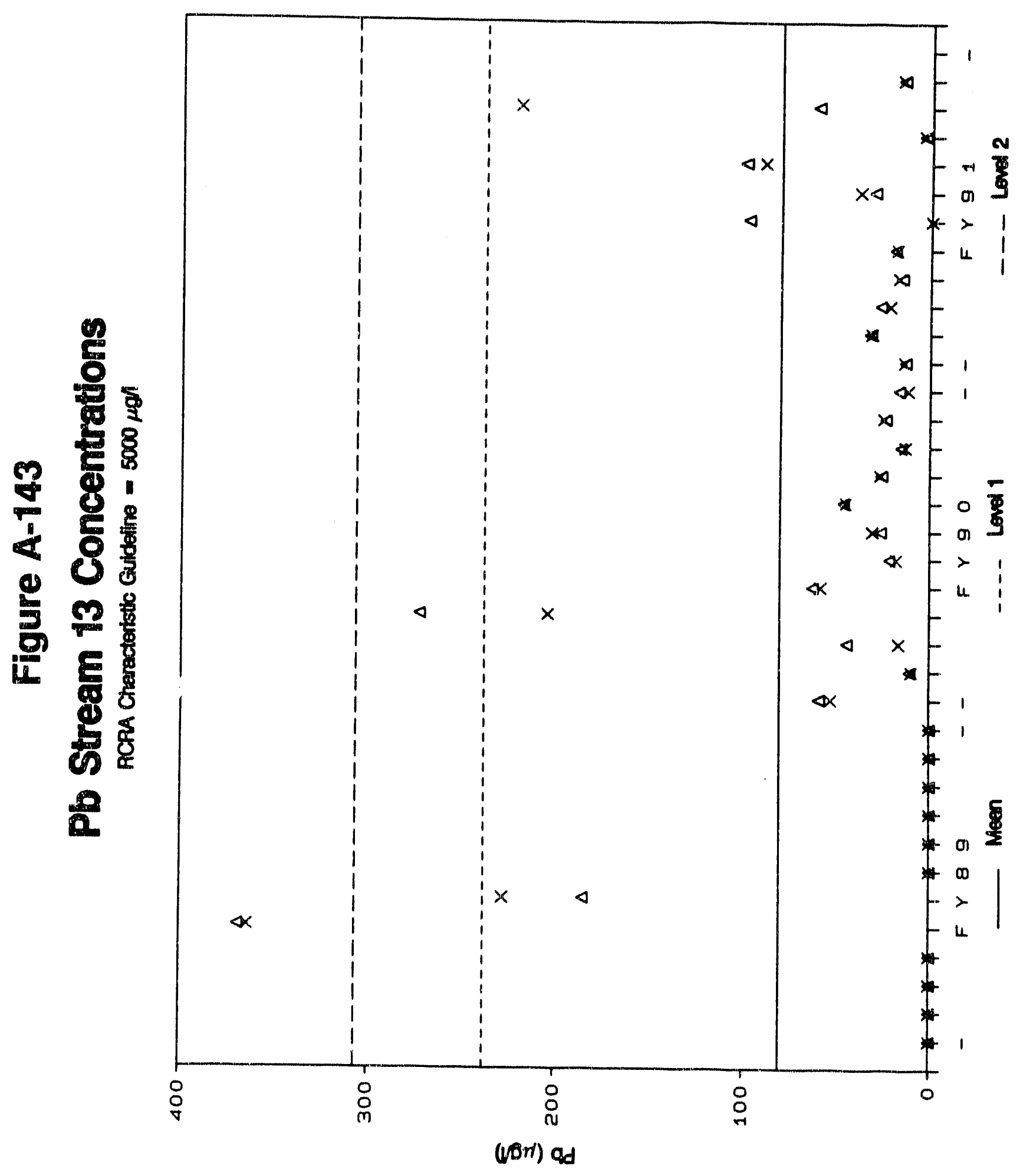




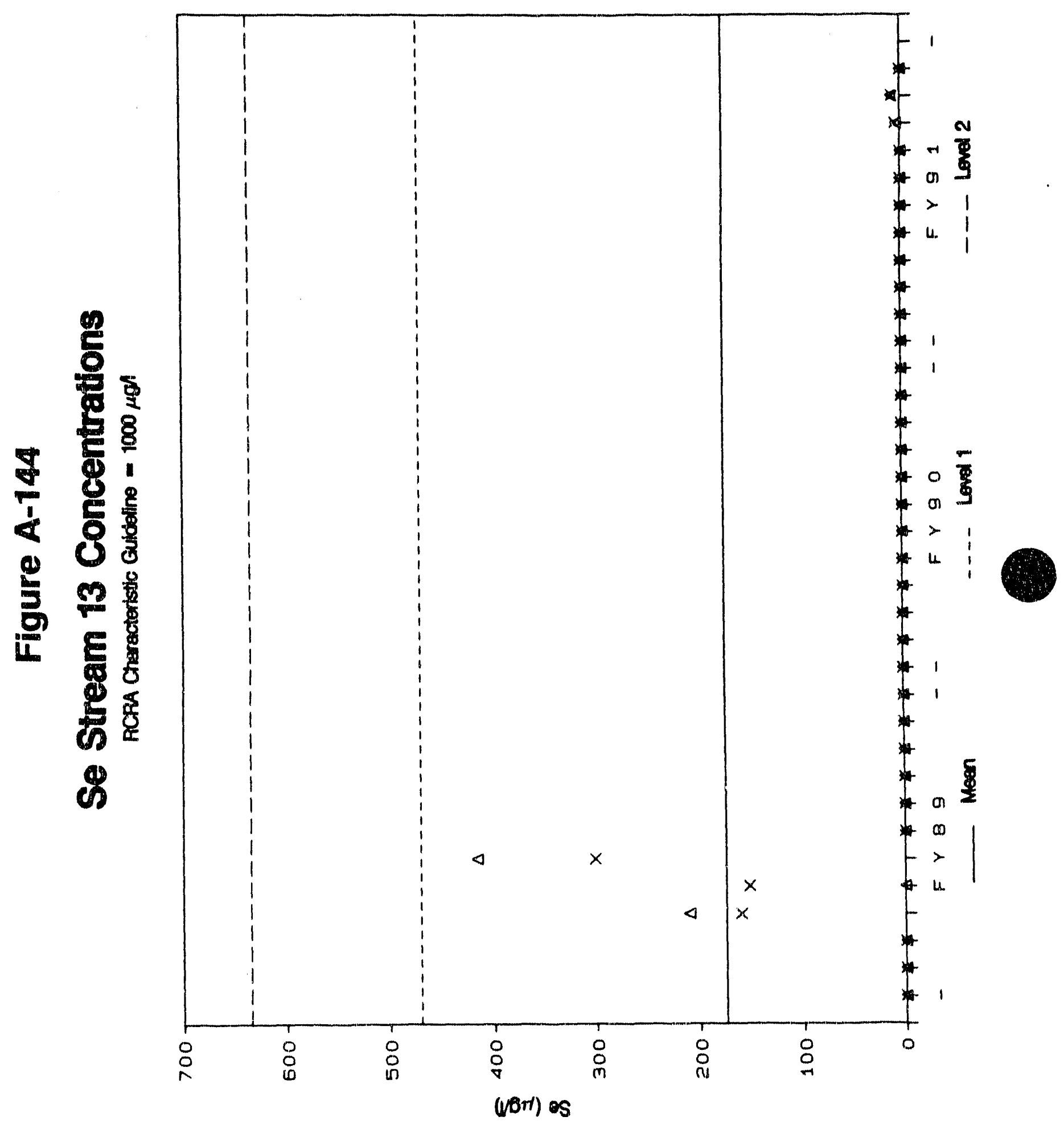




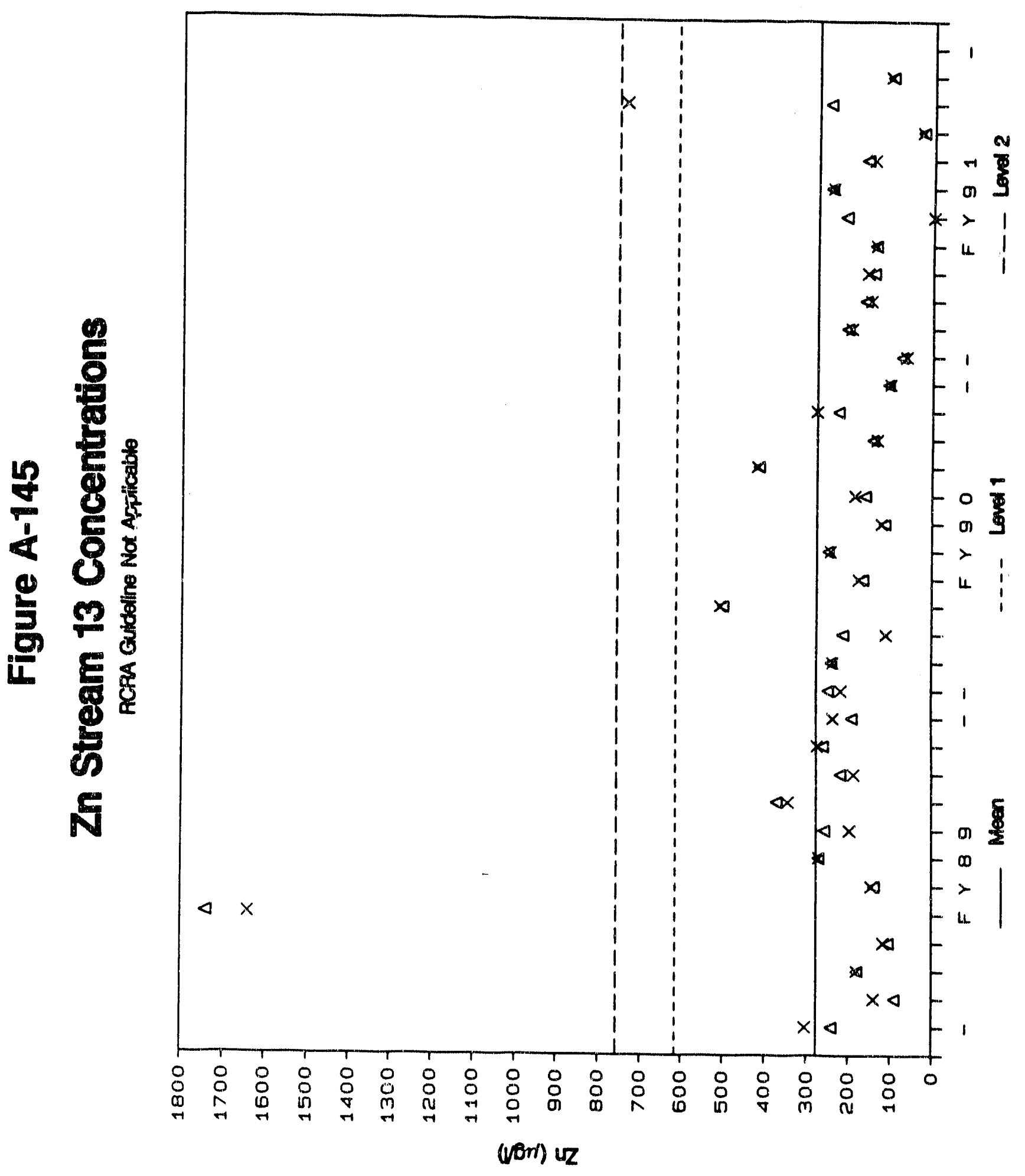




\section{Table A-41}

FY91 ENVIRONMENTAL MONITORING DATA

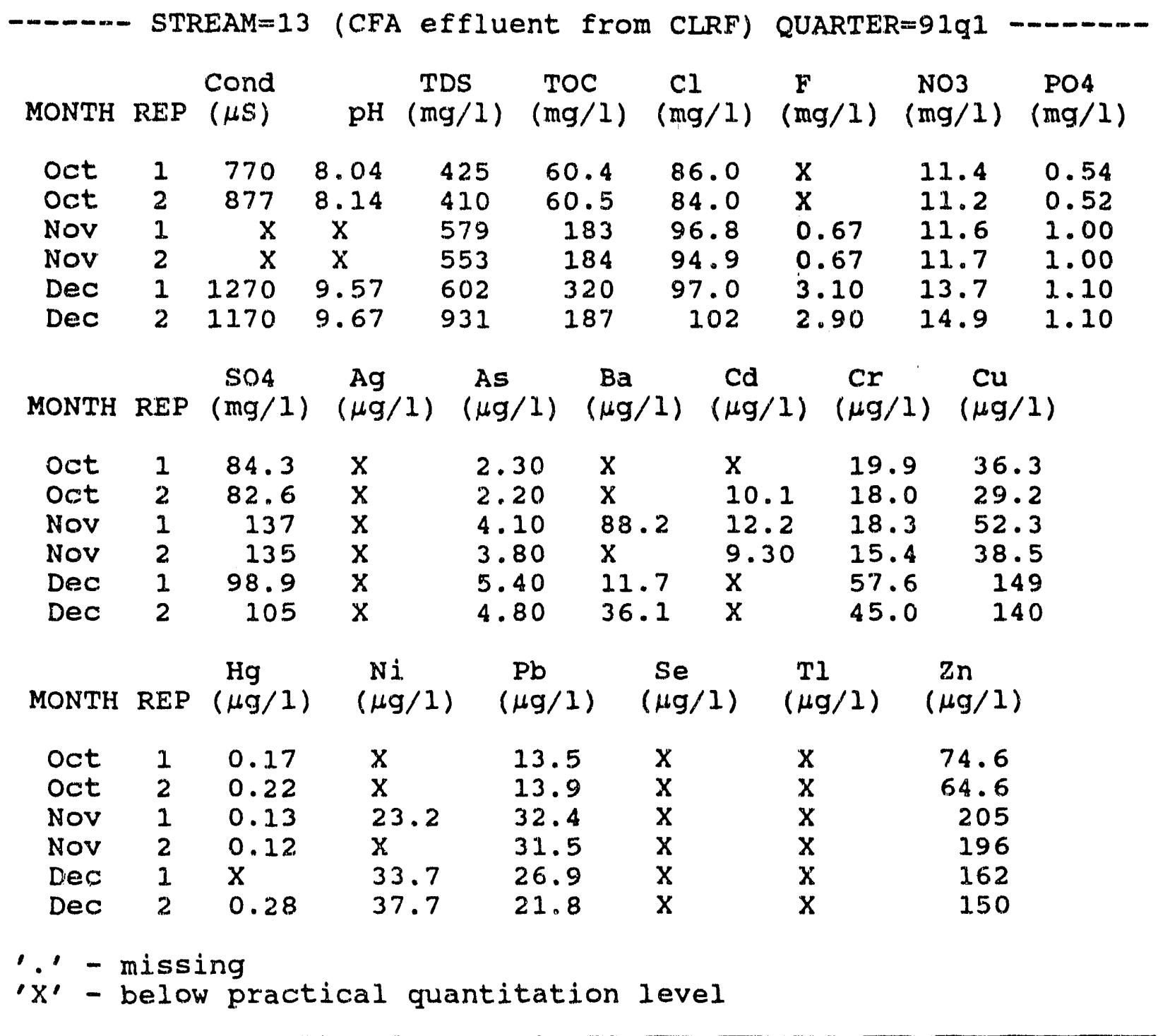




\section{Table A-42}

FY91 ENVIRONMENTAL MONITORING DATA

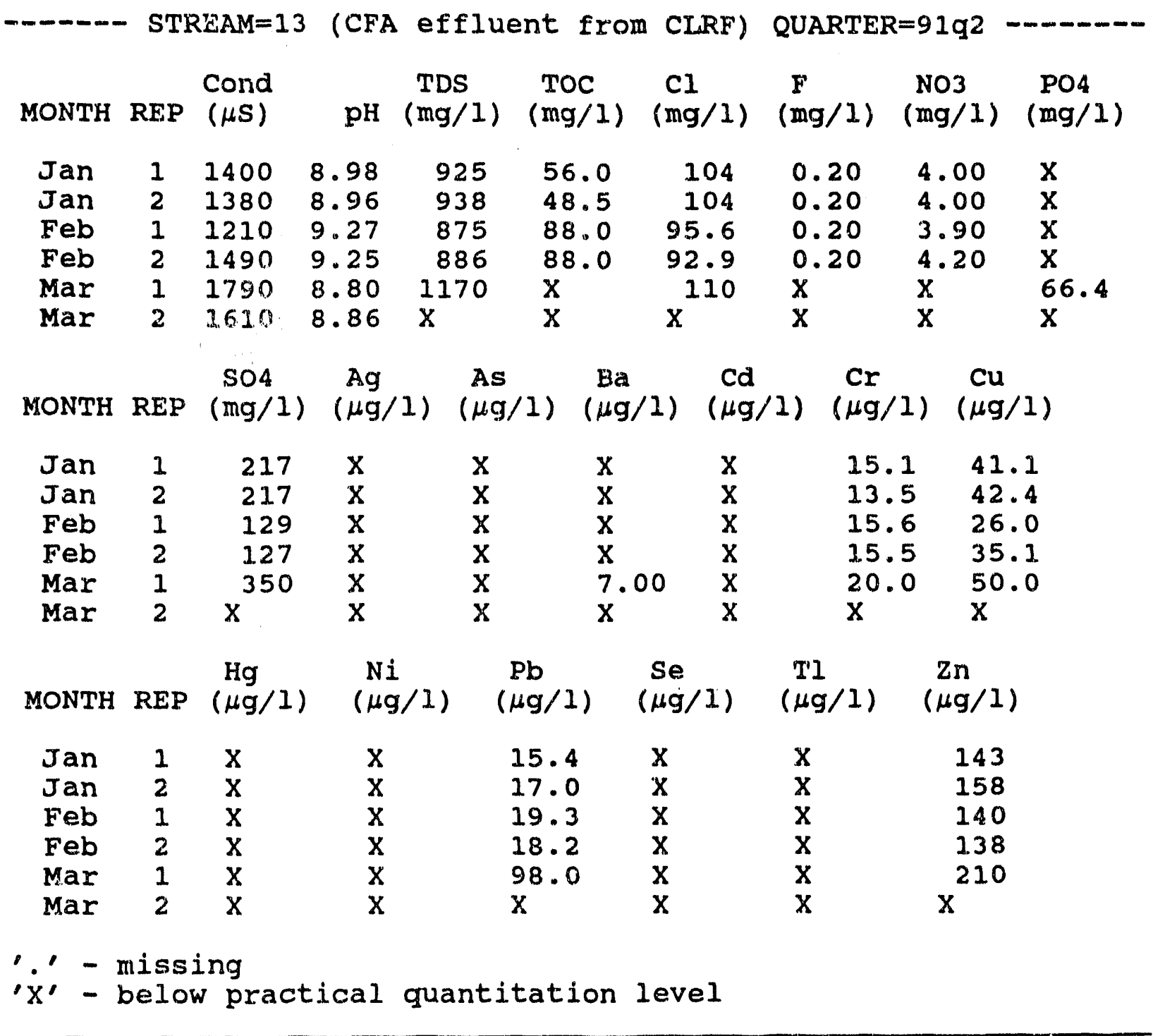




\section{Table A-43}

FY91 ENVIRONMENTAL MONITORING DATA

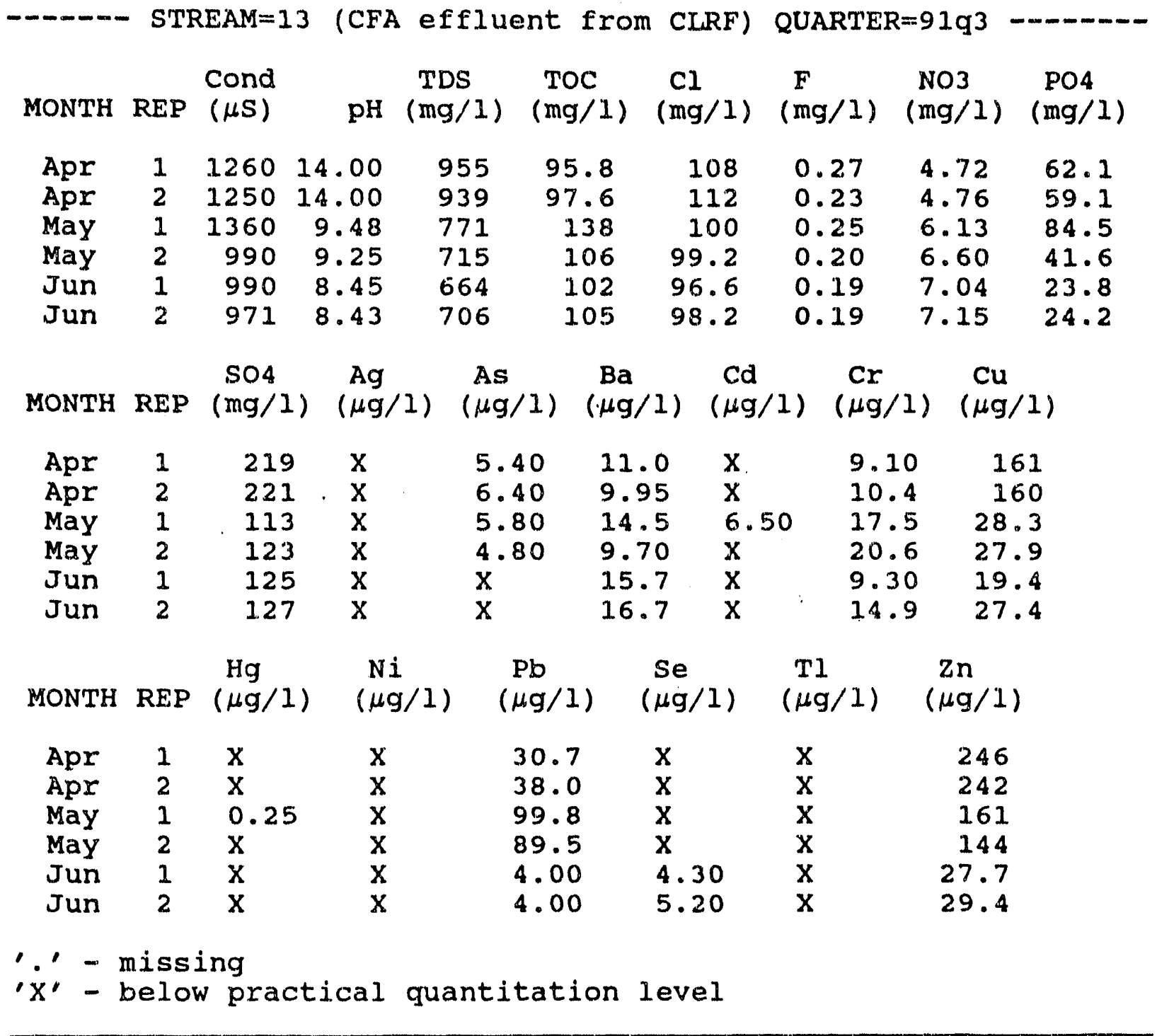




\section{Table A-44}

FY91 ENVIRONMENTAL MONITORING DATA

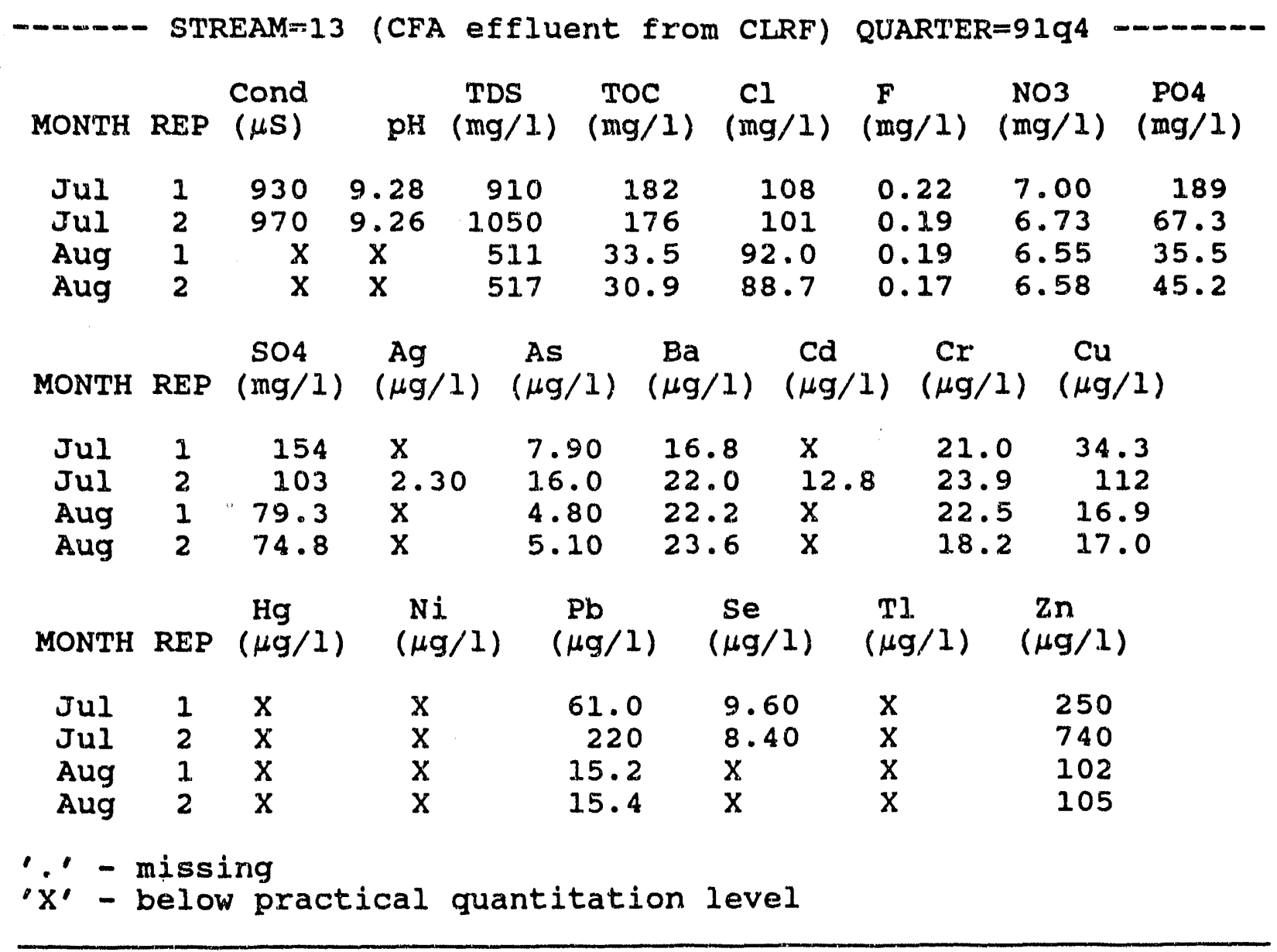




\section{Table A-45}

SUMMARY STATISTICS

STREAM=13 (CFA effluent from CLRF)

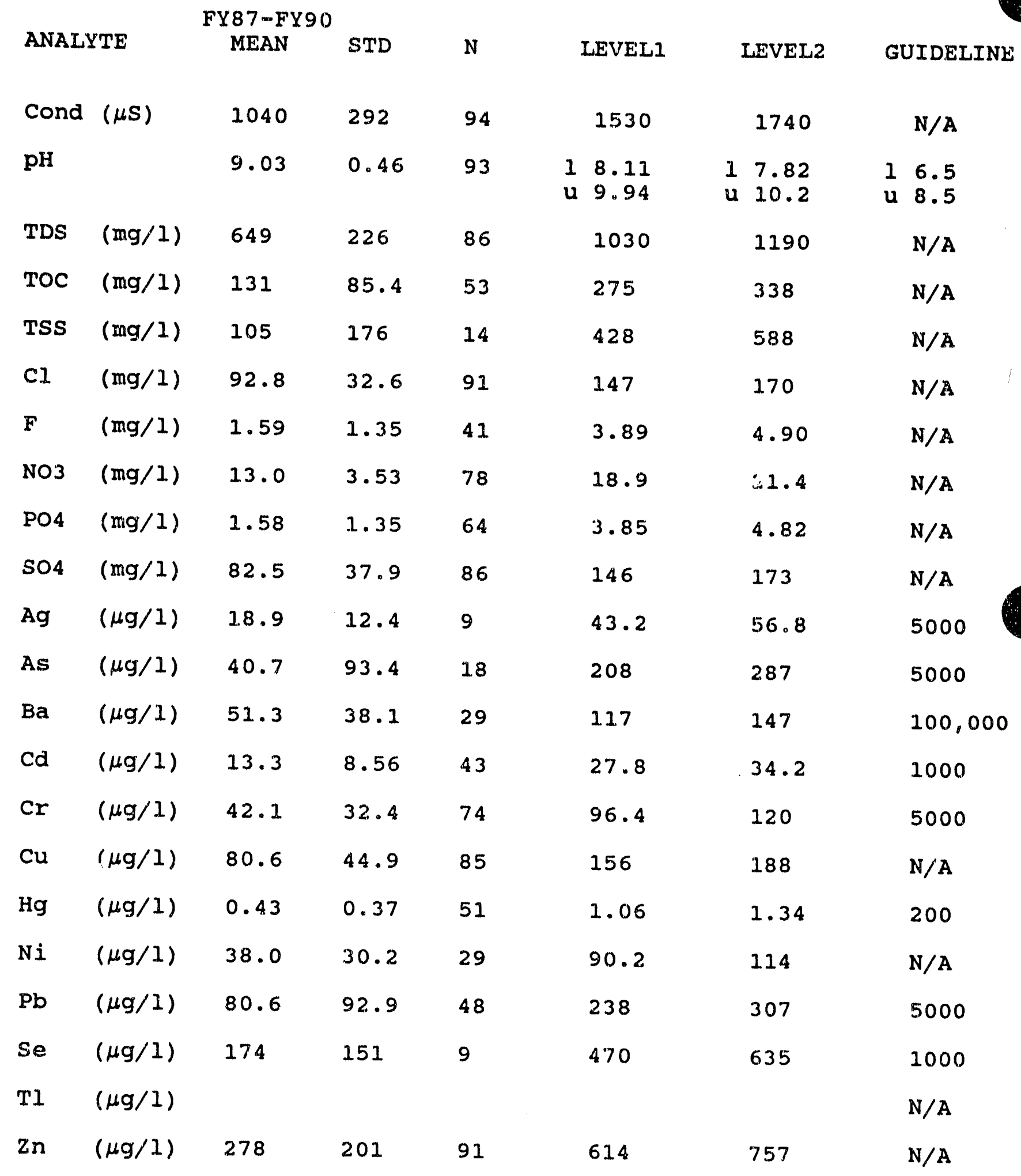

Guideline - RCRA Characteristic Guidelines N/A - not applicable 


\section{Table A-46}

QUARTERLY MEANS AND VARIANCE COMPONENTS

\section{STREAM $=13$ (CFA effluent from CLRF)}

\begin{tabular}{|c|c|c|c|c|c|c|c|c|c|}
\hline \multicolumn{2}{|c|}{ ANALYTE } & \multirow{2}{*}{$\begin{array}{l}\text { QTR } \\
91 q 1 \\
91 q 2 \\
91 q^{3} \\
91 q^{4}\end{array}$} & \multirow{2}{*}{$\begin{array}{r}\text { MEAN } \\
1022 \\
1480 \\
1137 \\
950\end{array}$} & \multirow{2}{*}{$\begin{array}{r}\text { STD } \\
\text { MEAN } \\
\\
198 \\
111 \\
81.5 \\
\text { - }\end{array}$} & \multirow{2}{*}{$\begin{array}{l}\text { STD } \\
\text { DAY } \\
\\
276 \\
166 \\
92.1 \\
.\end{array}$} & \multirow{2}{*}{$\begin{array}{l}\text { STD } \\
\text { REP } \\
73.2 \\
136 \\
151 \\
.\end{array}$} & \multirow{2}{*}{$\begin{array}{l}N \\
4 \\
6 \\
6 \\
2\end{array}$} & \multirow{2}{*}{$\begin{array}{c}\quad \text { N } \\
\text { MISS } \\
2 \\
0 \\
0 \\
0\end{array}$} & \multirow{2}{*}{$\begin{array}{c}\text { N } \\
\text { BPQI } \\
0 \\
0 \\
0 \\
4\end{array}$} \\
\hline cond & $(\mu s)$ & & & & & & & & \\
\hline $\mathrm{pH}$ & & $\begin{array}{l}91 q 1 \\
9192 \\
9193 \\
9194\end{array}$ & $\begin{array}{l}8.86 \\
9.02 \\
10.6 \\
9.27\end{array}$ & $\begin{array}{l}0.77 \\
0.13 \\
1.72 \\
.\end{array}$ & $\begin{array}{l}1.08 \\
0.22 \\
2.98 \\
.\end{array}$ & $\begin{array}{l}0.07 \\
0.03 \\
0.09 \\
.\end{array}$ & $\begin{array}{l}4 \\
6 \\
6 \\
2\end{array}$ & $\begin{array}{l}2 \\
0 \\
0 \\
0\end{array}$ & $\begin{array}{l}0 \\
0 \\
0 \\
4\end{array}$ \\
\hline TDS & $(\mathrm{mg} / \mathrm{l})$ & $\begin{array}{l}91 q 1 \\
91 q 2 \\
91 q 3 \\
91 q 4\end{array}$ & $\begin{array}{l}583 \\
959 \\
792 \\
747\end{array}$ & $\begin{array}{r}101 \\
81.0 \\
79.5 \\
233\end{array}$ & $\begin{array}{l}147 \\
135 \\
136 \\
326\end{array}$ & $\begin{array}{r}135 \\
8.51 \\
29.3 \\
70.1\end{array}$ & $\begin{array}{l}6 \\
b \\
6 \\
4\end{array}$ & $\begin{array}{l}0 \\
1 \\
0 \\
0\end{array}$ & $\begin{array}{l}0 \\
0 \\
0 \\
0\end{array}$ \\
\hline TOC & $(\mathrm{mg} / \mathrm{l})$ & $\begin{array}{l}91 q 1 \\
91 q 2 \\
91 q 3 \\
91 q 4\end{array}$ & $\begin{array}{r}166 \\
70.1 \\
107 \\
106\end{array}$ & $\begin{array}{l}56.4 \\
17.9 \\
7.56 \\
73.4\end{array}$ & $\begin{array}{r}89.9 \\
25.1 \\
9.22 \\
104\end{array}$ & $\begin{array}{l}54.3 \\
3.75 \\
13.1 \\
3.27\end{array}$ & $\begin{array}{l}6 \\
4 \\
6 \\
4\end{array}$ & $\begin{array}{l}0 \\
2 \\
0 \\
0\end{array}$ & $\begin{array}{l}0 \\
0 \\
0 \\
0\end{array}$ \\
\hline Tss & $(m g / 1)$ & $\begin{array}{l}91 q 1 \\
91 q 2 \\
91 q^{3} \\
91 q 4\end{array}$ & $\begin{array}{l}26.9 \\
27.6 \\
22.2 \\
19.8\end{array}$ & $\begin{array}{l}2.37 \\
7.67 \\
7.60 \\
3.75\end{array}$ & $\begin{array}{l}3.34 \\
11.9 \\
12.0 \\
3.67\end{array}$ & $\begin{array}{l}0.56 \\
6.10 \\
7.76 \\
5.41\end{array}$ & $\begin{array}{l}4 \\
5 \\
6 \\
4\end{array}$ & $\begin{array}{l}2 \\
1 \\
0 \\
0\end{array}$ & $\begin{array}{l}0 \\
0 \\
0 \\
0\end{array}$ \\
\hline Cl & $(\mathrm{mg} / \mathrm{l})$ & $\begin{array}{l}91 q 1 \\
9192 \\
9193 \\
9194\end{array}$ & $\begin{array}{r}93.5 \\
101 \\
102 \\
97.4\end{array}$ & $\begin{array}{l}4.35 \\
4.61 \\
3.89 \\
7.08\end{array}$ & $\begin{array}{l}7.36 \\
7.62 \\
6.61 \\
9.52\end{array}$ & $\begin{array}{l}2.33 \\
1.35 \\
1.79 \\
3.87\end{array}$ & $\begin{array}{l}6 \\
5 \\
6 \\
4\end{array}$ & $\begin{array}{l}0 \\
1 \\
0 \\
0\end{array}$ & $\begin{array}{l}0 \\
0 \\
0 \\
0\end{array}$ \\
\hline$F^{\prime}$ & $(\mathrm{mg} / \mathrm{l})$ & $\begin{array}{l}91 q 1 \\
91 q 2 \\
91 q^{3} \\
91 q^{4}\end{array}$ & $\begin{array}{l}1.83 \\
0.06 \\
0.22 \\
0.19\end{array}$ & $\begin{array}{l}1.16 \\
0.21 \\
0.02 \\
0.01\end{array}$ & $\begin{array}{l}1.65 \\
0.35 \\
0.02 \\
0.01\end{array}$ & $\begin{array}{l}0.10 \\
0.00 \\
0.03 \\
0.02\end{array}$ & $\begin{array}{l}4 \\
5 \\
6 \\
4\end{array}$ & $\begin{array}{l}0 \\
1 \\
0 \\
0\end{array}$ & $\begin{array}{l}2 \\
0 \\
0 \\
0\end{array}$ \\
\hline NO3 & $(\mathrm{mg} / \mathrm{I})$ & $\begin{array}{l}91 q] . \\
91 q 2 \\
91 q 3 \\
91 q^{4}\end{array}$ & $\begin{array}{l}12.4 \\
4.03 \\
6.07 \\
6.71\end{array}$ & $\begin{array}{l}0.95 \\
0.03 \\
0.70 \\
0.15\end{array}$ & $\begin{array}{l}1.60 \\
0.00 \\
1.20 \\
0.19\end{array}$ & $\begin{array}{l}0.50 \\
0.15 \\
0.20 \\
0.13\end{array}$ & $\begin{array}{l}5 \\
4 \\
6 \\
4\end{array}$ & $\begin{array}{l}0 \\
2 \\
0 \\
0\end{array}$ & $\begin{array}{l}0 \\
0 \\
0 \\
0\end{array}$ \\
\hline PO4 & $(m g / 1)$ & $\begin{array}{l}91 q 1 \\
91 q 2 \\
91 q^{3} \\
91 q^{4}\end{array}$ & $\begin{array}{l}0.88 \\
66.4 \\
49.2 \\
84.3\end{array}$ & $\begin{array}{l}0.18 \\
12.6 \\
43.9\end{array}$ & $\begin{array}{l}0.30 \\
18.0 \\
44.6\end{array}$ & $\begin{array}{l}0.01 \\
17.5 \\
61.1\end{array}$ & $\begin{array}{l}6 \\
1 \\
6 \\
4\end{array}$ & $\begin{array}{l}0 \\
0 \\
0 \\
0\end{array}$ & $\begin{array}{l}0 \\
5 \\
0 \\
0\end{array}$ \\
\hline SO4 & $(\mathrm{mg} / 1)$ & $\begin{array}{l}91 q 1 \\
91 q 2 \\
9193 \\
91 \mathrm{g4}\end{array}$ & $\begin{array}{l}107 \\
208 \\
155 \\
103\end{array}$ & $\begin{array}{l}15.4 \\
61.0 \\
32.7 \\
25.7\end{array}$ & $\begin{array}{r}26.6 \\
102 \\
56.6 \\
31.6\end{array}$ & $\begin{array}{l}2.71 \\
1.00 \\
4.24 \\
25.6\end{array}$ & $\begin{array}{l}6 \\
5 \\
6 \\
4\end{array}$ & $\begin{array}{l}0 \\
1 \\
0 \\
0\end{array}$ & $\begin{array}{l}0 \\
0 \\
0 \\
0\end{array}$ \\
\hline
\end{tabular}


Table A-46 (continued)

QUARTERLY MEANS AND VARIANCE COMPONENTS

STREAM=13 (CFA effluent from CLRF)

(continued)

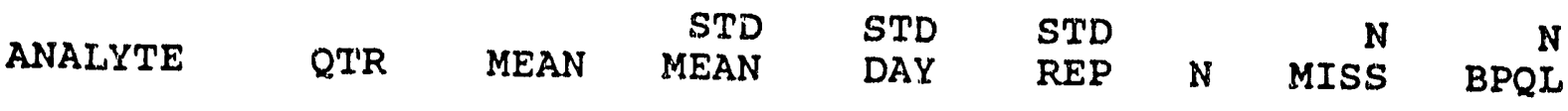

Ag $(\mu g / 1) \quad 91 q 1 \quad x$

$\begin{array}{llllllll}9191 & X & \cdot & : & : & 0 & 0 & 6 \\ 9192 & X & : & : & : & 0 & 0 & 6 \\ 9193 & X & : & : & : & 0 & 0 & 6 \\ 9194 & 2.30 & \cdot & : & : & 1 & 0 & 5\end{array}$

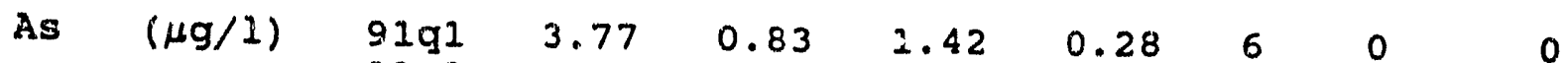

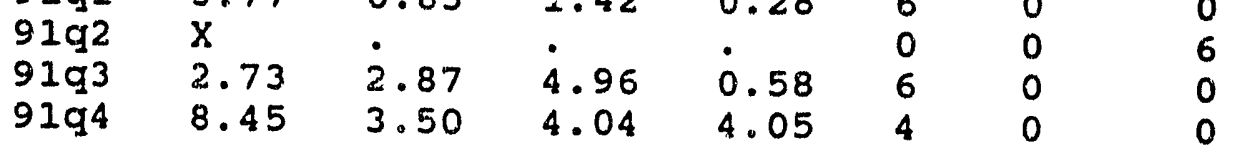

$\begin{array}{lllllllll}\mathrm{Ba} \quad(\mu g / 1) & 91 q 1 & 45.3 & . & . & . & 3 & 0 & 3 \\ & 91 q 2 & 7.00 & : & . & . & 1 & 0 & 5 \\ & 91 q^{3} & 12.9 & 1.70 & 2.57 & 2.05 & 6 & 0 & 0 \\ & 9194 & 21.1 & 1.75 & 1.58 & 2.69 & 4 & 0 & 0\end{array}$

Cd $(\mu g / 1) \quad 9 I q 1 \quad \ldots .5 \quad . \quad \begin{array}{lllllll}3 & 0 & 3\end{array}$

$9192 \quad . \quad . \quad 0 \quad 0 \quad 006$

$\begin{array}{lllllllllll}9193 & 6.50 & . & . & . & 1 & 0 & 5\end{array}$

Cr $(\mu \mathrm{g} / 1) \quad 9191 \quad 29.0 \quad 11.1 \quad 18.9 \quad 5.34$

\begin{tabular}{|c|c|c|c|c|c|c|}
\hline $\begin{array}{l}91 q 1 \\
91 q 2 \\
91 q 3 \\
91 q 4\end{array}$ & $\begin{array}{l}29.0 \\
15.9 \\
13.6 \\
21.4\end{array}$ & $\begin{array}{l}11.1 \\
1.57 \\
2.79 \\
1.05\end{array}$ & $\begin{array}{l}18.9 \\
2.55 \\
4.45 \\
0.00\end{array}$ & $\begin{array}{l}5.34 \\
0.80 \\
2.67 \\
2.59\end{array}$ & $\begin{array}{l}6 \\
5 \\
6 \\
4\end{array}$ & \\
\hline
\end{tabular}

$\begin{array}{llllllllll}\mathrm{Cu} & (\mathrm{Hg} / \mathrm{l}) & 91 \mathrm{q1} & 74.2 & 35.3 & 61.0 & 7.32 & 6 & 0 & 0\end{array}$

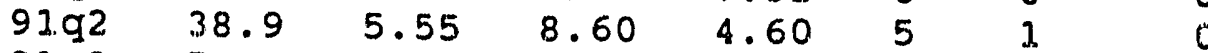

$\begin{array}{llllllll}9193 & 70.7 & 44.9 & 77.8 & 3.30 & 6 & 0 & 0\end{array}$

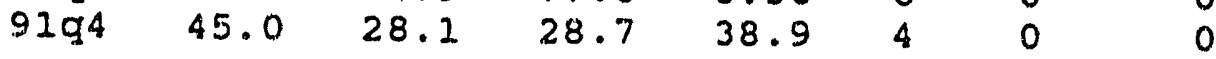

$\begin{array}{llllllllll}\mathrm{Hg}(\mu \mathrm{g} / 1) & 91 \mathrm{q}^{1} & 0.18 & 0.04 & 0.07 & 0.03 & 5 & 0 & \text { I }\end{array}$

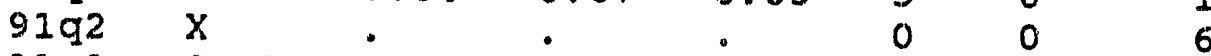

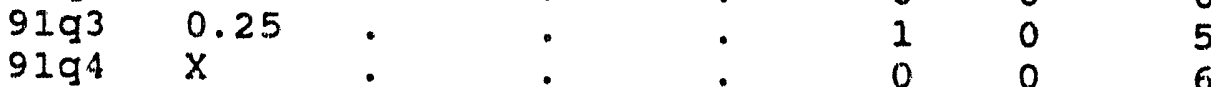

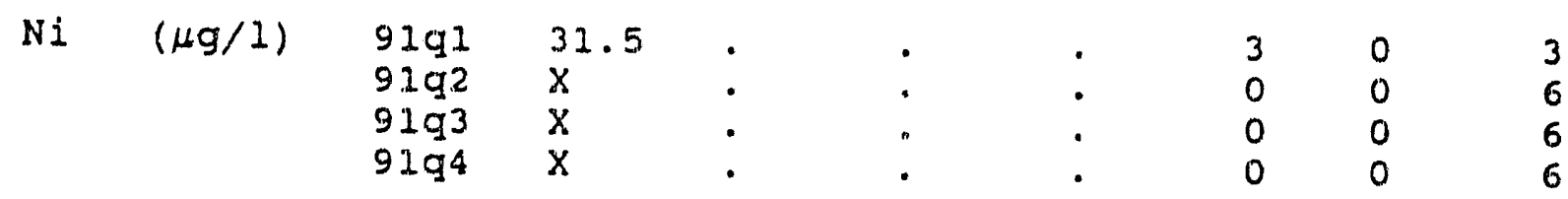

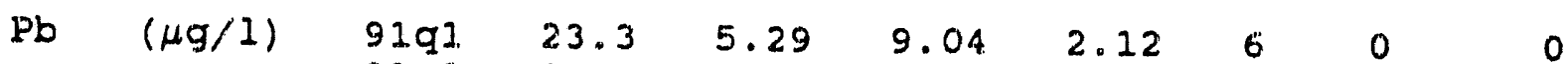

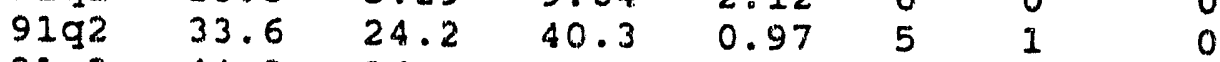

$\begin{array}{llllllll}9193 & 44.3 & 26.6 & 46.0 & 5.15 & 6 & 0 & 0 \\ 9194 & 77.9 & 62.6 & 68.4 & 79.5 & 4 & 0\end{array}$ 


\section{Table A-46 (continued)}

QUARTERLY MEANS AND VARIANCE COMPONENTS

\section{STREAM $=13$ (CFA effluent from CIRF)} (continued)

\begin{tabular}{|c|c|c|c|c|c|c|c|c|c|}
\hline \multicolumn{2}{|c|}{ ANALYTE } & Q'TR & MEAN & $\begin{array}{r}\text { STD } \\
\text { MEAN }\end{array}$ & $\begin{array}{l}\text { STD } \\
\text { DAX }\end{array}$ & $\begin{array}{l}\text { STD } \\
\text { REP }\end{array}$ & $\mathrm{N}$ & $\underset{\text { MISS }}{\mathrm{N}}$ & $\begin{array}{r}\mathrm{N} \\
\text { BPQL }\end{array}$ \\
\hline Se & $(\mu g / 1)$ & $\begin{array}{l}91 q 1 \\
9 I q 2 \\
91 q 3 \\
91 q 4\end{array}$ & $\begin{array}{l}X \\
X \\
4.75 \\
9.00\end{array}$ & $\dot{.}$ & $\begin{array}{l}\dot{\bullet} \\
\dot{\cdot}\end{array}$ & $\dot{:}$ & $\begin{array}{l}0 \\
0 \\
2 \\
2\end{array}$ & $\begin{array}{l}0 \\
0 \\
0 \\
0\end{array}$ & $\begin{array}{l}6 \\
6 \\
4 \\
4\end{array}$ \\
\hline TI & $(\mu \mathrm{g} / 1)$ & $\begin{array}{l}91 q 1 \\
91 q 2 \\
91 q 3 \\
91 q 4\end{array}$ & $\begin{array}{l}X \\
X \\
X \\
X\end{array}$ & $\dot{.}$ & $\begin{array}{l}\dot{\bullet} \\
\dot{\bullet}\end{array}$ & $\dot{.}$ & $\begin{array}{l}0 \\
0 \\
0 \\
0\end{array}$ & $\begin{array}{l}0 \\
0 \\
0 \\
0\end{array}$ & $\begin{array}{l}6 \\
6 \\
6 \\
6\end{array}$ \\
\hline $\mathrm{Zn}$ & $(\mu g / 1)$ & $\begin{array}{l}91 q 1 \\
91 q^{2} \\
91 q^{3} \\
91 q 4\end{array}$ & $\begin{array}{l}142 \\
158 \\
142 \\
299\end{array}$ & $\begin{array}{r}38.4 \\
19.9 \\
62.4 \\
196\end{array}$ & $\begin{array}{r}66.4 \\
32.7 \\
108 \\
216\end{array}$ & $\begin{array}{r}7.36 \\
7.57 \\
7.16 \\
245\end{array}$ & $\begin{array}{l}6 \\
5 \\
6 \\
4\end{array}$ & $\begin{array}{l}0 \\
1 \\
0 \\
0\end{array}$ & $\begin{array}{l}0 \\
0 \\
0 \\
0\end{array}$ \\
\hline
\end{tabular}




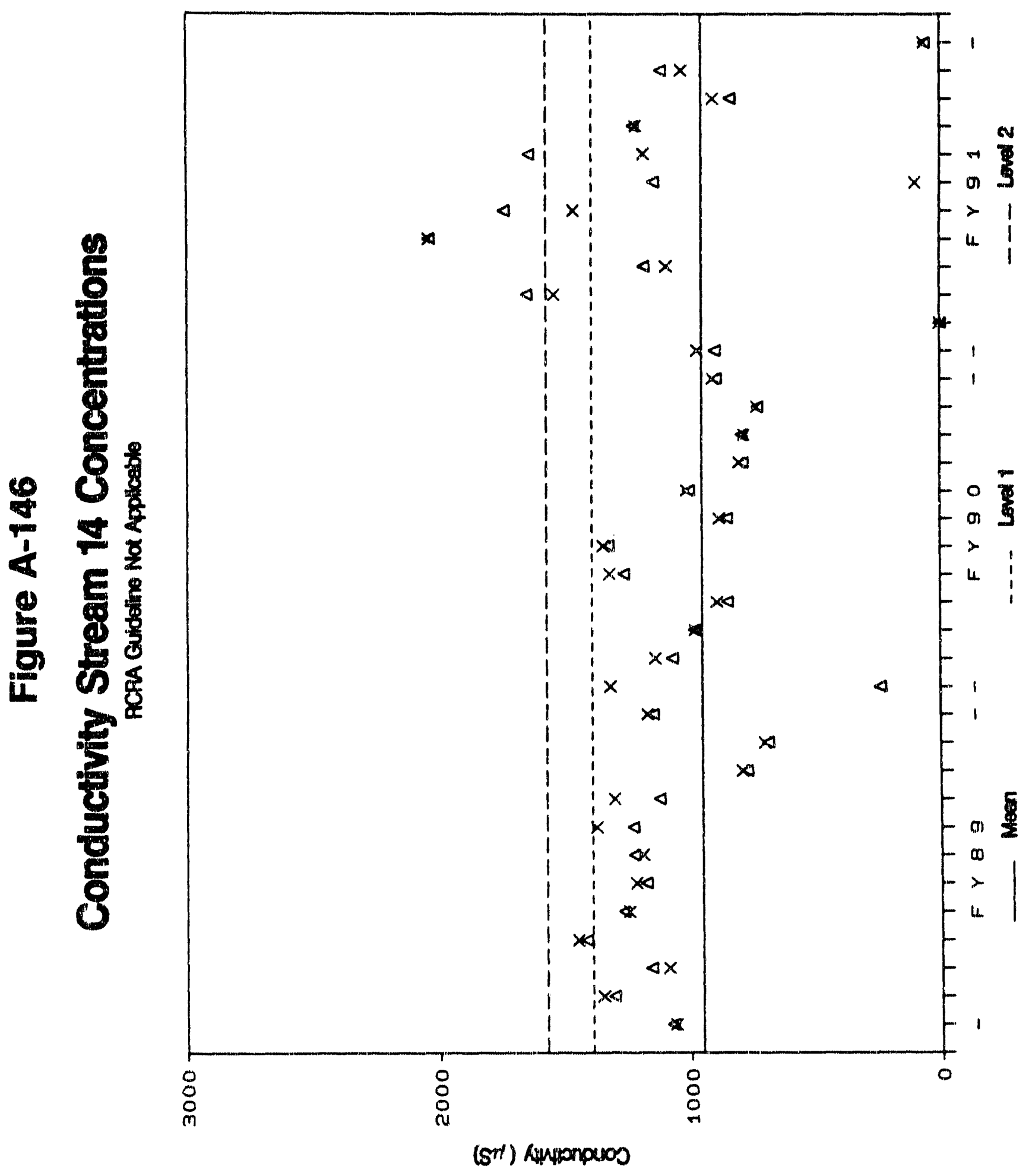




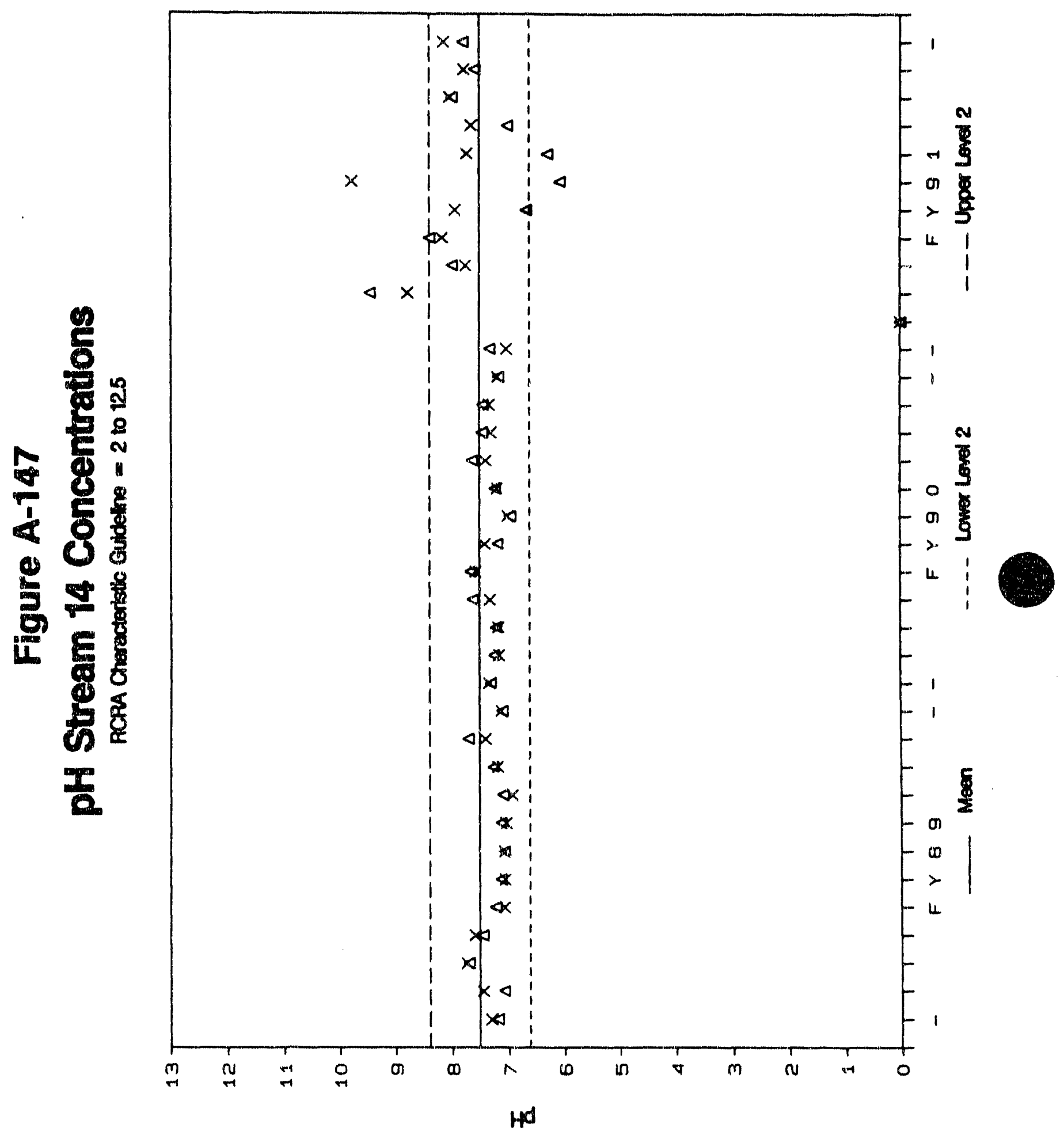




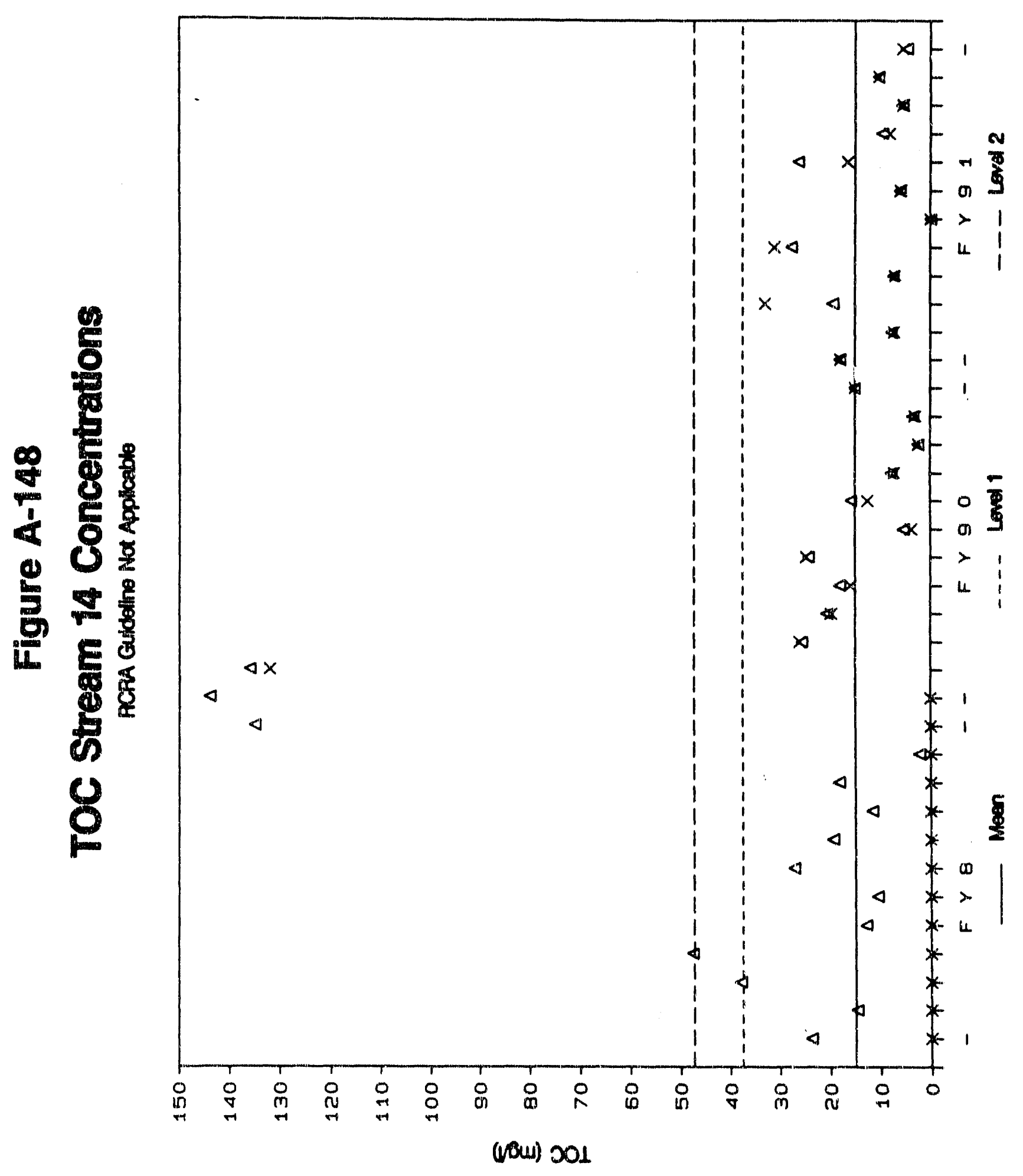




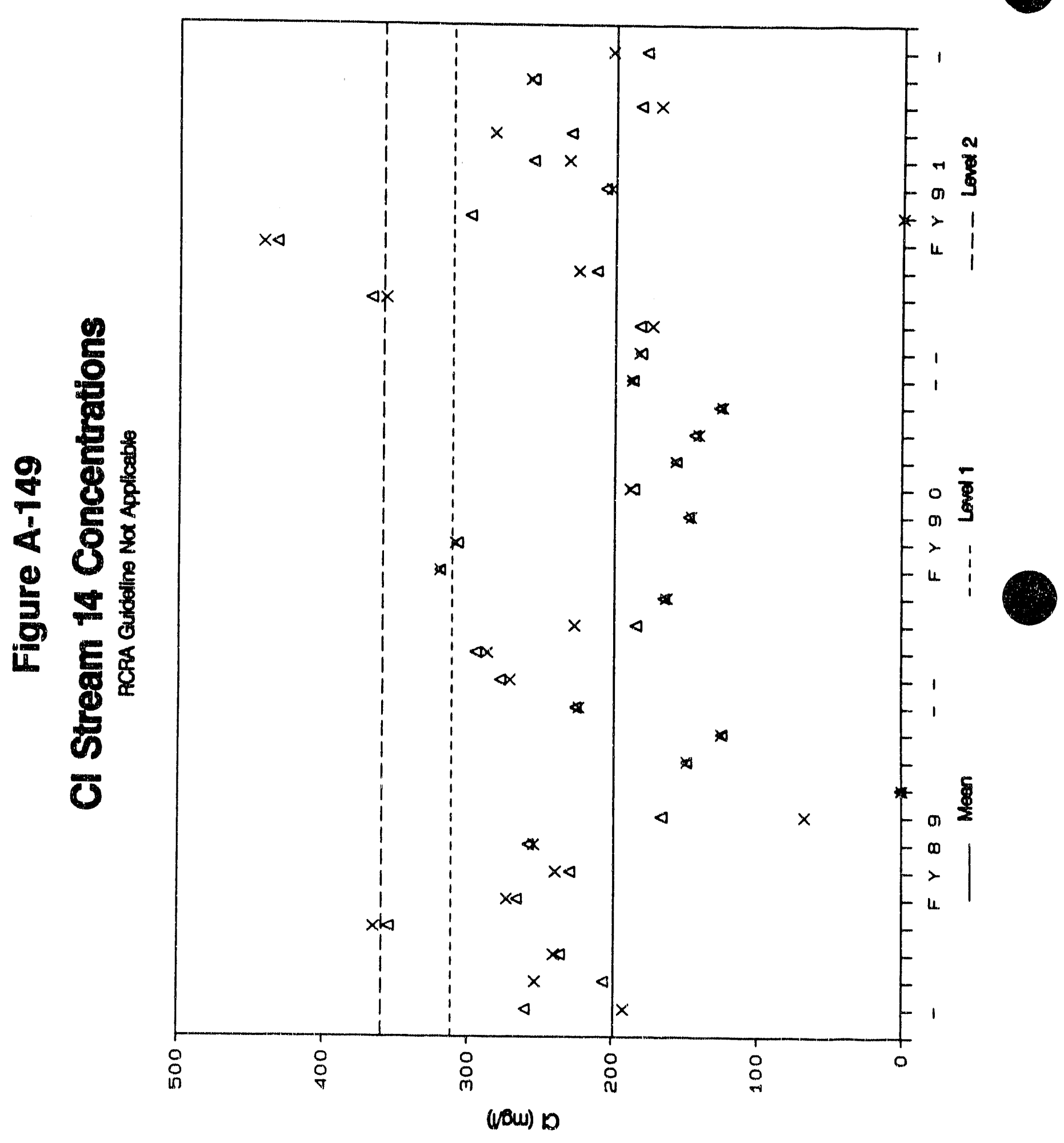




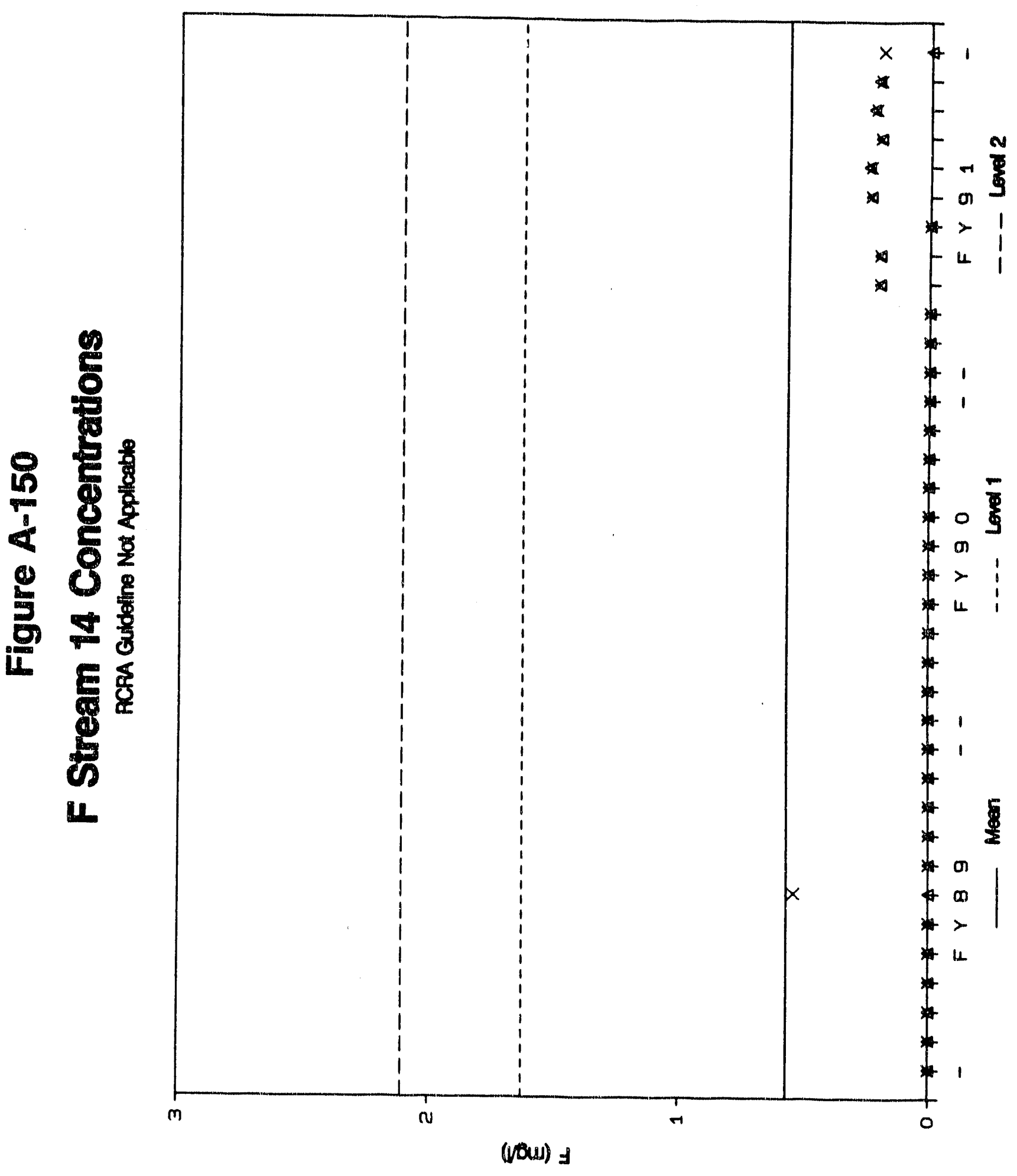




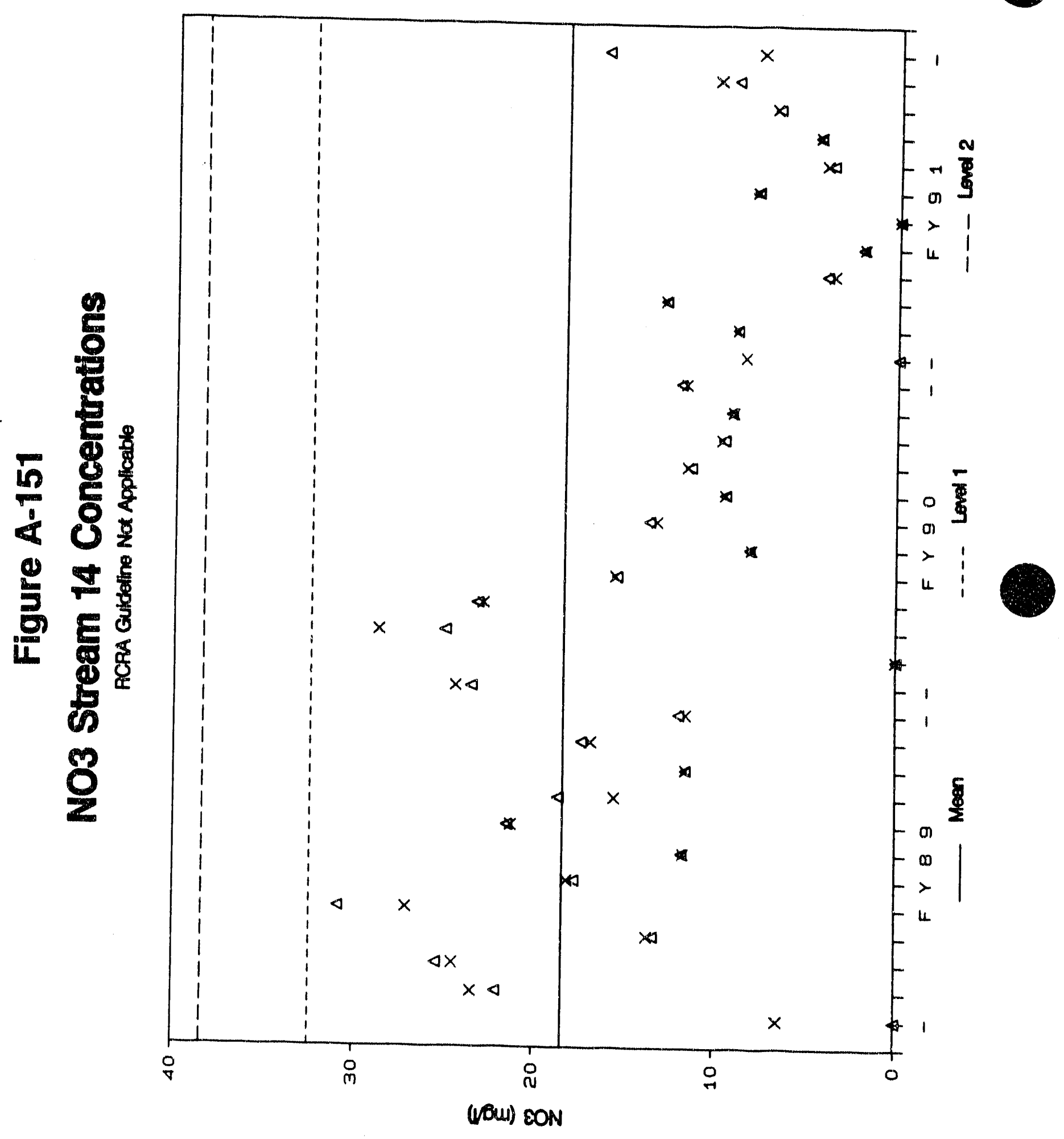




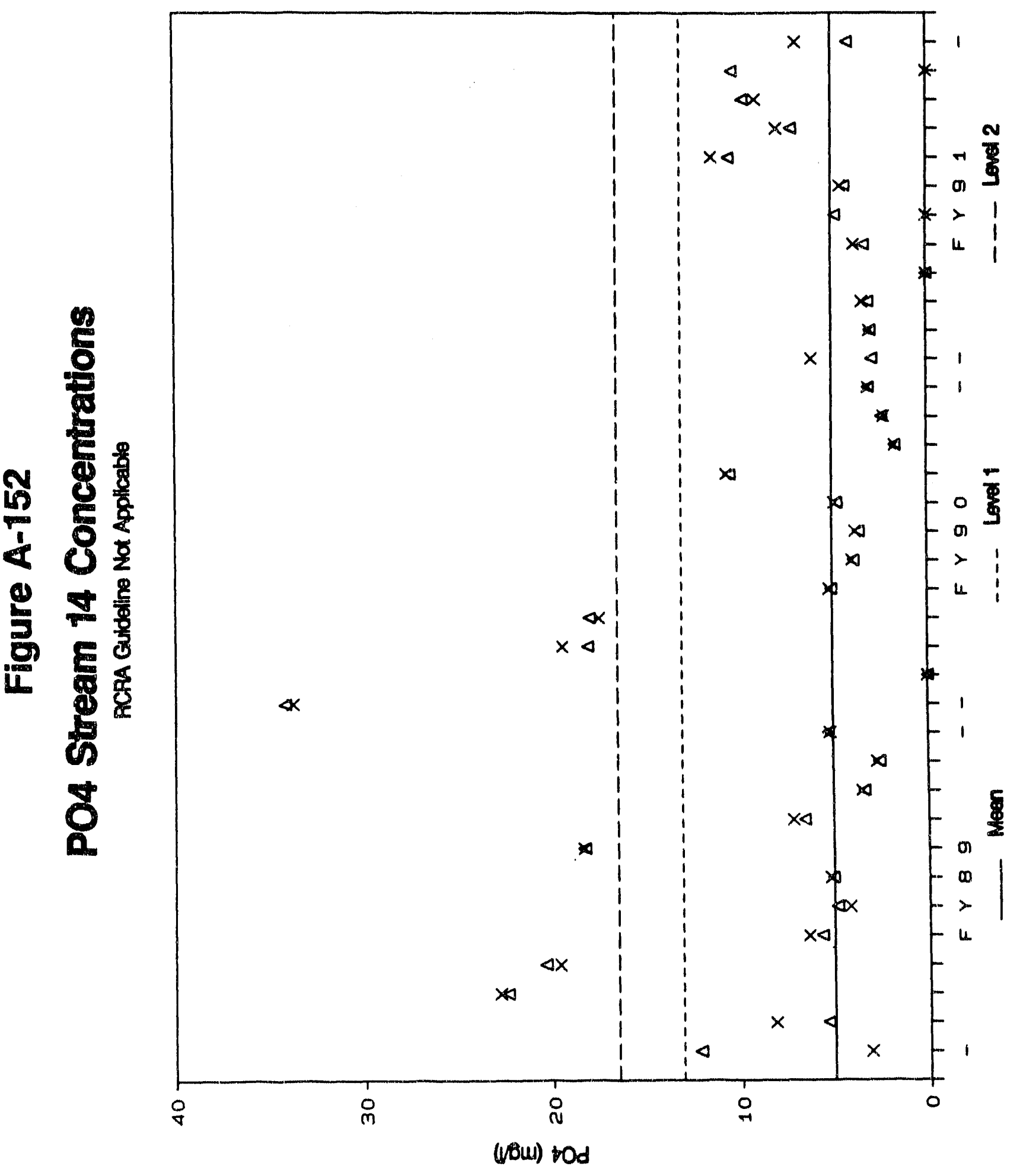




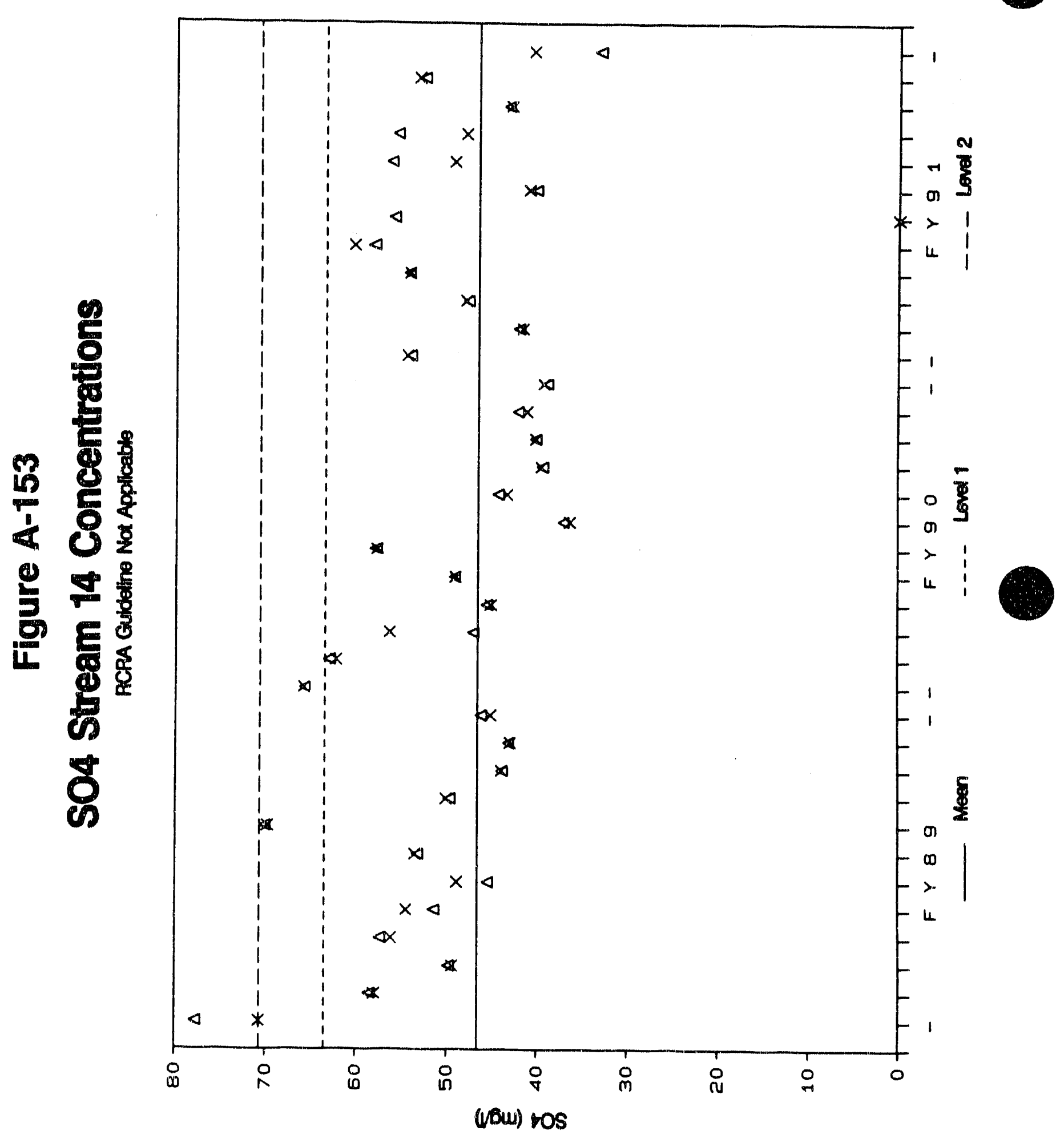




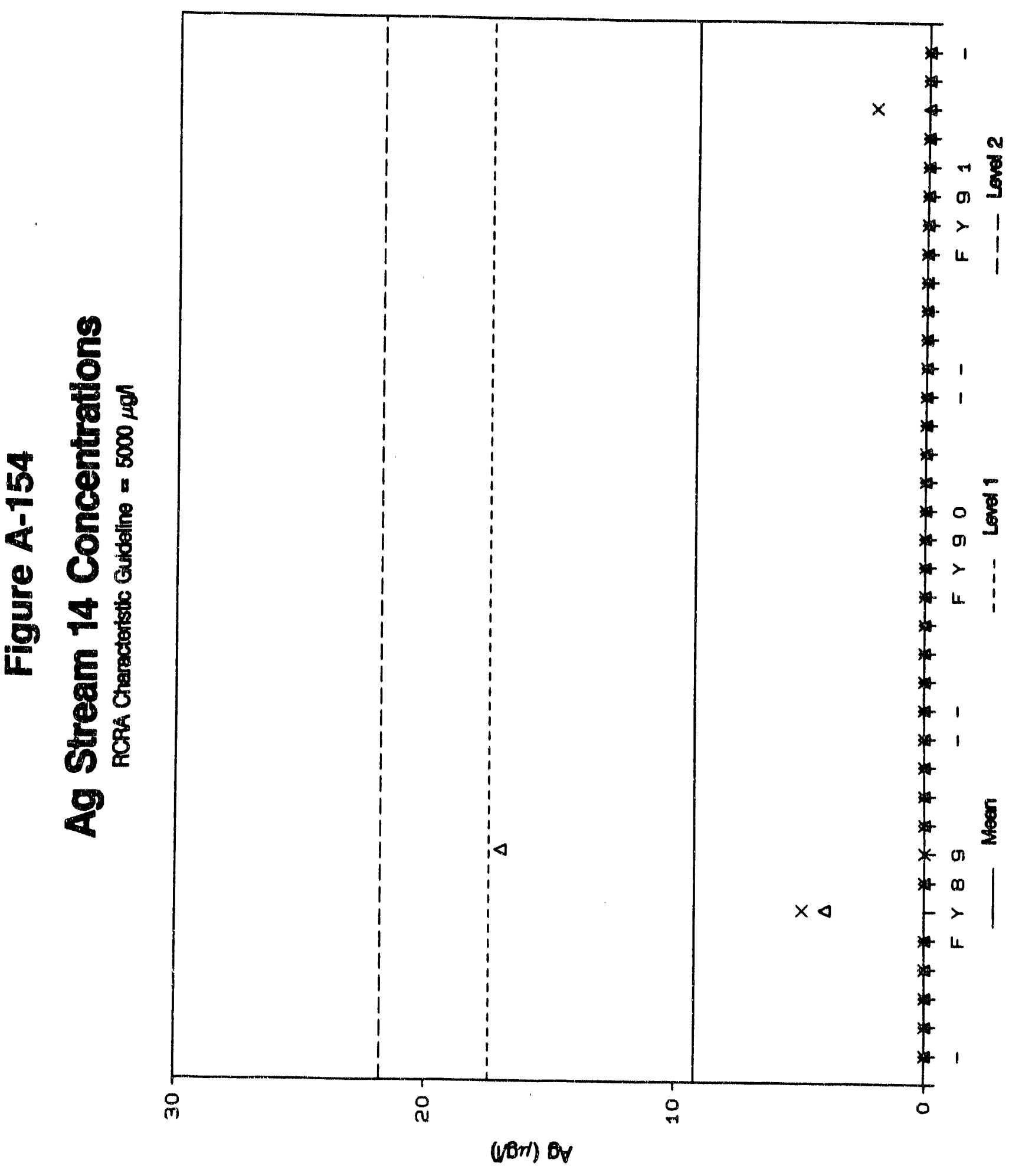




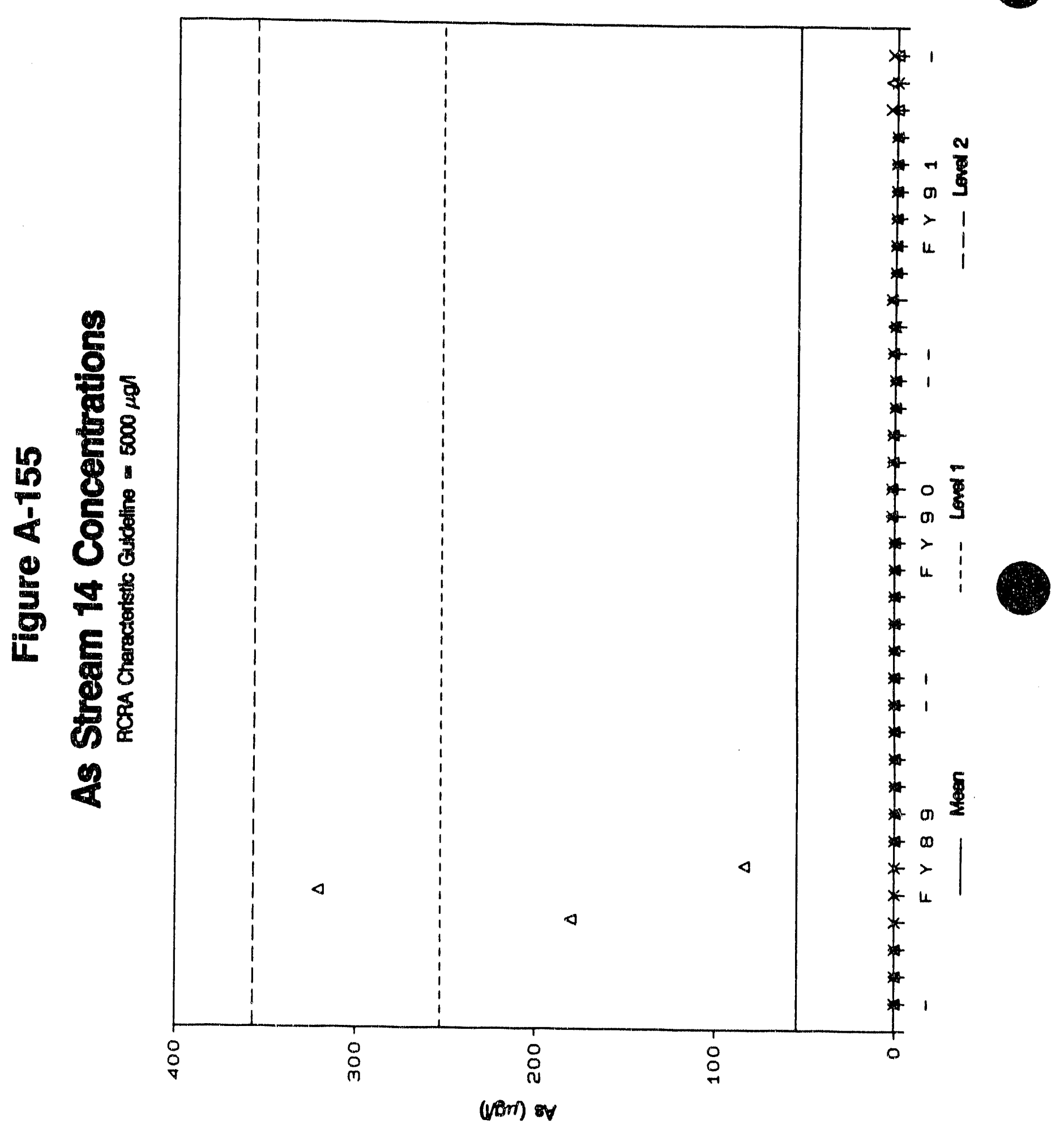




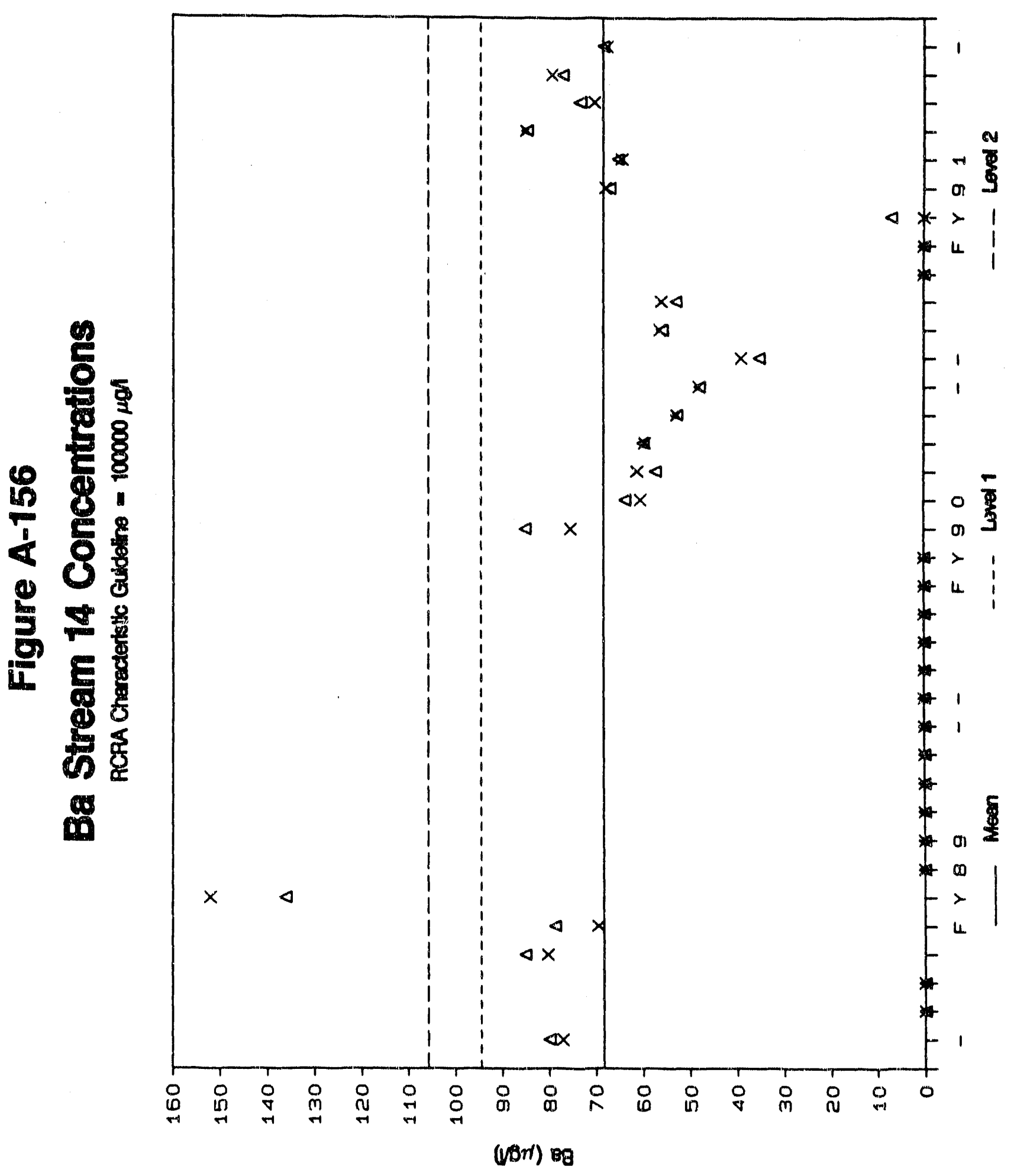




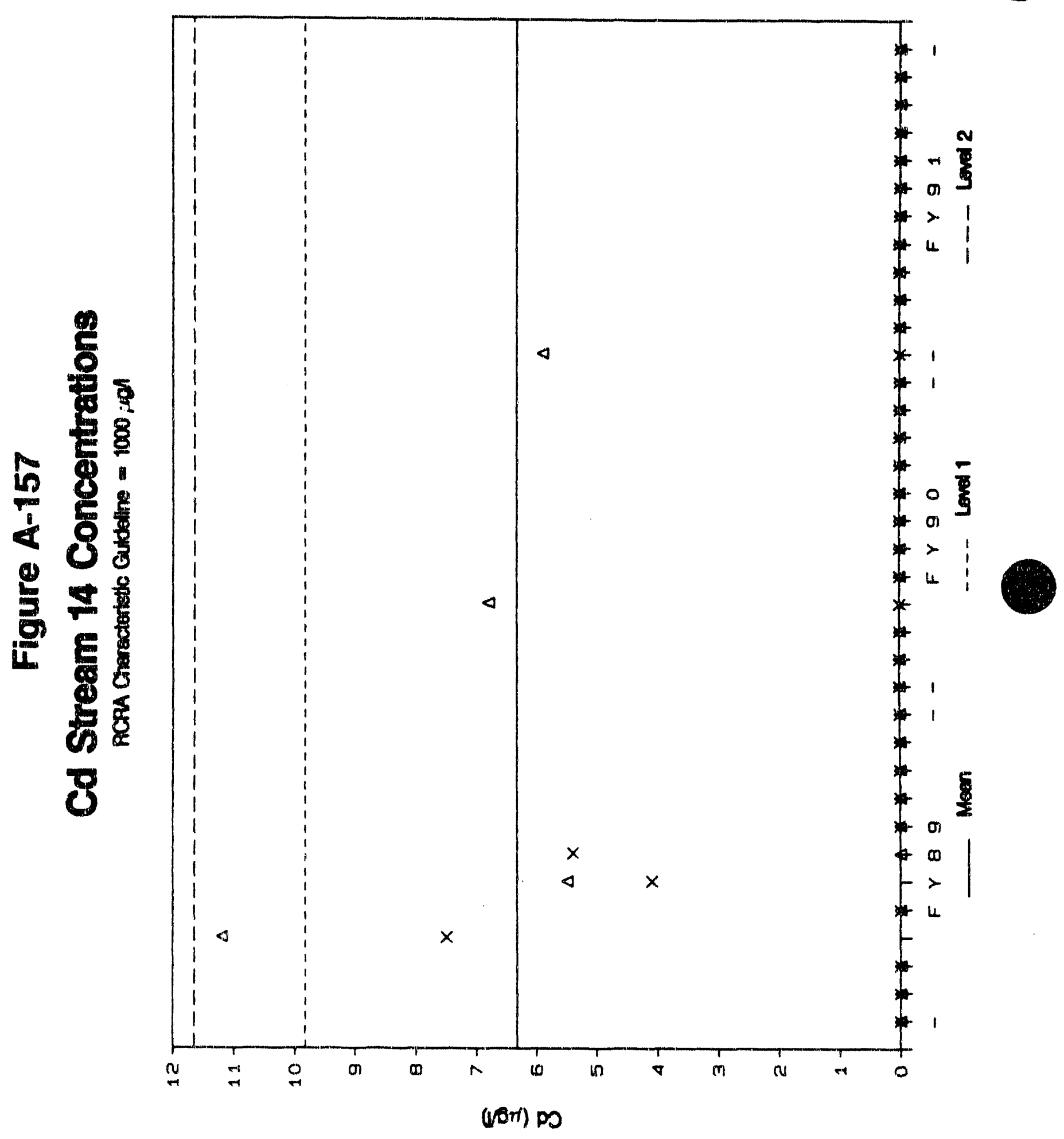




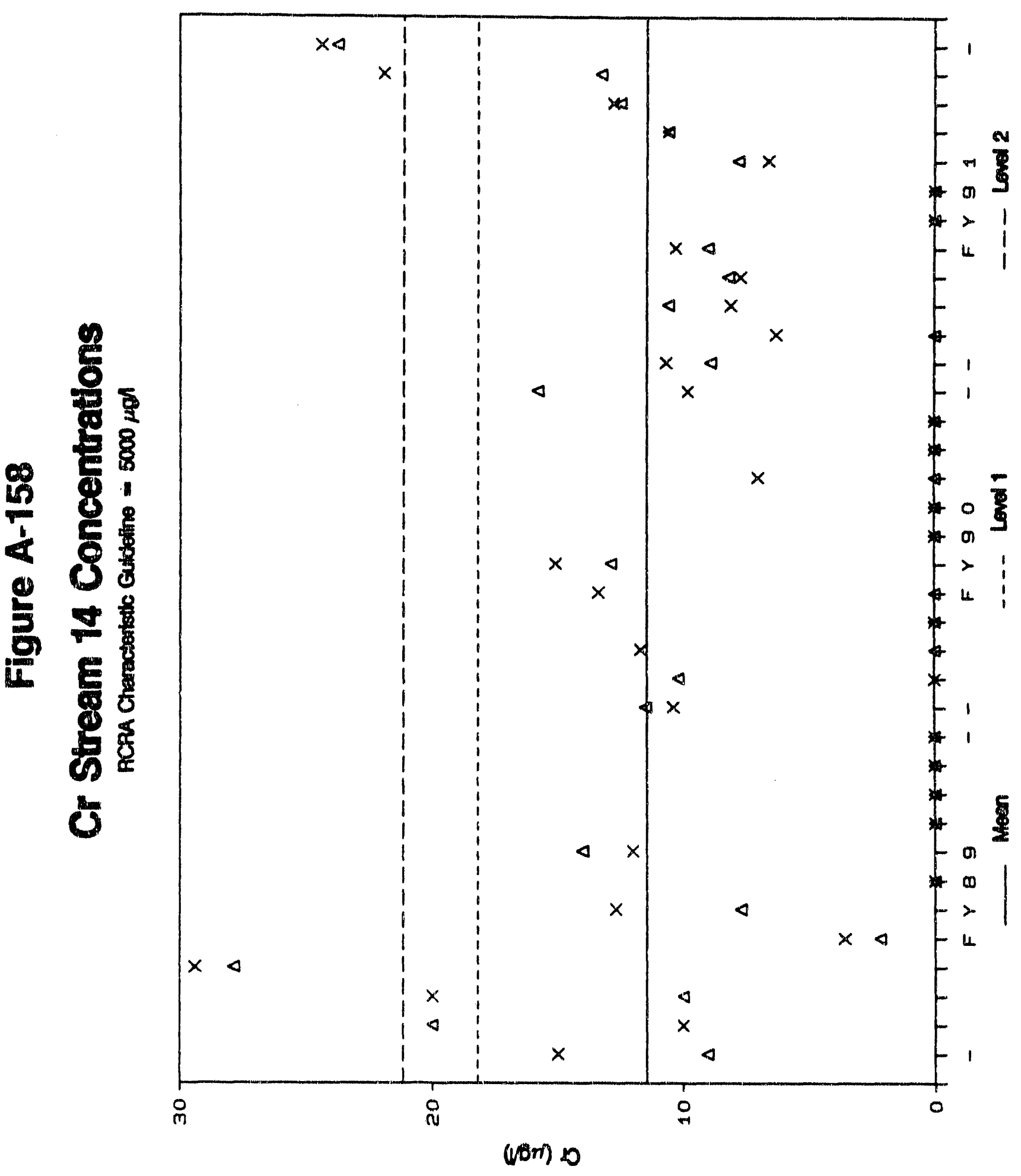




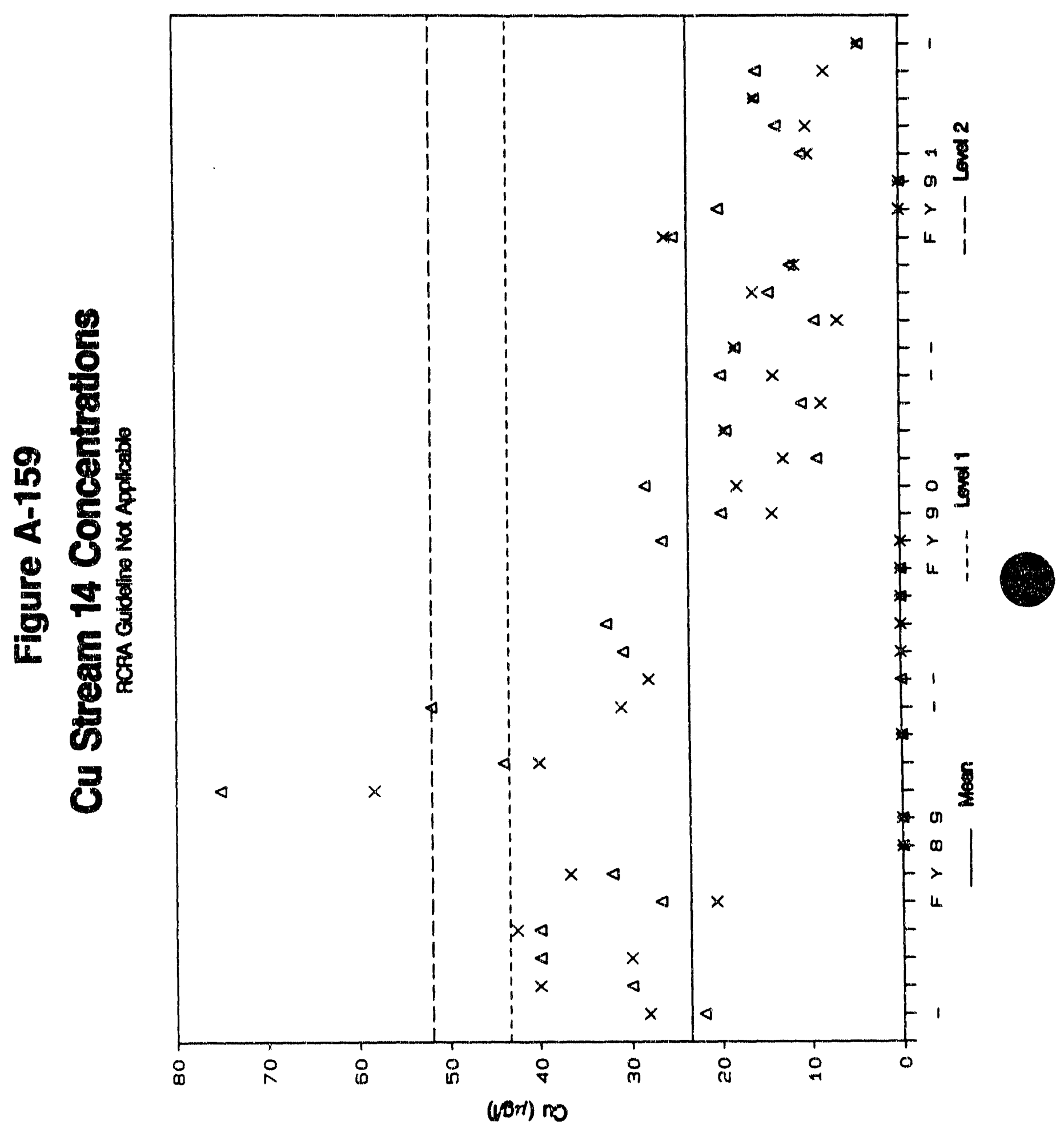




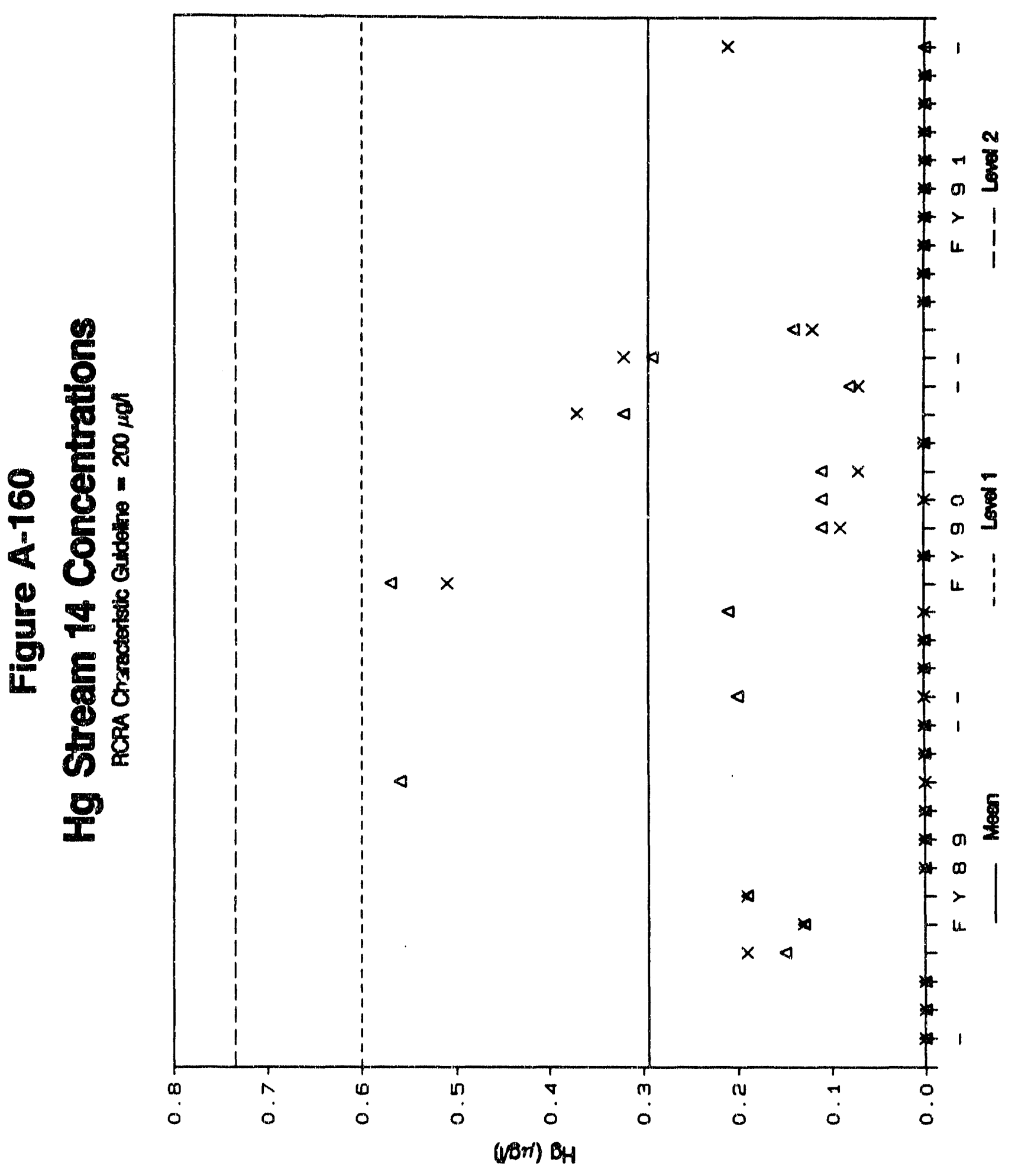




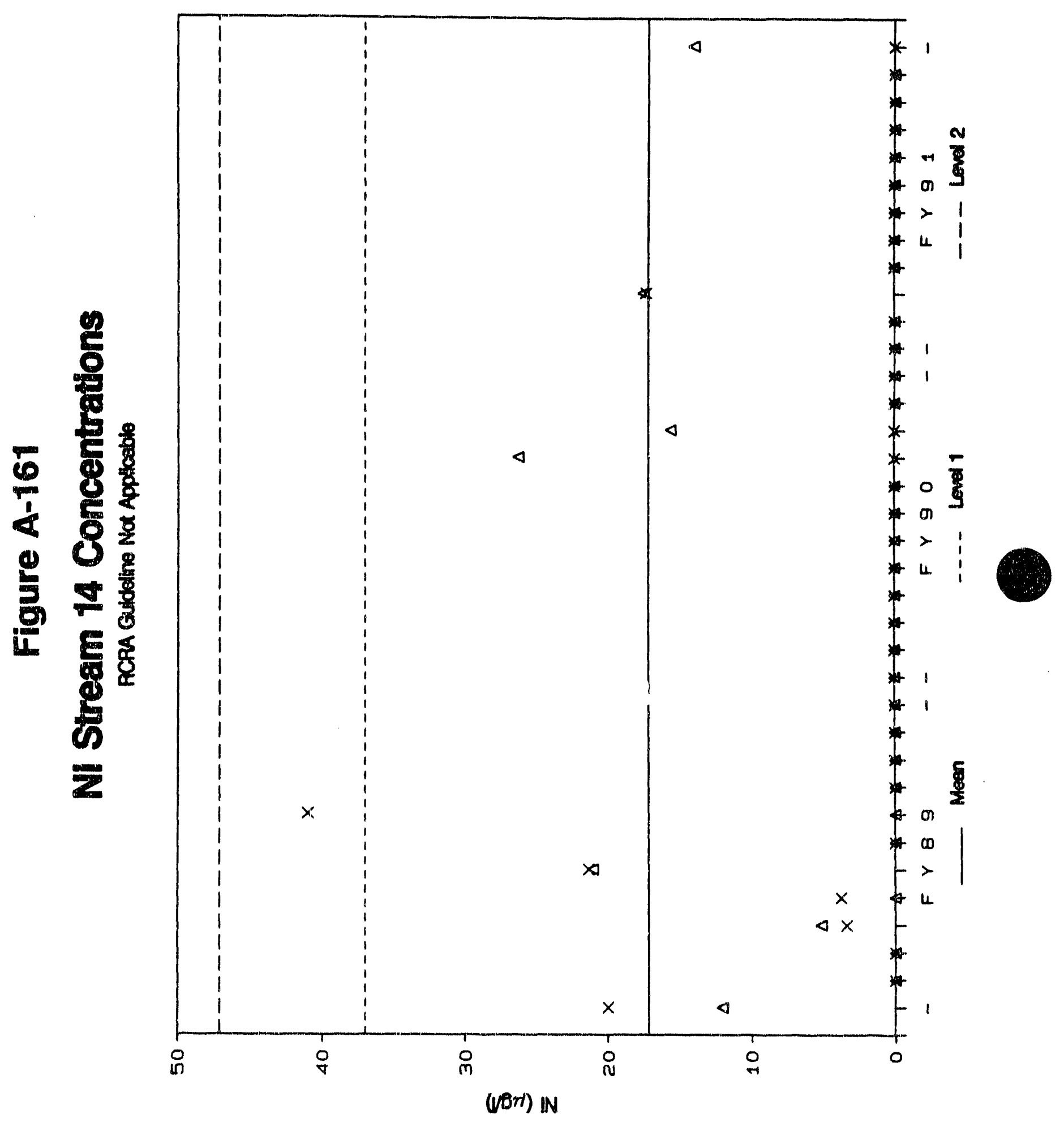




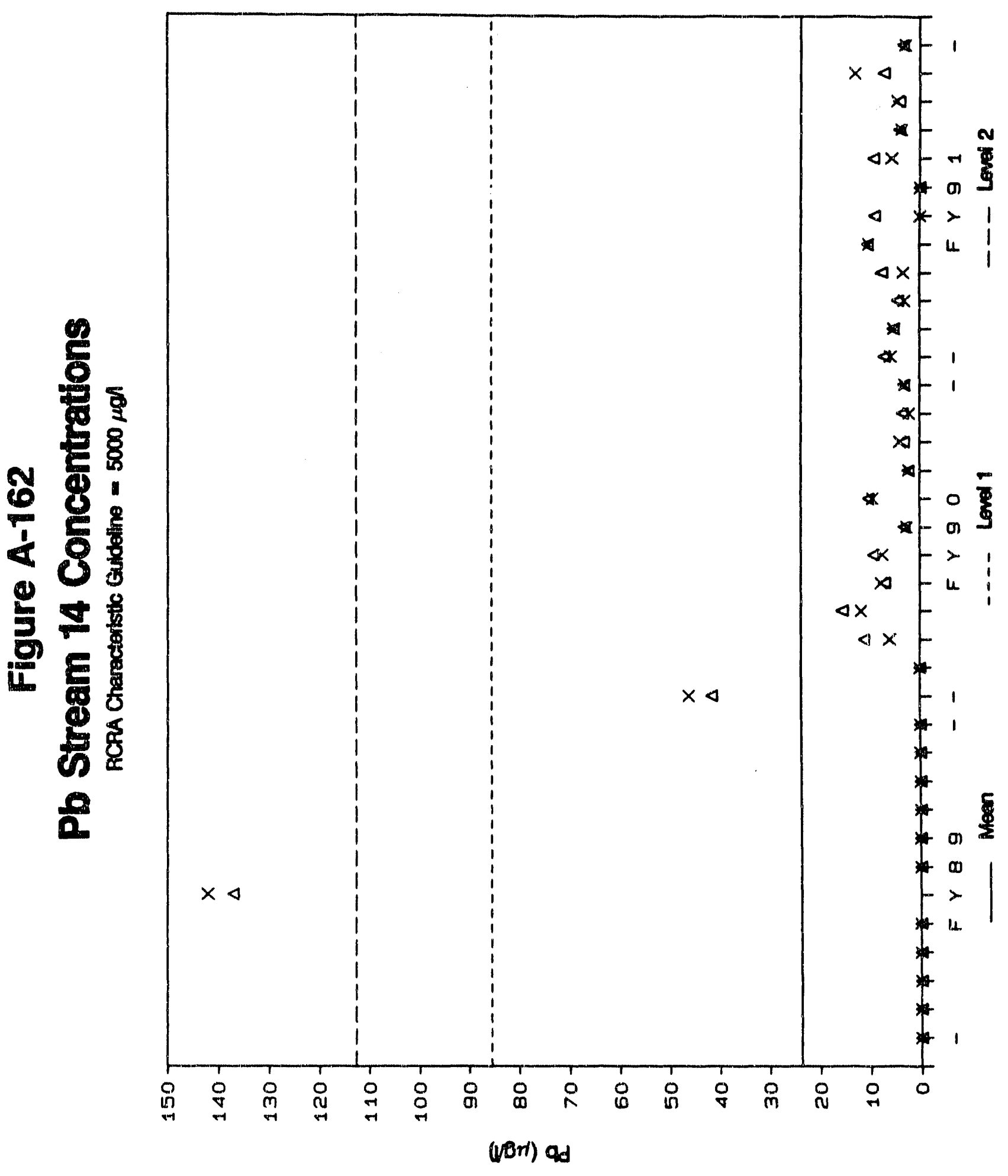




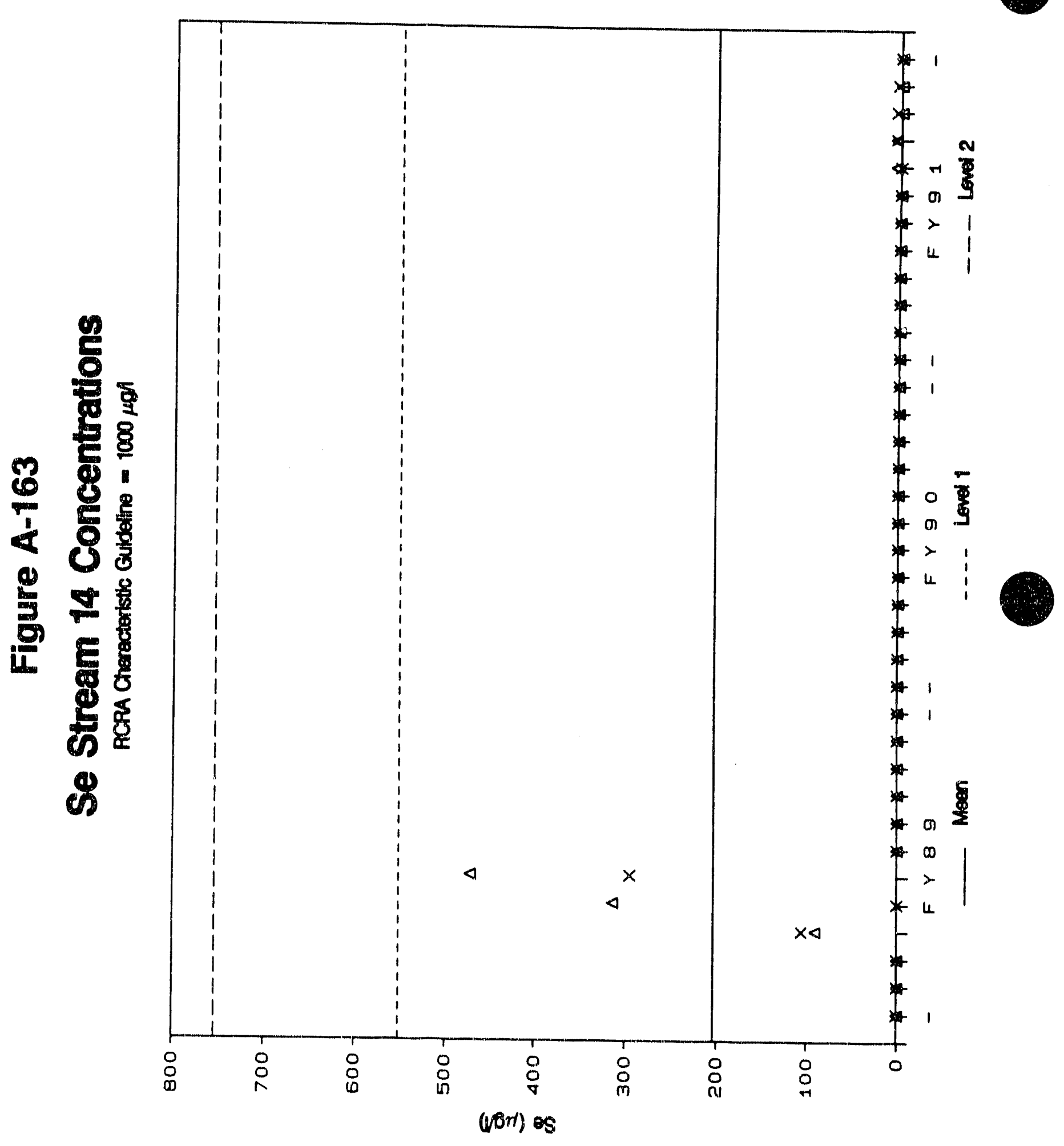




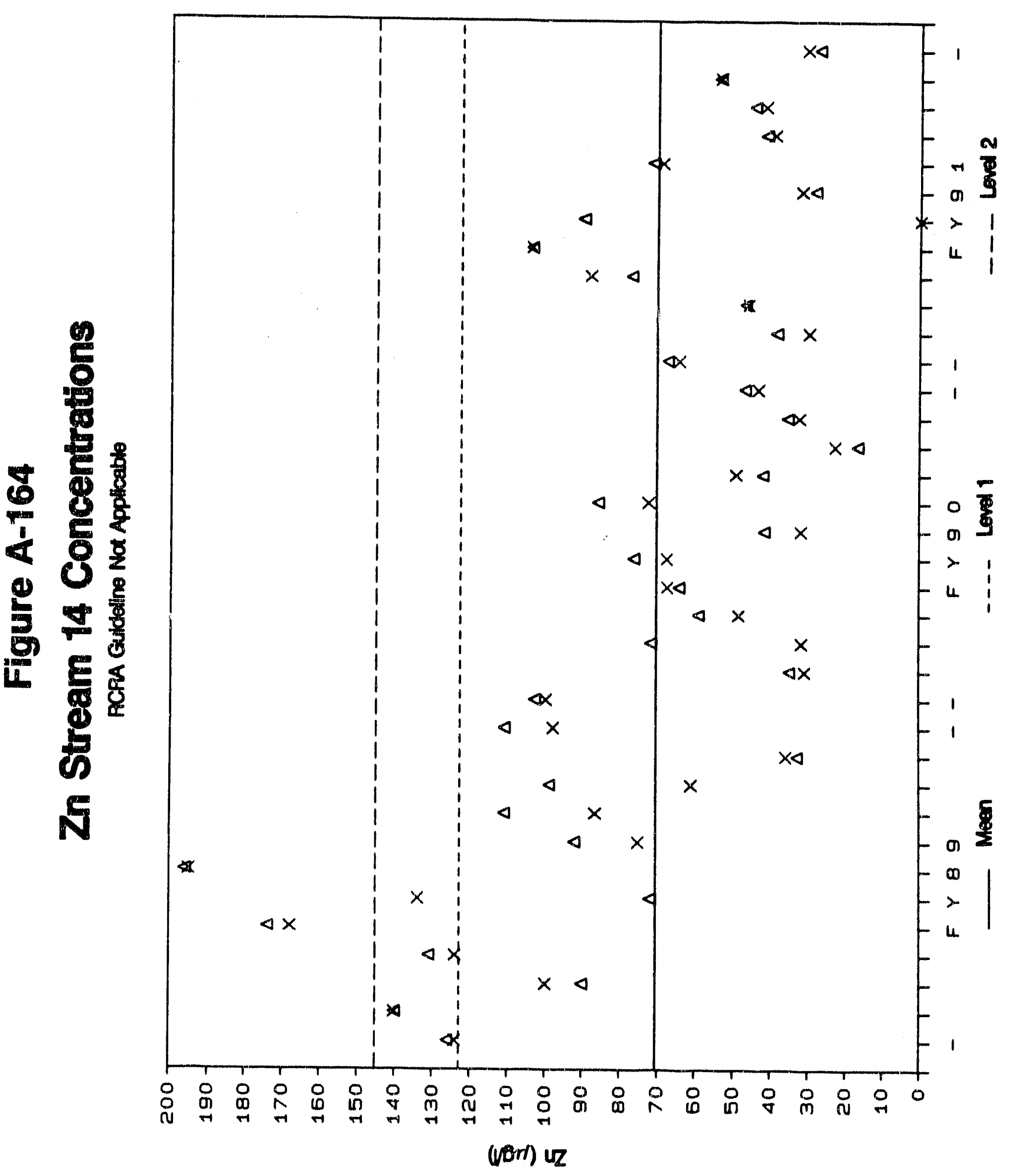




\section{Table A-47}

FY91 ENVIRONMENTAL MONITORING DATA

\begin{tabular}{|c|c|c|c|c|c|c|c|c|c|c|}
\hline \multirow{2}{*}{$\begin{array}{c}\text { MONTH } \\
\text { Oct } \\
\text { Oct } \\
\text { Nov } \\
\text { Nov } \\
\text { Dec } \\
\text { Dec }\end{array}$} & $\begin{array}{c}\text { REP } \\
\begin{array}{l}1 \\
2\end{array}\end{array}$ & $\begin{array}{l}\text { Cond } \\
(\mu S)\end{array}$ & $\mathrm{pH}$ & $\begin{array}{l}\text { TOC } \\
(\mathrm{mg} / \mathrm{l})\end{array}$ & $\frac{\mathrm{Cl}}{(\mathrm{mg} / \mathrm{l})}$ & \multicolumn{2}{|c|}{$\underset{(m g / l)}{F}$} & \multicolumn{2}{|c|}{$\begin{array}{l}\mathrm{NO}^{3} \\
(\mathrm{mg} / \mathrm{l})\end{array}$} & $\begin{array}{c}\mathrm{PO} 4 \\
(\mathrm{mg} / \mathrm{I})\end{array}$ \\
\hline & $\begin{array}{l}1 \\
2 \\
1 \\
2 \\
1 \\
2\end{array}$ & $\begin{array}{r}901 \\
971 \\
X \\
X \\
1650 \\
1540\end{array}$ & $\begin{array}{c}7.34 \\
7.04 \\
X \\
X \\
9.46 \\
8.79\end{array}$ & $\begin{array}{l}18.0 \\
17.9 \\
7.70 \\
7.20 \\
19.4 \\
33.0\end{array}$ & $\begin{array}{l}181 \\
182 \\
182 \\
173 \\
368 \\
358\end{array}$ & $\begin{array}{l}X \\
X \\
X \\
X \\
X \\
X\end{array}$ & & $\begin{array}{l}X \\
8.5 \\
8.5 \\
8.5 \\
12 . \\
12 .\end{array}$ & $\begin{array}{l}40 \\
90 \\
90 \\
.9 \\
.9\end{array}$ & $\begin{array}{l}2.90 \\
6.10 \\
3.00 \\
3.00 \\
3.10 \\
3.40\end{array}$ \\
\hline MONTH & REP & $\begin{array}{c}\mathrm{SO} 4 \\
(\mathrm{mg} / 1)\end{array}$ & $\begin{array}{l}\mathrm{Ag} \\
(\mu \mathrm{g} / 1)\end{array}$ & $\begin{array}{l}\text { As } \\
(\mu \mathrm{g} / 1)\end{array}$ & $\begin{array}{l}\mathrm{Ba} \\
(\mu \mathrm{g} / I)\end{array}$ & $\begin{array}{l}c d \\
(\mu g\end{array}$ & & $\begin{array}{l}\mathrm{Cr} \\
(\mu \mathrm{g} / 1)\end{array}$ & & $\begin{array}{l}u \\
g / 1)\end{array}$ \\
\hline $\begin{array}{l}\text { Oct } \\
\text { Oct } \\
\text { Nov } \\
\text { Nov } \\
\text { Dec } \\
\text { Dec }\end{array}$ & $\begin{array}{l}1 \\
2 \\
1 \\
2 \\
1 \\
2\end{array}$ & $\begin{array}{l}54.1 \\
54.5 \\
42.1 \\
41.7 \\
47.7 \\
48.0\end{array}$ & $\begin{array}{l}X \\
X \\
X \\
X \\
X \\
X\end{array}$ & $\begin{array}{l}1.20 \\
1.50 \\
1.10 \\
X \\
2.00 \\
2.20\end{array}$ & $\begin{array}{l}35.2 \\
39.0 \\
55.8 \\
56.4 \\
52.9 \\
56.0\end{array}$ & $\begin{array}{l}5 . \\
x \\
x \\
x \\
x \\
x\end{array}$ & & $\begin{array}{l}8.90 \\
10.7 \\
x \\
6.30 \\
10.6 \\
8.10\end{array}$ & & $\begin{array}{l}8.1 \\
8.2 \\
.30 \\
.80 \\
4.4 \\
6.1\end{array}$ \\
\hline MONTH & REP & $\begin{array}{l}\mathrm{Hg} \\
(\mu \mathrm{g} / \mathrm{I})\end{array}$ & $\stackrel{\mathrm{Ni}}{(\mu \mathrm{g} / 1)}$ & $\begin{array}{l}\mathrm{Pb} \\
(\mu \mathrm{g} / 1)\end{array}$ & $\begin{array}{r}\mathrm{Se} \\
(\mu g\end{array}$ & & $\begin{array}{l}\mathrm{T} 1 \\
(\mu \mathrm{g}\end{array}$ & (1) & $\begin{array}{r}\mathrm{Zn} \\
(\mu \mathrm{g}\end{array}$ & \\
\hline $\begin{array}{l}\text { Oct } \\
\text { Oct } \\
\text { Nov } \\
\text { Nov } \\
\text { Dec } \\
\text { Dec }\end{array}$ & $\begin{array}{l}1 \\
2 \\
1 \\
2 \\
1 \\
2\end{array}$ & $\begin{array}{l}0.29 \\
0.32 \\
0.14 \\
0.12 \\
X \\
X\end{array}$ & $\begin{array}{l}x \\
x \\
x \\
x \\
17.5 \\
17.3\end{array}$ & $\begin{array}{l}7.10 \\
5.90 \\
5.30 \\
5.20 \\
4.50 \\
3.20\end{array}$ & $\begin{array}{l}\mathrm{X} \\
\mathrm{X} \\
\mathrm{X} \\
\mathrm{X} \\
\mathrm{X} \\
\mathrm{X}\end{array}$ & & $\begin{array}{l}X \\
X \\
X \\
X \\
X \\
0 .\end{array}$ & & $\begin{array}{l}67 \\
64 \\
38 \\
30 \\
46 \\
46\end{array}$ & \\
\hline
\end{tabular}




\section{Table A-48}

FX91 ENVIRONMENTAL MONITORING DATA

\begin{tabular}{|c|c|c|c|c|c|c|c|c|c|c|c|}
\hline \multirow{2}{*}{$\begin{array}{c}\text { MONTH } \\
\text { Jan } \\
\text { Jan } \\
\text { Feb } \\
\text { Feb } \\
\text { Mar } \\
\text { Mar }\end{array}$} & \multirow{2}{*}{$\begin{array}{c}\text { REP } \\
\\
1 \\
2 \\
1 \\
2 \\
1 \\
2\end{array}$} & \multirow{2}{*}{$\begin{array}{l}\text { Cond } \\
(\mu S) \\
1180 \\
1090 \\
2040 \\
2040 \\
1740 \\
1460\end{array}$} & $\mathrm{pH}$ & $\begin{array}{l}\text { TOC } \\
(\mathrm{mg} / 1)\end{array}$ & \multicolumn{2}{|c|}{$\begin{array}{c}\mathrm{Cl} \\
(\mathrm{mg} / \mathrm{l})\end{array}$} & \multicolumn{2}{|c|}{$\underset{(m g / l)}{F}$} & \multicolumn{2}{|c|}{$\begin{array}{l}\text { NO3 } \\
(\mathrm{mg} / 1)\end{array}$} & $\begin{array}{c}\mathrm{PO4} \\
(\mathrm{mg} / \mathrm{l})\end{array}$ \\
\hline & & & $\begin{array}{l}8.00 \\
7.76 \\
8.40 \\
8.18 \\
6.69 \\
7.95\end{array}$ & $\begin{array}{l}7.40 \\
7.00 \\
27.7 \\
31.1 \\
x \\
x\end{array}$ & X & $\begin{array}{l}12 \\
24 \\
33 \\
42 \\
00\end{array}$ & $\begin{array}{l}0 \\
0 \\
0 \\
0 \\
X \\
X\end{array}$ & $\begin{array}{l}20 \\
20 \\
20 \\
20\end{array}$ & $\begin{array}{l}4.5 \\
3.5 \\
2.5 \\
1.5 \\
x \\
x\end{array}$ & $\begin{array}{l}00 \\
60 \\
00 \\
90\end{array}$ & $\begin{array}{l}X \\
X \\
3.31 \\
3.83 \\
4.85 \\
X\end{array}$ \\
\hline MONTH & REF & $\begin{array}{c}\mathrm{SO4} \\
(\mathrm{mg} / \mathrm{I})\end{array}$ & $\begin{array}{l}\mathrm{Ag} \\
(\mu \mathrm{g} / 1)\end{array}$ & $\underset{(\mu g / 1)}{R . s}$ & & $(1)$ & $\begin{array}{l}\mathrm{Cd} \\
(\mu \mathrm{g}\end{array}$ & & $\begin{array}{l}\mathrm{Cr} \\
(\mu g / 1)\end{array}$ & )$(\mathrm{H}$ & $\begin{array}{l}u \\
g / 1)\end{array}$ \\
\hline $\begin{array}{l}\text { Jan } \\
\text { Jan } \\
\text { Feb } \\
\text { Feb } \\
\text { Mar } \\
\text { Mar }\end{array}$ & $\begin{array}{l}1 \\
2 \\
1 \\
2 \\
1 \\
2\end{array}$ & $\begin{array}{l}54.3 \\
54.2 \\
58.2 \\
60.3 \\
56.0 \\
X\end{array}$ & $\begin{array}{l}X \\
X \\
X \\
X \\
X \\
X\end{array}$ & $\begin{array}{l}X \\
X \\
X \\
X \\
X \\
X\end{array}$ & $\begin{array}{l}x \\
x \\
x \\
x \\
7 \\
x\end{array}$ & 00 & $\begin{array}{l}X \\
X \\
X \\
X \\
X \\
X\end{array}$ & & $\begin{array}{l}8.20 \\
7.70 \\
9.00 \\
10.3 \\
X \\
X\end{array}$ & $\begin{array}{l}1 \\
1 \\
2 \\
2 \\
2 \\
3\end{array}$ & $\begin{array}{l}2.0 \\
1.5 \\
5.1 \\
6.0 \\
0.0\end{array}$ \\
\hline MONTH & REP & $\begin{array}{l}\mathrm{Hg} \\
(\mu g / 1)\end{array}$ & $\begin{array}{l}\mathrm{Ni} \\
(\mu \mathrm{g} / \mathrm{l})\end{array}$ & $\begin{array}{c}\mathrm{Pb} \\
(\mu \mathrm{g} /\end{array}$ & & $\begin{array}{r}\text { Se } \\
(\mu g\end{array}$ & & $\begin{array}{r}\mathrm{Tl} \\
(\mu \mathrm{g}\end{array}$ & /1) & $\begin{array}{l}\mathrm{Zn} \\
(\mu \mathrm{g})\end{array}$ & \\
\hline $\begin{array}{l}\text { Jan } \\
\text { Jan } \\
\text { Feb } \\
\text { Feb } \\
\text { Mar } \\
\text { Mar }\end{array}$ & $\begin{array}{l}1 \\
2 \\
1 \\
2 \\
1 \\
2\end{array}$ & $\begin{array}{l}X \\
X \\
X \\
X \\
X \\
X\end{array}$ & $\begin{array}{l}X \\
X \\
X \\
X \\
X \\
X\end{array}$ & $\begin{array}{l}7.6 \\
3.4 \\
10 . \\
10 . \\
9.0 \\
x\end{array}$ & & $\begin{array}{l}X \\
X \\
X \\
X \\
X \\
X\end{array}$ & & $\begin{array}{l}\mathrm{X} \\
\mathrm{X} \\
\mathrm{X} \\
\mathrm{X} \\
\mathrm{X} \\
\mathrm{X}\end{array}$ & & $\begin{array}{l}77 \\
88 \\
10 \\
10 \\
90 \\
\times\end{array}$ & \\
\hline
\end{tabular}




\section{Table A-49}

FY91 ENVIRONMENTAL MONITORING DATA

\begin{tabular}{|c|c|c|c|c|c|c|c|c|}
\hline MONTH & REP & $\begin{array}{l}\text { Cond } \\
(\mu \mathrm{S})\end{array}$ & $\mathrm{pH}$ & $\begin{array}{l}\text { TOC } \\
(\mathrm{mg} / 1)\end{array}$ & $\begin{array}{c}\mathrm{Cl} \\
(\mathrm{mg} / \mathrm{l})\end{array}$ & $\begin{array}{l}F \\
(m g / 1)\end{array}$ & $\begin{array}{c}\text { NO3 } \\
(\mathrm{mg} / \mathrm{l})\end{array}$ & $\begin{array}{c}\mathrm{PO4} \\
(\mathrm{mg} / \mathrm{l})\end{array}$ \\
\hline $\begin{array}{l}\text { Apr } \\
\text { Apr } \\
\text { May } \\
\text { May } \\
\text { Jun } \\
\text { Jun }\end{array}$ & $\begin{array}{l}1 \\
2 \\
1 \\
2 \\
1 \\
2\end{array}$ & $\begin{array}{r}1140 \\
99 \\
1640 \\
1180 \\
1220 \\
1210\end{array}$ & $\begin{array}{l}6.08 \\
9.78 \\
6.30 \\
7.74 \\
7.02 \\
7.66\end{array}$ & $\begin{array}{l}6.12 \\
5.97 \\
26.3 \\
16.5 \\
9.66 \\
8.23\end{array}$ & $\begin{array}{l}206 \\
202 \\
256 \\
231 \\
230 \\
283\end{array}$ & $\begin{array}{l}0.24 \\
0.24 \\
0.25 \\
0.24 \\
0.20 \\
0.20\end{array}$ & $\begin{array}{l}7.86 \\
7.84 \\
3.76 \\
4.07 \\
4.47 \\
4.43\end{array}$ & $\begin{array}{l}4.34 \\
4.56 \\
10.5 \\
11.4 \\
7.19 \\
7.96\end{array}$ \\
\hline
\end{tabular}

$\begin{array}{llllllll}\text { MONTH REP } & \mathrm{SO}^{2} & \mathrm{Ag} & \mathrm{As} & \mathrm{Ba} & \mathrm{Cd} & \mathrm{Cr} & \mathrm{Cu} \\ (\mu \mathrm{gg} / 1) & (\mu \mathrm{g} / 1) & (\mu \mathrm{g} / 1) & (\mu \mathrm{g} / 1) & (\mu \mathrm{g} / 1) & (\mu \mathrm{g} / 1)\end{array}$

$\begin{array}{lllllllll}\text { Apr } & 1 & 40.2 & X & X & 66.9 & X & X & X \\ \text { Apr } & 2 & 41.0 & X & X & 67.9 & X & X & X \\ \text { May } & 1 & 56.3 & X & X & 65.2 & X & 7.80 & 10.8 \\ \text { May } & 2 & 49.3 & X & X & 64.4 & X & 6.60 & 10.0 \\ \text { Jun } & 1 & 55.6 & X & X & 84.6 & X & 10.6 & 13.6 \\ \text { Jun } & 2 & 48.0 & X & X & 84.8 & X & 10.6 & 10.2\end{array}$

$\begin{array}{lllllll}\mathrm{MONTH} & \mathrm{Hg} & \mathrm{Ni} & \mathrm{Pb} & \mathrm{Se} & \mathrm{Tl} & \mathrm{Zn} \\ (\mu \mathrm{g} / 1) & (\mu \mathrm{g} / 1) & (\mu \mathrm{g} / 1) & (\mu \mathrm{g} / 1) & (\mu \mathrm{g} / 1) & (\mu \mathrm{g} / 1)\end{array}$

$\begin{array}{llllllll}\text { Apr } & 1 & X & X & X & X & X & 28.6 \\ \text { Apr } & 2 & X & X & X & X & X & 32.1 \\ \text { May } & 1 & X & X & 9.20 & 5.20 & X & 71.6 \\ \text { May } & 2 & X & X & 5.70 & X & X & 69.2 \\ \text { Jun } & 1 & X & X & 4.20 & 5.60 & 2.30 & 41.5 \\ \text { Jun } & 2 & X & X & 3.80 & 5.00 & X & 39.4\end{array}$

$'$ ' - missing

' $X$ ' - below practical quantitation level 


\section{Table A-50}

FY91 ENVIRONMENTAL MONITORING DATA

\begin{tabular}{|c|c|c|c|c|c|c|c|c|c|}
\hline \multicolumn{2}{|c|}{- STREAM $=14$} & \multirow{2}{*}{$\begin{array}{l}\text { (CFA S } \\
\text { Cond } \\
(\mu S)\end{array}$} & \multicolumn{2}{|c|}{ STP effluent $t$} & \multicolumn{2}{|c|}{ :o drain } & field) & \multicolumn{2}{|c|}{ QUARTER $=91 q 4$} \\
\hline MONTH & REP & & $\mathrm{pH}$ & $\begin{array}{l}\text { TOC } \\
(\mathrm{mg} / \mathrm{l})\end{array}$ & $\begin{array}{l}\mathrm{Cl} \\
\text { (mg }\end{array}$ & & $\underset{(m g / l)}{F}$ & $\begin{array}{c}\text { NO3 } \\
(\mathrm{mg} / \mathrm{l})\end{array}$ & $\begin{array}{c}\mathrm{PO4} \\
(\mathrm{mg} / \mathrm{l})\end{array}$ \\
\hline $\begin{array}{l}\text { Jul } \\
\text { Jul } \\
\text { Aug } \\
\text { Aug } \\
\text { Sep } \\
\text { Sep }\end{array}$ & $\begin{array}{l}1 \\
2 \\
1 \\
2 \\
1 \\
2\end{array}$ & $\begin{array}{r}840.0 \\
906.0 \\
1110.0 \\
1030.0 \\
61.1 \\
62.2\end{array}$ & $\begin{array}{l}8.00 \\
8.04 \\
7.60 \\
7.79 \\
7.81 \\
8.15\end{array}$ & $\begin{array}{l}5.66 \\
5.46 \\
10.4 \\
10.3 \\
4.76 \\
5.55\end{array}$ & & $\begin{array}{l}32 \\
58 \\
56 \\
8 \\
8 \\
1\end{array}$ & $\begin{array}{l}0.23 \\
0.22 \\
0.21 \\
0.20 \\
X \\
0.19\end{array}$ & $\begin{array}{l}6.75 \\
6.91 \\
9.01 \\
10.0 \\
16.3 \\
7.62\end{array}$ & $\begin{array}{l}9.76 \\
9.09 \\
10.3 \\
x \\
4.16 \\
6.92\end{array}$ \\
\hline MONTH & REP & $\begin{array}{c}\mathrm{SO} 4 \\
(\mathrm{mg} / \mathrm{l})\end{array}$ & $\begin{array}{l}\mathrm{Ag} \\
(\mu \mathrm{g} / 1)\end{array}$ & $\begin{array}{l}\text { As } \\
(\mu \mathrm{g} / I)\end{array}$ & & $/ 1)$ & $\frac{\mathrm{Cd}}{(\mu \mathrm{g} / 1)}$ & $\underset{(\mu g / 1)}{\operatorname{Cr}}$ & $\underset{(\mu g / I)}{C u}$ \\
\hline $\begin{array}{l}\text { Jul } \\
\text { Jul } \\
\text { Aug } \\
\text { Aug } \\
\text { Sep } \\
\text { Sep }\end{array}$ & $\begin{array}{l}1 \\
2 \\
1 \\
2 \\
1 \\
2\end{array}$ & $\begin{array}{l}43.3 \\
43.0 \\
52.6 \\
53.2 \\
33.1 \\
40.5\end{array}$ & $\begin{array}{l}x \\
2.10 \\
x \\
x \\
x \\
x\end{array}$ & $\begin{array}{l}x \\
3.50 \\
3.40 \\
x \\
x \\
2.40\end{array}$ & & $\begin{array}{l}3.2 \\
0.2 \\
6.8 \\
9.2 \\
8.2 \\
7.4\end{array}$ & $\begin{array}{l}X \\
X \\
X \\
X \\
X \\
X\end{array}$ & $\begin{array}{l}12.5 \\
12.8 \\
13.3 \\
21.9 \\
23.8 \\
24.4\end{array}$ & $\begin{array}{l}15.9 \\
15.9 \\
15.7 \\
8.20 \\
4.50 \\
4.50\end{array}$ \\
\hline MONTH & REP & $\begin{array}{l}\mathrm{Hg} \\
(\mu \mathrm{g} / 1)\end{array}$ & $\stackrel{\mathrm{Ni}}{(\mu \mathrm{g} / \mathrm{l})}$ & $\begin{array}{c}\mathrm{Pb} \\
(\mu \mathrm{g} /\end{array}$ & (1) & $\begin{array}{c}\text { Se } \\
(\mu \mathrm{g} /\end{array}$ & & $\begin{array}{l}\mathrm{Tl} \\
\mu \mathrm{g} / 1)\end{array}$ & $\underset{(\mu \mathrm{gn} / 1)}{\mathrm{Zn}}$ \\
\hline $\begin{array}{l}\text { Jul } \\
\text { Jul } \\
\text { Aug } \\
\text { Aug } \\
\text { Sep } \\
\text { Sep }\end{array}$ & $\begin{array}{l}1 \\
2 \\
1 \\
2 \\
1 \\
2\end{array}$ & $\begin{array}{l}X \\
X \\
x \\
X \\
X \\
0.21\end{array}$ & $\begin{array}{l}x \\
x \\
x \\
x \\
14.0 \\
x\end{array}$ & $\begin{array}{l}4.2 \\
4.6 \\
7.2 \\
13 . \\
3.5 \\
3.0\end{array}$ & $\begin{array}{l}20 \\
50 \\
20 \\
0 \\
50 \\
0\end{array}$ & $\begin{array}{l}x \\
4.8 \\
x \\
3.1 \\
x \\
X\end{array}$ & $\begin{array}{l}80 \\
10\end{array}$ & $\begin{array}{l}X \\
X \\
X \\
X \\
X \\
X \\
X\end{array}$ & $\begin{array}{l}44.6 \\
41.7 \\
53.9 \\
53.6 \\
27.6 \\
30.7\end{array}$ \\
\hline
\end{tabular}




\section{Table A-51}

SUMMARY STATISTICS

STREAM $=14$ (CFA STP effluent to drain field)

\begin{tabular}{|c|c|c|c|c|c|c|c|}
\hline \multicolumn{2}{|c|}{ ANALYTE } & \multicolumn{2}{|c|}{ FYB7-FY90 } & $\mathrm{N}$ & LEVELI & LEVEL2 & GUIDELINE \\
\hline Cond & $(\mu s)$ & 952 & 260 & 94 & 1390 & 1570 & $\mathrm{~N} / \mathrm{A}$ \\
\hline $\mathrm{pH}$ & & 7.52 & 0.33 & 96 & $\begin{array}{ll}1 & 6.85 \\
\mathrm{u} & 8.19\end{array}$ & $\begin{array}{ll}1 & 6.63 \\
u & 8.40\end{array}$ & $\begin{array}{ll}1 & 6.5 \\
u & 8.5\end{array}$ \\
\hline TOC & $(\mathrm{mg} / \mathrm{I})$ & 15.0 & 13.3 & 52 & 37.5 & 47.3 & $N / A$ \\
\hline $\mathrm{Cl}$ & $(\mathrm{mg} / \mathrm{I})$ & 199 & 67.6 & 94 & 312 & 360 & $N / A$ \\
\hline$F$ & $(\mathrm{mg} / \mathrm{l})$ & 0.57 & 0.61 & 27 & 1.63 & 2.11 & $\mathrm{~N} / \mathrm{A}$ \\
\hline NO3 & $(\mathrm{mg} / \mathrm{l})$ & 18.4 & 8.38 & 89 & 32.5 & 38.4 & $\mathrm{~N} / \mathrm{A}$ \\
\hline $\mathrm{PO} 4$ & $(\mathrm{mg} / \mathrm{l})$ & 5.06 & 4.81 & 88 & 13.1 & 16.5 & $N / A$ \\
\hline SO4 & $(\mathrm{mg} / 1)$ & 46.6 & 10.1 & 94 & 63.5 & 70.6 & $N / A$ \\
\hline Ag & $(\mu g / 1)$ & 9.18 & 4.37 & 11 & 17.5 & 2.8 & 5000 \\
\hline As & $(\mu g / 1)$ & 54.1 & 105 & 11 & 253 & 357 & 5000 \\
\hline $\mathrm{Ba}$ & $(\mu \mathrm{g} / 1)$ & 68.4 & 15.5 & 60 & 94.5 & 106 & 100,000 \\
\hline $\mathrm{Cd}$ & $(\mu g / 1)$ & 6.34 & 1.84 & 11 & 9.82 & 11.7 & 1000 \\
\hline $\mathrm{Cr}$ & $(\mu g / 1)$ & 11.4 & 3.98 & 47 & 18.2 & 21.1 & 5000 \\
\hline $\mathrm{Cu}$ & $(\mu g / 1)$ & 23.5 & 11.8 & 56 & 43.4 & 52.0 & $\mathrm{~N} / \mathrm{A}$ \\
\hline $\mathrm{Hg}$ & $(\mu \mathrm{g} / 1)$ & 0.29 & 0.18 & 38 & 0.60 & 0.73 & 200 \\
\hline $\mathrm{Ni}$ & $(\mu g / I)$ & 17.2 & 10.6 & 12 & 37.0 & 47.1 & $\mathrm{~N} / \mathrm{A}$ \\
\hline $\mathrm{Pb}$ & $(\mu g / I)$ & 23.6 & 36.2 & 39 & 85.5 & 113 & 5000 \\
\hline $\mathrm{Se}$ & $(\mu g / 1)$ & & & & & & 1000 \\
\hline $\mathrm{T} 1$ & $(\mu g / I)$ & & & & & & $\mathrm{N} / \mathrm{A}$ \\
\hline $\mathrm{Zn}$ & $(\mu g / 1)$ & 70.5 & 31.4 & 93 & 123 & 145 & $N / A$ \\
\hline
\end{tabular}




\section{Table A-52}

QUARTERLY MEANS AND VARIANCE COMPONENTS STREAM=14 (CFA STP effluent to drain field)

\begin{tabular}{|c|c|c|c|c|c|c|c|c|c|}
\hline \multicolumn{2}{|c|}{ ANALYTE } & QTR & MEAN & $\begin{array}{r}\text { STD } \\
\text { MEAN }\end{array}$ & $\begin{array}{l}\text { STD } \\
\text { DAY }\end{array}$ & $\begin{array}{l}\text { STD } \\
\text { REP }\end{array}$ & $\mathrm{N}$ & $\begin{array}{r}\mathrm{N} \\
\text { MISS }\end{array}$ & BPQI \\
\hline Cond & $(\mu S)$ & $\begin{array}{l}91 q 1 \\
91 q 2 \\
91 q 3 \\
91 q 4\end{array}$ & $\begin{array}{r}1266 \\
1592 \\
1082 \\
668\end{array}$ & $\begin{array}{l}330 \\
261 \\
238 \\
309\end{array}$ & $\begin{array}{l}464 \\
445 \\
248 \\
534\end{array}$ & $\begin{array}{r}65.2 \\
120 \\
465 \\
42.7\end{array}$ & $\begin{array}{l}4 \\
6 \\
6 \\
6\end{array}$ & $\begin{array}{l}2 \\
0 \\
0 \\
0\end{array}$ & $\begin{array}{l}0 \\
0 \\
0 \\
0\end{array}$ \\
\hline $\mathrm{pH}$ & & $\begin{array}{l}91 q 1 \\
91 q 2 \\
91 q 3 \\
91 q 4\end{array}$ & $\begin{array}{l}8.16 \\
7.83 \\
7.43 \\
7.90\end{array}$ & $\begin{array}{l}0.97 \\
0.28 \\
0.27 \\
0.10\end{array}$ & $\begin{array}{l}1.34 \\
0.31 \\
0.00 \\
0.14\end{array}$ & $\begin{array}{l}0.37 \\
0.53 \\
1.64 \\
0.16\end{array}$ & $\begin{array}{l}4 \\
6 \\
6 \\
6\end{array}$ & $\begin{array}{l}2 \\
0 \\
0 \\
0\end{array}$ & $\begin{array}{l}0 \\
0 \\
0 \\
0\end{array}$ \\
\hline TOC & $(\mathrm{mg} / \mathrm{l})$ & $\begin{array}{l}91 q 1 \\
91 q 2 \\
91 q^{3} \\
91 q^{4}\end{array}$ & $\begin{array}{l}17.2 \\
18.3 \\
12.1 \\
7.02\end{array}$ & $\begin{array}{l}5.43 \\
11.1 \\
4.71 \\
1.67\end{array}$ & $\begin{array}{l}8.54 \\
15.7 \\
7.64 \\
2.88\end{array}$ & $\begin{array}{l}5.56 \\
1.71 \\
4.04 \\
0.33\end{array}$ & $\begin{array}{l}6 \\
4 \\
6 \\
6\end{array}$ & $\begin{array}{l}0 \\
2 \\
0 \\
0\end{array}$ & $\begin{array}{l}0 \\
0 \\
0 \\
0\end{array}$ \\
\hline $\mathrm{Cl}$ & $(\mathrm{mg} / \mathrm{l})$ & $\begin{array}{l}91 q 1 \\
91 q 2 \\
91 q 3 \\
91 q 4\end{array}$ & $\begin{array}{l}241 \\
322 \\
235 \\
207\end{array}$ & $\begin{array}{l}61.2 \\
74.1 \\
15.8 \\
25.3\end{array}$ & $\begin{array}{r}106 \\
123 \\
21.4 \\
43.1\end{array}$ & $\begin{array}{l}5.51 \\
7.50 \\
24.0 \\
11.0\end{array}$ & $\begin{array}{l}6 \\
5 \\
6 \\
6\end{array}$ & $\begin{array}{l}0 \\
1 \\
0 \\
0\end{array}$ & $\begin{array}{l}0 \\
0 \\
0 \\
0\end{array}$ \\
\hline$F$ & $(\mathrm{mg} / \mathrm{l})$ & $\begin{array}{l}91 q 1 \\
91 q 2 \\
91 q 3 \\
91 q 4\end{array}$ & $\begin{array}{l}\mathrm{X} \\
0.06 \\
0.23 \\
0.04\end{array}$ & $\begin{array}{l}0.21 \\
0.01 \\
0.17\end{array}$ & $\begin{array}{l}0.35 \\
0.02 \\
0.09\end{array}$ & $\begin{array}{l}\dot{0} .00 \\
0.00 \\
0.40\end{array}$ & $\begin{array}{l}0 \\
5 \\
6 \\
6\end{array}$ & $\begin{array}{l}0 \\
1 \\
0 \\
0\end{array}$ & $\begin{array}{l}6 \\
0 \\
0 \\
0\end{array}$ \\
\hline NO3 & $(\mathrm{mg} / \mathrm{l})$ & $\begin{array}{l}91 q 1 \\
91 q 2 \\
91 q^{3} \\
91 q^{4}\end{array}$ & $\begin{array}{l}10.4 \\
2.87 \\
5.41 \\
9.44\end{array}$ & $\begin{array}{l}1.54 \\
0.93 \\
1.23 \\
1.48\end{array}$ & $\begin{array}{l}2.56 \\
1.30 \\
2.13 \\
0.47\end{array}$ & $\begin{array}{l}0.00 \\
0.21 \\
0.13 \\
3.57\end{array}$ & $\begin{array}{l}5 \\
4 \\
6 \\
6\end{array}$ & $\begin{array}{l}0 \\
2 \\
0 \\
0\end{array}$ & $\begin{array}{l}1 \\
0 \\
0 \\
0\end{array}$ \\
\hline PO4 & $(\mathrm{mg} / \mathrm{l})$ & $\begin{array}{l}91 q 1 \\
91 q 2 \\
91 q 3 \\
91 q 4\end{array}$ & $\begin{array}{l}3.58 \\
4.00 \\
7.67 \\
8.05\end{array}$ & $\begin{array}{l}0.46 \\
1.89 \\
1.54\end{array}$ & $\begin{array}{l}0.00 \\
3.25 \\
2.34\end{array}$ & $\begin{array}{l}1.31 \\
0.49 \\
1.42\end{array}$ & $\begin{array}{l}6 \\
3 \\
6 \\
5\end{array}$ & $\begin{array}{l}0 \\
0 \\
0 \\
1\end{array}$ & $\begin{array}{l}0 \\
3 \\
0 \\
0\end{array}$ \\
\hline SO4 & $(\mathrm{mg} / \mathrm{I})$ & $\begin{array}{l}91 q 1 \\
91 q 2 \\
91 q 3 \\
91 q^{4}\end{array}$ & $\begin{array}{l}48.0 \\
56.6 \\
48.4 \\
44.3\end{array}$ & $\begin{array}{l}3.58 \\
1.68 \\
3.91 \\
4.68\end{array}$ & $\begin{array}{l}6.20 \\
2.69 \\
6.08 \\
7.82\end{array}$ & $\begin{array}{l}0.26 \\
1.05 \\
4.23 \\
3.03\end{array}$ & $\begin{array}{l}6 \\
5 \\
6 \\
6\end{array}$ & $\begin{array}{l}0 \\
1 \\
0 \\
0\end{array}$ & $\begin{array}{l}0 \\
0 \\
0 \\
0\end{array}$ \\
\hline Ag & $(\mu g / 1)$ & $\begin{array}{l}91 q 1 \\
91 q 2 \\
91 q^{3} \\
91 q^{4}\end{array}$ & $\begin{array}{l}X \\
X \\
X \\
2.10\end{array}$ & $\dot{\dot{.}}$ & $\begin{array}{l}\dot{\bullet} \\
\dot{.}\end{array}$ & $\dot{.}$ & $\begin{array}{l}0 \\
0 \\
0 \\
1\end{array}$ & $\begin{array}{l}0 \\
0 \\
0 \\
0\end{array}$ & $\begin{array}{l}6 \\
6 \\
6 \\
5\end{array}$ \\
\hline
\end{tabular}


Table A-52 (continued)

QUARTERIY MEANS AND VARIANCE COMPONENTS

STREAM=14 (CFA STP effluent to drain field) (continued)

\begin{tabular}{|c|c|c|c|c|c|c|c|c|c|}
\hline \multicolumn{2}{|c|}{ ANALYTE } & QTR & MEAN & $\begin{array}{r}\text { STD } \\
\text { MEAN }\end{array}$ & $\begin{array}{l}\text { STD } \\
\text { DAY }\end{array}$ & $\begin{array}{l}\text { STD } \\
\text { REP }\end{array}$ & N & $\begin{array}{r}\mathrm{N} \\
\text { MISS }\end{array}$ & $\begin{array}{r}\mathbf{N} \\
\text { BPQI }\end{array}$ \\
\hline As & $(\mu g / 1)$ & $\begin{array}{l}91 q 1 \\
91 q 2 \\
91 q 3 \\
91 q 4\end{array}$ & $\begin{array}{l}1.60 \\
X \\
X \\
3.10\end{array}$ & $\begin{array}{l}0.31 \\
: \\
\dot{0}\end{array}$ & $\begin{array}{l}0.50 \\
\dot{0} \\
\dot{0}\end{array}$ & $\begin{array}{l}0.28 \\
: \\
:\end{array}$ & $\begin{array}{l}5 \\
0 \\
0 \\
3\end{array}$ & $\begin{array}{l}0 \\
0 \\
0 \\
0\end{array}$ & $\begin{array}{l}1 \\
6 \\
6 \\
3\end{array}$ \\
\hline $\mathrm{Ba}$ & $(\mu g / 1)$ & $\begin{array}{l}91 q 1 \\
91 q^{2} \\
91 q^{3} \\
91 q^{4}\end{array}$ & $\begin{array}{l}49.2 \\
7.00 \\
72.3 \\
72.5\end{array}$ & $\begin{array}{l}6.08 \\
\dot{6} .25 \\
2.97\end{array}$ & $\begin{array}{l}10.4 \\
10.8 \\
5.02\end{array}$ & $\begin{array}{l}2.02 \\
\dot{0} .53 \\
1.60\end{array}$ & $\begin{array}{l}6 \\
1 \\
6 \\
6\end{array}$ & $\begin{array}{l}0 \\
0 \\
0 \\
0\end{array}$ & $\begin{array}{l}0 \\
5 \\
0 \\
0\end{array}$ \\
\hline $\mathrm{cd}$ & $(\mu g / 1)$ & $\begin{array}{l}91 q 1 \\
91 q 2 \\
91 q^{3} \\
91 q^{4}\end{array}$ & $\begin{array}{l}5.90 \\
x \\
X \\
x\end{array}$ & $\dot{.}$ & $\dot{:}$ & $\begin{array}{l}\dot{ } \\
\dot{.}\end{array}$ & $\begin{array}{l}1 \\
0 \\
0 \\
0\end{array}$ & $\begin{array}{l}0 \\
0 \\
0 \\
0\end{array}$ & $\begin{array}{l}5 \\
6 \\
6 \\
6\end{array}$ \\
\hline $\mathrm{Cr}$ & $(\mu g / 1)$ & $\begin{array}{l}91 q 1 \\
91 q 2 \\
91 q 3 \\
91 q 4\end{array}$ & $\begin{array}{l}8.92 \\
5.04 \\
3.93 \\
18.1\end{array}$ & $\begin{array}{l}0.96 \\
5.67 \\
5.06 \\
3.32\end{array}$ & $\begin{array}{l}1.12 \\
9.43 \\
8.76 \\
5.17\end{array}$ & $\begin{array}{l}1.54 \\
0.70 \\
0.49 \\
3.52\end{array}$ & $\begin{array}{l}5 \\
5 \\
6 \\
6\end{array}$ & $\begin{array}{l}0 \\
1 \\
0 \\
0\end{array}$ & $\begin{array}{l}1 \\
0 \\
0 \\
0\end{array}$ \\
\hline $\mathrm{Cu}$ & $(\mu g / 1)$ & $\begin{array}{l}91 q 1 \\
91 q^{2} \\
91 q^{3} \\
91 q^{4}\end{array}$ & $\begin{array}{l}13.8 \\
18.9 \\
5.77 \\
10.8\end{array}$ & $\begin{array}{l}3.00 \\
4.65 \\
5.40 \\
3.34\end{array}$ & $\begin{array}{l}5.13 \\
7.73 \\
9.30 \\
5.37\end{array}$ & $\begin{array}{l}1.23 \\
0.51 \\
1.43 \\
3.06\end{array}$ & $\begin{array}{l}6 \\
5 \\
6 \\
6\end{array}$ & $\begin{array}{l}0 \\
1 \\
0 \\
0\end{array}$ & $\begin{array}{l}0 \\
0 \\
0 \\
0\end{array}$ \\
\hline $\mathrm{Hg}$ & $(\mu g / I)$ & $\begin{array}{l}91 q 1 \\
91 q^{2} \\
91 q^{3} \\
91 q^{4}\end{array}$ & $\begin{array}{l}0.22 \\
X \\
X \\
0.21\end{array}$ & $\begin{array}{l}0.09 \\
\dot{:} \\
\dot{0}\end{array}$ & $\begin{array}{c}0.12 \\
: \\
.\end{array}$ & $\begin{array}{c}0.02 \\
. \\
.\end{array}$ & $\begin{array}{l}4 \\
0 \\
0 \\
1\end{array}$ & $\begin{array}{l}0 \\
0 \\
0 \\
0\end{array}$ & $\begin{array}{l}2 \\
6 \\
6 \\
5\end{array}$ \\
\hline $\mathrm{Ni}$ & $(\mu g / I)$ & $\begin{array}{l}91 q 1 \\
91 q 2 \\
91 q 3 \\
91 q 4\end{array}$ & $\begin{array}{l}17.4 \\
X \\
X \\
14.0\end{array}$ & $\dot{\dot{.}}$ & $\dot{\dot{ }}$ & $\dot{:}$ & $\begin{array}{l}2 \\
0 \\
0 \\
1\end{array}$ & $\begin{array}{l}0 \\
0 \\
0 \\
0\end{array}$ & $\begin{array}{l}4 \\
6 \\
6 \\
5\end{array}$ \\
\hline $\mathrm{Pb}$ & $(\mu g / l)$ & $\begin{array}{l}91 q 1 \\
91 q 2 \\
91 q^{3} \\
91 q^{4}\end{array}$ & $\begin{array}{l}5.20 \\
8.12 \\
3.15 \\
5.92\end{array}$ & $\begin{array}{l}0.77 \\
1.61 \\
2.76 \\
2.12\end{array}$ & $\begin{array}{l}1.22 \\
2.18 \\
4.67 \\
3.26\end{array}$ & $\begin{array}{l}0.72 \\
2.10 \\
1.44 \\
2.38\end{array}$ & $\begin{array}{l}6 \\
5 \\
6 \\
6\end{array}$ & $\begin{array}{l}0 \\
1 \\
0 \\
0\end{array}$ & $\begin{array}{l}0 \\
0 \\
0 \\
0\end{array}$ \\
\hline se & $(\mu g / I)$ & $\begin{array}{l}91 q 1 \\
91 q^{2} \\
91 q^{3} \\
91 q^{4}\end{array}$ & $\begin{array}{l}x \\
x \\
5.27 \\
3.95\end{array}$ & $\begin{array}{l}\cdot \\
\dot{\cdot}\end{array}$ & $\dot{.}$ & $\begin{array}{l}\dot{.} \\
\dot{.}\end{array}$ & $\begin{array}{l}0 \\
0 \\
3 \\
2\end{array}$ & $\begin{array}{l}0 \\
0 \\
0 \\
0\end{array}$ & $\begin{array}{l}6 \\
6 \\
3 \\
4\end{array}$ \\
\hline
\end{tabular}


Table A-52 (continued)

QUARTERLY MEANS AND VARIANCE COMPONENTS

STREAM=14 (CFA STP effluent to drain field) (continued)

\begin{tabular}{|c|c|c|c|c|c|c|c|c|c|}
\hline \multicolumn{2}{|c|}{ ANALYTE } & QTR & MEAN & $\begin{array}{r}\text { STD } \\
\text { MEAN }\end{array}$ & $\begin{array}{l}\text { STD } \\
\text { DAY }\end{array}$ & $\begin{array}{l}\text { STD } \\
\text { REP }\end{array}$ & & $\begin{array}{r}\mathbf{N} \\
\mathbf{S S}\end{array}$ & $\begin{array}{r}\mathrm{N} \\
\text { BPQL }\end{array}$ \\
\hline TI & $(\mu g / 1)$ & $\begin{array}{l}91 q 1 \\
91 q 2 \\
91 q 3 \\
91 q 4\end{array}$ & $\begin{array}{l}0.66 \\
x \\
2.30 \\
x\end{array}$ & $\dot{\dot{ }}$ & $\dot{\dot{.}}$ & $\dot{\bullet}$ & $\begin{array}{l}1 \\
0 \\
1 \\
0\end{array}$ & $\begin{array}{l}0 \\
0 \\
0 \\
0\end{array}$ & $\begin{array}{l}5 \\
6 \\
5 \\
6\end{array}$ \\
\hline $\mathrm{zn}$ & $(\mu \mathrm{g} / 1)$ & $\begin{array}{l}91 q 1 \\
91 q 2 \\
91 q 3 \\
91 q 4\end{array}$ & $\begin{array}{l}48.9 \\
92.6 \\
47.1 \\
42.0\end{array}$ & $\begin{array}{l}9.23 \\
7.21 \\
12.0 \\
7.12\end{array}$ & $\begin{array}{l}15.8 \\
11.3 \\
20.8 \\
12.3\end{array}$ & $\begin{array}{l}3.76 \\
5.55 \\
1.93 \\
1.74\end{array}$ & $\begin{array}{l}6 \\
5 \\
6 \\
6\end{array}$ & $\begin{array}{l}0 \\
1 \\
0 \\
0\end{array}$ & $\begin{array}{l}0 \\
0 \\
0 \\
0\end{array}$ \\
\hline
\end{tabular}




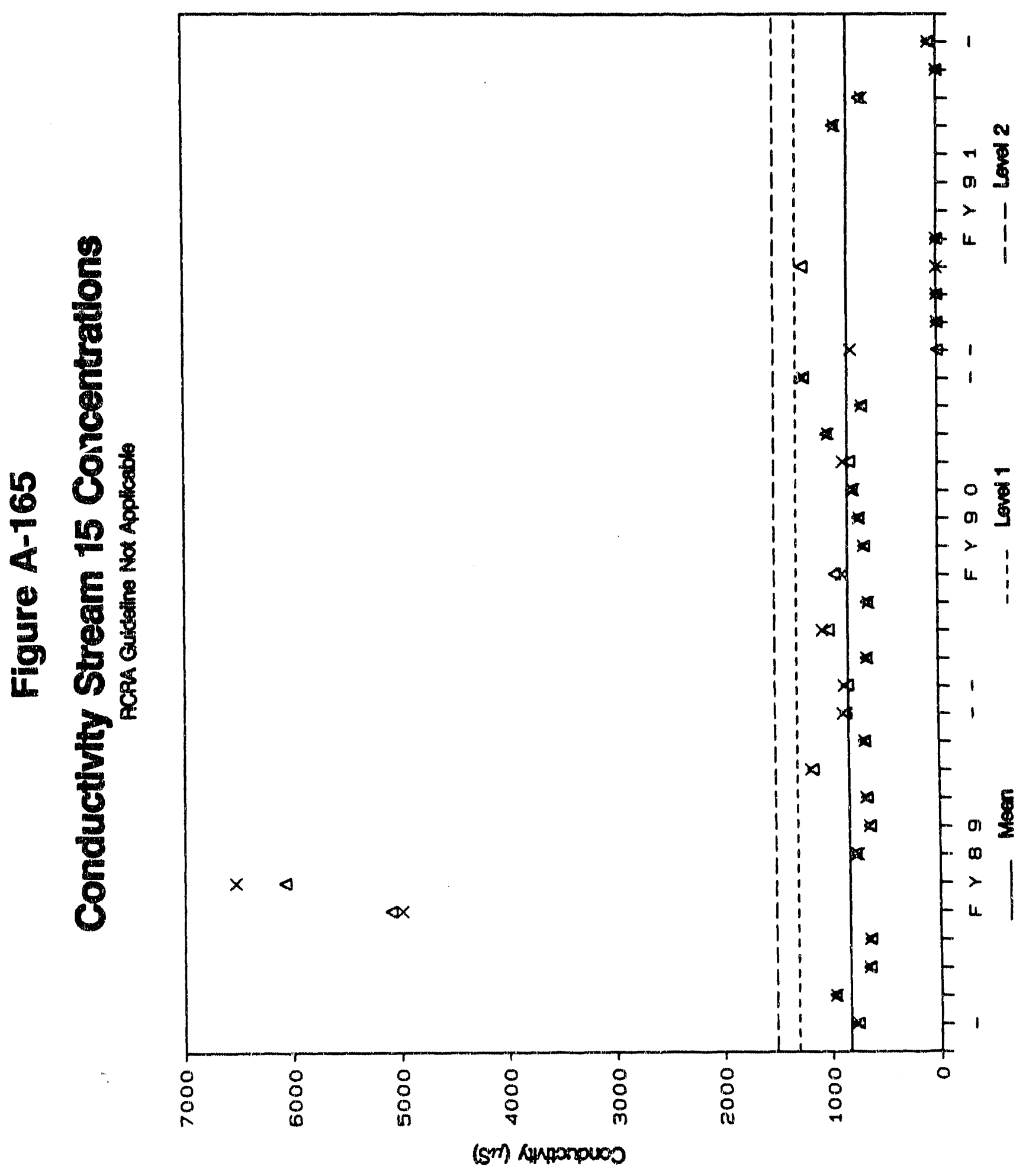




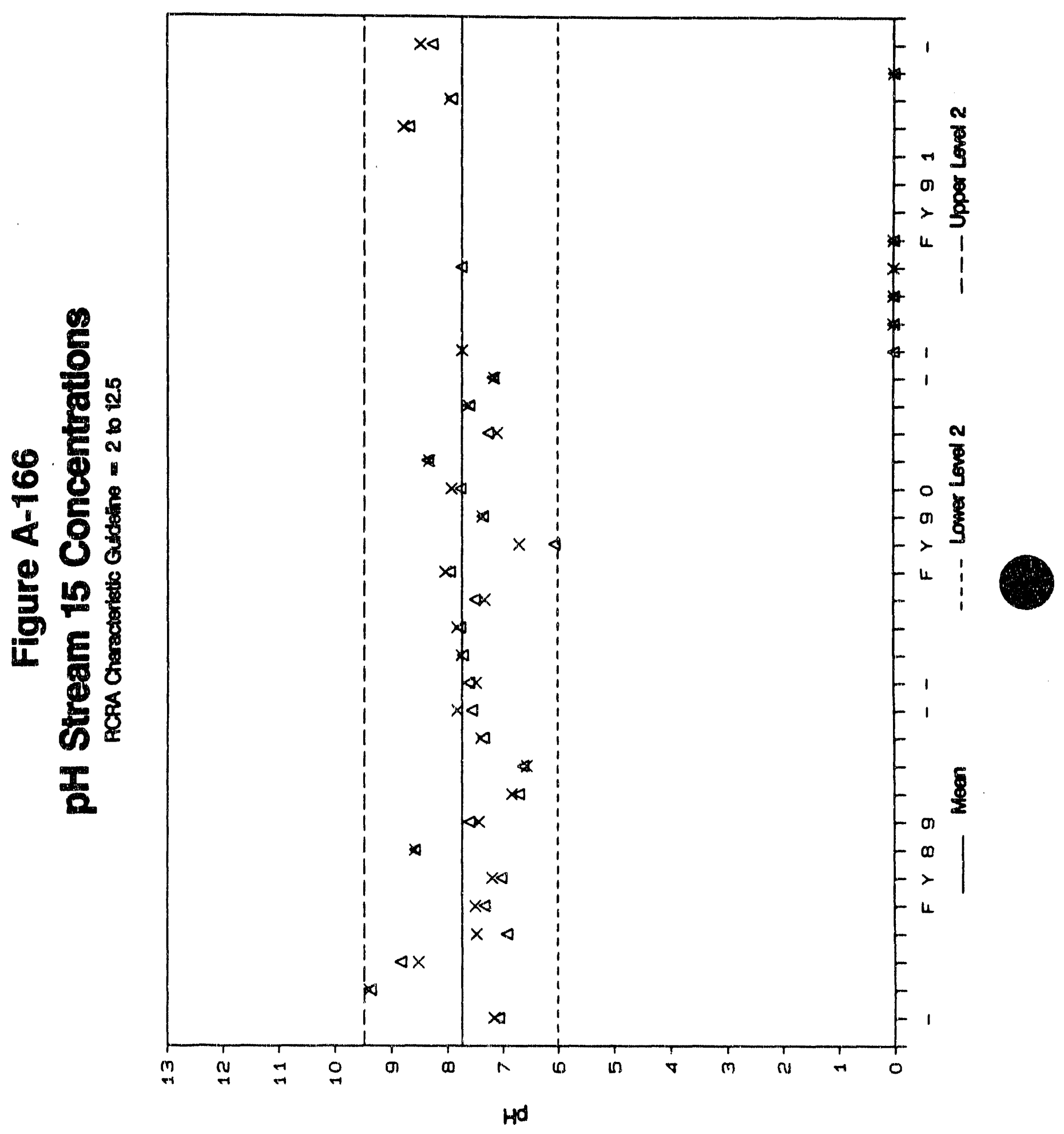

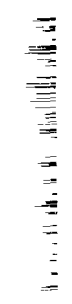




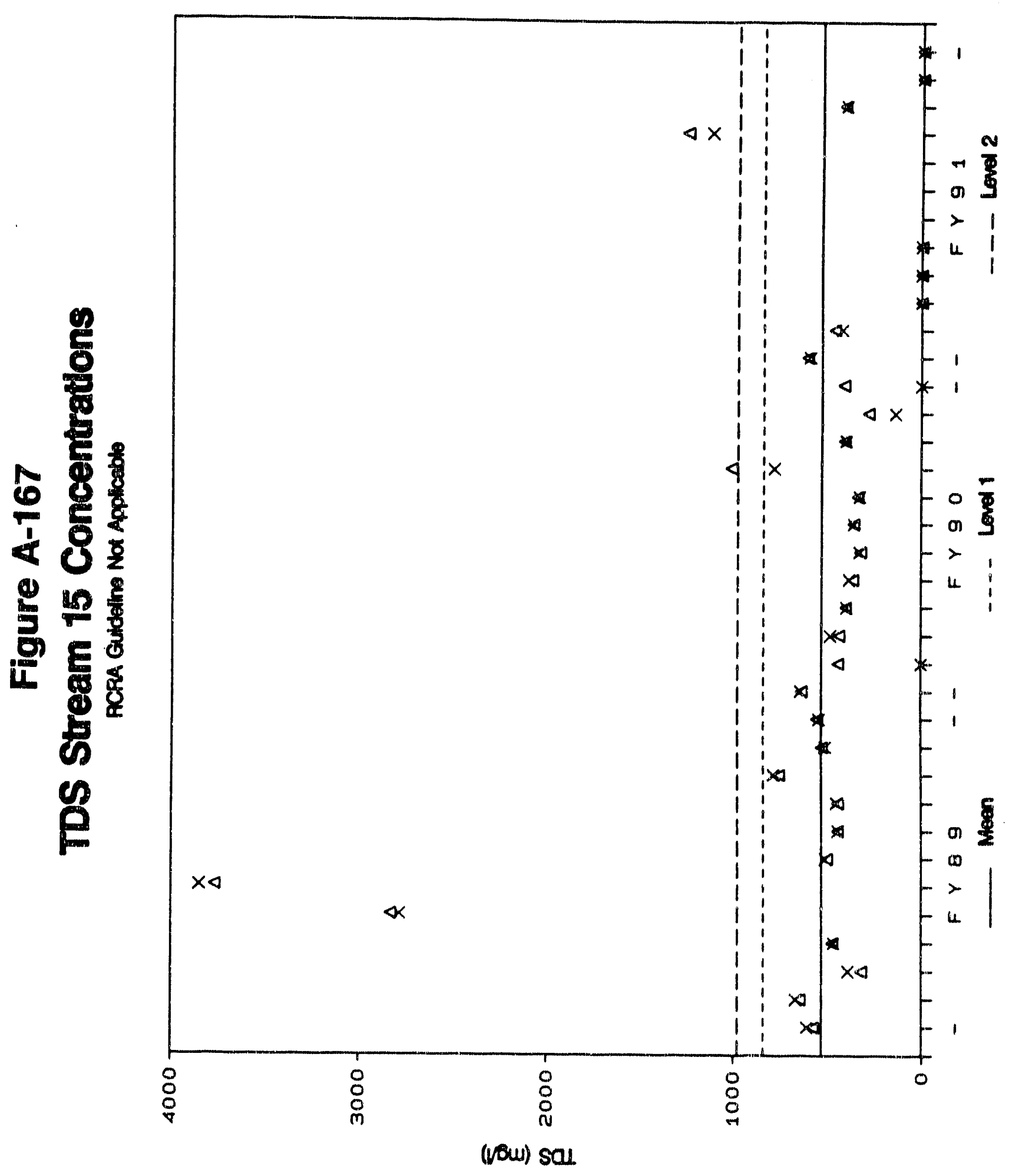




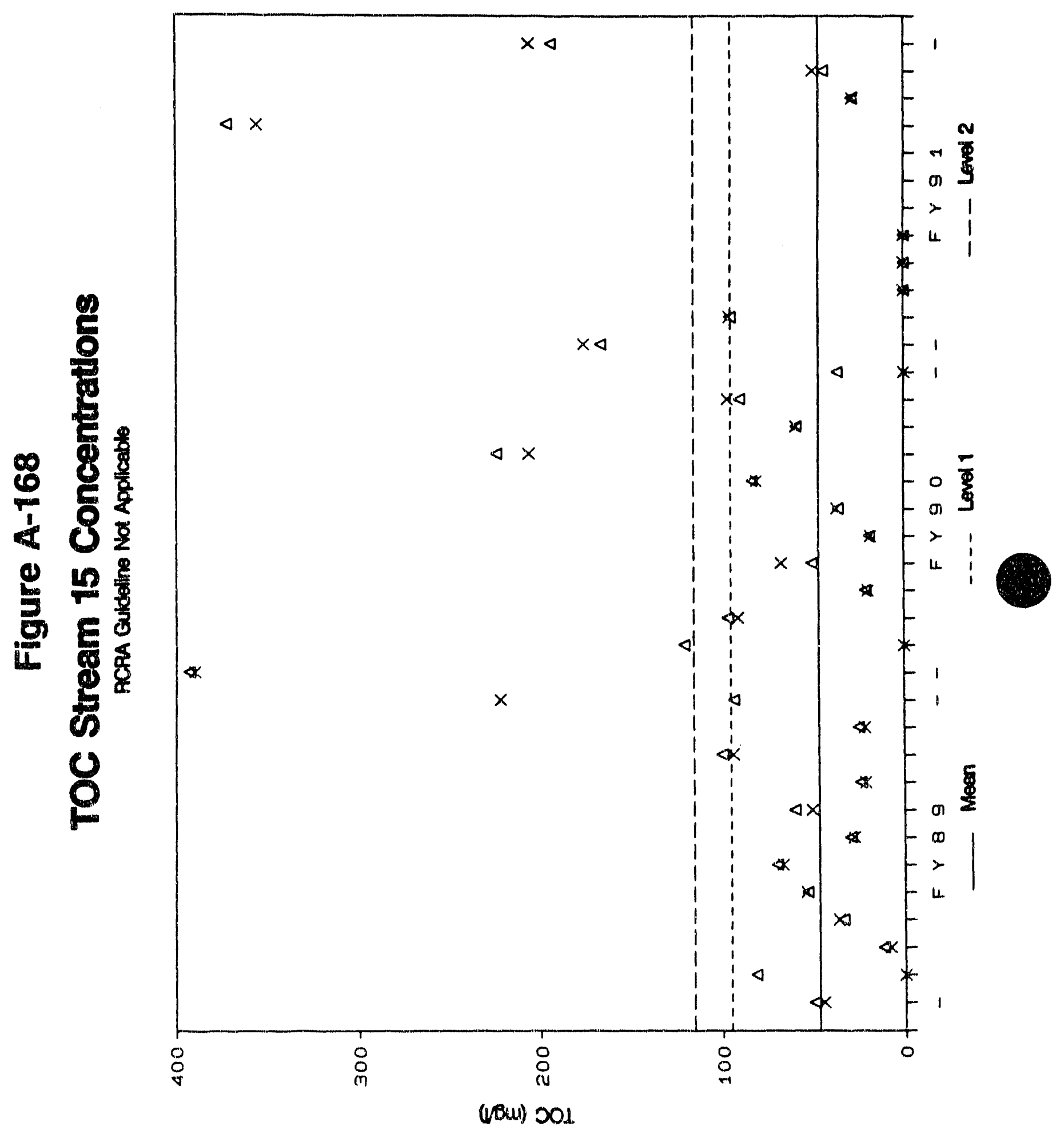




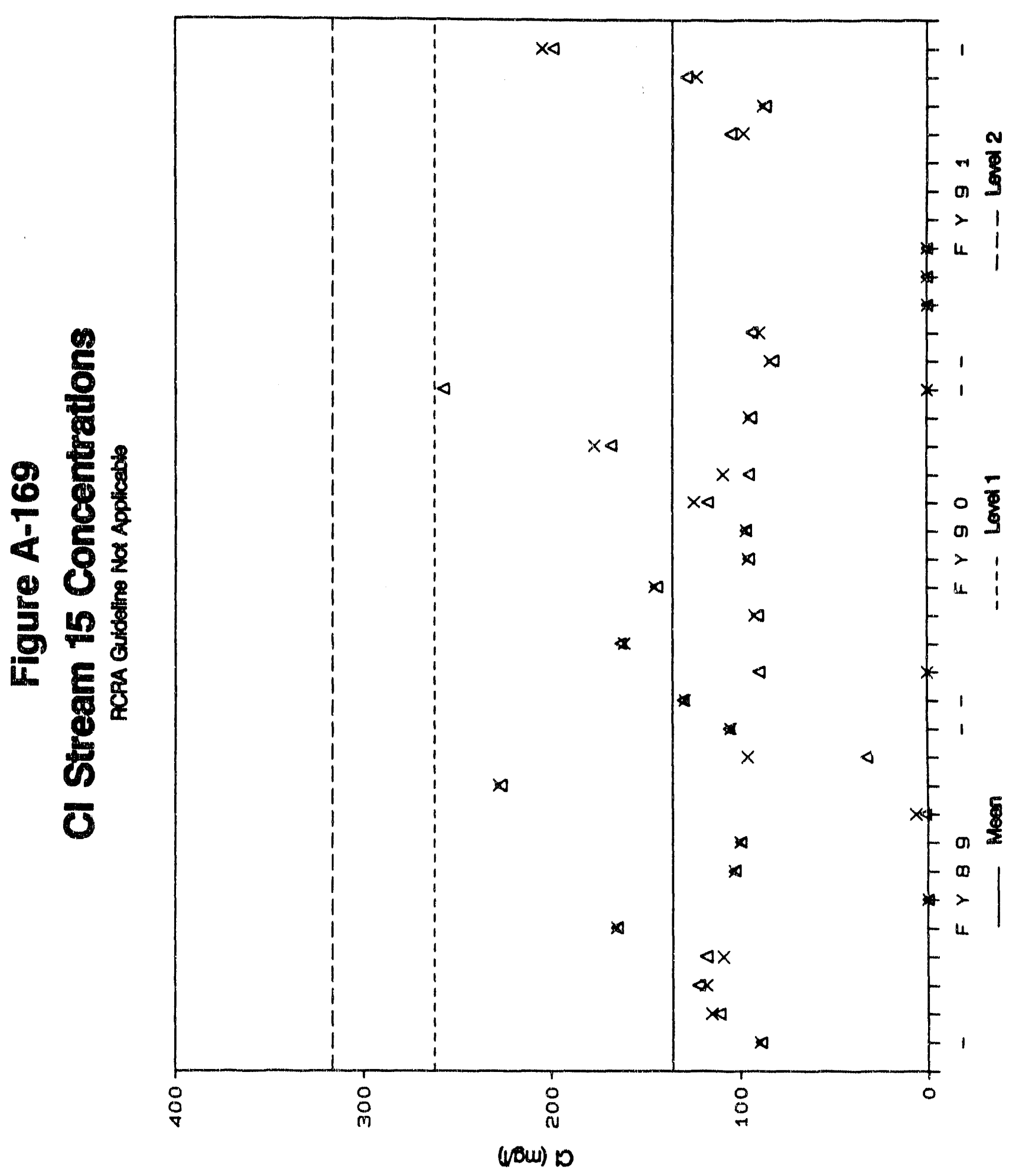



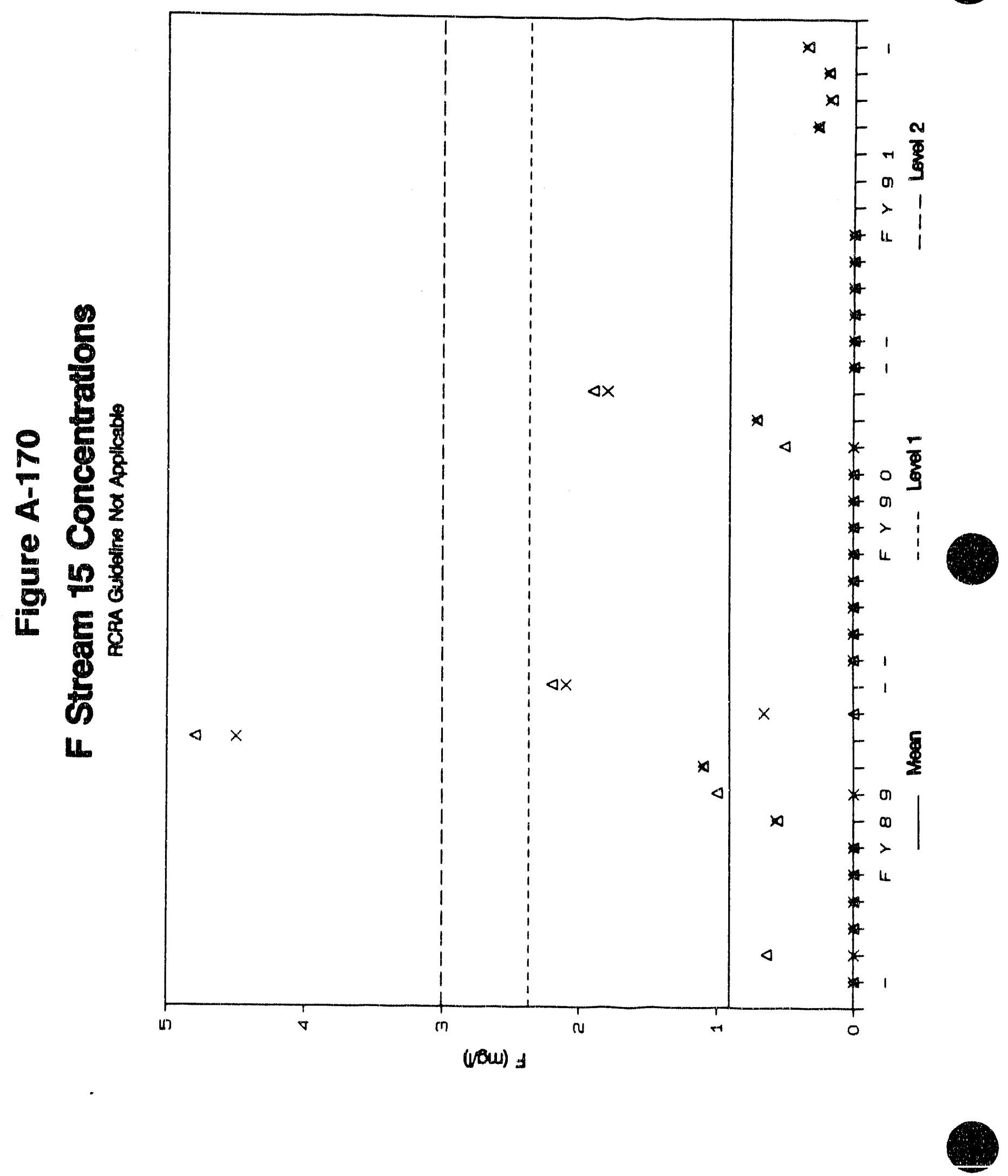


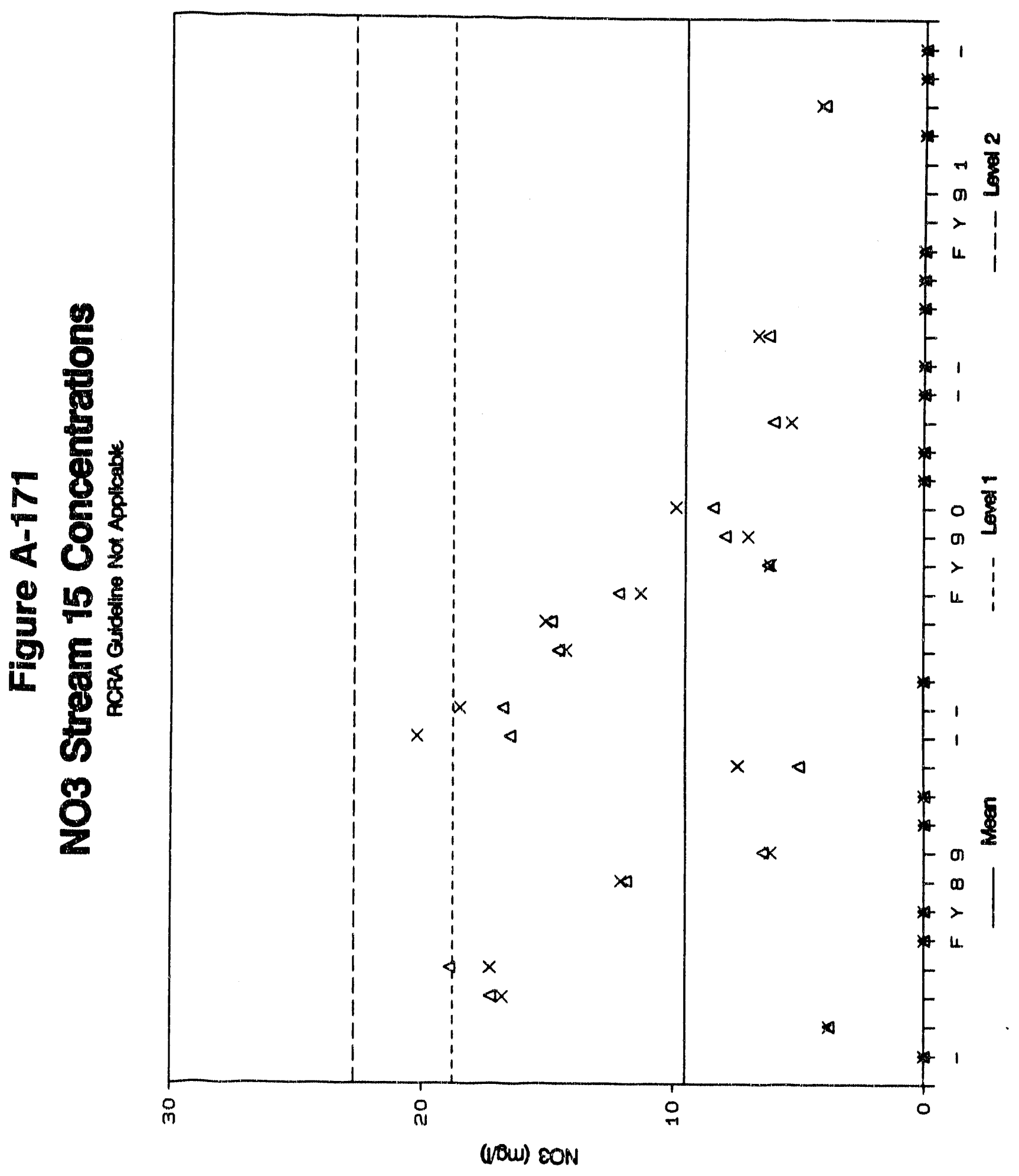




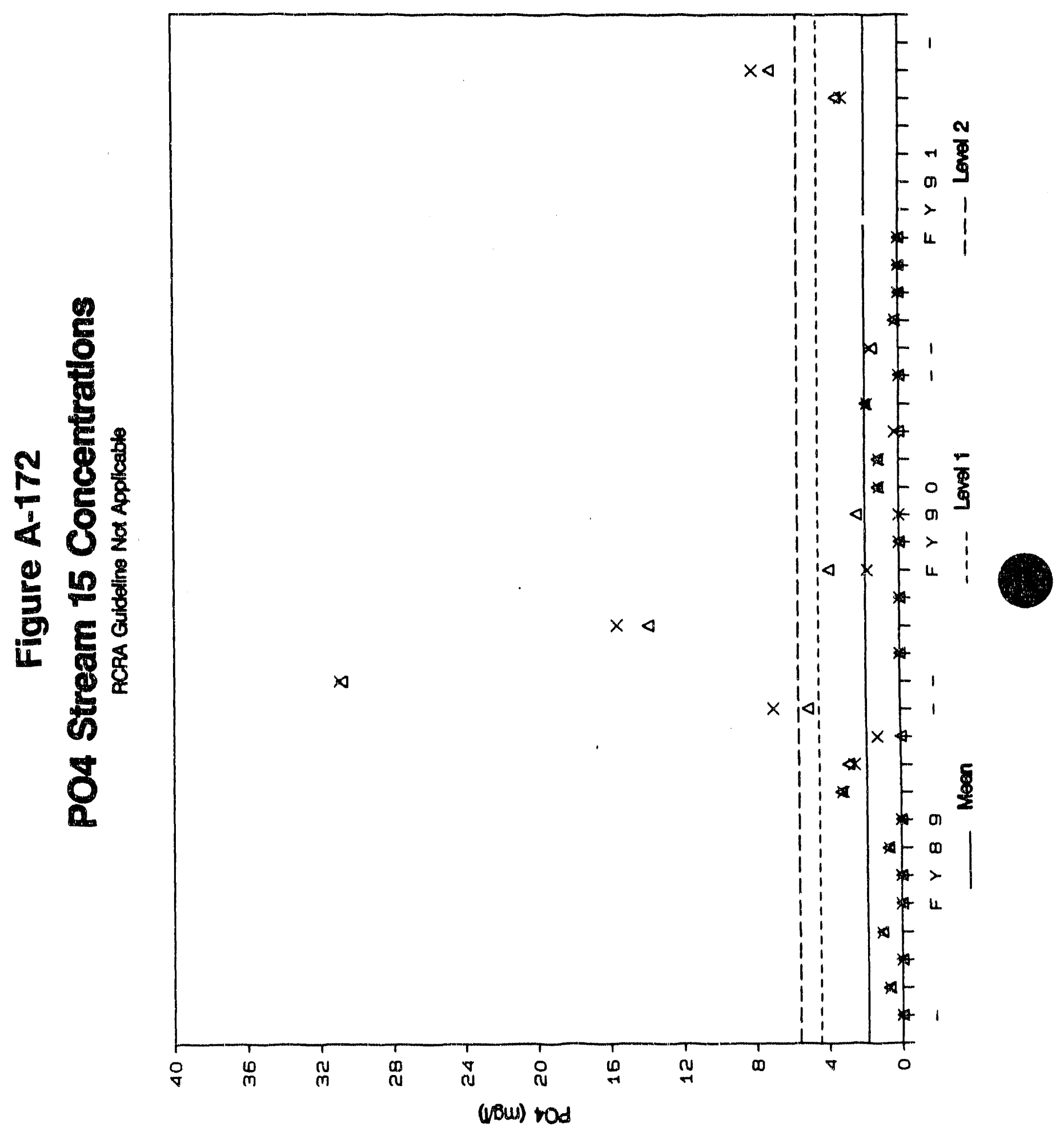




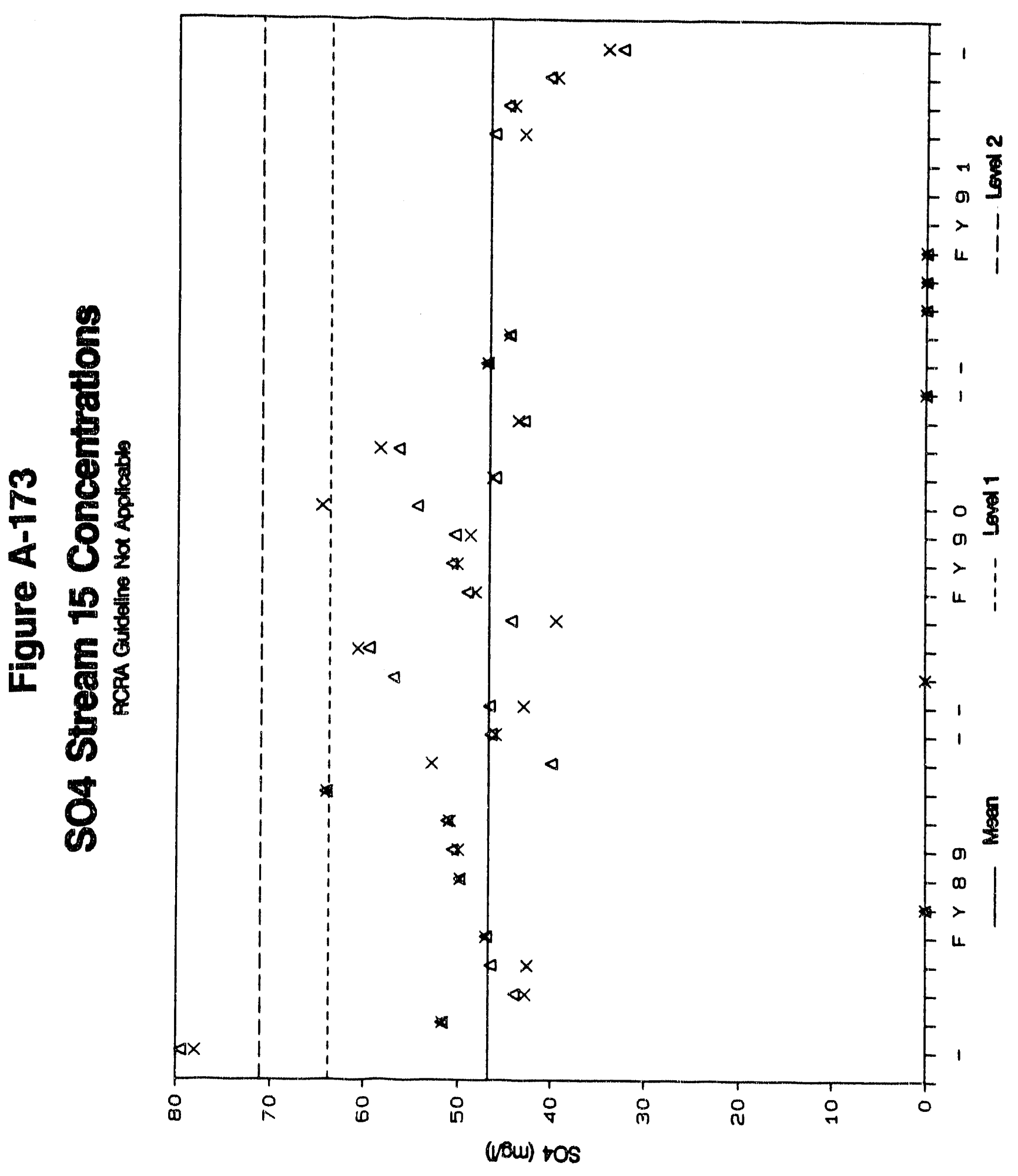




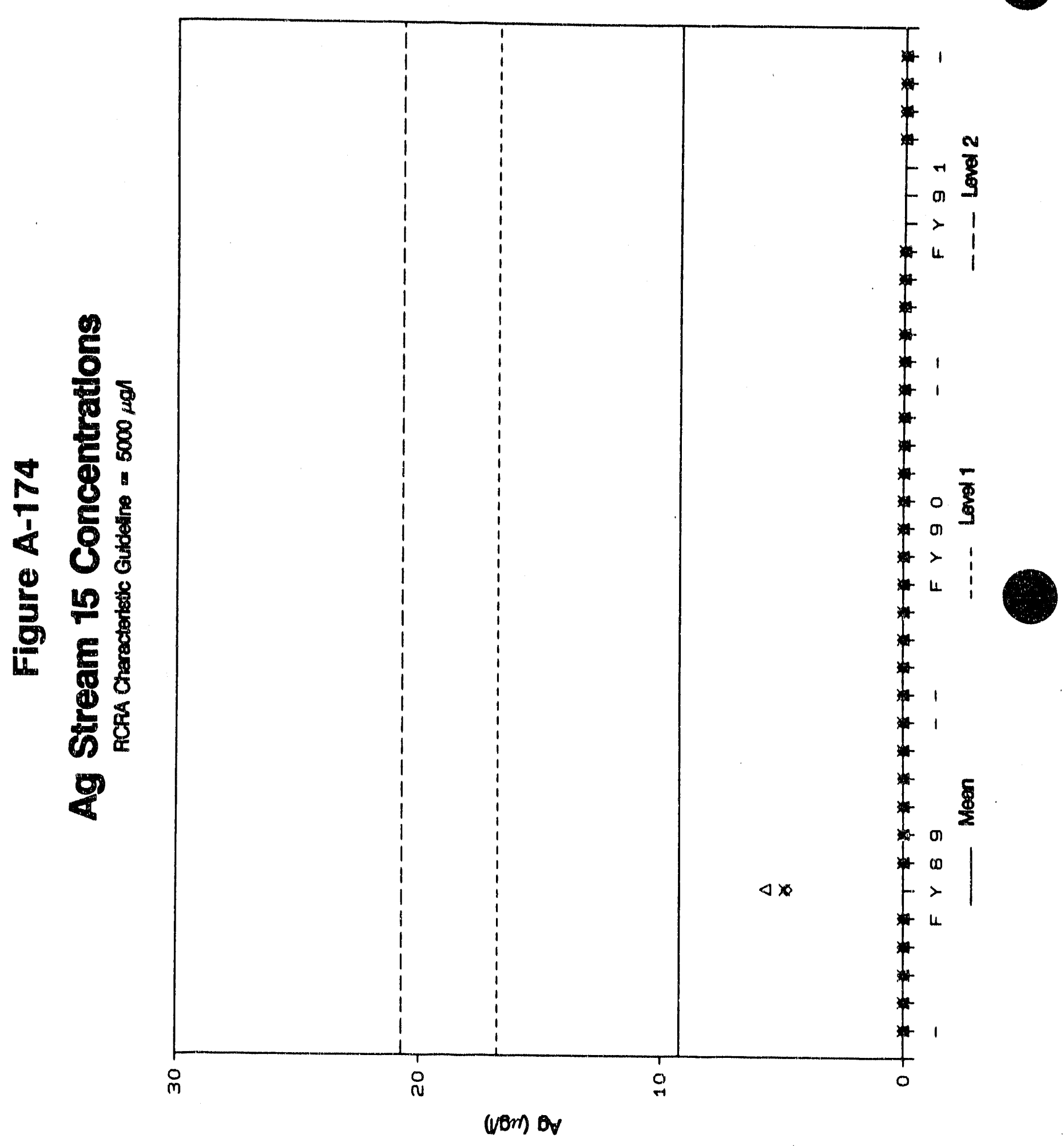

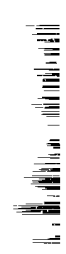




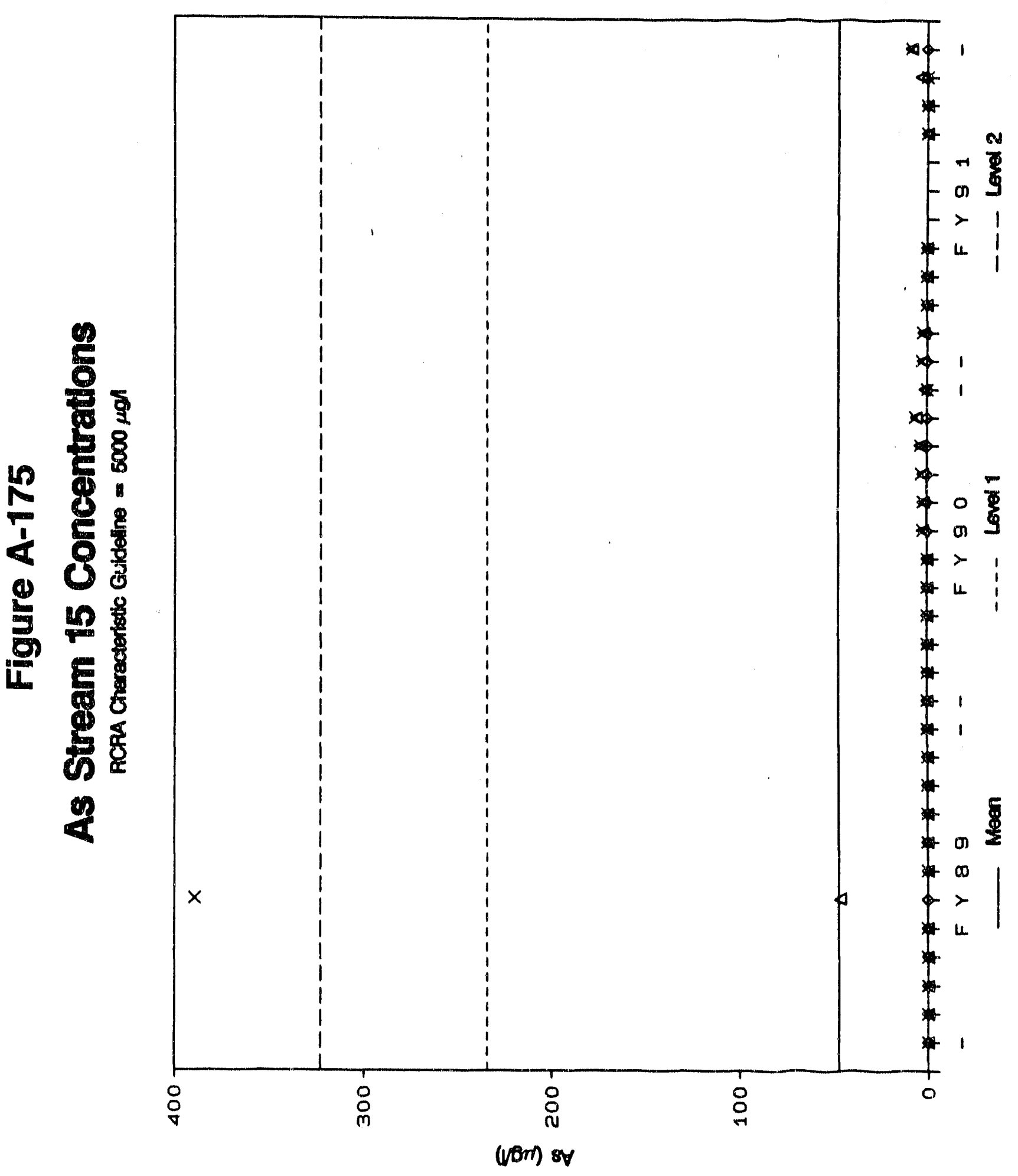




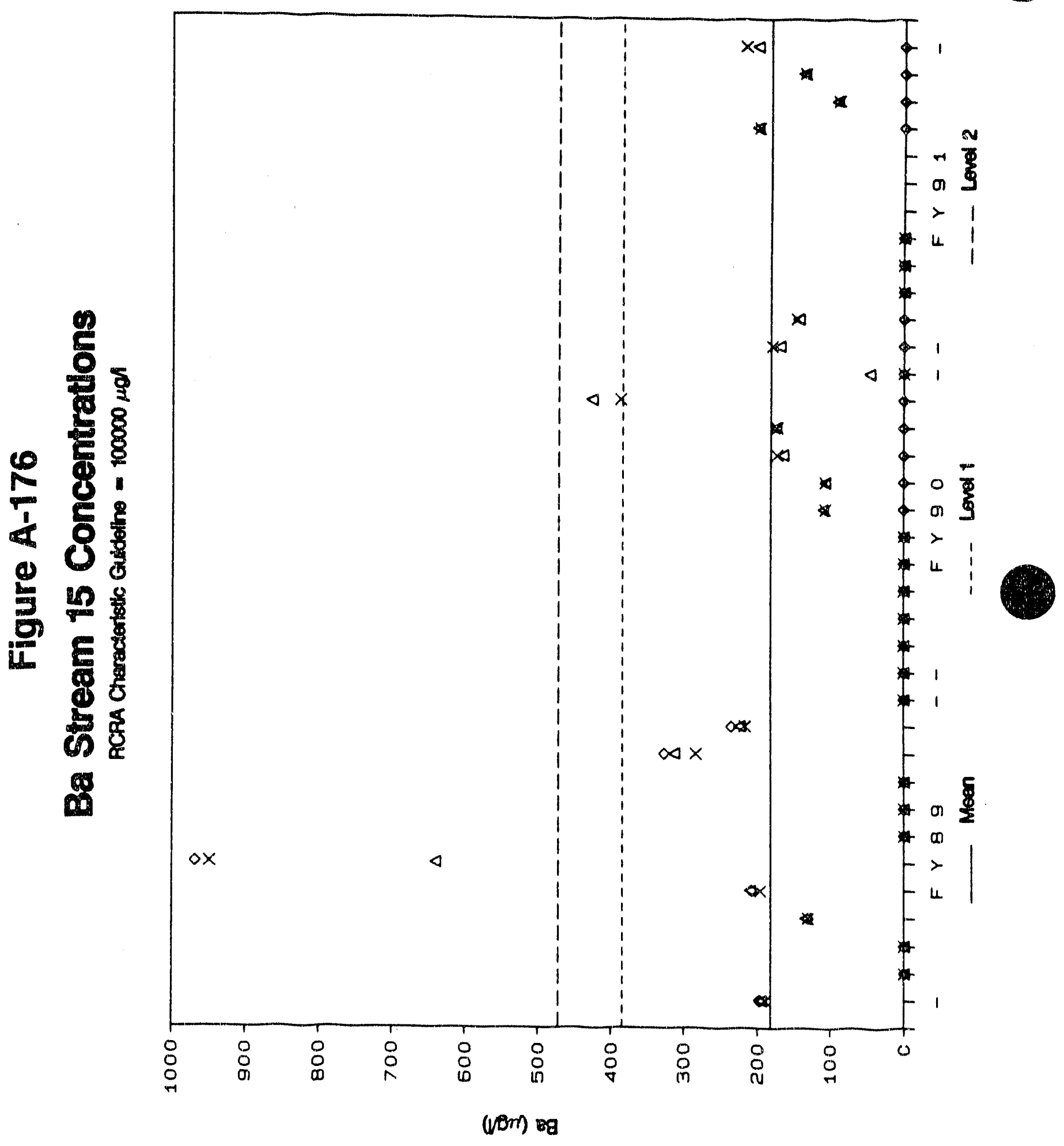

- 


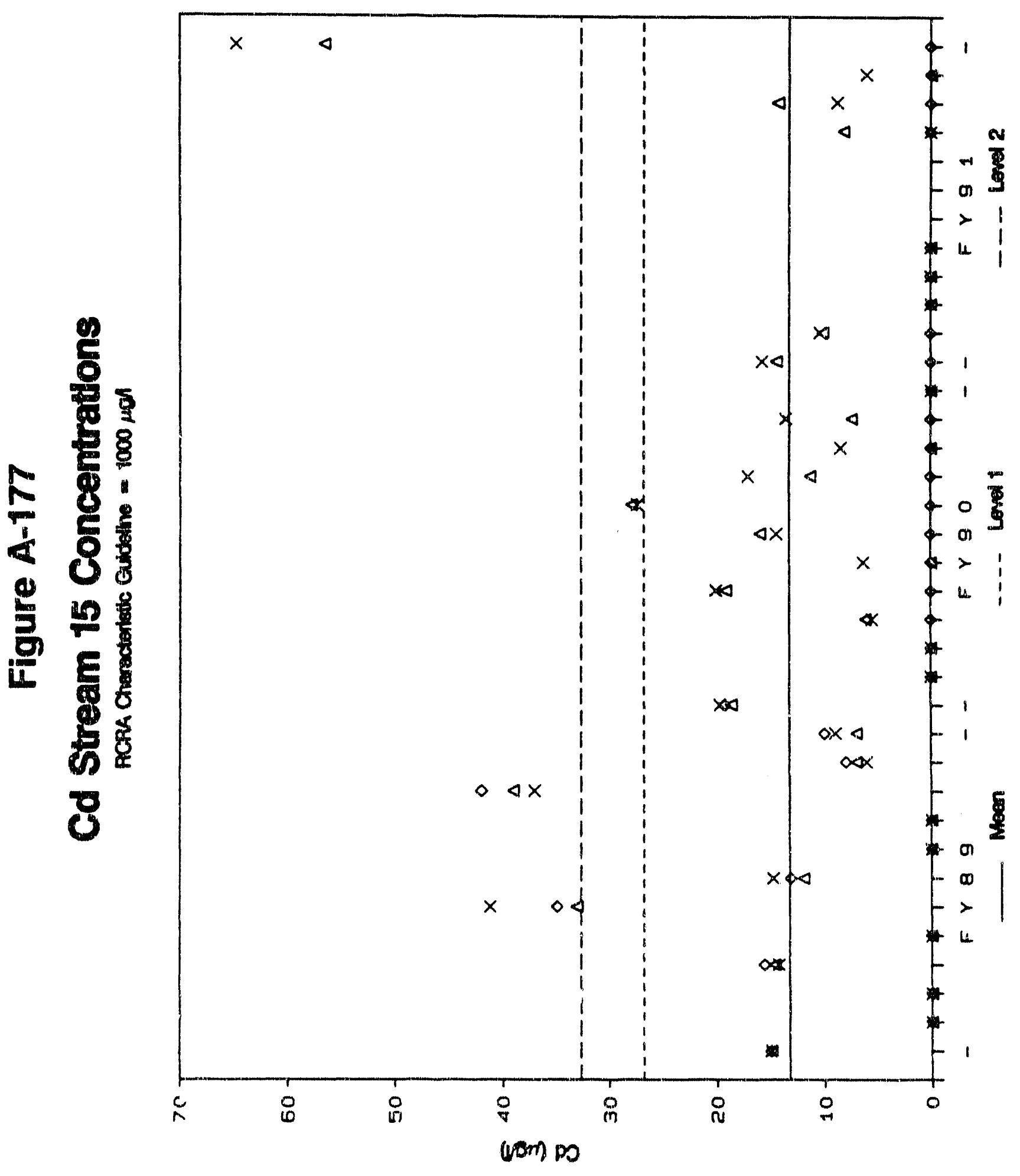




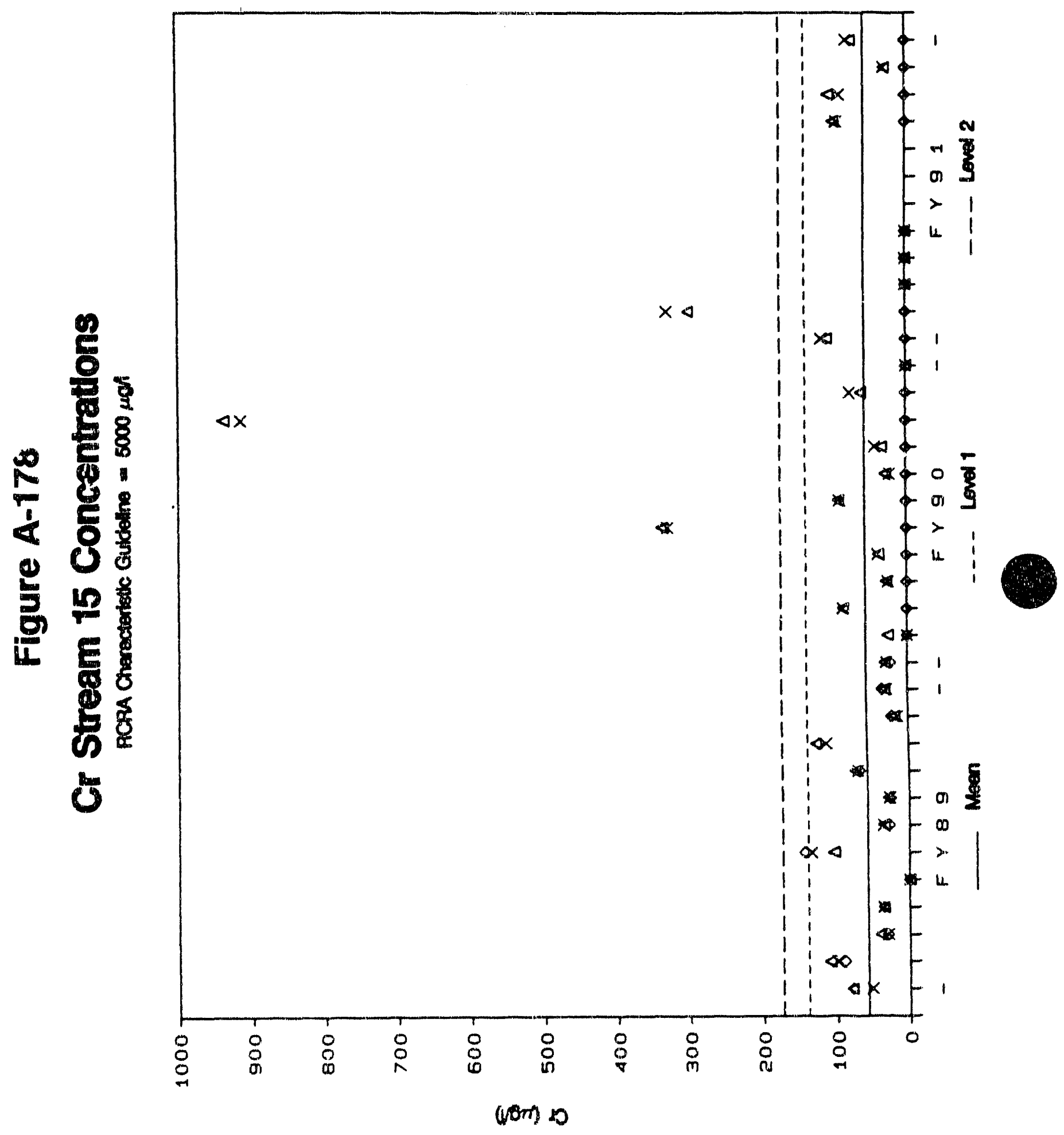




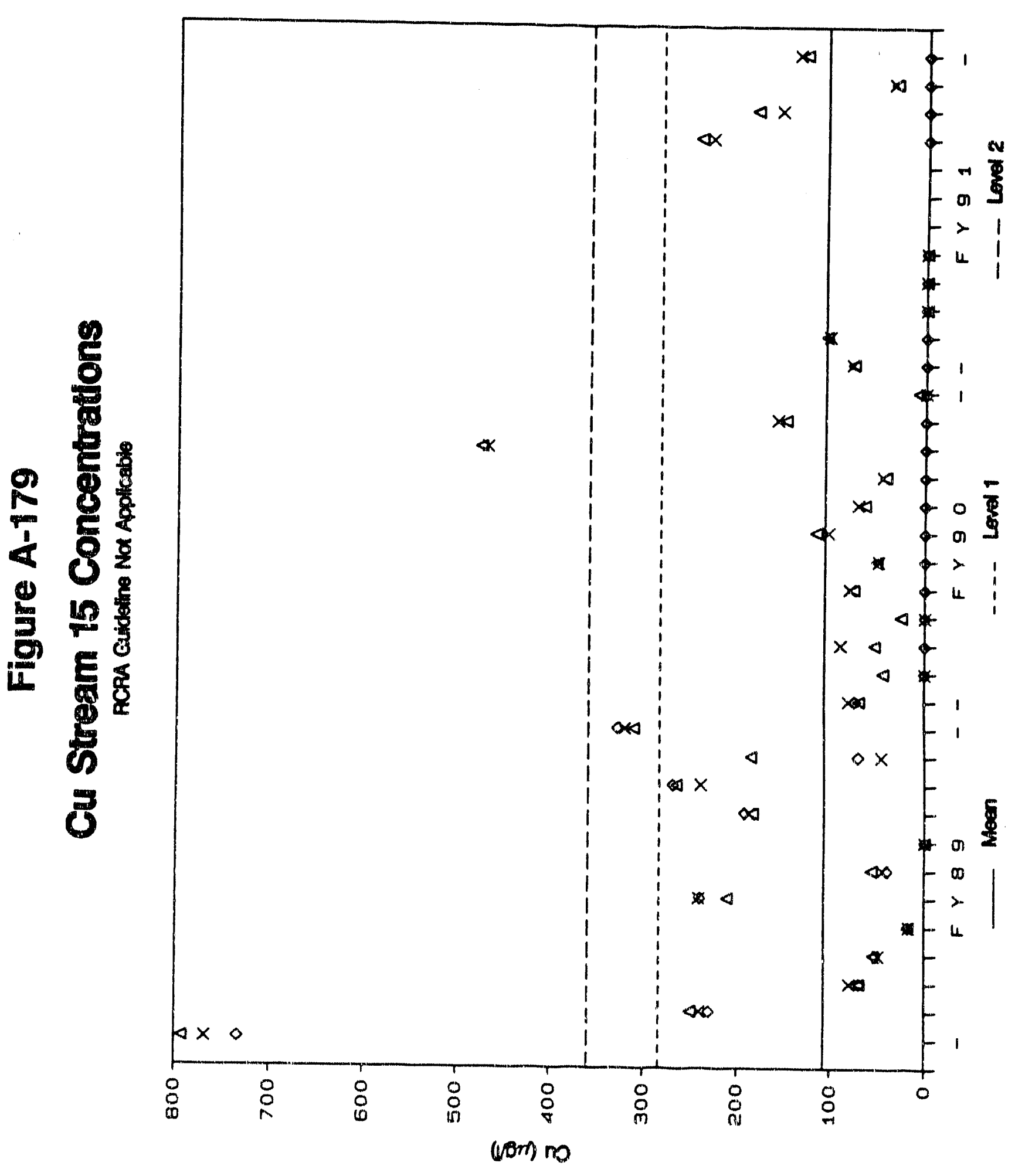




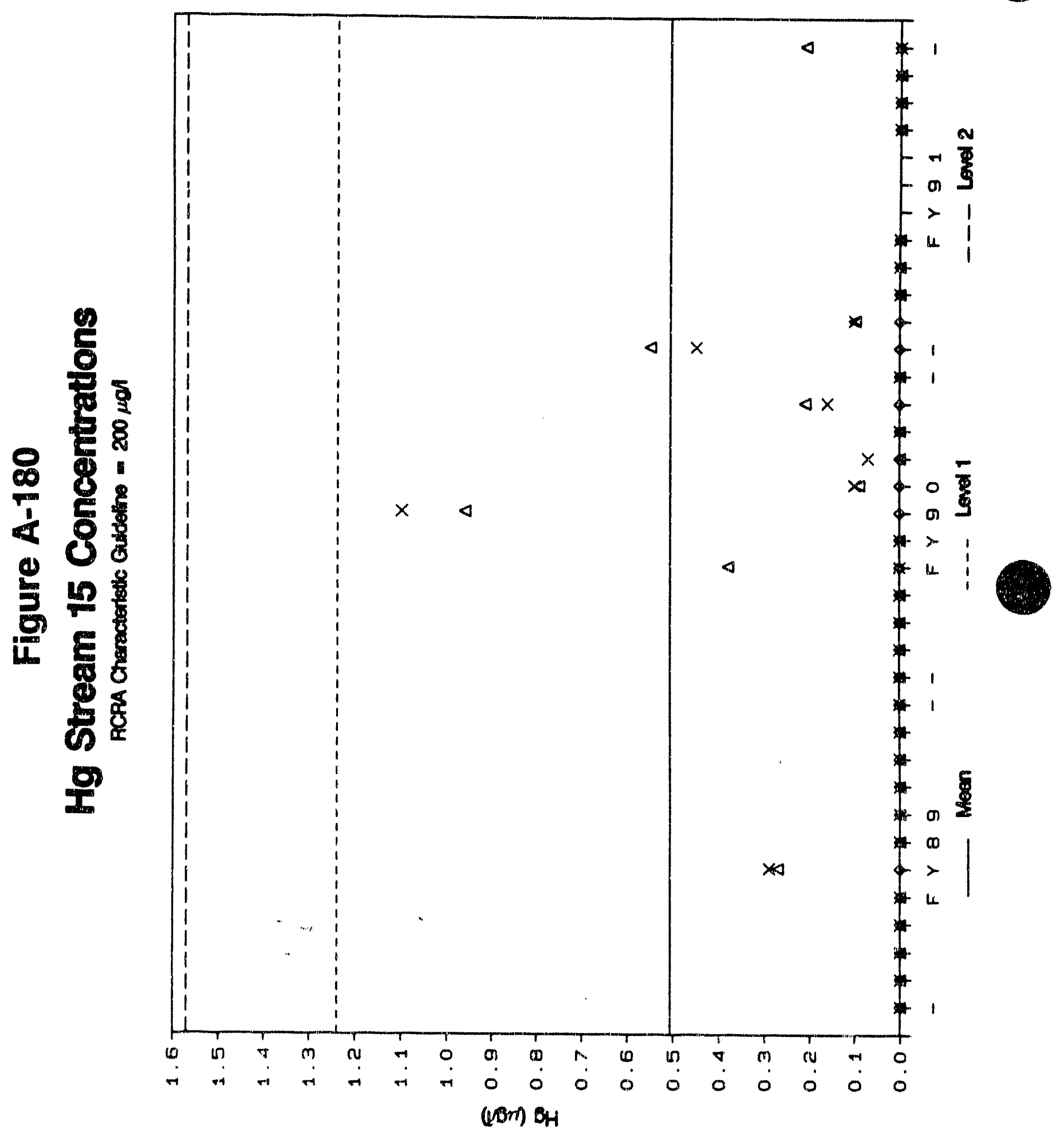

6 


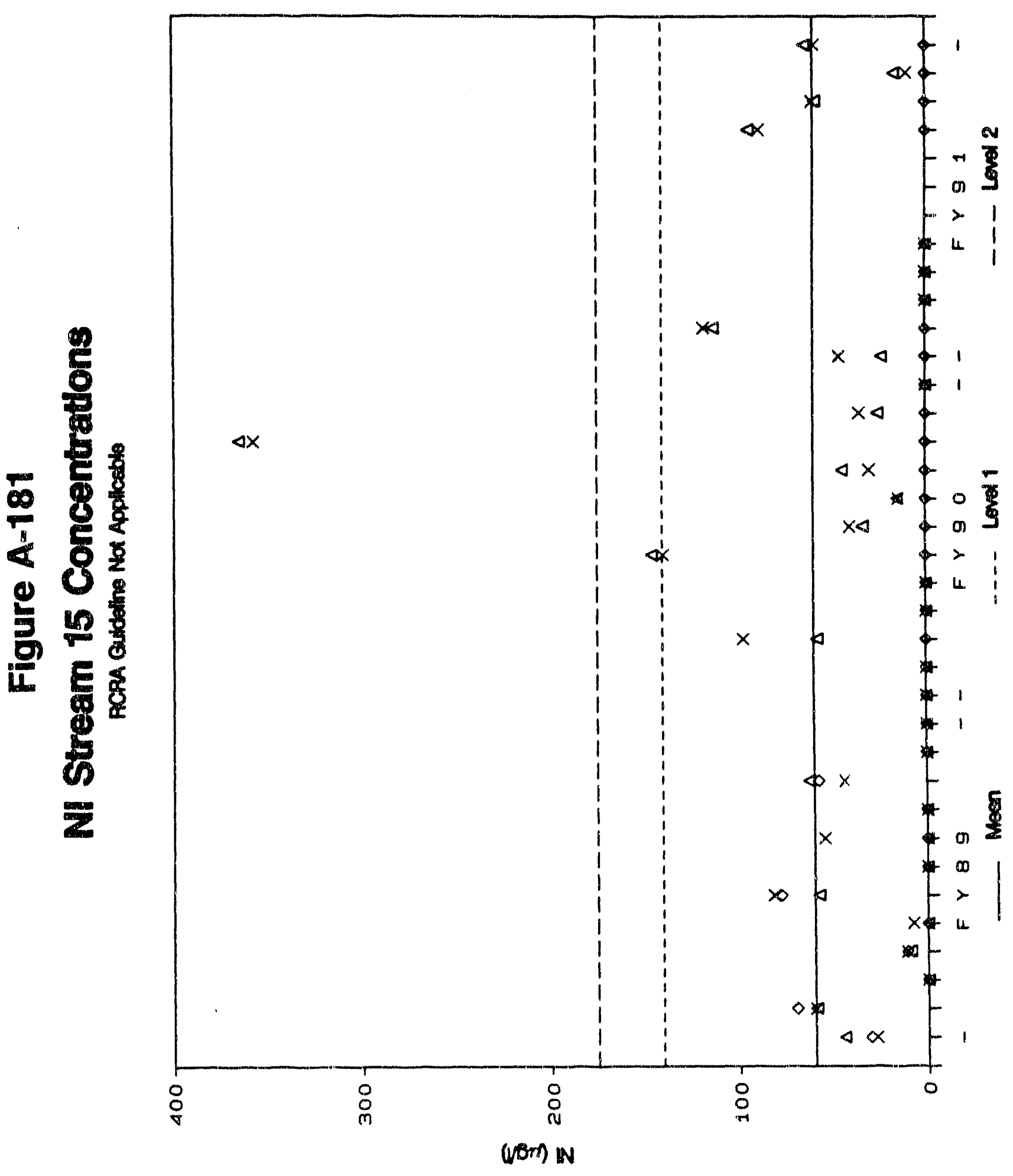




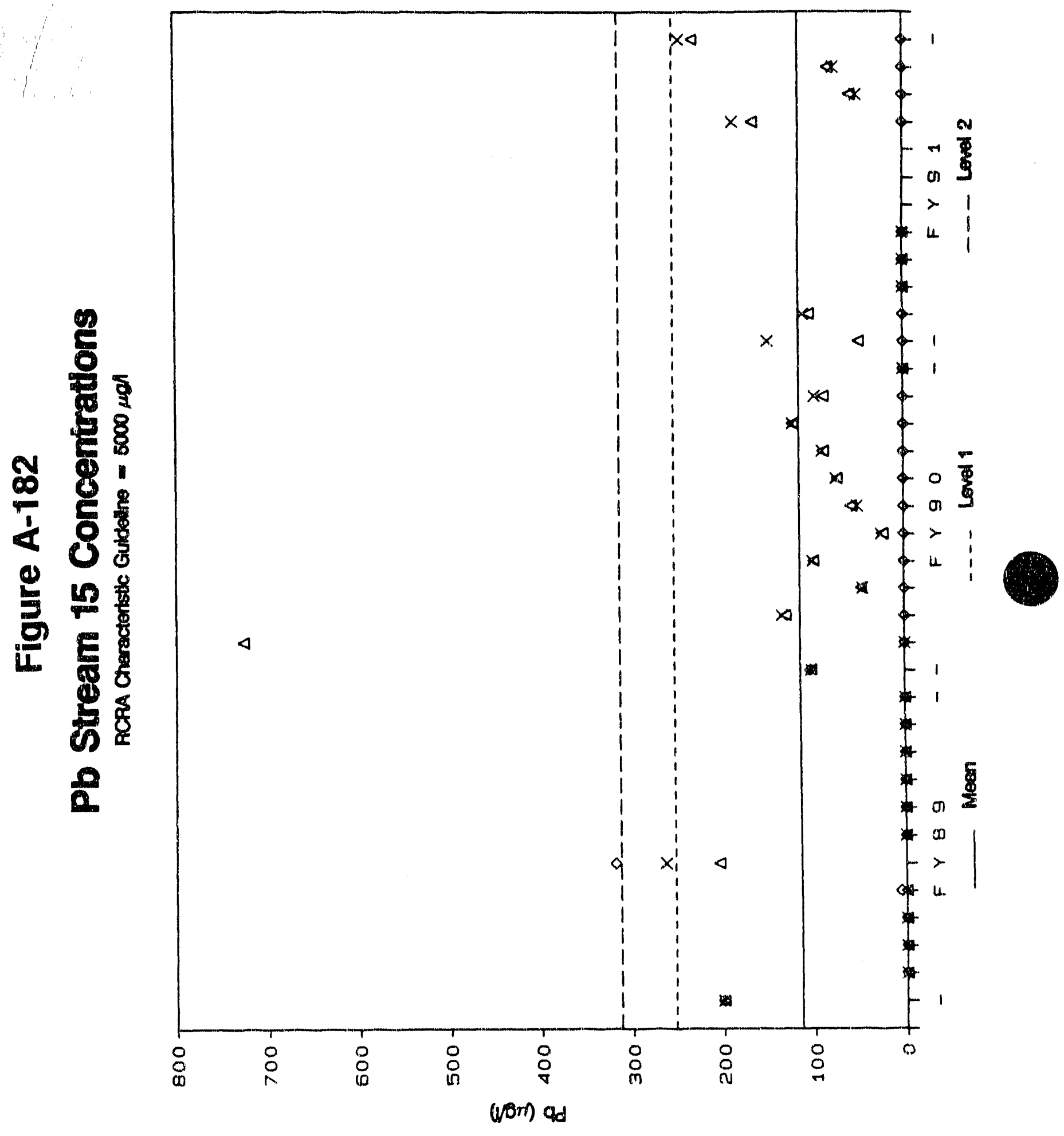




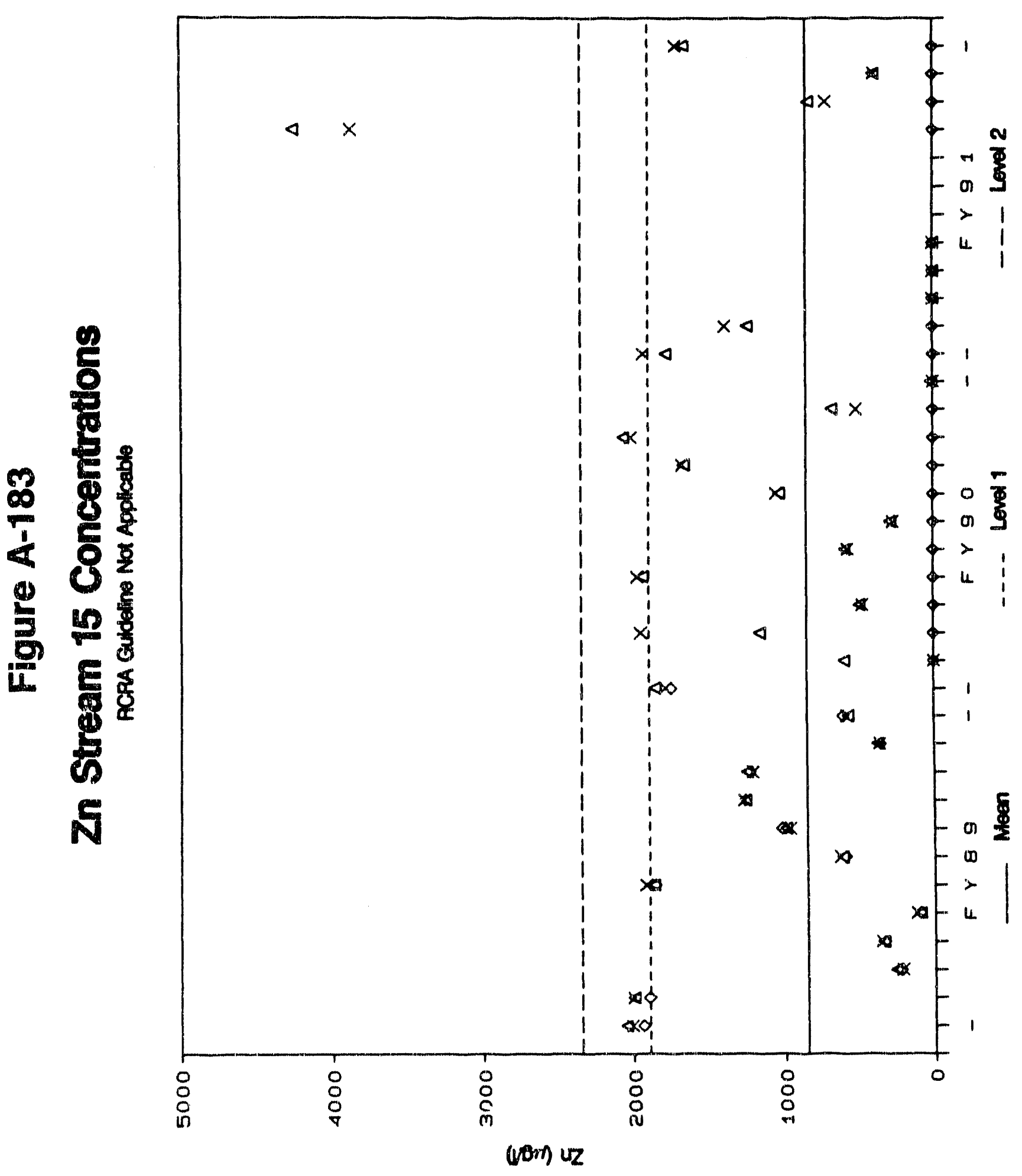




\section{Table A-53}

FY91 ENVIRONMENTAL MONITORING DATA

-- STREAM=15 (CFA oil/water separator effluent) QUARTER=91qI ---

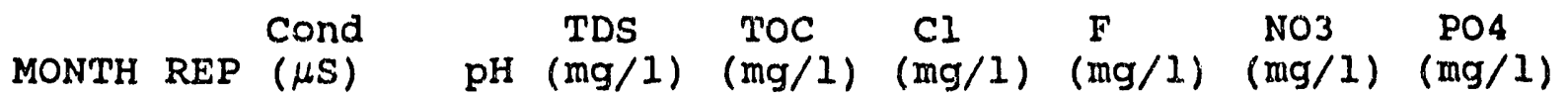

$\begin{array}{lrrrrrrrrr}\text { Oct } & 1 & \mathrm{X} & \mathrm{X} & 611 & 167 & 82.4 & \mathrm{X} & \mathrm{X} & 1.50 \\ \text { Oct } & 2 & 805 & 7.74 & 600 & 176 & 83.9 & \mathrm{X} & \mathrm{X} & 1.60 \\ \text { Nov } & 1 & \mathrm{X} & \mathrm{X} & 468 & 95.2 & 93.3 & \mathrm{X} & 6.20 & 0.27 \\ \text { Nov } & 2 & \mathrm{X} & \mathrm{X} & 429 & 95.8 & 89.5 & \mathrm{X} & 6.60 & 0.19 \\ \text { Dec } & 1 & \mathrm{X} & \mathrm{X} & \mathrm{X} & \mathrm{X} & \mathrm{X} & \mathrm{X} & \mathrm{X} & \mathrm{X} \\ \text { Dec } & 2 & \mathrm{X} & \mathrm{X} & \mathrm{X} & \mathrm{X} & \mathrm{X} & \mathrm{X} & \mathrm{X} & \mathrm{X}\end{array}$

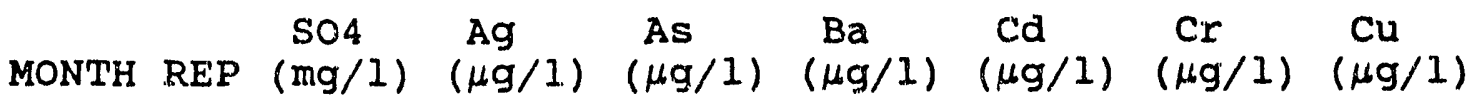

$\begin{array}{llllllllr}\text { Oct } & 1 & 47.1 & \mathrm{X} & 2.30 & 170 & 14.5 & 109 & 77.7 \\ \text { Oct } & 2 & 47.0 & \mathrm{X} & 2.80 & 180 & 15.8 & 116 & 78.8 \\ \text { Nov } & 1 & 44.7 & \mathrm{X} & 1.90 & 144 & 10.1 & 298 & 106 \\ \text { Nov } & 2 & 44.7 & \mathrm{X} & 2.20 & 146 & 10.4 & 328 & 103 \\ \text { Dec } & 1 & \mathrm{X} & \mathrm{X} & \mathrm{X} & \mathrm{X} & \mathrm{X} & \mathrm{X} & \mathrm{X} \\ \text { Dec } & 2 & \mathrm{X} & \mathrm{X} & \mathrm{X} & \mathrm{X} & \mathrm{X} & \mathrm{X} & \mathrm{X}\end{array}$

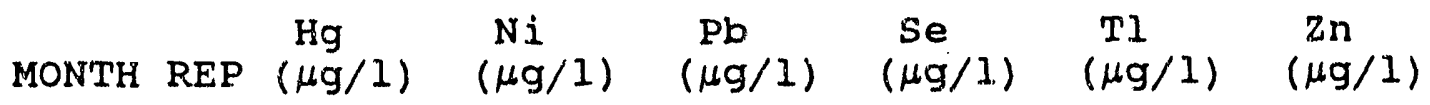

\begin{tabular}{|c|c|c|c|c|c|c|c|}
\hline oct & 1 & 0.55 & 24.0 & 48.8 & $x$ & $x$ & 1780 \\
\hline oct & 2 & 0.45 & 46.4 & 148 & $x$ & $\mathrm{x}$ & 1930 \\
\hline Nov & 1 & 0.10 & 113 & 103 & $X$ & 0.77 & 1240 \\
\hline Nov & 2 & 0.10 & 118 & 109 & $\mathrm{X}$ & $x$ & 1390 \\
\hline DeC & 1 & $\mathrm{x}$ & $X$ & $\mathrm{X}$ & $x$ & $x$ & $\mathrm{X}$ \\
\hline Deo & 2 & $\mathrm{x}$ & $\mathrm{x}$ & $\mathrm{x}$ & $x$ & $\mathrm{X}$ & $x$ \\
\hline
\end{tabular}

'.' - missing

' $x$ ' - below practical quantitation level 
Table A-54

FY91 ENVIRONMENTAL MONITORING DATA

-- STREAM=15 (CFA oil/water separator effluent) QUARTER=91q2 ---

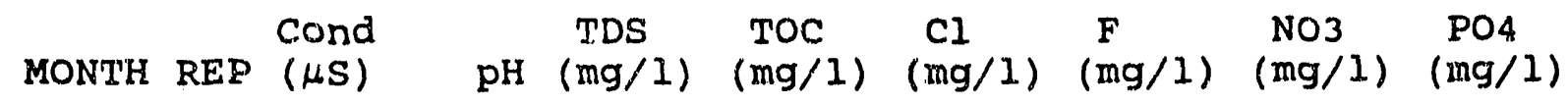

$\begin{array}{lrrrrrrrrr}\text { Jan } & 1 & 1250 & 7.75 & X & X & X & X & X & X \\ \text { Jan } & 2 & X & X & X & X & X & X & X & X \\ \text { Feb } & 1 & X & X & X & X & X & X & X & X \\ \text { Feb } & 2 & X & X & X & X & X & X & X & X\end{array}$

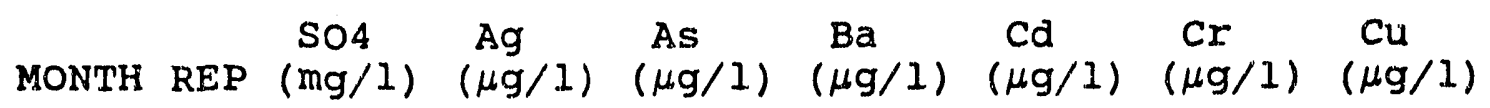

$\begin{array}{lllllllll}\text { Jan } & 1 & \mathrm{X} & \mathrm{X} & \mathrm{X} & \mathrm{X} & \mathrm{X} & \mathrm{X} & \mathrm{X} \\ \mathrm{Jan} & 2 & \mathrm{X} & \mathrm{X} & \mathrm{X} & \mathrm{X} & \mathrm{X} & \mathrm{X} & \mathrm{X} \\ \text { Feb } & 1 & \mathrm{X} & \mathrm{X} & \mathrm{X} & \mathrm{X} & \mathrm{X} & \mathrm{X} & \mathrm{X} \\ \text { Feb } & 2 & \mathrm{X} & \mathrm{X} & \mathrm{X} & \mathrm{X} & \mathrm{X} & \mathrm{X} & \mathrm{X}\end{array}$

$\begin{array}{lllllll}\mathrm{Kg} & \mathrm{Ni} & \mathrm{Pb} & \mathrm{Se} & \mathrm{Tl} & \mathrm{Zn} \\ \operatorname{MONTH} \operatorname{REP} & (\mu \mathrm{g} / 1) & (\mu \mathrm{g} / 1) & (\mu \mathrm{g} / 1) & (\mu \mathrm{g} / 1) & (\mu \mathrm{g} / 1) & (\mu \mathrm{g} / 1)\end{array}$

$\begin{array}{llllllll}\text { Jan } & 1 & X & X & X & X & X & X \\ \text { Jan } & 2 & X & X & X & X & X & X \\ \text { Feb } & 1 & X & X & X & X & X & X \\ \text { Feb } & 2 & X & X & X & X & X & X\end{array}$

$' . '$ - missing

' $X$ ' - below practical quantitation level 


\section{Table A-55}

-- STREAM=15 (CFA oil/water separator effluent) QUARTER=91q3 -.-

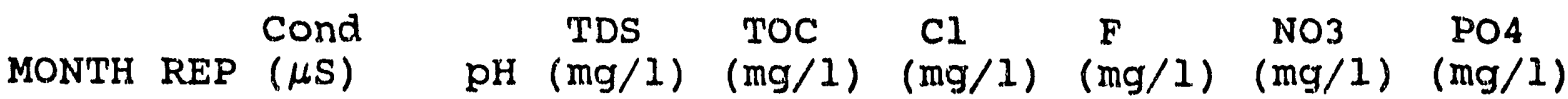

$\begin{array}{llllllrrrr}\text { Jun } & 1 & 960 & 8.69 & 1250 & 372 & 105 & 0.28 & X & 126 \\ \text { Jun } & 2 & 940 & 8.79 & 1120 & 356 & 98.0 & 0.27 & X & 122\end{array}$

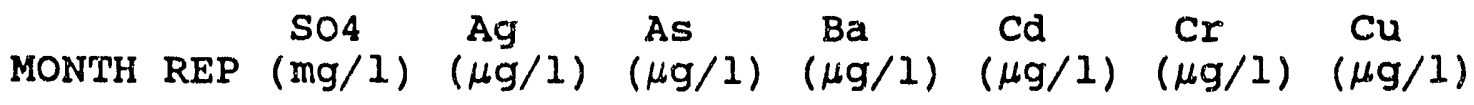

$\begin{array}{lllllllll}\text { Jun } & 1 & 46.4 & X & X & 201 & 8.20 & 99.5 & 243 \\ \text { Jun } & 2 & 43.1 & X & X & 198 & X & 95.2 & 230\end{array}$

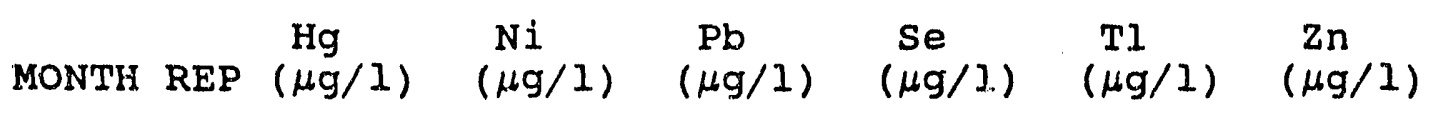

$\begin{array}{llllllll}\text { Jun } & 1 & X & 94.4 & 164 & 3.1 .0 & X & 4250 \\ \text { Jun } & 2 & X & 88.9 & 186 & X & 2.00 & 3870\end{array}$

'. - missing

' $\dot{X}$ ' - below practical quantitation level 


\section{Table A-56}

FY91 ENVIRONMENTAL MONITORING DATA

-- STREAM=15 (CFA oil/water separator effluent) QUARTER=91q4 --

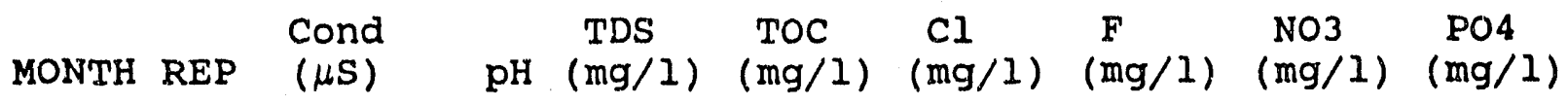

$\begin{array}{lccccccccc}\text { Jul } & 1 & 723.0 & 7.93 & 418 & 28.8 & 86.6 & 0.18 & 4.03 & 3.46 \\ \text { Jul } & 2 & 692.0 & 7.96 & 410 & 28.3 & 87.6 & 0.19 & 4.14 & 3.15 \\ \text { Aug } & 1 & X & X & X & 44.3 & 128 & 0.20 & X & 7.10 \\ \text { Aug } & 2 & X & X & X & 49.9 & 123 & 0.20 & X & 8.05 \\ \text { Sep } & 1 & 77.3 & 8.28 & X & 194 & 199 & 0.35 & X & 85.7 \\ \text { Sep } & 2 & 77.7 & 8.49 & x & 206 & 205 & 0.36 & X & 83.8\end{array}$

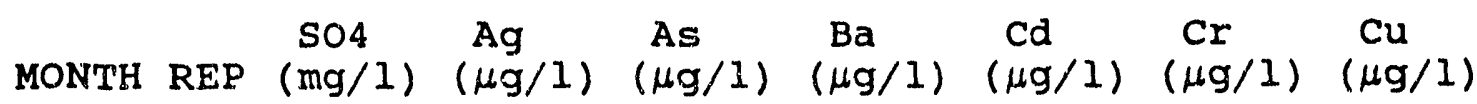

$\begin{array}{lllllrlrr}\text { Jul } & 1 & 44.9 & \mathrm{X} & \mathrm{X} & 91.9 & 14.3 & 106 & 182 \\ \text { Jul } & 2 & 44.2 & \mathrm{X} & \mathrm{X} & 89.0 & 8.80 & 91.4 & 156 \\ \text { Aug } & 1 & 40.4 & \mathrm{X} & 3.40 & 138 & \mathrm{X} & 29.0 & 34.5 \\ \text { Aug } & 2 & 39.6 & \mathrm{X} & \mathrm{X} & 136 & 6.00 & 29.5 & 36.4 \\ \text { Sep } & 1 & 32.6 & \mathrm{X} & 8.60 & 201 & 56.5 & 75.6 & 130 \\ \text { Sep } & 2 & 34.1 & \mathrm{X} & 8.30 & 217 & 64.8 & 82.0 & 137\end{array}$

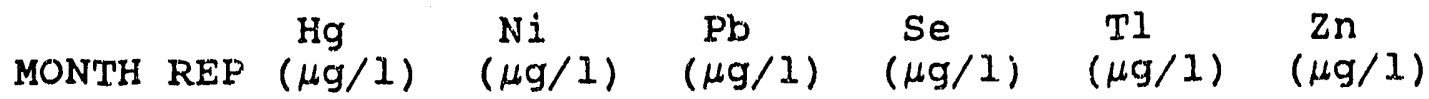

$\begin{array}{lllrrllr}\text { Jul } & 1 & X & 59.4 & 57.8 & 4.00 & X & 832 \\ \text { Jul } & 2 & X & 60.6 & 51.0 & 4.00 & X & 717 \\ \text { Aug } & 1 & X & 17.1 & 81.7 & X & X & 398 \\ \text { Aug } & 2 & X & 10.3 & 75.6 & X & X & 395 \\ \text { Sep } & 1 & 0.21 & 64.8 & 230 & X & X & 1660 \\ \text { Sep } & 2 & X & 59.8 & 245 & X & X & 1710\end{array}$

'. - missing

' $x$ ' - below practical quantitation level 
Tabie A-57

SUMMARY STATISTICS

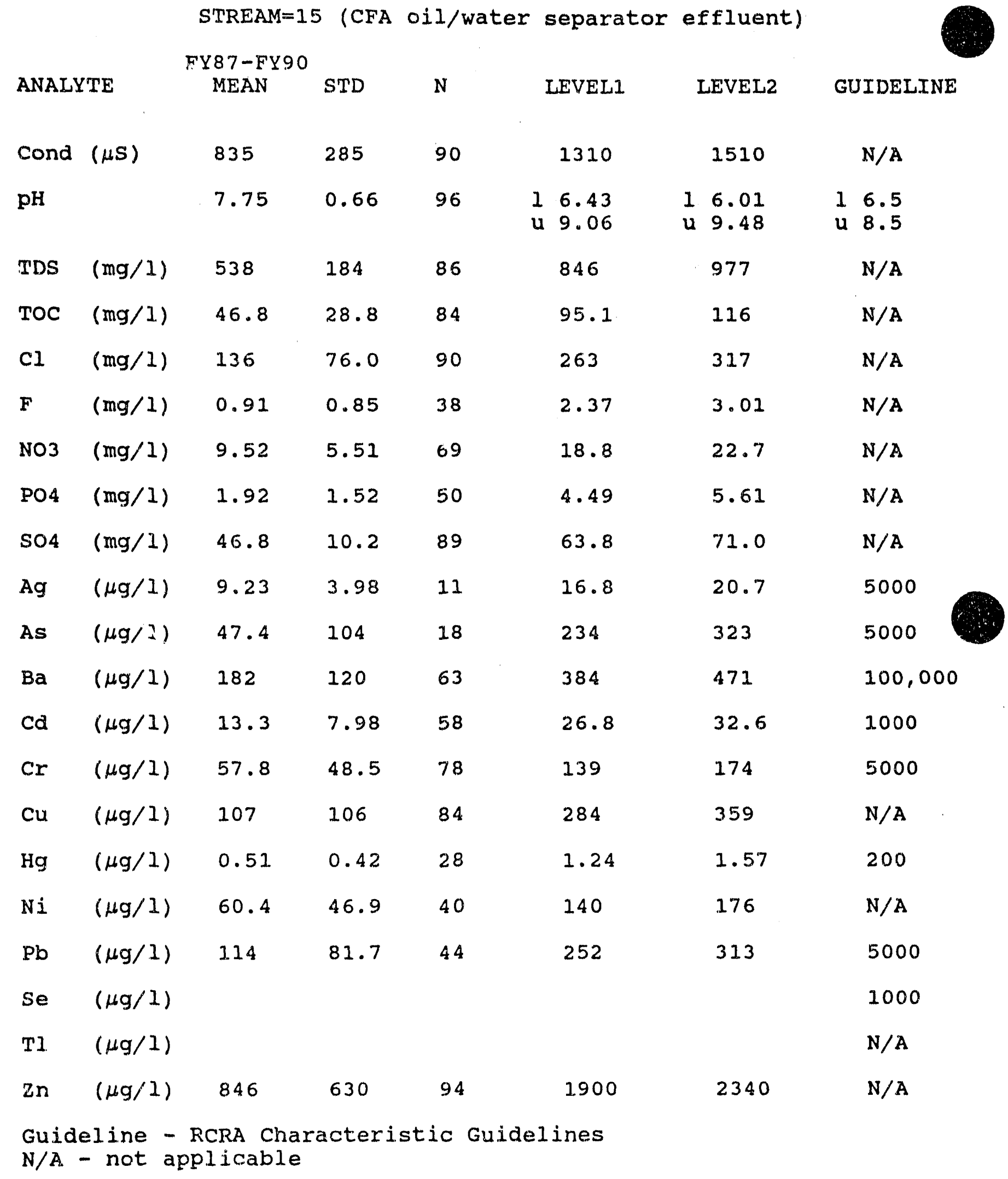




\section{Table A-58}

QUARTERLY MEANS AND VARIANCE COMPONENTS

STREAM=15 (CFA oil/water separator effluent)

\begin{tabular}{|c|c|c|c|c|c|c|c|c|c|}
\hline \multicolumn{2}{|c|}{ ANALYTE } & QTR & MEAN & $\begin{array}{r}\text { STD } \\
\text { MEAN }\end{array}$ & $\begin{array}{l}\text { STD } \\
\text { DAY }\end{array}$ & $\begin{array}{l}\text { S'TD } \\
\text { REP }\end{array}$ & $\mathrm{N}$ & $\underset{\text { MISS }}{\mathbf{N}}$ & BPQI \\
\hline Cond & $(\mu s)$ & $\begin{array}{l}91 . q 1 \\
91 . q 2 \\
91 . q 3 \\
91 q 4\end{array}$ & $\begin{array}{r}805 \\
1250 \\
950 \\
393\end{array}$ & $\begin{array}{l}\dot{\cdot} \\
\dot{*}\end{array}$ & $\begin{array}{l}\dot{1} \\
\dot{1} 45\end{array}$ & $\begin{array}{l}\dot{ } \\
i 5.5\end{array}$ & $\begin{array}{l}1 \\
1 \\
2 \\
4\end{array}$ & $\begin{array}{l}5 \\
0 \\
0 \\
2\end{array}$ & $\begin{array}{l}0 \\
5 \\
4 \\
0\end{array}$ \\
\hline $\mathrm{pH}$ & & $\begin{array}{l}91 q 1 \\
91 q 2 \\
91 q 3 \\
91 q 4\end{array}$ & $\begin{array}{l}7.74 \\
7.75 \\
8.74 \\
8.16\end{array}$ & $\begin{array}{l}\dot{ } \\
\dot{0} .22\end{array}$ & $\begin{array}{l}\dot{0} \\
0.30\end{array}$ & $\begin{array}{l}\dot{\dot{0}} \\
\dot{0} .11\end{array}$ & $\begin{array}{l}1 \\
1 \\
2 \\
4\end{array}$ & $\begin{array}{l}5 \\
0 \\
0 \\
2\end{array}$ & $\begin{array}{l}0 \\
5 \\
1 \\
0\end{array}$ \\
\hline TDS & $(\mathrm{mg} / \mathrm{l})$ & $\begin{array}{l}91 q 1 \\
91 q 2 \\
91 q 3 \\
91 q 4\end{array}$ & $\begin{array}{r}527 \\
x^{5185} \\
414\end{array}$ & $\begin{array}{l}78.5 \\
\dot{.} \\
.\end{array}$ & $\begin{array}{l}110 \\
\dot{0}\end{array}$ & $\begin{array}{l}20.3 \\
: \\
\dot{0}\end{array}$ & $\begin{array}{l}4 \\
0 \\
2 \\
2\end{array}$ & $\begin{array}{l}2 \\
0 \\
0 \\
0\end{array}$ & $\begin{array}{l}0 \\
6 \\
4 \\
4\end{array}$ \\
\hline TOC & $(\mathrm{mg} / \mathrm{l})$ & $\begin{array}{l}91 q 1 \\
91 q 2 \\
91 q 3 \\
91 q 4\end{array}$ & $\begin{array}{r}x^{134} \\
364 \\
91.9\end{array}$ & $\begin{array}{l}38.0 \\
\dot{5} \\
54.3\end{array}$ & $\begin{array}{l}53.6 \\
\dot{0} \\
94.0\end{array}$ & $\begin{array}{l}4.51 \\
\cdot \\
5.41\end{array}$ & $\begin{array}{l}4 \\
0 \\
2 \\
6\end{array}$ & $\begin{array}{l}2 \\
0 \\
0 \\
0\end{array}$ & $\begin{array}{l}0 \\
6 \\
4 \\
0\end{array}$ \\
\hline $\mathrm{Cl}$ & $(\mathrm{mg} / \mathrm{l})$ & $\begin{array}{l}91 q 1 \\
91 q^{2} \\
91 q^{3} \\
91 q 4\end{array}$ & $\begin{array}{l}87.3 \\
X \\
102 \\
138\end{array}$ & $\begin{array}{l}4.12 \\
\dot{3} 3.8\end{array}$ & $\begin{array}{l}5.65 \\
\dot{0} \\
58.4\end{array}$ & $\begin{array}{l}2.04 \\
\dot{3} \\
\dot{3} .21\end{array}$ & $\begin{array}{l}4 \\
0 \\
2 \\
6\end{array}$ & $\begin{array}{l}2 \\
0 \\
0 \\
0\end{array}$ & $\begin{array}{l}0 \\
6 \\
4 \\
0\end{array}$ \\
\hline$F$ & $(\mathrm{mg} / \mathrm{l})$ & $\begin{array}{l}91 q 1 . \\
91 q 2 \\
91 q 3 \\
91 q 4\end{array}$ & $\begin{array}{l}X \\
X \\
0.27 \\
0.25\end{array}$ & $\begin{array}{l}\dot{\dot{0}} \\
\dot{0} .05\end{array}$ & $\begin{array}{l}\dot{0} \\
\dot{0} .09\end{array}$ & $\begin{array}{l}\dot{0} \\
\dot{0} .01\end{array}$ & $\begin{array}{l}0 \\
0 \\
2 \\
6\end{array}$ & $\begin{array}{l}2 \\
0 \\
0 \\
0\end{array}$ & $\begin{array}{l}4 \\
6 \\
4 \\
0\end{array}$ \\
\hline NO3 & $(\mathrm{mg} / \mathrm{l})$ & $\begin{array}{l}91 q 1 \\
91 q 2 \\
91 q 3 \\
91 q 4\end{array}$ & $\begin{array}{l}6.40 \\
x \\
X \\
4.08\end{array}$ & $\begin{array}{l}\dot{\cdot} \\
\dot{\cdot}\end{array}$ & $\begin{array}{l}\dot{.} \\
\dot{.}\end{array}$ & $\dot{.}$ & $\begin{array}{l}2 \\
0 \\
0 \\
2\end{array}$ & $\begin{array}{l}2 \\
0 \\
0 \\
0\end{array}$ & $\begin{array}{l}2 \\
6 \\
6 \\
4\end{array}$ \\
\hline PO4 & $(\mathrm{mg} / \mathrm{l})$ & $\begin{array}{l}91 q 1 \\
91 q^{2} \\
91 q^{3} \\
91 q^{4}\end{array}$ & $\begin{array}{l}0.89 \\
\times \\
124 \\
31.9\end{array}$ & $\begin{array}{l}0.66 \\
\dot{2} \\
26.5\end{array}$ & $\begin{array}{l}0.93 \\
\dot{4} \\
45.8\end{array}$ & $\begin{array}{l}0.06 \\
\dot{0} \\
0.85\end{array}$ & $\begin{array}{l}4 \\
0 \\
2 \\
6\end{array}$ & $\begin{array}{l}2 \\
0 \\
0 \\
0\end{array}$ & $\begin{array}{l}0 \\
\epsilon \\
4 \\
0\end{array}$ \\
\hline SO4 & $(\mathrm{mg} / \mathrm{l})$ & $\begin{array}{l}91 q 1 \\
91 q^{2} \\
91 q^{3} \\
91 q^{4}\end{array}$ & $\begin{array}{l}45.9 \\
X \\
44.7 \\
39.3\end{array}$ & $\begin{array}{l}1.17 \\
\dot{3} \\
3.25\end{array}$ & $\begin{array}{l}1.66 \\
\dot{5} \\
5.61\end{array}$ & $\begin{array}{l}0.05 \\
\dot{0} \\
0.75\end{array}$ & $\begin{array}{l}4 \\
0 \\
2 \\
6\end{array}$ & $\begin{array}{l}2 \\
0 \\
0 \\
0\end{array}$ & $\begin{array}{l}0 \\
6 \\
4 \\
0\end{array}$ \\
\hline Ag & $(\mu g / 1)$ & $\begin{array}{l}91 q 1 \\
91 q^{2} \\
91 q^{3} \\
91 q^{4}\end{array}$ & $\begin{array}{l}x \\
x \\
x \\
x\end{array}$ & $\begin{array}{l}\dot{1} \\
\dot{ } \\
\dot{ }\end{array}$ & $\begin{array}{l}\dot{\bullet} \\
\dot{ } \\
.\end{array}$ & $\dot{\cdot}$ & $\begin{array}{l}0 \\
0 \\
0 \\
0\end{array}$ & $\begin{array}{l}2 \\
0 \\
0 \\
0\end{array}$ & $\begin{array}{l}4 \\
6 \\
5 \\
6\end{array}$ \\
\hline
\end{tabular}


Table A-58 (continued)

QUARTERLY MEANS AND VARIANCE COMPONENTS

STREAM=15 (CFA oil/water separator effluent) - - - - (continued)

\begin{tabular}{|c|c|c|c|c|c|c|c|c|c|}
\hline \multicolumn{2}{|c|}{ ANALYTE } & QTR & MEAN & $\begin{array}{r}\text { STD } \\
\text { MEAN }\end{array}$ & $\begin{array}{l}\text { STD } \\
\text { DAY }\end{array}$ & $\begin{array}{l}\text { STD } \\
\text { REP }\end{array}$ & $\mathrm{N}$ & $\begin{array}{r}\mathbf{N} \\
\text { MISS }\end{array}$ & $\stackrel{N}{\mathrm{~N}}$ \\
\hline As & $(\mu g / 1)$ & $\begin{array}{l}91 q^{1} \\
91 q^{2} \\
91 q^{3} \\
91 q^{4}\end{array}$ & $\begin{array}{l}2.30 \\
X \\
X \\
6.77\end{array}$ & $\begin{array}{l}0.25 \\
\dot{0} \\
.\end{array}$ & $\begin{array}{l}0.29 \\
\dot{\cdot} \\
\dot{0}\end{array}$ & $\begin{array}{l}0.29 \\
: \\
. \\
.\end{array}$ & $\begin{array}{l}4 \\
0 \\
0 \\
3\end{array}$ & $\begin{array}{l}2 \\
0 \\
0 \\
0\end{array}$ & $\begin{array}{l}0 \\
6 \\
6 \\
3\end{array}$ \\
\hline $\mathrm{Ba}$ & $(\mu g / 1)$ & $\begin{array}{l}91 q 1 \\
91 q 2 \\
91 q 3 \\
91 q 4\end{array}$ & $\begin{array}{r}160 \\
200 \\
145\end{array}$ & $\begin{array}{l}15.0 \\
\dot{3} 4.5\end{array}$ & $\begin{array}{l}20.9 \\
\dot{5} \\
59.5\end{array}$ & $\begin{array}{l}5.10 \\
\dot{0} \\
6.69\end{array}$ & $\begin{array}{l}4 \\
0 \\
2 \\
6\end{array}$ & $\begin{array}{l}2 \\
0 \\
0 \\
0\end{array}$ & $\begin{array}{l}0 \\
6 \\
4 \\
0\end{array}$ \\
\hline $\mathrm{Cd}$ & $(\mu g / 1)$ & $\begin{array}{l}91 q 1 \\
91 q^{2} \\
91 q^{3} \\
91 q^{4}\end{array}$ & $\begin{array}{l}12.7 \\
X \\
8.20 \\
24.2\end{array}$ & $\begin{array}{l}2.45 \\
\dot{18.5}\end{array}$ & $\begin{array}{l}3.43 \\
\dot{31} \\
\end{array}$ & $\begin{array}{l}0.67 \\
\dot{0} \\
6.06\end{array}$ & $\begin{array}{l}4 \\
0 \\
1 \\
6\end{array}$ & $\begin{array}{l}2 \\
0 \\
0 \\
0\end{array}$ & $\begin{array}{l}0 \\
6 \\
5 \\
0\end{array}$ \\
\hline $\mathrm{Cr}$ & $(\mu \mathrm{g} / \mathrm{I})$ & $\begin{array}{l}91 q 1 \\
91 q^{2} \\
91 q^{3} \\
91 q^{4}\end{array}$ & $\begin{array}{l}213 \\
x^{2} \\
97.3 \\
68.9\end{array}$ & $\begin{array}{l}100 \\
\dot{2} 0.6\end{array}$ & $\begin{array}{l}141 \\
35.5\end{array}$ & $\begin{array}{l}15.4 \\
\dot{6} \\
6.51\end{array}$ & $\begin{array}{l}4 \\
0 \\
2 \\
6\end{array}$ & $\begin{array}{l}2 \\
0 \\
0 \\
0\end{array}$ & $\begin{array}{l}0 \\
6 \\
4 \\
0\end{array}$ \\
\hline $\mathrm{Cu}$ & $(\mu g / 1)$ & $\begin{array}{l}91 q 1 \\
91 q 2 \\
91 q 3 \\
91 q 4\end{array}$ & $\begin{array}{l}91.4 \\
x \\
237 \\
113\end{array}$ & $\begin{array}{l}13.1 \\
\dot{3} 9.9\end{array}$ & $\begin{array}{l}28.5 \\
\dot{6} \\
68.7\end{array}$ & $\begin{array}{l}1.60 \\
i \\
i 1.0\end{array}$ & $\begin{array}{l}4 \\
0 \\
2 \\
6\end{array}$ & $\begin{array}{l}2 \\
0 \\
0 \\
0\end{array}$ & $\begin{array}{l}0 \\
6 \\
4 \\
0\end{array}$ \\
\hline $\mathrm{Hg}$ & $(\mu g / 1)$ & $\begin{array}{l}91 q^{1} \\
91 q^{2} \\
91 q 3 \\
91 q 4\end{array}$ & $\begin{array}{l}0.30 \\
X \\
X \\
0.21\end{array}$ & $\begin{array}{l}0.20 \\
\dot{.} \\
\dot{.}\end{array}$ & $\begin{array}{l}0.28 \\
. \\
.\end{array}$ & $\begin{array}{l}0.05 \\
\dot{.} \\
\dot{ }\end{array}$ & $\begin{array}{l}4 \\
0 \\
0 \\
1\end{array}$ & $\begin{array}{l}2 \\
0 \\
0 \\
0\end{array}$ & $\begin{array}{l}0 \\
6 \\
6 \\
5\end{array}$ \\
\hline $\mathrm{Ni}$ & $(\mu g / I)$ & $\begin{array}{l}91 q 1 \\
91 q^{2} \\
91 q^{3} \\
91 q^{4}\end{array}$ & $\begin{array}{l}75.3 \\
X \\
91.7 \\
45.3\end{array}$ & $\begin{array}{l}40.1 \\
\dot{15} .8\end{array}$ & $\begin{array}{l}56.2 \\
\cdot \\
\dot{2} 7.3\end{array}$ & $\begin{array}{l}1.1 .5 \\
\dot{3} \\
3.48\end{array}$ & $\begin{array}{l}4 \\
0 \\
2 \\
6\end{array}$ & $\begin{array}{l}2 \\
0 \\
0 \\
0\end{array}$ & $\begin{array}{l}0 \\
6 \\
4 \\
0\end{array}$ \\
\hline $\mathrm{Pb}$ & $(\mu g / 1)$ & $\begin{array}{l}91 q 1 \\
91 q 2 \\
91 q^{3}\end{array}$ & $x_{175}^{102}$ & $\begin{array}{l}3.80 \\
. \\
.\end{array}$ & $\begin{array}{l}0.00 \\
. \\
.\end{array}$ & $\begin{array}{l}49.7 \\
. \\
\text {. }\end{array}$ & $\begin{array}{l}4 \\
0 \\
2\end{array}$ & $\begin{array}{l}2 \\
0 \\
0\end{array}$ & $\begin{array}{l}0 \\
6 \\
4\end{array}$ \\
\hline & & $91 q 4$ & 124 & 57.4 & 99.3 & 7.17 & 6 & 0 & 0 \\
\hline $\mathrm{Se}$ & $(\mu g / 1)$ & $\begin{array}{l}91 q^{1} \\
91 q^{2} \\
91 q^{3} \\
91 q^{4}\end{array}$ & $\begin{array}{l}X \\
X \\
3.10 \\
4.00\end{array}$ & $\begin{array}{l}\dot{.} \\
\dot{.}\end{array}$ & $\dot{\dot{ }}$ & $\begin{array}{l}\dot{\bullet} \\
\dot{\cdot}\end{array}$ & $\begin{array}{l}0 \\
0 \\
1 \\
2\end{array}$ & $\begin{array}{l}2 \\
0 \\
0 \\
0\end{array}$ & $\begin{array}{l}4 \\
6 \\
5 \\
4\end{array}$ \\
\hline
\end{tabular}


Table A-58 (continued)

QUARTERLY MEANS AND VARIANCE COMPONENTS

STREAM=15 (CFA oil/water separator effluent) (continued)

\begin{tabular}{|c|c|c|c|c|c|c|c|c|c|}
\hline \multicolumn{2}{|c|}{ ANAI,YTE } & QTR & MEAN & $\begin{array}{r}\text { STD } \\
\text { MEAN }\end{array}$ & $\begin{array}{l}\text { STD } \\
\text { DAY }\end{array}$ & $\begin{array}{l}\text { STD } \\
\text { REP }\end{array}$ & N & $\begin{array}{r}\mathrm{N} \\
\text { MISS }\end{array}$ & $\stackrel{N}{B P Q L}$ \\
\hline rl & $(\mu g / 1)$ & $\begin{array}{l}91 q^{1} \\
91 q^{2} \\
91 q^{3} \\
91 q^{4}\end{array}$ & $\begin{array}{l}0.77 \\
x \\
2.00 \\
x\end{array}$ & $\begin{array}{l}\dot{\cdot} \\
\dot{\cdot}\end{array}$ & $\begin{array}{l}\dot{.} \\
\dot{.}\end{array}$ & . & $\begin{array}{l}1 \\
0 \\
1 \\
0\end{array}$ & $\begin{array}{l}2 \\
0 \\
0 \\
0\end{array}$ & $\begin{array}{l}3 \\
6 \\
5 \\
6\end{array}$ \\
\hline $2 n$ & $(\mu g / 1)$ & $\begin{array}{l}91 q 1 \\
91 q 2 \\
91 q 3 \\
91 q 4\end{array}$ & $\begin{array}{l}1585 \\
X \\
4060 \\
952\end{array}$ & $\begin{array}{l}270 \\
\cdot \\
382\end{array}$ & $\begin{array}{l}374 \\
\cdot \\
661\end{array}$ & $\begin{array}{l}106 \\
\dot{5} 1.2\end{array}$ & $\begin{array}{l}4 \\
0 \\
2 \\
6\end{array}$ & $\begin{array}{l}2 \\
0 \\
0 \\
0\end{array}$ & $\begin{array}{l}0 \\
6 \\
4 \\
0\end{array}$ \\
\hline
\end{tabular}




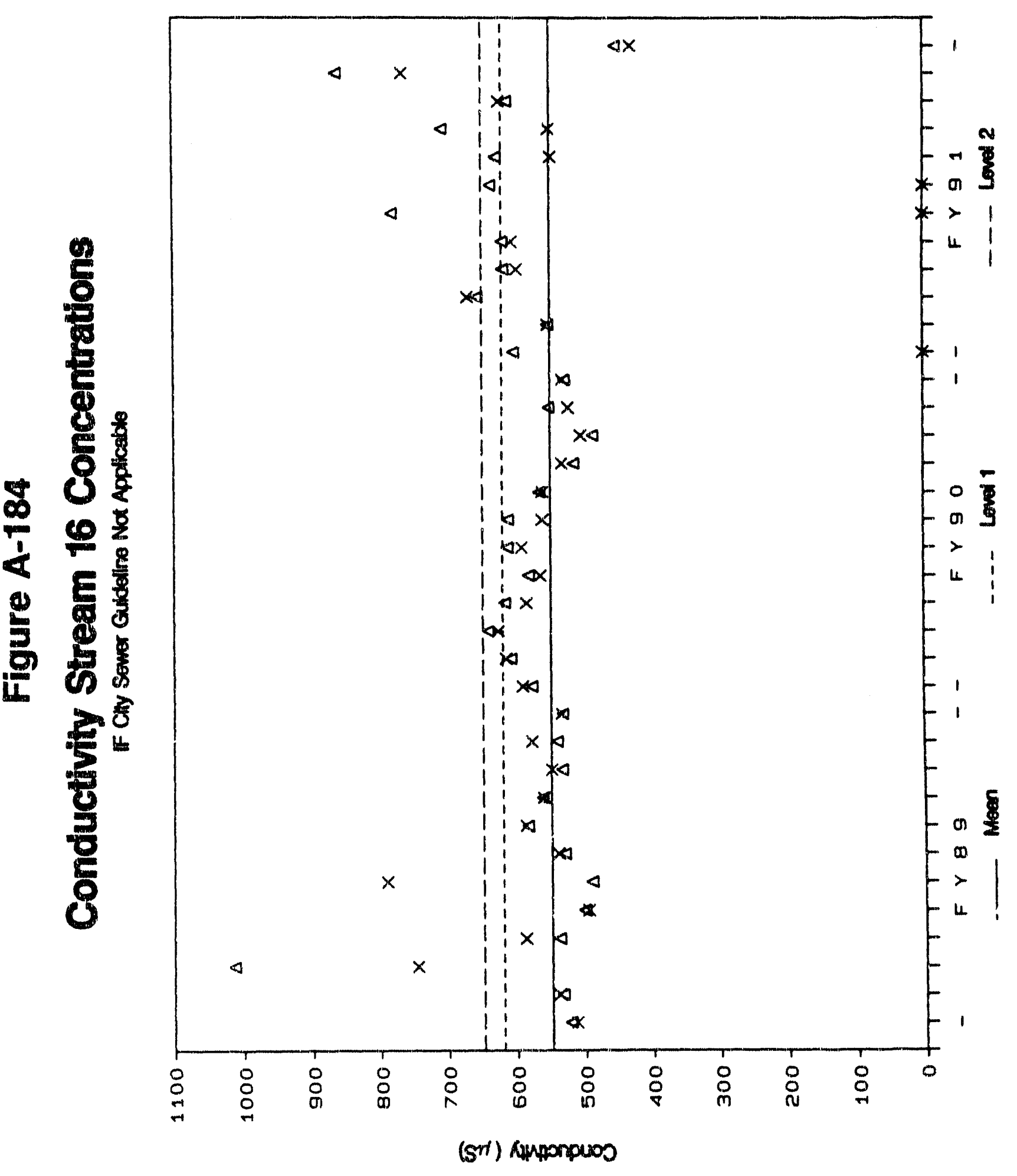




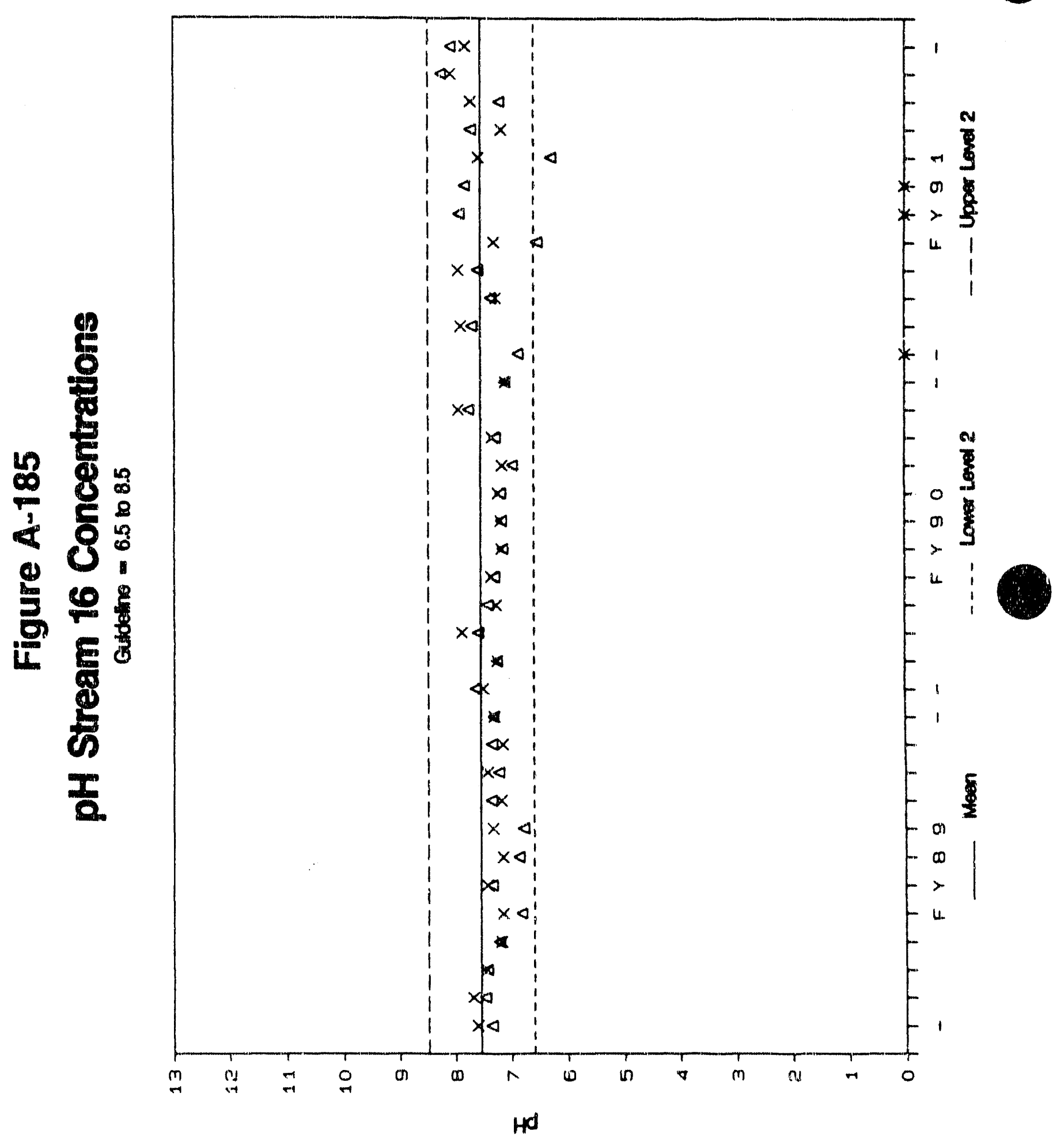




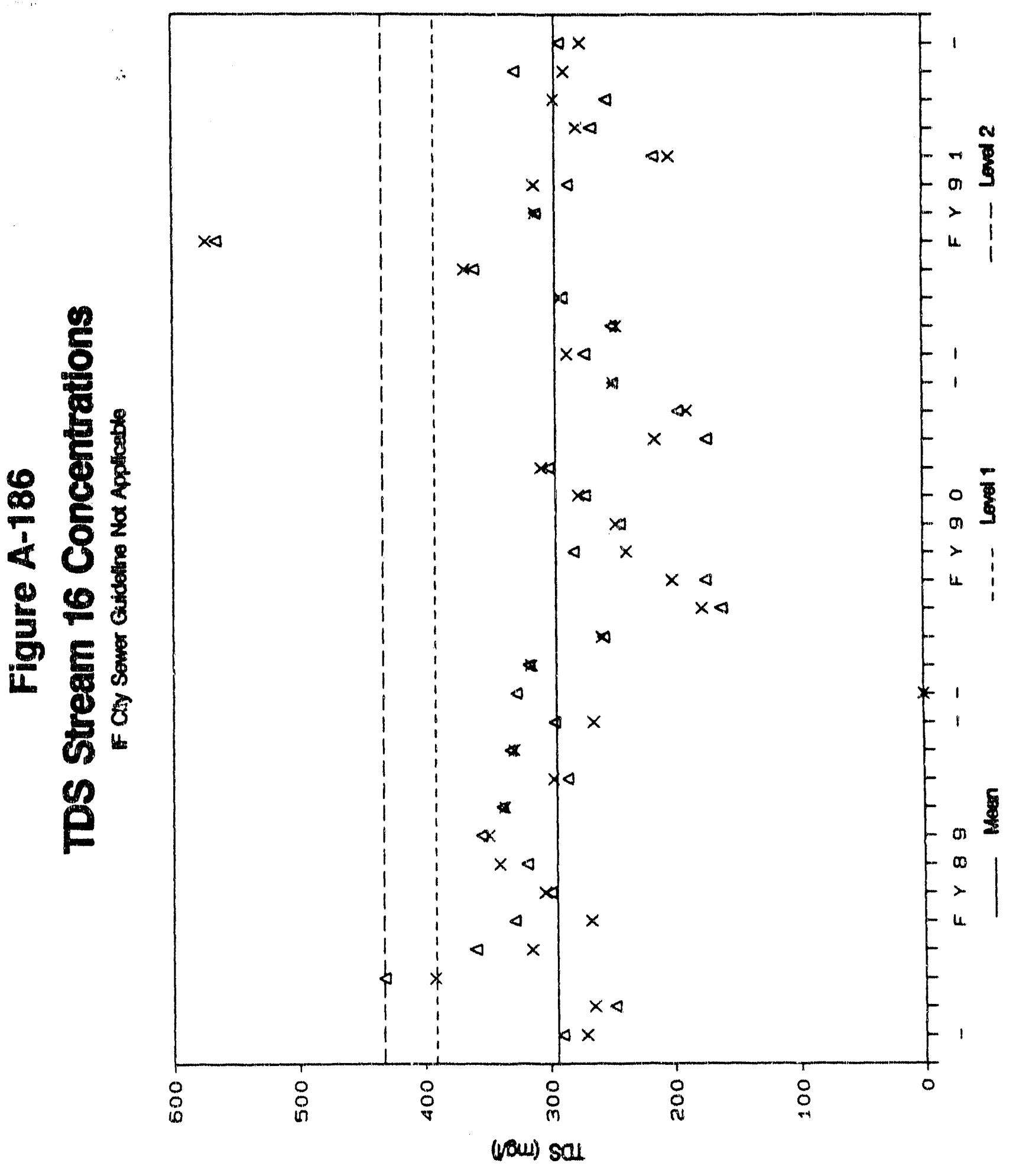




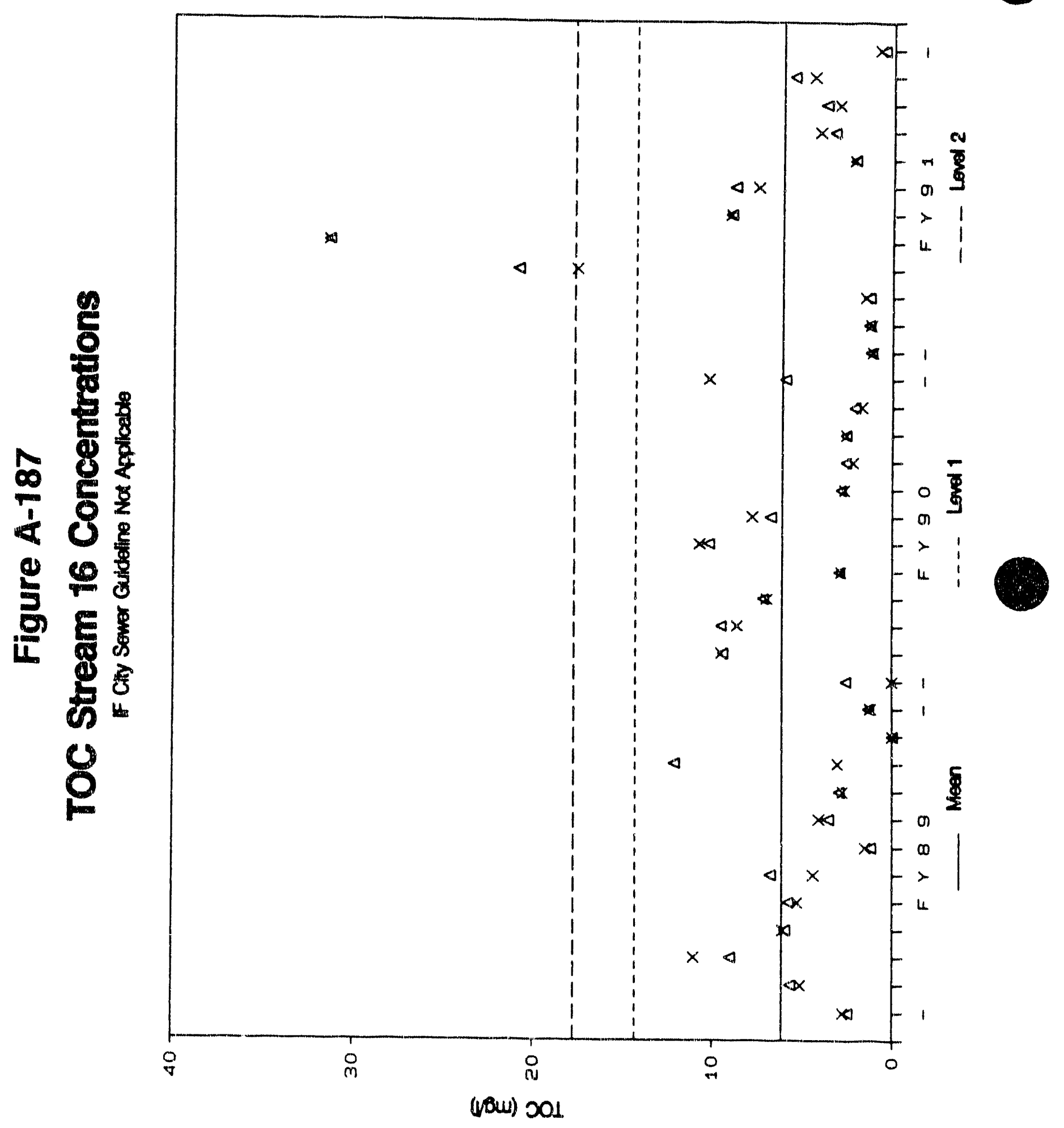




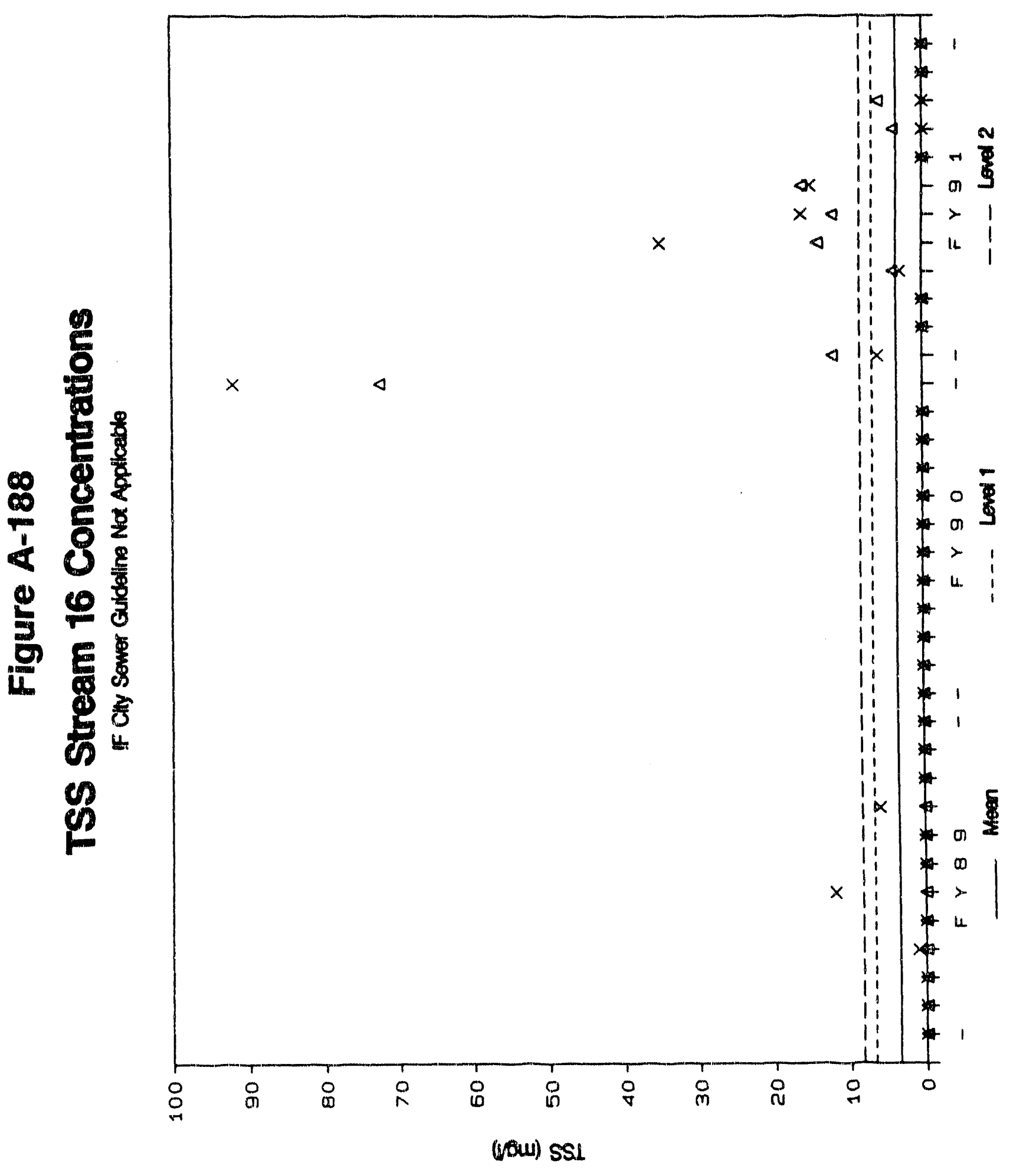




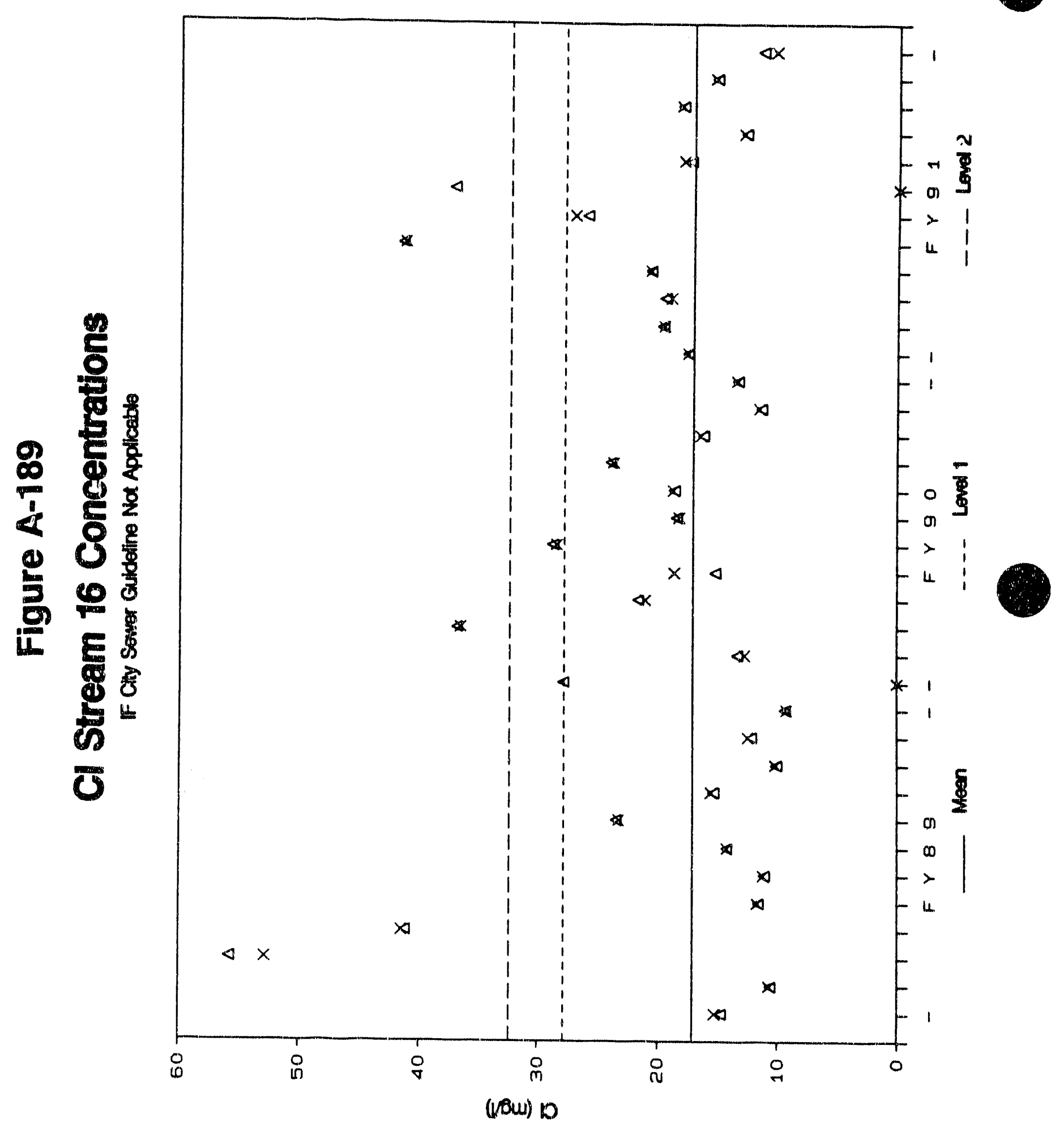




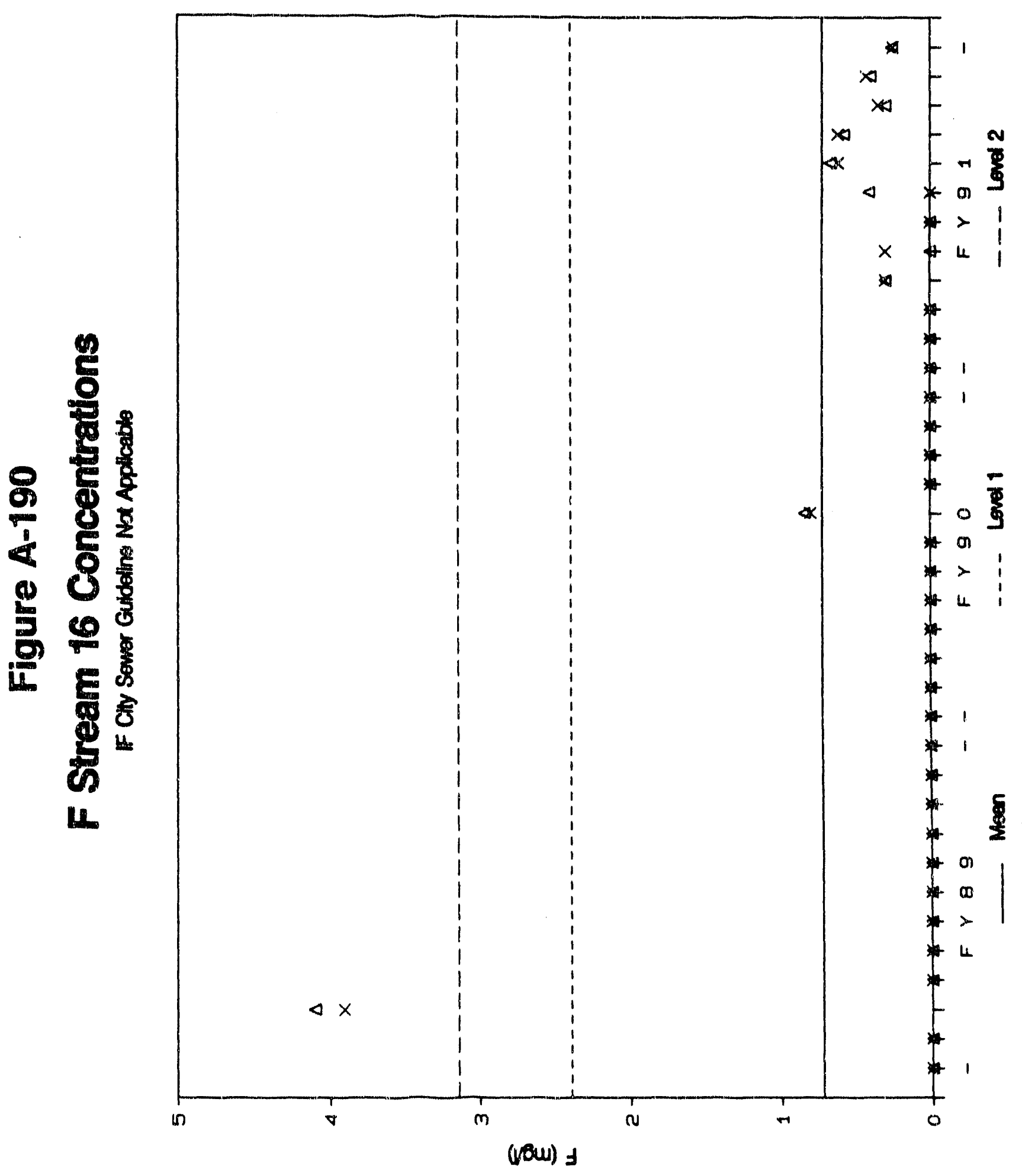



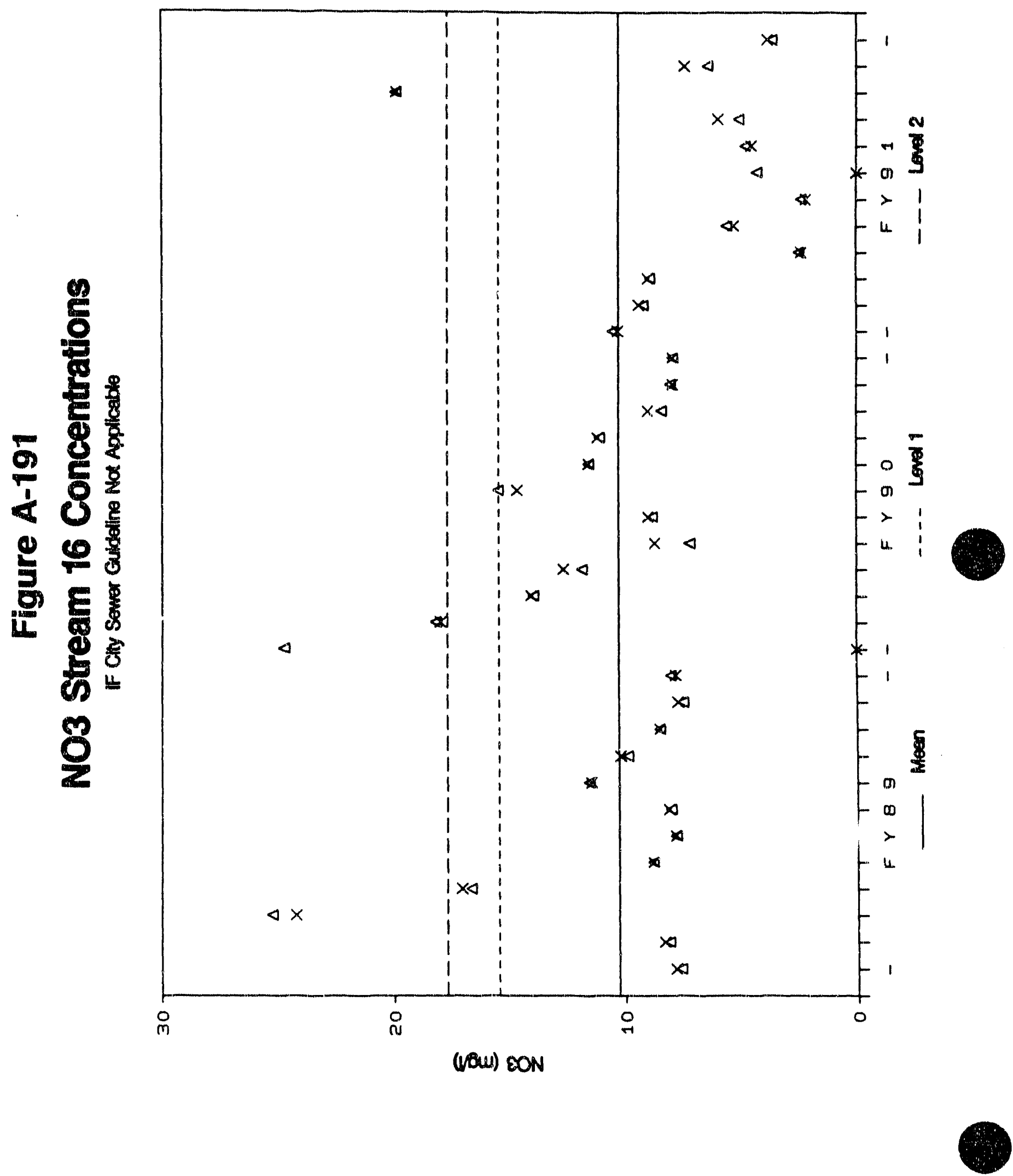


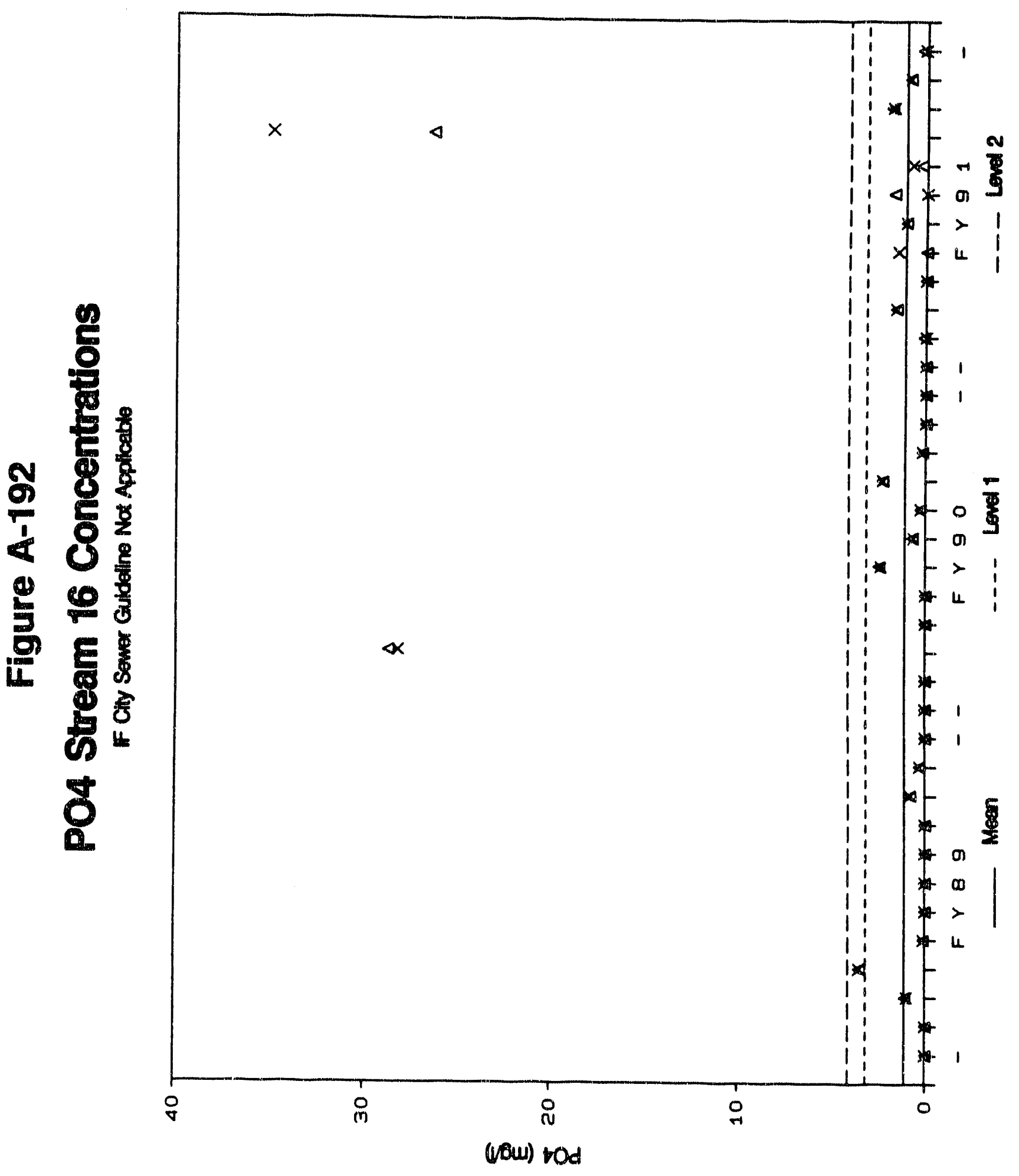




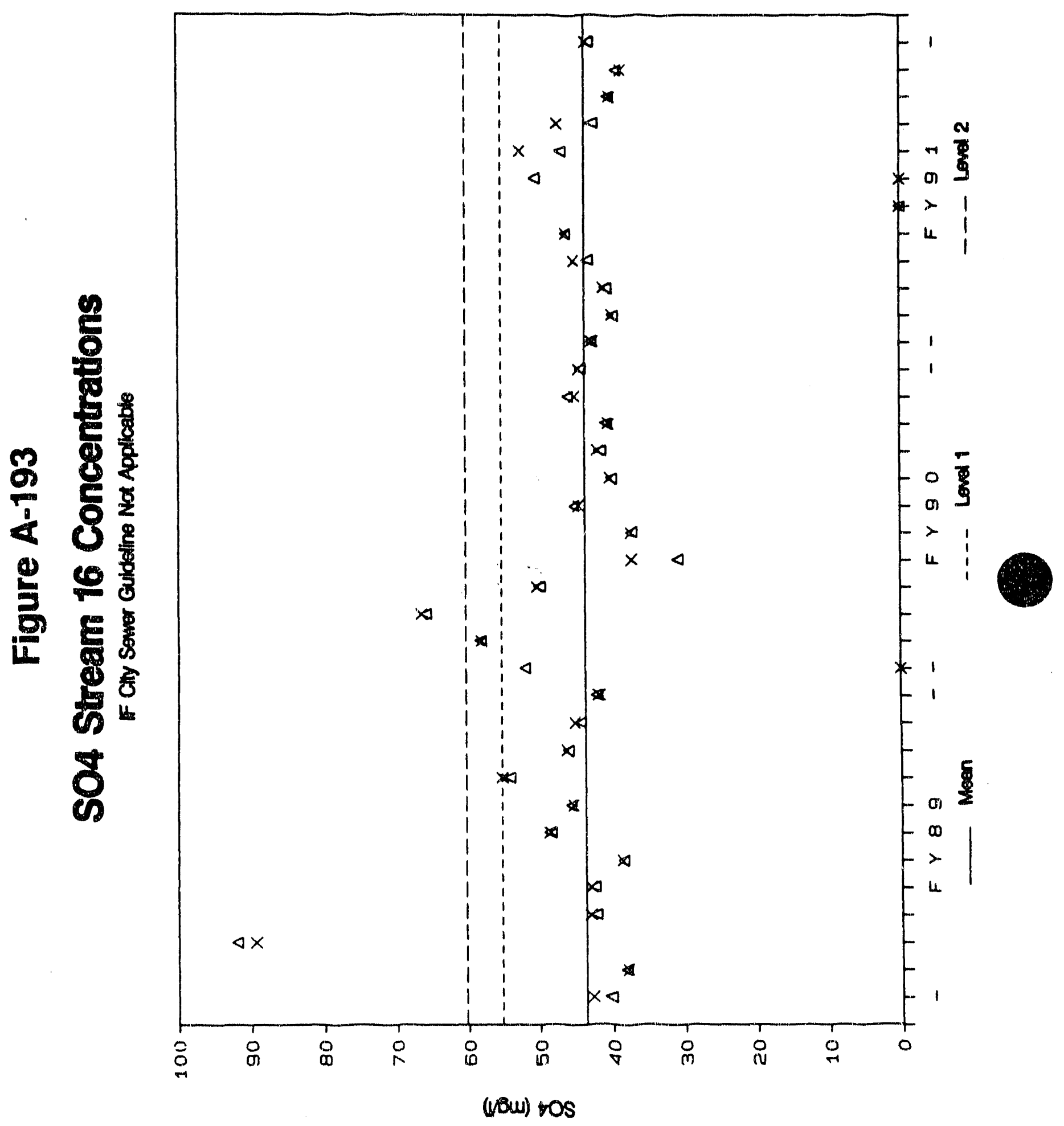




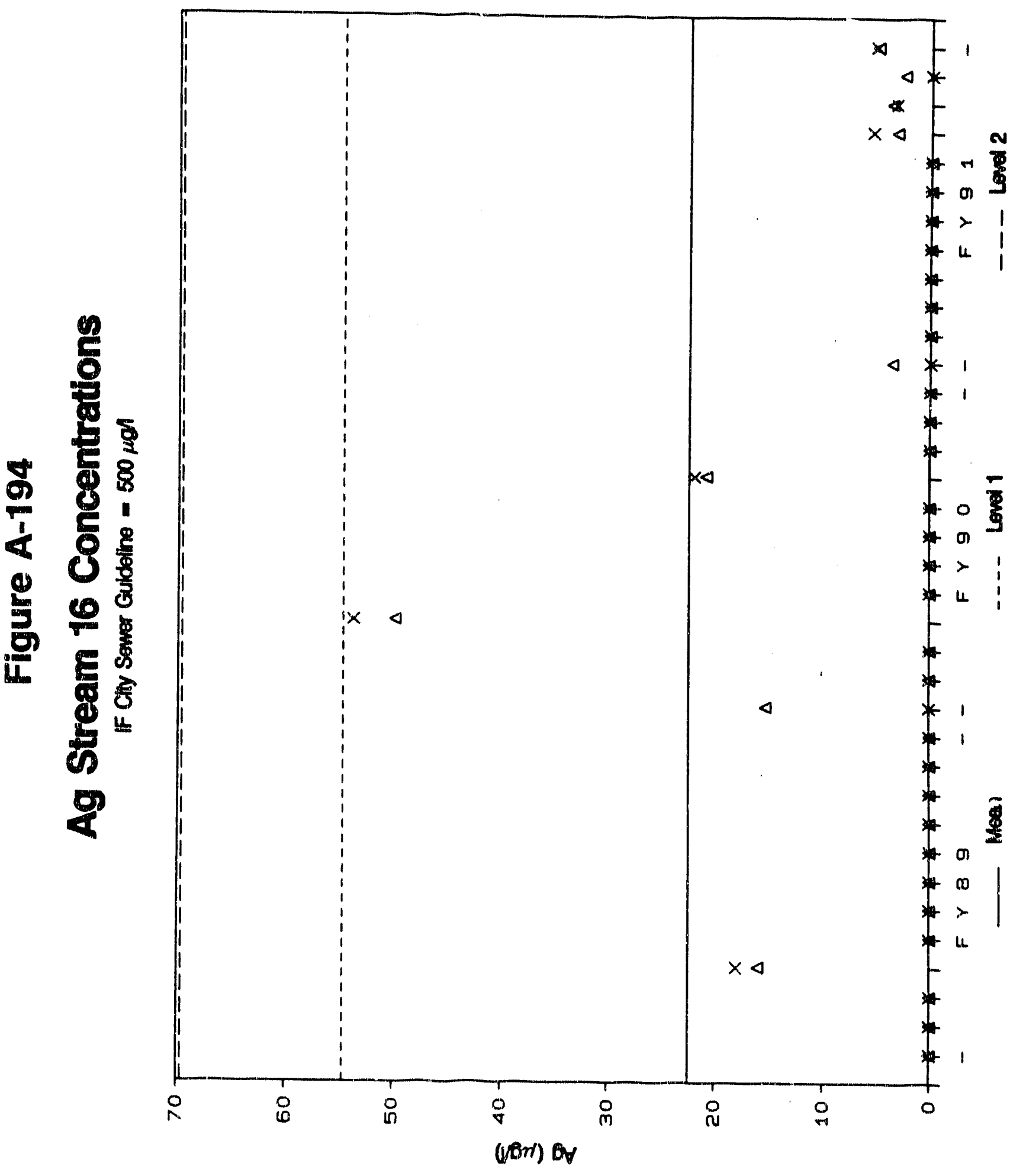




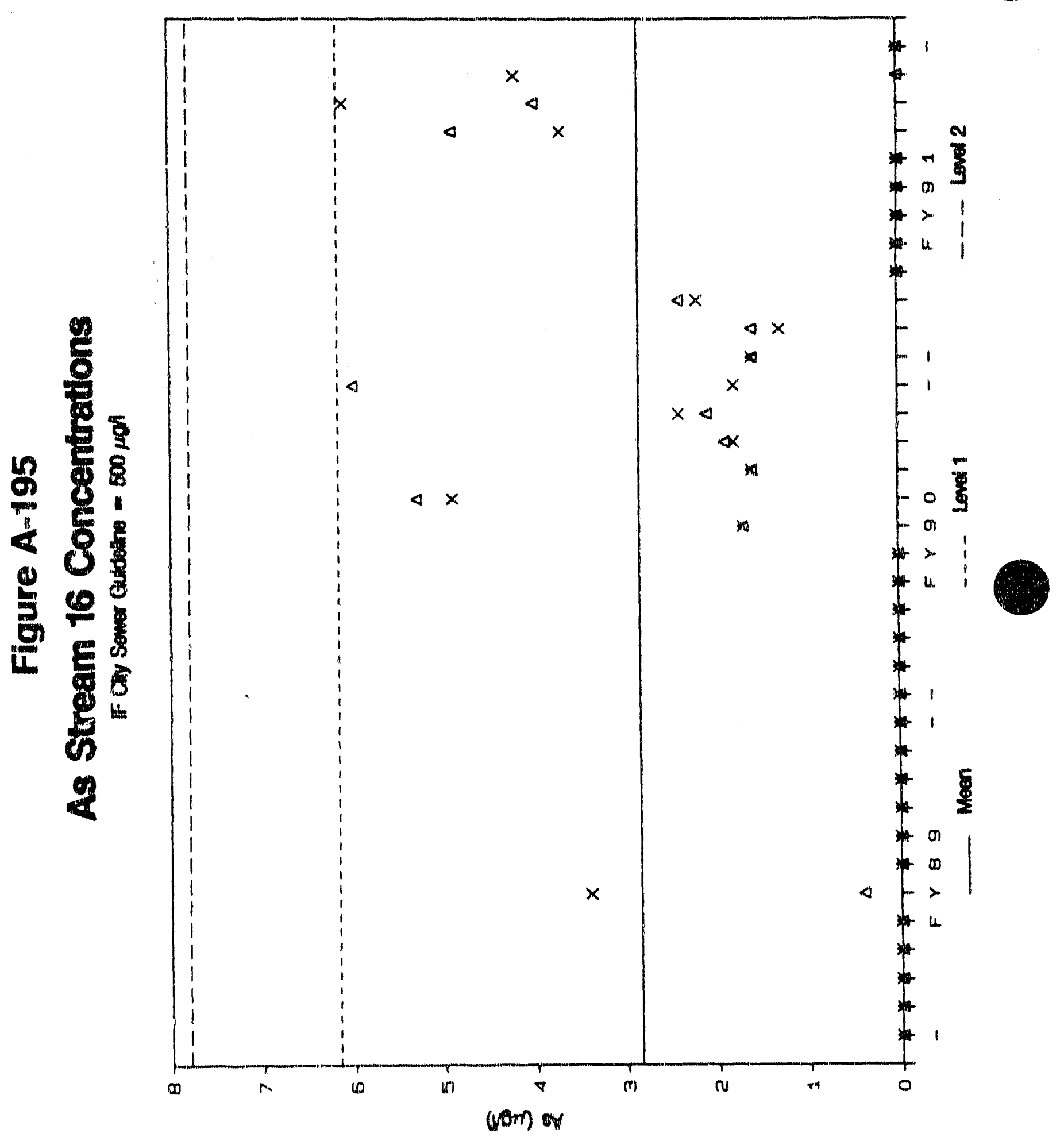




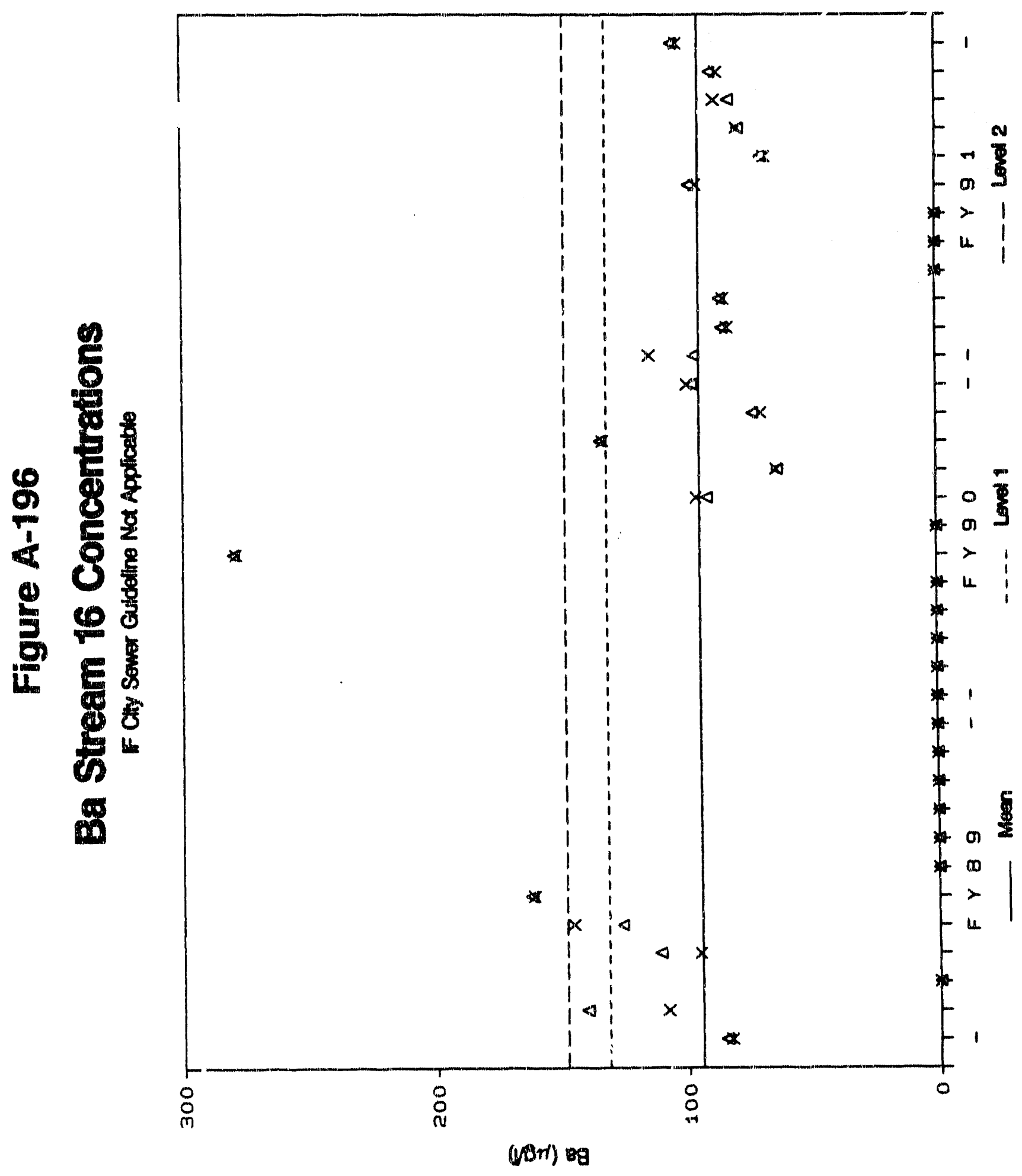




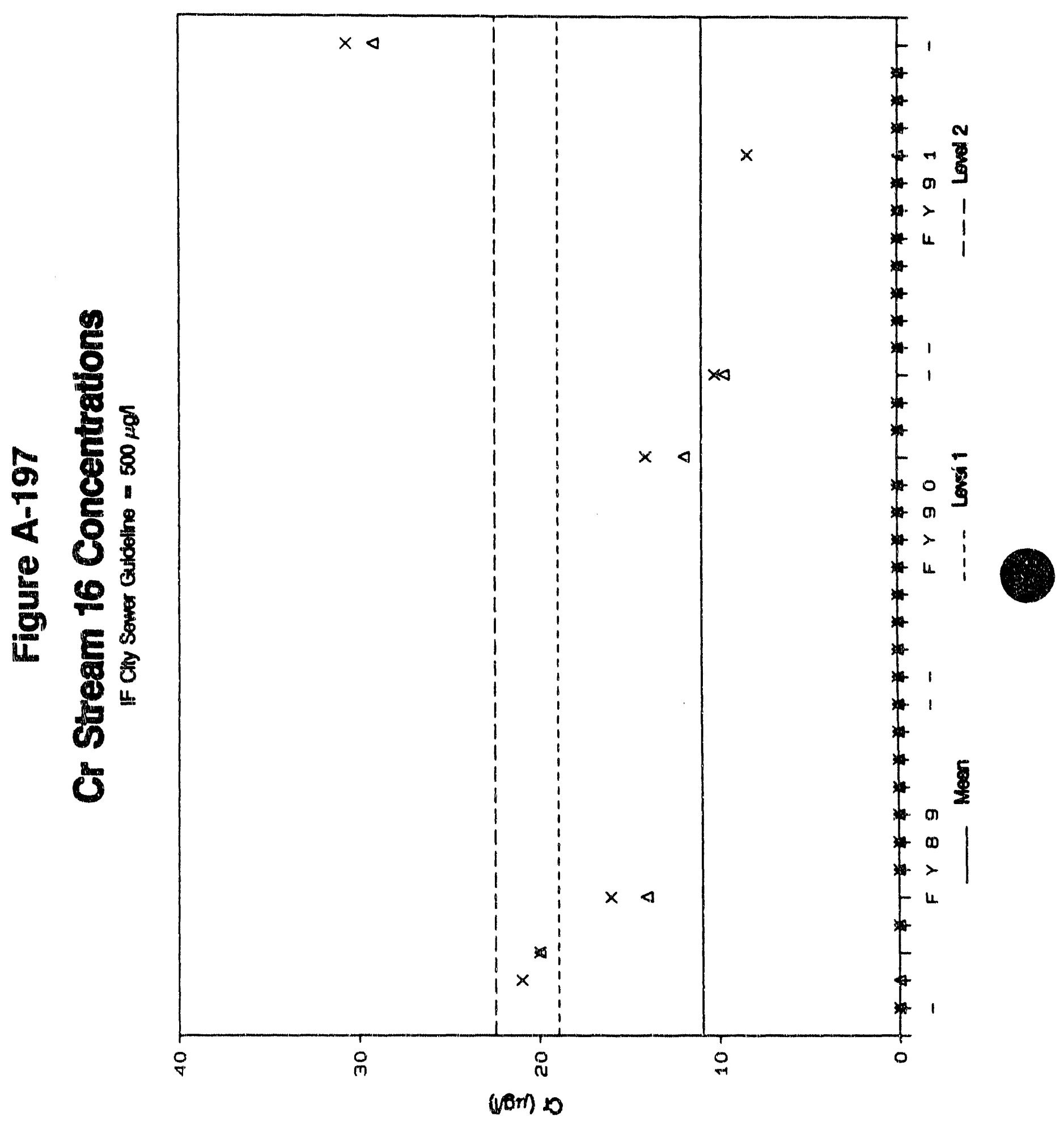




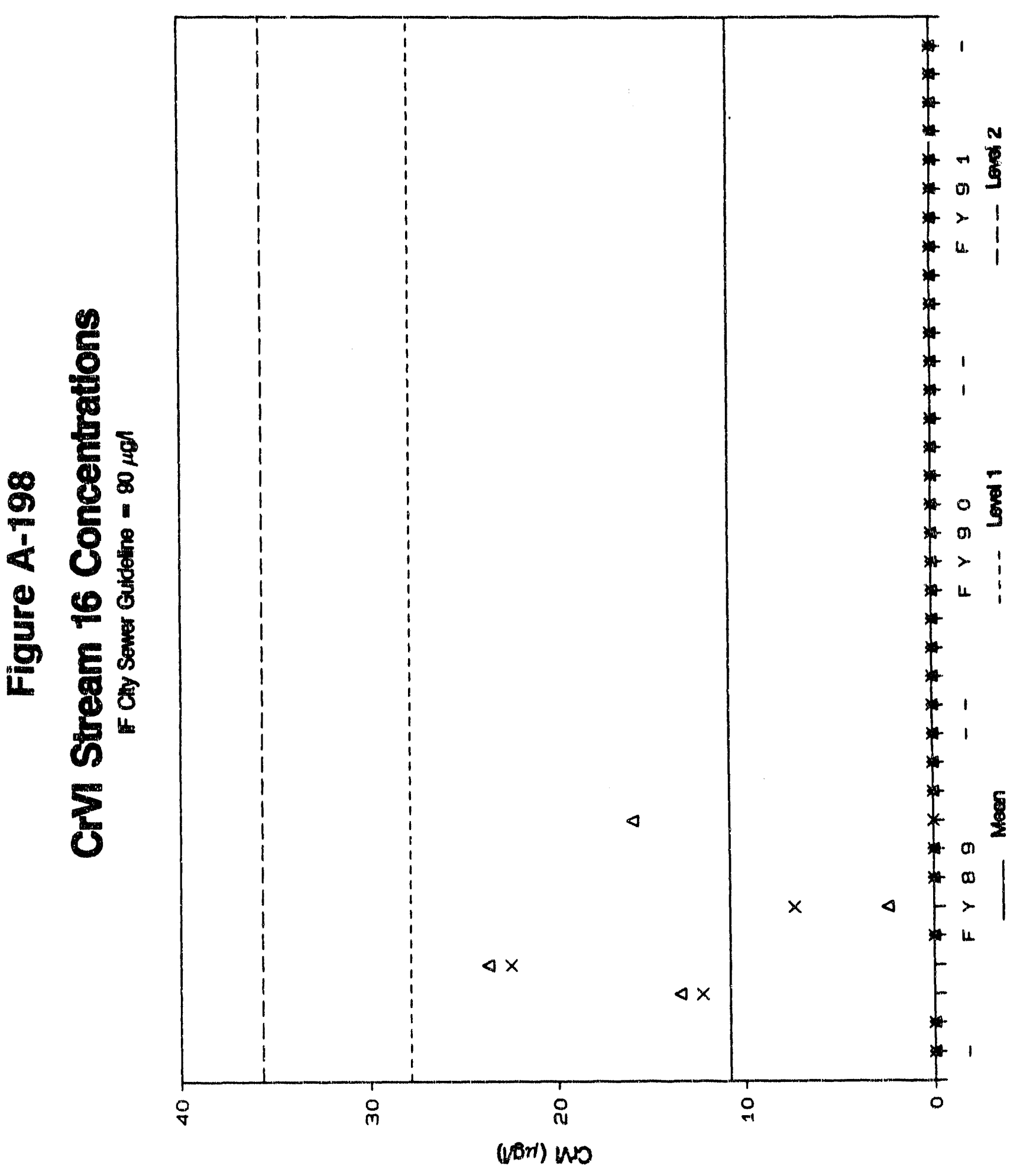




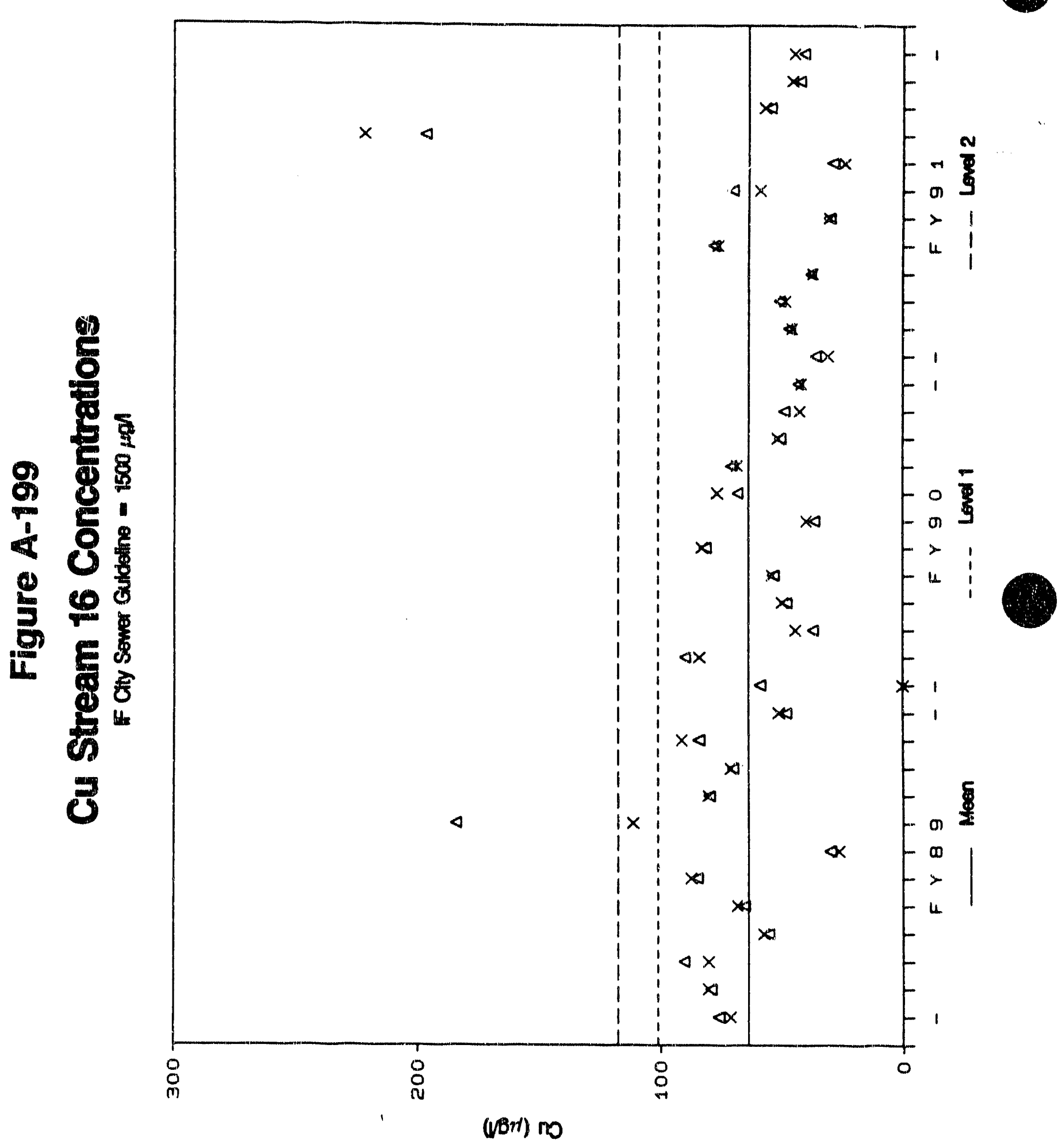




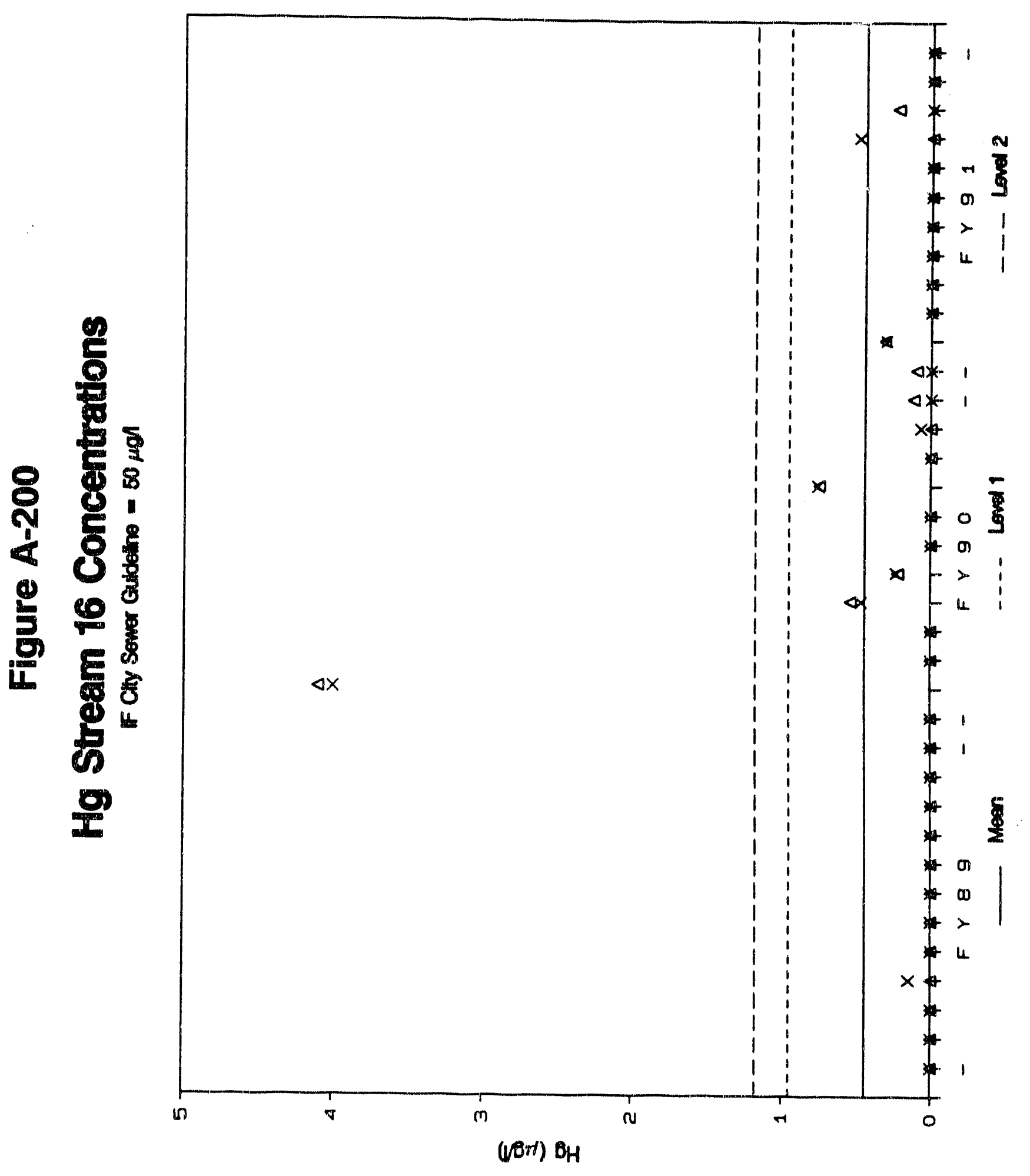




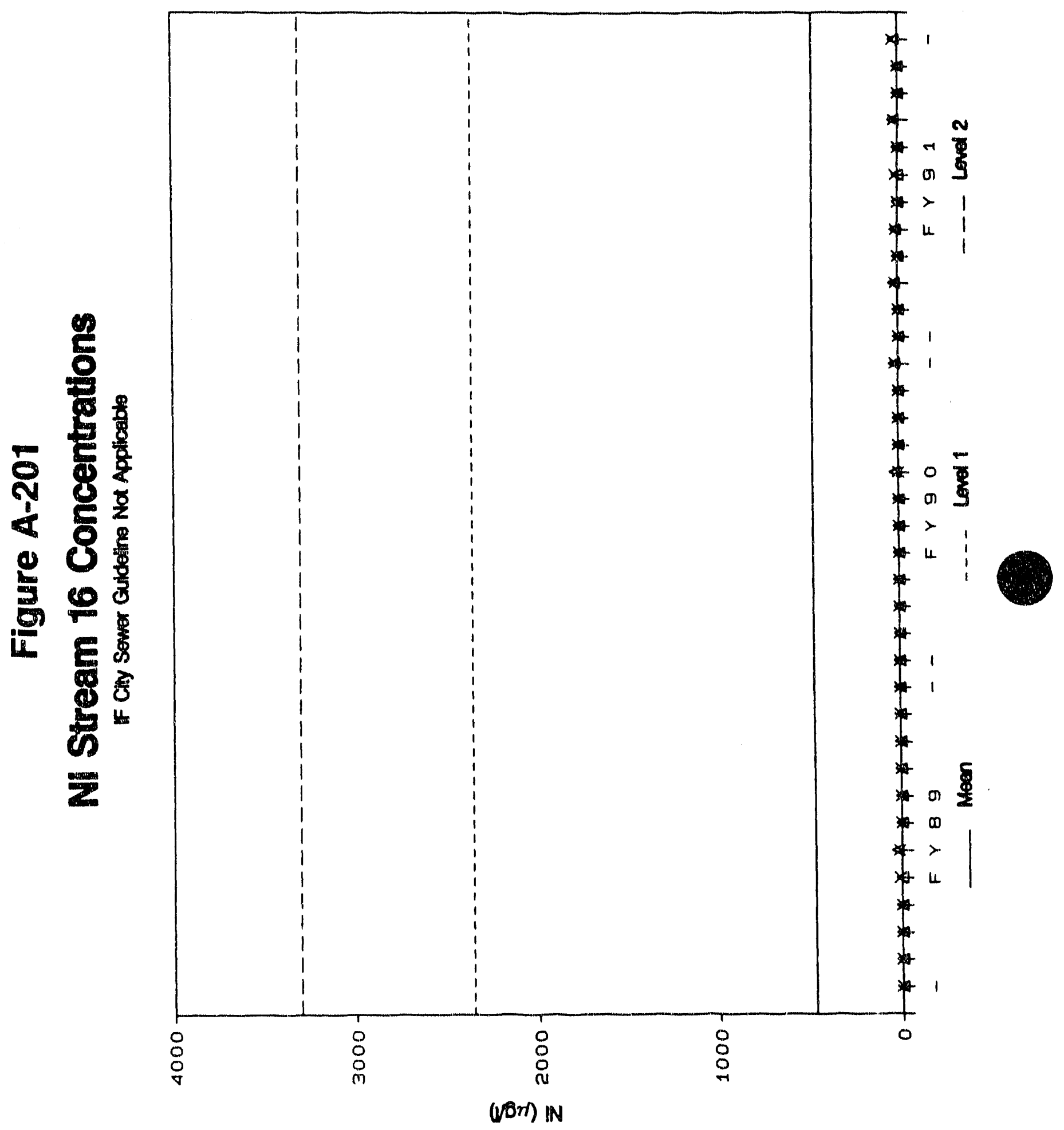




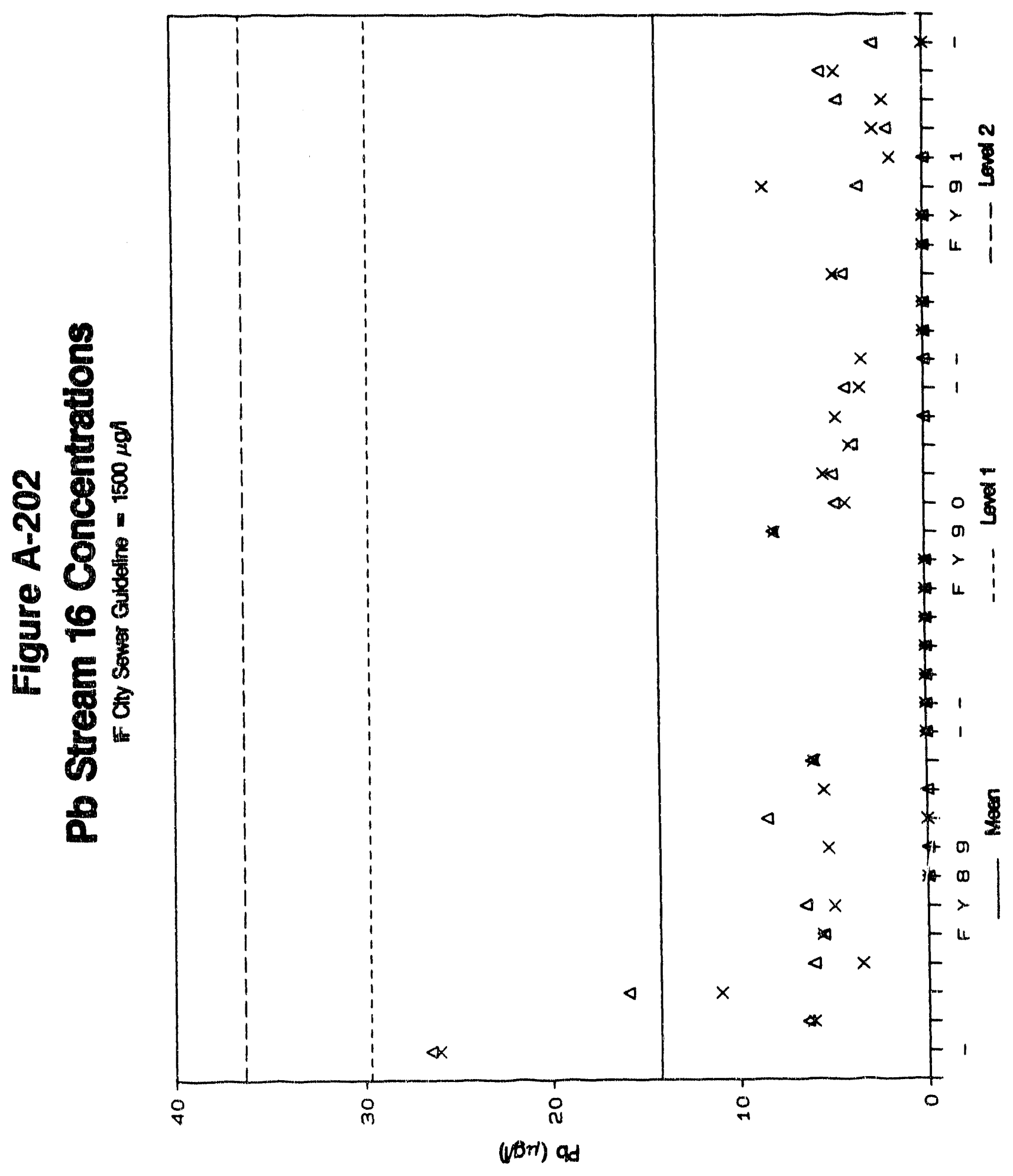




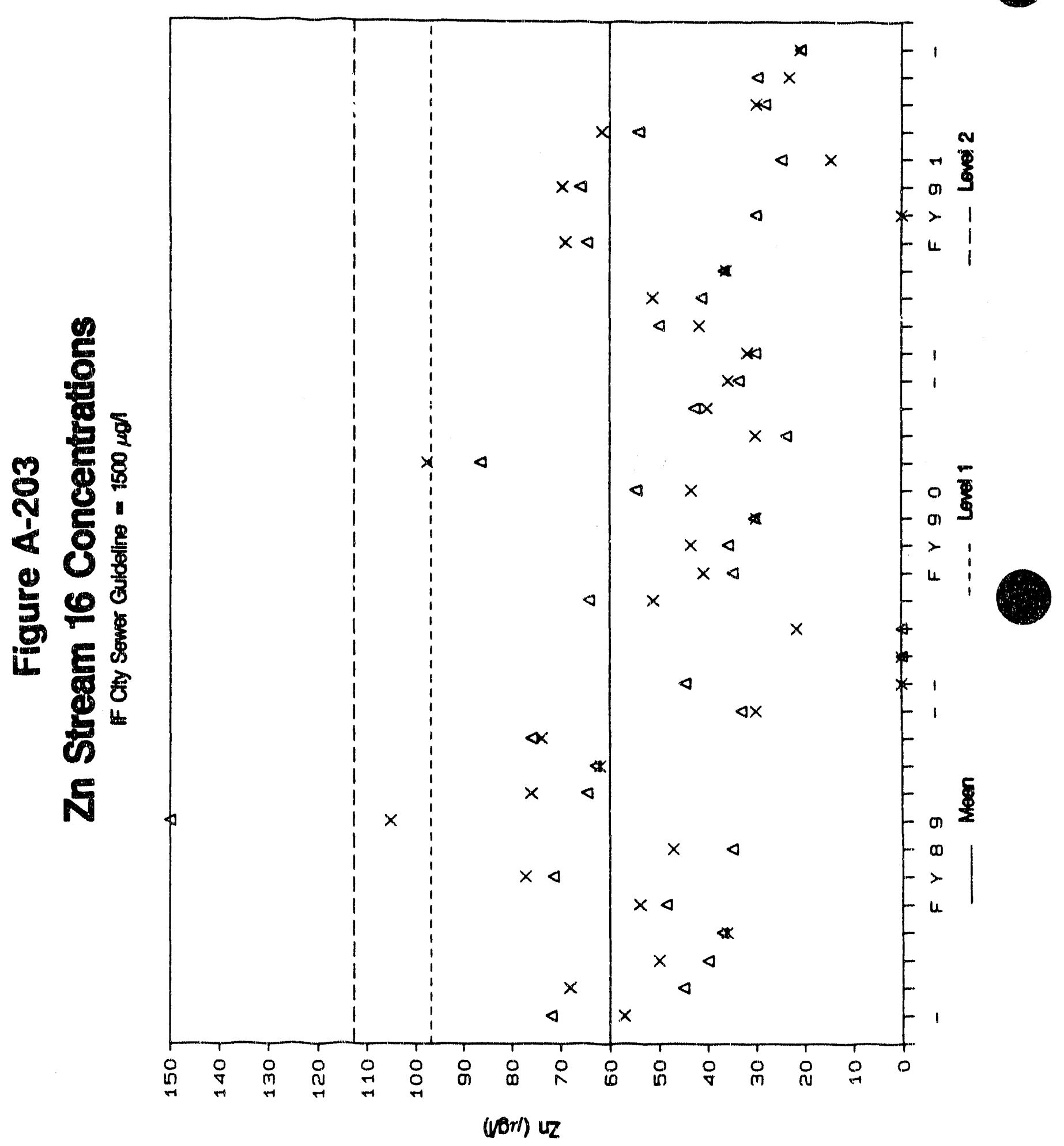




\section{Table A-59}

FY91 ENVIRONMENTAL MONITORING DATA

STREAM $=16$ (IRC waste effluent) QUARTER=91q2

$\begin{array}{ccccccccc}\text { MONTH } & \text { REP } & \begin{array}{c}\text { Cond } \\ (\mu S)\end{array} & \mathrm{pH} & \begin{array}{c}\mathrm{CN} \\ (\mathrm{mg} / 1)\end{array} & \begin{array}{c}\mathrm{TDS} \\ (\mathrm{mg} / 1)\end{array} & \begin{array}{c}\mathrm{TrJC} \\ (\mathrm{mg} / 1)\end{array} & \begin{array}{c}\mathrm{TSS} \\ (\mathrm{mg} / 1)\end{array} & \begin{array}{c}\mathrm{CI} \\ (\mathrm{mg} / 1)\end{array} \\ \mathrm{Jan} & 1 & 617 & 7.60 & \mathrm{X} & 360 & 20.9 & 4.00 & 20.7 \\ \mathrm{Jan} & 2 & 597 & 7.94 & \mathrm{X} & 367 & 17.6 & 3.00 & 20.7 \\ \text { Feb } & 1 & 618 & 6.54 & \mathrm{X} & 565 & 31.4 & 14.0 & 41.5 \\ \text { Feb } & 2 & 604 & 7.32 & \mathrm{X} & 573 & 31.4 & 35.0 & 41.3 \\ \text { Mar } & 1 & 780 & 7.93 & \mathrm{X} & 310 & 9.00 & 12.0 & 26.0 \\ \text { Mar } & 2 & \mathrm{X} & \mathrm{X} & \mathrm{X} & 310 & 9.00 & 16.0 & 27.0\end{array}$

$\begin{array}{lccccccc}\text { MONTH } & \text { REP } & \begin{array}{c}\mathrm{F} \\ (\mathrm{mg} / \mathrm{l})\end{array} & \begin{array}{c}\mathrm{NO3} \\ (\mathrm{mg} / 1)\end{array} & \begin{array}{c}\mathrm{PO4} \\ (\mathrm{mg} / \mathrm{l})\end{array} & \begin{array}{c}\mathrm{SO4} \\ (\mathrm{mg} / 1)\end{array} & \begin{array}{c}\mathrm{Ag} \\ (\mu \mathrm{g} / 1)\end{array} & \begin{array}{c}\mathrm{As} \\ (\mu \mathrm{g} / \mathrm{l}\end{array} \\ \text { Jan } & 1 & 0.30 & 2.50 & \mathrm{X} & 43.1 & \mathrm{X} & \mathrm{X} \\ \mathrm{Jan} & 2 & 0.30 & 2.40 & \mathrm{X} & 45.1 & \mathrm{X} & \mathrm{X} \\ \mathrm{Feb} & 1 & \mathrm{X} & 5.60 & \mathrm{X} & 46.3 & \mathrm{X} & \mathrm{X} \\ \text { Feb } & 2 & 0.30 & 5.30 & 1.53 & 46.3 & \mathrm{X} & \mathrm{X} \\ \text { Mar } & 1 & \mathrm{X} & 2.40 & 1.10 & \mathrm{X} & \mathrm{X} & \mathrm{X} \\ \text { Mar } & 2 & \mathrm{X} & 2.20 & 1.10 & \mathrm{X} & \mathrm{X} & \mathrm{X}\end{array}$

\begin{tabular}{|c|c|c|c|c|c|c|}
\hline MONTH & REP & $\begin{array}{l}\mathrm{Ba} \\
(\mu \mathrm{g} / \mathrm{l})\end{array}$ & $\frac{c d}{(\mu g / I)}$ & $\begin{array}{l}\mathrm{Cr} \\
(\mu \mathrm{g} / 1)\end{array}$ & $\begin{array}{l}\text { CrVI } \\
(\mu g / I)\end{array}$ & $\underset{(\mu \mathrm{g} / 1)}{\mathrm{Cu}}$ \\
\hline Jan & 1 & X & $x$ & $\mathrm{X}$ & $x$ & 37.5 \\
\hline Jan & 2 & $x$ & $x$ & $x$ & $\mathrm{x}$ & 36.7 \\
\hline Feb & 1 & $x$ & $x$ & $x$ & $x$ & 77.9 \\
\hline Feb & 2 & $\mathrm{x}$ & $x$ & $x$ & $x$ & 76.3 \\
\hline Mar & 1 & $x$ & $x$ & $x$ & $x$ & 30.0 \\
\hline Mar & 2 & $\mathrm{x}$ & $x$ & $\mathrm{x}$ & $x$ & 30.0 \\
\hline
\end{tabular}

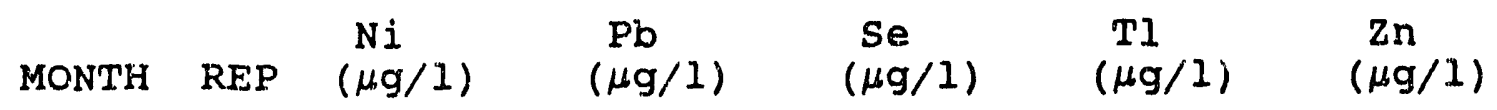

$\begin{array}{lllllll}\text { Jan } & 1 & X & 4.30 & X & X & 36.6 \\ \text { Jan } & 2 & X & 4.80 & X & X & 36.1 \\ \text { Feb } & 1 & 14.3 & X & X & X & 64.7 \\ \text { Feb } & 2 & 12.4 & X & X & X & 68.9 \\ \text { Mar } & 1 & X & X & X & X & 30.0 \\ \text { Mar } & 2 & X & X & X & X & X\end{array}$

'. - missing

' $\dot{X}$ ' - below practical quantitation level 
FY91 ENVIRONMENTAL MONITORING DATA

STREAM $=16$ (IRC waste effluent) QUARTER=91q3

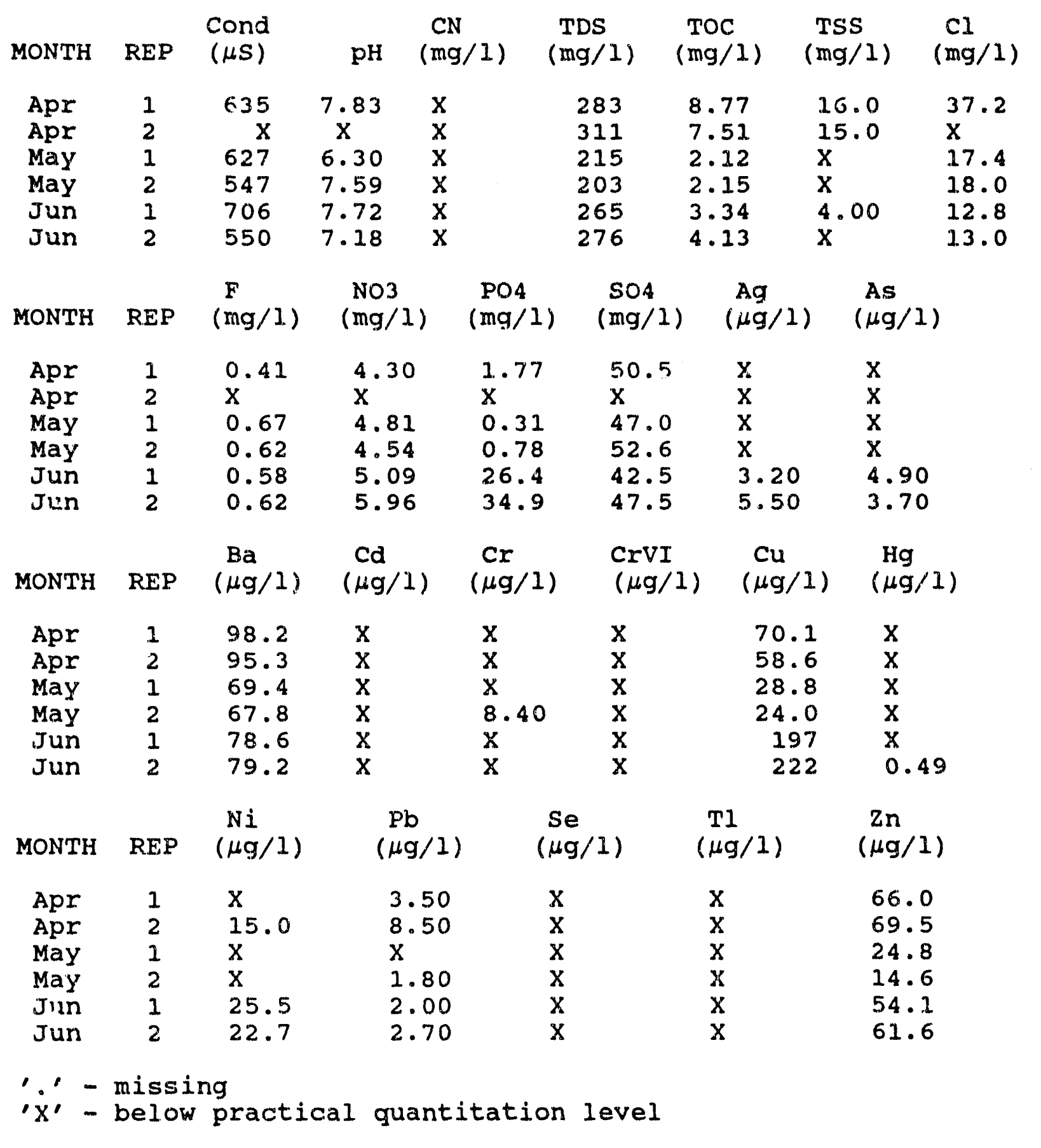




\section{Table A-61}

FY91 ENVIRONIENTAL MONITORING DATA

STREAM=16 (IRC waste effluent) QUARTER=9194

$\begin{array}{ccccccccc}\text { MONTH } & \text { REP } & \begin{array}{c}\text { Cond } \\ (\mu S)\end{array} & \mathrm{pH} & \begin{array}{c}\mathrm{CN} \\ (\mathrm{mg} / \mathrm{l})\end{array} & \begin{array}{c}\mathrm{TDS} \\ (\mathrm{mg} / 1)\end{array} & \begin{array}{c}\mathrm{TOC} \\ (\mathrm{mg} / 1)\end{array} & \begin{array}{c}\mathrm{TSS} \\ (\mathrm{mg} / 1)\end{array} & \begin{array}{c}\mathrm{Cl} \\ (\mathrm{mg} / 1)\end{array} \\ \text { JuI } & 1 & 612 & 7.22 & \mathrm{X} & 253 & 3.82 & 6.00 & 18.1 \\ \text { JuI } & 2 & 622 & 7.72 & \mathrm{X} & 295 & 3.05 & \mathrm{X} & 18.2 \\ \text { Aug } & 1 & 862 & 8.26 & \mathrm{X} & 326 & 5.54 & \mathrm{X} & 15.3 \\ \text { Aug } & 2 & 764 & 8.08 & \mathrm{X} & 286 & 4.44 & \mathrm{X} & 15.4 \\ \text { Sep } & 1 & 452 & 8.08 & \mathrm{X} & 290 & 0.56 & \mathrm{X} & 11.4 \\ \text { Sep } & 2 & 429 & 7.82 & \mathrm{X} & 273 & 0.78 & \mathrm{X} & 10.3\end{array}$

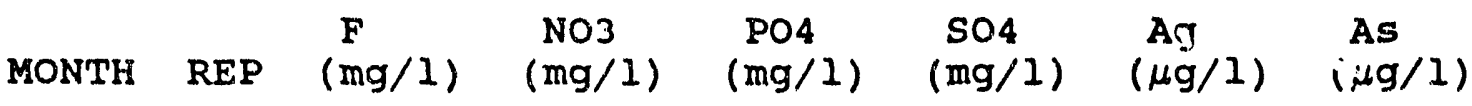

$\begin{array}{llllllll}\text { Jul } & 1 & 0.30 & 19.9 & 1.93 & 40.4 & 3.70 & 4.00 \\ \text { Jul } & 2 & 0.35 & 19.9 & 1.84 & 40.2 & 3.30 & 6.10 \\ \text { Aug } & 1 & 0.40 & 6.40 & 0.93 & 39.3 & 2.50 & X \\ \text { Aug } & 2 & 0.43 & 7.40 & 0.92 & 38.6 & X & 4.20 \\ \text { Sep } & 1 & 1.26 & 3.63 & 0.26 & 43.0 & 5.10 & X \\ \text { Sep } & 2 & 0.25 & 3.83 & 0.10 & 43.3 & 5.20 & X\end{array}$

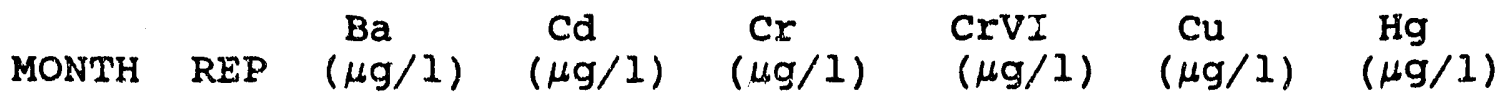

$\begin{array}{lrrlllll}\text { Jul } & 1 & 82.6 & X & X & X & 54.5 & 0.230 \\ \text { Jul } & 2 & 87.9 & X & X & X & 56.7 & X \\ \text { Aug } & 1 & 89.6 & X & X & X & 42.4 & X \\ \text { Aug } & 2 & 86.9 & X & X & X & 45.6 & X \\ \text { Sep } & 1 & 105 & 8.00 & 29.2 & X & 40.6 & X \\ \text { Sep } & 2 & 103 & 6.40 & 30.7 & X & 44.6 & X\end{array}$

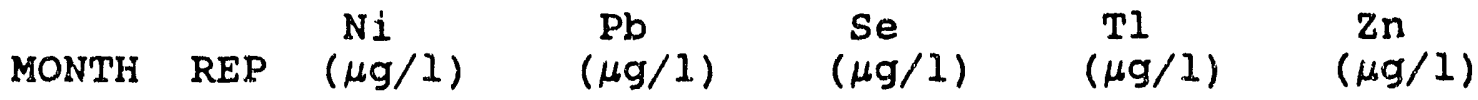

$\begin{array}{lllllll}\text { JuI } & 1 & \mathrm{X} & 4.60 & \mathrm{X} & \mathrm{X} & 28.1 \\ \text { JuI } & 2 & \mathrm{X} & 2.20 & \mathrm{X} & \mathrm{X} & 29.9 \\ \text { Aug } & 1 & \mathrm{X} & 5.50 & \mathrm{X} & \mathrm{X} & 29.7 \\ \text { Aug } & 2 & \mathrm{X} & 4.70 & \mathrm{X} & \mathrm{X} & 23.1 \\ \text { Sep } & 1 & 19.8 & 2.70 & \mathrm{X} & \mathrm{X} & 20.7 \\ \text { Sep } & 2 & 28.0 & \mathrm{X} & \mathrm{X} & \mathrm{X} & 20.8\end{array}$

$'$ ' - missing

' $X$ ' - below practical quantitation level 


\section{Table A-62}

SUMMARY STATISTICS

STREAM $=16$ (IRC waste effluent)

\begin{tabular}{|c|c|c|c|c|c|c|c|}
\hline ANALY & YTE & $\begin{array}{c}\text { FY87-FY9 } \\
\text { MEAN }\end{array}$ & STD & $\mathrm{N}$ & LEVELI & LEVEL2 & GUIDELIN \\
\hline Cond & $(\mu S)$ & 549 & 41.8 & 93 & 619 & 648 & $\mathbf{N} / \mathbf{A}$ \\
\hline $\mathrm{pH}$ & & 7.55 & 0.36 & 96 & $\begin{array}{ll}1 & 6.83 \\
u & 8.27\end{array}$ & $\begin{array}{ll}1 & 6.60 \\
u & 8.51\end{array}$ & $\begin{array}{ll}1 & 6.5 \\
u & 8.5\end{array}$ \\
\hline $\mathrm{CN}$ & $(\mathrm{mg} / \mathrm{l})$ & & & & & & 0.5 \\
\hline TDS & $(\mathrm{mg} / 1)$ & 294 & 58.0 & 93 & 391 & 432 & $N / A$ \\
\hline TOC & $(\mathrm{mg} / 1)$ & 6.17 & 4.85 & 88 & 14.3 & 17.7 & $N / A$ \\
\hline TSS & $(\mathrm{mg} / 1)$ & 3.57 & 1.74 & 14 & 6.76 & 8.35 & $\mathrm{~N} / \mathrm{A}$ \\
\hline $\mathrm{Cl}$ & $(\mathrm{mg} / \mathrm{l})$ & $17 \cdot 1$ & 6.42 & 91 & 27.9 & 32.4 & $\mathrm{~N} / \mathrm{A}$ \\
\hline $\mathbf{F}$ & $(\mathrm{mg} / \mathrm{l})$ & 0.73 & 0.97 & 31 & 2.39 & 3.1 .4 & $N / A$ \\
\hline NO3 & $(\mathrm{mg} / \mathrm{l})$ & 10.3 & 3.12 & 90 & 15.5 & 17.7 & $N / A$ \\
\hline PO4 & $(\mathrm{mg} / 1)$ & 1.14 & $1 \cdot 18$ & 30 & 3.17 & 4.08 & $\mathbf{N} / \mathbf{A}$ \\
\hline $\mathrm{SO} 4$ & $(\mathrm{mg} / 1)$ & 43.5 & 7.06 & 91 & 55.3 & 60.3 & $\mathrm{~N} / \mathrm{A}$ \\
\hline Ag & $(\mu g / 1)$ & 22.5 & 18.2 & 21 & 54.7 & 69.7 & 500 \\
\hline As & $(\mu g / 1)$ & 2.85 & 1.82 & 15 & 6.17 & 7.79 & 500 \\
\hline $\mathrm{Ba}$ & $(\mu \mathrm{g} / 1)$ & 94.3 & 22.2 & 62 & 132 & 148 & $N / A$ \\
\hline $\mathrm{Cd}$ & $(\mu g / 1)$ & & & & & & 500 \\
\hline $\mathrm{Cr}$ & $(\mu \mathrm{g} / 1)$ & 10.9 & 4.67 & 35 & 18.9 & 22.5 & 500 \\
\hline CrVI & $(\mu \mathrm{g} / 1)$ & 10.8 & 9.63 & 21 & 27.8 & 35.7 & 90 \\
\hline $\mathrm{Cu}$ & $(\mu g / 1)$ & 63.4 & 22.6 & 92 & 101 & 117 & 1500 \\
\hline $\mathrm{Hg}$ & $(\mu \mathrm{g} / 1)$ & 0.45 & 0.29 & 33 & 0.95 & 1.18 & 50 \\
\hline $\mathrm{Ni}$ & $(\mu \mathrm{g} / 1)$ & 476 & 1020 & 13 & 2360 & 3310 & 1500 \\
\hline $\mathrm{Pb}$ & $(\mu g / 1)$ & 14.3 & 9.17 & 62 & 29.7 & 36.4 & 1500 \\
\hline $\mathrm{Se}$ & $(\mu g / I)$ & & & & & & $N / A$ \\
\hline T1 & $(\mu g / 1)$ & & & & & & $\mathbf{N} / \mathbf{A}$ \\
\hline $\mathrm{Zn}$ & $(\mu \mathrm{g} / 1)$ & 60.0 & $22 \cdot 1$ & 90 & 96.8 & 113 & 1500 \\
\hline \multicolumn{8}{|c|}{$\begin{array}{l}\text { Guideline - city of Idaho Falls Sewer Regulatory Guidelines } \\
\text { N/A - not applicable } \\
\text { A-294 }\end{array}$} \\
\hline
\end{tabular}




\section{Table A-63}

FY91 ENVIRONMENTAL MONITORING VOLATILE ORGANICS DATA

\begin{tabular}{|c|c|c|c|}
\hline MONTH & REP & COMPOUND & $\begin{array}{c}\text { CONCENTRATION } \\
(\mu \mathrm{g} / 1)\end{array}$ \\
\hline $\begin{array}{l}\text { oct } \\
\text { oct } \\
\text { oct }\end{array}$ & $\begin{array}{l}1 \\
1 \\
1\end{array}$ & $\begin{array}{l}\text { Dibromochloromethane } \\
\text { Toluene } \\
\text { all other compounds }\end{array}$ & $\begin{array}{l}0.80 \\
0.87 \\
x\end{array}$ \\
\hline Nov & 1 & all compounds & $x$ \\
\hline Dec & 1 & all compounds & $\mathrm{x}$ \\
\hline
\end{tabular}




\section{Table A-64}

FY91 ENVIRONMENTAL MONITORING VOLATILE CRGANICS DATA STREAM=16 (IRC waste effluent) QUARTER=91q2 CONCENTRATION

MONTH REP CONPOUND

$\begin{array}{lll}\operatorname{Jan} & 1 & 1,1,1-\text { Trichloroethane } \\ \operatorname{Jan} & 1 & \text { all other compounds }\end{array}$

Feb 1 Acetone

Feb 1 Methylene Chloride

Feb 1 all other compounds

Mar 1 Toluene

Mar 1 all other compounds $(\mu g / 1)$

3.4

$\mathrm{X}$

$$
\begin{array}{r}
222.0 \\
3.0 \\
x
\end{array}
$$
4.0
$\mathrm{X}$

' $x$ ' - below practical quantitation level 


\section{Table A-65}

FY91 ENVIRONMENTAL MONITORING VOLATILE ORGANICS DATA

STREAM=16 (IRC waste effluent) QUARTER=91g3

MONTH REP COMPOUND

Apr 1 4-Penten-2-OL (TIC)

Apr 1 Acetone

Apr 1 all other compounds

$\begin{array}{lll}\text { May } & 1 & \text { Chloroform } \\ \text { May } & 1 & \text { all other compounds }\end{array}$

Jun 1 Acetone

Jun 1 all other compounds
CONCENTRATION

$(\mu g / 1)$

$$
\begin{array}{r}
100 \\
2300 \\
x
\end{array}
$$

7
$x$

48

' $X$ ' - below practical quantitation level

IIC - Tentatively Identified compound 


\section{Table A-66}

FY91 ENVIRONMENTAL MONITORING VOLATILE ORGANICS DATA

STREAM $=16$ (IRC waste effluent) QUARTER=91q4

MONTH REP COMPOUND CONCENTRATION

Jul 1 all compounds $\mathrm{x}$

Aug 1 Acetone 68

Aug 1 Ethanol (TIC) 8

Aug 1 Tetrahydofuran (TIC) 10

Aug 1 Toluene 10

Aug 1 Unknown (TIC) 2

Aug 1 all other compounds $x$

Sep 1 all compounàs $x$

' $X$ " - below practical quantitation level

TIC - Tentatively Identified Compound 


\section{Table A-67}

QUARTERLY MEANS AND VARIANCE COMPONENTS

STREAM=16 (IRC waste effluent)

\begin{tabular}{|c|c|c|c|c|c|c|c|c|c|}
\hline \multicolumn{2}{|c|}{ ANALYTE } & QTR & MEAN & $\begin{array}{r}\text { STD } \\
\text { MEAN }\end{array}$ & $\begin{array}{l}\text { STD } \\
\text { DAY }\end{array}$ & $\begin{array}{l}\text { STD } \\
\text { REP }\end{array}$ & $\mathrm{N}$ & $\begin{array}{r}N \\
\text { MISS }\end{array}$ & $\begin{array}{r}\mathrm{N} \\
\mathrm{BPQL}\end{array}$ \\
\hline \multicolumn{2}{|c|}{ Cond $(\mu S)$} & $\begin{array}{l}91 q 1 \\
9192 \\
9193 \\
9194\end{array}$ & $\begin{array}{l}606 \\
643 \\
613 \\
624\end{array}$ & $\begin{array}{r}36.9 \\
51.3 \\
8.07 \\
108\end{array}$ & $\begin{array}{r}61.3 \\
85.0 \\
0.00 \\
184\end{array}$ & $\begin{array}{l}7.00 \\
12.2 \\
87.7 \\
41.3\end{array}$ & $\begin{array}{l}5 \\
5 \\
5 \\
6\end{array}$ & $\begin{array}{l}1 \\
1 \\
1 \\
0\end{array}$ & $\begin{array}{l}0 \\
0 \\
0 \\
0\end{array}$ \\
\hline pH & & $\begin{array}{l}\text { 91q1 } \\
91 q 2 \\
9193 \\
9194\end{array}$ & $\begin{array}{l}7.43 \\
7.47 \\
7.32 \\
7.86\end{array}$ & $\begin{array}{l}0.26 \\
0.32 \\
0.23 \\
0.21\end{array}$ & $\begin{array}{l}0.43 \\
0.44 \\
0.00 \\
0.32\end{array}$ & $\begin{array}{l}0.11 \\
0.43 \\
0.70 \\
0.24\end{array}$ & $\begin{array}{l}5 \\
5 \\
5 \\
6\end{array}$ & $\begin{array}{l}1 \\
1 \\
1 \\
0\end{array}$ & $\begin{array}{l}0 \\
0 \\
0 \\
0\end{array}$ \\
\hline $\mathrm{CN}$ & $(\mathrm{mg} / \mathrm{l})$ & $\begin{array}{l}91 q 1 \\
91 \mathrm{q} 2 \\
91 \mathrm{q} \\
91 \mathrm{q} 4\end{array}$ & $\begin{array}{l}x \\
x \\
x \\
x\end{array}$ & $\dot{:}$ & $\begin{array}{l}\dot{.} \\
\dot{.}\end{array}$ & $:$ & $\begin{array}{l}0 \\
0 \\
0 \\
0\end{array}$ & $\begin{array}{l}0 \\
0 \\
0 \\
0\end{array}$ & $\begin{array}{l}6 \\
6 \\
6 \\
6\end{array}$ \\
\hline TDS & $(\mathrm{mg} / \mathrm{l})$ & $\begin{array}{l}91 q 1 \\
9192 \\
9193 \\
9194\end{array}$ & $\begin{array}{l}271 \\
414 \\
259 \\
287\end{array}$ & $\begin{array}{l}12.5 \\
78.9 \\
26.1 \\
9.66\end{array}$ & $\begin{array}{r}21.2 \\
137 \\
44.2 \\
0.00\end{array}$ & $\begin{array}{l}6.00 \\
4.34 \\
13.2 \\
24.7\end{array}$ & $\begin{array}{l}6 \\
6 \\
6 \\
6\end{array}$ & $\begin{array}{l}0 \\
0 \\
0 \\
0\end{array}$ & $\begin{array}{l}0 \\
0 \\
0 \\
0\end{array}$ \\
\hline TOC & $(\mathrm{mg} / \mathrm{I})$ & $\begin{array}{l}91 q 1 \\
91 q 2 \\
91 q 3 \\
9194\end{array}$ & $\begin{array}{l}1.27 \\
19.9 \\
4.67 \\
3.03\end{array}$ & $\begin{array}{l}0.07 \\
6.47 \\
1.80 \\
1.26\end{array}$ & $\begin{array}{l}0.10 \\
11.2 \\
3.08 \\
2.15\end{array}$ & $\begin{array}{l}0.10 \\
1.35 \\
0.61 \\
0.55\end{array}$ & $\begin{array}{l}6 \\
6 \\
6 \\
6\end{array}$ & $\begin{array}{l}0 \\
0 \\
0 \\
0\end{array}$ & $\begin{array}{l}0 \\
0 \\
0 \\
0\end{array}$ \\
\hline TSS & $(m g / 1)$ & $\begin{array}{l}9191 \\
9192 \\
9193 \\
9194\end{array}$ & $\begin{array}{l}9.00 \\
14.0 \\
11.7 \\
6.00\end{array}$ & $\begin{array}{l}\dot{6} .06 \\
\dot{\dot{b}}\end{array}$ & $\begin{array}{l}8.49 \\
\dot{5}\end{array}$ & $\begin{array}{l}8.74 \\
:\end{array}$ & $\begin{array}{l}2 \\
6 \\
3 \\
1\end{array}$ & $\begin{array}{l}0 \\
0 \\
0 \\
0\end{array}$ & $\begin{array}{l}4 \\
0 \\
3 \\
5\end{array}$ \\
\hline $\mathrm{Cl}$ & $(\mathrm{mg} / 1)$ & $\begin{array}{l}91 q 1 \\
91 q 2 \\
91 q 3 \\
9194\end{array}$ & $\begin{array}{l}18.8 \\
29.5 \\
19.7 \\
14.8\end{array}$ & $\begin{array}{l}0.63 \\
6.17 \\
6.76 \\
2.13\end{array}$ & $\begin{array}{l}1.08 \\
10.7 \\
11.3 \\
3.67\end{array}$ & $\begin{array}{l}0.21 \\
0.42 \\
0.32 \\
0.45\end{array}$ & $\begin{array}{l}6 \\
6 \\
5 \\
6\end{array}$ & $\begin{array}{l}0 \\
0 \\
1 \\
0\end{array}$ & $\begin{array}{l}0 \\
0 \\
0 \\
0\end{array}$ \\
\hline $\mathrm{F}$ & $(m g / l)$ & $\begin{array}{l}91 q 1 \\
91 q^{2} \\
91 q^{3} \\
91 q^{4}\end{array}$ & $\begin{array}{l}x \\
0.30 \\
0.58 \\
0.33\end{array}$ & $\begin{array}{l}\dot{0} \\
0.07 \\
0.05\end{array}$ & $\begin{array}{l}\dot{0} \\
0.11 \\
0.08\end{array}$ & $\begin{array}{l}\dot{j} \\
0.03 \\
0.02\end{array}$ & $\begin{array}{l}0 \\
3 \\
5 \\
6\end{array}$ & $\begin{array}{l}0 \\
0 \\
1 \\
0\end{array}$ & $\begin{array}{l}6 \\
3 \\
0 \\
0\end{array}$ \\
\hline $\mathrm{NO} 3$ & $(m g / 1)$ & $\begin{array}{l}91 q 1 \\
91 q 2 \\
91 q 3 \\
9194\end{array}$ & $\begin{array}{l}9.55 \\
3.40 \\
4.94 \\
10.2\end{array}$ & $\begin{array}{l}0.44 \\
1.03 \\
0.37 \\
4.95\end{array}$ & $\begin{array}{l}0.75 \\
1.77 \\
0.51 \\
8.56\end{array}$ & $\begin{array}{l}0.12 \\
0.15 \\
0.45 \\
0.42\end{array}$ & $\begin{array}{l}6 \\
6 \\
5 \\
6\end{array}$ & $\begin{array}{l}0 \\
0 \\
1 \\
0\end{array}$ & $\begin{array}{l}0 \\
0 \\
0 \\
0\end{array}$ \\
\hline
\end{tabular}




\section{Table A-67 (continued)}

QUARTERLY MEANS AND VARIANCE COMPONENTS

\section{STREAM $=16$ (IRC waste effluent)}

(continued)

\begin{tabular}{|c|c|c|c|c|c|c|c|c|c|}
\hline \multicolumn{2}{|c|}{ ANALYTE } & QTR & MEAN & $\begin{array}{r}\text { STD } \\
\text { MEAN }\end{array}$ & $\begin{array}{l}\text { STD } \\
\text { DAY }\end{array}$ & $\begin{array}{l}\text { STD } \\
\text { REP }\end{array}$ & $\mathrm{N}$ & MISS & BPQI \\
\hline PO4 & $(\mathrm{mg} / \mathrm{l})$ & $\begin{array}{l}91 q 1 \\
91 q 2 \\
91 q 3 \\
91 q 4\end{array}$ & $\begin{array}{l}1.11 \\
1.24 \\
12.8 \\
1.00\end{array}$ & $\begin{array}{l}\dot{1} \\
10.9 \\
0.49\end{array}$ & $\begin{array}{l}\dot{1} \\
17.9 \\
0.85\end{array}$ & $\begin{array}{l}\dot{1} \\
4.27 \\
0.07\end{array}$ & $\begin{array}{l}3 \\
3 \\
5 \\
6\end{array}$ & $\begin{array}{l}0 \\
0 \\
1 \\
0\end{array}$ & $\begin{array}{l}3 \\
3 \\
0 \\
0\end{array}$ \\
\hline SO4 & $(\mathrm{mg} / \mathrm{l})$ & $\begin{array}{l}91 q 1 \\
91 q 2 \\
91 q 3 \\
91 q 4\end{array}$ & $\begin{array}{l}41.1 \\
45.2 \\
48.0 \\
40.8\end{array}$ & $\begin{array}{l}0.80 \\
1.10 \\
1.76 \\
1.24\end{array}$ & $\begin{array}{l}1.39 \\
1.39 \\
0.89 \\
2.13\end{array}$ & $\begin{array}{r}0.15 \\
1.00 \\
3.75 \\
0.321\end{array}$ & $\begin{array}{l}6 \\
4 \\
5 \\
6\end{array}$ & $\begin{array}{l}0 \\
2 \\
1 \\
0\end{array}$ & $\begin{array}{l}0 \\
0 \\
0 \\
0\end{array}$ \\
\hline Ag & $(\mu g / 1)$ & $\begin{array}{l}91 q 1 \\
91 q 2 \\
91 q 3 \\
91 q 4\end{array}$ & $\begin{array}{l}3.60 \\
x \\
4.35 \\
2.97\end{array}$ & $\begin{array}{l}\dot{ } \\
\dot{1} .44\end{array}$ & $\begin{array}{l}\dot{ } \\
\dot{2} .12\end{array}$ & $\begin{array}{l}\dot{ } \\
i .84\end{array}$ & $\begin{array}{l}1 \\
0 \\
2 \\
6\end{array}$ & $\begin{array}{l}0 \\
0 \\
0 \\
0\end{array}$ & $\begin{array}{l}5 \\
6 \\
4 \\
0\end{array}$ \\
\hline As & $(\mu g / 1)$ & $\begin{array}{l}91 q 1 \\
91 q^{2} \\
91 q^{3} \\
91 q^{4}\end{array}$ & $\begin{array}{l}1.78 \\
x \\
4.30 \\
4.77\end{array}$ & $\begin{array}{l}0.26 \\
\dot{.} \\
\dot{5}\end{array}$ & $\begin{array}{l}0.44 \\
\dot{:} \\
:\end{array}$ & $\begin{array}{l}0.15 \\
: \\
:\end{array}$ & $\begin{array}{l}6 \\
0 \\
2 \\
3\end{array}$ & $\begin{array}{l}0 \\
0 \\
0 \\
0\end{array}$ & $\begin{array}{l}0 \\
6 \\
4 \\
3\end{array}$ \\
\hline $\mathrm{Ba}$ & $(\mu g / 1)$ & $\begin{array}{l}91 q 1 \\
91 q 2 \\
91 q 3 \\
91.44\end{array}$ & $\begin{array}{l}91.5 \\
x \\
81.4 \\
92.5\end{array}$ & $\begin{array}{l}6.83 \\
\dot{8} .22 \\
5.81\end{array}$ & $\begin{array}{l}10.6 \\
14.2 \\
9.91\end{array}$ & $\begin{array}{l}7.29 \\
i .37 \\
2.56\end{array}$ & $\begin{array}{l}6 \\
0 \\
6 \\
6\end{array}$ & $\begin{array}{l}0 \\
0 \\
0 \\
0\end{array}$ & $\begin{array}{l}0 \\
6 \\
0 \\
0\end{array}$ \\
\hline cd & $(\mu g / I)$ & $\begin{array}{l}91 q 1 \\
91 q 2 \\
91 q 3 \\
91 q^{4}\end{array}$ & $\begin{array}{l}X \\
X \\
X \\
7.20\end{array}$ & $\begin{array}{l}\dot{.} \\
\dot{.}\end{array}$ & $\begin{array}{l}\dot{ } \\
\dot{\bullet}\end{array}$ & $\dot{.}$ & $\begin{array}{l}0 \\
0 \\
0 \\
2\end{array}$ & $\begin{array}{l}0 \\
0 \\
0 \\
0\end{array}$ & $\begin{array}{l}6 \\
6 \\
6 \\
4\end{array}$ \\
\hline $\mathrm{Cr}$ & $(\mu g / I)$ & $\begin{array}{l}91 q 1 \\
91 q 2 \\
91 q 3 \\
91 q^{4}\end{array}$ & $\begin{array}{l}X \\
X \\
8.40 \\
30.0\end{array}$ & $\dot{:}$ & $\begin{array}{l}\dot{.} \\
\dot{.}\end{array}$ & $\begin{array}{l}\dot{.} \\
\dot{.}\end{array}$ & $\begin{array}{l}0 \\
0 \\
1 \\
2\end{array}$ & $\begin{array}{l}0 \\
0 \\
0 \\
0\end{array}$ & $\begin{array}{l}6 \\
6 \\
5 \\
4\end{array}$ \\
\hline CrVI & $(\mu \mathrm{g} / \mathrm{I})$ & $\begin{array}{l}91 q 1 \\
91 q^{2} \\
91 q 3 \\
91 q^{4}\end{array}$ & $\begin{array}{l}X \\
X \\
X \\
X\end{array}$ & $\dot{.}$ & $\begin{array}{l}\dot{ } \\
\dot{ }\end{array}$ & $\dot{:}$ & $\begin{array}{l}0 \\
0 \\
0 \\
0\end{array}$ & $\begin{array}{l}0 \\
0 \\
0 \\
0\end{array}$ & $\begin{array}{l}6 \\
6 \\
6 \\
6\end{array}$ \\
\hline $\mathrm{Cu}$ & $(\mu g / l)$ & $\begin{array}{l}91 q 1 \\
91 q 2 \\
91 q 3 \\
91 q 4\end{array}$ & $\begin{array}{r}42.9 \\
48.1 \\
100 \\
47.4\end{array}$ & $\begin{array}{l}5.05 \\
14.7 \\
55.8 \\
4.12\end{array}$ & $\begin{array}{l}8.59 \\
25.4 \\
96.3 \\
6.95\end{array}$ & $\begin{array}{l}2.27 \\
0.73 \\
11.4 \\
2.28\end{array}$ & $\begin{array}{l}6 \\
6 \\
6 \\
6\end{array}$ & $\begin{array}{l}0 \\
0 \\
0 \\
0\end{array}$ & $\begin{array}{l}0 \\
0 \\
0 \\
0\end{array}$ \\
\hline
\end{tabular}


Table A-67 (continued)

QUARTERLY MEANS AND VARIANCE COMPONENTS

STREAM $=16$ (IRC waste effluent) (continued)

\begin{tabular}{|c|c|c|c|c|c|c|c|c|c|}
\hline \multicolumn{2}{|c|}{ ANALYTE } & QTR & MEAN & $\begin{array}{r}\text { STD } \\
\text { MEAN }\end{array}$ & $\begin{array}{l}\text { STD } \\
\text { DAY }\end{array}$ & $\begin{array}{l}\text { STD } \\
\text { REP }\end{array}$ & $\mathrm{N}$ & $\begin{array}{r}\mathrm{N} \\
\text { MISS }\end{array}$ & $\stackrel{N}{N}$ \\
\hline $\mathrm{Hg}$ & $(\mu \mathrm{g} / 1)$ & $\begin{array}{l}91 q 1 \\
91 q 2 \\
91 q 3 \\
91 q 4\end{array}$ & $\begin{array}{l}0.24 \\
X \\
0.49 \\
0.23\end{array}$ & $\dot{.}$ & $\dot{.}$ & $\dot{\dot{ }}$ & $\begin{array}{l}3 \\
0 \\
1 \\
1\end{array}$ & $\begin{array}{l}0 \\
0 \\
0 \\
0\end{array}$ & $\begin{array}{l}3 \\
6 \\
5 \\
5\end{array}$ \\
\hline $\mathrm{Ni}$ & $(\mu g / I)$ & $\begin{array}{l}91 q 1 \\
91 q 2 \\
91 q 3 \\
91 q 4\end{array}$ & $\begin{array}{l}18.1 \\
13.4 \\
21.1 \\
23.9\end{array}$ & $\dot{.}$ & $\dot{.}$ & $\dot{.}$ & $\begin{array}{l}2 \\
2 \\
3 \\
2\end{array}$ & $\begin{array}{l}0 \\
0 \\
0 \\
0\end{array}$ & $\begin{array}{l}4 \\
4 \\
3 \\
4\end{array}$ \\
\hline $\mathrm{Pb}$ & $(\mu g / 1)$ & $\begin{array}{l}91 q 1 \\
91 q^{2} \\
91 q^{3} \\
91 q^{4}\end{array}$ & $\begin{array}{l}3.30 \\
4.55 \\
2.92 \\
2.95\end{array}$ & $\begin{array}{l}\dot{1} \\
i .64 \\
1.39\end{array}$ & $\begin{array}{l}\dot{ } \\
\dot{2} .30 \\
1.85\end{array}$ & $\begin{array}{l}\dot{0} \\
\dot{2} .36 \\
2.18\end{array}$ & $\begin{array}{l}1 \\
2 \\
6 \\
6\end{array}$ & $\begin{array}{l}0 \\
0 \\
0 \\
0\end{array}$ & $\begin{array}{l}5 \\
4 \\
0 \\
0\end{array}$ \\
\hline Se & $(\mu g / 1)$ & $\begin{array}{l}91 q^{1} \\
91 q^{2} \\
91 q^{3} \\
91 q^{4}\end{array}$ & $\begin{array}{l}X \\
X \\
X \\
X\end{array}$ & $\dot{\dot{.}}$ & $\begin{array}{l}\dot{\cdot} \\
\dot{\cdot}\end{array}$ & $\begin{array}{l}\dot{.} \\
\dot{.}\end{array}$ & $\begin{array}{l}0 \\
0 \\
0 \\
0\end{array}$ & $\begin{array}{l}0 \\
0 \\
0 \\
0\end{array}$ & $\begin{array}{l}6 \\
6 \\
6 \\
6\end{array}$ \\
\hline $\mathrm{Tl}$ & $(\mu g / l)$ & $\begin{array}{l}91 q 1 \\
91 q 2 \\
91 q 3 \\
91 q 4\end{array}$ & $\begin{array}{l}0.77 \\
x \\
x \\
x\end{array}$ & $\dot{\dot{ }}$ & $\dot{.}$ & $\dot{.}$ & $\begin{array}{l}1 \\
0 \\
0 \\
0\end{array}$ & $\begin{array}{l}0 \\
0 \\
0 \\
0\end{array}$ & $\begin{array}{l}5 \\
6 \\
6 \\
6\end{array}$ \\
\hline $\mathrm{Zn}$ & $(\mu \mathrm{g} / I)$ & $\begin{array}{l}91 q^{1} \\
91 q^{2} \\
91 q^{3} \\
91 q^{4}\end{array}$ & $\begin{array}{l}41.0 \\
36.0 \\
48.4 \\
25.4\end{array}$ & $\begin{array}{l}5.04 \\
17.8 \\
14.6 \\
2.44\end{array}$ & $\begin{array}{l}7.87 \\
27.3 \\
25.1 \\
3.73\end{array}$ & $\begin{array}{l}5.35 \\
20.5 \\
5.36 \\
2.79\end{array}$ & $\begin{array}{l}6 \\
6 \\
6 \\
6\end{array}$ & $\begin{array}{l}0 \\
0 \\
0 \\
0\end{array}$ & $\begin{array}{l}0 \\
0 \\
0 \\
0\end{array}$ \\
\hline
\end{tabular}




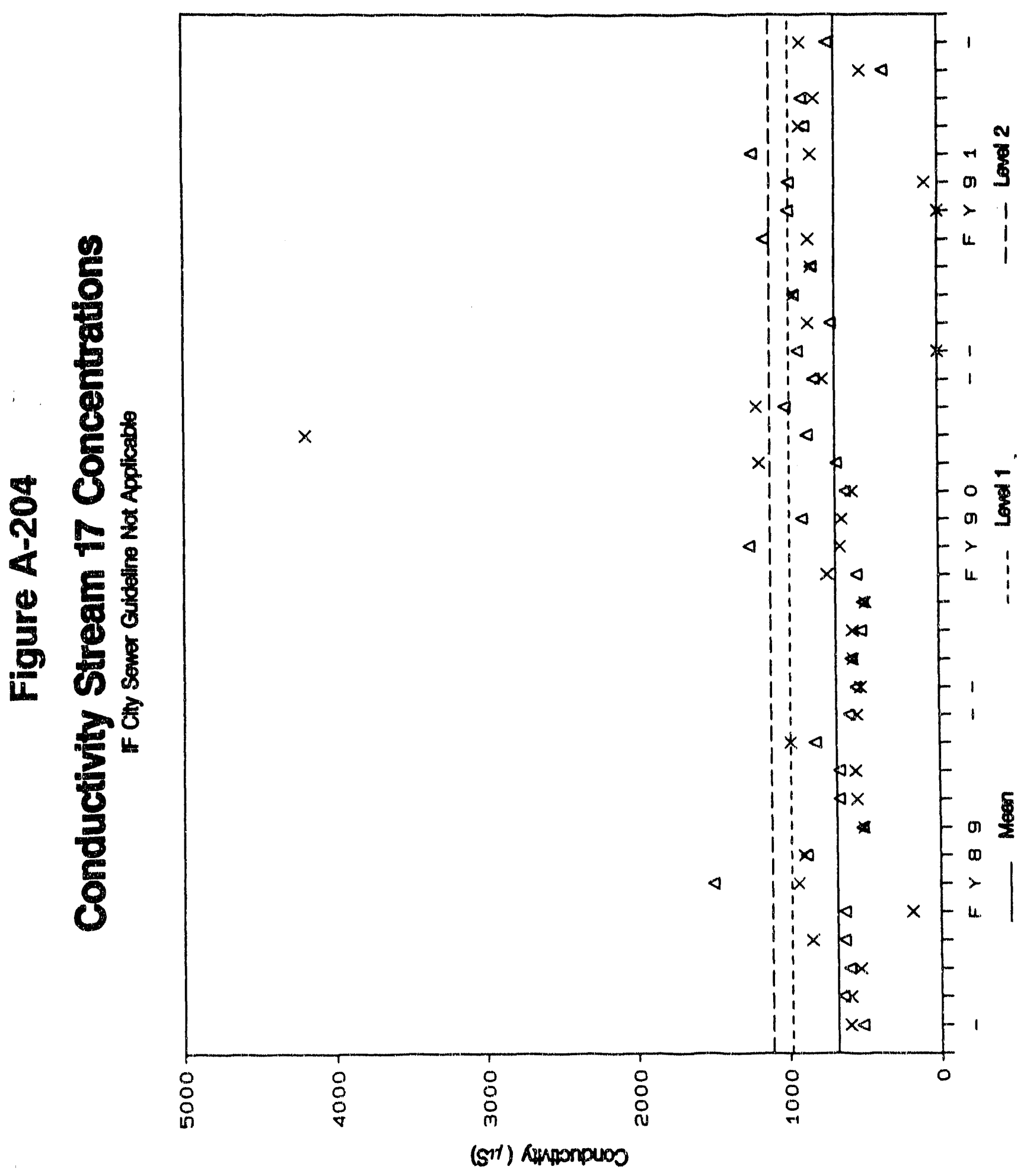




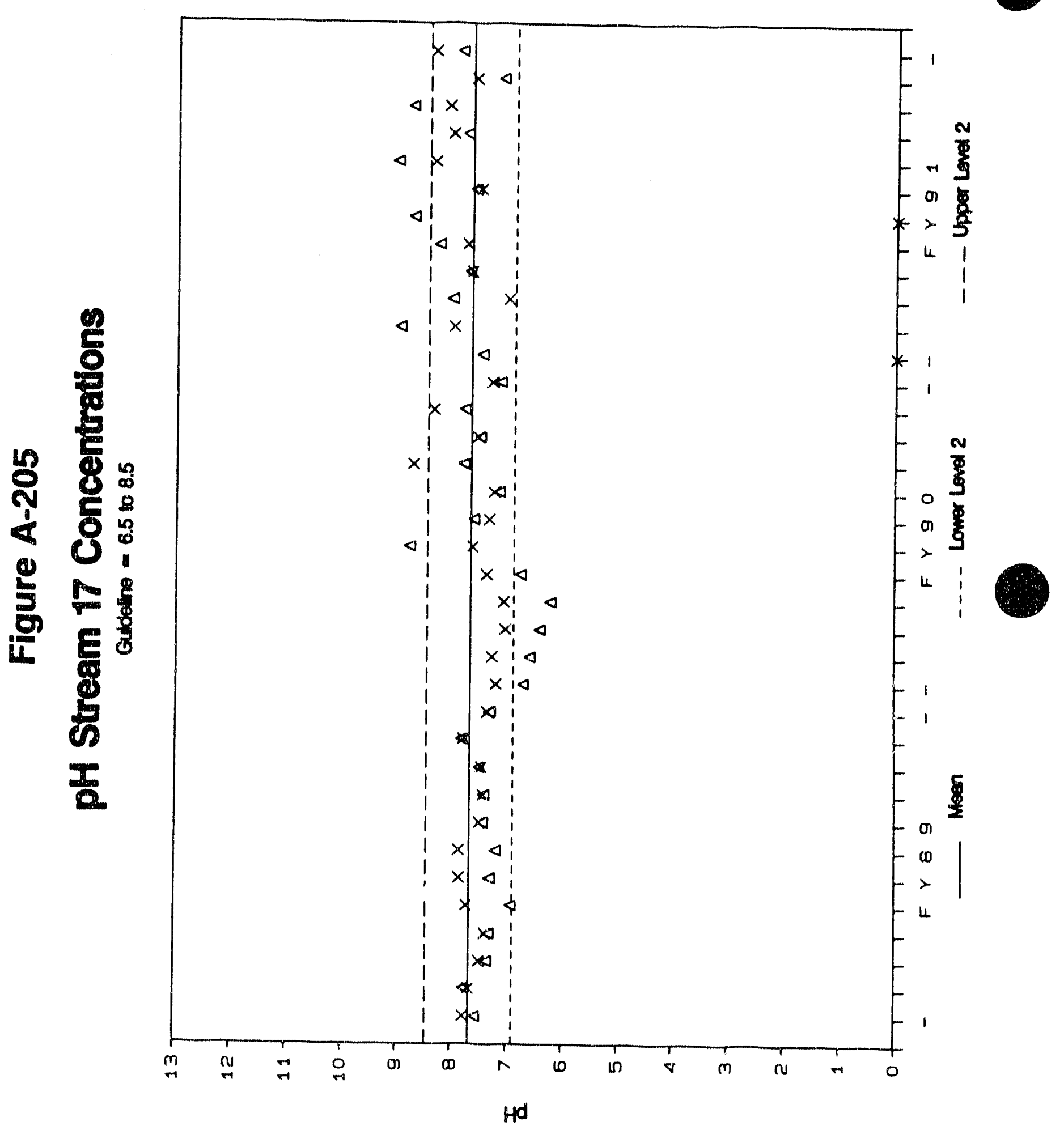




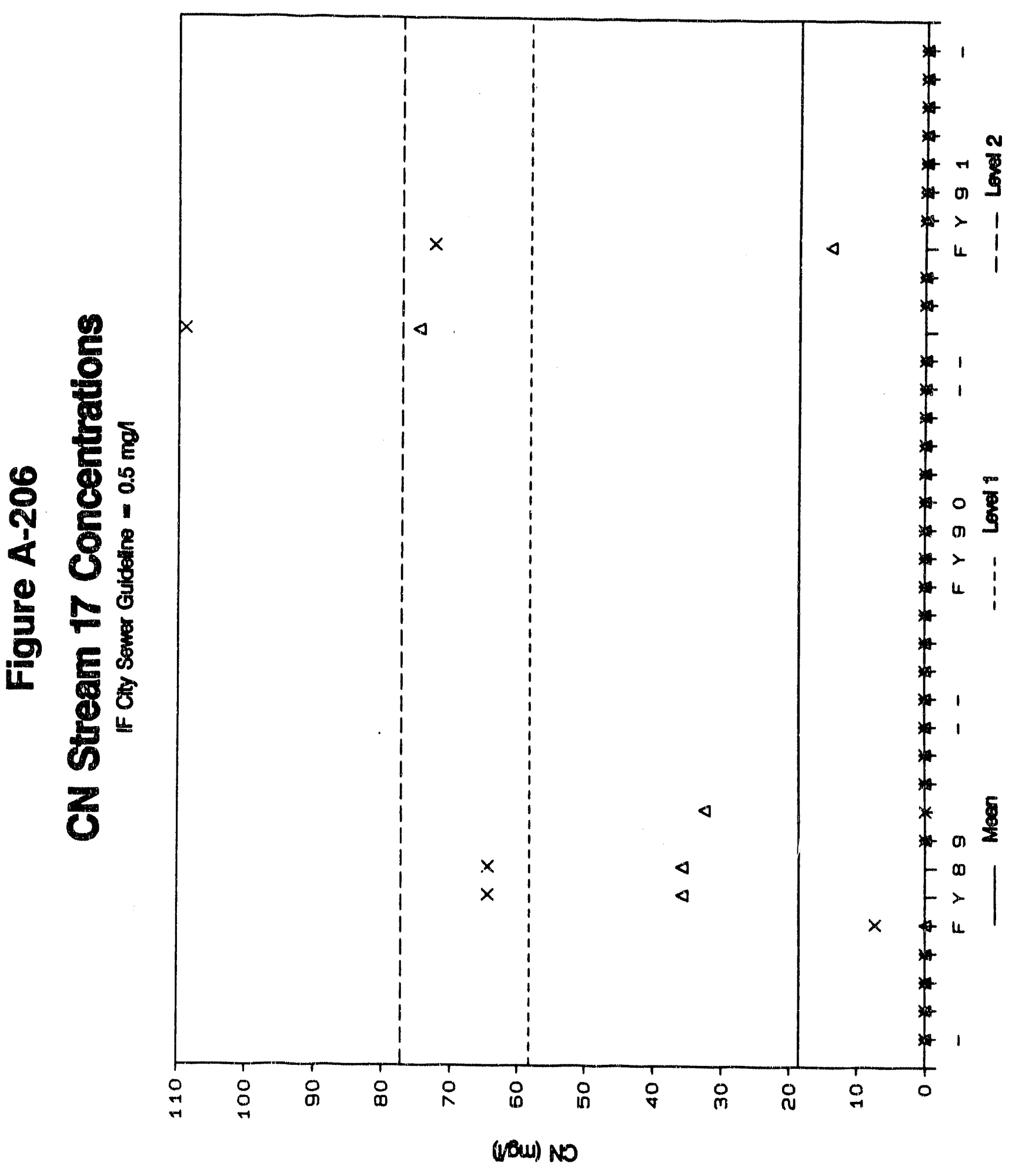




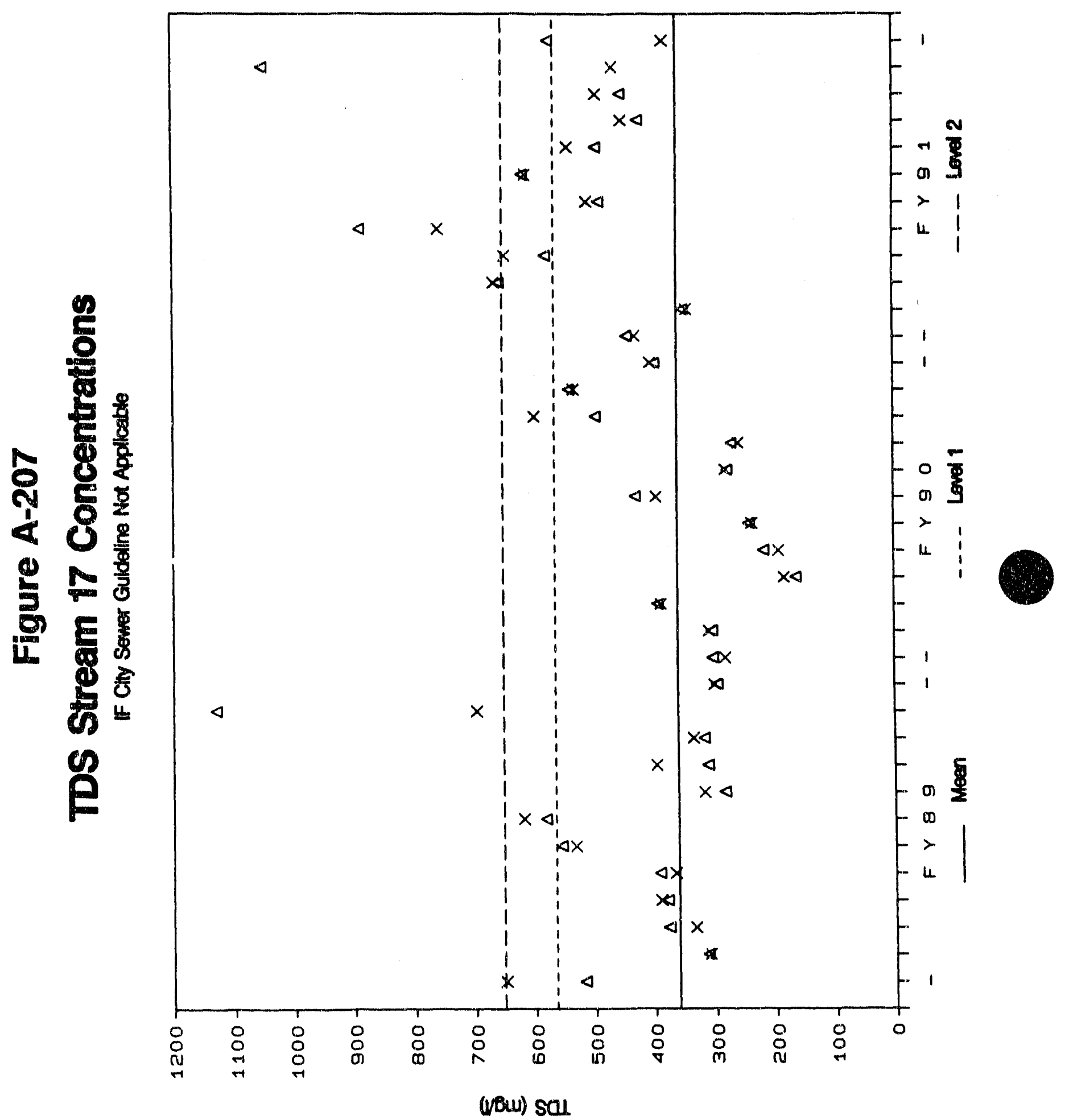




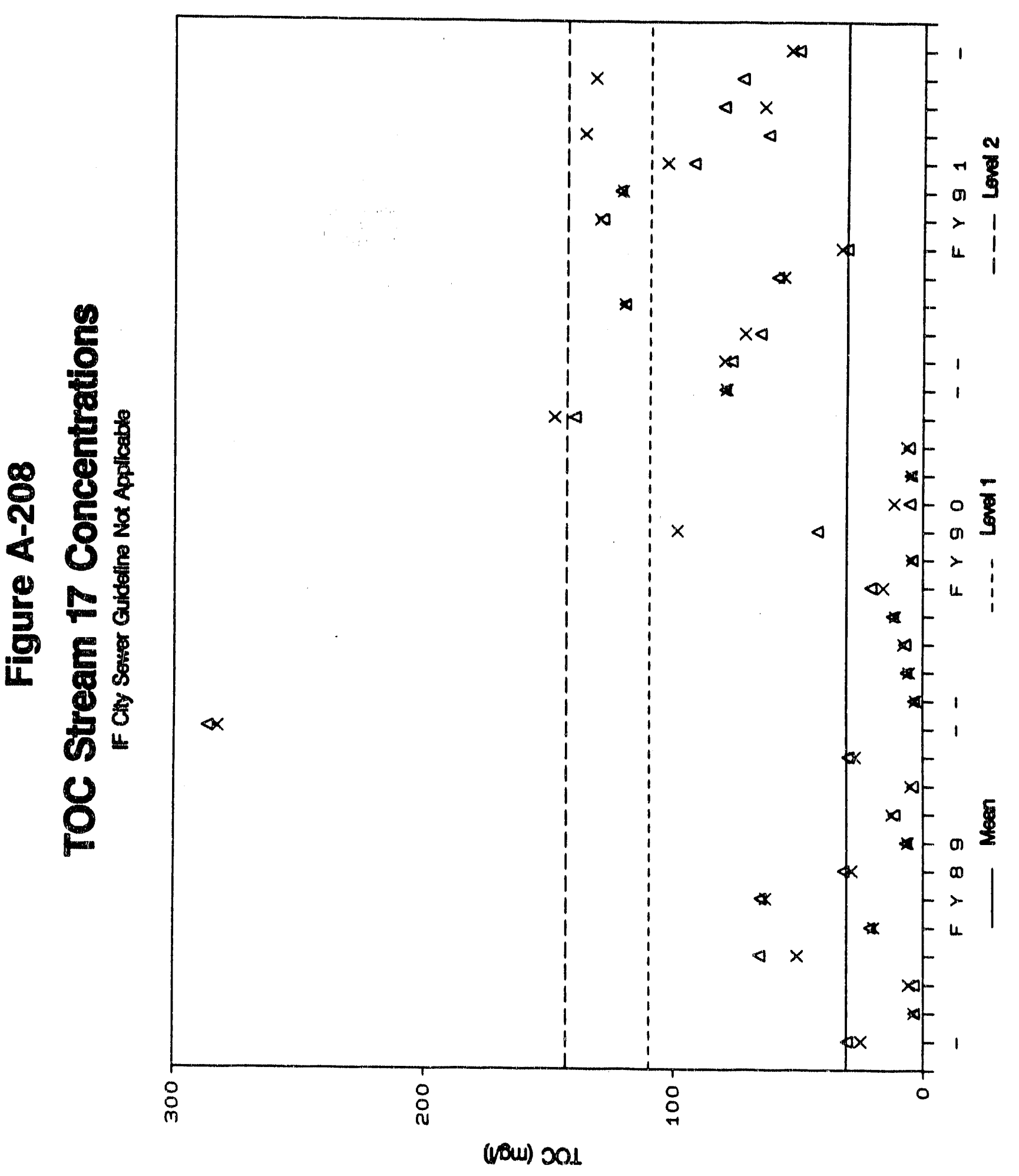




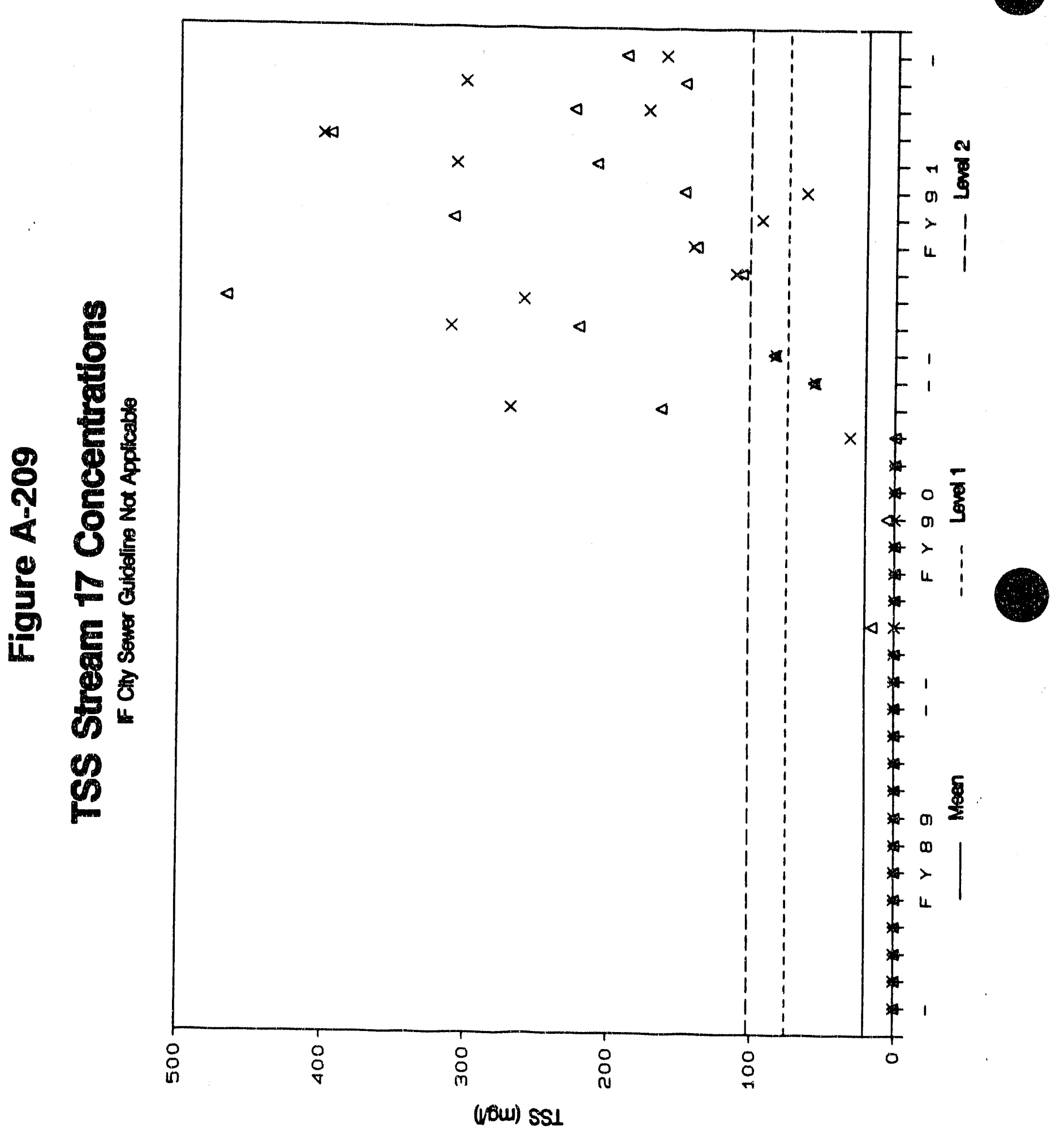




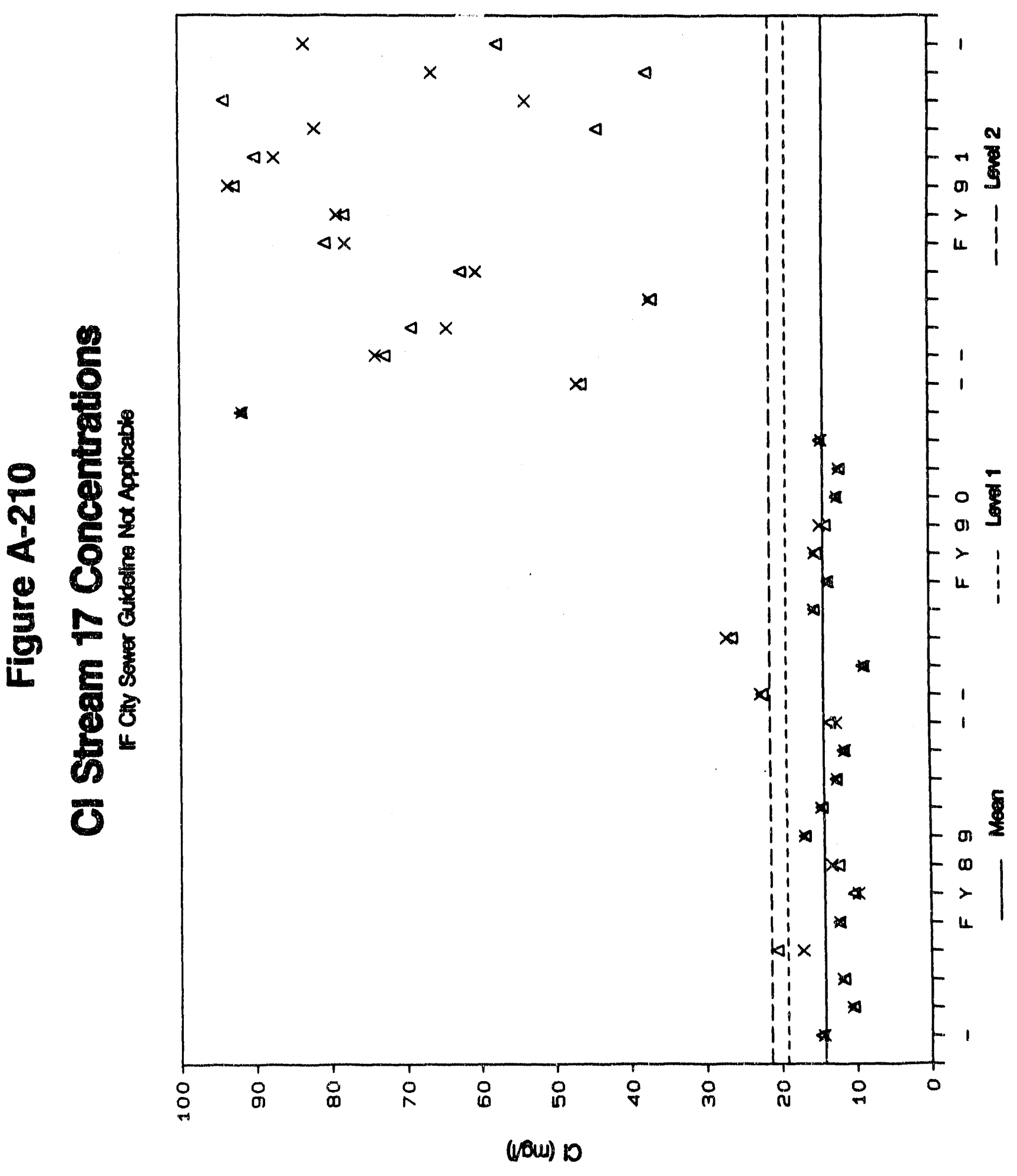




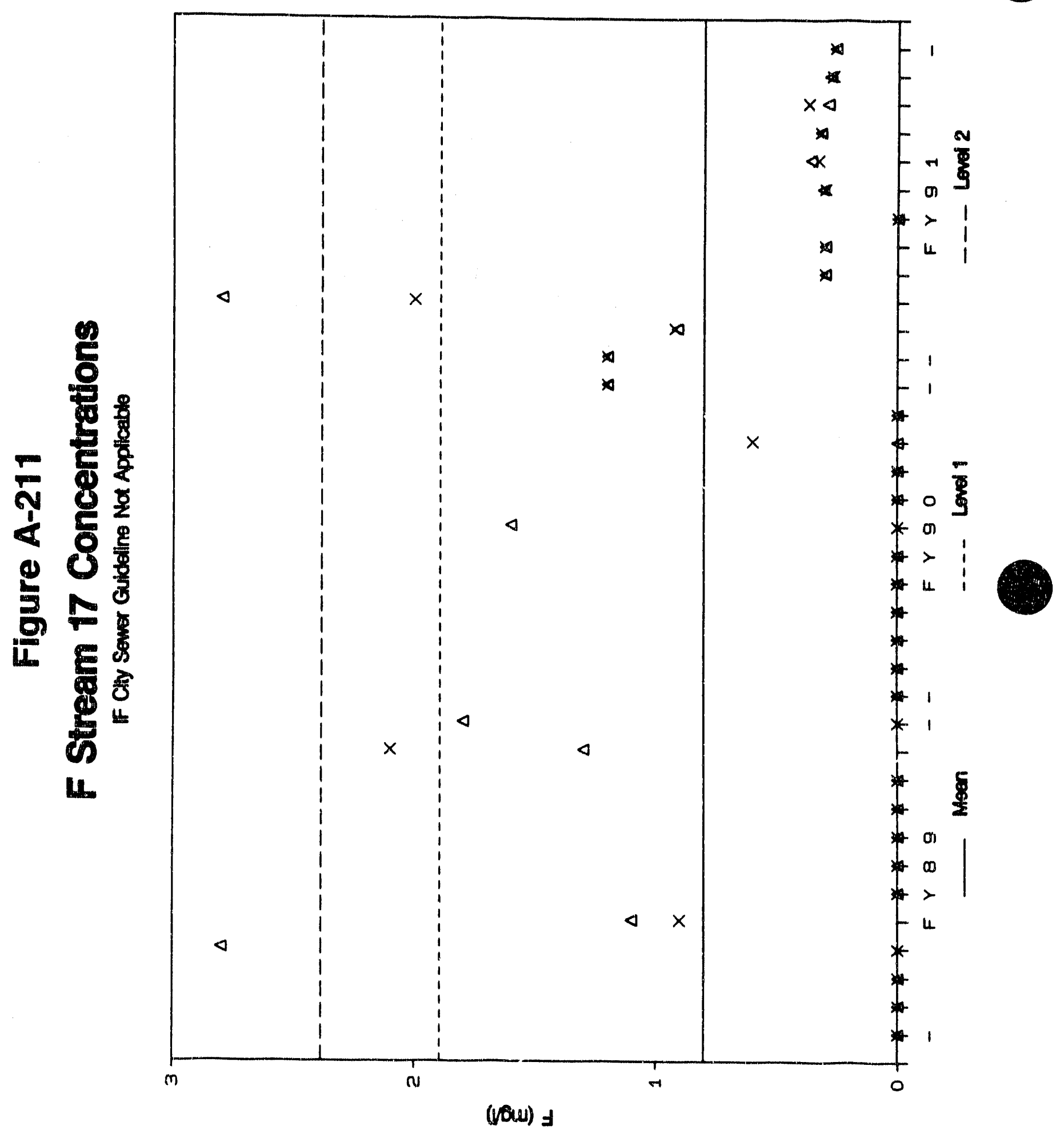

- 


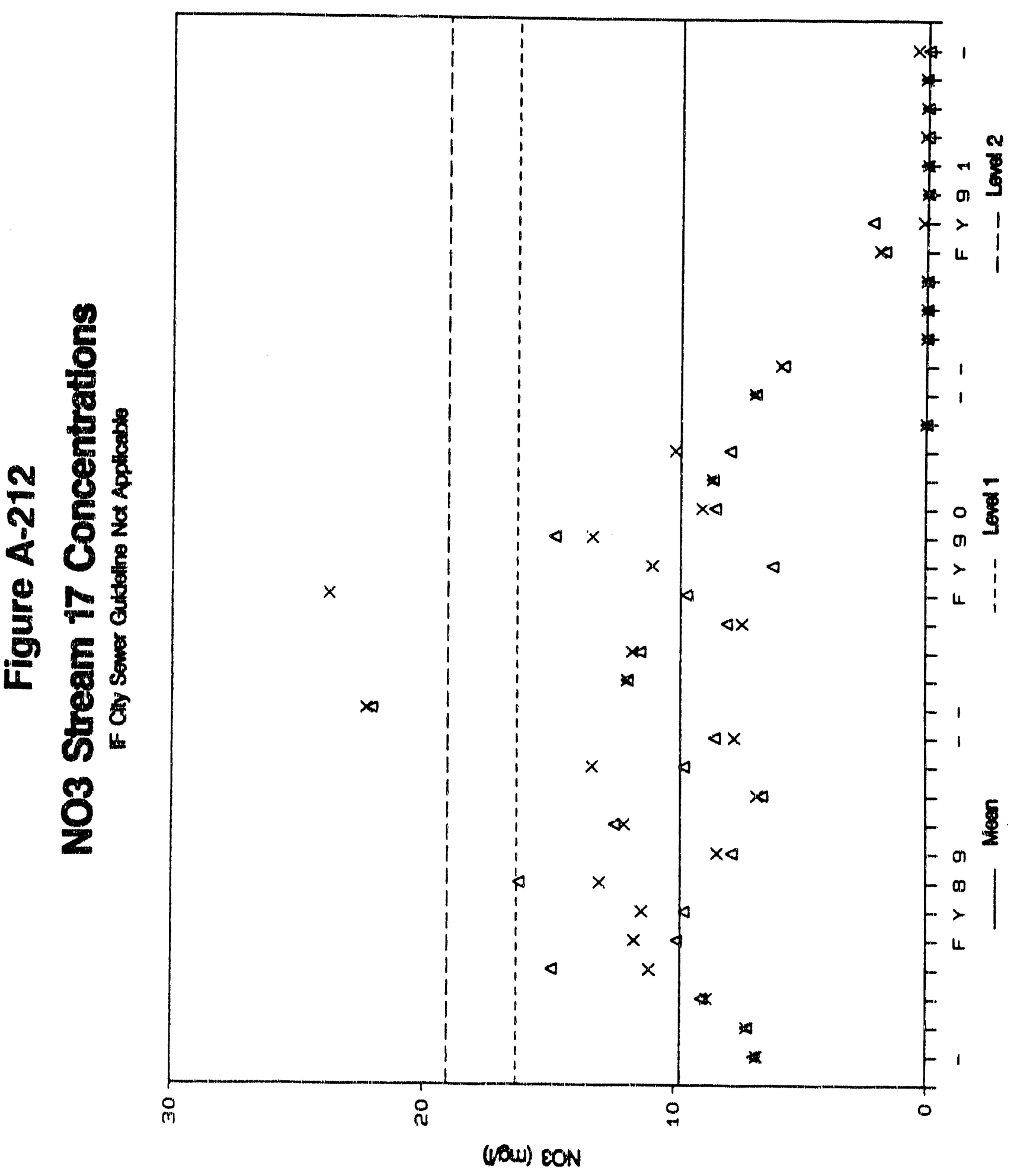




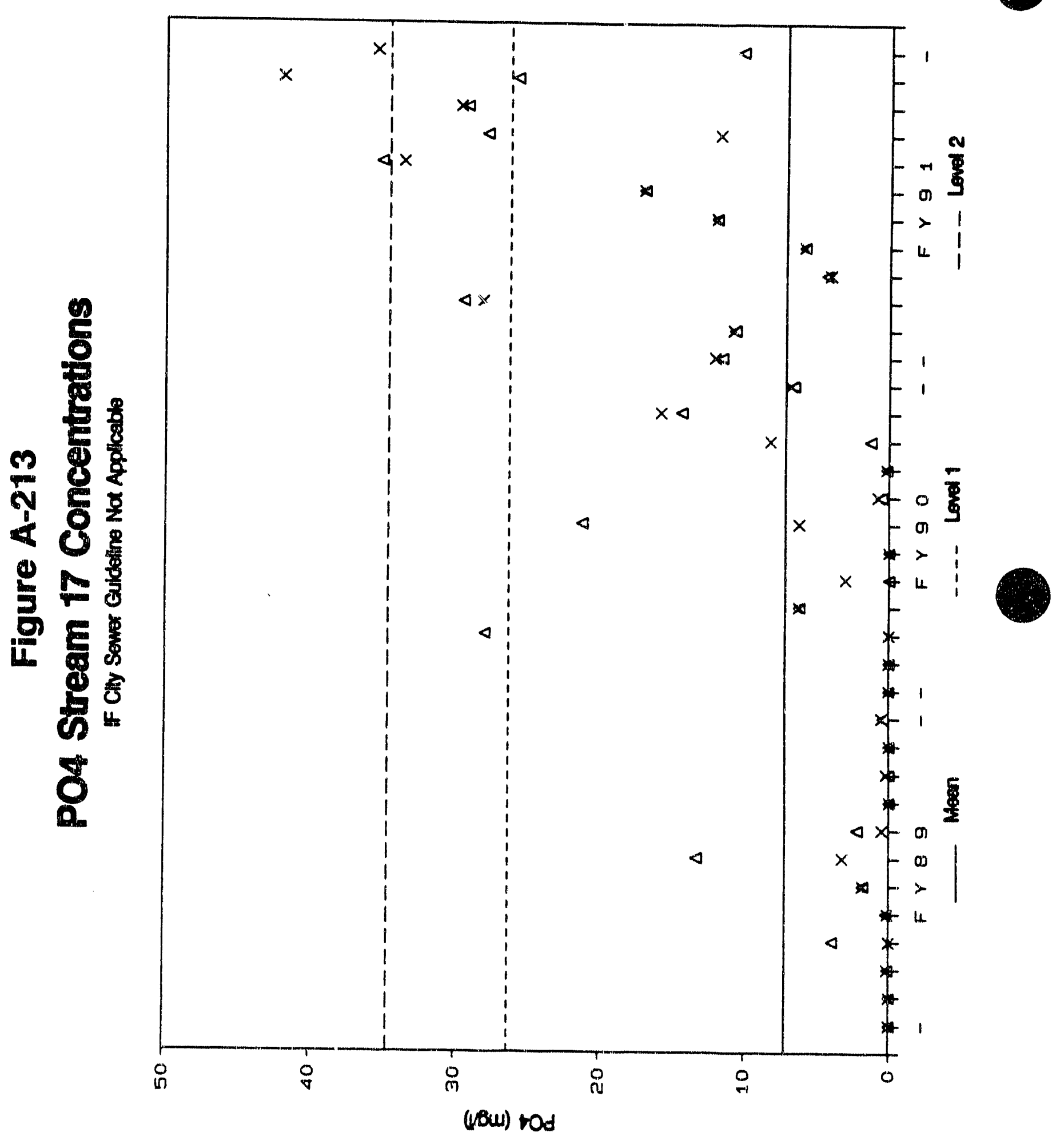




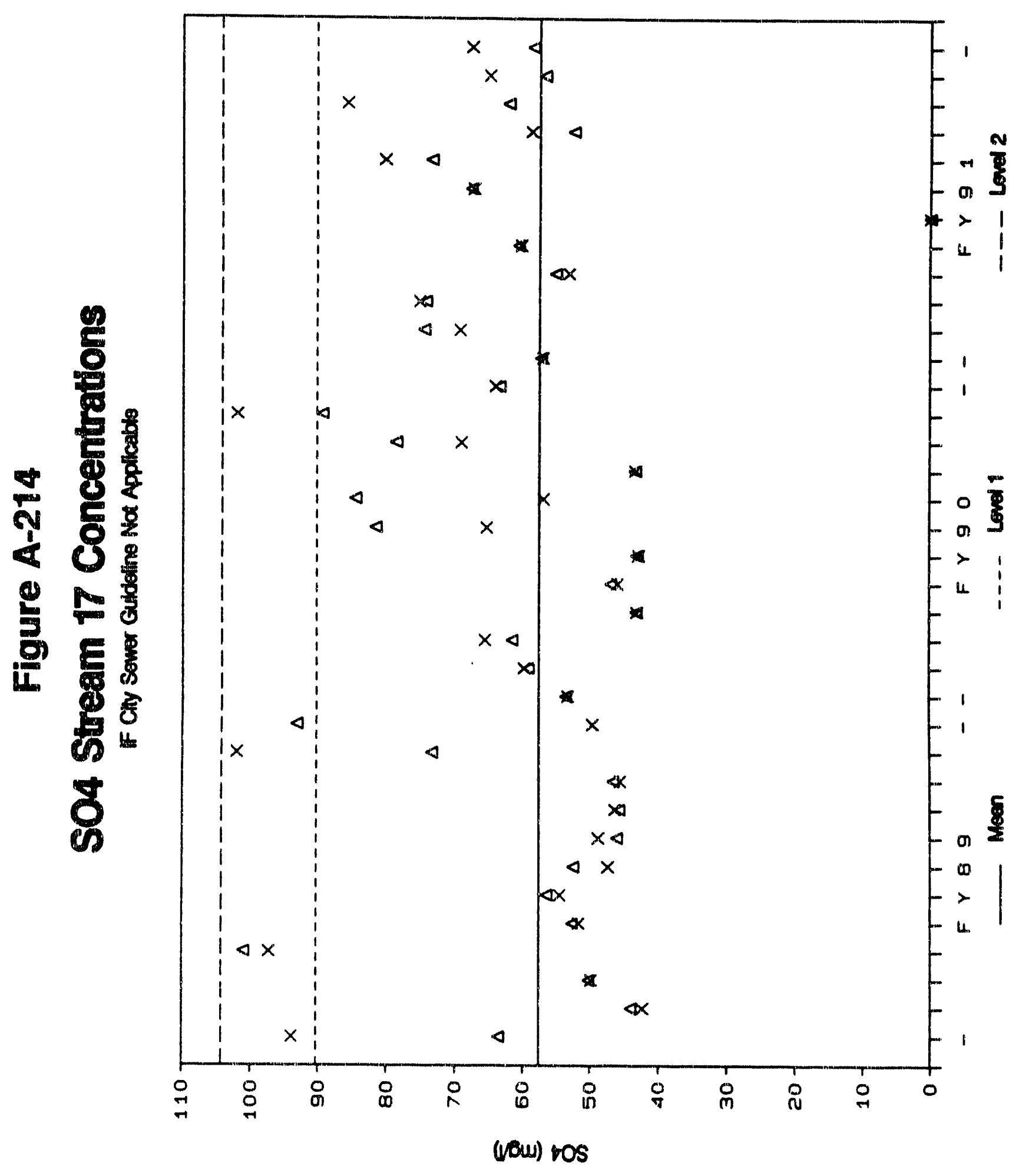




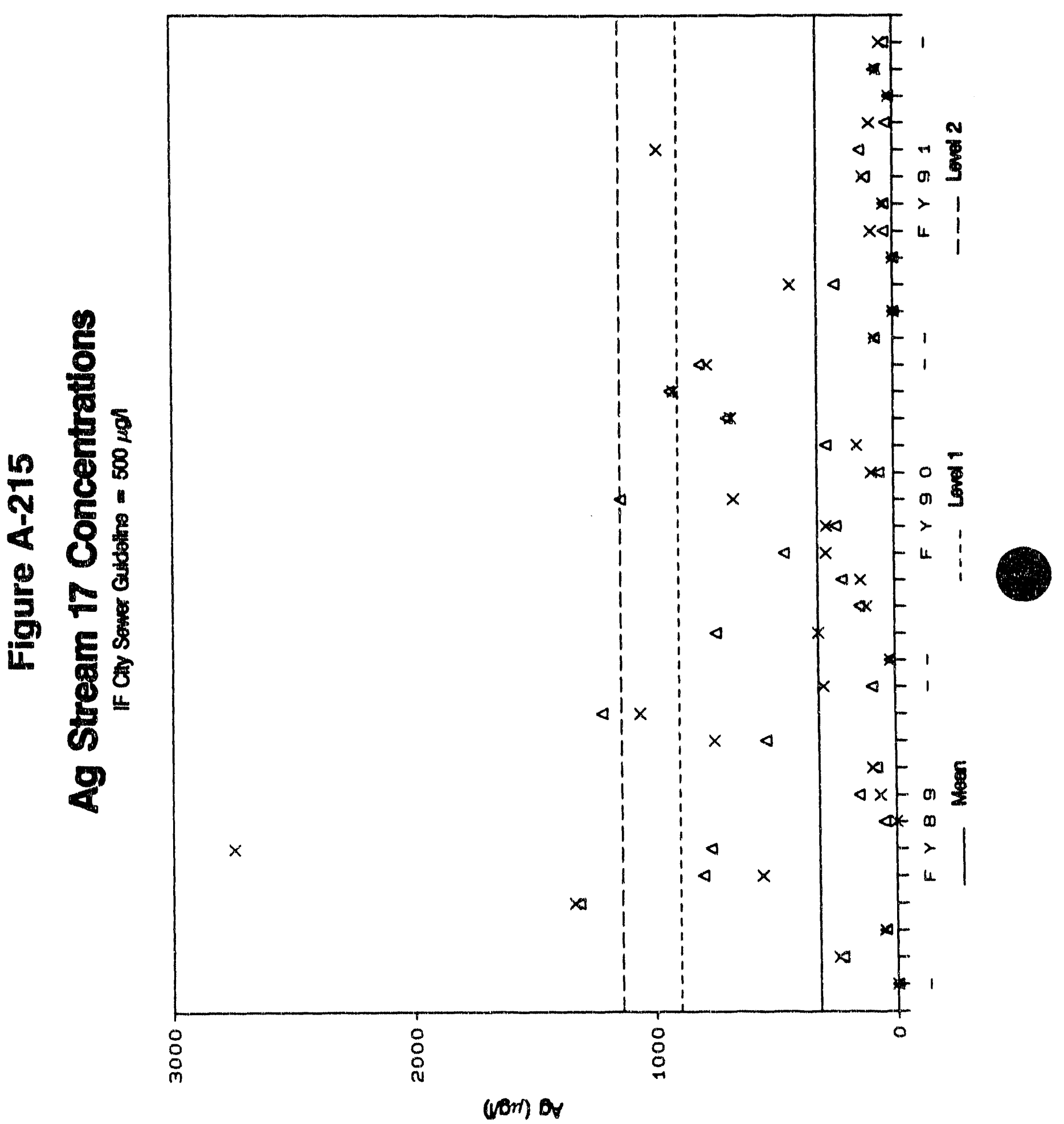




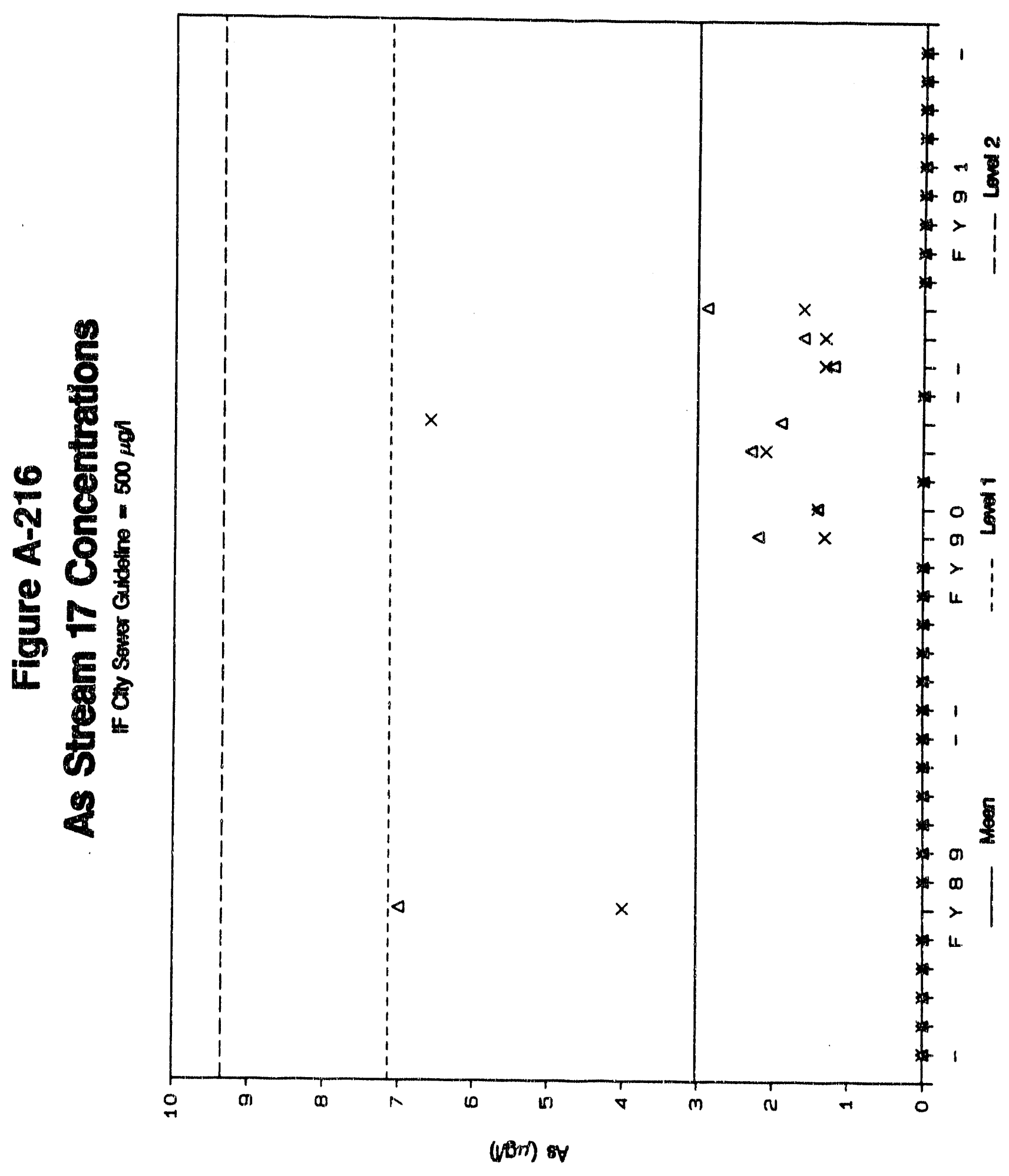




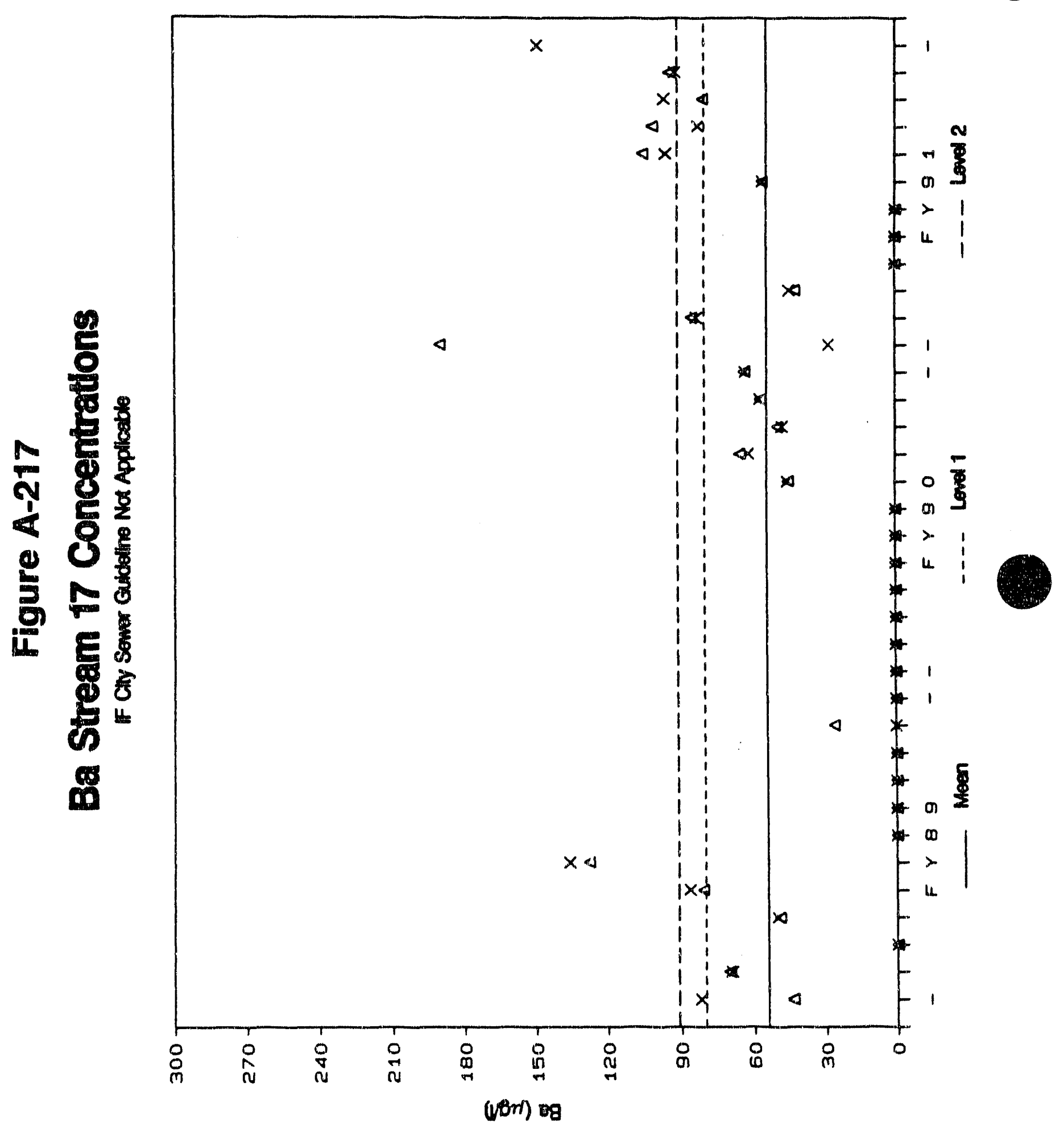




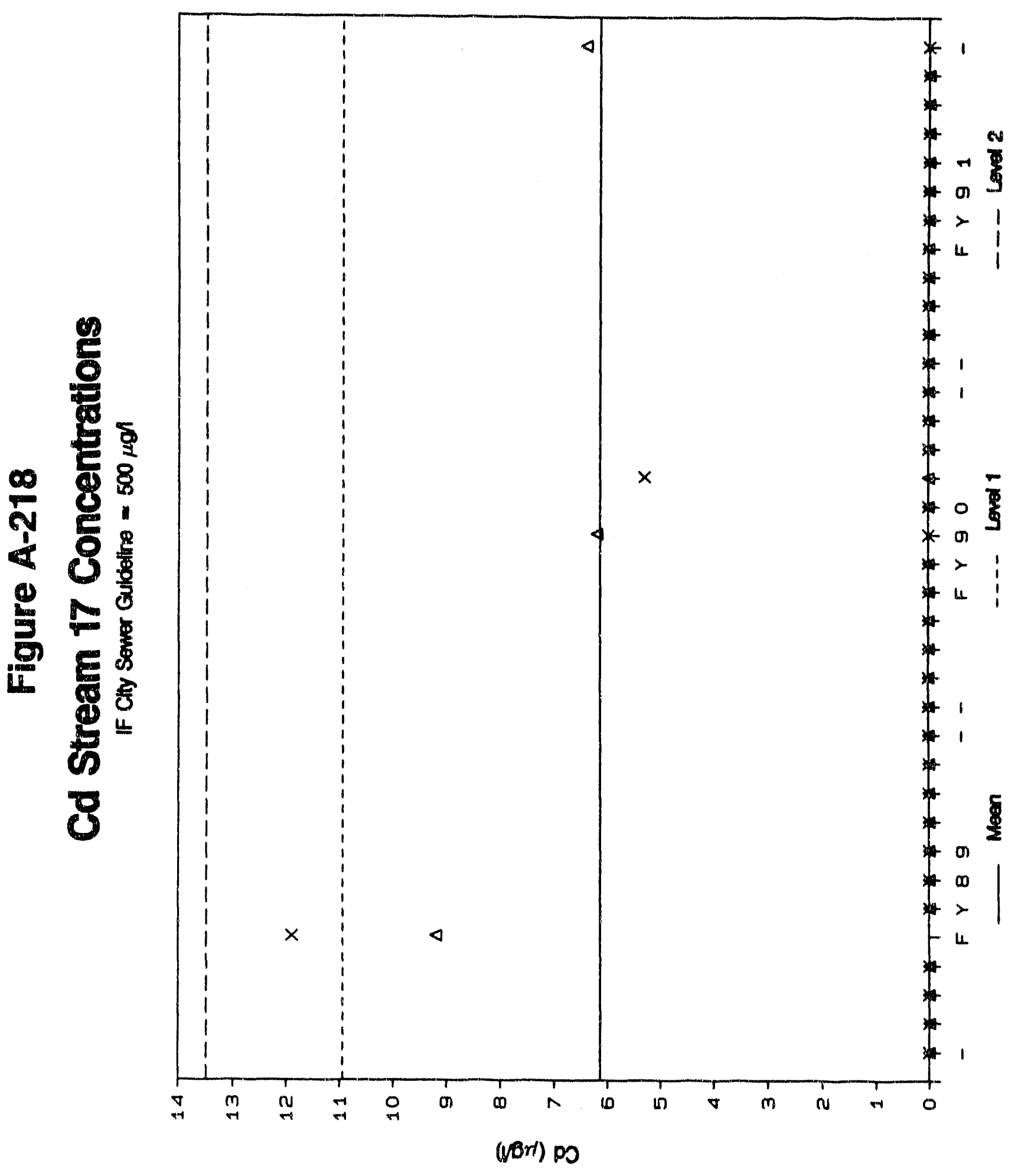




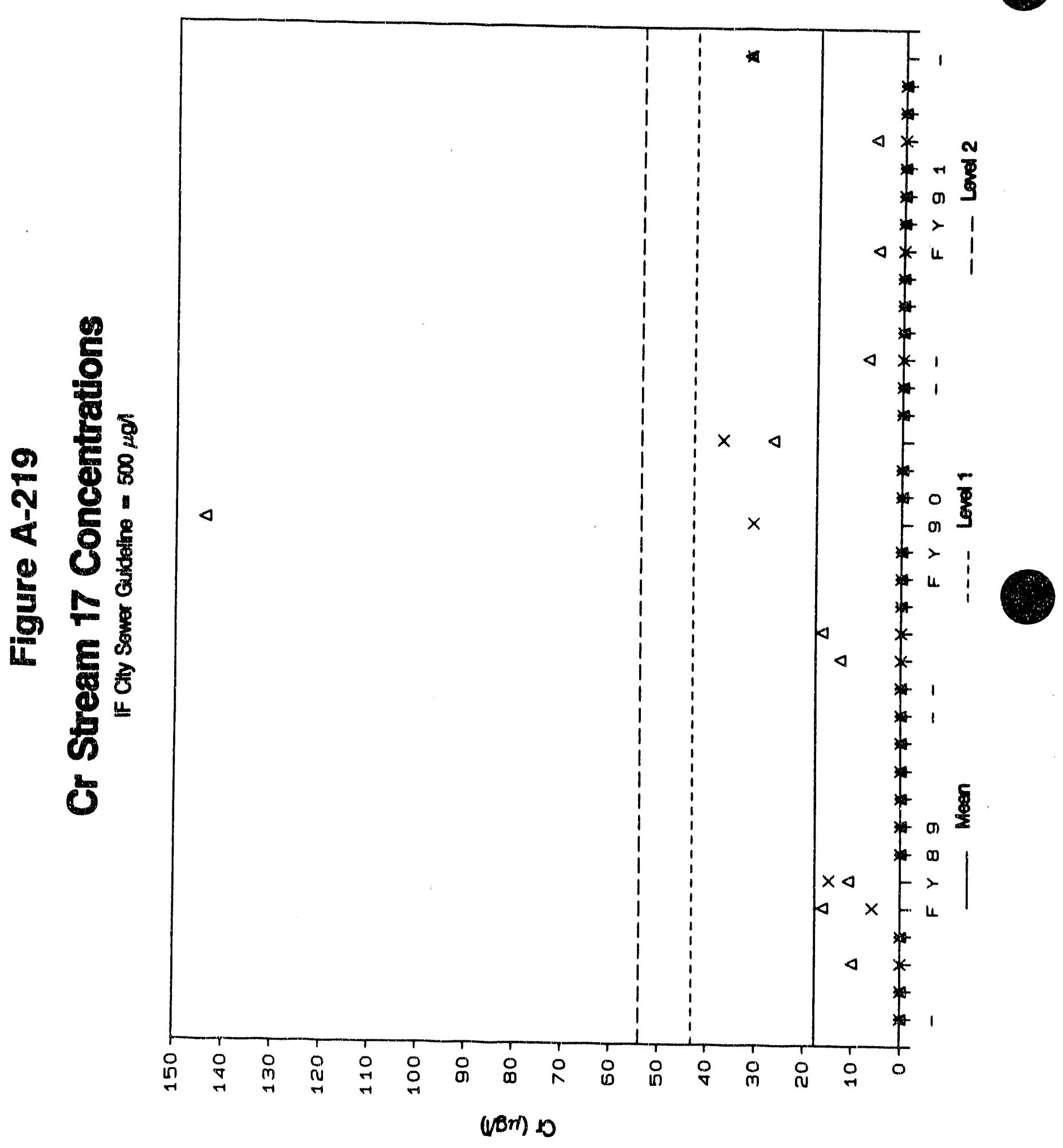




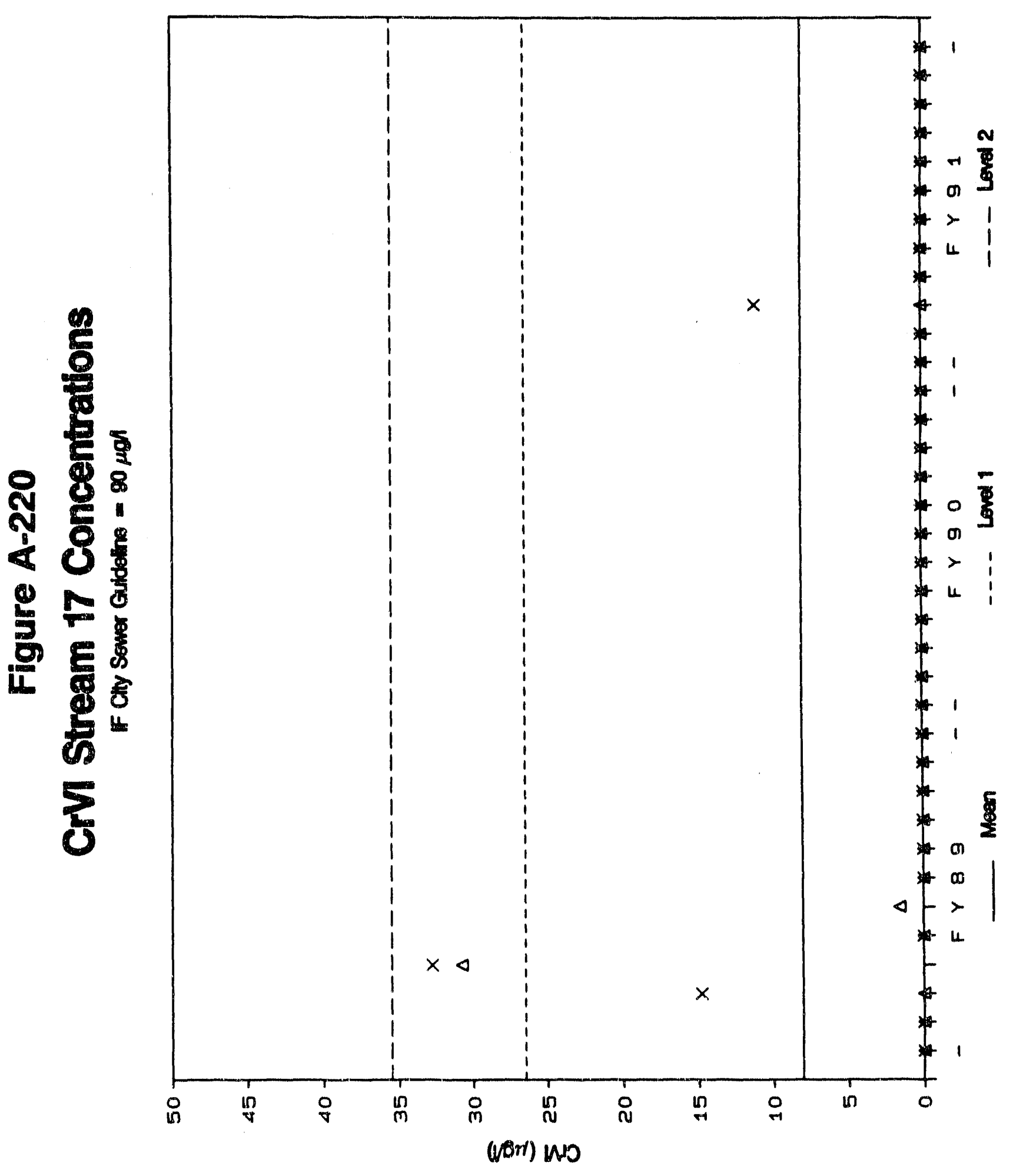

A- 319 


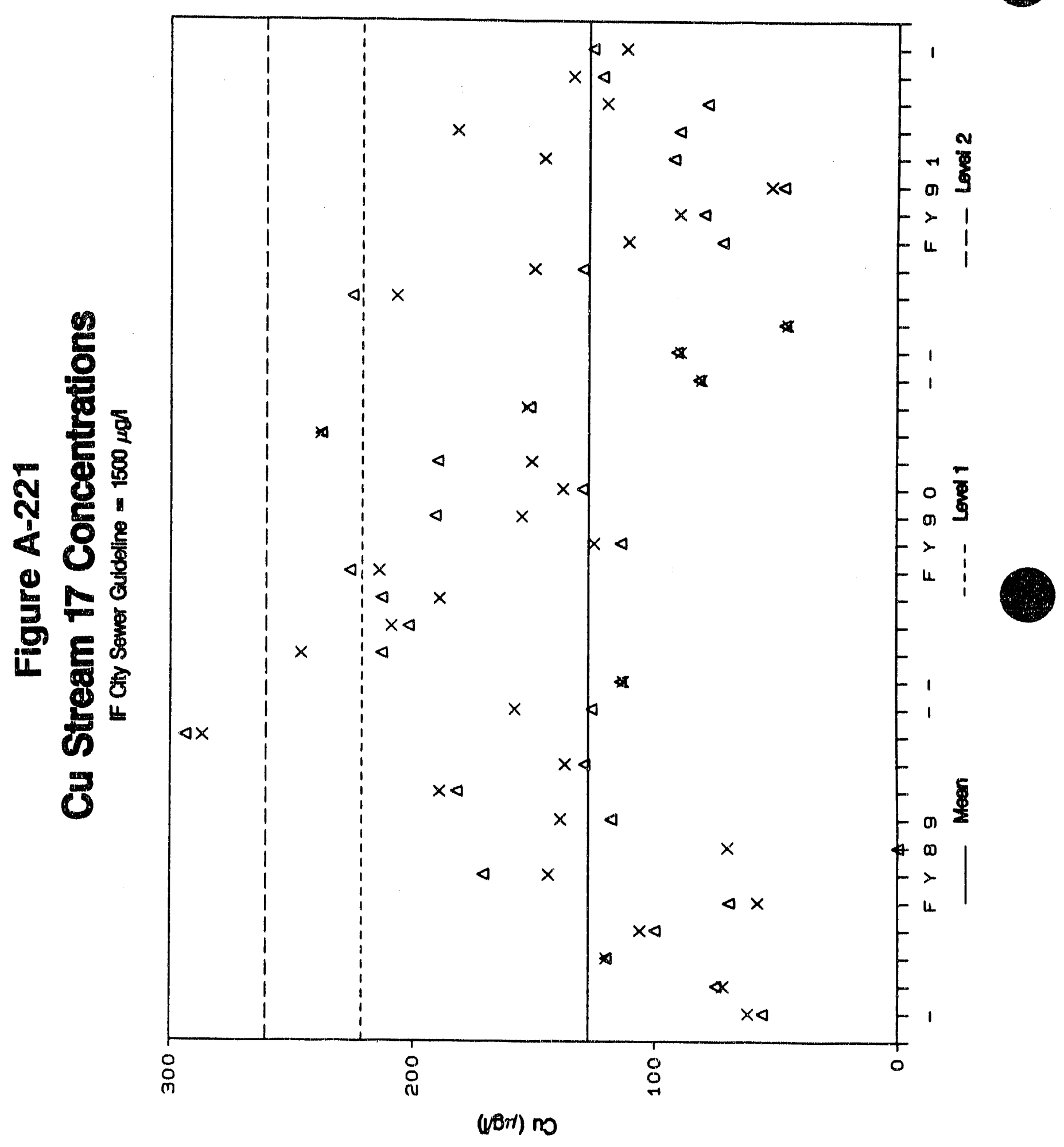




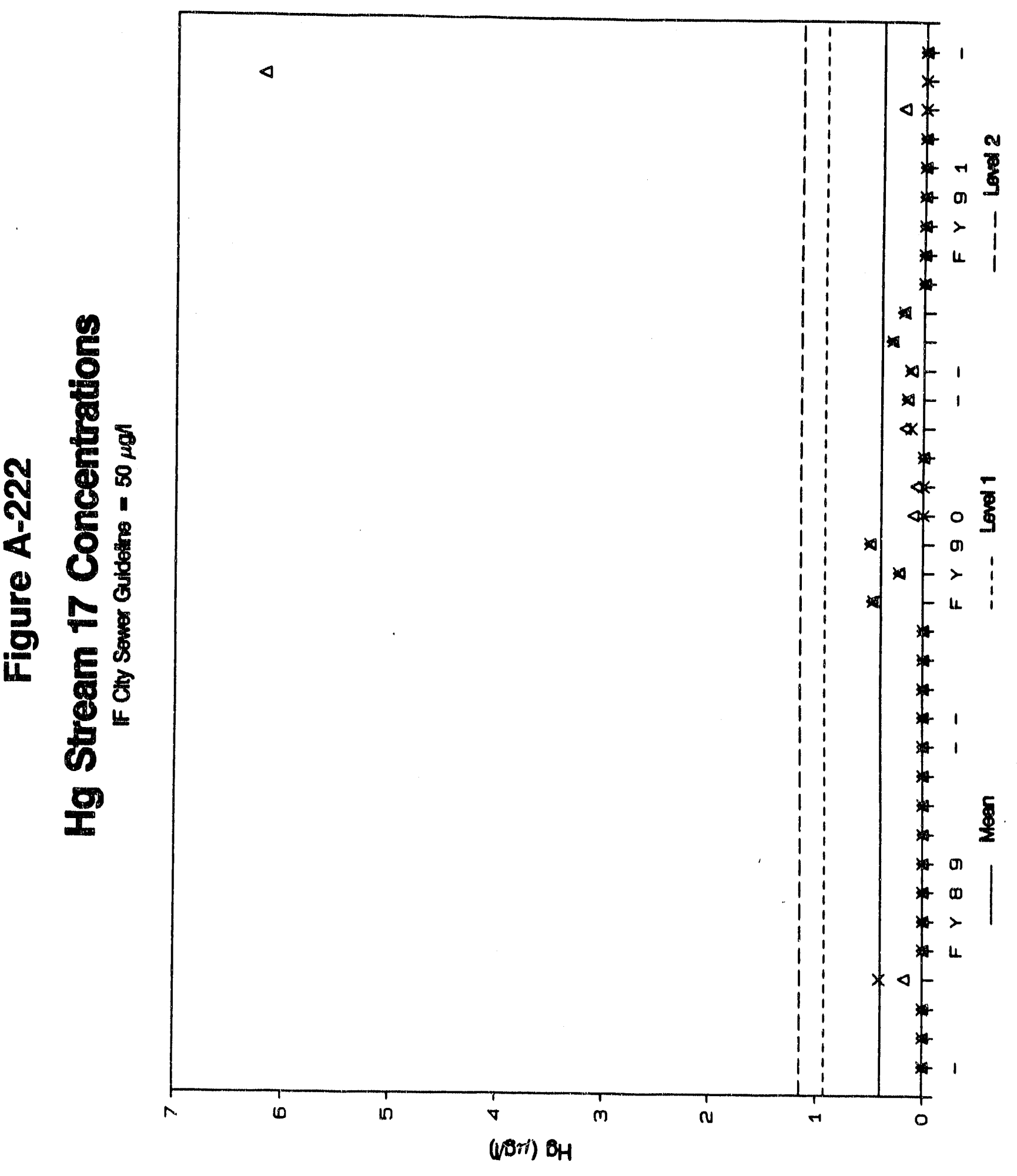




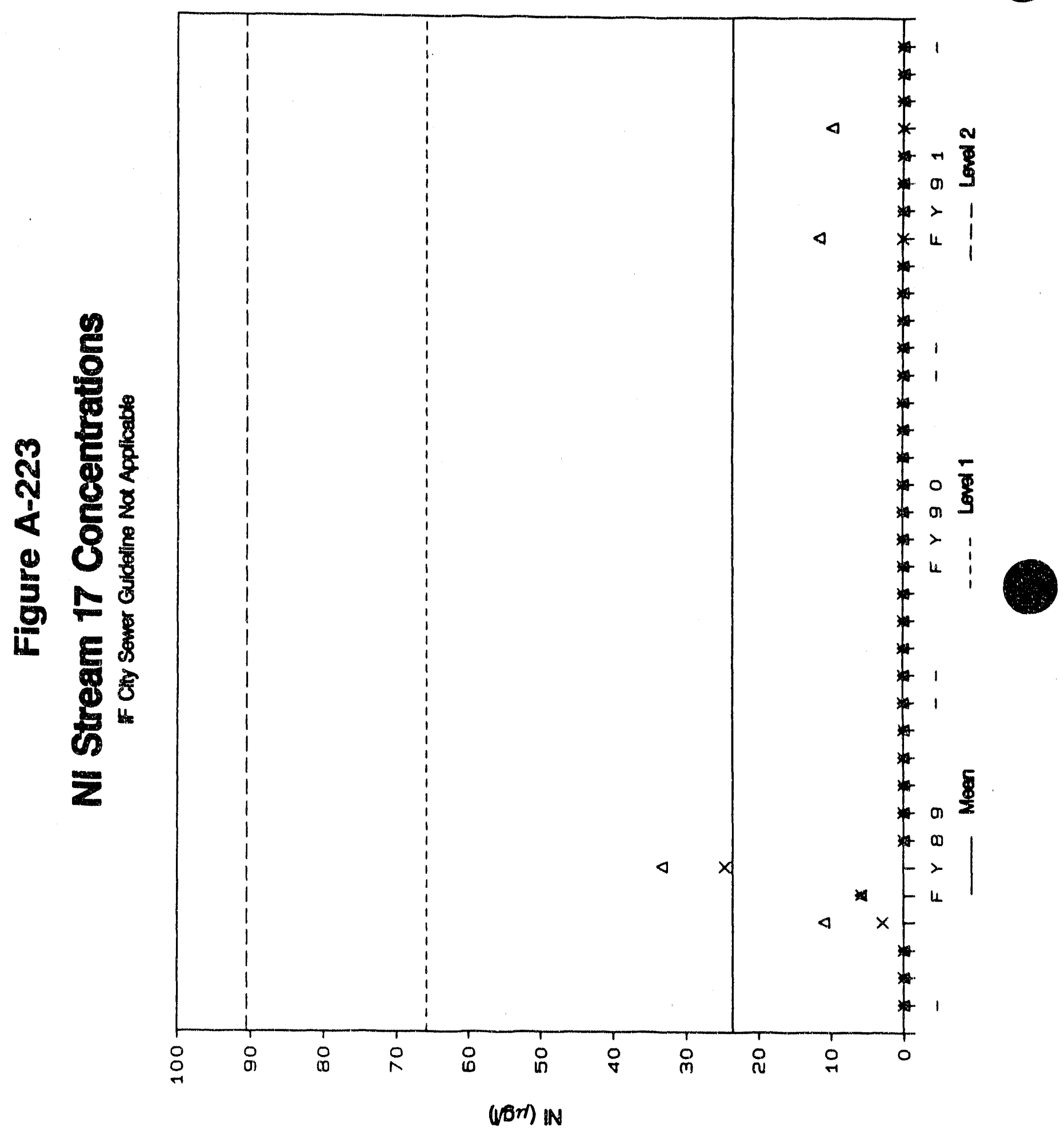




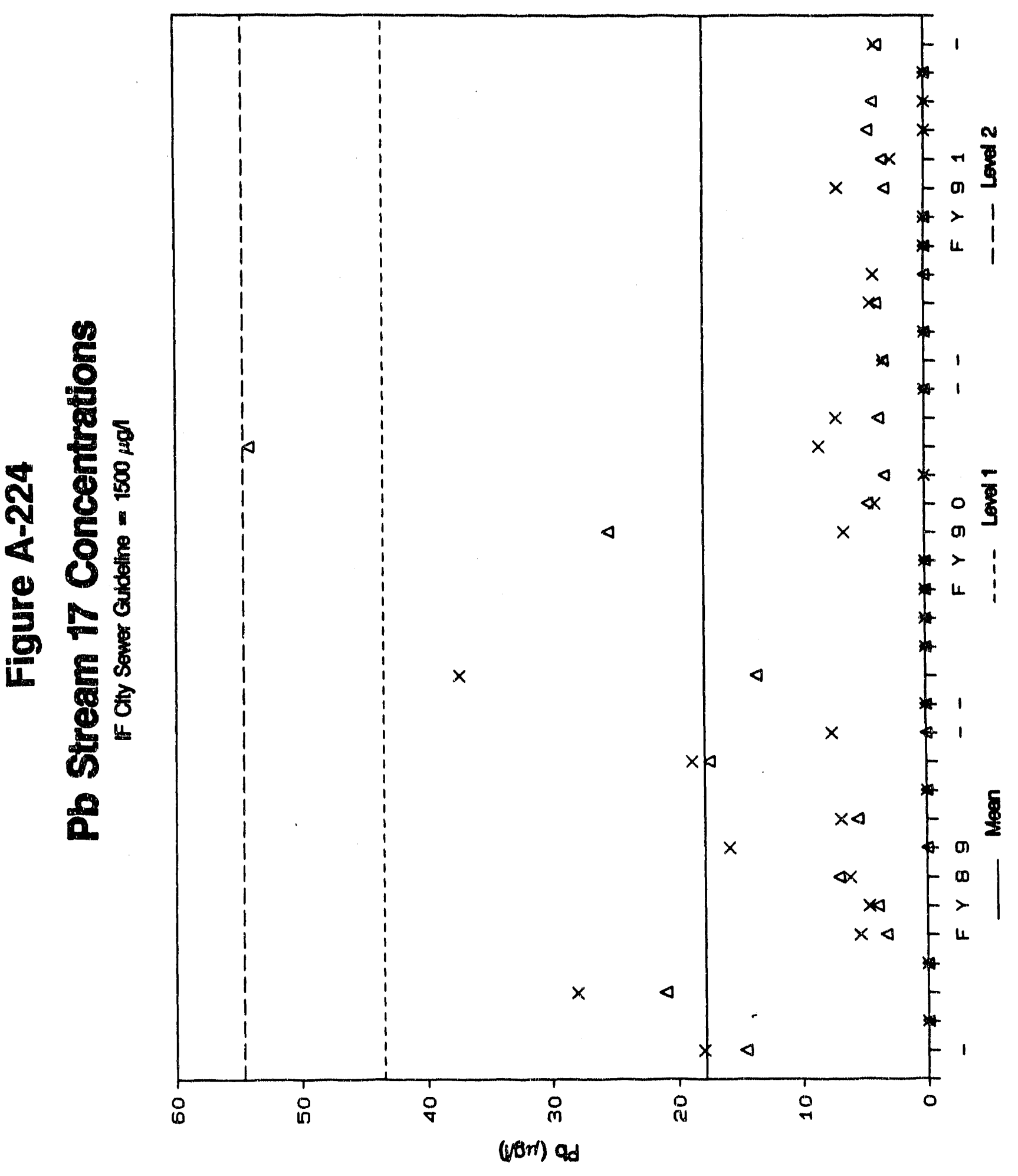




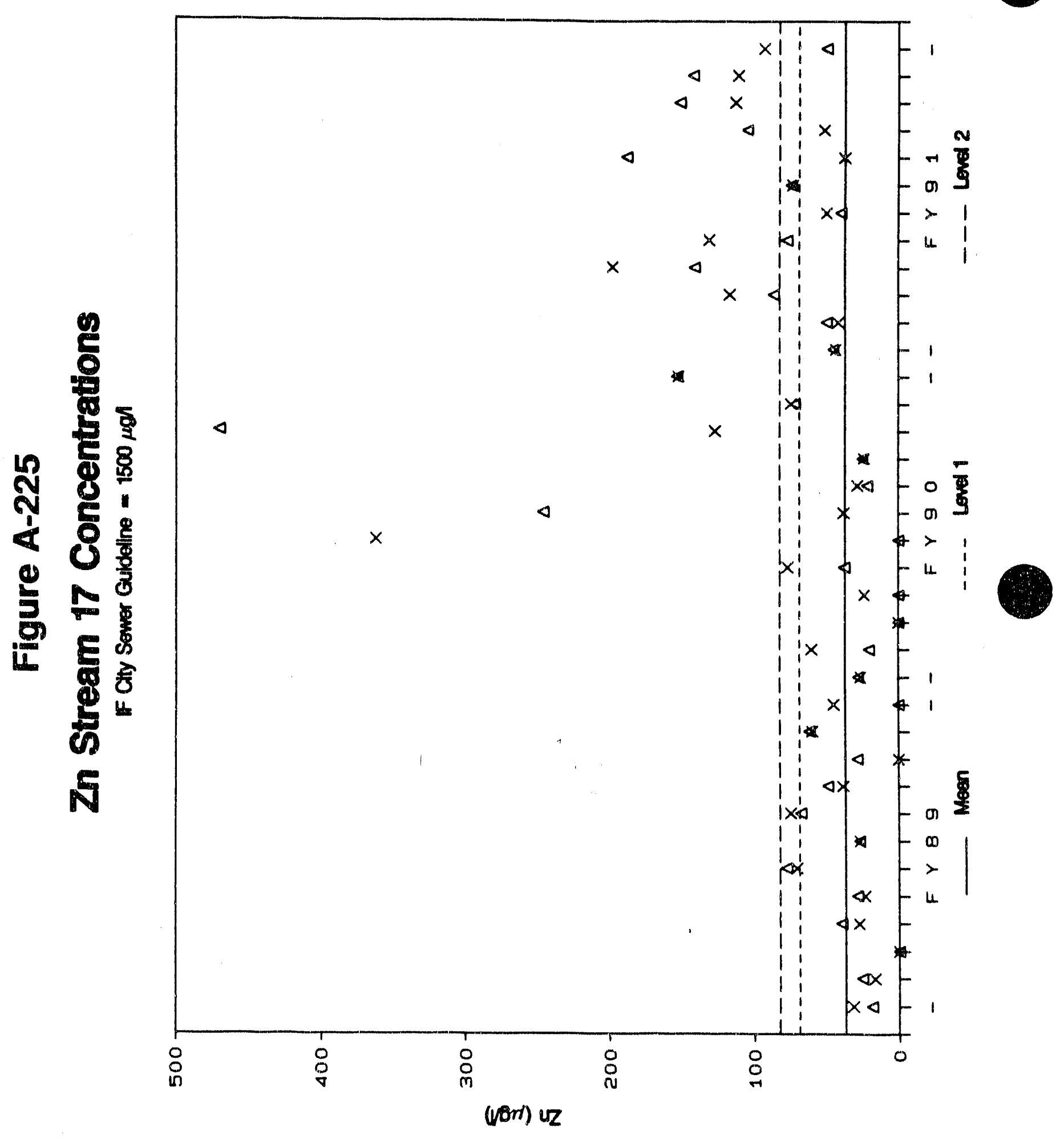




\section{Table A-68}

FX91 ENVIRONMENTAL MONITORING DATA

STREAM $=17$ (WCB waste effluent) QUARTER=91q1

\begin{tabular}{|c|c|c|c|c|c|c|c|c|}
\hline MONTH & REP & $\begin{array}{l}\text { Cond } \\
(\mu S)\end{array}$ & $\mathrm{pH}$ & $\underset{(\mathrm{mg} / 1)}{\mathrm{CN}}$ & $\begin{array}{l}\text { TDS } \\
\text { (mg/l) }\end{array}$ & $\begin{array}{l}\text { TOC } \\
(\mathrm{mg} / \mathrm{I})\end{array}$ & $\begin{array}{l}\text { TSS } \\
(\mathrm{ing} / \mathrm{d})\end{array}$ & $\frac{\mathrm{cl}}{(\mathrm{mg} / \mathrm{l})}$ \\
\hline $\begin{array}{l}\text { Oct } \\
\text { Oct } \\
\text { Nov } \\
\text { Nov } \\
\text { Dec } \\
\text { Dec }\end{array}$ & $\begin{array}{l}1 \\
2 \\
1 \\
2 \\
1 \\
2\end{array}$ & $\begin{array}{r}927 \\
x \\
715 \\
856 \\
955 \\
953\end{array}$ & $\begin{array}{l}7.48 \\
x \\
8.99 \\
8.00 \\
8.04 \\
7.02\end{array}$ & $\begin{array}{l}x \\
x \\
74.8 \\
109 \\
x \\
x\end{array}$ & $\begin{array}{l}444 \\
430 \\
351 \\
345 \\
658 \\
666\end{array}$ & $\begin{array}{r}77.0 \\
79.6 \\
65.4 \\
71.5 \\
120 \\
120\end{array}$ & $\begin{array}{r}85.0 \\
83.5 \\
222 \\
311 \\
468 \\
260\end{array}$ & $\begin{array}{l}72.8 \\
74.0 \\
69.2 \\
64.4 \\
37.0 \\
37.2\end{array}$ \\
\hline MONTH & REP & $\underset{(\mathrm{mg} / \mathrm{l})}{\mathrm{F}}$ & $\begin{array}{l}\mathrm{NO3} \\
(\mathrm{mg} / 1)\end{array}$ & $\begin{array}{l}\text { PO } \\
\text { (mg }\end{array}$ & $\begin{array}{c}\mathrm{SO4} \\
(\mathrm{mg} / \mathrm{l})\end{array}$ & $\underset{(\mu g / 1)}{\mathrm{Ag}}$ & $\begin{array}{r}\text { As } \\
(\mu g\end{array}$ & \\
\hline $\begin{array}{l}\text { Oct } \\
\text { Oct } \\
\text { Nov } \\
\text { Nov } \\
\text { Dec } \\
\text { Dec }\end{array}$ & $\begin{array}{l}1 \\
2 \\
1 \\
2 \\
1 \\
2\end{array}$ & $\begin{array}{l}1.20 \\
1.20 \\
0.91 \\
0.92 \\
2.80 \\
2.00\end{array}$ & $\begin{array}{l}5.70 \\
5.80 \\
X \\
X \\
X \\
X\end{array}$ & $\begin{array}{l}11 \\
12 \\
10 \\
10 \\
29 \\
28\end{array}$ & $\begin{array}{l}57.4 \\
57.1 \\
74.6 \\
69.3 \\
74.4 \\
75.3\end{array}$ & $\begin{array}{l}79.0 \\
78.7 \\
x \\
x \\
248 \\
430\end{array}$ & $\begin{array}{l}1.2 \\
1.2 \\
1 . \\
1.2 \\
2.5 \\
1 .\end{array}$ & \\
\hline
\end{tabular}

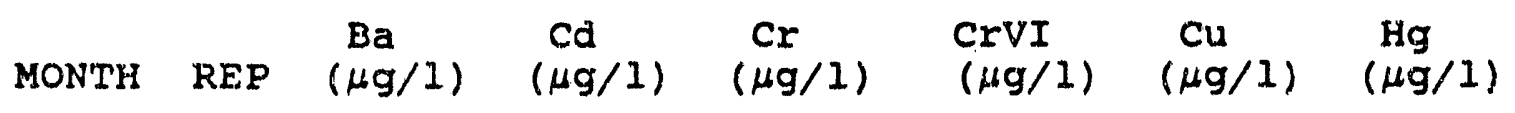

$\begin{array}{lrrlllrr}\text { Oct } & 1 & 190 & \mathrm{X} & 7.20 & \mathrm{X} & 91.1 & 0.12 \\ \text { Oct } & 2 & 28.2 & \mathrm{X} & \mathrm{X} & \mathrm{X} & 89.6 & 0.13 \\ \text { Nov } & 1 & 85.1 & \mathrm{X} & \mathrm{X} & \mathrm{X} & 46.8 & 0.31 \\ \text { Nov } & 2 & 83.1 & \mathrm{X} & \mathrm{X} & \mathrm{X} & 45.7 & 0.30 \\ \text { Dec } & 1 & 42.6 & \mathrm{X} & \mathrm{X} & \mathrm{X} & 225 & 0.20 \\ \text { Dec } & 2 & 44.6 & \mathrm{X} & \mathrm{X} & 11.1 & 207 & 0.19\end{array}$

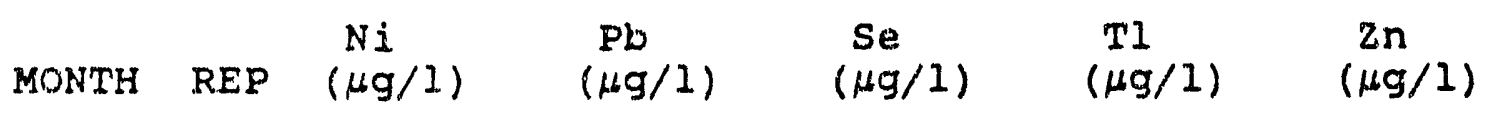

$\begin{array}{llllllr}\text { Oct } & 1 & X & 3.30 & X & X & 45.3 \\ \text { Oct } & 2 & X & 3.30 & X & X & 43.6 \\ \text { Nov } & 1 & X & X & X & X & 50.0 \\ \text { Nov } & 2 & X & X & X & X & 41.7 \\ \text { Dec } & 1 & X & 3.90 & X & X & 86.6 \\ \text { Dec } & 2 & X & 4.40 & X & X & 117\end{array}$

$'$ ' - missing

' $X$ ' - below practical quantitation level 


\section{Table A-69}

FY91 ENVIRONMENTAL MONITORING DATA

STREAM=17 (WCB waste effluent) QUARTER=91q2

$\begin{array}{lrrrlrrrr}\text { MONTH } & \text { REP } & \begin{array}{c}\text { Cond } \\ (\mu S)\end{array} & \mathrm{pH} & \begin{array}{c}\mathrm{CN} \\ (\mathrm{mg} / 1)\end{array} & \begin{array}{c}\text { TDS } \\ (\mathrm{mg} / 1)\end{array} & \begin{array}{c}\text { TOC } \\ (\mathrm{mg} / 1)\end{array} & \begin{array}{c}\text { TSS } \\ (\mathrm{mg} / 1)\end{array} & \begin{array}{c}\mathrm{Cl} \\ (\mathrm{mg} / 1)\end{array} \\ \text { Jan } & 1 & 837 & 7.72 & \mathrm{X} & 580 & 58.6 & 108 & 62.6 \\ \text { Jan } & 2 & 837 & 7.68 & \mathrm{X} & 648 & 56.0 & 112 & 60.5 \\ \text { Feb } & 1 & 1150 & 8.28 & 13.9 & 888 & 31.0 & 140 & 80.6 \\ \text { Feb } & 2 & 854 & 7.77 & 72.5 & 758 & 32.9 & 142 & 77.9 \\ \text { Mar } & 1 & 991 & 8.75 & \mathrm{X} & 490 & 129 & 310 & 78.0 \\ \text { Mar } & 2 & \mathrm{X} & \mathrm{X} & \mathrm{X} & 510 & 130 & 94.0 & 79.0\end{array}$

\begin{tabular}{|c|c|c|c|c|c|c|}
\hline MONTH & REP & $\underset{(m g / I)}{F}$ & $\begin{array}{l}\text { No3 } \\
\text { (mg/1) }\end{array}$ & $\begin{array}{l}\mathrm{PO4} \\
(\mathrm{mg} / \mathrm{l})\end{array}$ & $\begin{array}{l}504 \\
(m g / 1)\end{array}$ & $\underset{(\mu g / 1)}{\mathrm{Ag}}$ \\
\hline $\begin{array}{l}\text { Jan } \\
\text { Jan } \\
\text { Feb } \\
\text { Feb } \\
\text { Mar } \\
\text { Mar }\end{array}$ & $\begin{array}{l}1 \\
2 \\
1 \\
2 \\
1 \\
2\end{array}$ & $\begin{array}{l}0.30 \\
0.30 \\
0.30 \\
0.30 \\
x \\
x\end{array}$ & $\begin{array}{l}x \\
x \\
1.70 \\
1.90 \\
2.20 \\
0.15\end{array}$ & $\begin{array}{l}4.33 \\
4.08 \\
5.87 \\
5.87 \\
12.0 \\
12.0\end{array}$ & $\begin{array}{l}55.2 \\
53.2 \\
60.7 \\
60.3 \\
x \\
x\end{array}$ & $\begin{array}{l}X \\
X \\
38.6 \\
91.7 \\
40.0 \\
40.0\end{array}$ \\
\hline
\end{tabular}

\begin{tabular}{|c|c|c|c|c|c|c|}
\hline MONTH & REP & $\begin{array}{l}\mathrm{Ba} \\
(\mu \mathrm{g} / \mathrm{I})\end{array}$ & $\frac{\mathrm{cd}}{(\mu \mathrm{g} / 1)}$ & $\frac{\mathrm{Cr}}{(\mu \mathrm{g} / \mathrm{I})}$ & $\begin{array}{l}\text { CxVI } \\
(\mu g / I)\end{array}$ & $\frac{\mathrm{Cu}}{(\mathrm{kg} / \mathrm{I})}$ \\
\hline $\operatorname{Jan}$ & 1 & $x$ & $x$ & $x$ & $x$ & 130 \\
\hline Jan & 2 & $x$ & $\mathrm{x}$ & $x$ & $\mathrm{x}$ & 150 \\
\hline Feb & 1 & $\mathrm{x}$ & $\mathrm{x}$ & 5.30 & $\mathrm{x}$ & 72.3 \\
\hline Feb & 2 & $\mathrm{x}$ & $x$ & $x$ & $x$ & 111 \\
\hline Mar & 1 & $x$ & $\ddot{x}$ & $x$ & $x$ & 80.0 \\
\hline Mar & 2 & $\mathrm{x}$ & $\mathrm{x}$ & $x$ & $x$ & 90.0 \\
\hline
\end{tabular}

\begin{tabular}{|c|c|c|c|c|c|c|}
\hline MONTH & REP & $\begin{array}{l}\mathrm{Ni} \\
(\mu \mathrm{g} / \mathrm{l})\end{array}$ & $\begin{array}{l}\mathrm{Pb} \\
(\mu \mathrm{g} / 1)\end{array}$ & $\begin{array}{c}S e \\
(\mu g / 1)\end{array}$ & $\frac{T I}{(\mu g / I)}$ & $\begin{array}{l}\mathrm{Zn} \\
(\mu \mathrm{g} / 1)\end{array}$ \\
\hline $\operatorname{Jan}$ & 1 & $x$ & $x$ & $x$ & $x$ & 141 \\
\hline Jan & 2 & $\mathrm{x}$ & 4.10 & $\mathrm{X}$ & $\mathrm{X}$ & 198 \\
\hline Feb & 1 & 11.6 & $x$ & $x$ & $x$ & 77.3 \\
\hline Feb & 2 & $\mathrm{x}$ & $x$ & $x$ & $\mathrm{x}$ & 1.31 \\
\hline Mar & 1 & $x$ & $x$ & $x$ & $x$ & 40.0 \\
\hline Mar & 2 & $x$ & $x$ & $X$ & $\mathrm{X}$ & 50.0 \\
\hline
\end{tabular}

$'$ ' - missing

' $X '$ - below practical quantitation level 


\section{Table A-70}

FY91 ENVIRONMENTAL MONITORING DATA

STREAM=17 (WCB waste effluent) QUARTER=91q3

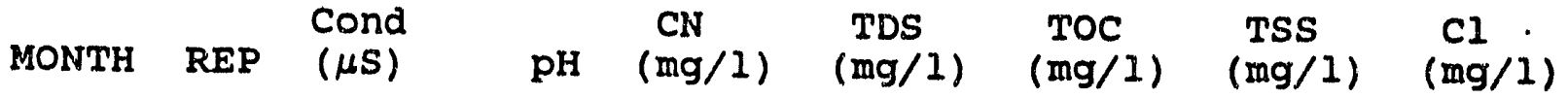

$\begin{array}{lrrrlrrrr}\text { Apr } & 1 & 989 & 7.62 & \mathrm{X} & 618 & 122 & 149 & 92.6 \\ \text { Apr } & 2 & 88 & 7.52 & \mathrm{X} & 613 & 121 & 63.0 & 93.4 \\ \text { May } & 1 & 1230 & 9.05 & \mathrm{X} & 495 & 92.4 & 210 & 89.9 \\ \text { May } & 2 & 840 & 8.36 & \mathrm{X} & 542 & 103 & 308 & 87.3 \\ \text { Jun } & 1 & 883 & 7.78 & \mathrm{X} & 424 & 62.5 & 396 & 44.2 \\ \text { Jun } & 2 & 914 & 8.04 & \mathrm{X} & 451 & 136 & 401 & 81.8\end{array}$

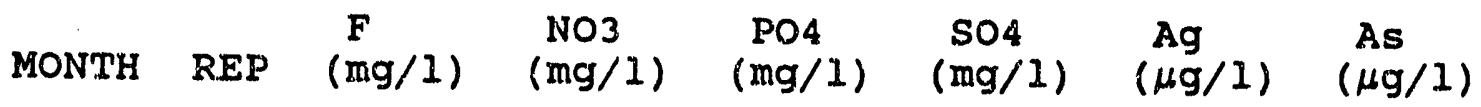

$\begin{array}{llllllrl}\text { Apr } & 1 & 0.31 & X & 17.0 & 67.7 & 117 & \text { X } \\ \text { Apr } & 2 & 0.30 & X & 17.0 & 67.3 & 126 & \text { X } \\ \text { May } & 1 & 0.36 & 0.06 & 35.2 & 73.5 & 141 & \text { X } \\ \text { May } & 2 & 0.33 & X & 33.7 & 80.3 & 981 & \text { X } \\ \text { Jun } & 1 & 0.32 & 0.03 & 27.9 & 52.5 & 30.9 & X \\ \text { Jun } & 2 & 0.32 & 0.11 & 11.8 & 58.8 & 97.9 & X\end{array}$

$\begin{array}{llllllll}\text { MONTH } & \operatorname{REP} & \mathrm{Ba} & \mathrm{Cd} & \mathrm{Cr} & \mathrm{CrVI} & \mathrm{Cu} & \mathrm{Hg} \\ (\mu \mathrm{g} / 1) & (\mu \mathrm{g} / 1) & (\mu \mathrm{g} / 1) & (\mu \mathrm{g} / 1) & (\mu \mathrm{g} / 1) & (\mu \mathrm{g} / 1)\end{array}$

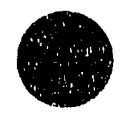

$\begin{array}{llrlll}\text { Apr } & 1 & 55.7 & \mathrm{X} & \mathrm{X} & \mathrm{X} \\ \text { Apr } & 2 & 55.7 & \mathrm{X} & \mathrm{X} & \mathrm{X} \\ \text { May } & 1 & 105 & \mathrm{X} & \mathrm{X} & \mathrm{X} \\ \text { May } & 2 & 95.7 & \mathrm{X} & \mathrm{X} & \mathrm{X} \\ \text { Jun } & 1 & 101 & \mathrm{X} & 6.00 & \mathrm{X} \\ \text { Jun } & 2 & 82.4 & \mathrm{X} & \mathrm{X} & \mathrm{X}\end{array}$

$\begin{array}{rr}47.6 & X \\ 52.3 & X \\ 92.7 & X \\ 146 & X \\ 90.4 & X \\ 182 & X\end{array}$

$\begin{array}{lclllll}\text { MONTH } & R E P & \begin{array}{c}\text { Ni } \\ (\mu \mathrm{g} / I)\end{array} & \begin{array}{c}\mathrm{Pb} \\ (\mu \mathrm{g} / I)\end{array} & \begin{array}{c}\mathrm{Se} \\ (\mu \mathrm{g} / I)\end{array} & \begin{array}{c}\mathrm{TI} \\ (\mu \mathrm{g} / 1)\end{array} & \begin{array}{c}\mathrm{Zn} \\ (\mu \mathrm{g} / I)\end{array} \\ \text { Apr } & 1 & \mathrm{X} & 3.20 & \mathrm{X} & \mathrm{X} & 74.3 \\ \text { Apr } & 2 & \mathrm{X} & 7.00 & \mathrm{X} & \mathrm{X} & 73.2 \\ \text { MaY } & 1 & \mathrm{X} & 3.40 & \mathrm{X} & \mathrm{X} & 188 \\ \text { May } & 2 & \mathrm{X} & 2.70 & \mathrm{X} & \mathrm{X} & 37.5 \\ \text { Jun } & 1 & 9.90 & 4.60 & \mathrm{X} & \mathrm{X} & 105 \\ \text { Jun } & 2 & \mathrm{X} & \mathrm{X} & \mathrm{X} & \mathrm{X} & 51.6\end{array}$

$'$ ' - missing

' $X '$ - below practical quantitation level 


\section{Table A-71}

FY91 ENVIRONMENTAL MONITORING DATA

STREAM $=17$ (WCB waste effluent) QUARTER=9194

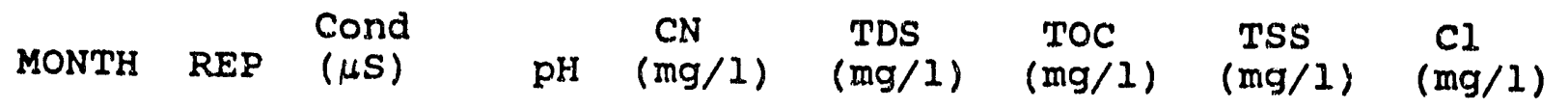

$\begin{array}{lllllrrrr}\text { Jul } & 1 & 900 & 8.78 & X & 453 & 80.3 & 226 & 94.0 \\ \text { Jul } & 2 & 818 & 8.11 & X & 494 & 64.0 & 174 & 53.8 \\ \text { Aug } & 1 & 366 & 7.15 & X & 1050 & 73.0 & 149 & 37.6 \\ \text { Aug } & 2 & 516 & 7.62 & X & 466 & 132 & 302 & 66.3 \\ \text { Sep } & 1 & 736 & 7.89 & X & 574 & 50.7 & 190 & 57.6 \\ \text { Sep } & 2 & 907 & 8.36 & X & 382 & 53.5 & 162 & 83.2\end{array}$

$\begin{array}{llllllll}\text { MONTH } & \operatorname{REP} & \underset{(\mathrm{mg} / 1)}{\mathrm{F}} & \mathrm{NO3} & \mathrm{PO} 4 & \mathrm{SO} & \mathrm{Ag} & \mathrm{As} \\ (\mathrm{mg} / 1) & (\mathrm{mg} / 1) & (\mathrm{mg} / 1) & (\mu \mathrm{g} / 1) & (\mu \mathrm{g} / 1)\end{array}$

$\begin{array}{llllllll}\text { Jul } & 1 & 0.29 & 0.04 & 29.3 & 62.2 & 19.7 & \mathrm{X} \\ \text { Jul } & 2 & 0.37 & 0.07 & 29.7 & 85.8 & 17.1 & \mathrm{X} \\ \text { Aug } & 1 & 0.28 & 0.09 & 25.8 & 56.8 & 77.7 & \mathrm{X} \\ \text { Aug } & 2 & 0.27 & 0.08 & 41.9 & 65.0 & 69.2 & \mathrm{X} \\ \text { Sep } & 1 & 0.26 & \mathrm{X} & 10.3 & 58.6 & 37.1 & \mathrm{X} \\ \text { Sep } & 2 & 0.26 & 0.43 & 35.5 & 67.6 & 54.7 & \mathrm{X}\end{array}$

$\begin{array}{lcclllll}\text { MONTH } & \mathrm{REP} & \begin{array}{c}\mathrm{Ba} \\ (\mu \mathrm{g} / 1)\end{array} & \begin{array}{c}\mathrm{Cd} \\ (\mu \mathrm{g} / 1)\end{array} & \begin{array}{c}\mathrm{Cr} \\ (\mu g / 1)\end{array} & \begin{array}{l}\mathrm{CrVI} \\ (\mu \mathrm{g} / 1)\end{array} & \begin{array}{c}\mathrm{Cu} \\ (\mu \mathrm{g} / 1)\end{array} & \begin{array}{c}\mathrm{Hg} \\ (\mu \mathrm{g} / 1)\end{array} \\ \text { Jul } & 1 & 80.4 & \mathrm{X} & \mathrm{X} & \mathrm{X} & 78.9 & 0.21 \\ \text { JuI } & 2 & 96.3 & \mathrm{X} & \mathrm{X} & \mathrm{X} & 120 & \mathrm{X} \\ \text { Aug } & 1 & 94.1 & \mathrm{X} & \mathrm{X} & \mathrm{X} & 122 & 6.20 \\ \text { Aug } & 2 & 91.9 & \mathrm{X} & \mathrm{X} & \mathrm{X} & 134 & \mathrm{X} \\ \text { Sep } & 1 & 1760 & 6.40 & 32.1 & \mathrm{X} & 126 & \mathrm{X} \\ \text { Sep } & 2 & 149 & \mathrm{X} & 31.8 & \mathrm{X} & 112 & \mathrm{X}\end{array}$

$\begin{array}{llccccc}\mathrm{MONTH} & \mathrm{REP} & \mathrm{Ni} & \mathrm{Pb} & \mathrm{Se} & \mathrm{TI} & \mathrm{Zn} \\ (\mu \mathrm{g} / 1) & (\mu \mathrm{g} / 1) & (\mu \mathrm{g} / \mathrm{I}) & (\mu \mathrm{g} / \mathrm{I}) & (\mu \mathrm{g} / \mathrm{I})\end{array}$

\begin{tabular}{|c|c|c|c|c|c|c|}
\hline Jul & 1 & $x$ & 4.20 & $x$ & $x$ & 151 \\
\hline Jul & 2 & $x$ & $x$ & $x$ & $x$ & 113 \\
\hline Aug & 1 & $x$ & $x$ & $x$ & $x$ & 142 \\
\hline Aug & 2 & $x$ & $x$ & $x$ & $x$ & 111 \\
\hline Sep & 1 & $x$ & 3.80 & $x$ & 1.10 & 49.8 \\
\hline Sep & 2 & $x$ & 4.00 & $x$ & $x$ & 93.1 \\
\hline
\end{tabular}

'.' - missing

' $X$ ' - below practical quantitation level 
Table A-72

SUMMARY STATISTICS

STREAM $=17$ (WCB waste effluent)

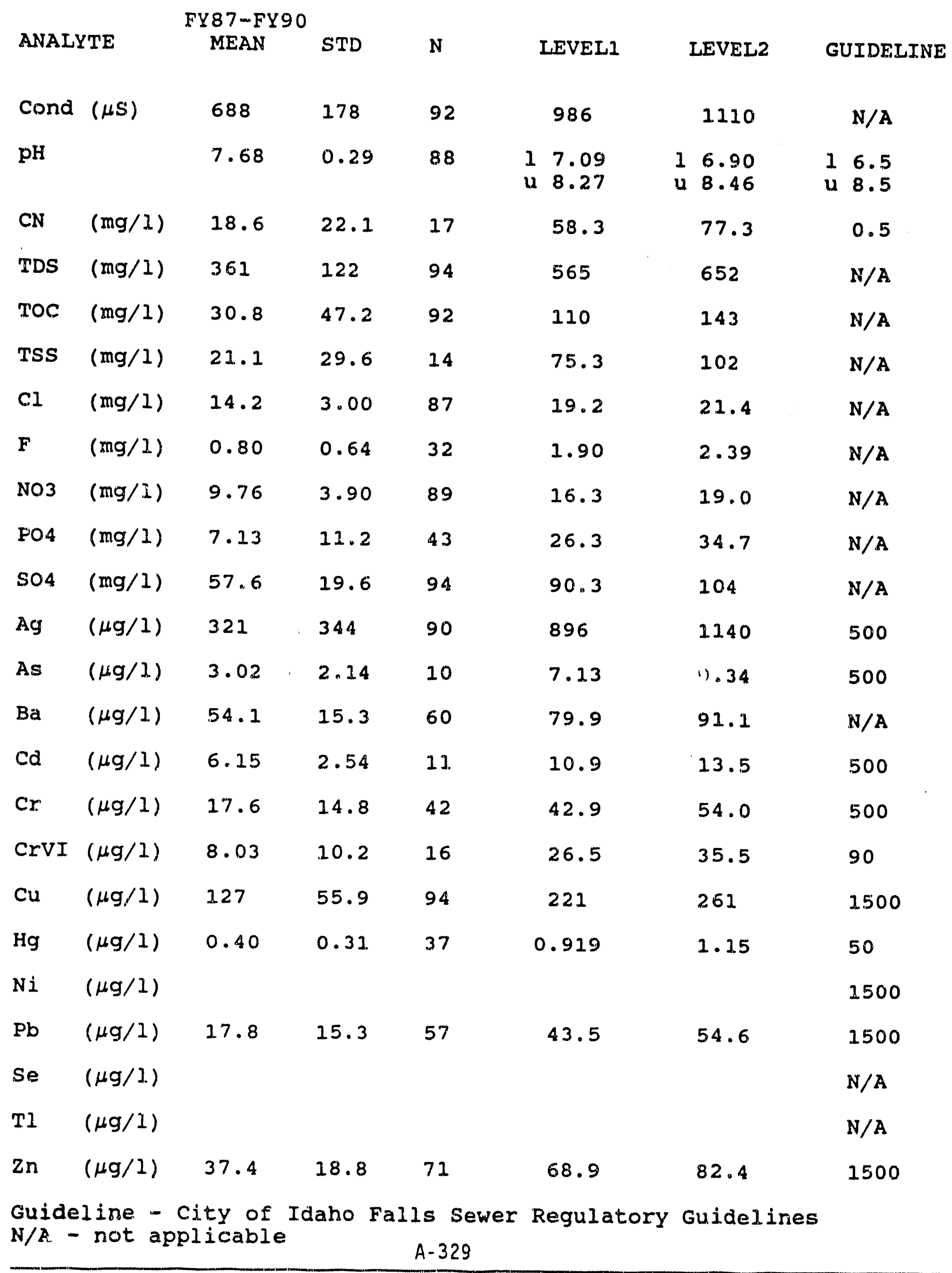




\section{Table A-73}

FY91 ENVIRONMENTAL MONITORING VOLATILE ORGANICS DATA

STREAM=17 (WCB waste effluent) QUARTER=9.1q1 CONCENTRATION

MONTH REP COMPOUND

Oct 1 Acetone

oct 1 Toluene

oct 1 all other compound.s

Nov 11 1,4-Dichlorobenzene

Nov 1 Acetone

Nov

Dec
1 all other compounds

1 all compounds $(\mu g / 1)$

$$
\begin{gathered}
98.50 \\
0.54 \\
X \\
4.60 \\
21.20 \\
X \\
X
\end{gathered}
$$

' $x$ ' - below practical quantitation level 


\section{Table A-74}

FY91 ENVIRONMENTAL MONITORING VOLATILE ORGANICS DATA

\section{STREAM $=17$ (WCB waste effluent) QUARTER=91q2}

MONTH REP COMPOUND

Jan 1 Acetone

Jan

Feb

Mar

Mar

Mar

Mar all other compounds

all compounds

1, 1, 1,-Trichloroethane Ethylbenzene

Toluene

all other compounds
CONCENTRATION

$(\mu \mathrm{g} / \mathrm{l})$

19.0

$\mathrm{X}$

$\mathrm{x}$

21.0

1.4

1.1

$\mathrm{x}$

' $X$ ' - below practical quantitation level 


\section{Table A-75}

FY91 ENVIRONMENTAL MONITORING VOLATILE ORGANICS DATA

STREAM=17 (WCB waste effluent) QUARTER=91q3

CONCENTRATION

\begin{tabular}{|c|c|c|c|}
\hline MONTH & REP & COMPOUND & $(\mu \mathrm{g} / \mathrm{I})$ \\
\hline Apr & 1 & Acetone & 110.0 \\
\hline Apr & 1 & Unknown (TIC) & 3.7 \\
\hline Apr & 1 & all other compounds & $\mathrm{x}$ \\
\hline May & 1 & all compounds & $\mathrm{x}$ \\
\hline $\begin{array}{l}\text { Jun } \\
\text { Jun }\end{array}$ & $\begin{array}{l}1 \\
1\end{array}$ & $\begin{array}{l}\text { Acetone } \\
\text { all compounds }\end{array}$ & $\begin{array}{l}7 \\
\times\end{array}$ \\
\hline
\end{tabular}

' $X$ ' - below practical quantitation level

TIC - Tentatively Identified compound 
Table A-76

FY91 ENVIRONMENTAL MONITORING VOLATILE ORGANICS DATA STREAM=17 (WCB waste effluent) QUARTER=91q4

MONTH REP COMPOUND

$\begin{array}{lll}\text { Jul } & 1 & \text { Acetone } \\ \text { Jul } & 1 & \text { all compounds }\end{array}$

Aug 1 Acetone

Aug

Sep

Sep

1

all other

Acetone

all other compounds
CONCENTRATION

$(\mu \mathrm{g} / \mathrm{l})$

25

$\mathrm{X}$

19

$\mathrm{X}$

25

' $X$ ' - below practical quantitation level 
Table A-77

QUARTERLY MEANS AND VARIANCE COMPONENTS

STREAM=17 (WCB waste effluent)

\begin{tabular}{|c|c|c|c|c|c|c|c|c|c|}
\hline ANALY & TE & QTR & MEAN & $\begin{array}{r}\text { STD } \\
\text { MEAN }\end{array}$ & $\begin{array}{l}\text { STD } \\
\text { DAY }\end{array}$ & $\begin{array}{l}\text { STD } \\
\text { REP }\end{array}$ & $\mathrm{N}$ & $\begin{array}{r}\mathbf{N} \\
\text { MISS }\end{array}$ & $\begin{array}{r}\mathbf{N} \\
\text { BPQL }\end{array}$ \\
\hline Cond & $(\mu S)$ & $\begin{array}{l}91 q 1 \\
91 q 2 \\
91 q 3 \\
91 q 4\end{array}$ & $\begin{array}{l}881 \\
934 \\
824 \\
707\end{array}$ & $\begin{array}{r}58.0 \\
54.5 \\
148 \\
134\end{array}$ & $\begin{array}{r}81.1 \\
0.00 \\
0.00 \\
220\end{array}$ & $\begin{array}{r}70.5 \\
148 \\
401 \\
98.7\end{array}$ & $\begin{array}{l}5 \\
5 \\
6 \\
6\end{array}$ & $\begin{array}{l}1 \\
1 \\
0 \\
0\end{array}$ & $\begin{array}{l}0 \\
0 \\
0 \\
0\end{array}$ \\
\hline $\mathrm{pH}$ & & $\begin{array}{l}91 q 1 \\
91 q 2 \\
91 q^{3} \\
91 q^{4}\end{array}$ & $\begin{array}{l}7.91 \\
8.04 \\
8.06 \\
7.99\end{array}$ & $\begin{array}{l}0.34 \\
0.29 \\
0.34 \\
0.31\end{array}$ & $\begin{array}{l}0.22 \\
0.43 \\
0.54 \\
0.47\end{array}$ & $\begin{array}{l}0.71 \\
0.26 \\
0.30 \\
0.39\end{array}$ & $\begin{array}{l}5 \\
5 \\
6 \\
6\end{array}$ & $\begin{array}{l}1 \\
1 \\
0 \\
0\end{array}$ & $\begin{array}{l}0 \\
0 \\
0 \\
0\end{array}$ \\
\hline $\mathrm{CN}$ & $(\mathrm{mg} / \mathrm{I})$ & $\begin{array}{l}91 q 1 \\
91 q^{2} \\
91 q^{3} \\
91 q^{4}\end{array}$ & $\begin{array}{l}91.9 \\
43.2 \\
X \\
X\end{array}$ & $\dot{.}$ & $\dot{.}$ & $\dot{\dot{ }}$ & $\begin{array}{l}2 \\
2 \\
0 \\
0\end{array}$ & $\begin{array}{l}0 \\
0 \\
0 \\
0\end{array}$ & $\begin{array}{l}4 \\
4 \\
6 \\
6\end{array}$ \\
\hline TDS & $(\mathrm{mg} / \mathrm{l})$ & $\begin{array}{l}91 q 1 \\
91 q^{2} \\
91 q^{3} \\
91 q^{4}\end{array}$ & $\begin{array}{l}482 \\
646 \\
524 \\
570\end{array}$ & $\begin{array}{l}93.4 \\
94.6 \\
51.5 \\
94.1\end{array}$ & $\begin{array}{r}162 \\
158 \\
87.7 \\
0.00\end{array}$ & $\begin{array}{r}7.02 \\
60.4 \\
22.2 \\
252\end{array}$ & $\begin{array}{l}6 \\
6 \\
6 \\
6\end{array}$ & $\begin{array}{l}0 \\
0 \\
0 \\
0\end{array}$ & $\begin{array}{l}0 \\
0 \\
0 \\
0\end{array}$ \\
\hline TOC & $(\mathrm{mg} / \mathrm{l})$ & $\begin{array}{l}91 q 1 \\
91 q^{2} \\
91 q^{3} \\
91 q^{4}\end{array}$ & $\begin{array}{r}88.9 \\
72.9 \\
106 \\
75.6\end{array}$ & $\begin{array}{l}15.8 \\
29.2 \\
7.69 \\
14.7\end{array}$ & $\begin{array}{l}27.3 \\
50.6 \\
0.00 \\
18.2\end{array}$ & $\begin{array}{l}2.71 \\
1.38 \\
30.3 \\
25.0\end{array}$ & $\begin{array}{l}6 \\
6 \\
6 \\
6\end{array}$ & $\begin{array}{l}0 \\
0 \\
0 \\
0\end{array}$ & $\begin{array}{l}0 \\
0 \\
0 \\
0\end{array}$ \\
\hline TSS & $(\mathrm{mg} / \mathrm{l})$ & $\begin{array}{l}91 q^{1} \\
91 q 2 \\
91 q^{3} \\
91 q^{4}\end{array}$ & $\begin{array}{l}238 \\
151 \\
255 \\
201\end{array}$ & $\begin{array}{l}82.0 \\
27.0 \\
84.5 \\
14.3\end{array}$ & $\begin{array}{r}126 \\
0.00 \\
141 \\
0.00\end{array}$ & $\begin{array}{l}92.4 \\
88.2 \\
53.3 \\
67.0\end{array}$ & $\begin{array}{l}6 \\
6 \\
6 \\
6\end{array}$ & $\begin{array}{l}0 \\
0 \\
0 \\
0\end{array}$ & $\begin{array}{l}0 \\
0 \\
0 \\
0\end{array}$ \\
\hline $\mathrm{Cl}$ & $(\mathrm{mg} / \mathrm{I})$ & $\begin{array}{l}91 q 1 \\
91 q^{2} \\
91 q^{3} \\
91 q^{4}\end{array}$ & $\begin{array}{l}59.1 \\
73.1 \\
81.5 \\
65.4\end{array}$ & $\begin{array}{l}11.2 \\
5.78 \\
9.35 \\
6.81\end{array}$ & $\begin{array}{l}19.3 \\
9.96 \\
12.0 \\
0.00\end{array}$ & $\begin{array}{l}2.02 \\
1.45 \\
15.4 \\
22.7\end{array}$ & $\begin{array}{l}6 \\
6 \\
6 \\
6\end{array}$ & $\begin{array}{l}0 \\
0 \\
0 \\
0\end{array}$ & $\begin{array}{l}0 \\
0 \\
0 \\
0\end{array}$ \\
\hline$F$ & $(\mathrm{mg} / \mathrm{l})$ & $\begin{array}{l}91 q 1 \\
91 q 2 \\
91 q^{3} \\
91 q^{4}\end{array}$ & $\begin{array}{l}1.50 \\
0.03 \\
0.32 \\
0.29\end{array}$ & $\begin{array}{l}0.45 \\
0.27 \\
0.01 \\
0.02\end{array}$ & $\begin{array}{l}0.75 \\
0.46 \\
0.02 \\
0.03\end{array}$ & $\begin{array}{l}0.33 \\
0.00 \\
0.01 \\
0.03\end{array}$ & $\begin{array}{l}6 \\
6 \\
6 \\
6\end{array}$ & $\begin{array}{l}0 \\
0 \\
0 \\
0\end{array}$ & $\begin{array}{l}0 \\
0 \\
0 \\
0\end{array}$ \\
\hline NO3 & $(\mathrm{mg} / \mathrm{l})$ & $\begin{array}{l}91 q 1 \\
91 q^{2} \\
91 q^{3} \\
91 q^{4}\end{array}$ & $\begin{array}{l}5.75 \\
0.92 \\
0.07 \\
0.12\end{array}$ & $\begin{array}{l}\dot{0.59} \\
\dot{0} .05\end{array}$ & $\begin{array}{l}\dot{0} .83 \\
\dot{0} .00\end{array}$ & $\begin{array}{l}\dot{0} .84 \\
0.19\end{array}$ & $\begin{array}{l}2 \\
6 \\
3 \\
6\end{array}$ & $\begin{array}{l}0 \\
0 \\
0 \\
0\end{array}$ & $\begin{array}{l}4 \\
0 \\
3 \\
0\end{array}$ \\
\hline
\end{tabular}




\section{Table A-77 (continued)}

\section{QUARTERLY MEANS AND VARIANCE COMPONENTS}

STREAM $=17$ (WCB waste effluent) (continued)

\begin{tabular}{|c|c|c|c|c|c|c|c|c|c|}
\hline \multicolumn{2}{|c|}{ ANALYTE } & QTR & MEAN & $\begin{array}{r}\text { STD } \\
\text { MEAN }\end{array}$ & $\begin{array}{l}\text { STD } \\
\text { DAY }\end{array}$ & $\begin{array}{l}\text { STD } \\
\text { REP }\end{array}$ & $\mathrm{N}$ & $\begin{array}{r}\mathrm{N} \\
\text { MISS }\end{array}$ & $\begin{array}{r}\mathbf{N} \\
\text { BPQL }\end{array}$ \\
\hline PO4 & $(\mathrm{mg} / \mathrm{I})$ & $\begin{array}{l}91 q 1 \\
91 q 2 \\
91 q 3 \\
91 q 4\end{array}$ & $\begin{array}{l}17.1 \\
7.36 \\
23.8 \\
28.7\end{array}$ & $\begin{array}{l}5.84 \\
2.37 \\
5.40 \\
3.18\end{array}$ & $\begin{array}{l}10.1 \\
4.10 \\
8.11 \\
0.00\end{array}$ & $\begin{array}{l}0.61 \\
0.10 \\
6.59 \\
12.2\end{array}$ & $\begin{array}{l}6 \\
6 \\
6 \\
6\end{array}$ & $\begin{array}{l}0 \\
0 \\
0 \\
0\end{array}$ & $\begin{array}{l}0 \\
0 \\
0 \\
0\end{array}$ \\
\hline SO4 & $(\mathrm{mg} / \mathrm{l})$ & $\begin{array}{l}91 q 1 \\
91 q 2 \\
91 q 3 \\
91 q 4\end{array}$ & $\begin{array}{l}68.0 \\
57.3 \\
66.7 \\
66.0\end{array}$ & $\begin{array}{l}5.45 \\
3.15 \\
6.15 \\
4.05\end{array}$ & $\begin{array}{l}9.31 \\
4.40 \\
10.3 \\
0.00\end{array}$ & $\begin{array}{l}2.20 \\
1.02 \\
3.79 \\
10.8\end{array}$ & $\begin{array}{l}6 \\
4 \\
6 \\
6\end{array}$ & $\begin{array}{l}0 \\
2 \\
0 \\
0\end{array}$ & $\begin{array}{l}0 \\
0 \\
0 \\
0\end{array}$ \\
\hline $\mathrm{Ag}$ & $(\mu g / I)$ & $\begin{array}{l}91 q 1 \\
91 q 2 \\
91 q 3 \\
91 q 4\end{array}$ & $\begin{array}{r}209 \\
31.7 \\
249 \\
45.9\end{array}$ & $\begin{array}{r}130 \\
22.1 \\
157 \\
15.9\end{array}$ & $\begin{array}{r}172 \\
35.0 \\
121 \\
26.9\end{array}$ & $\begin{array}{r}91.0 \\
21.7 \\
344 \\
8.05\end{array}$ & $\begin{array}{l}4 \\
6 \\
6 \\
6\end{array}$ & $\begin{array}{l}0 \\
0 \\
0 \\
0\end{array}$ & $\begin{array}{l}2 \\
0 \\
0 \\
0\end{array}$ \\
\hline As & $(\mu g / 1)$ & $\begin{array}{l}91 q 1 \\
91 q^{2} \\
91 q^{3} \\
91 q^{4}\end{array}$ & $\begin{array}{l}1.65 \\
x \\
x \\
x\end{array}$ & $\begin{array}{l}0.31 \\
\dot{0} \\
\dot{0}\end{array}$ & $\begin{array}{l}0.36 \\
\dot{0} \\
\dot{0}\end{array}$ & $\begin{array}{l}0.55 \\
\dot{\cdot} \\
\dot{\cdot}\end{array}$ & $\begin{array}{l}6 \\
0 \\
0 \\
0\end{array}$ & $\begin{array}{l}0 \\
0 \\
0 \\
0\end{array}$ & $\begin{array}{l}0 \\
6 \\
6 \\
6\end{array}$ \\
\hline $\mathrm{Ba}$ & $(\mu g / I)$ & $\begin{array}{l}91 q 1 \\
91 q 2 \\
91 q 3 \\
91 q^{4}\end{array}$ & $\begin{array}{l}78.9 \\
x \\
82.6 \\
379\end{array}$ & $\begin{array}{r}19.1 \\
13.7 \\
288\end{array}$ & $\begin{array}{r}0.00 \\
22.9 \\
180\end{array}$ & $\begin{array}{r}66.1 \\
8.49 \\
658\end{array}$ & $\begin{array}{l}6 \\
0 \\
6 \\
6\end{array}$ & $\begin{array}{l}0 \\
0 \\
0 \\
0\end{array}$ & $\begin{array}{l}0 \\
6 \\
0 \\
0\end{array}$ \\
\hline$c d$ & $(\mu g / 1)$ & $\begin{array}{l}91 q 1 \\
91 q^{2} \\
91 q^{3} \\
91 q^{4}\end{array}$ & $\begin{array}{l}X \\
X \\
X \\
6.40\end{array}$ & $\begin{array}{l}\dot{\bullet} \\
\dot{\bullet}\end{array}$ & $\dot{.}$ & $\dot{:}$ & $\begin{array}{l}0 \\
0 \\
0 \\
1\end{array}$ & $\begin{array}{l}0 \\
0 \\
0 \\
0\end{array}$ & $\begin{array}{l}6 \\
6 \\
6 \\
5\end{array}$ \\
\hline $\mathrm{Cr}$ & $(\mu \mathrm{g} / \mathrm{I})$ & $\begin{array}{l}91 q^{1} \\
91 q^{2} \\
91 q^{3} \\
91 q^{4}\end{array}$ & $\begin{array}{l}7.20 \\
5.30 \\
6.00 \\
32.0\end{array}$ & $\begin{array}{l}\dot{.} \\
\dot{\cdot}\end{array}$ & $\dot{.}$ & $\dot{.}$ & $\begin{array}{l}1 \\
1 \\
1 \\
2\end{array}$ & $\begin{array}{l}0 \\
0 \\
0 \\
0\end{array}$ & $\begin{array}{l}5 \\
5 \\
5 \\
4\end{array}$ \\
\hline CrVI & $(\mu g / 1)$ & $\begin{array}{l}91 q 1 \\
91 q^{2} \\
91 q^{3} \\
91 q^{4}\end{array}$ & $\begin{array}{l}11.1 \\
X \\
x \\
x\end{array}$ & $\dot{.}$ & $\dot{.}$ & $\begin{array}{l}\dot{.} \\
\dot{.}\end{array}$ & $\begin{array}{l}1 \\
0 \\
0 \\
0\end{array}$ & $\begin{array}{l}0 \\
0 \\
0 \\
0\end{array}$ & $\begin{array}{l}5 \\
6 \\
6 \\
6\end{array}$ \\
\hline $\mathrm{Cu}$ & $(\mu \mathrm{g} / 1)$ & $\begin{array}{l}91 q 1 \\
91 q^{2} \\
91 q^{3} \\
91 q^{4}\end{array}$ & $\begin{array}{l}118 \\
106 \\
102 \\
115\end{array}$ & $\begin{array}{l}50.9 \\
17.3 \\
26.4 \\
8.43\end{array}$ & $\begin{array}{l}87.9 \\
27.1 \\
33.9 \\
6.63\end{array}$ & $\begin{array}{l}7.39 \\
18.2 \\
43.3 \\
18.4\end{array}$ & $\begin{array}{l}6 \\
6 \\
6 \\
6\end{array}$ & $\begin{array}{l}0 \\
0 \\
0 \\
0\end{array}$ & $\begin{array}{l}0 \\
0 \\
0 \\
0\end{array}$ \\
\hline
\end{tabular}




\section{Table A-77 (continued)}

QUARTERLY MEANS AND VARIANCE COMPONENTS

STREAM $=17$ (WCB waste effluent) (continued)

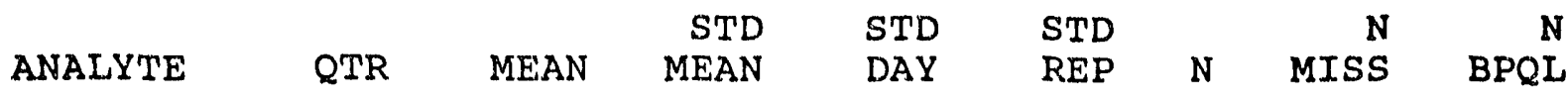

\begin{tabular}{|c|c|c|c|c|c|c|c|c|}
\hline $\mathrm{Hg}$ & $(\mu g / 1)$ & $\begin{array}{l}91 q 1 \\
91 q 2 \\
91 q 3 \\
91 q 4\end{array}$ & $\begin{array}{l}0.21 \\
x \\
x \\
3.20\end{array}$ & $\begin{array}{l}0.05 \\
\dot{0} \\
\dot{0}\end{array}$ & $\begin{array}{l}0.09 \\
\dot{0} \\
.\end{array}$ & $\begin{array}{l}0.01 \\
. \\
.\end{array}$ & $\begin{array}{l}6 \\
0 \\
0 \\
2\end{array}$ & $\begin{array}{l}0 \\
0 \\
0 \\
0\end{array}$ \\
\hline $\mathrm{Ni}$ & $(\mu \mathrm{g} / 1)$ & $\begin{array}{l}91 q^{1} \\
91 q^{2} \\
91 q^{3} \\
91 q^{4}\end{array}$ & $\begin{array}{l}x \\
11.6 \\
9.90 \\
x\end{array}$ & . & $\dot{.}$ & $\dot{.}$ & $\begin{array}{l}0 \\
1 \\
1 \\
0\end{array}$ & $\begin{array}{l}0 \\
0 \\
0 \\
0\end{array}$ \\
\hline
\end{tabular}

\begin{tabular}{|c|c|c|c|c|c|c|c|}
\hline$(\mu g / 1)$ & $\begin{array}{l}91 q 1 \\
91 q^{2} \\
91 q^{3} \\
91 q^{4}\end{array}$ & $\begin{array}{l}3.72 \\
4.10 \\
3.15 \\
4.00\end{array}$ & $\begin{array}{l}0.43 \\
i .10\end{array}$ & $\begin{array}{l}0.57 \\
0.00\end{array}$ & $\begin{array}{l}0.25 \\
3.12\end{array}$ & $\begin{array}{l}4 \\
1 \\
6 \\
3\end{array}$ & $\begin{array}{l}0 \\
0 \\
0 \\
0\end{array}$ \\
\hline$(\mu g / 1)$ & $\begin{array}{l}91 q 1 \\
91 q 2 \\
91 q 3 \\
91 q 4\end{array}$ & $\begin{array}{l}x \\
X \\
x \\
x\end{array}$ & $\begin{array}{l}\dot{.} \\
\dot{.}\end{array}$ & $\begin{array}{l}\dot{.} \\
\dot{.}\end{array}$ & . & $\begin{array}{l}0 \\
0 \\
0 \\
0\end{array}$ & $\begin{array}{l}0 \\
0 \\
0 \\
0\end{array}$ \\
\hline$(\mu g / 1)$ & $\begin{array}{l}91 q 1 \\
91 q 2 \\
91 q 3 \\
91 q 4\end{array}$ & $\begin{array}{l}X \\
X \\
X \\
1.10\end{array}$ & $\dot{.}$ & $\dot{.}$ & . & $\begin{array}{l}0 \\
0 \\
0 \\
1\end{array}$ & $\begin{array}{l}0 \\
0 \\
0 \\
0\end{array}$ \\
\hline$(\mu g / 1)$ & $\begin{array}{l}91 q 1 \\
91 q 2 \\
91 q 3 \\
91 q 4\end{array}$ & $\begin{array}{r}64.0 \\
106 \\
88.3 \\
110\end{array}$ & $\begin{array}{l}18.9 \\
36.0 \\
12.3 \\
19.3\end{array}$ & $\begin{array}{l}31.4 \\
58.0 \\
0.00 \\
27.6\end{array}$ & $\begin{array}{l}12.9 \\
32.2 \\
65.2 \\
26.7\end{array}$ & $\begin{array}{l}6 \\
6 \\
6 \\
6\end{array}$ & $\begin{array}{l}0 \\
0 \\
0 \\
0\end{array}$ \\
\hline
\end{tabular}

. - missing, too little data to calculate value

' $X$ ' - below practical quantitation level (BPQL) 


\section{Appendix B}

Statistical Methods 
-

-

-

\section{B-2}


APPENDIX B

STATISTICAL METHODS

THE EXPERIMENTAL OESIGN: A NESTED CLASSIFICATION

Quantifying the concentrations of analytes in potentially nonhomogeneous wastewater streams involves estimating (a) the average concentration of each analyte and (b) the variability in the concentration of each analyte.

The average of any given analyte concentration is subject to uncertainties that arise from sampling fluctuations. For example, water samples are drawn from effluent points of waste streams on different days; thus, a portion of the total variation in the analyte concentration is due to the day of sampling. Also within a given day, a number of water samples are drawn from the stream. Each sample is termed a replicate, and its average analyte concentration varies from that of other replicates drawn on that day. This replicate-to-replicate variation of the analyte concentration is called experimental error.

The importance of correctly partitioning the analyte concentration variation is this: subsequent water samples will be drawn from the waste streams on a monthly basis and will be declared as being within a controlled limit of analyte concentration or as being outside those controllea limits. The controlled limits are based directiy on a function of these variance components.

The statistical model used to determine these variance components is called a nested or hierarchical classification. The effects in the model (day and replicate) are random effects; thus the model is called a random madel. For example, days of sampling were chosen randomly, and no determination was made as to whether analyte concentrations on a specific day were significantly different from trose on another specified day. Using this model, any inferences are thus extended to all possible days in the population of days.

The data, analyte concentrations, were measured on a water sample replicate taken on a given day; the replicates are nested within days so each 
replicate $i s$ unique to a day. The object of using this model is to quantify the variance components in analyte concentration measurements.

THE STATISTICAL MODEL

\section{Notation}

Let

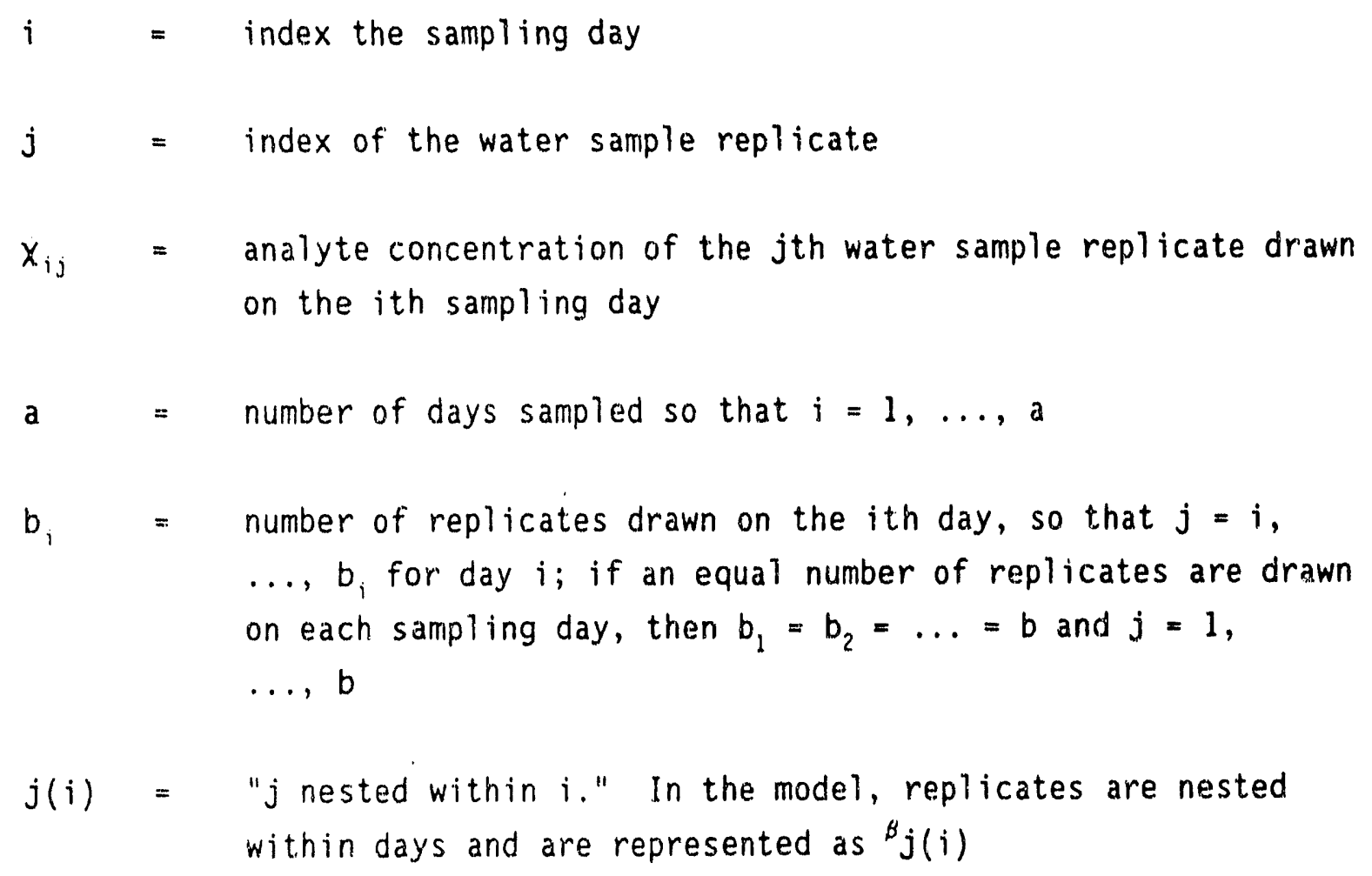

\section{The Model}

Analyte levels are modeled with the nested design as follows:

$$
x_{i}=\mu+\alpha_{i}+\beta_{j(i)}
$$

where 


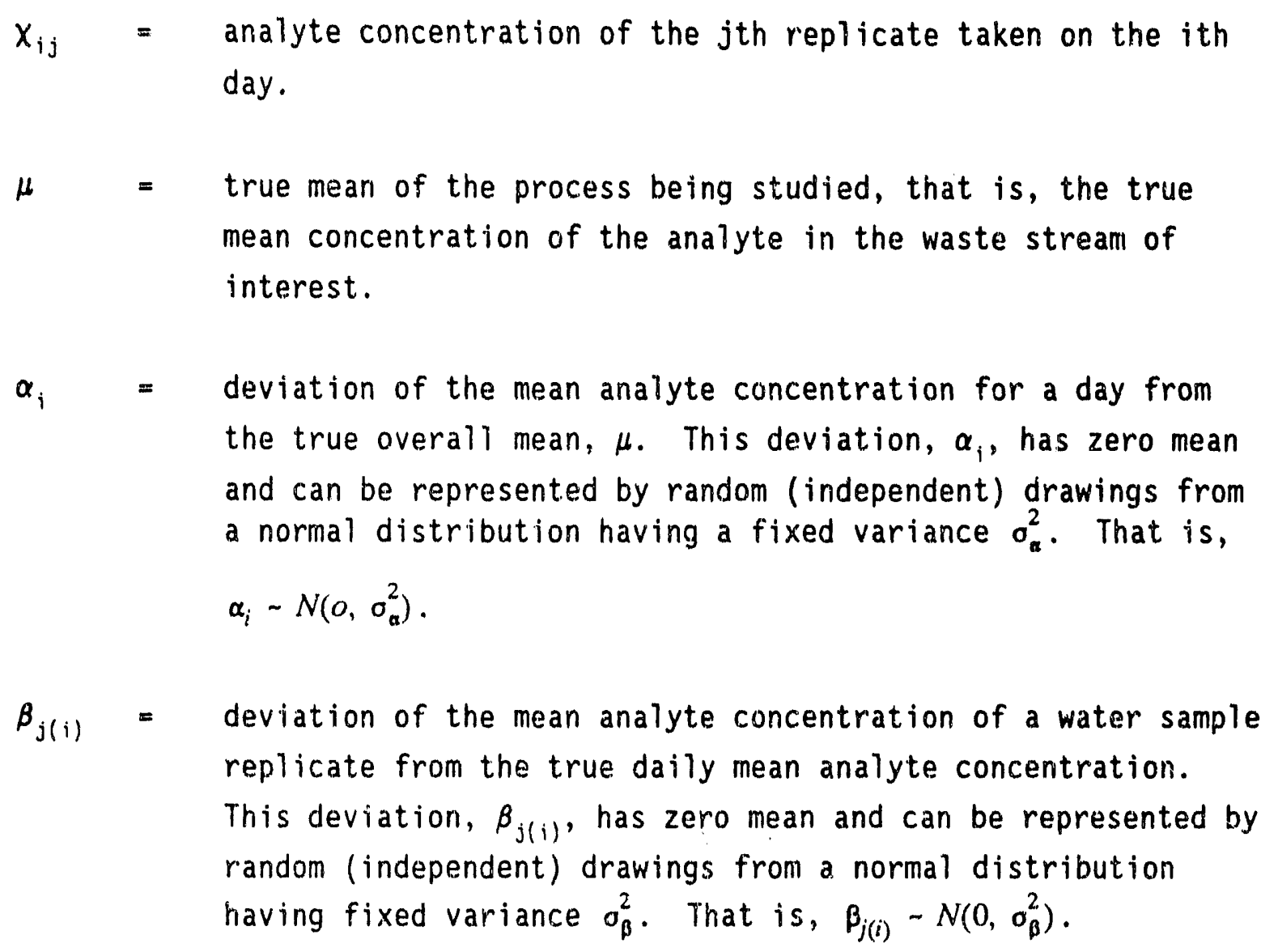

$\beta_{j(i)}=$ deviation of the mean analyte concentration of a water sample replicate from the true daily mean analyte concentration. This deviation, $\beta_{j(i)}$, has zero mean and can be represented by random (independent) drawings from a normal distribution having fixed variance $o_{\beta}^{2}$. That is, $\beta_{j(i)}-N\left(0, o_{\beta}^{2}\right)$.

FROM THE MODEL TO THE ANALYSIS OF VARIANCE

The following discussion includes dot notation $\left(x_{i}, x_{.}\right)$. The dot replaces a subscript and indicates that all values covered by the subscript have been summed. So:

$$
\begin{aligned}
& x_{i .}=\sum_{j} x_{i j} \\
& x_{\ldots .}=\sum_{i} \sum_{j} x_{i j}=\sum_{i} x_{i}
\end{aligned}
$$


The true parameters of the model are unknown but are estimated from the data. The model, as presented in the previous section, can be written in terms of deviations:

$$
\begin{aligned}
& x_{i j}=\quad \mu \quad+\quad \alpha_{i}+\beta_{j(1)} \\
& \text { Overall mean, Deviation of overall Deviation of } \\
& \text { estimated by mean from daily daily mean from } \\
& \bar{x} \text {. mean, estimated by } \\
& \bar{x}_{i .}-\bar{x}_{.} \\
& \text {replicate, } \\
& \text { estimated by } \\
& x_{i j}-\bar{x}_{i} \\
& =\bar{x}_{. .}+\left(\bar{x}_{i .}-\bar{x}_{. .}\right)+\left(x_{i j}-\bar{x}_{i .}\right)
\end{aligned}
$$

and can be rewritten as

$$
\left(x_{i j}-\bar{x}_{.}\right)=\left(\bar{x}_{i},-\bar{x}_{. .}\right)+\left(x_{i j}-\bar{x}_{i}\right)
$$

The sum of squared deviations from the overall mean is then

$$
\begin{aligned}
& \sum_{i}^{a} \sum_{j}^{b}\left(x_{i j}-\bar{x}_{. .}\right)^{2}=\sum_{i}^{a} \sum_{j}^{b}\left[\left(\bar{x}_{i .}-\bar{x}_{. .}\right)+\left(x_{i j}-\bar{x}_{i .}\right)\right]^{2} \\
& =\sum_{i}^{a} \sum_{j}^{b}\left(\bar{x}_{i}-\bar{x}_{.}\right)^{2}+\sum_{i}^{a} \sum_{j}^{b}\left(x_{i j}-\bar{x}_{i .}\right)^{2}
\end{aligned}
$$

No cross-product terms appear on the right hand side because the sums of deviations from means are restricted to be zero. The expression can be further reduced to 


$$
\sum_{i}^{a} \sum_{j}^{b}\left(x_{i j}-\bar{x}_{. .}\right)^{2}=b \sum_{i}^{a}\left(\bar{x}_{i}-\bar{x}_{.}\right)^{2}+\sum_{i}^{a} \sum_{j}^{b}\left(x_{i j}-\bar{x}_{i .}\right)^{2}
$$

where

$$
\begin{aligned}
& b \sum_{i}^{a}\left(\bar{x}_{i .}-\bar{x}_{. .}\right)^{2}=\text { the sum of squares for days } \\
& \sum_{i}^{a} \sum_{j}^{b}\left(x_{i j}-\bar{x}_{i .}\right)^{2}=\text { the sum of squares for replicates within days. }
\end{aligned}
$$

This partitioning or breakdown of variation characterized in the model is summarized in an analysis of variance (ANOVA) table. The object of the table is to identify and quantify the sources of variation in the analyte concentration measurements. The first column in the table identifies the accountable sources of variation. In this case, the first source is due to sampling day and the second is due to variation among replicates within a sampling day (experimental error).

The remaining columns in the ANOVA table quantify the respective sources of variation. These are the degrees of freedom (df), sums of squares (SS), mean square (MS), and expected mean square (EMS). The degrees of freedom for each sum of squares are the numbers by which the sum of squares are divided to obtain an unbiased estimate of the expected mean square. Thus, the mean square is SS/df. The table to be filled out is presented in Figure B-1.

\section{Expected Mean Square}

In statistical terms, the expected value of a statistic is the average value taken over an infinite number of similar samples. Consider the statistic $\bar{x}$, the sample mean. The expected or average value of $\bar{x}$ taken over 
Figure B-1. Analysis of variance table headings.

an infinite number of samples, written $E(\bar{\chi})$, is the population mean, $\mu_{\chi}$. That is,

$$
\begin{aligned}
E(\bar{\chi}) & =E\left(\frac{\sum_{i} x_{i}}{n}\right)=\frac{i}{n} E\left(\sum_{i} \chi_{i}\right)=\frac{1}{n}\left(\sum_{i} E\left(\chi_{i}\right)\right) \\
& =\frac{1}{n}\left(E \chi_{1}+E \chi_{2}+\ldots+E \chi_{n}\right)=\frac{1}{n} \cdot n \cdot E\left(\chi_{i}\right)=E \chi_{i}=\mu_{x}
\end{aligned}
$$

This follows since

$E(c)=c$, a constant

$E(C X)=C E(X)$

$E\left(X_{1}+X_{2}\right)=E X_{1}+E X_{2}$.

Another basic theorem of expected values is $E\left(x-\mu_{x}\right)^{2}=\sigma_{x}^{2}$, the variance of $x$. This is true since

$$
E\left(x-\mu_{x}\right)^{2}=E\left(\chi^{2}-2 x \mu_{x}+\mu_{x}^{2}\right)
$$


$=E \chi^{2}-E 2 \chi \mu_{\chi}+E \mu_{x}^{2}$

$=E \chi^{2}-2 \mu_{\chi} E \chi+\mu_{\chi}^{2}$ since 2, $\mu_{x}$, and $\mu_{x}^{2}$, are constants

$=E \chi^{2}-\mu_{\chi}^{2}$

$=\sigma_{x}^{2}$ by definition.

The expected value of the sample variance, $s^{2}$, is $\sigma^{2}$; symbolically

$E\left(s^{2}\right)=\left[\frac{\sum\left(x_{i}-\bar{\chi}\right)^{2}}{n-1}\right]=\sigma_{x}^{2}$.

The statistic $\sum_{i}\left(x_{i}-\bar{x}\right)^{2}$ is referred to as the sum of squares, or $\mathrm{SS}_{\bar{x}}$. It is actually the sum of squared deviations of the sample values from their sample mean. The expected value of $\mathrm{SS}_{x}$ is

$E\left(S S_{\chi}\right)=E\left[\sum_{i}(\chi-\bar{\chi})^{2}\right]=(n-1) \sigma_{\chi}^{2}$

where $n-1$ represents the degrees of freedom associated with the sum of squares. When a sum of squares is divided by its degrees of freedom, an average squared deviation from the mean is the result. This statistic, SS/df, is thus called the mean square, or MS, and the expected mean square, $E(M S)=E\left(\frac{S S}{d f}\right)$, is $\sigma^{2}$ of the variable being tested. 
The general equations for the expected mean squares of a balanced nested design with random terms are these:

$\begin{array}{ll}\text { Source } & \frac{E M S}{A} \\ & \sigma_{B}^{2}+b \sigma_{A}^{2} \\ B(A) & \sigma_{B}^{2}\end{array}$

\section{Variance Components}

Once the analysis of variance table has been completed, i.e. values for the mean squares have been determined and equations for the expected mean squares have been derived, the variance components can then be estimated. This is done simply by equating the expected mean squares to the mean squares and solving for $s_{A}^{2}$ and $s_{B}^{2}$. That is, for the balanced case as discussed in the previous section:

$\sigma_{B}^{2}$ is estimated by $s_{B}^{2}=M S[B(A)]$

$\sigma_{A}^{2}$ is estimated by $s_{A}^{2}=\frac{1}{b} \quad M S(A)-s_{B}^{2}$.

The total variability then is simply $s^{2}=s_{A}{ }^{2}+s_{B}^{2}$ and it can be said that 
$\frac{s_{A}^{2}}{s^{2}} \times 100 \%$ of the total variation is due to factor $A$

$\frac{s_{B}^{2}}{s^{2}} \times 100 \%$ of the total variation is due to factor $B$.

\section{Estimating the Variance of the Sample Mean from the Variance Components}

For FY-88, analyte concentration control charts are constructed based on FY-87 data. That is, a mean analyte concentration is plotted on a chart that has an upper limit based on the variance of the sample mean. If the plotted value is within the upper limit, the process is said to be in control. If the plotted value is above the upper limit, the process is out of control by definition and further investigation into the source of the problem is needed.

Therefore the variance of the sample mean, $s_{\bar{x}}^{2}$, is needed. the sample mean $\bar{y}$, is simply $\frac{\sum_{i} \sum_{j} x_{i j}}{a b}$, or the sum of all observations divided by the number of observations. But because our model states

$x_{13}=\mu+\alpha_{1}+\beta_{3(1)}$, it follows that

$(\bar{\chi}) \mu=\frac{1}{a b} \sum_{i}^{a} \sum_{j}^{b}\left[\mu+\alpha_{i}+\beta_{j(i)}\right]$.

The variance of $x$ is then derived as follows for the balanced case:

$\operatorname{Var}(\bar{\chi})=\operatorname{Var}\left\{\frac{1}{a b} \sum_{i}^{a} \sum_{j}^{b}\left[\mu+\alpha_{i}+\beta_{j(i)}\right]\right\}$ 


$$
\left.\begin{array}{l}
=\left(\frac{1}{a b}\right)^{2} \operatorname{Var} \sum_{i}^{a} \sum_{j}^{b}\left[\mu+\alpha_{i}+\beta_{j(i)}\right] \\
=\left(\frac{1}{a b}\right)^{2} \operatorname{Var} \sum_{i}^{a}\left[b \mu+b \alpha_{i}+\sum_{j}^{b} \beta_{j(i)}\right] \\
=\left(\frac{1}{a b}\right)^{2} \operatorname{Var}\left[a b \mu+b \sum_{i}^{a} \alpha_{i}=\sum_{i}^{a} \sum_{j}^{b} \beta_{j(i)}\right] \\
=\left(\frac{1}{a b}\right)^{2}\left[0=(b)^{2} a \sigma_{\alpha}^{2}+a b \sigma_{\beta}^{2}\right] \\
=\left(\frac{1}{a b}\right)^{2}\left\{(a b)^{2} \operatorname{Var}(\mu)+(b)^{2} \sum_{i}^{a} \operatorname{Var}\left(\alpha_{i}\right)+\sum_{i}^{a} \sum_{j}^{b} \operatorname{Var}\left[\beta_{j(i)}\right]\right.
\end{array}\right\}
$$

This form can be put in terms of the expected mean squares for days, $\sigma_{\beta}^{2}+b \sigma_{\alpha}^{2}$ such that $\operatorname{Var}(\bar{\chi})=\frac{1}{a b}\left(\sigma_{\beta}^{2}+b \sigma_{\alpha}^{2}\right)=\frac{1}{a b}$ EMS (days). Therefore, the variance of the sample mean can be estimated by $\frac{1}{a b}$ (MS days).

The derivation of the sample mean variance with unbalanced data is similar. Because the number of measurements may differ, that is $b_{i}$ may not 
necessarily equal $b$, the form for $\operatorname{Var}(\bar{x})$ cannot be reduced as neatly as in the balanced case. In the unbalanced case,

$\operatorname{Var}(\bar{\chi})=\frac{1}{n .^{2}}\left(n+\sum_{i} \sum_{j} n_{i j}^{2} \sigma_{\beta}^{2}+\sum_{i} n_{i}^{2} \sigma_{\alpha}^{2}\right)$

\section{EXAMPLE: ANALYSIS OF NESTED CLASSIFICATION OF TOC DATA}

In genera1, b replicate water samples were drawn from a waste stream on a days. The replicate water samples were then split into $n$ laboratory samples, upon which the analyte concentrations were measure. The model is:

$x_{i j}=\mu+\alpha_{i}+\beta_{j(i)}$

where

$$
\begin{aligned}
X_{i j}= & \text { analyte concentration of the } j \text { th water sample replicate taken } \\
& \text { on the ith sampling day. }
\end{aligned}
$$

The model assumes $\alpha_{i}-N\left(o, \sigma_{a}^{2}\right), \beta_{j(i)} \sim N\left(o, \sigma_{\beta}^{2}\right)$, and each of the $a_{i}$ and $\beta_{j(i)}$ are independent or random drawings from their respective normal distributions. Tabie B-1 summarizes the generalized analysis of variance (ANOVA) table for a nested classification with balanced data.

As an example, two water sample replicates were drawn from a waste stream on three days. TOC concentration $(\mathrm{mg} / \mathrm{L})$ were measured on these replicates. The data are presented in Table B-2. 
Table B-1. Generalized ANOVA table for nested classification with balanced data.

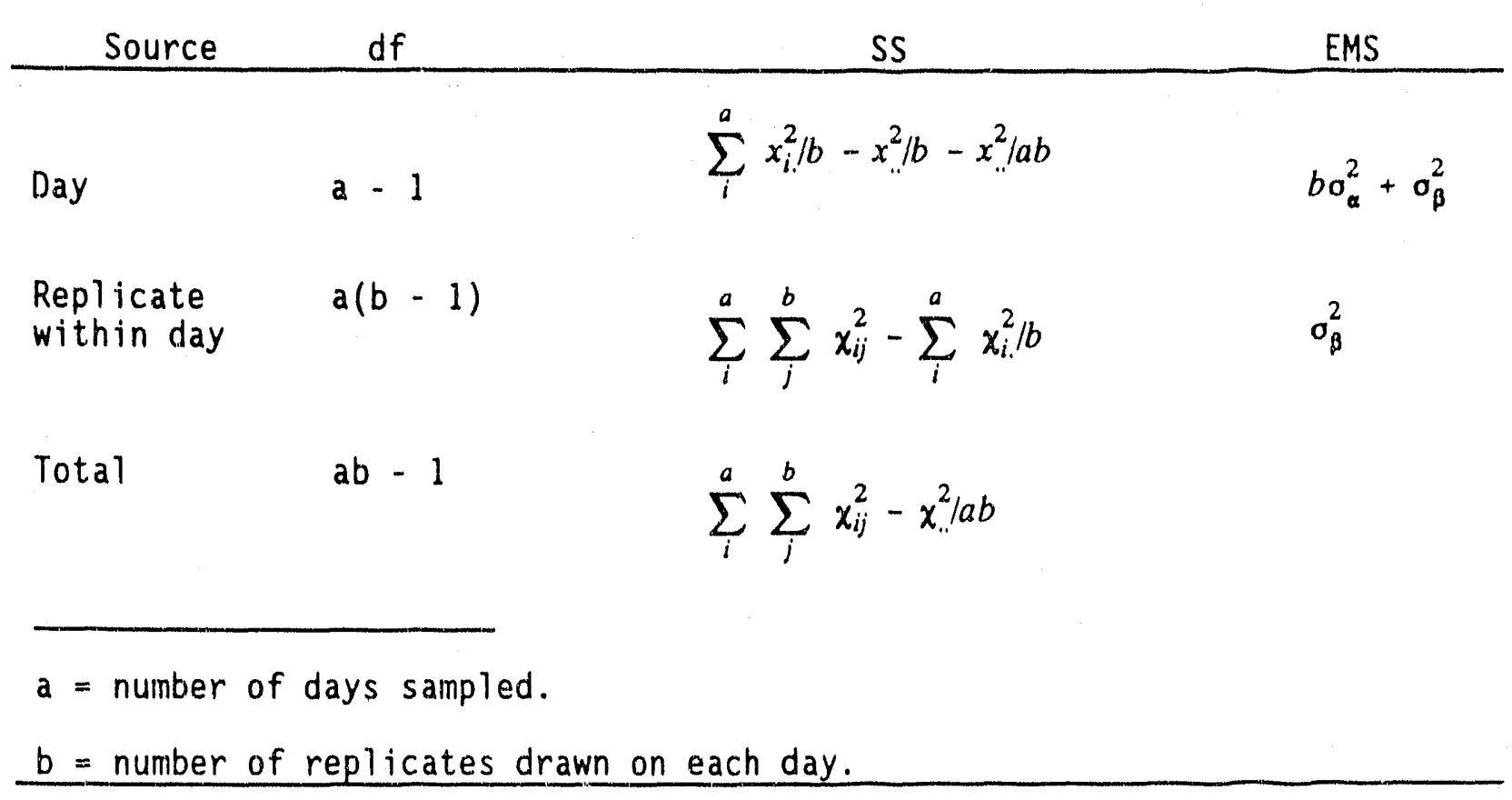

Table B-2. Balanced data set: levels of TOC (mg/L).

\begin{tabular}{|c|c|c|c|c|}
\hline $\begin{array}{l}\text { Day, } i \\
=1, \ldots, a \\
a=3 \text { days }\end{array}$ & $\begin{array}{c}\text { Replicate, } j \\
j=1, \ldots, b \\
b=2 \text { replicates }\end{array}$ & $\begin{array}{c}\text { Measurement } \\
x_{i j}\end{array}$ & $x_{i}$. & $x_{0}$ \\
\hline 1 & $\begin{array}{l}1 \\
2\end{array}$ & $\begin{array}{l}3.15 \\
3.22\end{array}$ & 6.37 & - \\
\hline 2 & $\begin{array}{l}1 \\
2\end{array}$ & $\begin{array}{l}2.17 \\
1.52\end{array}$ & 3.69 & - \\
\hline 3 & $\begin{array}{l}1 \\
2\end{array}$ & $\begin{array}{l}1.08 \\
1.17\end{array}$ & 2.25 & 12.31 \\
\hline
\end{tabular}


The sums of squares and degrees of freedom calculations are:

Source $=$ day

$$
\begin{aligned}
d f & =a-1=3-1=2 \\
S S & =\left(6.37^{2}+3.69^{2}+2.25^{2}\right) / 2-(12.31)^{2} / 6 \\
& =29.63-25.26 \\
& =4.37 .
\end{aligned}
$$

\section{Source $=$ replicate with in day}

$$
\begin{aligned}
\mathrm{df} & =a(b-1)+3(2-1)=3 \\
\text { SS } & =\left(3.15^{2}+\ldots+1.17^{2}\right)-29.63 \\
& =29.85-29.63 \\
& =0.22 .
\end{aligned}
$$

Table B-3 presents the results of this analysis in ANOVA table form.

From the variance components, it can be said that of the overall variability, $\mathrm{s}^{2}$ :

$$
\begin{aligned}
& \left(s_{\beta}^{2} / s_{\text {total }}^{2}\right)=100 \%=(0.07 / 0.78)=100 \%=9 \% \text { is due to analyte } \\
& \text { concentration differences in } \\
& \text { replicates taken within a day } \\
& \left(s_{\mathfrak{a}}^{2} / s_{\text {total }}^{2}\right)=100 \%=(0.71 / 0.78)=100 \%=91 \% \text { is } \\
& \text { due to analyte measurement } \\
& \text { differences over days. }
\end{aligned}
$$


Table B-3. ANOVA results of balanced TOC data set.

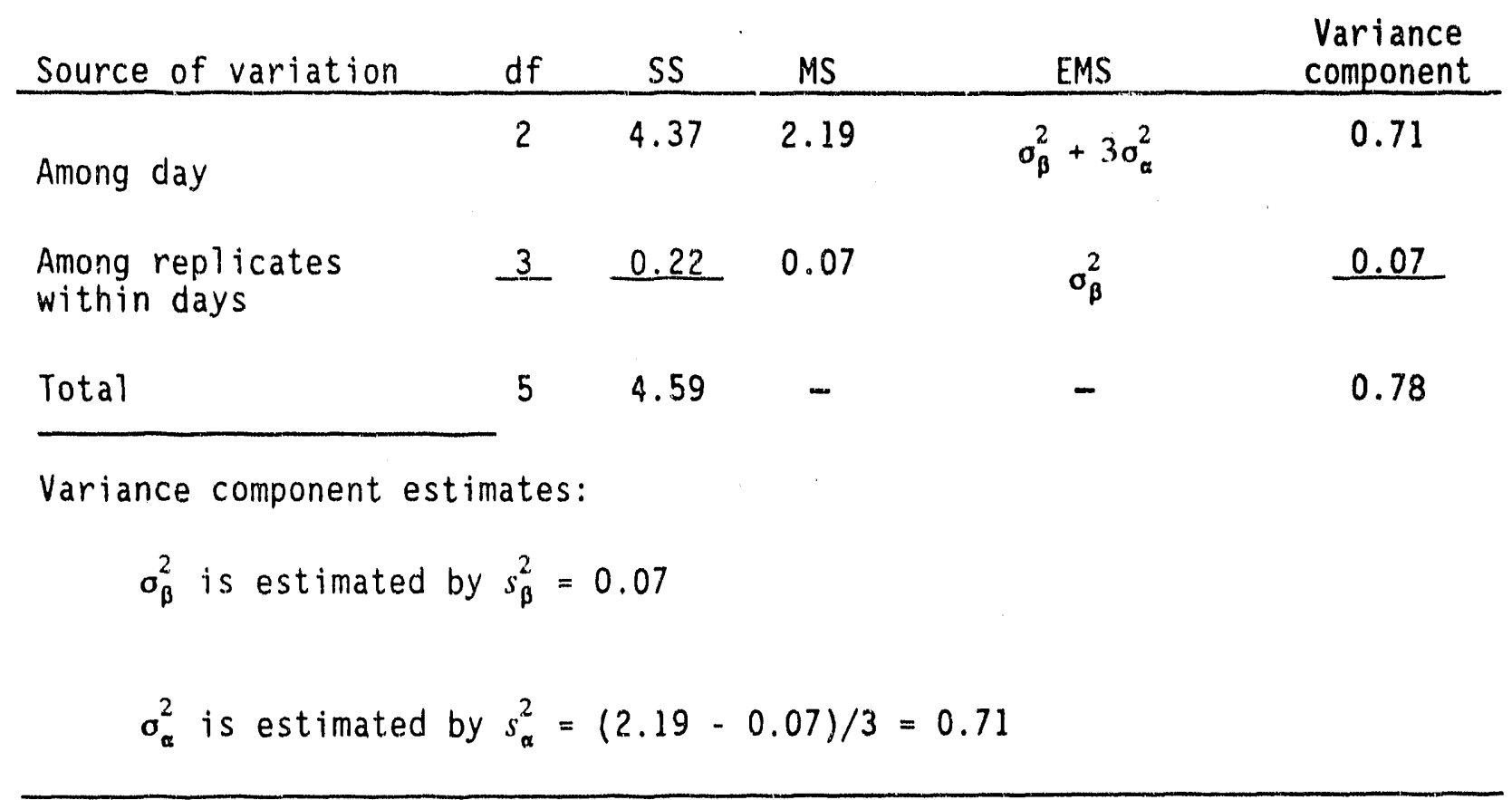

The variance of the sample mean is

$$
\begin{aligned}
\operatorname{Var}(\bar{\chi}) & =\frac{1}{a b}\left(\sigma_{\beta}^{2}+B \sigma_{\alpha}^{2}\right)=\frac{1}{a b} \text { EMS (days) and is estimated by } \\
s_{\bar{\chi}}^{2} & =\frac{1}{a b}[M S(\text { days })] \\
& =\frac{1}{6}(2.19) \\
& =0.365 .
\end{aligned}
$$


The control chart is a statistical tool used primarily to study a continuous process. The process of concern here is the concentration of an analyte in various wastewater streams. Even under uniform conditions, the analyte concentration is a varying process. The purpose of plotting values on a control chart is to keep track of the process so that management can identify high concentrations indicating that investigation and/or action is necessary.

Among the analyte concentrations, as mentioned, there is bound to be variation; this variation is natural to the process and is due to random or chance causes. If variation in the process is due only to chance causes, the process is said to be in control. If, however, a significant deviation detected in the process can be traced to an assignable cause, the process is said to be out of control, and attempts are made to identify and eliminate the cause.

If an excessively high analyte concentration is detected, it can be attributed to either (a) an assignable cause or (b) a chance cause. If it is the former, change may have taken place in the underlying prccess. Given a set of data, control chart nethods can be used to determine the cause.

The basic concept of control charts is not complicated. Any varying quantity forms a distribution if chance causes are present. The quantity therefore has a mean and variance. Consider the quantity $\bar{x}$; it has a normal distribution parameterized by a mean and variance. (See Figure B-2.) Very few points of this distribution lie in the tail regio... Specifically, 0.00135 or $0.135 \%$ of the area under this curve is beyond $\bar{x}+30$ and $\bar{x}-30$. So, if variability in the process is due only to chance cause, there is approximately one out of a thousand chances that a value will be larger than 


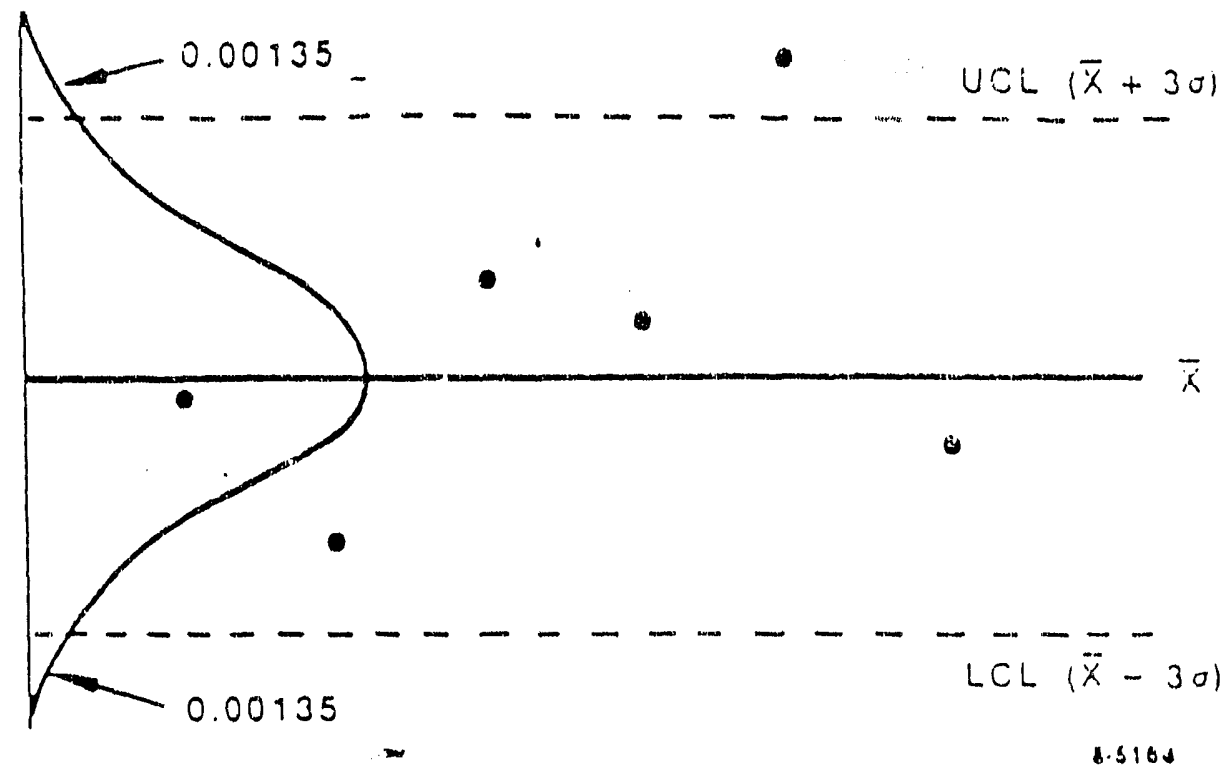

Figure B-2. Basic concept of an $x$ control chart.

$\bar{x}+30$. Although it is possible for an analyte concentration to be this large due simply to chance cause, it is much more likely that it would be high due to an assignable cause.

Thus, the decision rule is this: if a value is outside the determined limits, generally $\bar{x}=30$, it typically has an assignable cause. The process is said to be out of control until the cause is identified and eliminated. If a value is plotted within the determined concentration, it is concluded that no reliable evidence of an assignable cause is present. The process is then said to be in control.

A process in control has obvious advantages over a nonmonitored process:

- The process is free from variation worth identifying. Hence, there is no reason to tamper with it.

- Quality performance is improved simply because the act of getting a process in control entails eliminating assignable causes.

- The process becomes predictabie; in cases, decreasing the trequency of illspection or sampling may be justified. 


\section{No Set Standards versus Given Standards}

The methods used to construct a control chart depend on the situation. For example, if nothing is known about the population under consideration, it is determined whether the sampling results are homogeneous (e.g., whether they come from a single population). Statistically, the purpose is to test the hypothesis that $k$ samples are from a single population with constant but unknown parameter. It is common to apply this reasoning until the process is under control. Therefore, the limits or standards are set by the process itself and subsequent sampling results are compared to these determined standards. It must be assumed that no conditions are changed from the preliminary state to the subsequent sampling.

However, if set standards or limits are given and need not be determined, the object is to compare observed parameter estimates to the set standards. Statistically, the hypothesis tested is that the samples are all drawn from a population with known parameters.

\section{Subgroups}

Sampling from the population is a critical step in the control chart method. Sample results plotted on a chart need to represent subgroups that are as homogeneous as possible. If an assignable cause is present, it is much more likely to show up in differences between subgroups. If the subgroups are not homogeneous, that is, if large differences exist among members of the subgroup, results due to an assignable cause may be iess evident.

The subgroups for this program are water samples drawn on a quarterly basis from wastewater streams. For some of the analytes under consideration, that is, those whose standards are not set, their processes will be studied for one year in order to eliminate as many assignable causes as possible. With one year of data analyzed, it is hoped that the processes wili be left with variation due only to chance causes. Four quarterly sets of samples will be drawn and analyzed; the results will then be used to construct control charts undét stâtilizeú curuatitiuns. 


\section{Constructing the $\bar{x}$ Control Chart}

A control chart for the process average, $\bar{x}$, has a center line $(\mathrm{CL})$ and two upper control limits (ULI and UL2). These are constructed as follows:

$C L=\overline{\bar{\chi}}=$ analyte concentration averaged over four quarters

UL1 $=\overline{\bar{\chi}}+2 s /\left(C_{4} n\right)=$ upper concentration l limit

UL2 $=\overline{\bar{\chi}}+3 s /\left(C_{4} n\right)=$ upper concentration 2 limit where

$C_{4}=\left(\frac{2}{n-1}\right) 1 / 2 \frac{\left(\frac{n-2}{2}\right) !}{\left(\frac{n-3}{2}\right) !}$.

Only for $\mathrm{pH}$ are lower control limits constructed:

$L L 1=\bar{\chi}-2 s /\left(\begin{array}{ll}C_{4} & n\end{array}\right)=$ lower concentration 1 limit

$L L \hat{\imath}=\bar{x}-3 s /\left(C_{4} \quad n\right)=$ lower concentration 2 limit.

The variance estimate, $s^{2}$, is calculated depending on the homogeneity of the quarterly variances. First, therefore, the variances $s_{i}^{2}$ are tested over $k$ quarters to determine if each $s_{1}^{2}$ estimates the same theoretical variance $\sigma^{2}$. 
A test for homogeneous variances was developed by M. S. Bartlett in 1937 . The test, referred to as Bartlett's M Test, assumes the variances are from normal distributions and, secondly, that random samples were drawn independently from the populations.

The null hypothesis of equal variances is tested without the requirement that the $k$ estimates are based on equal sample sizes. The test statistic is $M=(N-k) \ln \left(s_{p}^{2}\right)-\sum_{i}\left(n_{i}-1\right) \ln \left(s_{i}^{2}\right)$.

Under the null hypothesis, $M$ is distributed approximately $\chi^{2}$ with $k-1$ degrees of freedom.

$M$ is biased upward, so if the calculated value exceeds the critical $x^{2}$ value, an adjustment is necessary. Specifically, $M$ is divided by

$C=1+\frac{1}{3(k-1)}\left(\sum_{i} \frac{1}{n_{i}-1}-\frac{1}{N-k}\right)$

and is still compared to $x_{k}^{2}-1$.

If the sample sizes are all equal, that is $n_{1}=n_{2}=\ldots=n_{k}=n$, the formulas are simplified:

$s_{p}^{2}=\sum_{i} s_{i}^{2} / k$

$M=(N-k) \ln \left(s_{p}^{2}\right)-(n-1) \cdot \sum_{i} \ln \left(s_{i}^{2}\right)$ 
$C=1+\frac{1}{3(k-1)}\left(\frac{k}{n-1}-\frac{1}{N-k}\right)$.

If the test statistic $M / C$ does exceed the critical value, it is concluded that at least one of the $k$ estimates is not estimating the same theoretical variance, $\sigma^{2}$. If the $k$ quarterly variance estimates, $s_{i}^{2}$, do not prove to be different, the estimates can then be pooled into a single estimate, $s_{p}^{2}$. The pooling simply weights each variance estimate, $s_{i}^{2}$, by its sample size $n_{i}$ :

$$
\begin{aligned}
s_{p}^{2} & =\frac{\left(n_{1}-1\right) s_{1}^{2}+\left(n_{2}-1\right) s_{2}^{2}+\ldots+\left(n_{k}-1\right) s_{k}^{2}}{\left(n_{1}-1\right)+\left(n_{2}-1\right)+\ldots+\left(n_{k}-1\right)} \\
& =\sum_{i}\left(n_{i} \cdot 1\right) s_{i}^{2} /(N-k)
\end{aligned}
$$

where

$$
N=\sum_{i} n_{i}
$$

If variance estimates show evidence of not estimating the same theoretical variance, $\sigma^{2}$, and if a particular transformation is appropriate, then the data are transformed. The transformed data are then tested again for equal variances. If these estimates are still nonhomogeneous, then a variance is calculated by pooling all of the data over all quarters and determining a single estimate, $s^{2}$. That is: 
$s^{2}=\sum_{\text {quarters }} \sum_{\text {days }} \sum_{\text {reps }}\left(x_{i j k}-\overline{\bar{\chi}}\right)^{2} /(N-1)$

and this estimate is used in forming the control limits.

\section{Qut-of-Control Criteria}

It is natural for a process in control to exhibit the following characteristics:

- Most points are near the center line.

- A few points are spread out and approach the three sigma control limits.

- None of the points exceed the three sigma control limits.

If any of the following situations occur, it is unnatural for the process, and an assignable cause should be sought:

- A single point outside the three sigma control limits

- Two out of three successive points outside the two sigma warning limits

- Seven out of eight points in a row on one side of the center line.

It is possible to apply more rules than those listed above. Too many rules, however, will result in a higher probability of incorrect conclusions, such as saying the process is out of control when it is not. 


\section{REFERENCES}

1. American Society for Testing and Materials, ASTM Manual on Presentation of Data and Control Chart Analysis STP 150, 4th Revision, Philadelphia: ASTM, 1976.

2. A. J. Duncan, Quality Control and Industrial Statistics, Homewood, Illinois: Richard D. Irwin, Inc., 1965.

3. 0. J. Dunn and V. A. Clark, Applied Statistics: Analysis of Variance and Regression, New York: John Wiley \& Sons, 1974.

4. C. R. Hicks, Fundamental Concepts in the Design of Experiments, New York: Holt, Rinehart and Winston, 1973.

5. Stat-A-Matrix Seminars, Practical Application of Statistics to Quality Control, 1984.

6. R. G. Steel and J. H. Torrie, Principles and Procedures of Statistics, New York: McGraw-Hill Book Co., Inc., 1960. 


\section{GLOSSARY OF STATISTICAL TERMS}

Analys is of Variance (ANOVA-A statistical technique used to divide the total variation of the measured variable into meaningful components.

Assignable Cause-An identifiable event in a process that in some way changes the population and affects the control charts. An assignable cause may, for example, result in a change in the process average and/or variability and/or may cause cyclic or erratic shifts in the process.

Balanced-An experimental design is "balanced" when there exists an equal number of observations for every combination of the classification factors, e.g., the same number of replicates within each day sampled.

Chance Cause or Random Cause-Slight, natural changes in conditions of the process causing random variation in the population. This variation is the sum of the effects of all chance causes; no major part of the total variation can be traced to a single cause.

Components of Variance-See variance components.

Control chart-A statistical device used primarily for the study and control of repetitive processes. The chart typically is based on the upper and/or lower control limits; points outside these limits signal a process "out of control."

Controlled Process-A process for which the control chart shows no points outside the limits and no nonrandom variation within the limits.

Degrees of Freedom-The number of "free" elements associated with a mathematical expression. The degrees of freedom for a variance estimate are, in general, the number of observations used minus the number of parameters that must be estimated from the data in order to calculate the estimated variance. For example, 
$\sum_{i}^{n}\left(x_{i}-\bar{x}\right)^{2}$

has $n-1$ degrees of freedom since there are $n$ observations and one parameter, $\mu$, is being estimated by $\bar{\chi}$.

Deviation-Difference; typically refers to difference in an observation and its mean, $\bar{x}-\mu$ or $s-\bar{x}$.

Expected Mean Square (EMS)-The mathematical expected value of the mean square.

Expected Value-The average value of a variable or statistic if it were calculated from an infinite number of equal-sized samples from a given population, denoted $E(X)$.

Experimental Error-Variation among experimental units.

Experimental Unit-The smallest unit of experimental material that can be treated independently of all other units, e.g., replicates.

Hierarchical Design-An experimental design in which the units are classified in a nested manner, e.g., replicates are chosen within days.

Mean-Average or expected value of a variable.

Of a sampie, $\bar{x}=\sum_{i}^{n} x_{i} / n$.

Of a population, $\mu=E(x)$.

Mean Square-An unbiased estimate of a population variance. Determined by dividing the sum of squared deviations by its degrees of freedom, $M S=S S / d f$. ivested Uesiun-see nierarchicai design. 
Pooled Variance-A weighted average of $k$ sample variances, $s_{1}^{2}, s_{2}^{2}, \ldots, s_{k}^{2}$, with weights proportional to the degrees of freedom for the $s_{i}^{2}$.

$s_{p}^{2}=\sum_{i}^{n}\left(n_{i}-1\right) s_{i}^{2} / \sum_{i}^{n}\left(n_{i}-1\right)$

Random Effects Model-An experimental design in which the concentrations of the factors (such as days) are randomly selected and inferences are extended to all concentrations of the populations (of which the observed concentrations are random samples). In a random model, the experimenter is not usually interested in testing hypotheses, setting confidence limits, or making contrasts; rather he is interested in estimating components of variance.

Replicate-A repetition of the experiment. When a concentration of a factor appears more than once, the experiment is said to be replicated.

Sample-A subset of measurements taken from a population, usually on which to make inferences about the population. Unless otherwise specified, it is assumed to be a random sample from the population.

Source of variation in an ANOVA-The experimental characteristics or factors of a variable to which portions of the total variability are attributed.

Standard Deviation-Square root of the variance.

Statistical Hypothesis-An assumption about a population being sampled.

Subgrouping-Dividing a group of items into smaller subsets of the group so that the subgroups are as homogeneous as possible. To be of analytical value, the segregation of the items should be such that if assignable causes are present, they will be common to all elements of one or more subgroups. 
Sum of Square-Sum of squared deviations from the mean, $\sum\left(x_{i}-\bar{x}\right)^{2}$; used in the ANOVA table as a method of partitioning the total variability into portions due to identified sources.

Variance-The expectation of $(x-\mu)^{2}$ where $\mu$ is the mean of the random variable $x$.

of a sample, $s^{2}=\sum_{i}^{n}\left(\chi_{i}-\bar{\chi}\right)^{2} / n-1$.

of a population, $\sigma^{2}=E(x-\mu)^{2}$.

Variance Components-An analysis used to estimate the proportion of overall variance contributed by each factor in the model. 

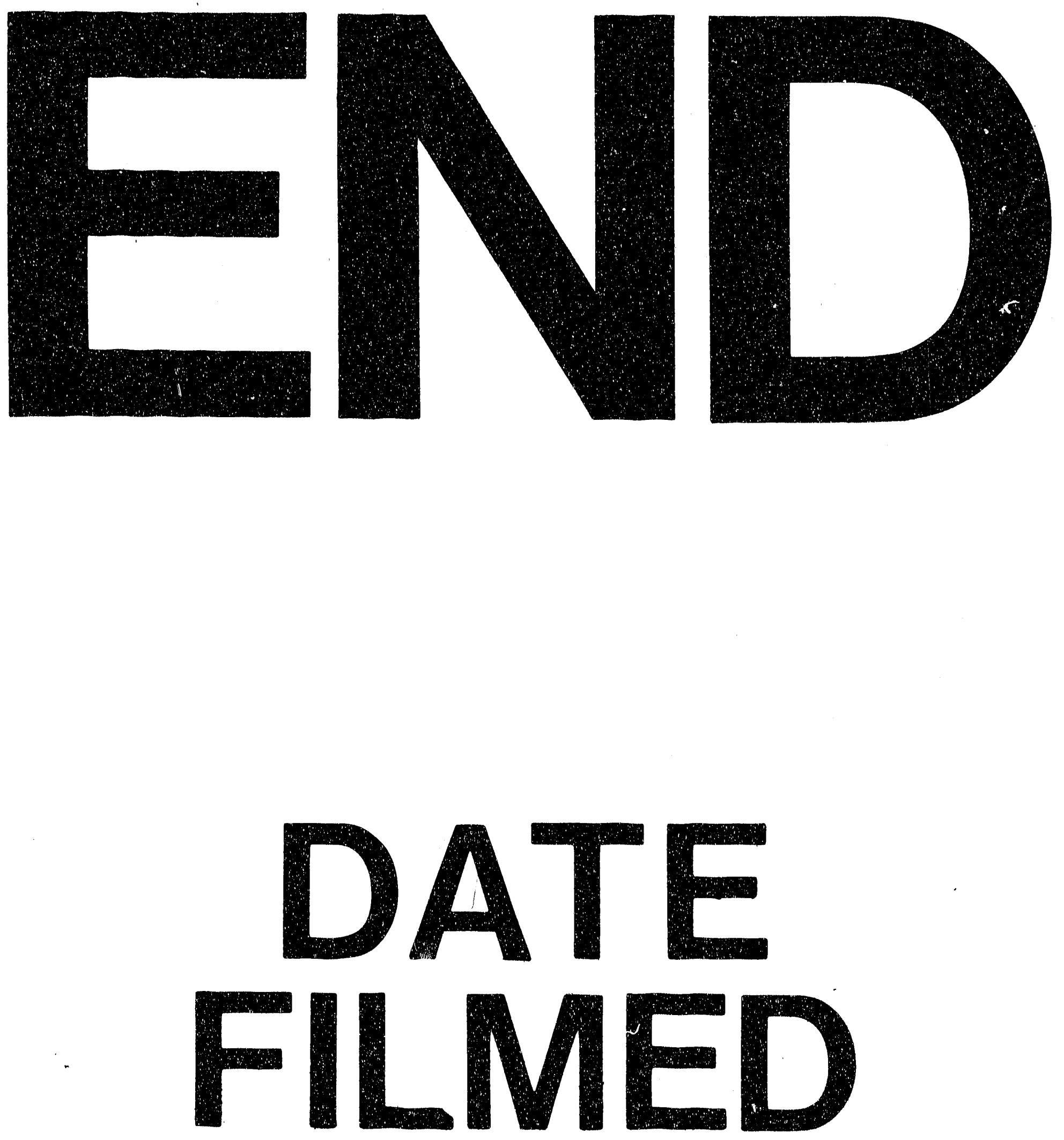

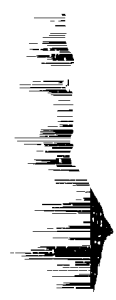

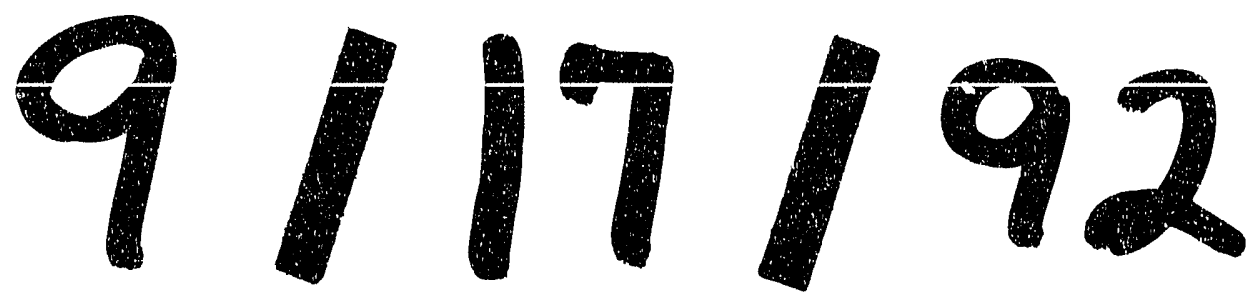




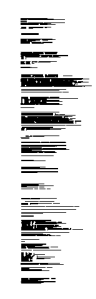

